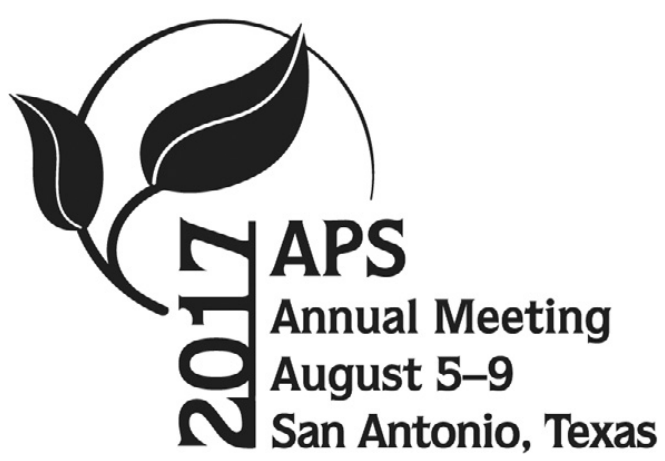

\title{
2017 APS Annual Meeting Abstracts of Presentations
}

\begin{abstract}
APS Annual Meeting in San Antonio, Texas, U.S.A., August 5-9, 2017. Recommended format for citing annual meeting abstracts, using the first abstract below as an example, is as follows: Serrano, M., and Robertson, A. E. 2017. The effect of cold stress on damping-off of soybean caused by Pythium sylvaticum. (Abstr.) Phytopathology 107:S5.1. https://doi.org/10.1094/PHYTO-107-12-S5.1
\end{abstract}

The abstracts are published for citation purposes. They were not reviewed by the Phytopathology Editorial Board and were not edited by the APS editorial staff. Please send questions or comments to aps@scisoc.org.

https://doi.org/10.1094/PHYTO-107-12-S5.1

(C) 2017 The American Phytopathological Society

The effect of cold stress on damping-off of soybean caused by Pythium sylvaticum M. SERRANO, A. E. Robertson, Iowa State University, Ames, IA, USA

Pythium sylvaticum is an oomycete that causes damping-off of soybean (Glycine max) particularly when cool weather occurs soon after planting. The objective of these experiments was to study the role of cold stress in the development of the disease. Soybean seeds cv. IA 2094 were planted in cups that were inoculated with the pathogen, placed at $18^{\circ} \mathrm{C}$, and then subjected to 96 hours of cold stress $\left(4^{\circ} \mathrm{C}\right.$ or $\left.10^{\circ} \mathrm{C}\right)$ at different times after planting $(0,24,48$, 96, 144 and 192 hours). Emergence was lowest when cold stress occurred 48 or 96 hours after planting and $P$. sylvaticum was present. To further understand the effect of temperature we evaluated solute leakage of seeds, mycelial growth and sporangia germination of $P$. sylvaticum at $4{ }^{\circ} \mathrm{C}, 10^{\circ} \mathrm{C}$ and $18^{\circ} \mathrm{C}$. Mycelial growth was slower but the pathogen was still able to grow at $4^{\circ} \mathrm{C}$, solute leakage was greater from seeds imbibed at $4^{\circ} \mathrm{C}$ and more sporangia of $P$. sylvaticum germinated in the presence of those seed exudates. These results indicate that periods of cold stress soon after planting increase the susceptibility of soybean to damping-off. We propose that at low temperatures seed exudation increases, which stimulates greater germination of $P$. sylvaticum sporangia and may contribute to reduced emergence due to damping-off. The results from these research contribute in the understanding of factors involved in the occurrence of damping-off caused by $P$. sylvaticum.

Genetics, genomics, and transcriptomics reveal candidate avirulence/virulence effectors in Pyrenophora teres f. teres J. RICHARDS (1), V. Koladia (1), R. S. Brueggeman (1), T. L. Friesen (2), (1) North Dakota State University, Fargo, ND, USA; (2) USDA ARS, Fargo, ND, USA

Pyrenophora teres f. teres, a necrotrophic fungal pathogen of barley and causal agent of net form net blotch, utilizes a repertoire of effector molecules to facilitate disease. Previous research identified 19 QTL associated with virulence/avirulence on 10 barley lines in a fungal mapping population derived from a cross between isolates FGOH04Ptt-21 (North Dakota) and BB25 (Denmark), of which, three QTL contributed a large effect to the phenotypic variation $\left(\mathrm{R}^{2}>30 \%\right)$. Reference quality genomes of each parental isolate were developed via Pac-Bio sequencing and RNAseq analyses conducted to identify candidate effectors underlying the major QTL. Two loci, designated Pin1 and Beel are hypothesized to be virulence loci, as analysis of QTL effects in progeny isolates appear additive. RNAseq analysis revealed single polymorphic, small secreted proteins underlying Pinl and Beel, exhibiting $\sim 4.5 \mathrm{x}$ and $\sim 4 \mathrm{x}$ upregulation post-inoculation, respectively. A third locus, Tifl, appears to condition avirulence in a qualitative manner. The genomic region underlying Tifl in the avirulent isolate BB25 contains an $\sim 100 \mathrm{~kb}$ expansion, containing 23 genes with low or no homology to annotated genes in the FGOH04Ptt-21 genome. A candidate gene within this region containing a predicted transmembrane domain was identified to be upregulated $\sim 34 \mathrm{x}$ at 48 hours post-inoculation. Pin1, Beel, and Tifl candidate genes are currently being targeted for functional validation.

Volatiles from LOX10 promote maize susceptibility to Colletotrichum graminicola by hijacking jasmonic and salicylic acids antagonism Z. GORMAN (1), S. Christensen (2), Y. He (1), Y. Yan (3), E. J. Borrego (1), M. V. Kolomiets (1), (1) Texas A\&M University, College Station, TX, USA; (2) Chemistry Unit, Center of Medical, Agricultural, and Veterinary Entomology, USDA, Gainesville, TX, USA; (3) Nanjing Agricultural University, Nanjing, CHINA

Green leaf volatiles (GLVs) constitute an important class of lipoxygenase (LOX)-derived, stress-inducible molecules that are paramount in mediating plant-pathogen interactions. GLVs modulate plant defenses by inducing biosynthesis of the phytohormone, jasmonic acid (JA) that results in substantial defense against insects and necrotrophic pathogens, leading to the pervasive view that GLVs only increase resistance to pathogens. Contrary to this view, we show that the GLV-deficient maize lipoxygenase mutant, lox10, has enhanced resistance to anthracnose disease caused by the fungal pathogen, Colletotrichum graminicola. The JA-deficient double mutant, opr 7 opr 8 , exhibits similar resistance to C. graminicola, suggesting lox 10 mutant resistance is related to JA signaling. To further investigate the underlying reasons for LOX10-mediated susceptibility to this pathogen, phytohormone, metabolite, and gene expression analyses were conducted on wild-type (WT), lox 10 mutants, and opr 7 opr8 double mutants. Results revealed that lox 10 
and $\operatorname{opr} 7$ opr 8 mutants have little to no amounts of JA. Accordingly, both mutants displayed increased levels of salicylic acid (SA), the classical phytohormone that provides defense against hemibiotrophic pathogens and is antagonistic with JA. Altogether, these results suggest that LOX10 mediates susceptibility to $C$. graminicola by suppressing SA through increased GLV-mediated JA biosynthesis.

Bioprospecting rhizobacteria to improve drought resistance in grasses

M. JOCHUM (1), S. P. Gaire (1), G. Niu (2), Y. K. Jo (1), (1) Texas A\&M University, College Station, TX, USA; (2) Texas A\&M University Agricultural Research and Extension Center at El Paso, El Paso, TX, USA

Water is the main limiting resource in global agriculture, restricting yields in approximately $70 \%$ of arable farmlands. Therefore, in an effort to influence the phytobiome to increase drought resistance in grasses, we developed a high-throughput screening method to bioprospect beneficial rhizobacteria from bermudagrass growing in the semiarid climate of West Texas. Bermudagrass samples were exposed to varying levels of water deficiency in a greenhouse, followed by sub-selection for drought resistance. Rhizosphere bacteria were isolated onto a selective medium and screened by inoculation onto the roots of bermudagrass cv. Champion and rice cv. Nipponbare. This screen successfully isolated and characterized two novel plant growthpromoting rhizobacteria strains Bacillus sp. 12D6 and Enterobacter sp. 12i. These two strains of rhizobacteria contributed the greatest alleviation of host drought phenotype based on survivability, root and shoot biomass, relative water content, and ROS production when compared to the untreated control. These strains and future discoveries from this project can potentially be used as a probiotic treatment for grasses in drought seasons and help to increase our understanding of influencing the phytobiome for improved host resistance to abiotic stress.

Resistance to Sclerotinia sclerotiorum in soybean deciphered by transcriptome and metabolome analyses A. RANJAN PHD (1), D. L. Smith (2), M. Kabbage (2), (1) University of Wisconsin Madison, Madison, WI, USA; (2) University of Wisconsin, Madison, WI, USA

Sclerotinia sclerotiorum, a necrotrophic fungal pathogen with a broad host range, causes a devastating disease on soybean called Sclerotinia stem rot (SSR) that can lead to yield losses as high as 50-60\%. Resistance mechanisms against SSR are poorly understood, thus hindering the commercial deployment of SSR resistant varieties. We used a dual approach utilizing RNA-sequencing and GC-MS based metabolomics to decipher the molecular mechanisms governing resistance to S. sclerotiorum in soybean. Transcripts and metabolites of resistant and susceptible highly inbred soybean lines were analyzed in a time course manner. RNA-sequencing analysis revealed differential expression of key receptor kinases, transcription factors, signal transduction, stress responsive, jasmonic and phenylpropanoid pathway related genes. Phytohormones estimation identified early accumulation of JA-Ile $((+)-7-i s o-J a s m o n o y l-L-i s o l e u c i n e)$, a bioactive jasmonate in the resistant interaction. Importantly, aromatic and aliphatic amino acids along with phenylpropanoid pathway intermediates and antimicrobial compounds were highly accumulated in the resistant interaction, suggesting their key role in resistance. Indeed, we found that phenylpropanoid pathway intermediates such as 4-hydroxybenzoate, ferulic acid, and caffeic acid were highly accumulated in the resistant line. In vitro assays showed that ferulic and caffeic acids affected S. sclerotiorum growth and development. This dual approach reveals key defense strategies in soybean against $S$. sclerotiorum, and highlights the importance of the phenylpropanoid pathway in this hostmicrobe interaction.

Identifying functions of effectors and small RNAs involved in wheat-rust interactions

S. R. RAMACHANDRAN (1), C. Yin (1), N. A. Mueth (2), K. Tanaka (3), S. H. Hulbert (1), (1) Dept. of Plant Pathology, Washington State University, Pullman, WA, USA; (2) Washington State University, Pullman, WA, USA; (3) Department of Plant Pathology, Washington State University, Pullman, WA, USA

Efficient management of wheat rust diseases is challenging since Puccinia spp. can rapidly alter their effector repertoires in response to recognition by resistance genes. Although many putative effectors have been predicted, functional studies have been limited due to the lack of a suitable effector delivery system for wheat. Here we identified two Pseudomonas strains for delivering rust effectors. Twenty effector proteins from $P$. graminis and $P$. stiriiformis were screened and nine effectors capable of suppressing host defense responses were identified. Further, we analyzed the small RNA profiles of two wheat cultivars differentially susceptible to $P$. striiformis to understand changes in gene regulation following infection. A total of 163 novel miRNAs and 182 known miRNAs were identified using 12 small RNA libraries from two cultivars - Louise and Penawawa infected with $P$. striiformis. 145 miRNAs targeted wheat genes, while 69 miRNAs targeted fungal genes. Of these miRNAs, 53 were differentially expressed $(P<0.05)$ between the two infected cultivars that varied in their response to rust disease. The majority of fungal targets coded for small, secreted proteins, providing preliminary evidence for cross-kingdom control of gene regulation. Overall, this study contributes to the current repository of wheat effectors and miRNAs; and provides novel information on the yet to be characterized roles for proteins and microRNAs in the wheat-rust interactions.

Influence of biotic and abiotic factors on common scab severity and pathogenic Streptomyces spp. populations in agricultural fields C. GOYER (1), S. Neupane (1), S. Whitney (1), B. Zebarth (1), M. Chantigny (2), D. Burton (3), (1) Agriculture and Agri-Food Canada, Fredericton, NB, CANADA; (2) Agriculture and Agri-Food Canada, Quebec, QC, CANADA; (3) Dalhousie University, Truro, NS, CANADA

Control of common scab is highly variable due to a poor understanding of the effect of soil and environmental conditions on the pathogen populations. This study aimed at determining the biotic (soil microbial communities) and abiotic factors (soil physico-chemical properties) influencing common scab severity and pathogenic Streptomyces spp. populations. The experiment was carried out in nine field sites located in Eastern Canada with historically relatively low (control) and high (CS infested) severity of common scab symptoms. Common scab severity varied from $7 \%$ to $70 \%$ of lesion coverage in $\mathrm{CS}$ infested plots while control plots had less than $5 \%$ lesion coverage. Soil test potassium and magnesium and $\mathrm{pH}$ were positively correlated $(\mathrm{r}=0.41$ to $0.45)$ with disease severity while soil test iron and phosphorus $(r=-0.28$ to -0.40$)$ were negatively correlated. The dominant bacterial phyla included Proteobacteria (30\%), Acidobacteria (18\%) and Actinobacteria (14\%) while Ascomycota (63\%), Basidiomycota (15\%) and Zygomycota (13\%) were the three dominant fungal phyla. A significant correlation between disease severity in the CS infested plots, and the diversity of the microbial community, measured using Shannon index, was observed for the bacterial but not the fungal community. The results suggest that soil properties and diversity of bacterial communities may influence disease severity, however further investigations are needed to assess causality.

A Greenhouse System to Study the Effect of Soil pH on Fusarium wilt of Tomato caused by Fusarium oxysporum f. sp. lycopersici Race 3 C. J. LAND (1), H. Adkison (2), R. Willis (1), G. E. Vallad (3), (1) University of Florida, Wimauma, FL, USA; (2) Univ of Florida GCREC, Wimauma, FL, USA; (3) Gulf Coast Research and Education Center, University of Florida, Wimauma, FL, USA

The relationship between soil pH and soil borne pathogens has been well documented for various cropping systems. In the 1970's, Jones and Woltz demonstrated in field trials that raising soil $\mathrm{pH}$ with hydrated lime suppressed Fusarium wilt of tomato caused by Fusarium oxysporum f. sp. lycopersici (FOL) race 2. Using a large pot greenhouse study, we evaluated whether a FOL race 3 isolate would react to increased soil $\mathrm{pH}$ in a similar manner. Experiments used pots fabricated from $20 \mathrm{~cm}$ diameter PVC pipe cuffs sealed on the bottom with heavy-duty nylon screen. Soil treatments consisted of a pasteurized field soil (Myakka fine sand), mixed with or without hydrated lime ( $20 \mathrm{~g} /$ pot). In addition, each soil was mixed with FOL prepared from a sterile cornmeal:sand medium to a final level of $10^{1}$ to $10^{4} \mathrm{cfu} / \mathrm{g}$. Four-week old tomato seedlings were transplanted and rated 9 and $11 \mathrm{weeks}$ after planting (WAP). Increasing soil $\mathrm{pH}$ from an average of 6.4 to 7.5 with lime reduced disease incidence by $36 \%(P=0.0400)$ and $26 \%(P=0.0037)$, at 9 and 11 WAP, respectively. Symptomatic plants growing in limed soils also exhibited reduced foliar symptoms compared to non-treated soils. No differences in the recovery of total Fusarium from soil or tomato roots were observed between soil treatments. A Real Time PCR technique is being 
developed to better measure FOL levels in soil and in planta. This system will be implemented to further study the effects of lime on the Fusarium lifecycle.

Dissection of the APIP6-mediated ubiquitin-proteasome pathway in rice immunity against Magnaporthe oryzae

Y. NING (1), G. L. Wang (1,2), (1) Institute of Plant Protection, Chinese Academy of Agricultural Sciences, Beijing, CHINA; (2) The Ohio State University, Columbus, OH, USA

The ubiquitin-proteasome system (UPS) is one of the most important protein turnover mechanisms that regulate growth, development and responses to abiotic and biotic stresses. We previously found that the Magnaporthe oryzae effector AvrPiz-t suppresses the E3 ligase activity of the RING-type ligase APIP6 in rice. In return, APIP6 ubiquitinates and degrades AvrPiz-t. Genetic studies revealed that APIP6 is involved in the PAMP-triggered immunity (PTI) against M. oryzae. Recently, we found that APIP6 interacts with OsELF3-2 in rice, the ortholog of ELF3 in Arabidopsis. The expression of OsELF3-2 is induced in both incompatible and compatible rice-M. oryzae interactions. Compared with the wild type plants, the oself3-2 mutant and RNAi plants have a significant increase in flg22- and chitin-induced ROS generation, and confer enhanced resistance to a compatible $M$. oryzae isolate, which is similar with the phenotype of the AvrPiz- $t$ ectopic expression lines in rice but opposite with that of the APIP6 RNAi plants. Co-expression assays in rice protoplasts and Nicotiana benthamiana leaves indicate that APIP6 promotes OsELF3-2 degradation, suggesting that OsELF3-2 is a substrate of APIP6. Taken together; these results demonstrate that the APIP6-mediated UPS plays an important role in immunity to M. oryzae through OsELF3-2 in rice.

Rice plants exhibiting bacterial blightresistance at high temperature suppress abiotic response

S. COHEN (1), H. Liu (2), C. Argueso (1), C. M. Vera Cruz (3), V. M. Verdier (4), J. E. Leach (1), (1) Colorado State University, Fort Collins, CO, USA; (2) Institute of Crop Sciences, Chinese Academy of Agricultural Sciences, Beijing, CHINA; (3) International Rice Research Institute, Los Baños, Laguna, PHILIPPINES; (4) IRD, Cirad, Univ Montpellier, IPME, Montpellier, FRANCE

During some plant-pathogen interactions, heat stress increases host susceptibility, a phenomenon which could pose a serious risk to global food security in light of climate variability. Despite the importance of this phenomenon, little is known about the underlying molecular mechanisms. To better understand host plant responses during simultaneous heat and pathogen stress, we conducted a transcriptomics experiment for rice plants infected with Xanthomonas oryzae during high temperature stress. Differential gene expression analysis revealed that plants exhibiting resistance at high temperature downregulated abiotic stress responses, while plants in the susceptible interaction did the opposite. This trend suggests that during simultaneous heat and disease stresses, disease resistant plants prioritize response to pathogen over the response to abiotic stress. Hormone stress was also notably perturbed due to simultaneous stresses, with plants exhibiting resistance repressing the abscisic acid signaling pathway at high temperature. Future research will focus on understanding how these pathways contribute to increased plant disease at high temperature.

\section{A performance-based approach to the detection of Acidovorax citrulli in cucurbit seed samples} K. D. KLEINHESSELINK, Monsanto, Woodland, CA, USA

Acidovorax citrulli $(A c)$ is a causal agent of bacterial fruit blotch (BFB) which is a continuing threat to cucurbit fruit production and can be seed-borne. Strains of $A c$ can infect several species in the family of Cucurbitaceae, with significant losses reported in melon and watermelon. Current NSHSapproved seed health testing methods include a seedling grow-out and several molecular pre-screening methods. Through an initiative driven by seven private companies through the International Seed Health Initiative (ISHI-Veg) the PCR-based pre-screening methods have been harmonized to generate a single reference method built on the concepts of performance-based testing. Validation components of sensitivity, specificity, accuracy and robustness were demonstrated through collaborative research resulting in a method with $100 \%$ detection across a collection of $517 \mathrm{Ac}$ isolates and a limit of detection of 237 bacterial cells per 5,000 seed sample. These criteria were used to establish performance targets which allows for flexibility in nucleic acid extraction and PCR chemistries due to the robustness of the method. This enables individual laboratories to optimize and improve methods over time while maintaining a stringent commitment to the delivery of high-quality seed to the vegetable industry.

A comparative study of three detection techniques for Leifsonia xyli subsp. $x y l i$, the causal pathogen of sugarcane ratoon stunting disease Q. WU (1), Y. B. Pan (2), L. Xu (1), D. Zhou (1), M. P. Grisham (2), Y. Su (1), J. Guo (1), Y. Que (1), (1) Fujian Agriculture and Forestry University, Fuzhou, CHINA; (2) USDA-ARS, SRU, Houma, LA, USA

Sugarcane ratoon stunting disease (RSD) caused by the bacterium Leifsonia xyli subsp. $x y l i(L x x)$ is responsible for significant yield losses worldwide. The $L x x$ bacteria are very small and difficult to isolate, culture, and detect. In this study, conventional polymerase chain reaction (PCR), $L x x$-loop mediated isothermal amplification ( $L x x$-LAMP), and real-time quantitative PCR (RT-qPCR) were utilized to specifically detect the presence of the pathogen in $L x x$-infected juice of YG18 and $L x x$-pMD18-T recombinant plasmids. The results showed that the techniques were specific for $L x x$ and provided great reproducibility. When DNA from $L x x$-infected juice was used as a template, the $L x x$-LAMP was 10- and 100-fold more sensitive than RTqPCR and conventional PCR, respectively. When $L x x$-pMD18-T recombinant plasmids were used as the template, $L x x$-LAMP was as sensitive as RTqPCR, but was 10 times more sensitive than conventional PCR. Based on our lab's $L x x$-LAMP detection system, adding $0.4 \mu \mathrm{M}$ of loop primers (LF/LP) can accelerate the reaction and reduce the total time required. In addition, the amount of Bst DNA polymerase was further optimized to $6.0 \mathrm{U}$ per reaction. The results will help sugarcane diagnostic labs further improve $L x x$ detection.

Growth of Legume Species Under Variable Zinc Regimes and Different Mycorrhizal Fungi M. L. PAWLOWSKI (1), G. L. Hartman (2), (1) Univ of Illinois Urbana Champaign, Urbana, IL, USA; (2) USDA-ARS at the University of Illinois, Urbana, IL, USA

Zinc (Zn) is an essential plant micronutrient that is an integral component of enzymes, membrane integrity, and binding of radicals. Zn deficiency widely occurs in plants resulting in poor plant growth and deficiencies in humans. Zn deficiency in plants can be caused by low levels of $\mathrm{Zn}$ in soil or $\mathrm{Zn}$ fixation in soils that may inversely be related to phosphorus $(\mathrm{P})$ in soil. $\mathrm{Zn}$ toxicity may be less common but may occur in some situation often related to industrial wastes. Over-fertilization of $\mathrm{P}$ can immobilize $\mathrm{Zn}$ and restrict root growth and root colonization of arbuscular mycorrhizal (AM) fungi, which facilitate uptake of immobile minerals such as $\mathrm{P}$ and $\mathrm{Zn}$. The objective of this study was to evaluate the impact of different species of mycorrhizal fungi on alleviating $\mathrm{Zn}$ deficiency and toxicity in legumes. This experiment was conducted in a growth chamber as a 3-factor completely randomized design with three replicates. The three factors included AM fungal species, legume species, and $\mathrm{Zn}$ treatments. Common bean, cowpea, and soybean were inoculated with three different AM fungal species. Mycorrhizal and nonmycorrhizal plants were treated with a modified low-P Hoagland's solution with no $\mathrm{Zn}, 0.001 \mathrm{x} \mathrm{Zn}, \mathrm{Zn}$ or 100x Zn. Treatment effects were measured by above and below ground biomass, mycorrhizal colonization, and Zn leaf content. Results from this study will be further developed once analyses are complete. This information will further our understanding of this 3-way interaction and provide insights to the benefits of mycorrhizal fungi on $\mathrm{Zn}$ uptake and plant growth.

\section{Ecology and epidemiology of Helminthosporium solani and Colletotrichum coccodes on potato in the San Luis Valley of Colorado} F. J. LICHTNER, K. D. Broders, Colorado State University, Fort Collins, CO, USA

Helminthosporium solani and Colletotrichum coccodes which cause silver scurf and black dot of potato, continue to have a negative economic impact on potato production in the San Luis Valley of Colorado. These diseases are often misdiagnosed, but a thorough assessment of fields and harvested tuber 
has not been conducted in the region. Soil properties are also known to influence the persistence and the viability of pathogen inoculum year to year. Therefore, the objective of this study was to evaluate the incidence of silver scurf and black dot in potato production systems and the impact of soil properties on pathogen abundance. We sampled 56 fields and 64 tubers for the incidence of $H$. solani and C. coccodes, and performed a multivariate analysis to determine the effect of soil properties on $H$. solani and C. coccodes inoculum. Of the 56 soils sampled 56 and 53 were positive by qPCR for $H$. solani and C. coccodes, respectively. However, there was significantly greater quantities of $C$. coccodes than $H$. solani in the soil. Disease incidence of $H$. solani and C. coccodes on tubers were $48 \%$ and $98.4 \%$, respectively. The first year of sampling showed percent silt and organic matter content were strong indicators of the presence $C$. coccodes, while soil $\mathrm{pH}$ and Fe content were important factors predicting the presence of $H$. solani in the soil. Predictive and quantitative understanding of this fungal disease complex will require in vitro experimentation and regional surveying.

Expanding the scope of a single pathogen survey to solve the mystery of the ailing red raspberries J. E. WEILAND (1), C. Benedict (2), I. A. Zasada (1), B. Beck (1), A. Davis (1), A. Peetz (3), K. Graham (1), R. R. Martin (1), J. K. S. Dung PhD (4), A. J. Reyes Gaige (5), L. D. Thiessen (6), (1) USDA ARS, Corvallis, OR, USA; (2) WSU, Bellingham, WA, USA; (3) USDA ARS HCRU, Corvallis, OR, USA; (4) Oregon State University, Madras, OR, USA; (5) Oregon State University, Corvallis, OR, USA; (6) North Carolina State University, Raleigh, NC, USA

Washington state produces almost $60 \%$ of the processed raspberries ( $\$ 79 \mathrm{M}$ value) in the U.S. Production is severely limited by Raspberry Bushy Dwarf Virus (RBDV) and the soilborne pathogens Phytophthora rubi and Pratylenchus penetrans. However, in 2012, growers began noticing plants with unusual symptoms that were not attributed to any of these pathogens. Starting in late summer, individual primocanes or entire plants would suddenly wilt and die. Adjacent plants were often chlorotic and stunted. Early diagnostic work indicated that Verticillium dahliae was the culprit at three locations, but analyses of soil populations often produced conflicting results depending on the lab and detection method. Growers became concerned that this pathogen was a previously undiagnosed problem for the industry. Therefore, a survey was conducted to determine if $V$. dahliae was common in production fields, to determine if $V$. dahliae was associated with disease, and to compare the sensitivity of two detection methods (selective media versus qPCR) for quantifying $V$. dahliae in the soil. The presence of $P$. rubi, P. penetrans, and RBDV was also assessed. In 2013 to 2014, primocanes, roots, and soil were collected from 51 disease sites and 20 healthy sites within 24 production fields. Results show that $V$. dahliae is common and that qPCR is more sensitive than plating for detection. But, the main question remains, which pathogen(s) are causing the disease? Come find out!

Effects of N-P-K fertilizer rates on the interaction between Fusarium virguliforme and soybean cyst nematode on soybean P. OKELLO (1), S. Osborne (2), F. M. Mathew (1), (1) South Dakota State University, Brookings, SD, USA; (2) USDA Agricultural Research Service, Brookings, SD, USA

Fusarium virguliforme (sudden death syndrome pathogen) and soybean cyst nematode (SCN) are important pathogens of soybean (Glycine max) in South Dakota. In this study, the effect of fertilizer rates on the interaction between $F$. virguliforme and SCN on soybean was examined in the greenhouse using two cultivars, SCN susceptible cv. Williams 82 and SCN resistant cv. Jack. Two N-P-K fertilizer rates-starter (15:15:15) and high (50:80:110) were used on each of the three pathogen treatments $(F$. virguliforme alone, SCN alone, and concomitant inoculation of SCN and $F$. virguliforme) and a non-inoculated control. Pre-germinated soybean seeds were planted in cone-tainers filled with steam pasteurized sand-clay soil mixture, which were premixed with either fertilizer rates. For inoculating soybean seedlings, either $F$. virguliforme-infested sorghum seeds and/or $2000 \mathrm{SCN}$ eggs $/ \mathrm{mL}$ were applied to the respective treatments. After 35 days, root rot severity caused by $F$. virguliforme and SCN reproduction were evaluated. Root rot severity caused by $F$. virguliforme increased significantly $(P<0.001)$ in the presence of $\mathrm{SCN}$ and fertilizer rates. However, $\mathrm{SCN}$ reproduction was significantly reduced $(P<0.001)$ by fertilizer rates and $F$. virguliforme. Our study suggests that soybean farmers should manage fertility applications in their fields infected with $F$. virguliforme and $\mathrm{SCN}$.

Rapid diagnostic tools for soilborne pathogens of strawberry

A. BURKHARDT (1), M. Ramon (1), S. T. Koike (2), F. N. Martin (1), (1) USDA ARS, Salinas, CA, USA; (2) Univ of California, Salinas, CA, USA

The ability to rapidly identify and quantify soilborne strawberry pathogens in plants and soil allows growers to make decisions about what to plant and how to manage diseases in a field. Therefore, we are developing rapid assays using TaqMan real-time PCR and recombinase polymerase amplification (RPA) to diagnose pathogens that severely damage strawberry, including Verticillium dahliae, Fusarium oxysporum f. sp. fragariae, and Macrophomina phaseolina. These assays provide results in hours (TaqMan) or in as little as 20 minutes (RPA) using DNA extracted from either infected plant tissue or soil. For M. phaseolina, genomic sequencing, assembling, and comparing multiple isolates has identified a genotype-specific locus to detect isolates which have been found to only infect strawberry. This locus was used to develop an RPA assay with a sensitivity of 200 fg that has been shown to be specific on nearly 100 samples. A nested qPCR TaqMan assay is also being optimized to detect a low level of M. phaseolina microsclerotia in the soil. A published locus specific for a majority of $F$. oxysporum $f$. sp. fragariae isolates has been translated to an RPA assay ( 200 fg sensitivity) and is being developed into a TaqMan assay for soil quantification. Additional loci are being developed to detect all $F$. oxysporum f. sp. fragariae isolates. For $V$. dahliae, a published TaqMan assay for soil has been transitioned into an RPA for infected crown tissue with a sensitivity of $200 \mathrm{fg}$.

\section{Variation in phenylpropanoid intermediates associated with adaptation to abiotic stress in the perennial tree species Prunus persica and $P$. armeniaca}

A. O. CONRAD (1), T. Zhebentyayeva (2), M. Staton (3), J. M. Audergon (4), V. Decroocq (5), Z. Liu (6), C. D. Dardick (7), C. D. Nelson (8), A. Abbott (1), (1) University of Kentucky, Lexington, KY, USA; (2) Clemson University, Clemson, SC, USA; (3) University of Tennessee, Knoxville, TN, USA; (4) INRA, Avignon, FRANCE; (5) INRA, Bordeaux, FRANCE; (6) USDA ARS, Kearneysville, WV, USA; (7) Appalachian Fruit Research Station, USDA, Agricultural Research Service, Kearneysville, WV, USA; (8) USDA-Forest Service, Southern Research Station, Lexington, KY, USA

Phenological traits, e.g. dormancy, are mechanisms by which perennial tree species have adapted to stressful conditions that are unfavorable for growth. While there are many mechanisms by which trees adapt and respond to stress, the phenylpropanoid pathway in particular is known to be associated with tree stress response. In this study, Prunus persica (peach) and P. armeniaca (apricot) were used as models to explore the relationship between phenylpropanoids and adaptive trait variation. Concentrations of eight phenylpropanoid intermediates were measured throughout dormancy in floral buds of trees that differed genotypically and phenotypically in chill requirement (peach) and bloom date (apricot). There was a significant interaction effect of time and genotype on the concentration of each of the compounds (mixed ANOVA, $P<0.05$ ). Moreover, concentrations of the flavonoid aglycones, apigenin, kaempferol, and quercetin, dropped precipitously in high chill peach and late flowering apricot, around the endodormancy-ecodormancy transition and at the time when buds began to show signs of blooming (e.g. presence of sepals and petals). These results suggest a potential role of phenylpropanoid intermediates in mediating the resumption of growth and development following winter dormancy. A better understanding of tree responses to stress, will be critical for tree improvement programs aimed at identifying and breeding trees more suitable for rapidly changing environments.

Extracting Genomic DNA from Root-Knot Nematode Infested Soil using $\mathrm{Fe}_{3} \mathrm{O}_{4}$ Super Paramagnetic Nanoparticles A. M. GORNY (1), X. Wang (2,3), S. J. Pethybridge (4), (1) Cornell University, Plant Pathology \& Plant Microbe Biology Section, Geneva, NY, USA; (2) USDA-ARS, Robert W. Holley Center for Agriculture and Health, Ithaca, NY, USA; (3) Cornell University, School of Integrative Plant Science, Ithaca, NY, USA; (4) Cornell University, Geneva, NY, USA 
Quantifying the level of plant-parasitic nematodes in field soil prior to planting using a DNA-based soil test could result in a more accurate estimation of crop damage or yield loss observed at harvest. Incorporation of this information into management decisions may promote conservative use or targeted application of pesticides, reducing the environmental impact and cost of production. The isolation of DNA from soil is a critical first step in molecular quantification, and standard techniques for extracting DNA from soil include phenol-chloroform based methods and commercial kits. However, these are often time consuming, produce hazardous waste, are cost prohibitive, and may only be designed for isolating DNA from small volumes of soil. The variability in pathogen population density estimations may be greatly reduced when larger volumes of soil are analyzed. Recently, the use of super paramagnetic nanoparticles has been successful in genomic DNA isolation, providing a quicker, easier, and more economical protocol than traditional kit or phenol-chloroform based methods. Here, a method using $\mathrm{Fe}_{3} \mathrm{O}_{4}$ super paramagnetic nanoparticles for the isolation of genomic DNA from soil was investigated for its utility in quantification of plant-parasitic nematodes directly from soil. DNA was isolated from $100 \mathrm{~g}$ of soil inoculated with a known quantity of Meloidogyne hapla (root-knot nematode). The method was optimized, and several permutations assaying the volume of nanoparticles used and number of nematodes in the soil were trialed. Resultant DNA was assessed for quantity and quality using spectroscopy, fluorometry, and PCR technologies. Trade-offs between parameter optimization and DNA quality and quantity are discussed.

Effect of moisture at physiological maturity on soybean seed infection by Phomopsis longicolla and Cercospora flagellaris J. C. RUPE, R. Holland, A. Steger, University of Arkansas, Fayetteville, AR, USA

Phomopsis seed decay (caused by Phomopsis longicolla) infects soybean in two stages: infection of the plant and pods throughout the season and infection of seed from pod infections after physiological maturity (PM). To determine if enhancing moisture at PM increases seed infection, four soybean lines were planted at Kibler, AR, in 2014 and 2015. Pods from the lower half of the plant were removed just as they began to yellow and either assayed immediately for $P$. longicolla and Cercospora flagellaris, the causal agent of purple seed stain, or placed in a moist chamber for three days at room temperature and then assayed. Infection levels were determined by surface disinfesting seeds and pods and incubating on potato dextrose agar for seven days. Fungi were identified by culture appearance. Pod infections ranged from $15-88 \%$ for $P$. longicolla and ranged from $0-8 \%$ for $C$. flagellaris. Seed infection by $P$. longicolla at PM ranged from $0-13 \%$, but increased to $19-67 \%$ after $3 \mathrm{~d}$ in the moist chamber. Seed infection by $C$. flagellaris at PM ranged from $2-38 \%$ and from $2-32 \%$ after $3 \mathrm{~d}$ in the moist chamber. At harvest, seed infection by $P$. longicolla on the lower half of the plant ranged from $1-71 \%$ and by C. flagellaris from 3-27\%. Seed infection at harvest by Colletotrichum spp. and by Fusarium equisetti ranged from 0-12\% and from 1$17 \%$, respectively, but infection by these pathogens was not observed at PM. There were significant differences between soybean lines and years.

Anaerobic soil disinfestation to manage soilborne disease complexes in protected culture tomato production A. L. TESTEN, S. A. Miller, The Ohio State University OARDC, Wooster, OH, USA

A soilborne disease complex consisting of Verticillium wilt (Verticillium dahliae), black dot root rot (Colletotrichum coccodes), corky root rot (Pyrenochaeta lycopersici), and root knot nematodes (Meloidogyne sp.) threatens the productivity and profitability of protected culture production of tomato in Ohio. Anaerobic soil disinfestation (ASD) with one of three carbon sources (wheat bran, molasses, or ethanol) was tested as a method to manage this soilborne disease complex in soils from two Ohio sites. ASD with wheat bran or ethanol amendments led to significantly reduced tomato root rot severity, and nematode galling damage was significantly reduced following ASD with any carbon source. The use of ethanol as a carbon source in ASD decreased the incidence of fungal pathogen isolation from roots. Bacterial community diversity decreased following ASD with wheat bran in all soils tested. The relative abundance of Clostridia increased in soils following treatment with any ASD carbon source, while the relative abundance of Firmicutes increased significantly following ASD with wheat bran. The relative abundance of Acidobacteria, Actinobacteria, Chloroflexi, and Planctomycetes decreased following ASD, regardless of carbon source. These findings provide insight into the impacts of ASD on microbial communities and will be used to optimize ASD as a tool for Midwestern protected culture tomato production.

Effect of tillage and cultivar on sudden death syndrome and yield of soybean in Iowa Y. R. KANDEL, L. F. S. Leandro, D. S. Mueller, Iowa State University, Ames, IA, USA

Reduced-tillage has become a common practice of soybean farming in the Midwestern U.S. Field trials were established in a field with a history of sudden death syndrome (SDS; caused by Fusarium virguliforme) in Iowa in 2011, and evaluated for five consecutive years, to determine the impact of long-term tillage on SDS and yield. The study site contains mainly webster clay loam, bemis moraine, and nicollet loam soil, with 1 to $3 \%$ slopes that are poorly drained. The experiment was laid out in a split split-plot design with four replicates. The main plot factor was tillage (no till both crops, no till corn and chisel plow soybean, and disc corn and chisel plow soybean, respectively), and each main plot was divided into subplots of corn or soybeans (in a 2-year rotation). Each subplot was again divided into sub-subplots where two soybean cultivars, susceptible and moderately resistant to SDS, were planted each year. Root rot and SDS disease index (FDX) differed among years, as some years were more favorable for the disease than the others. However, tillage did not affect any parameters, including yield, in any year $(\mathrm{P}>0.05)$. Cultivar effect was significant for each parameter occasionally. Compared to the susceptible cultivar, the moderately resistant cultivar had 61\% less root rot in 2015; 61 and $62 \%$ less FDX in 2014 and 2015 , respectively, and $21 \%$ greater yield in 2015 . These data suggest planting resistant cultivars can be an effective management tactic but tillage is not an effective option for SDS management.

An intriguing taste of 30 years of coevolution: Monitoring Coffee Leaf Rust (Hemileia vastatrix Berk. \& Br) in Colombia C. A. ANGEL (1), M. Cristancho (1,2), A. Jaramillo (1), A. L. Gaitan (1), (1) CENICAFE, Colombia, Manizales, COLOMBIA; (2) Bioinformatics and Computational Biology of Colombia -BIOS, Manizales, COLOMBIA

Coffee leaf rust (CLR, Hemileia vastatrix) is the most important disease of coffee worldwide. Yield and quality losses can reach $23 \%$ to $50 \%$ in susceptible varieties. Before CLR arrived to Brazil in 1970, CENICAFÉ - Colombia initiated a breeding program using the susceptible C. arabica cv. Caturra mainly and Timor Hybrid (TH) as resistant parent. The results were multiline resistant varieties: "Colombia" was released in 1982 (one year before CLR was found in Colombia), "Tabi" a taller variety in 2002, "Castillo ${ }^{\mathbb{}}$ " in 2005, and "Cenicafé 1" in 2016, the first three being planted in 74\% out of 932,000 ha of coffee in Colombia. From the initial breeding, a set of 112 experimental materials with diverse CLR responses were planted between 1995 and 1999 to monitor disease progress in five localities. Six CLR coffee differentials to assess race development, and 52 TH materials were also planted. CLR was measured every four months following Eskes \& Toma-Braghini (1981) incidence diagrams. Deviation analyses of historicals for temperature, rainfall, and solar bright and CLR progress per each location are supporting isolates adaptation and different disease patterns. Climate events such as "La Niña" in 2008-2009, and weather variations increased CLR epidemics in 2011-2012 and 2014-2015 as well, favoring new races establishment. By 2016 , between $36 \%$ to $88 \%$ of materials had low-incomplete CLR resistance or lost it depending on the locality, all differentials were infected, $28 \%$ of TH materials did not have CLR and 34\% had high-incomplete CLR resistance, indicating pathogen coevolution and adaptation.

Design and operational considerations for use of UV and red light for suppression of plant diseases under field conditions D. M. GADOURY (1), A. Bierman (2), M. Rea (2), A. Stensvand (3), A. Suthaparan (4), R. Borba Onofre (5), (1) Cornell University, Geneva, NY, USA; (2) Lighting Research Center, Rensselaer Polytechnic Institute, Troy, NY, USA; (3) Norwegian Institute of Bioeconomy Research, Ås, NORWAY; (4) Norwegian University of Life Sciences, Ås, NORWAY; (5) University of Florida - Gulf Coast Research and Education Center, Wimauma, FL, USA

Diverse plant pathogens have been suppressed by ultraviolet (UV) light, both alone and in combination with select portions of the visible light spectrum in recent studies. Stationary UVB lamps or UVB lamps borne on a slow-moving boom (e.g. $0.03 \mathrm{~km} / \mathrm{h}$ ) have been used to apply UV doses approximating 
50 to $200 \mathrm{~J} / \mathrm{m}^{2} /$ day. Operational speeds closer to $3.0 \mathrm{~km} / \mathrm{h}$ (100X the foregoing) are needed to adapt this technology to field applications involving conventionally driven tractors. Reciprocity of UVB effects extrapolated from longer exposure time/ lower intensity treatments were confirmed at target intensity and times for UVC field treatments. We used a dense reflectorized hemicylindrical array of multiple UVC fluorescent lamps to yield sufficient intensity and multiple reflectance angles to maximize exposure of all plant surfaces in preliminary trials in a commercial strawberry field in Florida. Dense arrays of high intensity red $(663 \mathrm{~nm}$ peak irradiance) LEDs were required for synergistic activity with short-duration/high intensity UV treatments. Neither UVB fluorescent lamps nor currently available UV-LEDs produced sufficient energy in the biologically relevant wavebands (250 to $300 \mathrm{~nm}$ ) to allow their use in units moving at speeds significantly above $0.06 \mathrm{~km} / \mathrm{h}$, but might be amenable for autonomous or robotic applications in field plantings at greatly reduced speeds.

\section{Identifying the most efficient agroecosystems for potato production in Nariño, Colombia P. URIBE, C. A. Benavides, D. Calvache, Corporación Colombiana de Investigación Agropecuaria, Pasto, COLOMBIA}

With global warming knocking on our doors, its impact on agricultural systems are feared to be severe and the likelihood of the outcome will largely depend on how these systems adapt to the new reality. In general three responses can be foreseen to happen: either, there is a shift of the agricultural frontier, new varieties adapted to the changing conditions are developed or a switch to other crops suitable for the new environment is made. This reality is perhaps more complicated in developing countries where the resilience of agricultural systems is not always present. In the case of Colombia, this is a big concern, but a departmental sponsored project might have an answer. The project, "Technological and Productible Improvement of the Potato System in the Department of Nariño" was developed with the changing conditions caused by global warming in mind and has as its main objectives to improve the technological know-how of potato producers in the Department of Nariño and to have them to be more competitive and sustainable in the long term. This four year, US $\$ 2.76$ Million project aims to give solutions to 1.5 million people that in the region, directly or indirectly derive their income from the potato production. The project gathers data from five interlaced objectives and here we are presenting data from mostly the first objective, which aims to identify the most efficient agroecosystems to grow potatoes within the producing areas of Nariño.

A Multi-state Coordinated Project to Evaluate Integrated Management Strategies for Fusarium Head Blight and Deoxynivalenol in Wheat J. D. SAlGADO (1), P. A. Paul (2), K. A. Ames (3), G. C. Bergstrom (4), C. A. Bradley PhD (5), E. Z. Byamukama (6), J. A. Cummings (7), V. Chapara (8), M. Chilvers (9), R. Dill-Macky (10), A. J. Friskop (11), P. Gautam (12), N. M. Kleczewski (13), L. V. Madden (2), E. A. Milus (14), M. Nagelkirk (15), J. Ransom (16), K. R. Ruden (17), J. Stevens (18), S. N. Wegulo (19), K. A. Wise (20), D. Yabwalo (21), (1) The Ohio State University, Wooster, OH, USA; (2) Ohio State Univ, Ohio Agricultural Research and Development Center, Dept of Plant Pathology, Wooster, OH, USA; (3) University of Illinois, Urbana, IL, USA; (4) Cornell University, Ithaca, NY, USA; (5) University of Kentucky, Princeton, KY, USA; (6) South Dakota State Univ, Brookings, SD, USA; (7) Cornell University, Trumansburg, NY, USA; (8) NDSU, Langdon, ND, USA; (9) Michigan State University, East Lansing, MI, USA; (10) University of Minnesota, St Paul, MN, USA; (11) Department of Plant Pathology, North Dakota State University, Fargo, ND, USA; (12) Bayer CropScience, Beaver Crossing, NE, USA; (13) University of Delaware, Newark, DE, USA; (14) University of Arkansas, Salida, CO, USA; (15) Michigan State Univ Ext, Sandusky, MI, USA; (16) North Dakota State University, Fargo, ND, USA; (17) Bayer Crop Science, Bruce, SD, USA; (18) University of Nebraska, Lincoln, NE, USA; (19) Univ of Nebraska, Lincoln, NE, USA; (20) Purdue University, West Lafayette, IN, USA; (21) South Dakota State University, Brookings, SD, USA

The integration of cultivar resistance with a fungicide application at anthesis is the most effective approach for minimizing wheat grain yield and quality losses due to Fusarium head blight (FHB) and deoxynivalenol (DON). However, local weather and field conditions often prevent fungicides from being applied at anthesis. In 2014 and 2015 we evaluated the integrated effects of cultivar resistance (susceptible [S], moderately susceptible [MS] and moderately resistant $[\mathrm{MR}]$ ) and application timing of the fungicide Prosaro (untreated or treated at anthesis or 2 to 7 days post-anthesis) on FHB and DON in 27 trials across 12 states, representing four market classes of wheat. Mean FHB index and DON in the untreated susceptible check (reference program) ranged from 3 to $54 \%$ and from 1.9 to $33 \mathrm{ppm}$, respectively, across trials. Combinations of Prosaro with a MS or MR cultivar were consistently more effective than fungicide or resistance alone at reducing FHB and DON, with percent control relative to the reference program ranging from 4 to $99 \%$ for index and 11 to $89 \%$ for DON on the MS cultivars and from 42 to $99 \%$ for FHB index and 32 to $93 \%$ for DON on MR cultivars. When evaluated across trials, post-anthesis applications were as effective as anthesis applications, particularly on MR cultivars. Results from a meta-analysis to quantify the effects of location-specific factors on the magnitude of the observed responses will be presented.

\section{Use of the disease severity index for null hypothesis testing}

K. S. P. CHIANG (1), C. H. Bock (2), (1) Division of Biometrics, Department of Agronomy, National Chung Hsing University, Taichung, TAIWAN; (2) USDA ARS, Southeastern Fruit and Tree Nut Research Laboratory, Byron, GA, USA

A disease severity index (DSI) is a single number for summarizing a large amount of disease severity information. It is used to indicate relative resistance of cultivars, to relate disease severity to yield loss, or to compare treatments. The DSI has most often been based on a special type of ordinal scale data defining a series of consecutive numeric ranges, generally based on the percent scale. The objective of this work is to explore the effects both of different ordinal scales (those having equal or unequal interval widths) and of the selection of values for scale intervals (the ordinal score for the interval range or the midpoint value) on the null hypothesis test for treatment comparisons. A simulation approach was employed to approximate the mechanisms governing the estimation process. Subsequently, a meta-analysis was used to compare two treatments, demonstrating that using quantitative ordinal rating scores or the midpoint values of interval yielded very comparable results with respect to the power of hypothesis testing. The main factor determining the power of the hypothesis test is the nature of the interval widths (equal or unequal), not the selection of values for ordinal scale intervals (score or midpoint value). Although the percent scale is preferable, the results provide a framework for developing improved research methods where the use of ordinal scales in conjunction with a DSI is preferred for comparing disease severities.

\section{Assessment and utilization of risk factors in predicting the development of soybean sudden death syndrome} M. ROTH, Z. Noel, J. Wang, A. Byrne, M. Chilvers, Michigan State University, East Lansing, MI, USA

Sudden death syndrome (SDS) of soybean is caused by the fungal pathogen Fusarium virguliforme (Fv) in the United States. Successful management strategies to control SDS have been inconsistent, leading to devastating outbreaks. Few fungicides have shown efficacy against Fv, and genetic resistance through breeding of quantitative trait loci have yielded only partially resistant cultivars. The ability to predict SDS development could provide growers with valuable information for making important SDS management decisions, like using effective fungicides or partially resistant cultivars. The aim of this study was to identify risk factors that may allow accurate predictions about the severity SDS development. In 2014 and 2015, we collected data from soybean fields naturally infested with Fv and soybean cyst nematodes (SCN) to identify potential risk factors, including Fv DNA quantity and and SCN counts at planting and post-harvest, and identify correlations to late season SDS ratings. At-planting soil distributions of Fv DNA quantity had a significant positive correlation to the spatial distribution of late season foliar SDS in both years. Similarly, at-planting soil distributions of SCN had a significant positive correlation to late season foliar SDS in 2015, but not 2014. Interestingly, Fv quantities in seedling roots had inconsistent correlations to late season foliar SDS symptoms, indicating that Fv abundance within roots may not be indicative of SDS severity.

Spline regression providing the area under the sensitivity curve as an alternative for the $\mathbf{E C}_{50}$ values on fungicide resistance studies P. D. S. F. LICHTEMBERG (1), W. Zeviani (2), T. J. Michailides (1), (1) University of California - Davis, Parlier, CA, USA; (2) Universidade Federal do Paraná, Curitiba, BRAZIL 
The use of effective concentration of a fungicide that inhibits $50 \%$ of a pathogen's growth $\left(\mathrm{EC}_{50}\right)$ is of major importance in fungicide resistance studies. This value is used to monitor the efficacy of fungicides and the shift of pathogen sensitivity before the occurrence of field control failure. Sensitivity values are quantified by dose-response curves with different fungicide concentrations. The usage of linear regression on log-log scale of variables to estimate $\mathrm{EC}_{50}$ is not always suitable. Problems to determine $\mathrm{EC}_{50}$ values include: absence of linear relation between fungicide dose and inhibition which makes adjustments difficult, higher doses are not enough to reveal half inhibition, and exclusion of the mean colony diameter when the dose is zero. To overcome these problems it is proposed to use the area under the sensitivity curve (AUSC) by using the cubic spline regression. Cubic splines are functions constructed of piecewise third-order polynomials which pass through a set of knots. Their flexibility and numerical integration can be used to compute the AUSC. In our study, we use a data set of mycelial growth obtained from nine doses ranging from 0 to $122.8 \mu \mathrm{g} / \mathrm{ml}$ to determine the Alternaria alternata sensitivity to three SDHI fungicides. We observed a significant linear correlation between $\mathrm{EC}_{50}$ and AUSC values $\left(\mathrm{r}^{2}=82.5 \%\right)$. Additionally, we could determine the AUSC for all 765 tested isolates, while the traditional $\mathrm{EC}_{50}$ method allowed us to identify only $72 \%$ of the tested isolates.

Validation of a Florida strawberry anthracnose fruit rot (AFR) warning system in Iowa

X. ZHANG (1), J. C. Batzer (1), X. Li (1), N. Peres (2), M. L. Gleason (1), (1) Iowa State University, Ames, IA, USA; (2) Gulf Coast Research and Education Center; University of Florida, Wimauma, FL, USA

Field validation of a Florida disease-warning system for strawberry anthracnose fruit rot (AFR), caused by Colletotrichum acutatum sensu lato, was conducted in Iowa during 2012 to 2014. The inputs to the warning system are measurements of air temperature and leaf wetness duration; an algorithm developed at University of Florida was used to predict two levels (moderate and high) of infection risk. A factorial combination of two spray timing methods (the warning system and prescheduled, calendar-based timing) and two fungicides (captan and pyraclostrobin) were compared, along with an unsprayed control treatment, in replicated field trials with day-neutral strawberries (cv. Tristar) during three growing seasons, following artificial inoculation with the pathogen at the start of the fruit maturation period. In the unsprayed control, AFR disease incidence ranged among seasons from $3.64 \%$ to $57.3 \%$. The calendar-based treatments and warning system-based treatments both provided significant control of AFR incidence compared to the unsprayed control during the two seasons that experienced substantial disease pressure. The AFR warning system saved an average of 1.7 sprays annually compared to calendar-based treatments, with no loss of AFR control. However, nonlinear mixed model analyses showed that under high disease pressure in the late growing season, the warning system may underestimate the disease risks. Therefore, a lower threshold should be used in such a situation to allow more fungicide spray. These results provided evidence that the Florida warning system can be valuable for helping Midwest strawberry growers control AFR with less reliance on fungicide sprays, but certain adjustments are needed for the late season.

\section{A model bacterial community of maize roots}

B. NIU (1), J. Paulson (2,3), X. Zheng (2,4), R. Kolter (1), (1) Department of Microbiology and Immunobiology, Harvard Medical School, Boston, MA, USA; (2) Department of Biostatistics and Computational Biology, Dana-Farber Cancer Institute, Boston, MA, USA; (3) Department of Biostatistics, Harvard T. H. Chan School of Public Health, Boston, MA, USA; (4) Department of Mathematics, Shanghai Normal University, Shanghai, CHINA

Plant-associated microbes are crucial for the health of their hosts. However, the high complexity of plant microbiomes challenges detailed studies to define experimentally the mechanisms underlying the beneficial effects of such microbiota on plant hosts and the dynamics of community assembly. Using host-mediated selection, we obtained a greatly simplified synthetic bacterial community consisting of seven strains (Enterobacter cloacae, Stenotrophomonas maltophilia, Ochrobactrum pituitosum, Herbaspirillum frisingense, Pseudomonas putida, Curtobacterium pusillum and Chryseobacterium indologenes) representing three of the four most dominant phyla found in maize roots. In planta and in vitro, this model community inhibited the phytopathogenic fungus Fusarium verticillioides indicating a clear benefit to the host. By utilizing a selective culture-dependent method to track the abundance of each strain we investigated the role that each plays in community assembly on roots of axenic maize seedlings. Only the removal of E. cloacae led to the complete loss of the community and C. pusillum took over. This suggests that E. cloacae plays the role of keystone species in this model ecosystem. Thus, our synthetic seven-species community has the potential to serve as a useful system to dissect the beneficial effects of root microbiota on hosts and explore how bacterial interspecies interactions affect root microbiome assembly under laboratory conditions in future.

Impact Network Analysis of Avocado Laurel Wilt Disease

R. CHOUDHURY (1,2,3), J. H. Crane (4), D. Carrillo (4), E. Evans (4), J. Wasielewski (5), R. C. Ploetz (4), K. A. Garrett (2,3,6), (1) University of Florida Plant Pathology Department, Gainesville, FL, USA; (2) Institute for Sustainable Food Systems, Gainesville, FL, USA; (3) Emerging Pathogens Institute, Gainesville, FL, USA; (4) University of Florida - Tropical Research and Education Center, Homestead, FL, USA; (5) University of Florida/IFAS Extension, Homestead, FL, USA; (6) University of Florida, Gainesville, FL, USA

Laurel wilt (LW) disease is a serious threat to woody plants in the Lauraceae in both natural and agricultural ecosystems. It is caused by the ascomycete Raffaelea lauricola and is vectored by several ambrosia beetles. In addition to vectors, spread via rootgrafts is especially important in avocado orchards. The pathogen causes wilting, dieback, and death of the trees shortly after infection. Recommended control in avocado orchards entails early detection of LW symptomatic trees, immediate roguing, and prophylactic fungicide treatment. Adoption of a new technology or management practice depends on the economic benefits and the ease with which the practice can be incorporated into the grower's current routine. Impact network analysis is a framework for modeling the impact of new disease control methods in socioeconomic networks of communication and influence, linked to biophysical networks of epidemic spread. We use impact network analysis to estimate the relative effects of different control strategies on disease progress, accounting for both within- and between-grove spread. Early detection and rapid eradication of affected trees allow for reduced within-grove spread of the pathogen. Management strategies that reduce between-grove spread slow the overall epidemic. Managing LW is critical to sustainable Florida avocado production, as well as protecting production elsewhere in the US, Mexico, Central America, and the Caribbean.

Functional diversity of citrus microbiomes correlates with Huanglongbing disease symptom severity R. BLAUSTEIN, J. Silfa-Cifuentes, G. Lorca, M. Teplitski, University of Florida, Gainesville, FL, USA

Microbiomes can bolster host productivity by providing protection against pathogens, among other mechanisms. To investigate associations between citrus microbiome function and Huanglongbing (HLB) disease progression, microbial communities of leaves and roots from citrus trees ( $\mathrm{n}=70$ ) that varied in HLB symptom severity were characterized with 16S Illumina sequencing and metagenomes were predicted with PICRUSt. The taxonomically rich communities contained abundant core members, some over-represented site-specific members, and a diverse set of low-abundance variable taxa. The microbiota and the metagenomes were strongly associated with the presence of HLB symptoms. Substantial variation between the functional potential of healthy and diseased microbiomes was explained by abundances of genes encoding KEGG pathways for ribosomes, DNA repair proteins, $\mathrm{ABC}$ transporters, two-component systems, secretion systems, and various forms of metabolism. Genes involved in nucleotide and energy metabolism were crucial to the health-state of leaves, while those associated with carbohydrate, lipid, xenobiotic, and cofactor metabolism were crucial to that of roots. Interestingly, the rank abundances of KEGG pathways in the respective microbiomes were still highly conserved. This may have been due to the core members encoding core functions and/or to functional redundancies across certain variable taxa. Key microbiome functions that were identified may be important for citrus health.

The effects of cultivar, production system, and nursery on the composition of the rhizosphere microbiome of cultivated rhododendrons in Oregon Z. FOSTER (1,2), J. E. Weiland (1), C. Scagel (1), N. Grunwald (1), (1) USDA ARS, Corvallis, OR, USA; (2) Oregon State University, Corvallis, OR, USA 
The composition of plant microbiomes influences important agricultural processes such as nutrient absorption and plant health. Plant genotype and environment affect the microbiome, but the nature and relative importance of these effects are not well understood. We evaluated the effect of host genotype, nursery, and production system (potted versus in-ground planting) on the composition of the fungal and oomycete rhizosphere microbiome of rhododendrons in Oregon nurseries. Rhizosphere and roots were sampled from randomly selected, potted and in-ground plants of 3 host cultivars at 4 nurseries. ITS1 amplicons were sequenced using the Illumina MiSeq. Sequences were clustered and assigned to taxa using vsearch. Organism trophic mode was inferred using the FUNGuild database. Diversity statistics, ordination plots, and heat trees were calculated in R with vegan and metacoder. There was little difference in alpha diversity between potted and in-ground plants, but nurseries that grew plants with both production systems had higher diversity overall. Cultivar did not account for observed differences in community composition; however, nursery and production system had a significant effect. Ordination results suggest that production system had the dominant effect on microbiome composition, followed by a moderate effect from nursery, and no effect from cultivar. Most fungal species were predicted to be saprotrophs (50\%), followed by pathogens (12\%) and symbionts (6\%).

A Meta-Analysis of Maize Yield Response to Azoxystrobin and Propiconazole as Influenced by Foliar Disease Intensity and Application Timing F. DALlA LANA (1), A. E. Robertson (2), C. A. Bradley PhD (3), D. L. Smith (4), D. A. Shah (5), K. A. Wise (6), L. V. Madden (7), P. Esker (8), S. Conley (9), P. A. Paul (7), (1) Ohio State University, Wooster, OH, USA; (2) Iowa State University, Department of Plant Pathology, Ames, IA, USA; (3) University of Kentucky, Princeton, KY, USA; (4) University of Wisconsin, Madison, WI, USA; (5) Kansas State University, Manhattan, KS, USA; (6) Purdue University, West Lafayette, IN, USA; (7) Ohio State Univ, Ohio Agricultural Research and Development Center, Dept of Plant Pathology, Wooster, OH, USA; (8) Univ de Costa Rica, San Jose, COSTA RICA; (9) Purdue Univ, West Lafayette, IN, USA

The benefits of using foliar fungicides in hybrid field maize in the US Corn Belt continue to be debated among producers and researchers. We examined this question from the standpoint of the effects of single- and dual-mode-of-action fungicides and application timing on yield through a network metaanalysis of data from 119 field experiments. Mean yield differences (D) between each treatment and an untreated check were estimated as effect sizes and formed the basis for comparing yield responses among five fungicide treatments (T). The treatments were azoxystrobin (151 to $154 \mathrm{~g}$ a.i./ha) and propiconazole (122 g a.i./ha) applied either individually at R1 (T1 and T2, respectively) or as a premix (72 + $120 \mathrm{~g}$ a.i./ha) at R1 (T3), R2 (T4) or R3 (T5). The effects of baseline foliar disease severity as a categorical (low $[\leq 5 \%]$, high [ $>5 \%]$, or none [no data]) moderator variable on yield was also evaluated. D varied among treatments, with the R1 applications resulting in the higher yield increases than R2 and R3 applications. D was the highest for T1 $(279.3 \mathrm{~kg} / \mathrm{ha})$, followed by T3 $(263.5 \mathrm{~kg} / \mathrm{ha}), \mathrm{T} 2(243.69 \mathrm{~kg} / \mathrm{ha}), \mathrm{T} 4(171.16 \mathrm{~kg} / \mathrm{ha})$ and T5 $(45.33 \mathrm{~kg} / \mathrm{ha}) . \mathrm{T} 4 \mathrm{and}$ T5 did not result in statistically significant yield increases relative to the check $(P>0.05)$. Baseline foliar disease severity had a significant effect on yield response to T1, T2, and T3, but not T4 and T5, with D being the highest (399 to $405 \mathrm{~kg} / \mathrm{ha}$ ) in the high disease severity category for the T1 treatment.

\section{"I heard it through the grapevine": Assessing effects of information diffusion on disease recognition and response within the Florida pepper industry}

J. FULTON (1), C. Lapaire Harmon (2), S. Turner (1), K. A. Garrett (3), (1) University of Florida, Gainesville, FL, USA; (2) Univ of Florida PDC, Gainesville, FL, USA; (3) Plant Pathology Department, University of Florida, Gainesville, FL, USA

Detection of chronic and emerging pathogens is essential for a safe and dependable food supply and stakeholders also require a thorough understanding of disease risk to prepare for and respond to these challenges. For these reasons, the National Plant Diagnostic Network (NPDN) was established in 2002 to coordinate growers, local diagnosticians, and state regulatory officials in early recognition and response to plant pathogens. However, since the establishment of NPDN, there has been no explicit study of the structure of the network, nor process of information dissemination, so policymakers lack knowledge regarding the efficiency of information dissemination, the factors influencing communication amongst network partners, and characteristics of surveillance gaps. To begin an investigation of this network, an analysis of information dissemination throughout the Florida bell pepper industry has begun to characterize the network of individuals engaged in information exchange about diseases, identify surveillance gaps, and learn which factors influence network participation. Survey respondents were recruited from among growers, commercial agents, diagnosticians, and extension agents. A model of the system was generated and simulations of introduced pathogens were used to identify the system vulnerabilities to emerging pathogens in local production. Greater knowledge of the NPDN's function across regional and individual farm levels supports policies directed at improving food security.

\section{Impact of Long-Term Tillage and Fertility Treatments on Soil Microbial Communities}

A. Y. SROUR (1), J. Hackman (2), R. Cook (3), J. P. Bond (4), A. M. Fakhoury (4), (1) Southern Illinois Univ, Carbondale, IL, USA; (2) Southern Illinois University Carbondale, Carbondale, IL, USA; (3) North Carolina State University, Raleigh, NC, USA; (4) Southern Illinois University, Carbondale, IL, USA

Tillage practices and fertility treatments influence soil edaphic and biological properties. In particular, conventional tillage may result in loss of organic matter and in turn may reduce soil productivity. In this study, the effects of long-term conventional tillage and no-till practices in combination with three fertility treatments (No fertilization, N-only, and NPK) on soil microbial communities were investigated on a somewhat poorly drained soil near Belleville, IL. The field study was established almost 45 years ago to determine the effect of tillage and fertilizer management on corn and soybean yield, and soil abiotic factors. The soil microbial community structure was assessed using high-throughput sequencing on the MiSeq platform. Barcoded amplicon libraries targeting members of bacteria, fungi, fusaria and oomycetes were developed and analyzed at the taxonomy and trait levels. Results of principal components analysis based on soil microbial communities profiles were in agreement with yield data collected; NPK samples clustered separately from the control and N-only samples and yielded better than the latter two. Multivariate analysis revealed that the incidence of mycorrizhal fungi and Fusarium oxysporum were correlated with no-till, whereas that of Fusarium solani and several other fusaria increased in conventional tillage.

\section{Effect of Copper Nanomaterials on the Soil Microbiome}

A. L. STRAYER (1), J. C. Hong (2), Y. Y. Liao (1), M. Young (3), S. Santra (4), E. N. Rosskopf (2), S. Timilsina (5), G. E. Vallad (1,6), J. B. Jones (5), M. Paret (1,7), (1) University of Florida, Department of Plant Pathology, Gainesville, FL, USA; (2) USDA ARS, United States Horticultural Research Laboratory, Fort Pierce, FL, USA; (3) 3NanoScience Technology Center and Burnett School of Biomedical Science, UCF, Orlando, FL, USA; (4) University of Central Florida, Orlando, FL, USA; (5) Department of Plant Pathology, University of Florida, Gainesville, FL, USA; (6) Gulf Coast Research and Education Center, University of Florida, Wimauma, FL, USA; (7) North Florida Research and Education Center, University of Florida, Quincy, FL, USA

Due to high biocidal properties, copper-based compounds have been utilized to control plant pathogens for centuries. In a previous study, three novel copper nanomaterials (CS-Cu, MV-Cu, and FQ-Cu) were identified as potential alternatives to commercially available copper bactericides for the management of bacterial spot of tomato (Xanthomonas spp.). However, little is known about the potential impact of the copper nanomaterials on the environment. Thus, the objective of this study was to compare the ability of CS-Cu, MV-Cu, FQ-Cu, Kocide ${ }^{\circledR} 3000$, and copper-mancozeb to cause changes in bacterial population dynamics in the soil. Soil was collected from a tomato production field in Quincy, FL and divided into $10 \mathrm{~cm}$ diameter pots, and incubated at $28^{\circ} \mathrm{C}$ in a growth chamber. The soil microbiome was investigated by extracting bacterial DNA from soil samples taken at 1 and 7 days post-treatment, and using length-heterogeneity PCR. Non-metric multidimensional scaling analysis of all treatments revealed that the $200 \mu \mathrm{g} / \mathrm{ml}$ concentration of metallic copper from CS-Cu (CS200) at 1 day post-treatment resulted in the greatest shift in the soil microbiome in comparison to the non-treated control. Bacterial amplicon populations from the CS200 treated soil only share $70.7 \%$ average similarity with the non-treated control. Although the CS-Cu nanomaterial altered bacterial populations in the soil microbiome, the microbiome reverted to its original state at 7 days posttreatment. 
Validation of Models for Predicting the Daily Infection Risk of Cucurbit Host Types by Pseudoperonospora cubensis

K. N. NEUFELD (1), A. P. Keinath (2), P. Ojiambo (1), (1) North Carolina State University, Raleigh, NC, USA; (2) Coastal Research and Education Center, Clemson University, Charleston, SC, USA

Models have been developed for predicting the daily infection risk of cucumber by Pseudoperonospora cubensis based on day temperature (dT) and hours of relative humidity $>80 \%$ (RH80) over a 24 - and 48 -h period. These models were validated to determine their accuracy and utility in guiding management decisions for controlling cucurbit downy mildew in squash and cantaloupe. Field experiments were conducted in Clayton, North Carolina and Charleston, South Carolina, where squash and cantaloupe plants were exposed to prevailing weather conditions and downy mildew severity was rated 7 days after exposure. Predictor variables, dT and RH80, were calculated over each exposure period and used to predict the risk of infection, which was subsequently compared to observed data. The 24-h model was consistently more accurate than the 48-h model in predicting the infection risk for the two host types. Area under the receiver operating characteristic curve for the 24-h model ranged from 0.75 to 0.81 (maximum 1.0), with a correct classification rate of 0.69 to 0.74 across the two hosts. The 24 -h model correctly identified periods when infection occurred $81-84 \%$ of the time and periods without infection $58-67 \%$ of the time. Optimal decision thresholds developed based on estimates of economic damage and management costs showed that the benefit of using the 24-h model for making disease management decisions was greatest at low levels of disease.

\section{Emerging plant pathogens on ornamental crops in south Florida}

G. SANAHUJA SOlSONA (1), P. Lopez (2), S. N. Suarez (2), E. V. Campoverde (3), E. Evans (4), A. J. Palmateer (5), (1) Tropical REC, Homestead, FL, USA; (2) University of Florida, Homestead, FL, USA; (3) University of Florida Extension Miami-Dade, Homestead, FL, USA; (4) University of Florida - Tropical Research and Education Center, Homestead, FL, USA; (5) Bayer Environmental Science, Homestead, FL, USA

The state of Florida is geographically at risk for the introduction of new plant pathogens and insect pests due to it's location, subtropical climate, and agro tourism industry. Florida's hot and humid climate is highly favorable for newly introduced plant pathogens to become established and the movement of plant material through the ornamental industry creates an effective means for long distance dispersal. The University of Florida's Extension Plant Diagnostic Clinic has reported six new ornamental diseases in 2016. Accurate identification and characterization of new plant pathogen introductions and the diseases they cause is the first and most important step in successful and effective disease management. This presentation provides examples of several newly emerging diseases affecting plants in the Florida landscape and in commercial production. One fungal plant pathogen, Bipolaris oryzae first reported causing brown spot disease on rice leaves in the United States has recently been reported on two new ornamental hosts plants including a popular bromeliad hybrid, Aechmea tayoensis and a tropical foliage plant, Strelitzia nicolai. Notably, glyceraldehyde-3-phosphate dehydrogenase (GPD) sequences extracted from B. oryzae isolates causing disease on A. tayoensis and $S$. nicolai were a $99 \%$ match with an isolate of $B$. oryzae previously reported causing disease on Panicum virgatum in New York. This is an excellent example of the potential for fungal pathogens to infect new plant host species.

\section{Climatic Variables Related to the Development of Peanut Foliar Pathogens}

W. ELWAKIL (1,2), R. Barocco (1), N. S. Dufault (3), (1) University of Florida, Gainesville, FL, USA; (2) University of Florida, GAINESVILLE, FL, USA; (3) Univ of Florida, Gainesville, FL, USA

Peanut production in the southeastern U.S. is significantly impacted by the foliar diseases early leaf spot (ELS; Cercospora arachidicola), late leaf spot (LLS; Cercosporidium personatum), and rust (Puccinia arachidis). Previous research shows that climatic factors, especially rainfall, can impact foliar disease development. The objective of this study was to assess effects of rainfall and temperature on peanut foliar disease development in Florida. Field plots of the cultivar Georgia-06G were planted in June 2014, 2015, and 2016. Florida 1-10 scale was used for in-field ratings, and a 12-leaflet sample per plot was collected on a biweekly schedule 55 to 112 days after planting. Weather data was obtained from a station located $\sim 0.8 \mathrm{~km}$ from the plots. ELS was dominant ( $42 \%$ peak incidence) in 2015 when average total daily rainfall $(0.56 \mathrm{~cm})$ was the highest; however, disease incidence was more evenly distributed in 2014 and 2016 with rainfall values of 0.35 and $0.30 \mathrm{~cm}$, respectively. AUDPC values based on the Florida 1-10 scale were positively correlated $(\mathrm{r}=0.96, \mathrm{p}=<0.01)$ with total seasonal rainfall and were correlated $(\mathrm{r}=0.77, \mathrm{p}=<0.01)$ with average temperature (only $0.64^{\circ} \mathrm{C}$ difference between minimum and maximum). This data shows the importance of rainfall in the development of foliar peanut diseases; however, further research is currently being conducted to examine these trends across more locations in Florida and in relation to biweekly weather variables.

\section{Detection of virulent races from international populations of Puccinia graminis $\mathrm{f}$. sp. tritici}

P. D. OLIVERA FIRPO (1), L. J. Szabo (2), D. Luster (3), Y. Jin (4), (1) University of Minnesota, St. Paul, MN, USA; (2) USDA ARS, St Paul, MN, USA; (3) USDA ARS FDWSRU, Fort Detrick, MD, USA; (4) USDA-ARS Cereal Disease Laboratory, Saint Paul, MN, USA

Wheat stem rust, caused by Puccinia graminis f. sp. tritici (Pgt) is a re-emerging disease, posing a new threat to wheat production worldwide exemplified by epidemics caused by races in the Ug99 race group in Eastern Africa. In response to this new threat, a global pathogen surveillance system was established under the Borlaug Global Rust Initiative (BGRI) to detect and monitor Ug99 and other races virulent to commercially important resistance genes. The USDA-ARS Cereal Disease Laboratory, in collaboration with multiple international partners, has played a critical role in the discovery and characterization of stem rust races with significant virulence combinations. During the past five years, we analyzed over $1,000 P g t$ samples from countries in East Africa, Asia, Europe, and South America. Phenotypic and genotypic analyses enabled us to identify new variants with virulence to previously effective resistance genes and monitor the spread of the Ug99 race group. We characterized the non-Ug99 races responsible for local epidemics in Ethiopia and Germany, and identified hotspots of phenotypic and genotypic diversity from Pgt populations in Georgia and Kazakhstan. Relevant races are selected for screening of US and international germplasm to identify resistant wheat breeding lines.

\section{Meta-Analysis of a Web-Based Disease Forecast System for Control of Anthracnose and Botrytis Fruit Rots of Strawberry in Southeastern United States}

L. CORDOVA (1), L. V. Madden (2), A. Amiri (3), G. Schnabel (4), N. Peres (5), (1) University of Florida, Wimauma, FL, USA; (2) Ohio State Univ, Ohio Agricultural Research and Development Center, Dept of Plant Pathology, Wooster, OH, USA; (3) Washington State University, Wenatchee, WA, USA; (4) Clemson University, Clemson, SC, USA; (5) Gulf Coast Research and Education Center; University of Florida, Wimauma, FL, USA

Anthracnose fruit rot (AFR), caused by Colletotrichum acutatum, and Botrytis fruit rot (BFR), caused by Botrytis cinerea, are major strawberry diseases in Southeastern US. Its effective management traditionally relied on weekly fungicide sprays. However, many growers have been using the Strawberry Advisory System (StAS) to improve timing and reduce the number of fungicide applications. StAS is a decision support system for forecasting AFR and BFR outbreaks based on environmental conditions. Thirty-nine trials were conducted from 2009 to 2014 in Florida and South Carolina strawberry commercial fields to determine the effectiveness of the StAS compared to a calendar-based spray program for AFR and BFR management. Meta-analysis was performed to quantify the treatment effects on four effect sizes, all based on the difference in response variables for both treatments in each trial. The mean difference in BFR and AFR incidence, yield, and number of marketable fruit between the StAS and the calendar-based treatment was not significantly different from $0(P<0.05)$. However, the use of the StAS reduced fungicide application per season in $50 \%$ compared to the calendarbased treatment. Effect sizes were not influenced by location or environment favorability for disease development. These findings indicate that the use of the StAS in commercial fields is effective in controlling fruit rot diseases, with no reduction in yield, while substantially reducing fungicide applications. 
Endophytic fungi associated with Populus roots: The Ilyonectria complex

A. ROJAS (1), H. L. Liao (2,3), G. Bonito (4), K. M. Hameed (1), P. E. Busby (1,5), I. Grigoriev (6), C. Schadt (7), J. Labbe (7), G. Tuskan (7), R. J. Vilgalys (1), (1) Duke University, Durham, NC, USA; (2) University of Florida, quincy, FL, USA; (3) University of Florida, Quincy, FL, USA; (4) Michigan State University, East Lansing, MI, USA; (5) Oregon State University, Corvallis, OR, USA; (6) US DOE Joint Genome Institute, Walnut Creek, CA, USA; (7) Oak Ridge National Laboratory, Oak Ridge, TN, USA

Populus is emerging as an important model tree genetic system for studies aimed at understanding forest ecosystems and the interactions of these with the associated microbiota. Populus species are known to establish associations with arbuscular and ectomycorrhizal fungi which vary depending on the host species. Recent studies have unlocked a great diversity of fungal endophytes in Populus, associated with different plant tissues. The ecological and physiological roles of fungal endophytes in the Populus-microbe interaction have not been fully characterized. We describe a novel approach using multilocus sequence typing (MLST) approach using PacBio and the downstream analysis to genotype individuals in single sequencing run, providing enough loci to characterize a species complex. This approach was applied to Ilyonectria complex, an endophyte present in cottonwood (Populus trichocarpa and Populus deltoides), but also associated with black-foot disease on grapevines. The methodology could be applied to study the phylogenetics and systematics of other fungal groups. In addition, genomes of Populus root-associated fungal endophytes are being sequenced. The data extracted from these genomes provides insight into the lifestyle strategies of various endophyte species, and provides a framework for metatranscriptomic approaches to understand the role of endophytic fungi.

Development and deployment of a strawberry disease advisory system in North Carolina

F. LOUWS (1), R. Mahfuz (2), G. Buol (3), G. Wilkerson (3), R. Boyles (4), (1) North Carolina State University, Raleigh, NC, USA; (2) West Virginia University, Morgantown, WV, USA; (3) NC State University, Raleigh, NC, USA; (4) SE Climate Science Center, Raleigh, NC, USA

Botryis cinerea ( $B c$; Botrytis fruit rot) and Colletotrichum acutatum ( $\mathrm{Ca}$; anthracnose fruit rot [AFR]) are the most important fruit rot pathogens limiting strawberry production in $\mathrm{NC}$ and surrounding states. $B c$ occurs annually during cooler wet conditions. $C a$ inoculum arrives with transplants, occurs sporadically and can reach epidemic levels in warm wet weather resulting in 100\% fruit rot. Multiple IPM practices are employed to minimize losses but the majority of growers rely on frequent fungicide applications during bloom and harvest. However, when weather conditions are not conducive to disease, applications may not be needed, and reduced use may limit lost efficacy due to emerging pathogen resistance to the most efficacious products. Field trials demonstrated the viability of a weather-based advisory system in NC. For example, three advisory-based sprays suppressed AFR as well as 68 calendar sprays in a two year study. The advisory system was integrated into the ipm.ncsu.edu portal and uses real-time leaf wetness and temperature data from 33 sites across the state. Growers choose a location most representative of their site. Risk indices are provided for the past 10 days and forecast the next 5-7 days. Risk levels guide management decisions and are linked to a Strawberry IPM guide, updated annually by regional plant pathologists, and available at smallfruits.org. Feedback suggests the system is useful for grower decisions to optimize fungicide use and efficacy.

Disease dynamics of Verticillium wilt fungi in the biocontrol of Ailanthus altissima

K. WICKERT (1), M. T. Kasson (2), (1) West Virginia University, Morgantown, WV, USA; (2) West Virginia University, Division of Plant and Soil Sciences, Morgantown, WV, USA

Ailanthus altissima is a highly invasive exotic tree species. In 2002, a vascular wilt was discovered in Ailanthus stands in Pennsylvania. Isolation from symptomatic trees revealed the causal agent as Verticillium nonalfalfae. Since its discovery, studies have investigated its potential as a biocontrol focusing on efficacy, host specificity and disease transmission. As efforts to commercialize $V$. nonalfalfae intensify, several key questions need to be addressed: (1) does the naturally co-occurring and less virulent Verticillium dahliae, which also infects Ailanthus, alter Verticillium wilt progression; (2) how do environmental conditions associated with hardiness zones influence the individual contributions of each of the two Verticillium spp.; and (3) does continued disease pressure in long-established disease epicenters select for tolerant / resistant Ailanthus genotypes that correlate with morphological differences? Preliminary data from stands with co-occurring Verticillium spp. may indicate that synergistic interactions are facilitating enhanced disease progression. With regard to putative tolerance / resistance, re-visitation to sites inoculated with V. nonalfalfae from 2006-2008, where the fungus continues to infect trees, revealed established asymptomatic intermediate size saplings. Re-inoculation, symptom development and death of these residual saplings would indicate these stems merely escaped the initial infection and, as such, are not more tolerant or resistant to the disease. Conversely, failure of disease development would support tolerance and invite genotypic and morphological (vessel size and tyloses formation) comparisons to help explain differences in susceptibility.

Hok/Sok toxin-antitoxin system in Erwinia amylovora: "To be, or not to be" J. PENG, G. W. Sundin, Michigan State University, East Lansing, MI, USA

Fire blight is a devastating disease of apple and pear trees caused by the bacterial pathogen Erwinia amylovora. E. amylovora can grow rapidly on flowers, spread systemically, and cause severe yield losses. Current disease management of fire blight has been challenged by antibiotic resistance in some regions. The toxin-antitoxin (TA) system has recently shown potential as an antimicrobial target in bacteria. In Escherichia coli, plasmid R1 encodes a Hok/Sok TA system. Hok is a "poison" protein and Sok is a small RNA that acts as the corresponding "antidote". A TBLASTN search identified a previously unknown hok homolog on the chromosome of $E$. amylovora ATCC 49946 with $87 \%$ amino acid identity to hok from R1. The antitoxin small RNA Sok, was identified through BLASTN search and verified using a 5' RACE assay. Over-expression of hok in vitro caused distinct killing of E. amylovora presumably because of irreversible damage to the cell membrane. This phenotype was partially reversed by co-overexpression of sok. Deletion of hok-sok did not have any significant effects on virulence factors, i.e. exopolysaccharide amylovoran or motility, and the mutant strain maintained full virulence on immature pear. This is the first report of a TA system in E. amylovora. Hok/Sok is hypothesized to be involved in stress responses, e.g. heat stress and antibiotic stress, and further effects of this system on bacterial physiology and host-pathogen interactions are under investigation.

Radiant energy thresholds and spectral distribution of light as regulatory factors in sporulation of Erysiphe necator

T. MCCANN (1), M. D. Carter (1), D. M. Gadoury (1), M. Rea (2), (1) Cornell University, Geneva, NY, USA; (2) Lighting Research Center, Rensselaer Polytechnic Institute, Troy, NY, USA

The obligate biotroph Erysiphe necator causes powdery mildew of grapevine. Many powdery mildew species produce conidia at a near-constant rate once colonies reach an age of sporulation competency. However, E. necator produces a single conidium per conidiophore per $24 \mathrm{hr}$ cycle, requires light to initiate the process, and thus provides an ideal model to study regulatory effects of light on sporulation. Leaf disks inoculated with $E$. necator were grown to an age of sporulation competency and exposed to (i) midday sunlight; (ii) red (peak $\sim 660 \mathrm{~nm}$, Full Width Half Maximum=19 nm, average irradiance $=62.25 \mathrm{~W} / \mathrm{m}^{2}$ ); (iii) blue (peak $\sim 447.5 \mathrm{~nm}, \mathrm{FWHM}=21 \mathrm{~nm}$, average irradiance $=38.65 \mathrm{~W} / \mathrm{m}^{2}$ ); and (iv) mixed blue and red (average irradiance $=50.30 \mathrm{~W} / \mathrm{m}^{2}$ ). Production of conidia was assessed microscopically, and maturity of conidia was confirmed by micromanipulation to physically detach conidia from conidiophores. Sporulation was initiated following as little as $30 \mathrm{sec}$ of midday sun exposure, and after 60 seconds of blue, or mixed red+blue. Red light treatments required significantly longer exposure time to initiate sporulation. Differential responses to specific wavelengths implies an operational system of photoreceptors, it may explain diurnal patterns of airborne spore dose, allows investigation of circadian rhythms, and opens opportunities to manipulate and possibly disrupt sporulation using solid state lighting to suppress disease. 
Identification of bacterial leaf spot disease caused by Xanthomonas campestris pv. vitians on lettuce in Taiwan W. L. DENG (1,2), Y. C. Huang (2), J. Y. Tzeng (2), (1) Agricultural Biotechnology Center, Taichung, TAIWAN; (2) National Chung Hsing University, Taichung, TAIWAN

In 2011-2012, a bacterial leaf spot disease was observed on lettuce plants in the nurseries of Yulin County, Taiwan. Characteristic symptoms were water-soaked circular, dark brown spots at the margin of the leaves. On heavily infected leaves, individual black lesions may expand and coalesce into larger lesions and eventual leaf blight. Eleven bacterial strains were isolated from infected lettuce leaves from the two nurseries, and five of them were identified as Xanthomonas campestris pv. vitians (Xcv) based on morphological, physiological, biochemical, and pathogenicity tests, as well as the analyses of fatty acid methyl ester (FAME) and nucleotide sequences of $16 \mathrm{~S} r R N A$ gene, internal transcribed spacer sequence (ITS), and gyr $B$. Four commercial lettuce cultivars (Lactuca sativa L. cv. L143, cv. General, cv. Green Romaine, and cv. Grand Rapid) purchased from Known-You Seed Co. (Taiwan) were evaluated and shown to be susceptible to Xcv; additionally, the bacteria can elicit necrotic spots on tomato and pepper by spray inoculation, demonstrating that the Xcv strains can also infect tomato and pepper plants. Xcv can be transmitted via seeds, use of certified disease-free seeds and transplants and other cultural practices are the primary means of controlling the BLS disease.

Feeling the heat: Towards predicting pitch canker outbreaks in the context of climate change T. QUESADA (1), J. Hughes (1), K. Smith (2), P. James (1), K. Shin (1), C. Staub (1), M. Marsik (1), J. A. Smith (1), (1) University of Florida, Gainesville, FL, USA; (2) USDA Forest Service, Gainesville, FL, USA

The pitch canker pathogen, Fusarium circinatum, thrives under high temperatures and humidity. It affects most pine species and may cause episodic outbreaks that result in high economic losses to the timber industry. As climate conditions shift towards warmer temperatures, pitch canker disease may become an important problem in the future. Selection of resistant pine genotypes would help overcome potential outbreaks, but requires many years to achieve. To anticipate which strains may be more likely to cause future outbreaks, F. circinatum isolates were obtained from 15 samples from Florida and Georgia, and growth, sporulation, and spore germination was recorded at 25,28 , and $31^{\circ} \mathrm{C}$. In addition, pathogenicity tests were conducted in which significant variation was observed. The four most pathogenic isolates were donated to the USDA Resistance Screening Center in Asheville, to be used in their screening tests, as the strains routinely used in their tests are less virulent than these new strains. A survey of spore production throughout the growing season was also conducted at three Florida locations, where spore traps were placed and weather data were recorded. Spore production was determined by quantitative PCR using $F$. circinatum-specific primers, averaging between 18 and $114 \mathrm{pg}$ among locations. The integration of weather data with F. circinatum will allow development of epidemiological models to predict pitch canker outbreaks under future conditions.

Occurrence of pepper stem black spot disease caused by Pectobacterium carotovorum subsp. brasiliense in Guangdong of China X. SHE, Z. He, Y. Tang, L. Yu, Plant Protection Research Institute, Guangdong Academy of Agricultural Sciences, Guangzhou, CHINA

Pepper (Capsicum spp.) is a major vegetable crop in China. In 2015, a bacterial disease of pepper, exhibiting black spot on stem symptom, was found in Guangdong Province, China. The diseased incidence reached more than $75 \%$ in the field. 15 diseased plants with typical symptoms were collected from the field. Total 33 bacterial isolates were isolated from the stem tissue of the diseased plants on nutrient agar medium. Four isolates were randomly selected for pathogenicity assays. Pepper plants at the six- to eight-leaf stage were inoculated by sprinkling their stems with bacterial suspension $\left(3 \times 10^{8}\right.$ $\mathrm{CFU} / \mathrm{ml}$ ), respectively. After 3 days post inoculation (dpi), the inoculated pepper plants exhibited the same symptoms as the diseased pepper plants in the field. Using the universal bacterial $16 \mathrm{~S}$ rDNA primer set 27f/1541R, approximately 1,400 bp-fragments were amplified from the four isolates respectively. The sequences of the 4 fragments were identical, and had $99 \% \sim 100 \%$ sequence identity with $16 \mathrm{~S}$ rDNA of Pectobacterium carotovorum subsp. brasiliense 1033 (GenBank accession JF926720). Furthermore, using the specific primers of Pectobacterium carotovorum subsp. brasiliense, the 322-bp specific fragments can be amplified from the all of four isolates, respectively. It is concluded that pepper stem black spot disease in Guangdong, China was caused by Pectobacterium carotovorum subsp. brasiliense.

Three complete genome sequences of novel Xanthomonas citri strains from Texas carry atypical PthA alleles and unusual large plasmids A. MUNOZ BODNAR (1), G. Santillana (2), V. A. Mavrodieva (3), Z. Liu (4), M. K. Nakhla (3), D. W. Gabriel (1), (1) University of Florida, Gainesville, FL, USA; (2) APHIS, Beltsville, MD, USA; (3) USDA-APHIS-PPQ-S\&T-CPHST, Beltsville, MD, USA; (4) USDA APHIS PPQ, Riverdale, MD, USA

In 2015, backyard lime (Citrus spp.) trees in Texas showing typical citrus canker symptoms were confirmed positive for Xanthomonas citri subsp. citri. This confirmation triggered a regulatory response since the disease has been absent from the state since 1940s. The complete genomes of three strains from Texas were determined by PacBio Single Molecule, Real-Time (SMRT) sequencing. The genomes were 5,330,822 bp, 5,341,733 bp and 5,337,252 bp in size with an average read length of 15,756 bp, 15,261 bp and 16,590 bp, respectively. Bioinformatic analyses indicated that the new strains were related to the Florida $\mathrm{A}^{\mathrm{w}}$ strain. However, all three strains carried nearly identical and unusual large plasmids $(123557 \mathrm{bp}, 123557 \mathrm{bp}$, and $129139 \mathrm{bp}$, respectively), that matched contigs in the NCBI Whole Genome Shotgun (WGS) database from some X. citri subsp citri, X. fuscans pv. fuscans and $X$. axonopodis pv. manihotis strains. These plasmids contain a region of ca. $39 \mathrm{kB}$ similar to Type 4 conjugational transfer genes, indicating recent horizontal transfer into $X$. citri. All three Texas strains carried predicted functional pthA homologs with 18.5 tandem repeats each instead of the typical 17.5 repeats and a second homolog predicted to be nonfunctional. Atypically for citrus canker causing strains, two of the Texas strains carried both $p t h A$ homologs on the chromosome. Specific primers to distinguish the Texas strains from other canker-causing strains were designed and evaluated.

First report of phytoplasmas subgroups 16SrIII-B, 16SrIII-J and 16SrVII-B in Brassica rapa, a weed specie present in brassica crops in Brasil T. BANZATO (1), I. Bedendo (2), (1) Universidade de São Paulo, Piracicaba, BRAZIL; (2) ESALQ-USP, Piracicaba, BRAZIL

In Brazil, were observed in brassica production fields, plants of mustard-field (Brassica rapa) showing intense shoots proliferation, besides small sized leaves and flowers. Since these symptoms were similar to those induced by phytoplasmas, an investigation was performed aiming to confirm this suspicion. Twenty symptomatic plants were collected in commercial field. Total DNA was extracted and used in nested PCR assays conducted with universals primers R16mF2/mR1, SN910601/SN011119 and F16F2n/R2. The amplification of 1,2kb genomic fragments from the $16 \mathrm{Sr}$ RNA in $63 \%$ symptomatic samples, showed a constant association between presence of phytoplasmas and diseased plants. No amplifications was obtained for asymptomatics plants. Products generated by nested PCR with primers F16F2n/R2 were cloned and sequenced. The sequences were analysed using iPhyClassifier, an online phytoplasma classification tool, which revealed that phytoplasma present in affected plants belonged to subgroups 16SrIII-B, 16SrIII-J and 16SrVII-B. Based on the results, mustard-field plants was evidenced as a weed species that harbor distinct phytoplasmas, which were previously described in cabbage, broccoli and cauliflower cultivated in the same geographical area. Thus, these findings reveal mustard-field as a new host for the three strains and suggest this weed as a reservoir, ensuring the phytoplasmas survival, as well as a source of inoculum of pathogens for commercial vegetable crops.

Differences in Fusarium species in brown midrib sorghum and in air populations in production fields D. L. FUNNELL-HARRIS (1), E. Scully (2), S. Sattler (1), P. O’Neill (1), (1) USDA-ARS, Lincoln, NE, USA; (2) USDA-ARS, Manhattan, KS, USA

Several Fusarium species cause sorghum [Sorghum bicolor (L.) Moench] grain mold, resulting in deterioration and mycotoxin production in the field and during storage. Fungal isolates from air (2005-2006), and from leaves and grain from wild-type and brown midrib (bmr)-6 and 12 sorghum plants (2002-2003) at two locations were collected. Plants containing $b m r$ have reduced lignin content, altered cell wall composition and different levels of 
phenolic intermediates, compared with wild-type. Multilocus maximum likelihood analysis identified two Fusarium thapsinum operational taxonomic units (OTUs). One OTU was identified at greater frequency in grain and leaves of $b m r$ and wild-type plants, but was infrequently detected in air. Nine Fusarium graminearum/Gibberella zeae species complex OTUs were identified: one was detected at low levels in grain and leaves while the rest were only detected in air. Wright's $F$-statistic $\left(F_{S T}\right)$ indicated that fungal air populations differentiated between locations during crop anthesis, but did not differ during vegetative growth, grain development and maturity. $F_{S T}$ also indicated that the Fusarium population from wild-type grain was differentiated from those in either bmr6 or bmr12 grain at the first location but at the second location, populations from wild-type and bmr6 grain were more similar. Thus, impairing monolignol biosynthesis in $b m r$ plants substantially effected Fusarium populations but the environment also had a strong influence.

\section{Survey of the natural infection by Erwinia amylovora in Korea}

C. S. OH (1), D. H. Park (2), (1) Kyung Hee Univ, Yongin, KOREA; (2) Kangwon National University, Chuncheon, KOREA

Apple and pear trees are apparently main host to fire blight disease, causing by Erwinia amylovora on the basis of economic loss. Beside these two important hosts, 129 species in 37 genera of the family Rosaceae have been reported as host and also there are possibilities that other plants may be susceptible to this disease. Since occurrence of fire blight had been reported in 2015 in Korea, there is no performance to determine whether rosaceous plants are host around orchard of apple and pear in Korea. Thus, we have collected 149 samples of 23 species in 14 genera from May to September, 2016. The sampling sites were involved $100 \mathrm{~m}$ regions far from orchards of Anseong, Chonan, and Jecheon, which occurred areas at last year. The pathogen is detected based on colony-PCR with primer sets (AMSbL/AMSbR) and AgriStrip (Bioreba, Switzerland). At results, pathogen has been detected from Chinese quince (Chaenomeles sinensis (Thouin) Koehne) by either colony-PCR and AgriStrip so that Chinese quince is apparent host for E. amylovora in Korea. Interestingly, isolate from David Peach (Prunus davidiana Franch.) is showed positive reaction via AgriStrip but we could not developed amplificon by colony-PCR with several time tries. All others samples except Chinese quince and David Peach, however, did not produce any positive sign by either methods. Therefore, we can concluded that Chinese quince is host tested samples in this study for E. amylovora in Korea and suggest that eradication program of fire blight must be consider rogueing of Chinese quince.

\section{Population dynamics of Phytophthora rubi indicate high rates of migration between states and nurseries in the Pacific Northwestern United} States

J. TABIMA (1), I. A. Zasada (2), N. Grunwald (2), (1) Oregon State University, Corvallis, OR, USA; (2) USDA ARS, Corvallis, OR, USA

The Pacific Western US is the main producer of red raspberry (Rubus idaeus) in the world. The most common plant pathogen causing disease in raspberry fields in the PNW is Phytophthora rubi, an oomycete plant pathogen known to cause red root disease in red raspberry. $P$. rubi is found in $90 \%$ of raspberry crops in the state of Washington, resulting in millions of dollars in losses to the berry industry of the United States. A recent study showed an absence of population structure in $P$. rubi across the PNW, indicating that constant migration might be taking place. To study the population dynamics of $P$. rubi, we used genotyping-by-sequencing (GBS) to characterize diversity and study population structure and migration between West coast states of the US. To determine interconnectivity and migration between states we evaluated several demographic scenarios using DIYABC. Our results show that P. rubi forms a single population in the PNW, and has high rates of migration between geographic locations. Analysis of directed migration show that nurseries in the state of Washington are sinks of disease from nurseries in California and Oregon, most likely due to the transport of contaminated soil and plant material. These findings pose a concern for the nursery industry, indicating that the pathogen might be moving into regions with high red raspberry production.

Characterizing Optimum Conditions for the Lolium pathotype of Magnaporthe oryzae to Infect and Cause Wheat Blast K. B. MILLS (1), P. A. Paul (2), L. V. Madden (2), (1) Ohio State University, Wooster, OH, USA; (2) Ohio State Univ, Ohio Agricultural Research and Development Center, Dept of Plant Pathology, Wooster, OH, USA

Wheat blast is an emerging disease in South America and Asia caused by the Triticum pathotype of Magnaporthe oryzae (MoT). Although MoT has not yet been reported in North America, the closely related M. oryzae Lolium pathotype (MoL) is commonly found in wheat growing regions and could pose a threat under certain environmental conditions. Multifactorial experiments were conducted under controlled conditions to evaluate the effects of different combinations of temperature $(20,25$, and $30 \mathrm{C}), 100 \%$ relative humidity $(\mathrm{RH})$ duration $(0,3,6,12,24$, and $48 \mathrm{~h})$, inoculum concentration $(2 \mathrm{e} 2$, $2 \mathrm{e} 3,2 \mathrm{e} 4$, and $2 \mathrm{e} 5$ spores $/ \mathrm{ml}$ ), and growth stage (Feekes 10.1-3, 10.5, 10.5.1, and 10.5.4) on MoL infection and blast development. MoL infected and blast developed under all conditions evaluated, but results from linear mixed model regression analyses showed that response to inoculum density was significantly greater $(P<0.05)$ when infections occurred at Feekes 10.5.1 (anthesis) or 10.5.4 than between Feekes 10.1 and 10.5. When infected at anthesis, there was a significant positive linear relationship between arcsine square root blast severity (arcSEV) and high RH duration, but slopes for this relationship varied among temperatures. The rate of increase in arcSEV per hour increase in $100 \% \mathrm{RH}$ was significantly greater at 25 and $30^{\circ} \mathrm{C}$ than at $20^{\circ} \mathrm{C}$. Similar trends were observed for blast incidence. These results are invaluable for ongoing efforts to assess the risk posted by MoL to US wheat.

\section{Role of spring cankers in bacterial spot epidemics of peach in North Carolina} D. F. RITCHIE, NC State University, Raleigh, NC, USA

Xanthomonas arboricola pv. pruni (Xap) causes bacterial spot of Prunus spp. infecting fruit, leaves and twigs. Following the description of the disease in the early 1900s, research focused on understanding the epidemiology, particularly the role of spring cankers (sgck) as a source of primary inoculum. Significance of the role of spring cankers for overwintering inoculum still remains somewhat uncertain. In North Carolina, occurrence, frequency, and association of spring cankers with epidemics over multiple years, having a range of disease severity, was analyzed using linear regression. Spring cankers develop mid-winter to early spring as blackish bark areas on the previous year's twig growth associated with the terminal and lateral buds. Cankers were observed as early as January on highly to moderately susceptible peach cultivars most commonly on twigs in the tree canopy center. Isolations from spck consistently yielded abundant, pure cultures of Xap bacteria until about 6-8 weeks after bloom when the cankers were colonized by other microbes. During a 6-yr period, average number of sgck on pruned trees varied from 2.03-5.81 per tree (214 trees). The season's first leaf lesions were associated with sgck, but the subsequent leaf and fruit disease severity did not always correlate with frequency of sgck. Autumn copper sprays compared to late winter/spring copper sprays reduced the frequency of sgck but did not always translate to less disease during the season.

\section{Identification and Characterization of Pantoea ananatis as a Cause of Etiolation on Perennial Ryegrass} G. Z. Achenef, J. A. ROBERTS, University of Maryland, College Park, MD, USA

Bacterial etiolation and subsequent decline impacts multiple cool-season turfgrasses. In 2016, an outbreak of etiolation occurred on a golf course fairway comprised of perennial ryegrass (Lolium perenne L.) and annual bluegrass (Poa annua L.). Symptomatic tissue was surface sterilized in $0.6 \% \mathrm{NaOCl}$, cut with a sterile scalpel and examined for bacterial streaming at 400X magnification. Suspected bacteria were plated onto nutrient agar $+1 \%$ sucrose using an Autoplate Spiral Plater. Sequencing of the $16 \mathrm{~S}$ subunit in prominent colonies identified the causal agent as Pantoea ananatis. Etiolation screening was conducted on perennial ryegrass seeds $\left(20-30\right.$ pot $\left.^{-1}\right)$, planted in media $(85 \%$ sand $/ 15 \%$ peat). Plants were irrigated daily and fertilized every $14 \mathrm{~d}$ using $1 \frac{1}{2}$ Strength Hoagland's. Inoculations ( $P$. ananatis-1, P. ananatis-2, X. translucens, or none) were arranged in a randomized complete block design with 4 replications. Treatments were applied 2 wks post germination by cutting turf with sterile scissors dipped into a suspension $\left(10^{9} \mathrm{CFUs}\right.$ $\mathrm{ml}^{-1}$ ) of each bacterial treatment. Turf was evaluated every 5-7 days for etiolation and turf quality. Both $P$. ananatis strains resulted in greater etiolation compared to non-inoculated, yet both had less etiolation than the $X$. translucens positive control. Inoculations with $P$. ananatis-2 had greater turfgrass density compared to other treatments. Results illustrate that additional bacterial species can infect perennial ryegrass. 
Simultaneous fine mapping and locus characterization of the Andean $\boldsymbol{U} \boldsymbol{r}-4$ and Mesoamerican $\boldsymbol{U} \boldsymbol{r}$ - 5 rust resistance genes in Common bean O. P. HURTADO-GONZALES (1), G. Valentini (2), T. Gilio (2), Q. Song (1), M. Pastor-Corrales (1), (1) Soybean Genomics \& Improvement Lab, BARC-West, ARS-USDA, Beltsville, MD, USA; (2) Universidade Estadual de Maringá, Maringa, PR, BRAZIL

Bean rust, caused by the hyper variable Uromyces appendiculatus fungus, is a destructive disease of common bean in the Americas and Africa. Effective management of bean rust is accomplished by pyramiding complementary disease resistance genes into single cultivars. Combining $U r-4$ with $U r-5$ confers broad-spectrum resistance to $95 \%$ of the races of $U$. appendiculatus maintained in ARS-Beltsville. However, pyramiding these genes is difficult due to the epistatic interaction between $U r-4$ and $U r-5$. Conversely, co-dominant molecular markers facilitate the combination of these genes. We report here the simultaneous fine mapping of the $U r-4$ and $U r-5$ genes to obtain tightly-linked and highly accurate markers for marker-assisted selection of both loci. A total of $393 \mathrm{~F}_{2}$ plants from the Early Gallatin $(U r-4) \times$ Mexico $309(U r-5)$ cross was phenotyped with various races of $U$. appendiculatus. Bulk segregant analysis combined with SNP genotyping using the BARCBEAN6K 3 SNP chip with 5,398 SNPs, identified the genomic region containing the $U r-4$ and $U r-5$ genes in chromosome Pv06 and Pv04 of the common bean, respectively. Targeted SSR and KASP markers were developed to accurately map the location of both genes using recombinant $\mathrm{F}_{2: 3}$ families. Our analysis established that while $U r-4$ was located in a region of high recombination on the long arm of Pv06, Ur-5 was positioned in a cold-spot for recombination on the short arm of Pv04. Markers tightly linked to these genes useful for marker-assisted selection are being developed and validated. Moreover, candidate disease resistance genes were also identified in the small genomic region containing both loci.

Soybean resistance locus $R h g 1$ can confer resistance in diverse plant families to multiple cyst nematode species K. J. BUTLER (1), J. Smith (1), S. Chen (2), X. Wang (3), A. F. Bent (1), (1) University of Wisconsin-Madison, Department of Plant Pathology, Madison, WI, USA; (2) Cornell University, School of Integrative Plant Science, Ithaca, NY, USA; (3) USDA-ARS, Robert W. Holley Center for Agriculture and Health, Ithaca, NY, USA

Cyst nematodes consistently threaten agricultural production, causing billions of dollars of loss globally. The Rhgl resistance locus of soybean is the most popular resistance source used against soybean cyst nematode. We hypothesized that this resistance locus may be able to function in different plant families, conferring resistance to their respective cyst nematode parasites. Transgenic Arabidopsis and potato plants were generated that express the three soybean genes of the complex Rhgl locus. These plants exhibited elevated resistance to Heterodera schachtii and Globodera spp., respectively. The transgenic plants were subjected to additional molecular and phenotypic characterization. While root length was slightly reduced in Arabidopsis lines, equal numbers of nematodes can penetrate the roots, though not all develop. Additionally, native alpha-SNAP proteins were shown to be able to compensate for toxic Rhgl alpha-SNAP proteins. PR-1-associated defenses were not constitutively elevated in the transgenic Arabidopsis. The efficacy of Rhgl in Brassicaceae and Solanaceae plants demonstrates that this locus can function across plant families and suggests biotechnological approaches for managing cyst nematodes in systems where natural resistance is rare or ineffective. Furthermore, Rhgl apparently evolved to interfere with one or more components of nematode virulence that are conserved across the cyst nematode Heteroderinae subfamily.

Phytoplasma survey in Pennsylvania

E. V. NIKOLAEVA (1), K. A. Peter (2), J. Lesperance (3), T. Jones (4), S. Costanzo (5), R. E. Davis (6), (1) Pennsylvania Department of Agriculture, Harrisburg, PA, USA; (2) Penn State University, Biglerville, PA, USA; (3) PA Dep of Agriculture, Harrisburg, PA, USA; (4) PSU, Harrisburg, PA, USA; (5) USDA-APHIS-PPQ-S\&T-CPHST, Beltsville, MD, USA; (6) USDA ARS Molecular Plant Pathology Lab, Beltsville, MD, USA

In 2013-2016, the PA Department of Agriculture conducted an exotic phytoplasmas survey with support of Farm Bill funds. Phytoplasma identification was made based on sequencing of $16 \mathrm{~S}$ rDNA and using $i$ PhyClassifier for group-subgroup and $\mathrm{Ca}$. Phytoplasma species assignments. None of the USDA regulated phytoplasma species has been found in PA, however several known phytoplasma species have been detected. Ca. Phytoplasma pruni (16SrIIIA group, X-Disease subgroup) has been found in PA apple, peach and grape samples. Four out of 274 tested apple trees have been found infected with Apple X-Disease. Positive trees exhibited abnormally small fruits, leaf curl and premature reddening. Sixteen out of 191 tested peach samples were found positive for X-Disease and expressed symptoms of shot-holed leaves and chlorosis. X-Disease phytoplasma has been detected in one grape sample with symptoms of veinal chlorosis and rolling of leaves. Pear Decline Phytoplasma ( $\mathrm{Ca}$. Phytoplasma pyri, $16 \mathrm{SrX}$ group) has been detected from three out of 12 pear trees. Five peach trees were found to be infected with unknown Ash Yellows-like phytoplasma. Positive peach trees exhibited more severe damage than X-Disease infected ones with symptoms ranging from premature discolored small leaves to dieback of branches and death of the trees. The nucleotide sequence of the $16 \mathrm{~S}$ rDNA from new phytoplasma showed $98 \%$ similarity to $\mathrm{Ca}$. Phytoplasma fraxini. Significant differences in nucleotide sequences of tuf and $\operatorname{Sec} A$ genes of the new phytoplasma from typical $C a$. Phytoplasma fraxini suggest that this strain may represent a new phytoplasma species. This report could have important epidemiological and plant health quarantine consequences for the fruit tree industry.

Impact of Macrophomina phaseolina infection on plastid- and cell- membrane integrity and lipid based signaling capacity of sorghum stalk tissues A. Y. BANDARA, S. Liu, C. R. Little, Kansas State University, Manhattan, KS, USA

Galactolipids (monogalactosyldiacylglycerol, MGDG; digalactosyldiacylglycerol, DGDG) are major components of the plastid membrane. Higher MGDG/DGDG ratios can lead to loss of membrane integrity. Phosphatidylserine (PS) is an integral component of plasma membrane while oxidized lipids (ox-lipids) and free fatty acids (oxylipins) are important as signaling molecules. An RNASeq experiment revealed the Macrophomina phaseolina's (MP) ability to significantly down-regulate a digalactosyldiacylglycerol synthase gene (Sb05g003730; converts MGDG to DGDG) and two lypoxygenase genes (Sb01g011040, Sb06g031350; dioxygenation of polyunsaturated FA) while significantly up-regulating a PS synthase gene (Sb09g027850) of a MP susceptible sorghum genotype (Tx7000), but not in a resistant genotype (SC599) at 7 days post-inoculation. To confirm these findings, lipids were extracted from stalk tissues of MP- and mock-inoculated SC599 and Tx7000 and the lipidomes were profiled with an electrospray ionization-TQMS. MP significantly increased the MGDG/DGDG ratio of Tx7000 (CON $=1.1, \mathrm{MP}=1.4, P=0.0004)$ while it decreased the ox-lipid content $\left(\%\right.$ normalized mass spectral signal $\left.\mathrm{mg}^{-1}\right)(\mathrm{CON}=0.18, \mathrm{MP}=0.14, P=0.02)$. Contrary to the transcriptional data, MP-inoculated Tx7000 had a lower PS content $(\mathrm{CON}=1.9, \mathrm{MP}=1.6, P=0.01)$. Results revealed the harmful impacts of MP on plastid- and cell- membrane integrity of Tx7000 and the impeded signaling capacity of Tx7000 under MP infection.

New sources of soybean cyst nematode resistance in 'Forrest' soybean identified through forward and reverse genetics Z. ZHOU (1), N. Lakhssassi (1), S. Liu (1), S. Bekal (1), V. Colantonio (1), K. Lambert (2), A. Barakat (3), K. Meksem (1), (1) Southern Illinois University, Carbondale, IL, USA; (2) University of Illinois, Urbana, IL, USA; (3) University of South Dakota, Vermillion, SD, USA

Soybean cyst nematode (SCN, Heterodera glycines Ichinohe) is the most economically destructive pathogen for soybean production worldwide. Although planting resistant cultivars is the primary management practice to control this pathogen, the mechanism of soybean [Glycine max (L.) Merr.] resistance to SCN remains unknown. In Peking-type soybean cultivar (cv.) 'Forrest', rhgl and Rhg4 are two major quantitative trait loci (QTL) controlling resistance to SCN. Ethyl methanesulfonate (EMS) mutagenized 'Forrest' populations have been extensively and successfully used in both forward and reverse genetics approaches to study the function of SCN resistance genes in soybean. In this study, a total of 193 'Forrest' mutant families was screened for their SCN female index (FI), from which 18 mutants were found susceptible to SCN (FI>10\%). Five of the 18 mutants carried mutations in the GmSHMT08, a gene at Rhg4 locus contributing SCN resistance, while four of them carried no mutations in both GmSHMT08 and GmSNAP18, a gene conferring SCN resistance at rhgl locus. Our findings suggest that other gene(s) may play a role of SCN resistance in 'Forrest'. The 
next-generation sequencing (NGS) is considered to identify EMS-induced mutations in novel candidate SCN resistance genes. Using a recombinant inbred line (RIL) to conduct SCN phenotype screening, GmSNAP11, a member of GmSNAP gene family sharing highly structural similarity with GmSNAP18, were identified to contribute to an additive resistance to SCN. Combined with the expression profiles and structural analyses, it is confirmed as a novel minor SCN resistance gene.

\section{Macrophomina phaseolina infection down-regulates phytosterol biosynthesis in stalk tissues of a charcoal rot susceptible sorghum genotype} A. Y. BANDARA, S. Liu, C. R. Little, Kansas State University, Manhattan, KS, USA

Phytosterols (PS) are integral components of the lipid bilayer in plants and regulate membrane fluidity. Pathogen-induced changes in membrane PS (campesterol; sitosterol, ST; and stigmasterol, SG) composition influence the outcome of plant-pathogen interactions. Further, ST to SG conversion is triggered by ROS. An RNASeq experiment revealed Macrophomina phaseolina's (MP) ability to significantly down-regulate four cycloartenol synthase genes (Sb06g015960, Sb08g019310, Sb08g019300, Sb08g019290; net log2 fold down-regulation = -11.5), six cycloartenol-C-24-methyltransferase genes (Sb01g004280, Sb01g004290, Sb01g004295, Sb01g004300, Sb01g004310, Sb09g029600; -26.8), a cycloeucalenol cycloisomerase gene (Sb09g002170; -2.2), and two cytochrome P450 51 genes (Sb05g022370, Sb08g002250; -5.9) (involved in PS precursor synthesis), in a charcoal rot susceptible sorghum genotype (Tx7000), but not in a resistant genotype (SC599) at seven days post inoculation. To confirm these findings, lipids were extracted from stalk tissues of MP- and mock-inoculated SC599 and Tx7000 and the lipidomes were profiled with ESI-MS/MS. MP significantly reduced the PS content $\left(\%\right.$ normalized mass spectral signal $\left.\mathrm{mg}^{-1}\right)$ of Tx7000 $(P=0.002)$ while increasing its SG/ST ratio $(P=0.006)$. Neither of these was significantly affected in SC599 by MP inoculation. Results suggested the lethal impact of MP on membrane fluidity of Tx7000 and the strong oxidative stress experienced by Tx7000 under MP infection.

Characterization and marker development for three resistance loci to the spinach downy mildew pathogen C. FENG (1), B. H. Bluhm (1), J. C. Correll (2), (1) University of Arkansas, Fayetteville, AR, USA; (2) Univ of Arkansas, Fayetteville, AR, USA

Downy mildew of spinach, caused by the obligate pathogen Peronospora farinosa f. sp. spinaciae (Pfs), is the most economically important disease of spinach. Growing resistant cultivars is the most economical way to manage downy mildew, particularly for organic spinach production, which is approaching $50 \%$ in the US. Breeding resistance cultivars is the primary objective of all spinach breeding programs. Resistance to downy mildew is typically controlled by single dominant genes, and 11 resistant loci have been hypothesized in spinach. Three resistant loci, $R P F 1, R P F 2$ and $R P F 3$ have been genetically characterized and segregate as a dominant resistance gene; each locus provides resistance to more than 10 of the 16 described races of Pfs. Bulked segregant analysis, examination of resistance gene analog sequences, and genotyping by sequencing approaches were employed to develop markers for the $R P F 1, R P F 2$ and $R P F 3$ loci. Thirteen, four and five markers have been developed for these R loci, respectively. One SNP marker was found to be associated with all three loci, indicating that these three resistant loci are linked. The markers could be used to pyramid resistant loci to improve the durability of downy mildew resistance in spinach. The genetic distances among these loci will be estimated, which will affect the strategies for deployment of these resistance loci.

Genetics underlying intra-species antagonism in Pseudomonas syringae and the potential for bacterial pathogen control K. HOCKETT, Pennsylvania State University, University Park, PA, USA

With the decreasing effectiveness of antibiotics and other chemical agents against bacterial pathogens, there is a need to develop new, robust control strategies. Selectively toxic proteins, termed bacteriocins, are potential alternatives. Eighteen pathovars of the phytopathogen Pseudomonas syringae were screened for bacteriocin-mediated inhibition using an agar overlay method, with diverse killing spectra recovered. The majority of killing activity was genetically linked to a bacteriophage-derived bacteriocin (tailocin). Screening $P$. syringae pv. actinidiae harboring characterized transposon insertions and sequencing spontaneous tailocin-resistant mutants in pv. glycinea and pv. phaseolicola backgrounds recovered mutations in lipopolysaccharide (LPS) biosynthesis genes, likely indicating that LPS serves as the tailocin receptor. Alterations in LPS biosynthesis were confirmed directly by SDS-PAGE separation. Two lines of evidence suggest bacteriocin-mediated control may be effective. Resistance conferring mutations in pv. actinidiae simultaneously result in acquired sensitivity to an alternative, non-tailocin bacteriocin. Additionally, pv. glycinea and pv. phaseolicola tailocin-resistant mutants exhibit reduced ability to proliferate when infiltrated directly into their host plants (soy and green bean, respectively) compared to the wild type parent strains. Such trade-offs could be exploited to develop bacteriocins as targeted, robust control strategies.

Lose the effector or die tryin': Novel quantitative resistance loci for bacterial blight

A. I. HUERTA (1), E. Delorean (1), A. Bossa-Castro (1), C. Raghavan (2), R. Corral (1), V. M. Verdier (3), H. Leung (4), J. E. Leach (1), (1) Colorado State University, Fort Collins, CO, USA; (2) International Rice Research Institute, Metro Manila 1301, PHILIPPINES; (3) IRD, Cirad, Univ Montpellier, IPME, Montpellier, FRANCE; (4) Intl Rice Research Inst, Metro Manila, PHILIPPINES

Bacterial blight (BB), caused by Xanthomonas oryzae pv. oryzae (Xoo), is the single most destructive bacterial disease of rice. The optimal agronomic practice to manage the disease is deployment of resistant varieties. However, effective and durable disease resistance for BB is a continuous challenge due to the pathogen's evolution and adaptation on cultivated varieties. Key to Xoo pathogenicity and virulence are Transcription Activator-Like (TAL) effectors, which activate expression of host susceptibility genes. TAL effectors differently contribute to a strain's virulence, and some are essential to pathogen fitness. We hypothesize that effective and durable disease resistance is attainable by targeting important virulence factors in the bacterium. To test this hypothesis we took advantage of the indica Multi-Parent Advanced Generation Inter-Cross (MAGIC) inbred lines and screened them for resistance against Xoo strains PXO99 ${ }^{\mathrm{A}}$ and PXO99A carrying the virulence factor TAL7b (+TAL7b). Transgressive segregation for resistance in the MAGIC lines was observed to both PXO99A and PXO99 ${ }^{\mathrm{A}}+\mathrm{TAL} 7 \mathrm{~b}$, suggesting the presence of resistance for BB. Genome-wide association and interval mapping analysis revealed 13-disease resistance QTL, six specific to PXO99 ${ }^{\mathrm{A}}+\mathrm{TAL} 7 \mathrm{~B}$ and seven to PXO99 ${ }^{\mathrm{A}}$. The top ranking predicted gene target for TAL 7b correlated to a QTL on chromosome 8. Altogether, our data suggest that the TAL7b target is a disease susceptibility gene that contributes to Xoo fitness in rice and that the resistance locus identified may harbor polymorphisms in the TAL7b gene target, disabling activation of the susceptibility gene. Future research will focus on the mechanism underlying this novel resistance phenotype.

Viral metagenomics uncovers virus diversity in wild and cultivated blueberry (Vaccinium corymbosum) N. SAAD (1), R. I. Alcalá-Briseño (1), P. Harmon (1), J. Polston (1), J. Olmstead (2,3), (1) Department of Plant Pathology, University of Florida, Gainesville, FL, USA; (2) Department of Horticultural Science, University of Florida, Gainesville, FL, USA; (3) Driscoll's, Watsonville, CA, USA

Southern highbush blueberry (interspecific hybrids of Vaccinium corymbosum L.) is cultivated near wild $V$. corymbosum as well as closely related species in Florida. The expansion of blueberry cultivation into new areas in Florida and deployment of new cultivars which may contain viruses can potentially increase the diversity of viruses in wild and cultivated $V$. corymbosum. In this study, viral diversity in wild and cultivated blueberries ( $V$. corymbosum) was described using a metagenomic approach. RNA viromes from $V$. corymbosum plants collected from six locations (two cultivated and four wild) in north central Florida were generated by sequencing (Illumina HiSeq 2000) and analyzed using a bioinformatic analysis pipeline. De novo assembled contigs obtained from viromes from both commercial and wild sites produced sequences with homologies to plant virus species from a diverse range of families (Amalgaviridae, Caulimoviridae, Endornaviridae, Geminiviridae, Ophioviridae, Partitiviridae, Virgaviridae). De novo assembled contigs with homology to species in the family Betaflexiviridae were found in viromes only from commercial sites, while contigs with homology to species in the Bromoviridae and Reoviridae were found in viromes only from wild sites. Three complete viral genomes, Blueberry latent 
virus (Amalgavirus), Blueberry mosaic associated virus (Ophiovirus) and a new Tepovirus species, were assembled de novo from the virome obtained from a commercial site. To the best of our knowledge, this is the first study that compares viral diversity in wild and cultivated blueberry using a metagenomic approach.

Whole genome sequences reveal polymorphisms in bacteriocin genes of Xanthomonas perforans in Florida

J. KLEIN (1), S. Timilsina (1), P. Abrahamian (2), G. V. Minsavage Jr. (1), N. Potnis (3), J. B. Jones (1), G. E. Vallad (2), E. M. Goss (1), (1) Department of Plant Pathology, University of Florida, Gainesville, FL, USA; (2) Gulf Coast Research and Education Center, University of Florida, Wimauma, FL, USA; (3) Department of Entomology and Plant Pathology, Auburn University, Auburn, AL, USA

Bacterial spot is an economically important disease of tomato. Worldwide, this disease is caused by four species of Xanthomonas: X. euvesicatoria, X. gardneri, X. perforans, and X. vesicatoria. In Florida, X. euvesicatoria (Xe) was the sole causal agent of bacterial spot until 1991 when $X$. perforans $(X p)$ was detected. Since it was first detected, $X p$ quickly replaced populations of $X e$ in Florida. Previous studies have shown that $X p$ outcompetes $X e$ via the production of three bacteriocins $(\mathrm{BcnA}, \mathrm{BcnB}, \mathrm{BcnC})$. We compared genome sequences of $X p$ strains collected in Florida from 1991 to 2016 and observed polymorphisms in essential open reading frames of $\mathrm{Bcn} A$ and $\mathrm{BcnB}$, but observed no variation in $\mathrm{BcnC}$. We investigated the impact of these polymorphisms using inhibition assays on Petri plates and confirmed a reduction in the ability of $X p$ strains to inhibit $X e$. $X p$ strains that do not produce $\mathrm{BcnA}$ or BcnB remain insensitive to these bacteriocins produced by other $X p$ strains. However, loss of bacteriocin production could result in $X e$ becoming re-established on tomato in Florida.

The interplay between apoptosis and autophagy in the pathogenicity of Sclerotinia sclerotiorum Y. LI, M. B. Dickman, Texas A\&M University, College Station, TX, USA

Programmed cell death (PCD) mediates both resistance and susceptible responses during plant-microbe interactions. Apoptosis and autophagy are two principal cell death regimes widely characterized in animal and to a lesser extent in plants. The "hemi-necrotrophic" fungus Sclerotinia sclerotiorum $(S s)$, induces an apoptotic-like cell death (apoptosis), in part via the secreted toxin oxalic acid (OA), which is required for disease. The OA-deficient mutant (Ss A2) is non-pathogenic and exhibits a restricted autophagic cell death (autophagy), acting as a defense response in Arabidopsis Col-0. To further examine these responses, we pretreated Col-0 with the autophagy inducer Tunicamycin (TM) prior to pathogen challenge and monitored induction of autophagy by MDC staining while apoptosis was blocked. Consistently, overexpression of $C E D-9$ in Col-0, an anti-apoptotic Bcl-2 family member from Caenorhabditis elegans, can suppress apoptosis but curiously, trigger autophagy, thus confer resistance to wild-type $S s$ 1980. Conversely, inhibition of autophagy by using autophagy mutants ( $\operatorname{atg} 7$ and $\operatorname{atg} 8$ ) and treatment with the autophagy inhibitor (Wortmannin), can increase apoptosis and result enhanced susceptibility to $S s$ A2. The expression of $A T G s$ showed most of genes highly induced by $S s$ A2, were significantly down-regulated in Col-0 by Ss 1980 . Collectively, we suggest tipping the balance between apoptosis and autophagy can dictate the outcome of Sclerotinia-Arabidopsis interaction.

Current status of Tomato chlorotic spot virus in Florida and the Caribbean

S. T. ADKINS (1), S. Hutton (2), C. Estevez de Jensen (3), J. E. Funderburk (4), W. W. Turechek (5), (1) USDA ARS USHRL, Fort Pierce, FL, USA; (2) University of Florida, Wimauma, FL, USA; (3) University of Puerto Rico, Agro-environmental Sciences, Mayaguez, PR, USA; (4) University of Florida, Quincy, FL, USA; (5) USDA ARS SAA SPP, Fort Pierce, FL, USA

Damaging outbreaks of Tomato chlorotic spot virus (TCSV), an emerging thrips-vectored tospovirus, and several invasive species of thrips are significantly impacting vegetable and other crops in Florida and the Caribbean. Host and geographic ranges of TCSV are continuing to expand in this region. Development of effective strategies requires a multi-pronged approach against TCSV and its key vectors, including western flower thrips and common blossom thrips. The $S w-5$ gene for resistance to Tomato spotted wilt virus (TSWV), a related tospovirus, also confers resistance to TCSV. New hybrids containing this gene are currently being evaluated in south Florida field trials. Epidemic development is being characterized through intensive sampling of commercial pepper fields. Viral incidence data is being used to characterize the spatial and temporal progress of the virus. Corresponding flower samples are being collected for thrips identification and to characterize the relationship between thrips density and disease incidence. New qRTPCR protocols have been developed for rapid, quantitative and specific identification of TCSV. Symptomatic plant samples tested to date have been infected primarily by TCSV although TSWV is also occasionally detected. Collectively, this information will lead to development of management strategies that are better focused on disrupting virus and/or thrips biology.

\section{Plasmid transfer by conjugation in Xylella fastidiosa} L. BURBANK, D. C. Stenger, USDA-ARS, Parlier, CA, USA

Recombination and horizontal gene transfer have been implicated in the adaption of Xylella fastidiosa (Xf) to infect a wide variety of different plant species. There is evidence that certain strains of Xf carry native plasmids equipped with transfer and mobilization genes, suggesting conjugation as a mechanism of horizontal gene transfer in some instances. Xf strains M23 (subspecies fastidiosa) and Riv5 (subspecies multiplex) carry two operons, tra and $t r b$, on a $38 \mathrm{~kb}$ plasmid. The tra and trb operons, which encode a conjugative Type IV secretion system, are also are present in $X f$ Dixon (subspecies multiplex) and several other isolates of subspecies fastidiosa. This suggests that certain $X f$ strains have the ability to transfer DNA via a conjugation mechanism, and that this transfer can occur between strains from different subspecies. Using either M23 (fastidiosa) or Dixon (multiplex) as the donor strain and Temecula (fastidiosa) as the recipient strain, plasmid transfer was characterized using the mobilizable broad host range vector pBBR5pemIK. Transfer of plasmid pBBR5pemIK was observed under in vitro conditions with both donor strains, and was dependent on both tra and trb operon functions. The possibility of plasmid transfer by conjugation in the natural environment would have implications for horizontal gene transfer between different strains of Xf that may be present in the same location and/or in the same vector or host.

Strain specificity of helper components encoded by Potato virus $\boldsymbol{Y}$

S. MONDAL, S. Gray, Cornell University, Ithaca, NY, USA

In recent years, the recombinant strains of Potato virus $Y\left(\mathrm{PVY}^{\mathrm{NTN}}\right.$ and $\left.\mathrm{PVY} \mathrm{Y}^{\mathrm{N} O}\right)$ have steadily replaced their nonrecombinant parent, $\mathrm{PVY} \mathrm{O}^{\mathrm{O}}$, in the U.S. potato crop. Previous studies showed a transmission bias for recombinant strains when acquired sequentially with PVY ${ }^{\mathrm{O}}$, regardless of acquisition order. To understand this bias, the nonstructural helper component (HC) and infectious virus particles were separated, mixed in various combinations, and fed to green peach aphids using Parafilm ${ }^{\circledR}$ sachets. Following a 20 min acquisition access period, groups of 10-12 aphids were transferred to potato seedlings for a $24 \mathrm{~h}$ inoculation access period. Treatments included all possible combinations of $\mathrm{HC}$ and purified virus (PV) from PVY $\mathrm{PVY}^{\mathrm{N}: \mathrm{O}}$, and PVY $\mathrm{PV}^{\mathrm{NTN}}$. Plants were assayed 21-28 days post inoculation by TAS-ELISA. When heterologous or homologous combinations of PV and HC of PVY ${ }^{\mathrm{N}: O}$ and $\mathrm{PVY}^{\mathrm{NTN}}$ were mixed and fed immediately to aphids, transmission from any treatment was $<20 \%$, except the PVY ${ }^{\mathrm{NTN}} \mathrm{PV}-\mathrm{PVY}^{\mathrm{N}: 0} \mathrm{HC}$ combination $(>60 \%)$. Following a 3-4 h incubation of PV with $\mathrm{HC}$ at $4{ }^{\circ} \mathrm{C}$, transmission efficiency of the all combinations increased to $>70 \%$ except the PVY $\mathrm{N}: \mathrm{O} P \mathrm{PV}-$ $\mathrm{PVY}^{\mathrm{NTN}} \mathrm{HC}$ combination, which remained $<20 \%$. These data indicate a strain specific function of HC, and suggest that formation of a HC-PV protein complex prior to aphid acquisition facilitates transmission. Ongoing research is determining transmission of $\mathrm{HC}-\mathrm{PV}$ combinations of $\mathrm{PVY}^{\mathrm{O}}-\mathrm{PVY} \mathrm{N}^{\mathrm{N}: \mathrm{O}}$ and $\mathrm{PVY}^{\mathrm{O}}-\mathrm{PVY}^{\mathrm{NTN}}$. 
Two RNA viruses as tools for the co-expression of proteins in the same cells of infiltrated plants M. R. MENDOZA, B. D. Shaw, H. B. Scholthof, Texas A\&M University, College Station, TX, USA

RNA plant viral vectors represent tools that allow high level expression of valuable proteins in a relatively short time. For certain purposes it could be desirable to express two or more proteins in a single cell but that is often not possible when using a single virus vector or when the host induces rapid silencing. In this instance, a co-expression strategy is required for the simultaneous delivery by two non-competitive and silencing-insensitive viruses. Here we describe the use of two RNA viruses, Tomato bushy stunt virus (TBSV) and Tobacco mosaic virus (TMV) as agro-launchable virus vector systems that both express suppressors to minimize the effects of Argonaute2-mediated silencing. To co-express proteins we used TBSV and TMV each expressing green fluorescent protein (GFP) of different sizes, in addition to co-infecting TBSV-GFP with TMV expressing red fluorescent protein (RFP). The results in Nicotiana benthamiana and tomato demonstrated that these two vectors expressed their cargo in the same plant, same leaves, and in the same cells. Therefore, co-expression by these two vectors provides a potential biotechnological platform for the expression of proteins that need to form oligomers for activity.

Antimicrobial activities of cationic antimicrobial peptides immobilized on plant virus-like particles R. W. HAMMOND (1), N. Kovalskaya (1), N. Kreger (1), J. Mowery (2), C. Gulbronson (2), J. Hammond (3), (1) USDA ARS Molecular Plant Pathology Laboratory, Beltsville, MD, USA; (2) USDA ARS Electron and Confocal Microscopy Unit, Beltsville, MD, USA; (3) USDA ARS Floral and Nursery Plant Research Laboratory, Beltsville, MD, USA

Antimicrobial peptides have broad utility in agriculture and medicine for the control of diseases caused by gram-positive and gram-negative bacteria, fungi, and protozoan parasites, among others. Small cationic peptides on the order of 10-30 amino acids, in general, are expensive to synthesize and may not exhibit sufficient antimicrobial activity at concentrations required in intra- and intercellular environments and on surfaces. Anchoring the peptide in an assembled, dense array may increase the effective concentration and increase antimicrobial activity. In our laboratory, we have developed multifunctional, self-assembling isometric virus-like particle protein cages composed of marafivirus or ilarvirus capsid proteins. In this study, we displayed antimicrobial peptides with differing activity spectra on the surface of the virus-like particles. The peptides were displayed either as a fusion with the amino terminus or in a predicted surface loop of the capsid protein. The particles were produced in, and purified from, either bacterial or plant expression platforms. Electron microscopy revealed the generation of isometric particles (marafivirus) and pleiomorphic particles (ilarvirus). The antimicrobial activity of the tethered peptides was assayed against several animal and plant pathogens in this work.

Characterization of Bacterial Endophytes and Their Applications

C. MEI (1,2), R. Chretien (1), S. Yan (1), J. Carey (1), Y. He (1), S. Lowman (1,2), (1) Institute for Advanced Learning and Research, Danville, VA, USA; (2) Department of Horticulture, Virginia Tech, Blacksburg, VA, USA

Bacterial endophytes live inside plants and represent a largely unexplored resource for enhancing sustainable agricultural production. We have demonstrated significant growth promotion of switchgrass by the bacterial endophytes Burkholderia phytofirmans (PsJN) and Pantoea agglomerans (PaKM) under in vitro, growth chamber, greenhouse and field conditions, particularly in low fertility soil. Currently, we have established a library of more than 400 bacterial endophytes isolated from plants grown in various environments and characterized their abilities with respect to $\mathrm{N}$ fixation, $\mathrm{P}$ solubilization, auxin and siderophore production and ACC deaminase activity, as well as antifungal and antibacterial activities. In the agricultural sector, we are exploring growth enhancement and disease resistance of various crops important to the region such as tobacco, grapevine, strawberry, tomato and tall fescue. In the environmental sector, we are focusing on bioremediation of polychlorinated biphenyls (PCBs) and polycyclic aromatic hydrocarbons $(\mathrm{PAH})$ by screening bacterial endophytes isolated from plants grown in contaminated areas in enrichment experiments. Then we test potential bacterial endophytes for the ability to degrade biphenyl (an analog of PCBs) or pyrene (a PAH) in vitro by quantification with Ultra Performance Liquid Chromatography or GC-MS. Finally, we inoculate switchgrass with potential bacterial endophytes and grow in contaminated soil for remediation.

Viral delivery of a gene editing tool for transient screening of gene function

W. B. CODY (1), H. B. Scholthof (1), T. E. Mirkov (2), (1) Texas A\&M University, College Station, TX, USA; (2) Texas A\&M Agrilife, Weslaco, TX, USA

The CRISPR/Cas9 gene editing platform has been adapted as a transient screening device in biological model systems, but there are currently limited tools available for plant pathologists. Here, we used the Tobacco mosaic virus viral vector, TRBO, to deliver biologically active single guide RNA (sgRNA) constructs in Nicotiana benthamiana by measuring the presence of genomic indels (inserts and deletions) when co-delivered with Cas 9 through agroinfiltration. Indel percentages averaged $\sim 70 \%$ within 7 days post-inoculation (dpi) when targeting the $m g f p 5$ coding region of GFP-expressing $N$. benthamiana 16c plants. High editing efficiencies translated to a knockdown in GFP production and green fluorescence in 16c plants leaves. The $N$. benthamiana paralogs Argonaute 1-H and Argonaute 1- $L$ were targeted using one sgRNA construct, which created indels within both of the native genes. Similar indel efficiencies were observed when $\mathrm{NbAGO1}$ and $m g f p 5$ sgRNAs were co-delivered (multiplexed) using a single TRBO delivery construct. Additionally, we used TRBO to deliver an RNA transcript with a sgRNA adjoining a GFP protein coding region, which successfully created both indels in the genomic $m g f p 5$ target and viral based protein overexpression. These results show that TRBO-sgRNA-Cas9 co-infiltration provides a transient gene knockout system that can be used for functional genetic studies.

TAL effector-mediated induction of a SWEET sugar transporter gene confers susceptibility to bacterial blight of cotton L. SHAN (1), K. Cox (2), (1) Dept. Plant Pathology \& Microbiology, Texas A\&M University, College Station, TX, USA; (2) Dept. Plant Pathology and Microbiology, Texas A\&M University, College Station, TX, USA

The interaction between cotton and Xanthomonas citri subsp. malvacearum $(X \mathrm{~cm})$, which causes bacterial blight of cotton (BBC), is among the first examples to study classical gene-for-gene resistance in plants. $\mathrm{BBC}$ is also among the devastating diseases of cotton worldwide, in particular with recent outbreaks in the United States. The quantitative trait loci and recessive inheritance of certain BBC resistance, the complex genome and tetraploid nature of this important crop, as well as limited molecular and genetic tools, make the isolation of cotton resistance $(R)$ or susceptibility $(S)$ genes challenging via traditional map-based cloning. We have deployed single molecule real-time sequencing of $\mathrm{Xcm}$ strains and assembled the whole repertoires of transcription activator-like (TAL) effectors, which are key pathogenicity determinants in xanthomonads. By combining gene expression profiling of cotton expressing a specific TAL effector and code-assisted bioinformatics prediction of TAL effector binding element (EBE), we identified GhSWEET10, encoding a sugar transporter, as an $S$ gene to BBC. The TAL effector from Xcm massively induces GhSWEET10 expression by directly binding to the EBE of its promoter. Activation of GhSWEET10 by designer TAL effectors restored Xcm virulence, and silencing of GhSWEET10 in cotton reduced its susceptibility to $X \mathrm{~cm}$ infection. A sucrose FRET sensor assay demonstrated that GhSWEET10 is a functional sugar transporter. The work provides the first example of identifying $S$ target in a tetraploid species and suggests that pathogen-induced GhSWEETS contribute to disease progression by transporting sugar into apoplast.

Unraveling the cyclic-di-GMP mediated transcriptional regulation of amylovoran production in Erwinia amylovora R. R. KHARADI, G. W. Sundin, Michigan State University, East Lansing, MI, USA

Cyclic-di-GMP (c-di-GMP) is a ubiquitous bacterial second messenger that signals the transition from a motile lifestyle to an attached lifestyle in the form of biofilms. Diguanylate cyclase enzymes (encoded by $d g c$ genes) are involved in the synthesis of c-di-GMP and phosphodiesterase enzymes 
(encoded by pde genes) are involved in its degradation. In Erwinia amylovora, c-di-GMP is known to positively regulate the production of the most abundant exopolysaccharide, amylovoran, which is also a pathogenicity factor. However, the mechanism of c-di-GMP-mediated transcriptional regulation remains elusive. We mapped the promoter region of ams $G$, the first gene of the amylovoran biosynthesis operon, and subdivided the ca. 750 $\mathrm{bp}$ region based on the location of the transcriptional start site and a binding sequence for the major regulatory proteins RcsA and RcsB. These smaller sequence fragments were cloned into a GFP reporter transcriptional fusion vector, and the fluorescence activity of these probes was measured under varying intracellular levels of c-di-GMP. We observed a strong positive correlation between the intracellular concentration of c-di-GMP and the level of transcriptional regulation imposed on the ams $G$ promoter. Further, we screened a library of transposon mutants generated using these reporter strains in order to identify potential effectors that transcriptionally regulate $a m s G$, or an $a m s G$ regulator, after binding to c-di-GMP.

A truncated Xanthomonas oryzae TAL effector required for effector triggered immunity suppression in an American heirloom rice cultivar A. C. READ (1), F. Rinaldi (1), M. Hutin (1), Y. Q. He (2), L. R. Triplett (3), A. J. Bogdanove (1), (1) Cornell University, Ithaca, NY, USA; (2) Guangxi Univ, Nanning, CHINA; (3) Connecticut Agricultural Experiment Station, New Haven, CT, USA

Xanthomonas oryzae pv. oryzicola (Xoc) causes bacterial leaf streak (BLS) of rice. The first BLS resistance locus, Xo1, was recently identified from American heirloom rice cultivar Carolina Gold Select. Xo1 responds to many TAL effector variants resulting in robust effector triggered immunity (ETI). Although both African and Asian strains of Xoc encode and deliver TAL effectors that can be recognized, Xo1 only provides resistance to African strains. Our group recently demonstrated that the failure to elicit ETI by Asian strains is due to the delivery of a truncated TAL effector (truncTALE) by these strains resulting in suppression of resistance. Analysis of available sequence data revealed the presence of truncTALEs in most $X$. oryzae strains and noted conserved features that may be required for ETI suppression. We found that truncTALEs lack the hallmark DNA binding ability of full-length TAL effectors and do not appear to require a nuclear localization signal (NLS) to function. A study published shortly after ours found that the NLS is required for truncTALE suppression of bacterial blight resistance gene Xa1. Here we summarize our current knowledge of this novel class of effector and provide new insight into truncTALE sub-cellular localization and function. Further characterization of this ETI suppressing effector will guide resistance gene discovery and engineering efforts against truncTALE deploying Xanthomonas strains.

Binding properties of cercosporin and their role in symptoms of Cercospora leaf blight in soybean B. WARD (1), C. L. Robertson (1), P. Abhayawardhana (1), M. Macnaughtan (2), R. W. Schneider (2), (1) Louisiana State University Agricultural Center, Baton Rouge, LA, USA; (2) Louisiana State University, Baton Rouge, LA, USA

Cercospora leaf blight (CLB) is a serious disease of soybeans in the Mid South of the U.S., Brazil, and Argentina. The disease is caused by three species of Cercospora that produce the red photoactivated toxin, cercosporin. This toxin alone is responsible for disease progression from symptomless to purple/bronzed leaves to blighted leaves. Along with generation of reactive oxygen species, the toxin also binds to various metals found in plants. Bound forms of these cercosporin-metal complexes were analyzed for binding strength via various chemical tests. These complexes were also applied to leaf disks and assessed for symptom development after 5 days. Cercosporin bound to iron and aluminum resulted in less cellular damage and different symptoms than unbound cercosporin. Cercosporin bound to manganese resulted in higher cellular damage. Furthermore, these complexes exhibited siderophore-like properties when incorporated into minimal media broth, which was used to culture Cercospora cf. flagellaris. Findings from these studies help to explain results from field experiments in which several metals were applied as foliar sprays with some suppressing symptoms of CLB and others enhancing disease severity.

Dynamic expression of T3SS genes in single cells of Dickeya dadantii during the interaction with potato

Z. CUI (1,2), X. Yuan (3), Q. Zeng (1), C. H. Yang (2), (1) Department of Plant Pathology \& Ecology, The Connecticut Agricultural Experiment Station, New Haven, CT, USA; (2) Department of Biological Sciences, University of Wisconsin-Milwaukee, Milwaukee, WI, USA; (3) Institute of Plant Protection, Jiangsu Academy of Agricultural Sciences, Nanjing, CHINA

Dickeya dadantii is a bacterial pathogen and causal agent of soft rot on potato and other tuber producing plants. The type III secretion system (T3SS), which induces plant cell death, is an important virulence factor in $D$. dadantii. The expression of T3SS in is induced in plant apoplast or in Hrp-inducing minimal medium (Hrp-MM). Despite the understanding of induction conditions, how individual cells in a bacterial population respond to the induction signal is not well understood. Our previous studies revealed that when cultured in Hrp-MM, D. dadantii express the T3SS genes in a bi-stable manner: only a small proportion of cells highly expresses T3SS genes, whereas the rest of the population does not express these genes. In this study, we further characterized the T3SS gene expression in single cells of $D$. dadantii under in vivo conditions, using potato as a model host. Using a dual fluorescence reporter and single-cell techniques, we proved that the expression of T3SS genes in $D$. dadantii is also bistable in potato apoplast. Furthermore, we characterized the dynamics of "T3SS On" and "T3SS Off" populations at different infection stages and locations. More cells are in the "T3SS On" population at the initial infection stage than in the late infection stage in potato leaves, and more cells are in the "T3SS On" population when inoculated in stems than in leaves. No "T3SS On" cells were observed in tuber. These results implicate that the T3SS bistability might be important for $D$. dadantii to adapt the hostile host environment.

\section{A change in perspective: PAMP triggered immunity as told by Pseudomonas syringae pv. tomato} A. LOVELACE, B. Kvitko, University of Georgia, Athens, GA, USA

The recognition of conserved Pathogen-Associated Molecular Patterns (PAMPs), such as bacterial flagellin, by Pattern Recognition Receptor (PRRs) membrane proteins activates an immune response that confers broad defense against many microbes. When pre-induced, PAMP triggered immunity (PTI) restricts the growth of pathogenic bacteria such as Pseudomonas syingae pv. tomato (Pto) in the apoplastic space. Although the plant cellular processes involved in flagellin/FLS2 signaling have been well studied, the mechanisms by which PTI suppresses further colonization of the apoplastic space by Pto are poorly understood. The objective of this study is to use Pto as a probe to better understand the mechanism(s) of PTI. Pto was exposed to pre-induced immune activated (Flg22) and naïve (Mock) Arabidopsis leaves for 1, 3, and 5 hours before extraction using a newly developed method to access the Pto transcriptome in planta. Principal component analysis of transcriptome profiles between treatments show separation of Flg22 and Mock samples after one hour of exposure. Differential expression of genes in Pto exposed to these two conditions reveal that virulence genes and sugar metabolism are downregulated whereas chemotaxis, motility, and nutrient uptake are upregulated by exposure to immune defenses. Expression profile of Pto exposed to immune defenses is suggestive of entering stationary phase with consistent levels of expression for major cellular processes.

Epiphyllous growth and conidiation of Hordeum spp. endophytes and the conidial dissemination potential M. YI, C. A. Young, The Samuel Roberts Noble Foundation, Ardmore, OK, USA

Epichloë species (Clavicipitaceae, Ascomycota) are systemic fungal symbionts that associate with many cool-season grasses widely distributed around the world. Their association often provides beneficial effects to their host grasses, promoting persistence and enhanced growth. Sexual Epichloë species can be horizontally transmitted among individual plants and asexual and some sexual species are vertically transmitted infecting the host seeds by colonizing reproductive tillers. The role of conidia that form on the leaf surfaces of some endophyte-infected plants has not been well characterized, although there is evidence they may play a role in horizontal transmission. Scanning electron microscopy, optical microscopy, and confocal laser scanning microscopy was used to evaluate several asexual Epichloë species that naturally colonize Hordeum bogdanii and H. brevisubulatum for their growth and colonization characteristics. Conidiogenous cells and conidia from epiphytically growing mycelia were observed on the leaf surface in addition to the endophytic intercellular growth. The frequency of epiphyllous hyphae and the conidiation development varied among different Hordeum 
spp. endophytes. The viability and infectivity of the conidia generated on the host grass will be further tested to evaluate the potential for horizontal transmission by asexual conidia of Hordeum spp. endophytes.

Potential to manage date palm black scorch disease in the United Arab Emirates using a resident actinomycete isolate K. A. EL-TARABILY (1), S. Abuqamar (2), E. E. Saeed (3), Z. Salmin (4), (1) United Arab Emirates University, Al-Ain, UAE; (2) United Arab Emirates University, Al Ain, UAE; (3) UAE University, Al-Ain, UAE; (4) UAE Univeristy, Al-Ain, UAE

Among the diseases affecting date palms, the black scorch disease caused by Thielaviopsis punctulata, is known to cause considerable losses in date production, world-wide. While the fungicide Cidely ${ }^{\mathbb{E}}$-top was found to effectively inhibit the mycelial growth of the fungus both in vitro and in vivo, the use of biocontrol agents (BCAs) is considered to be preferable as they have minimum impact on economic and environmental aspects. Isolates of actinomycetes obtained from the rhizosphere soil of healthy date palm cultivated in the United Arab Emirates (UAE) were screened against $T$. punctulata. Among the BCAs tested that showed antagonistic effects in vitro and in greenhouse experiments against this fungus, an isolate of Streptomyces sp. showed consistently high efficacy when applied before or at the same time of inoculation with T. punctulata, compared with BCA or fungicide application after inoculation. The antagonistic activity of the actinomycete isolate against $T$. punctulata, was mainly due to the production of diffusible antifungal metabolites that inhibited hyphal growth of the fungus. Altogether, the pathogenicity tests which indicated the reductions in disease severity indices and the definition of the mode of action, confirmed that the BCA can not only control black scorch disease symptoms, but also prevent the spread of the fungus, as a potential practical method to improve disease containment in date palm plantations. This is the first report of an actinomycete naturally occurring in UAE and the region showing considerable promise as a BCA against the causal fungus of black scorch disease on date palm.

Immunity at cauliflower hydathodes controls infection by Xanthomonas campestris pv. campestris

A. CERUTTI (1), A. Jauneau (2), M. C. Auriac (2), E. Lauber (1), Y. Martinez (2), S. Chiarenza (3), N. Leonhardt (3), R. Bethomé (1), L. Noel (1), (1) LIPM Laboratoire des Interactions Plantes Micro organismes, UMR CNRS / INRA 2594/441, Castanet-Tolosan, FRANCE; (2) Institut Fédératif de Recherche 3450, Plateforme Imagerie, Castanet-Tolosan, FRANCE; (3) UMR7265, Laboratoire de Biologie du Développement des Plantes, Saint-Paullez-Durance, FRANCE

Hydathodes are water pores found on leaves of a wide range of vascular plants and are the sites of guttation. They are found in direct contact with vascular discontinuities and represent one of the entry pathways for vascular pathogens. Xanthomonas campestris pv. campestris (Xcc), an epiphytic bacterium, is able to naturally enter inside hydathodes and spread within plants xylem, causing black rot disease of Brassicaceae. Xcc infects economically important crops such as cabbage but also the model plant Arabidopsis thaliana. Understanding bacterial behavior at hydathodes and immune responses in this tissue is essential to design knowledge-informed control strategies against this pathogen. We report here the detailed anatomy of cauliflower and Arabidopsis hydathodes and describe their infection by Xcc, thus recapitulating natural infection and black rot disease symptoms. Preinvasive immune mechanisms were investigated by studying the ability of hydathode pores to respond to stimuli such as ABA, light or the flg22 flagellin peptide, a well-characterized elicitor of plant basal immunity. Results showed that hydathode pores respond to ABA and light but not to flg22. Postinvasive immunity was shown to limit pathogen growth in the epithem and is actively suppressed by the type III secretion system and its effector proteins. Altogether, these results give a detailed anatomic description of Brassicaceae hydathodes and advance our understanding of hydathode immunity.

Biological control of root and stem rot of soybean caused by Rhizoctonia solani using chitinolytic and ACC deaminase producing yeasts K. A. EL-TARABILY (1), S. Abuqamar (2), A. Alkhajeh (3), (1) United Arab Emirates University, Al-Ain, UAE; (2) United Arab Emirates University, Al Ain, UAE; (3) UAE Univeristy, Al-Ain, UAE

Twenty-five yeast isolates obtained from soybean rhizosphere were screened for the production of cell-wall degrading enzymes (chitinase and $\beta$-1,3glucanase) using mycelial (Rhizoctonia solani) fragment agar. Only seven isolates showed exceptional chitinolytic activity in vitro by producing chitinase and $\beta$-1,3-glucanase and caused lysis of $R$. solani hyphae, the causal agent of root and stem rot of soybean in the United Arab Emirates (UAE). None of the seven isolates produced volatile antibiotics, diffusible antifungal metabolites, hydrogen cyanide or siderophores. These isolates were subsequently tested in vitro for their ability to produce 1-aminocyclopropane-1-carboxylic acid (ACC) deaminase, the immediate precursor of the stress hormone ethylene. Under greenhouse conditions, only four cell-wall degrading yeast isolates capable of producing high levels of ACC deaminase, significantly reduced disease incidence of root and stem rot disease compared to the three yeast isolates which did not produce ACC deaminase. The application of the four ACC deaminase-producing yeast isolates resulted in the reduction of the endogenous levels of ACC and promoted soybean growth compared with plants treated with the three non-ACC deaminase producing yeast isolates. The results clearly showed that these yeasts could substitute azoxystrobin which is the currently recommended fungicide for $R$. solani diseases. This is the first successful use of yeasts as plant growth promoters and biocontrol agents against $R$. solani diseases. This study also demonstrated the ability of ACC deaminase-producing cell-wall degrading yeasts to control root and stem rot and to promote soybean growth under stress conditions caused by $R$. solani.

\section{Multiple resistance in fungal plant pathogens: Selection schemes and impact on disease control management strategies S. TORRIANI, G. Scalliet, H. Sierotzki, Syngenta Crop Protection, Stein, SWITZERLAND}

Several fungicide classes are used to control a fungal disease. Fungicide classes inhibit independent biochemical targets and are often applied in mixtures or in spray program during the season. By sexual recombination or accumulation of independent mutations, multiple resistant individuals can be selected. Each resistance is characterized by different resistance factors, fitness, frequency and impact on field performance. Multiple resistances have been observed in various pathogens including Zymoseptoria tritici, Plasmopara viticola and Botrytis cinerea. Z. tritici evolved resistance to MBC, DMI, QoI and SDHI fungicides, but also might express weak multidrug characteristics. P. viticola developed resistance to PA, QoI, CAA and cymoxanil. B. cinerea showed targeted and multidrug resistance towards a range of fungicide classes. Whereas frequency of single resistance is high, the combined resistance might remain at lower frequencies and can be managed through appropriate spray applications and good agronomic practices. Multiple fungicide resistance can follow different evolutionary directions in response to the selection imposed by the spray strategies (alternation/mixtures), the number of registered products, the characteristics of each fungicide and the putative contribution of multidrug resistance. A better understanding of how multiple resistances evolve is beneficial to frame the impact to disease control and to propose sound new anti-resistance strategies.

Biological control of Agrobacterium rhizogenes using Pseudomonas strains C. CHAGAS DE FREITAS (1), C. G. Taylor (2), (1) Ohio State University, Wooster, OH, USA; (2) The Ohio State University, Wooster, OH, USA

Agrobacterium rhizogenes is the bacterial causative agent for hairy root-disease in plants. A. rhizogenes harbors a root-inducing (Ri) plasmid which contains a region of DNA that is transferred (T-DNA) from the bacteria into the host chromosomes. After T-DNA incorporation, the transformed plant cell grows and divides to produce a transgenic root. These transgenic roots alter the plant source-sink relationship to favor root growth over shoot or fruit production. Since chemical control of bacterial diseases via antibiotics has limited effectiveness is highly regulated, we seek alternative ways to control this disease. We have been studying the ability of certain Pseudomonas strains to inhibit the growth of $A$. rhizogenes. In our in vitro experiments, we identified 14 Pseudomonas strains that were able to inhibit $A$. rhizogenes growth on agar plates. These 14 strains were selected for in planta assays (on Kalanchoe and soybean). Three Pseudomonas strains (1B1, 93G8 and 48G9) were able to reduce hairy root formation by $95 \%$ as compared to controls. 
We also examined Agrobacterium persistence in Pseudomonas treated soybean and tomato cuttings. Pseudomonas treatments were able to reduce Agrobacterium numbers by nearly 1000 -fold. Taken together, these results suggest that the certain Pseudomonas strains have the ability to inhibit $A$. rhizogenes growth and disease development and may be useful in as biocontrol agents in the prevention of hairy-root disease.

Complex Patterns of Resistance to Respiration Inhibitors Revealed among Corynespora cassiicola Isolates from Tomato in Florida K. J. MACKENZIE (1), A. Wen (2), G. E. Vallad (3), H. Adkison (4), L. L. Hellu (1), (1) Gulf Coast Research \& Education Center, Wimauma, FL, USA; (2) University of Florida, Wimauma, FL, USA; (3) Gulf Coast Research and Education Center, University of Florida, Wimauma, FL, USA; (4) Univ of Florida GCREC, Wimauma, FL, USA

Target spot caused by Corynespora cassiicola is one of the most economically important foliar and fruit diseases of tomato in Florida. From 2015 to 2017, 105 isolates collected from 7 tomato producing counties in Florida were evaluated for sensitivity to 7 respiration inhibitor fungicides, including quinone outside inhibitors (QoI) and succinate dehydrogenase inhibitors (SDHI), based on mycelial growth inhibition (MGI), spore germination (SG), and sequence-based analyses. Nearly $90 \%$ of the isolates were resistant to the QoI fungicides azoxystrobin and fenamidone based on MGI and SG with $\mathrm{EC}_{50}>10 \mathrm{ppm}$. Additional sequence-based screening of cytB confirmed the presence of the G143A mutation in all but one of the QoI resistant isolates identified by MGI. Based on MGI, $75.2 \%$ of the C. cassiicola isolates exhibited reduced sensitivity to one or more of the tested SDHI fungicides, including boscalid, penthiopyrad, fluxapyroxad, benzovindiflupyr, and fluopyram. Cross-resistance to all 5 SDHI fungicides was evident in one isolate, while $39 \%$ of the isolates exhibited reduced sensitivity to all but fluopyram. In planta assays and sequence analysis of sdhB, C, and D subunits from representative isolates are being peformed to further corroborate MGI results. Enhanced knowledge of fungicide resistance among C. cassiicola isolates will improve the management of target spot and help limit further development of fungicide resistance in Florida.

An in vitro study of the inhibitory effect of Pseudomonas spp. on important greenhouse ornamental pathogens

D. E. MARTIN (1), C. G. Taylor (2), F. Peduto Hand PhD (3), (1) The Ohio State University, Columbus, OH, USA; (2) The Ohio State University, Wooster, OH, USA; (3) Ohio State Univ, Dept of Plant Pathology, Columbus, OH, USA

Biological control is becoming a common practice in greenhouse production of ornamentals due to emerging pathogen resistance to fungicides and consumer preference. In this study, 45 bacterial strains across 9 species of Pseudomonas were tested in vitro for inhibition of 7 common ornamental pathogens, including Pythium cryptoirregulare, Phytophthora cryptogea, Rhizoctonia solani, Sclerotinia sclerotiorum, Thielaviopsis basicola, Colletotrichum acutatum and Sclerotium rolfsii. Using a dual plating method, a $5 \mathrm{~mm}$ mycelium plug of each pathogen was individually placed in the center of a PDA plate and a single colony of a 2-day old Pseudomonas culture was streaked in a vertical line $2.5 \mathrm{~cm}$ from either side of the plug. Inhibition was recorded as the distance from the center of the agar plug to the growing edge of the mycelium, parallel (R1) and perpendicular (R2) to the bacterium. R2 measurements along with an inhibition index, calculated as $I=(\mathrm{R} 1-\mathrm{R} 2) / \mathrm{R} 1 * 100$, were analyzed by one-way ANOVA and the means separated using Fisher's LSD. Pythium and Rhizoctonia were the most responsive pathogens to inhibition by Pseudomonas, particularly P. chlororaphis, $P$. brassicacearum, and $P$. protegens, whose inhibitory effect was comparable to the positive control, cycloheximide. Selected bacterial strains will be further tested in planta for disease suppression ability. By characterizing these strains, this work aims to ultimately promote the use of biocontrol in greenhouse production.

The detection and characterization of QoI-resistant pathogens causing ascochyta blight of pulse crops in Montana A. OWATI, Montana State University, Bozeman, MT, USA

Ascochyta blight $(\mathrm{AB})$ of pulse crops (chickpea, field pea, and lentils) causes yield loss in Montana, where 1.2 million acres was planted to pulses in 2016. Pyraclostrobin, a Quinone outside Inhibitor (QoI) fungicide is used widely for the management of AB in pulse crops. However, a mutation in the cytochrome $b$ gene has been reported to confer resistance. To evaluate QoI resistance, a total of 1000 Ascochyta isolates were screened. Out of these, $10 \%$ from chickpea, $81 \%$ were from dry peas, and $9 \%$ from lentil seed lots (chickpea $=17$, pea $=131$, and lentil $=21$ ) from 23 counties in Montana. Analysis of cDNA and protein sequences of isolates indicated resistant isolates contained the G143Amutation in the cytochrome $b$ gene, which commonly confers complete resistance to all QoI fungicides. A multiplex TaqMan assay was developed to discriminate QoI-resistant and sensitive isolates. Using this assay, QoI fungicide-resistant isolates of Didymella rabiei were detected in Daniels, McCone, and Valley Counties MT, from seed produced in 2015 and 2016. In vivo greenhouse studies indicated that isolates with the G143A mutation caused significantly higher amounts of disease than sensitive isolates on pyraclostrobin-treated chickpea plants ( $\mathrm{p}$-value $=0.001)$. These results imply that $\mathrm{AB}$ control may be inadequate at locations where resistant isolates were found, and resistant isolates may be spread via seed.

Managing an oomycete community: Fungicide sensitivity and evolution of resistance to ethaboxam

Z. NOEL (1), A. Rojas (2), J. L. Jacobs (1), M. Chilvers (1), (1) Michigan State University, East Lansing, MI, USA; (2) Duke University, Durham, NC, USA

Seedling diseases caused by oomycetes pose a significant threat to soybean production. In 2011 and 2012, over 80 oomycete species were found to be associated with soybean seedlings. Over half of those species were found to be pathogenic. Evaluation of these species for fungicide sensitivity is important for management. Fungicide amended medium assays are slow, labor intensive and expensive. A high-throughput assay to evaluate fungicide sensitivity of many oomycete isolates at once was developed using optical density measurements of macerated mycelial fragments. Z'-factor was used as a quality control statistic. The assay was utilized to evaluate the sensitivity of 81 oomycete species to mefenoxam and ethaboxam. Of the isolates tested, $87.5 \%$ had an $\mathrm{EC}_{50}<1 \mu \mathrm{g} \mathrm{ml}^{-1}$ and only one Phytopythium isolate had an $\mathrm{EC}_{50}>10 \mu \mathrm{g} \mathrm{ml}^{-1}$ mefenoxam. For ethaboxam, $61.7 \%$ of isolates tested had an $\mathrm{EC}_{50}<1 \mu \mathrm{g} \mathrm{ml}^{-1}$, whereas, species within Pythium clades A, B and E had EC $50 \geq 20 \mu \mathrm{g} \mathrm{ml}^{-1}$ ethaboxam. This suggested that reduced sensitivity to ethaboxam may be inherent and possibly related phylogenetically. Therefore, we investigated the evolutionary history and mechanism of resistance to ethaboxam. Phylogenies indicated that species with reduced sensitivity to ethaboxam followed a convergent evolutionary pattern and had evolved three separate times. Two different transversion mutations lead to the same amino acid change in the target gene of lineages with reduced sensitivity to ethaboxam.

Biological control of Fusarium root rot on row crops in the Great Plains using PGPR and Trichoderma species L. P. PARIKH, M. Eskelson, A. O. Adesemoye, University of Nebraska Lincoln, North Platte, NE, USA

Biological control agents (BCAs) for managing plant pathogens is a very important component of integrated disease management. The use of BCAs have become more relevant with the potential for many pathogens to develop resistance against chemical fungicides. Some BCAs are very specific to certain pathosystems. The present investigation was undertaken to identify plant growth promoting rhizobacteria (PGPR) - Bacillus sp. and Burkholderia sp. and Trichoderma species that have broad spectrum effectiveness against various species of Fusarium. Potential BCAs were recovered as endophytes or from the rhizosphere of healthy wheat and corn crops using standard procedures. From our collection, 21 strains of PGPR and 26 strains of Trichoderma indigenous to Nebraska were selected in preliminary in vitro and in planta screening against 7 virulent strains of Fusarium, collected in a previous study. Two layers of screening identified lead BCA strains that were further studied in the greenhouse in a randomized complete block design. Five Trichoderma, two Burkholderia ambifara and one Bacillus strains showed broad spectrum activity against Fusarium isolates. Biocontrol effectiveness of BCAs ranged from 16-76\% for bacteria and $40-90 \%$ for Trichoderma sp. Effective strains would be further tested under field conditions. 
Variation in endophytic and epiphytic bacteria populations isolated from cultivated small grains

F. CRUTCHER, S. Hoesel, Montana State University, Sidney, MT, USA

In Montana, Fusarium head blight (FHB) and deoxynivalenol (DON) contamination, caused by Fusarium graminearum, is emerging as a threat to small grains production as more corn is introduced into rotations. There are several control methods for FHB including fungicide applications and rotations with pulses, however, the occurrence and intensity of this disease is highly variable. Thus, new technologies such as bacterial biocontrol agents are needed. The aim of this work was to select for bacterial endophytes and epiphytes from wheat, barley, durum, and oats grown in fields with a history of FHB to assess the diversity, distribution, and potential integrated pest management applications of these bacteria. Heathy leaves and florets from production fields were collected and sampled for bacteria growing within and on the plant surface. These bacteria, identified using biochemical and sequencing techniques, included many gram negative and gram positive species. However, the vast majority were Bacillus and Pseudomonas, both known to be involved in plant-microbe induced resistance. These results provide candidates for future evaluation as potential management tools against FHB in small grain crops.

\section{Fungicide Resistant Phenotypes in Botrytis cinerea from Mandarin and Their Impact on Control of Gray Mold on Stored Mandarin Fruit in California}

S. SAITO (1), C. L. Xiao (2), (1) USDA ARS, Parlier, CA, USA; (2) USDA-ARS, Parlier, CA, USA

Gray mold caused by Botrytis cinerea is an emerging postharvest disease affecting stored mandarin fruit in California. To develop effective control programs, phenotypes of resistance to four citrus postharvest fungicides were determined. One hundred $B$. cinerea isolates each in 2015 and 2016 were obtained from decayed fruit collected from packinghouses and tested for resistance to the fungicides. Sensitivity to azoxystrobin was examined based on the point mutation in the $c y t b$ gene using PCR, while sensitivities to fludioxonil, pyrimethanil and thiabendazole were examined on fungicide-amended media. For azoxystrobin, $83 \%$ and $98 \%$ of the isolates were resistant in 2015 and 2016 , respectively. Based on the in vitro fungicide tests, $71 \%$ and $93 \%$ were resistant to pyrimethanil, and $63 \%$ and $68 \%$ were resistant to thiabendazole in 2015 and 2016, respectively. No fludioxonil resistance was detected in both years. Five fungicide resistant phenotypes were detected, and the most common phenotype was multiple resistance to the three fungicides, accounting for 59 and $65 \%$ in 2015 and 2016, respectively. Of the $200 \mathrm{~B}$. cinerea isolates, $5 \%, 23.5 \%$, and $62 \%$ were resistant to 1,2 or 3 classes of fungicides, respectively. Efficacy tests were conducted to evaluate if the fungicides at label rates control various resistant phenotypes on fruit. Most fungicides failed to control gray mold on mandarin fruit inoculated with respective fungicide resistant phenotypes. Our results suggest that alternative control methods need to be integrated into existing decay control programs to target this emerging disease on mandarin fruit.

\section{Effect of Contans WG and burial of sclerotia on survival of sclerotia of Sclerotinia sclerotiorum in the Columbia Basin of central Washington} J. Weber, L. DU TOIT, Washington State University, Mount Vernon, WA, USA

White mold, caused by Sclerotinia sclerotiorum, is the main disease affecting sunflower seed crops in central Washington. A microplot trial was initiated in fall 2015 near Ephrata, WA to assess the effects of drenching Contans WG (Coniothyrium minitans, a mycoparasite of S. sclerotiorum) at 0, 0.56, and $4.48 \mathrm{~kg} / \mathrm{ha}$ on survival of sclerotia on the soil surface or buried $15 \mathrm{~cm}$ deep. Sclerotia viability was tested at $\sim 2$ month intervals. In plots not drenched with Contans WG, $<10 \%$ of buried sclerotia were viable after 7 months vs. $>70 \%$ for surface sclerotia. Contans WG reduced survival of surface sclerotia, not buried sclerotia, but only 12 months after application (from $99 \%$ at application to $42 \pm 8,6 \pm 3$, and $1 \pm 1 \%$ in plots with $0,0.56$, and $4.48 \mathrm{~kg}$ Contans WG/ha, respectively). Results were similar for a repeat trial initiated in spring 2016 . Contans WG also was applied by spray boom at 0 and $4.48 \mathrm{~kg} / \mathrm{ha}$ to residues in large, replicated plots after harvest of a sunflower seed crop with $>50 \%$ incidence of white mold. Viability of sclerotia in buried residues decreased faster than that of sclerotia in surface residues ( $73 \pm 4$ vs. $88 \pm 2 \%$, respectively, after 5 months). The Contans WG application had no effect on survival of sclerotia in infested crop residues or sclerotia sampled from soil after residues had decomposed. In summary, Contans WG only reduced survival of sclerotia on the soil surface, and was never as effective as burial at reducing sclerotium survival.

Evolutionary relatedness and sources of US lineages of Phytophthora infestans (Mont.) de Bary A. C. SAVILLE, J. Ristaino, North Carolina State University, Raleigh, NC, USA

The oomycete Phytophthora infestans is an important pathogen on potato and tomato crops worldwide. It primarily reproduces asexually and forms clonal lineages. We compared the population structure of 18 of the 24 documented US lineages to lineages from Europe, South America, and Mexico. Structure analysis, neighbor joining trees, and principal component analyses of 12 microsatellite loci indicated that many recent US genotypes (US-7, US-8, US-11, US-22, US-24) shared significant allelic diversity with lineages from Mexico, while the US-1 lineages clustered with US-1 isolates from Peru. The US-8, US-14, and US-24 lineages, predominantly virulent on potato, and the US-21 and US-22 lineages, predominantly virulent on tomato, formed two distinct and separate monophyletic clades. The US-23 lineage, currently the most prevalent lineage detected in the US, shared allelic diversity primarily with isolates from Bolivia and Brazil and not Mexico. Mexican lineages showed evidence of multiple ancestral recombination events. A survey of the presence of RXLR effector PiAVR2 across all samples revealed the presence of lineages that carried either PiAVR2, its resistancebreaking variant PiAVR2-like, or both, suggesting lineages have experienced different levels of selection to the $R 2$ gene in potato. These findings suggest populations of $P$. infestans in the US are the result of introductions from both South America and Mexico.

Prospective endophyte antagonists of Verticillium dahliae isolated from three field-grown cucurbit cultivars in western WA L. TYMON (1), P. Morgan (1), D. A. Inglis (2), (1) Washington State University-NWREC, Mount Vernon, WA, USA; (2) Washington State UniversityMount Vernon NWREC, Mount Vernon, WA, USA

Endophytes (ENDs) are fungi and bacteria that live asymptomatically within plant tissue and protect plants from root pathogens. In western WA, cucurbits are high-value specialty crops and are susceptible to Verticillium dahliae $(V d)$. In June 2016 moderately susceptible pumpkin cv. 'Cinnamon Girl' (CG), susceptible watermelon cv. 'Sugar Baby' (SB) and resistant squash cv. 'Tetsukabuto' (Tet) were transplanted into a field infested with Vd. Roots were excavated, surface disinfested, and the tips, midroots, and crowns plated onto corn meal and modified potato dextrose agars. In total, 603 fungal ENDs were recovered. The highest number was isolated from 'CG' (225 ENDs, 37\% of total) while the lowest was from 'SB' (161 ENDs, 27\% of total). 'CG' and 'SB' had the highest number isolated from root crowns while 'Tet' had the highest number from root tips. Isolates were grouped into one of 37 morphotypes. Amplification and sequencing of the ITS region of select morphotype isolates revealed Actinomucor, Boeremia, Clonostachys, Fusarium, and Mortierella. Clonostachys and Fusarium have exhibited antagonism to Rhizoctonia and Fusarium spp., respectively, in previous studies Antagonism of recovered END isolates to $V d$ currently is being assessed in vitro as well as in plantae. $V d$ is a poor competitor in soil and a poor root colonizer when certain ENDs are present. The higher number of ENDs colonizing the root tips may be a factor in the resistance that 'Tet' exhibits towards $V d$.

\section{A new multilocus genotype of Phytophthora nicotianae found in isolates from pineapple heart rot disease}

M. F. RATTI (1), M. Ascunce (1), J. Landivar (2), E. M. Goss (1), (1) Department of Plant Pathology, University of Florida, Gainesville, FL, USA; (2) Facultad de Ingeniería Marítima, Ciencias Biológicas Oceánicas y Recursos Naturales, ESPOL, Guayaquil, ECUADOR

Phytophthora nicotianae is an Oomycete pathogen that causes diseases in $\sim 225$ plant genera, thus standing out among other Phytophthora species in its geographic distribution, host range and economic impact. In pineapple (Annanas comosus), P. nicotianae causes heart rot disease, which significantly reduces crop yield due to plant mortality. Recent studies suggest that genotypes of this pathogen are host-specific to some extent; however, it is not clear 
how many host-specific lineages of the pathogen exist. The objective of this study was to determine the level of intra-specific genetic variation of $P$. nicotianae causing heart rot disease of pineapple in Ecuador. In addition, we aimed to compare the genotypes found on pineapple to lineages previously reported in citrus, tobacco and ornamentals. Thirty $P$. nicotianae colonies were collected from infected leaves of four farms during an outbreak in the 2016 rainy season in Santo Domingo province, Ecuador. We extracted DNA from single-spore isolates and confirmed them to be $P$. nicotianae by sequencing the cox 2 gene. We genotyped the isolates using seven simple sequence repeat (SSR) loci. These loci revealed a single lineage that was distinct from those previously reported. These results support the hypothesis of host specialization of $P$. nicotianae in intensive farm systems and contribute to the understanding of the global population structure of this important pathogen.

Identification of bioactive secondary metabolites from a Bacillus amyloliquefaciens strain for control of fungal plant pathogens T. Qu (1), Y. Yin (2), L. Xiao (2), S. TIAN (1), P. Ji (1), (1) University of Georgia, Tifton, GA, USA; (2) Qingdao Agricultural University, Qingdao, CHINA

Endophytic bacteria were isolated from medicinal plant Albizzia julibrissin and evaluated for control of fungal plant pathogens. Among the bacteria screened, an isolate, designated H6, inhibited growth of Fusarium oxysporum, F. graminearum, Gaeumannomyces graminis and a number of other pathogens. The bacterial isolate was identified as Bacillus amyloliquefaciens by morphological characteristics, biochemical and physiological tests, and $16 \mathrm{~S}$ rRNA gene sequence analysis. Ethyl acetate extract of the bacterial isolate exhibited potent antifungal activity in in vitro studies against various phytopathogenic fungi. The crude extract was separated by silica gel chromatography and thin-layer chromatography, and ten fractions (F.1-F.10) were obtained. Two fractions which exhibited active antimicrobial activities were silylated, and compounds with bioactive property in the fractions were identified by gas chromatograph-mass spectrometer (GC-MS). Bioactive metabolites identified included aromatic acids, cyclic dipeptides, and fatty acids. The broad-spectrum antifungal activity of the bacterial isolate and its metabolites indicated that the endophytic bacterium could be a promising biological control agent for plant diseases caused by fungal pathogens.

\section{Population Structure of the oomycete soilborne pathogen Phytophthora capsici in North Carolina} L. M. Quesada, C. H. PARADA ROJAS, North Carolina State University, Raleigh, NC, USA

Phytophthora capsici is a devastating oomycete plant pathogen that affects solanaceous, cucurbitaceous, fabaceous, and other crops in the United States (US) and worldwide. Previous studies have documented high genetic variation in US populations and geographic stratification since $P$. capsici has limited dispersal resulting in isolated field populations. Despite its impact in vegetable production, $P$. capsici population structure in North Carolina (NC) is unknown. In this study, more than 120 isolates of $P$. capsici collected from different regions of NC were assayed for mefenoxam, fluopicolide, dimethomorph, and oxathiapiprolin resistance and genotyped using eleven microsatellite loci and fragment analysis. Preliminary data suggest that fungicide resistance is more common in some NC counties than others. Both mating types A1 and A2 are present across NC, but A2 P. capsici isolates are the most common throughout the state. Population analyses revealed that the greatest genetic differentiation occurs when isolates are grouped by location more so than when grouped by host, mating type, or fungicide sensitivity. Our results indicate that $P$. capsici is highly diverse in NC, but isolate genotypes remain structured by location; therefore, deployment of fungicides and resistant cultivars must account for spatial distribution of genetic and phenotypic diversity.

Temporal and host driven variation of Pythium and Globisporangium populations in chrysanthemum and geranium M. F. PROANO (1), C. Ayala (2), A. Chiriboga (2), P. Garrido (3), E. Diaz (1), S. M. Marek PhD (1), H. A. Melouk (4), M. L. Daughtrey (5), C. D. Garzon PhD (1), (1) Oklahoma State University, Stillwater, OK, USA; (2) Universidad de las Fuerzas Armadas ESPE, Sangolqui, ECUADOR; (3) AGROCALIDAD, Quito, ECUADOR; (4) USDA ARS, Stillwater, OK, USA; (5) Cornell University, Long Island, NY, USA

Multiple Pythium and Globisporangium species cause damping off of seedlings, seed rot, stem lesions, and root rot in greenhouse ornamental crops. To identify the diversity of species present in floricultural greenhouses located in Long Island, New York, two hundred seven isolates were collected from Chrysanthemum L. plants in 2014. Analysis of DNA sequences based on ITS region identified fifteen species of Globisporangium $(\mathrm{n}=4)$ and Pythium $(\mathrm{n}=11)$. The G. irregulare s.l. complex $(\mathrm{n}=120)$ were the most prevalent plant pathogenic species, which agrees with results of previous studies on Pelargonium spp. and other spring crops (2011-2013). Moderate to high genetic structure was found between greenhouse populations for each species of the G. irregulare complex. Analysis of Molecular Variance (AMOVA), Principal Coordinates Analysis (PCoA) and minimum spanning networks (MSN) suggest local sources of inoculum as well as shared sources of inoculum for Pythium diseases of chrysanthemum. When genotypes of G. irregulare s.1. from Geranium were associated by MSN over different seasons and years (2011-2013), closely related genotypes occurred in the same location over different years, suggesting inoculum may have survived in greenhouse populations from year to year. Scouting propagative materials and immediate eradication of symptomatic plants, as well maintaining good greenhouse sanitation practices are needed to minimize movement and survival of inoculum in floricultural greenhouses.

\section{Screening Mycotoxins For Quorum Inhibition In A Biocontrol Bacterial Endophyte}

\section{W. BACON (1), D. Hinton (2), (1) USDA ARS, Athens, GA, USA; (2) Richard Russell Research Center, Athens, GA, USA}

Bacterial endophytes are used as biocontrol organisms for plant pathogens such as the maize endophyte Fusarium verticillioides and its production of fumonisin mycotoxins. However, such applications are not always predictable and efficient. Bacteria communicate via cell-dependent signals, which are referred to as quorum sensing resulting from the production of metabolites that regulate a cascade of gene expressions resulting in biocontrol activity. The assumed role for mycotoxins is to act as defensive metabolites thus serving as protection for fungi from biotic antagonisms and as such do not interact with the daily metabolic requirements of the producing fungus. Recently, quorum-sensing inhibitors have been isolated from several fungi, including Fusarium species, three of which are mycotoxins. Thus, we postulate that other mycotoxins are also inhibitors of quenching metabolites aimed at preventing key biochemical activities of biocontrol bacteria within intercellular spaces. Biosensor bacteria have been developed as useful assays for both quorum sensing and inhibitory activities. In order to test this hypothesis, we determined the quorum inhibitory activity of some common mycotoxins using two strains of biosensor bacteria, Chromobacterium violaceum, and Agrobacterium tumefaciens to access their role in inhibiting developmental controls of the bacterium endophyte Bacillus mojavensis.

Biological control of aflatoxins in maize and groundnut through use of aflasafe products developed for Ghana D. Agbetiameh (1,2), A. ORTEGA-BELTRAN (1), R. Awuah (2), J. Atehnkeng (3), P. J. Cotty (4), R. Bandyopadhyay (1), (1) International Institute of Tropical Agriculture, Ibadan, NIGERIA; (2) Kwame Nkrumah University of Science and Technology, Kumasi, GHANA; (3) International Institute of Tropical Agriculture, Lilongwe, MALAWI; (4) USDA-ARS, University of Arizona, Tucson, AZ, USA

Use of atoxigenic Aspergillus flavus isolates in biocontrol formulations allows producing maize and groundnut with reduced aflatoxin content. For Ghana, two biocontrol products, aflasafe GH01 and aflasafe GH02, were developed through extensive examination of fungi associated with maize and groundnut. Each product contains four atoxigenic A. flavus strains belonging to genotypes widely distributed across Ghana. In 2015 and 2016 , efficacy of each product was independently evaluated in $>100$ maize and groundnut fields located in several districts of the Northern, Upper East, and Upper West regions. Proportions of atoxigenic strains composing each product were assessed in soils before application, and soils and grains at harvest. Aflatoxin content was determined in treated and untreated crops. Significant $(P<0.05)$ displacement of toxigenic fungi occurred in all years, regions, and crops. Crops from aflasafe-treated fields contained significantly $(P<0.05)$ less aflatoxins (range $=98 \%$ to $100 \%$ less). The large majority of crops from treated 
fields could have been commercialized in both local and international food and feed premium markets. Results indicate that aflasafe GH01 and aflasafe GH02 are efficient, cost-effective, environmentally safe tools for aflatoxin mitigation. Adopting use of aflasafe products throughout Ghana has the potential to improve the health of Ghanaians, and both income and trade opportunities of farmers, aggregators, distributors, and retailers.

Unraveling historical shifts in Pseudoperonospora cubensis populations in the U.S. that resulted in the 2004 cucurbit downy mildew epidemic A. RAHMAN (1), E. Wallace (2), J. A. Crouch (3), F. N. Martin (4), L. Quesada-Ocampo (5), (1) NCSU, Raleigh, NC, USA; (2) North Carolina State University, Raleigh, NC, USA; (3) Mycology and Nematology Genetic Diversity and Biology Laboratory, USDA-ARS, Beltsville, MD, USA; (4) USDA ARS, Salinas, CA, USA; (5) Department of Plant Pathology North Carolina State University, Raleigh, NC, USA

The biotrophic oomycete pathogen Pseudoperonospora cubensis causes cucurbit downy mildew (CDM) on a plethora of Cucurbitaceae hosts. Following a devastating outbreak in 2004, CDM re-emerged as a major foliar disease on cucurbit crops in the United States (US). Prior to this outbreak, CDM was managed by host resistance in cucumber and fungicide applications in other cucurbits, both of which became ineffective or limited in efficacy. To investigate shifts in US P. cubensis populations that resulted in the 2004 epidemic we used mitochondrial genome sequencing and multilocus sequence analysis to examine more than 450 recent (post-2004) and 300 herbarium (pre-2004) samples. Our findings indicate that $P$. cubensis has two distinct clades with host specialization. Interestingly, Clade I was predominant before 2004 in the US regardless of cucurbit host, while samples collected after 2004 exhibited a mix of Clade I and II isolates with Clade II isolates being specialized on Cucumis sativus, C. melo, and Cucurbita foetidissima. Moreover, analysis of mitochondrial genomes allowed for development of a PCR marker that differentiates Clade I and Clade II, providing a means to assess crop infection risk by a particular isolate. We are expanding our investigation to determine the phylogenetic relationship among $P$. cubensis isolates from within and outside of US. Our findings will provide significant insight into population shifts that resulted in the 2004 CDM outbreak in the US.

Genic copy number variation reveals an unexpected mosaic of varying ratios of diploidy and triploidy among isolates of Phytophthora infestans B. J. KNAUS (1), J. Tabima (2), S. Shakya (3), H. S. Judelson (4), N. Grunwald (5), (1) USDA ARS, Corvallis, OR, USA; (2) Oregon State University, Corvallis, OR, USA; (3) OREGON STATE UNIVERSITY, Corvallis, OR, USA; (4) University of California, Riverside, CA, USA; (5) USDA-ARS, Horticultural Crops Research Unit, Corvallis, OR, USA

For almost as long as the potato has been cultivated, it has also been repeatedly subject to severe epidemics caused by the Irish Potato Famine pathogen, Phytophthora infestans (Mont.) de Bary. Fundamental to understanding the genetic architecture of traits such as fungicide resistance and mating type determination is knowledge of how many copies of each gene are expected (i.e., is a gene diploid, triploid, tetraploid or higher ploid). We used high throughput sequencing, from previously published sources as well as our own sequencing, to infer gene copy number for each of the annotated $P$. infestans genes. Instead of observing individuals that were predominantly diploid or triploid we observed individuals that represented a full spectrum of intermediate states from diploid to triploid. Comparison of gene copy number among samples did not result in large regions of conserved copy number but instead presented a surprisingly plastic genome. Previous perspectives that individuals are diploid at the species' center of diversity and higher ploid in clonal epidemics are shown to be more ambiguous than previously thought. These results indicate that no simple assumption about gene copy number can be made within $P$. infestans and highlights the importance of inference of copy number prior to genetic analysis. Our findings present new perspectives on $P$. infestans as an organism with great variability in how many copies each gene it may contain.

Antagonistic potential of biocontrol bacteria against Sclerotinia sclerotiorum causing lettuce drop A. D. SANABRIA, C. G. Taylor, S. A. Miller, The Ohio State University, Wooster, OH, USA

The plant pathogen Sclerotinia sclerotiorum constitutes a major phytosanitary problem in lettuce production, causing losses of 5 to $70 \%$. This phytopathogen overwinters as sclerotia surviving in the field for more than 5 years. In order to identify potential biocontrol agents for the sustainable management of lettuce drop, the antagonism of 45 bacterial strains (one Streptomyces sp., two Bacillus spp., and 42 Pseudomonas spp.) against $S$. sclerotiorum was tested using the dual culture method. Bacteria were streaked transversely $2.5 \mathrm{~cm}$ from both sides of the fungal pathogen inoculum plug on Potato-Dextrose-Agar medium and incubated at room temperature. The percentage of S. sclerotiorum mycelial growth compared to the non-treated control, and the number of sclerotia produced in Petri plates were evaluated. Both Bacillus strains reduced mycelial growth and totally inhibited sclerotia production, while three Pseudomonas strains (48G9, 14B11 and 48D8) were able to reduce mycelial growth more than $70 \%$ and number of sclerotia more than $80 \%$. The Streptomyces strain tested did not reduce fungal growth or sclerotia production. The results suggest that these Bacillus and Pseudomonas strains may have potential to reduce Sclerotinia inoculum in the field.

\section{Serenade ASO: A Sustainable Agricultural Solution Providing Multiple Modes of Action Against Bacterial Pathogens} M. IOTT, Bayer, West Sacramento, CA, USA

Growers are in need of integrated solutions to combat a multitude of bacterial diseases which account for billions of dollars in crop losses globally each year. Biological products are a flexible option for growers’ agricultural spray programs in both organic and conventional systems. Serenade ASO ${ }^{\circledR}$, a registered biological product by Bayer, is a unique tool in providing efficacy against a variety of bacterial pathogens such as Pseudomonas syringae pv tomato (PSDMTM), Xanthomonas campestris pv. vesicatoria (XANTAV), Clavibacter michiganensis (CORBMI), and Erwinia amylovora (ERWIAM). Research continues to expand on the three main modes of action for Serenade $\mathrm{ASO}^{\circledR}$ on bacterial pathogens: direct contact activity from antibacterial biological compounds, induced systemic resistance, and antagonistic competition. For direct contact activity, new approaches, such as predictive genomics, have been utilized to help identify potential antibacterial biological chemistry. Induced systemic responses through Serenade ASO $^{\circledR}$ applications include maintained stomata closure and efficacy on foliar diseases via drench applications. The third mode of action is demonstrated by Serenade's active ingredient, Bacillus subtilis stain QST 713, through antagonistic competition against ERWIAM on pome flowers. By utilizing several complimentary modes of action, Serenade $\mathrm{ASO}^{\circledR}$ proves to be a sustainable solution for growers around the world by managing bacterial disease while expanding their integrated programs.

\section{Growth medium affects physiology and virulence of Phytophthora infestans sporangia}

\section{S. P. Patev (1), W. E. FRY (2), (1) Clark University, Worcester, MA, USA; (2) Cornell University, Ithaca, NY, USA}

Our lab has observed that the medium on which $P$. infestans is grown can strongly influence the results of experiments with sporangia produced on those media. The first observation was that sporangia produced from pure cultures (culture sporangia) on agar (Rye B or Pea) medium were more sensitive to solar radiation than were sporangia produced on leaflet lesions (leaflet sporangia). The second observation was that arachidonic acid induced resistance in potatoes to culture sporangia, but not to leaflet sporangia. To quantify the effect of "source" of sporangia on the virulence of those sporangia, we conducted inoculation studies in the field and in moist chambers. Leaflet sporangia produced three- to twenty-times as much disease per sporangium as did culture sporangia. In order to develop hypotheses concerning mechanism, gene expression in both types of sporangia was assessed. The ratio of expression of an RxLR effector to expression of INF1 differed more than 50-fold in the two types of sporangia. The ratio declined gradually as an isolate was obtained from leaflets and then passaged on agar medium. Thus, we conclude that for $P$. infestans, leaflet sporangia are physiologically and pathogenically different from culture sporangia, and leaflet sporangia should be used for the most accurate assessment of host-pathogen interactions under natural/agricultural conditions. 
Presence-absence polymorphism and balancing selection of RxLR genes in Phytophthora clade 1c species S. SHAKYA (1), B. J. Knaus (2), J. Tabima (1), H. S. Judelson (3), N. Grunwald (2), (1) OREGON STATE UNIVERSITY, Corvallis, OR, USA; (2) USDA ARS, Corvallis, OR, USA; (3) University of California, Riverside, CA, USA

Plant pathogens, including Phytophthora, secrete effector proteins which suppress PAMP triggered immunity \& enable them to colonize host cells. One distinct class of effectors in Phytophthora is known as RxLR effectors. Recognition of effectors (Avr genes) by resistance genes (R-gene) triggers immunity in the host. Loss of RxLR genes has been described as one of the mechanisms to evade recognition by R-genes. Therefore, it is important to identify conserved RxLR/Avr effectors to deploy R-genes for breeding purposes. Here, we identify core RxLR genes from three clade 1c Phytophthora species: P. infestans, P. mirabilis and P. ipomoeae by mapping whole genome sequence reads to the T30-4 reference genome. Genes were considered absent using a breadth of coverage (BOC) value of zero. BOC is defined as the proportion of bases of a gene that was re-sequenced at least once. We found 369 conserved RxLRs and 194 RxLRs with presence-absence polymorphism. Out of 369 conserved RxLRs, 364 genes had at least one variant. The 364 conserved RxLR genes were tested for selection using Tajima's D. We found 39 P. infestans, 27 P. mirabilis and 20 P. ipomoeae RxLR genes under balancing selection. None of the genes were under positive selection in $P$. mirabilis; however, $3 P$. infestans and $1 P$. ipomoeae genes were under positive selection. Our results provide insights into the evolution of core RxLR genes in Phytophthoraclade1c species and suggest predominance of balancing selection.

Effects of seed treatment with biocontrol agents and gibberellic acid on seedling disease, plant growth, yield and quality in organic rice X. ZHOU, Texas A\&M AgriLife Research, Beaumont, TX, USA

Stand loss caused by seedling disease complex (Rhizoctonia solani, Pythium spp., Fusarium spp., etc.) is one of the most important factors limiting organic rice production in the US. Field trials were conducted to determine the effects of seed treatment with biocontrol agents and gibberellic acid (GA, a plant growth regulator) on seedling disease complex, plant growth, yield and quality in two rice varieties under organic production system in Texas. Seed treatment with any of the three biocontrol agents, Sonata (Bacillus pumilus), Integral (B. subtilis) and BioEnsure (fungal endophytes), significantly improved plant stand and numerically increased grain yield on Presidio or XL753. Seed treatment with BioEnsure also improved milling quality. GA seed treatment significantly increased plant height at the stages of seedling and maturity. The hybrid variety XL753 had higher yield (42\% increase) and better milling quality compared to Presidio. These results demonstrate for the first time that seed treatment with the biocontrol agents can reduce stand loss caused by the seedling disease complex and that seed treatment with GA can improve plant growth in rice under organic production system.

Application of Genotyping-by-Sequencing in the development of downy mildew resistant lima bean cultivars T. MHORA (1), C. Scanlan (1), N. F. Gregory (1), E. Ernest (2), S. Polson (1), R. J. Wisser (1), T. A. Evans (1), N. M. Donofrio (1), (1) University of Delaware, Newark, DE, USA; (2) University of Delaware, Georgetown, DE, USA

Downy mildew of lima bean, caused by the oomycete Phytophthora phaseoli, is one of the leading causes of yield loss in the mid-Atlantic region. Enhancing cultivar resistance by gene pyramiding is an effective management strategy. A combination of phenotyping and genotyping using molecular markers to detect resistance to race $\mathrm{F}$ of $P$. phaseoli led to a hypothesis that there may be alternative race $\mathrm{F}$ resistance genes in our 256-accession diversity panel. To test this hypothesis, genotyping-by-sequencing (GBS) was optimized for lima bean to characterize a diversity panel and better understand the underlying cause of this trait. Using in silico digest on the Phaseolus vulgaris genome, the enzyme pair of BsrI/CviQI uniformly sampled the genome. To assess the performance of this enzyme pair in GBS and to develop filters for genotype data from the diversity panel, two $\mathrm{F}_{2}$ populations were genotyped. Cluster analysis of the sequence reads supported our in silico results, with the anticipated number of loci $(1,150-2,200)$ being found per chromosome, in addition to 14,000 unique loci which did not map to the P. vulgaris genome. A bioinformatics pipeline (RedRep) was used to score SNPs across these loci. This led to the identification of 1,400 SNPs in the two biparental populations, as well as a set of filters developed for use with the diversity panel data. Developments on cultivar resistance and characterization of the diversity panel will be shared at this meeting.

Evaluation of the antagonistic activity of native Trichoderma isolates from Texas citrus orchards against Phytophthora nicotianae M. GURUNG (1), F. Gurung (1), C. Simpson (1), J. L. Hernandez (2), V. Ancona PhD (1), (1) Texas A\&M Kingsville Citrus Center, Weslaco, TX, USA; (2) Instituto Politecnico Nacional Centro de Biotecnologia Genomica, Reynosa, MEXICO

Trichoderma spp. is a common fungus found in cultivated soils and possesses antifungal activity against many phytopathogens. The aim of this study was to isolate and evaluate indigenous Trichoderma species for their antagonistic activity against Phytophthora nicotianae, for their potential use as biocontrol agents of citrus foot and root rot in Texas. Twenty eight Trichoderma strains were isolated from soils collected from six orchards across the Texas citrus producing region. Trichoderma isolates were evaluated in vitro for their antifungal activity against four $P$. nicotianae strains isolated from grapefruit orchards located across the citrus producing area of the state. All Trichoderma isolates showed antagonistic activity against $P$. nicotianae by competing for nutrients and space, however only one isolate (Ray1715) showed strong mycoparasitism. Sequencing of the ITS region confirm that Trichoderma isolate Ray 1715 was $T$. asperellum. Co-infection experiments of citrus seedlings with $P$. nicotianae and T. asperellum showed that strain Ray1715 protected seedlings by preventing infection and promoting seedling growth. These findings support the further evaluation of indigenous Trichoderma spp. as a biocontrol agent against citrus phytopathogens.

Identification and characterization of Phytophthora isolates from citrus orchards of South Texas

S. CHAUDHARY (1,2,3), V. Ancona PhD (2), C. Barbola (4), (1) Texas A\&M University, College Station, TX, USA; (2) Texas A\&M Kingsville Citrus Center, Weslaco, TX, USA; (3) South Texas College, McAllen, TX, USA; (4) Texas A\&M University-Kingsville, Citrus Center, Weslaco, TX, USA

Foot rot and root rot caused by Phytophthora species are prevalent diseases in commercial citrus orchards of South Texas. There is a need for comprehensive study to identify and characterize Phytophthora isolates in the orchards. Ninety four isolates, were collected from soil and infected roots from 30 citrus orchards located across the three citrus producing counties in the Lower Rio Grande Valley of Texas. ITS sequence generated based on primers ITS4 and ITS6, exhibit 98 to $100 \%$ identity to Phytophthora nicotianae for all the isolates tested. This was also supported by morphological characteristics such as colony motif, sporangium and oogonium shape and size. Thirty Isolates were tested for mating type with known A1 and A2, $P$. nicotianae testers. Twenty two and eight isolates were identified as A2 and A1 mating type respectively. Thirty isolates of $P$. nicotianae were also screened for sensitivity to mefenoxam on $10 \%$ clarified V8 agar at 1 a.i. $\mu \mathrm{g} \mathrm{mL}^{-1}$. Six isolates were inhibited less than $50 \%$, twenty-two isolates were inhibited $50 \%$ to $90 \%$ and two isolates were inhibited more than $90 \%$ and were marked as insensitive, intermediate sensitive and sensitive respectively We conclude from this study that prevalent species in the commercial citrus groves is $P$. nicotianae and most of the isolates tested, are intermediate or insensitive to mefenoxam at 1 a.i. $\mu \mathrm{g} \mathrm{mL}^{-1}$. Occurrnce of both mating types in the orchards, can have an implication for the management of the Phytophthora diseases in the region.

Characterization of the interaction between biological control agent ZJU60 and Fusarium graminearum J. WANG, Y. Chen*, N. Yang, Z. Ma, Institute of Biotechnology, Zhejiang University, Hangzhou, CHINA

F. graminearum is the predominant pathogenic fungus causing Fusarium head blight (FHB) disease. Biological control with beneficial bacterial agents is an alternative option to control this disease. In this study, we isolated a Pseudomonas sp. strain ZJU60 from wheat rizhosphere, which showed strong antagonistic activity towards F. graminearum and more than $60 \%$ biocontrol efficacy to FHB disease in field trials. Combining with genomic sequence and MALDI-TOF-MS, the ZJU60 was identified as the Pseudomonas chlororaphis subsp. piscium. ZJU60 contains 15 predicted secondary metabolism 
pathways. Among of them, deletion of the phenazine-1-carboxamide (PCN) biosynthesis cluster was totally abolished the antifungal activity, indicating that PCN is the essential antifungal compound. ZJU60 can form the air-liquid interface pellicle or submerged biofilm in the various media. Moreover, it can colonize the $F$. graminearum mycelium and form biofilm on it. To further investigate the antifungal mechanism of PCN, we conducted a chemical genetic assay with the yeast mutant library and found that PCN could interfere with four biological pathways in the yeast, including cell cycle, oxidative stress, cellular morphogenesis and multidrug transport. A total of 21 mutants of these pathways revealed significantly increased sensitivity to PCN. Intriguingly, deletion mutants of these 21 orthologs in F. graminearum did not show distinct variation of susceptible to PCN. These results indicate that the pathways targeted by PCN in filamentous fungi are different from those of yeast. The underlying mechanisms of antifungal activity towards filamentous fungi by PCN have also been determined.

Biological seed treatments for Soybean Cyst Nematode (Heterodera glycines) management

W. ALJAAFRI (1), G. W. Lawrence (2), V. Klink (2), S. Lu (2), D. Long (3), K. S. Lawrence (4), (1) Mississippi State University, Starkville, MS, USA; (2) Mississippi State University, Mississippi State, MS, USA; (3) Albaugh, LLC, Olive Branch, MS, USA; (4) Auburn University, Auburn, AL, USA

One strategy for $H$. glycines management is with biological control. A test was conducted in the greenhouse at Mississippi State University to determine the efficacy of selected biological products. Treatments included seeds treated with ALB EXP Bacteria 1, 2, \& 3, Burkholderia sp. alone and in combination with Bacterial metabolite, Saponin, and Harpin, a standard Abamectin and an untreated control. All seeds were treated by Albaugh, LLC. The study included effects on plant growth and nematode life stage development. Seeds were placed in $2.54 \mathrm{~cm}$ depressions in a steam sterilized sand: soil mix in $10 \mathrm{~cm}$ dia clay pots. H. glycines, $2500 \mathrm{eggs}$, was added on top of the seed for each treatment. The test was arranged in a RCB with 5 replications and ran for 60 days. At harvest, no negative effects were recorded from any treatment on soybean growth. Seed treatments significantly reduced eggs and cysts of $H$. glycines compared with the untreated control. Seed treatments were similar in efficacy to the standard, Abamectin. $H$. glycines $\mathrm{J} 2$ numbers were significantly lower in the seed treatments compared with the control except in treatments ALB EXP Bacteria 1 and 2. When two systemic acquired resistant products were added to Burkholderia sp., both cyst and egg numbers were lower compared to Burkholderiaalone. Future research will focus on stacking different modes of action to enhance nematicidal activity.

Use of KASP markers to screen the Pisum sativum single plant core collection and commercial varieties for resistance to Pea seed-borne mosaic virus

L. D. Porter, K. D. SWISHER, USDA-ARS, Prosser, WA, USA

Kompetitive allele-specific PCR (KASP) markers were previously developed to distinguish two resistance alleles of the translation initiation factor (eIF4E) in Pisum sativum against Pea seed-borne mosaic virus (PSbMV) pathotype P-1. Endpoint genotyping was conducted using KASP markers to separate PSbMV-susceptible lines from those comprising one of the two eIF4E-resistance alleles. Using these markers, the 325 lines of the $P$. sativum single plant core collection (SPCC) and 46 commercial varieties were screened. Genotypes were correlated to phenotypic and serological assay results obtained for PSbMV from greenhouse trials. Of the 325 single plant lines, 22 were resistant to the PSbMV pathotype P-1 in the greenhouse, showing no visual PSbMV symptoms and testing negative for PSbMV by ELISA. Of these 22 resistant lines, 15 were identified with the eIF4E resistant W62L amino acid mutation, and seven with the eIF4E resistant S78 amino acid deletion using the KASP markers. Two SPCC lines showed a mixed population of PSbMV-susceptibility and W62L resistance, both by ELISA and KASP marker analysis. Of the 46 commercial varieties screened, five showed resistance to PSbMV pathotype P-1 in the greenhouse and tested negative by ELISA, and were all found to have the eIF4E-resistant W62L amino acid mutation based on the KASP markers. This genotyping screen of the SPCC and commercial varieties provides breeders with information regarding PSbMV-resistance in their programs.

Field-testing potential biological control agents for enhanced Fusarium head blight mitigation A. L. BEHARI, G. Kuldau, Pennsylvania State University, University Park, PA, USA

The fungal disease Fusarium head blight (FHB) affects wheat and other cereal production worldwide. In addition to a reduction in grain quality and weight, this disease results in the contamination of grain with mycotoxins, including deoxynivalenol (DON). Current FHB mitigation includes the use of moderately resistant varieties of wheat and fungicides, but even in conjunction, these strategies provide limited control when environmental conditions support disease development. This research sought to identify potential biological control agents for enhanced FHB mitigation, specifically looking for microbes from soil, silage, and plant material, with the abilities to degrade DON and inhibit Fusarium graminearum. Through a co-culturing assay, six $F$. graminearum inhibiting bacteria have been isolated and identified preliminarily in the genera Bacillus and Brevibacillus. These bacteria have also been shown to inhibit macroconidia germination to varying degrees. Samples incubated with DON as a sole carbon source have resulted in the isolation of microbes that can potentially degrade DON. Based on additional inhibition assays and chromatography to confirm DON degrading abilities, potential biocontrol agents will be selected for a field experiment measuring disease incidence and severity, Fusarium damaged kernels, and DON levels in FHB inoculated susceptible and moderately resistant wheat cultivars. Researching biocontrol for FHB mitigation can potentially introduce a new tool into FHB IPM programs, allowing for FHB disease control to be flexible and compatible within breeding focused mitigation of other cereal diseases.

Host protein interaction network associated with the non-incorporated form of the potato leafroll virus RTP identified using mass spectrometry S. L. DEBLASIO (1), A. R. Rebelo (2), R. Johnson (3), S. Gray (4,5), M. MacCoss (6), M. Cilia (2,4,5), (1) USDA-ARS, Ithaca, NY, USA; (2) Boyce Thompson Institute, Ithaca, NY, USA; (3) Department of Genome Sciences, University of Washington, Seattle, WA, USA; (4) Emerging Pest and Pathogen Research Unit, USDA-ARS, Ithaca, NY, USA; (5) Section of Plant Pathology and Plant-Microbe Biology, Cornell University, Ithaca, NY, USA; (6) Department of Genomics Sciences, University of Washington, Seattle, WA, USA

Potato leafroll virus (PLRV) is an aphid-transmitted plant virus in the family Luteoviridae that infects solanaceous crops. Its +ssRNA genome is protected by an icosahedral-shaped virion composed of 180 subunits of coat protein (CP), the major capsid component. Sporadic, translational readthrough of a stop codon at the end of the CP gene produces a readthrough protein (RTP), which exists as two forms, a minor structural protein that is incorporated into virions to regulate systemic movement and transmission, and a non-incorporated form, which restricts virus movement to the phloem. We used affinity-purification mass spectrometry coupled to reverse genetics using viral mutants to identify protein-protein interactions among virus and host proteins that are specific to virions from those that interact with the non-incorporated form of the RTP. By quantifying differences in the protein interaction networks of wild type PLRV and a mutant where the CP stop codon has been deleted, we have evidence showing that the non-incorporated RTP forms complexes with a distinct class of host proteins, viral RNA and several nonstructural viral proteins including P3a, a newly discovered PLRV protein involved in systemic movement. Our analysis has also revealed a class of plant proteins involved in vesicle trafficking whose interaction with PLRV is dependent on virion assembly. Characterization of the function of these host proteins in the context of a PLRV infection is ongoing.

Paecilomyces lilacinus in Nacobbus aberrans control in tomato cv. San Marzano

R. GARCIA-VELASCO, E. A. Chavarro-Carrero, Universidad Autónoma del Estado de México, Tenancingo, MEXICO

Nacobbus aberrans is the causal agent of one of the main diseases in tomato crops in Mexico, for its control inorganic nematicides are used, search and evaluation of new biological products is a necessity for the management of this disease. The aim of this research was to evaluate the effect of Paecilomyces lilacinus application in Nacobbus aberrans control in tomato cv. San Marzano. The research was performed in a completely randomized design with seven treatments and ten replicates in a controlled growth chamber at $26^{\circ} \mathrm{C}$ and 12 light hours. Treatment 1 consisted of uninoculated tomato seedlings, treatment 2 of inoculated seedlings (2000 J2 and 1000 eggs), treatment 3, 4 and 6 of seedlings inoculated and treated with products based on 
Paecilomyces lilacinus $\left(\right.$ Nemaroot $^{\circledR}$, Nematicida PI ${ }^{\circledR}$ and BioAct ${ }^{\circledR}$, respectively), treatment 5 and 7 of substrate (Peat Moss ${ }^{\circledR}$ and Agrolite $\left.{ }^{\circledR} 1: 1\right)$ inoculated and treated with fenamiphos and fluensulfone respectively and then planted with tomato seedlings. Fresh weight of root and foliage, stem length and gall index were evaluated. Analysis of variance and LSD test $(\mathrm{p}=0.05)$ were performed. The fresh weights and stem length were lower in the inoculated plants correlating with its high gall index (72\%), treatments 3, 4 and 6 presented the lowest gall indexes with 20,8 and $14 \%$ respectively, seedlings transplanted in the substrate treated with fenamiphos showed symptoms of phytotoxicity and 14 days later died, finally the seedlings in the substrate with fluensulfone presented a gall index of $30 \%$. These results suggest that Paecilomyces lilacinus represents an alternative for Nacobbus aberrans control in tomato crops.

Genetic and functional analysis of $v a$ resistance durability to Potato virus $\boldsymbol{Y}$ in tobacco

V. MICHEL (1), E. Julio (2), J. Cotucheau (2), F. Dorlhac de Borne (2), L. Glais (3), E. Jacquot (4), V. Decroocq (1), T. Candresse (1), S. GermanRetana (1), (1) INRA Bordeaux, UMR 1332 BFP, Villenave d'Ornon, FRANCE; (2) Imperial Tobacco, Bergerac, FRANCE; (3) FN3PT/RD3PT, Paris, FRANCE; (4) INRA, Montpellier Cedex, FRANCE

Many recessive resistances against potyviruses are mediated by eukaryotic translation initiation factors (eIF4E). In tobacco, the $v a$ resistance gene commonly used to control PVY corresponds to a large (almost 1Mb) deletion affecting a copy of eIF4E (S10760) [Julio, E. et al., 2014, Plant Mol. Bio. Rep.]. We compared the resistance durability conferred by various types of mutations affecting the $\$ 10760$ gene (large deletions, Frameshift, or EMS nonsense mutations). Thirteen tobacco genotypes were challenged with ten PVY isolates according to their biological and molecular characteristics. The "large deletion" genotypes display the largest resistance spectrum and durability towards the 10 PVY isolates, whereas, the "Frameshift" and "EMS nonsense" mutants display the less durable resistance, with rapid and frequent apparition of resistance-breaking PVY isolates. Upon further investigations, genetic and transcriptomic analyses revealed that resistance durability is strongly influenced by a complex genetic locus which contains two eIF4E copies, one of which is truncated in the most stable genotypes, while the other is differentially expressed between the tested varieties. RNASeq transcriptomic data and Q-PCR experiments demonstrate that the expression level of this second copy, T021658, is positively correlated with resistance durability. This suggests that T021658 might act as a decoy, in a virus-plant non-productive interaction and limiting the ability of PVY to evolve towards resistance-breaking. Taken together these results show that $v a$ durability can be explained by redundancy/competition effects in the eIF4E gene family, similar to what was recently shown in tomato [Gauffier, C. et al., 2016, Plant J.].

\section{Characterization of a symptom determinant of grapevine fanleaf virus suggests a novel function for its RNA-dependent RNA polymerase} L. J. OSTERBAAN, M. Fuchs, Cornell University, Geneva, NY, USA

Fanleaf degeneration is one the most devastating viral diseases of grapevine. It is caused by a number of viruses of the genus Nepovirus (family Secoviridae), including Grapevine fanleaf virus (GFLV). These viruses have a bipartite $(+)$ sense RNA genome encapsidated in isometric particles and are transmitted by dagger nematodes. Grapevines infected with GFLV exhibit a range of symptoms such as fan-like leaf morphology, mosaics, vein yellowing, shortened internodes and premature death. Though GFLV is one of the most well characterized grapevine viruses, the mechanisms underlying symptom development remain poorly understood. Previous research revealed that symptom determinant(s) of GFLV strain GHu on Nicotiana benthamiana, a systemic herbaceous host, map to the 3'-end of the RNA-dependent RNA polymerase $\left(1 \mathrm{E}^{\mathrm{Pol}}\right)$ coding region. Mutagenesis of amino acids within this region of protein $1 \mathrm{E}^{\mathrm{Pol}}$ followed by infectivity studies in planta ruled out several residues unique to strain GHu as being individually responsible for symptom development. Unexpectedly, some of the corresponding chimeric viruses failed to establish systemic infections in $N$. benthamiana, suggesting that this region may also be involved in systemic movement, a function not previously ascribed to the $1 \mathrm{E}^{\mathrm{Pol}}$ protein. These results highlight the multi-functional nature of this GFLV protein and advance our understanding of molecular interactions that enable infection and symptom development by GFLV.

New products for managing lesion nematode on Easter lilies

B. B. WESTERDAHL (1), D. D. Giraud (2), (1) University of California, Davis, Davis, CA, USA; (2) University of California, Eureka, CA, USA

California and Curry County, Oregon, USA. Lesion nematode, Pratylenchus penetrans, infestation of soil and roots seriously affects growth of field grown bulbs. During two years of field trials, commercial formulations of essential oils and a nematode parasitic fungus were compared to the control and a chemical standard (1,3-dichloropropene plus metam sodium [FU]) applied preplant followed by Thimet [TH] at planting to determine their value in the management of lesion nematode, and in improving plant health. The essential oil products Duogard, EF400, EF300, and Cinnamite were tested as preplant dips to bulblet planting stock. MeloCon (the fungus Paecilomyces lilacinu)s was tested as a soil treatment applied at-planting. Trials were statistically evaluated at $\mathrm{P}=0.05$. The essential oils and MeloCon were tested either alone, in combination with $\mathrm{TH}$ at planting, at planting following FU, or in combination with TH at planting following FU. In the first trial, 16 treatments had a greater bulb circumference than the control. In the second trial, 20 had a greater bulb circumference than the control. Thirteen treatments outperformed the control in both years. Nine treatments in the first trial, and 22 in the second trial had lower levels of lesion nematode within roots at harvest than the control. Nine treatments performed consistently over the two years of trials.

Potential of native yeasts from Washington vineyards as biocontrol agents against Botrytis bunch rot of grape

X. WANG (1), E. Kramer (1), D. A. Glawe (1), T. D. Murray (1), D. M. Weller (2), P. A. Okubara (2), (1) Washington State University, Pullman, WA, USA; (2) USDA-ARS Wheat Health, Genetics and Quality Research Unit, Pullman, WA, USA

Public concerns about the use of chemical fungicides against Botrytis bunch rot and the emergence of fungicide resistance in the pathogen Botrytis cinerea can be partially alleviated using biocontrol strategies. The objectives of this study were to evaluate native yeast strains for their ability to colonize 'Thompson Seedless' grape berries, inhibit $B$. cinerea growth in vitro, and suppress disease symptoms on berries. Eleven yeasts strains, isolated from Washington vineyards, were identified as Aureobasidium pullulans var. pullulans, Candida saitoana, Curvibasidium pallidicorallinum, Metschnikowia chrysoperlae, Metschnikowia aff. pulcherrima, Meyerozyma guilliermondii, Saccharomyces cerevisiae and Wickerhamomyces anomalus. All yeast strains showed rapid multiplication on grapes berries by two days after inoculation. Variation in virulence on berries was observed among $B$. cinerea isolates from Washington State vineyards. Consistent symptom suppression occurred on berries treated with A. pullulans P01A006, Met. chrysoperlae P34A004, P40A002, Met. pulcherrima P01A016, P01C004, Mey. guilliermondii P34D003, and S. cerevisiae HNN11516. Biolog profiling of nutrient utilization of yeast and $B$. cinerea strains revealed separated metabolic groups, which provide new information for improving the efficacy of the biocontrol system. Further characterization of native yeasts in vineyards will contribute to an understanding of bunch rot management and coevolution of local pathogens and native biocontrol agents.

Mapping Loci that Control Tuber and Foliar Symptoms Caused by PVY in Autotetraploid Potato (Solanum tuberosum L.) W. L. DA SILVA (1), S. Gray (2,3), W. De Jong (4), (1) Cornell University, Ithaca, NY, USA; (2) USDA, Agricultural Research Service, Ithaca, NY, USA; (3) Cornell University/USDA-ARS, Ithaca, NY, USA; (4) Cornell Univ, Ithaca, NY, USA

Potato tuber necrotic ringspot disease (PTNRD) is a tuber deformity associated with infection by the tuber necrotic strain of potato virus $\mathrm{Y}$ (PVYNTN) PTNRD negatively impacts tuber quality, marketability, and poses a serious threat to seed and commercial potato production worldwide. PVY ${ }^{\mathrm{NTN}}$ symptoms differ in the cultivars Waneta and Pike; Waneta expresses severe PTNRD and foliar mosaic with vein and leaf necrosis, whereas Pike does not express PTNRD and mosaic is the only foliar symptom. To map loci that influence tuber and foliar symptoms, $244 \mathrm{~F}_{1}$ progeny of a cross between Waneta and Pike were inoculated with isolate PVY ${ }^{\mathrm{NTN}}$-NY090029 and also genotyped using 12808 Potato SNP markers. Foliar symptom type and 
severity were monitored for 10 weeks, while tubers were evaluated for PTNRD expression at harvest and again after 60 days in storage. Pairwise correlation analyses indicate a strong association between PTNRD and veinal necrosis $(\tau=0.4195)$. QTL analyses revealed major-effect QTLs on chromosomes IV and V for mosaic, IV for PTNRD, and V for foliar-necrosis symptoms. Locating QTLs associated with PVY-related symptoms provides a foundation for breeders to develop markers that can be used to screen out potato clones with undesirable phenotypes, e.g., those likely to develop PTNRD or to be symptomless carriers of PVY.

Thermo-stability, dose effects and shelf-life of antifungal compounds produced by the symbiotic bacterium Xenorhabdus szentirmaii S. Hazir (1), D. I. Shapiro-Ilan (2), C. H. BOCK (3), L. G. Leite (4), (1) Faculty of Arts and Sciences, Department of Biology, Adnan Menderes University, Aydin, TURKEY; (2) USDA/ARS, Southeastern Fruit and Tree Nut Research Laboratory, Byron, GA, USA; (3) USDA ARS, Southeastern Fruit and Tree Nut Research Laboratory, Byron, GA, USA; (4) Instituto Biologico, APTA, Campinas, BRAZIL

Xenorhabdus spp bacteria are associated with Steinernematid nematodes and produce antifungal metabolites that protect nematode-infected cadavers from fungal colonization. Previous work demonstrated concentrated or cell-free metabolites of $X$. szentirmaii were toxic to fungal phytopathogens. We prepared cell free extracts by filtration and heating, determining 1. thermo-stability, 2. dose-response, and 3. shelf-life of antifungal metabolites of $X$. szentirmaii against Monilinia fructicola (cause of brown rot of peach and other stone fruit) and Glomerella cingulata (cause of antharacnose). Thermostability was determined by autoclaving bacterial culture broths $\left(121^{\circ} \mathrm{C}\right.$ and $15 \mathrm{psi}$ for 15 minutes $)$. Bacterial culture broths were filtered $(0.22 \mathrm{~mm}$ Millipore filter) to remove bacterial cells. Subsequent growth of phytopathogens was measured on amended potato dextrose agar (PDA) containing $10 \%$ of the supernatants from the autoclaved or filtered samples. Autoclaving had no impact on antifungal activity. Autoclaved or filtrated supernatants were tested monthly for 9 months: activity was not altered when stored at room temperature, $4^{\circ} \mathrm{C}$ or $-20^{\circ} \mathrm{C}$. A dose-response study $(10,20,40,60,80$ and $100 \%$ supernatant-containing metabolite) demonstrated that a greater dose of supernatant increased antifungal activity. The supernatant of $X$. szentirmaii has potential as a bio-fungicide. The results demonstrate the metabolite(s) are thermo-stable, they have a long shelf-life.

Role of lysine residues of the Magnaporthe oryzae effector AvrPiz-t in effector and PAMP-triggered immunity

P. BAI (1), C. H. Park (1), G. Shirsekar (2), P. Songkumarn (1), M. D. Bellizzi (1), G. L. Wang (1), (1) The Ohio State University, Columbus, OH, USA; (2) Max Planck Institute for Developmental Biology, Tuebingen, GERMANY

Magnaporthe oryzae is an important fungal pathogen for both rice and wheat. How $M$. oryzae effectors modulate plant immunity is not fully understood. Our published research shows that the $M$. oryzae effector AvrPiz-t targets the host ubiquitin-proteasome system to manipulate plant defense, in return, AvrPiz-t is ubiquitinated by the two rice ubiquitin E3 ligases APIP6 and APIP10 for degradation. To find the precise role of lysine residues in AvrPiz-t ubiquitination and stability, we mutated all the six lysines into arginines in AvrPiz-t and generated the lysine deficient mutant LL-AvrPiz-t. We found that the LL-AvrPiz-t protein became more stable than the wild-type AvrPiz-t when co-expressed with APIP10 in Nicotiana benthamiana. In contrast to the resistance phenotype to $M$. oryzae isolate RB22-AvrPiz- $t$, Piz-t-carrying plants showed disease lesions when inoculated with isolate RB22- $L L$ AvrPiz-t. Moreover, Piz-t:HA plants inoculated with RB22-LL-AvrPiz- $t$ had significantly less accumulation of Piz-t:HA protein comparing with that in the RB22-AvrPiz- $t$ inoculated plants. Ectopic expression of AvrPiz- $t$ in rice Nipponbare background led to 50\% reduced reactive oxygen species (ROS) generation by chitin treatment comparing to Nipponbare, whereas there is only $20 \%$ reduction in $L L-A v r P i z-t$ transgenic plants. The $A v r P i z-t$ transgenic plants also showed bigger lesion size than the $L L-A v r P i z-t$ plants when inoculated with the virulence isolate RB22. Altogether, our results show that lysine residues of AvrPiz-t are required for its stability and function in Piz-t-mediated resistance and host basal defense.

Localization and functional study of Ustilago hordei avirulence effector UhAVR1 using barley and Nicotiana benthamiana plant systems A. P. MONTENEGRO ALONSO (1), G. Bakkeren (2), (1) University of British Columbia, Vancouver, CANADA; (2) Agric \& Agi Food Canada, Summerland, BC, CANADA

UhAVR1 is a 190-aa protein of the barley smut fungal pathogen Ustilago hordei (Uh). It has a predicted 19-aa signal peptide (SP) and this candidate effector was identified as an avirulence factor triggering an HR within $48 \mathrm{hrs}$ after infection (hpi) of cv. Hannchen having resistance gene Ruhl. To decipher its virulence function, host target and host response triggered upon its recognition, transcriptomic analyses and localization studies were performed. Barley seedlings from cvs. Odessa ( $r u h 1)$ and Hannchen (Ruhl), representing compatible and incompatible interactions respectively, were inoculated with teliospores from wild type and an UhAVRI deletion mutant. UhAvrl expression was quantified via ddPCR during infection from 24 hpi till heading showing expression only at the early biotrophic stage and peaking between 72 and 96 hpi. Global transcriptome analysis via RNA-seq at 48 hpi revealed 151 differentially expressed genes (DEGs) in Odessa including kinases, transcription factors, chloroplast genes among others, whereas in Hannchen 173 DEGs were detected including peroxidases, $R$ genes, and chitinases, among others. To identify putative interactors, localization experiments were performed. In heterologous Nicotiana benthamiana $(\mathrm{Nb})$, chimeric UhAVR1-SP, C-terminally tagged with eGFP, was delivered by Agrobacterium tumefaciens into infiltrated leaves. Confocal microscopy revealed localization to cytosolic bodies at 24 and 48 hpi, compared to free eGFP control which was found in the nucleoplasm and cytosol. Protein analysis from leaf samples at the same time points by Western blots using antiGFP and anti-UhAVR1 antibodies, confirmed the expression of full length chimeric mature UhAVR1 protein of $46 \mathrm{kDa}$.

Characterization of an Aspergillus flavus population from Argentina and its potential use as biocontrol agents for mycotoxins in maize B. X. Camiletti (1), J. Moral (2), A. K. Torrico (3), C. M. Asensio (1), M. D. L. P. Giménez Pecci (3), E. I. Lucini (1), T. J. MICHAILIDES (4), (1) Universidad Nacional de Córdoba, Córdoba, ARGENTINA; (2) Univ De Cordoba/ Univ Davis, Parlier, CA, USA; (3) Instituto Nacional de Tecnologia Agropecuaria, Córdoba, ARGENTINA; (4) University of California - Davis, Parlier, CA, USA

Species of genus Aspergillus contaminate more than $30 \%$ of maize kernels grown in Argentina with mycotoxins, mainly aflatoxin and cyclopiazonic acid (CPA). Biocontrol products formulated with atoxigenic strains of $A$. flavus are well known as an effective method to reduce this contamination. Here, we characterized an A. flavus population from two maize regions of Argentina and evaluate its atoxigenic strains as potential biocontrol agents (BCA). The entire group of strains (84) was tested for aflatoxin and CPA production on inoculated maize kernels. The fungal population was characterized according to its vegetative compatibility groups (VCGs). Co-inoculation tests were conducted using toxigenic and atoxigenic strains to evaluate the potential use of the latter as BCAs. A $33 \%$ of the strains produced aflatoxin (from 0.53 to $25.05 \mu \mathrm{g} / \mathrm{g}$ ) and $83 \%$ of them produced CPA (from 0.04 to $21.67 \mu \mathrm{g} / \mathrm{g}$ ). Only $17 \%$ of our isolates neither produced aflatoxins nor CPA. The 57 non-aflatoxigenic strains formed six VCGs, with AC1 and AC5 being the dominant groups with a frequency of 58 and $35 \%$, respectively. All the atoxigenic strains showed aflatoxin and CPA reductions, ranging from 53.8 to $83.4 \%$ and from 59.8 to $96.9 \%$, respectively. Members of AC1 group showed a greater aflatoxin reduction than members of AC5 ( 72.8 vs $65.3 \%$ ) but no differences were found for CPA. This is the first report on atoxigenic isolates of $A$. flavus with a potential use as BCAs in maize crops in Argentina.

\section{A unique group of CLE effectors from cyst nematodes in the Globodera genus}

P. Lang (1), S. Chen (1), I. A. Zasada (2), M. G. Mitchum (3), X. WANG (1,4), (1) Cornell University, School of Integrative Plant Science, Ithaca, NY, USA; (2) USDA ARS, Corvallis, OR, USA; (3) University of Missouri, Columbia, MO, USA; (4) USDA-ARS, Robert W. Holley Center for Agriculture and Health, Ithaca, NY, USA

Cyst nematodes (Globodera and Heterodera spp.) are among the most devastating plant pathogens causing significant crop losses worldwide. During infection, cyst nematodes secrete CLAVATA3/ESR (CLE)-like effector proteins that act as mimics of plant CLEs to modulate plant developmental pathways required for successful parasitism. We previously studied a small group of multi-domain CLE effectors from G. rostochiensis. To explore sequence and functional conservation of nematode CLEs, we cloned 23 new CLE sequences from G. pallida, G. ellingtonae, and G. tabacum. As 
reported for G. rostochiensis $C L E \mathrm{~s}$, the majority of these newly cloned CLEs were found to encode multi-domain CLE proteins. When combining with previously reported sequences, in total 34 Globodera CLE sequences have been obtained, which encode CLE proteins that likely give rise to 37 CLE peptides when proteolytically processed in planta. We performed phylogenetic analysis on the 37 Globodera CLE peptides with plant CLEs and functionally characterized a few Globodera CLEs using in planta assays. Our results indicate that these Globodera CLEs have redundant as well as divergent functions. We also performed an alanine scanning experiment and identified several residues in a representative dodeca-CLE peptide as critical to the root growth suppression activity and the binding of the potato CLV2 receptor. The collection of these Globodera CLEs provides a basis for further evolutionary and functional studies.

Sensitivity of biocontrol agents to common soybean seed treatment fungicide active ingredients

J. V. LACEY (1), A. Kachroo PhD (1), A. M. Fakhoury (2), C. A. Bradley PhD (3), (1) University of Kentucky, Lexington, KY, USA; (2) Southern Illinois University, Carbondale, IL, USA; (3) University of Kentucky, Princeton, KY, USA

Potential biological control organisms were obtained from a previous multi-state study in which isolates were obtained from soybean roots growing in fields. Fourteen of these isolates, which were 4 isolates of Trichoderma hamatum, 5 isolates of $T$. harziarum, 2 isolates of $T$. virens, 2 isolates of Mortierella elongata, and 1 isolate of Bionectria ochroleuca, were tested for their sensitivity to six fungicide active ingredients that can be found in common fungicide seed treatment products. Each fungicide tested represented a different chemistry class, which were pyraclostrobin (quinone outside inhibitor), prothioconazole (demethylation inhibitor), sedaxane (succinate-dehydrogenase inhibitor), fludioxonil (phenylpyrrole), thiabendazole (methyl benzimidazole carbamate), and metalaxyl (phenylamide). Plates with potato dextrose agar were amended with each fungicide at a rate of $1 \mathrm{ppm}$, and a $5 \mathrm{~mm}$ plug of an actively growing culture was placed at the center. Sensitivity of the isolates were assessed by the percent of mycelial growth inhibition: $[1-\{($ Growth on amended $) /($ Growth on non-amended $)\} \times(100)]$. Each isolate varied in its sensitivity to select fungicides. The results of this analysis indicate that some organisms found within the rhizosphere can be used in conjunction with select fungicide seed treatments to offer enhanced diseased protection through biological means.

Integration and biological effect of the filamentous phage $\phi$ Rs551 on its Ralstonia solanacearum host A. A. AHMAD (1,2), Q. Huang (1), (1) FNPRU, US National Arboretum, USDA/ARS, Beltsville, MD, USA; (2) MINIA University, EL-MINIA, EGYPT

Ralstonia solanacearum is a soil-borne plant pathogenic bacterium causing serious diseases in a wide range of economically important crops around the world including tomato and potato. Its race 3 biovar 2 strains cause devastating potato brown rot and are listed as select agent pathogens due to their potential threat to U.S. agriculture. Previously, we reported the isolation and characterization of an active filamentous phage $\phi$ Rs551 from the supernatant of $R$. solanacearum race 3 biobar 2 strain UW551 grown under normal culture conditions. Genome analysis revealed that $\phi R s 551$ is the first isolated virion that contains both a putative integrase encoded by its ORF13 and a type- 2 phage repressor encoded by its ORF14. In this study, we determined how $\phi$ Rs551 integrates into its host bacterium and the biological effect of its infection. We found that $\phi$ Rs 551 integrated into the $R$. solanacearum host strain by site-specific recombination between a core phage attachment site $a t t P$ and the bacterial attachment site $a t t B$, mediated by $\phi R$ s551's ORF13. This is based on our discovery that when the ORF13 and the core attP site of $\phi$ Rs551 were either deleted individually or in combination, phage integration was not observed. Infection of a R. solanacearum host strain by $\phi$ Rs551 resulted in different colony morphology and reduced motilities of the bacterium. Interestingly, infection by $\phi$ Rs 551 also resulted in reduced virulence. This is in contrast to the enhanced or loss of virulence caused by other $R$. solanacearum filamentous phages, suggesting a possible role played by the type-2 phage repressor in $\phi R s 551$.

\section{Dissecting the pathogenicity mechanisms of Phytophthora palmivora}

M. TIAN (1), R. L. Gumtow (1), N. Navet (1), D. Wu (1), S. Schornak (2), J. Y. Uchida (1), (1) University of Hawaii at Manoa, Honolulu, HI, USA; (2) Sainsbury Laboratory, University of Cambridge, Cambridge, UNITED KINGDOM

Phytophthora palmivora is a broad host range oomycete that infects many economically important plant species, such as papaya and cacao. To dissect its pathogenicity mechanisms on papaya, which is very rich in plant defensive cysteine protease papain, we identified four putative cystatin-like extracellular protease inhibitors (PpmEPICs) from P. palmivora transcriptome. Among them, PpmEPIC8 was shown to be highly induced during infection and a strong inhibitor of papain. PpmEPIC8 mutants generated via CRISPR/Cas9 gene editing exhibited increased papain sensitivity of $i n$ vitro growth and reduced pathogenicity, suggesting that PpmEPIC8 plays a significant role in P. palmivora infection of papaya by inhibiting papain. In addition, we are characterizing a cytoplasmic effector that is highly conserved in plant pathogenic oomycetes and designated as Oomycete RxLR Conserved Effector 1 (ORCE1). ORCE1 homologs exhibit remarkable degree of amino acid sequence conservation and are under purifying selection suggesting that they play fundamental roles in parasitizing their host plants. Overexpression of ORCE1 in P. palmivora increased virulence. P. palmivora ORCE1 (PpmORCE1) was highly expressed in certain developmental stages and during infection. We are generating PpmORCE1 mutants via gene editing to further determine its role in development and pathogenicity. Dozens of host proteins were identified as potential targets of ORCE1 using yeasttwo-hybrid. During the above studies, we have established easy and effective genetic modification tools for P. palmivora. These studies will help dissect infection mechanisms of $P$. palmivora and other oomycetes, which in turn help us develop effective disease control strategies.

\section{Controlling soilborne pathogens by use of biologicals}

B. ESPEJO (1), E. Gachango (2), M. Twizeyimana (3), S. Ronyak (4), K. Tyson (4), K. Craig (4), P. E. Hammer PhD (4), (1) AgBiome, Inc, Research Triangle Park, NC, USA; (2) AgBiome, Inc., Research Triangle Park, NC, USA; (3) AgBiome Inc., Durham, NC, USA; (4) AgBiome, Research Triangle Park, NC, USA

AgBiome has built a core collection of sequenced microbes from plant-related environmental sources. The microbes are screened in high-throughput bioassays against a broad range of fungal pathogens, insect pests, and plant-parasitic nematodes. Activity against soilborne pathogens, Rhizoctonia solani and Pythium aphanidermatum, is assessed in the lab and greenhouse through screening paradigms involving treatment of cucumber seeds with biological strains. The treated seeds are planted in soil inoculated with either $R$. solani or P. aphanidermatum, and strain activity is evaluated by observing seedling emergence and rate of infection. In 2016, several hits from the lab and greenhouse-based assays were selected and will be tested in the field this year. Trends in activity will be monitored for broad spectrum, including activity against Pythium spp., Rhizoctonia solani and Fusarium spp. on soybean.

Phytophthora hijacks host BAG7 and bZIP28 resulting in sequestration in endoplasmic reticulum for compatibility via effector-mediated BiP accumulation

M. JING (1), Y. Li (1), Y. Wang (2), M. B. Dickman (1), (1) Texas A\&M University, College Station, TX, USA; (2) Nanjing Agric Univ, Nanjing, Jiangsu, CHINA

The endoplasmic reticulum (ER) is a central hub for stress responses to pathogens as well environmental Phytophthora species secrete a large array of specific effector proteins to manipulate host innate immunity. Phytophthora uses an essential effector, Avh262 to stabilize Binding immunoglobulin Proteins (BiPs) in the ER, that act as negative regulators of plant resistance to Phytophthora and ER stress-induced cell death (ERCD), can result in attenuated plant defense responses. However, little is known about how Phytophthora hijack the host BiPs to regulate the ER machinery for successful infection. Here we show Avh262 interacts with both AtBAG7, an ER-localized cochaperone that helps maintain for the maintenance of the unfolded protein response, and bZIP28, an ER membrane-tethered transcription factor. The translocation of AtBAG7 and bZIP28 from the ER to the nucleus is required for activating the downstream pathways. We found AtBAG7 negatively regulates plant resistance to Phytophthora, and bZIP28 plays a positive 
role. The culture filtrate of Phytophthora triggers the translocation of AtBAG7 and bZIP28 from the ER to the nucleus, which can be prevented by the Avh262-mediated accumulation of BiPs. In conclusion, Phytophthora pathogens secrete effectors to accumulate high level of BiPs, which retains AtBAG7 and bZIP28 in the ER lumen, resulting in suppressing the ERCD pathway and blocking expression of downstream defense genes, beneficial to infection.

Effect of a contact sterilant on apple flower microbial populations and impacts on biological control of fire blight by Aureobasidium pullulans S. M. SLACK (1), S. Gebben (1,2), B. Begley (1), C. A. Outwater (1), G. W. Sundin (1), (1) Michigan State University, East Lansing, MI, USA; (2) University of Florida, Immokalee, FL, USA

Due to concerns of antibiotic resistance along with increased interest in organic agriculture, there is a need for novel strategies to protect orchards from fire blight (Erwinia amylovora) outbreaks. Organic apple producers rely on copper and biological control agents to suppress fire blight outbreaks at bloom. In Michigan and the Eastern United States however, there has been little success historically using biological agents to manage fire blight, mainly due to humid climates that favor rapid growth and infection by E. amylovora. Humidity also likely increases the size and diversity of the apple flower microbial community which may hinder the effectiveness of the biological control agent due to competition. In a four-year study, we used a contact sterilant (Oxidate) to clear the apple flower niche prior to application of Blossom-Protect, a biological control agent containing two strains of $A$. pullulans. We also examined the efficacy of various application numbers and timings of Blossom Protect in controlling blossom blight. Cultural populations of bacteria, total fungi, and yeast were evaluated in all treatments. Although the Oxidate application did significantly reduce culturable microbial populations, increases in A. pullulans populations on flowers were not observed in all experiments. The Oxidate treatment also did not result in further reductions of blossom blight compared to comparable Blossom Protect treatments without Oxidate.

Differential antibiosis of hydrolytic enzymes and metabolites by Paraguayan isolates of Trichoderma spp. against Macrophomina phaseolina F. A. Villalba Silvero (1), A. D. SANABRIA (2), M. E. Flores-Giubi (1), J. E. Barua (1), (1) Universidad Nacional de Asuncion, Central, PARAGUAY; (2) Paraguayan Institute of Agrarian Technology (IPTA), Central, PARAGUAY

Several agricultural crops are affected by soilborne pathogens like the phytopathogenic fungus Macrophomina phaseolina that causes charcoal rot disease. In this work was evaluated the antagonistic ability of 25 Paraguayan isolates of Trichoderma spp. against M. phaseolina isolated from soybean (Glycine $\max$ (L.) Merr.) and sesame (Sesamum indicum L.). Direct confrontations in dual cultures were performed for both isolates of M. phaseolina, FCQ26 (sesame) and FCQ39 (soybean), against 25 Trichoderma isolates. The growth of the phytopathogens was measured every 24 hours and the Percentages of Growth Inhibition (PGI) were calculated. For the antibiosis assay, Trichoderma strains were grown on cellophane and dialysis membranes (cutoff, $10 \mathrm{kDa}$ ) to allow the diffusion of Trichoderma extracellular compounds into the medium. After the removal of the membranes containing the mycelia, the effect of such hydrolytic enzymes plus metabolites (cellophane) or only metabolites (dialysis) on the growth of both isolates of $M$. phaseolina was determined. In the confrontation assay, all Trichoderma isolates exerted inhibition on the growth of M. phaseolina FCQ26 and FCQ39. Trichoderma isolates FCQ36, FCQ37, and FCQ45 showed higher antagonism against $M$. phaseolina FCQ26; while against M. phaseolina FCQ39 seven Trichoderma isolates showed high antagonistic capacity. In the antibiosis assay, it was observed that the metabolites exert inhibition on the growth of both phytopathogen isolates and that when these molecules were in the presence of hydrolytic enzymes, in some cases the antibiosis was increased, in other cases remained the same and in others decreased antibiosis.

Bioplastic seed coating formulations combining pesticides with biocontrol isolates to control agricultural pests H. K. ABBAS (1), C. Accinelli (2), W. T. Shier (3), (1) USDA ARS BCPRU, Stoneville, MS, USA; (2) University of Bologna, Bologna, ITALY; (3) University of Minnesota, College of Pharmacy, Minneapolis, MN, USA

Film-coating of agronomic seeds is a rapidly developing technology that generally uses petroleum-derived polymers and synthetic pesticides. However, serious consideration has recently been given to using renewable source polymers and reducing use of synthetic pesticides. We evaluated the feasibility of film-coating agronomic seeds with a starch-based bioplastic formulation, and combining conventional pesticides with beneficial microorganisms. Corn and canola seeds were film-coated with bioplastic formulations containing the insecticide imidacloprid, the fungicide metalaxyl-M, the growth promoting fungus Trichoderma harzianum or a non-aflatoxigenic biocontrol isolate of the fungus Aspergillus flavus. Bioplastic coating did not affect germination of either seed type in vitro or in soil. Bioplastic film-coating reduced dust-off from coated seeds by up to $95 \%$ compared to coating with a commercial polymer. This is important, particularly when neonicotinoid insecticides are included. When spores of the biocontrol fungus, $T$. harzianum, were incorporated into bioplastic coatings, growth of corn and canola seedlings was significantly stimulated (i.e., shoot and root lengths of corn seedlings were $29 \%$ and $44 \%$ longer, than in uncoated seeds, respectively; similar results were observed with canola). No growth stimulatory effects were observed with bioplastic coatings containing the biocontrol $A$. flavus isolate. Preliminary field studies indicated that coating corn seeds with bioplastic and spores of non-aflatoxigenic $A$. flavus biocontrol isolate significantly reduced aflatoxin contamination in harvested seed. More extensive studies are in progress in corn growing areas of USA and Southern Europe.

Serenade $^{\circledR}:$ Research advances in soil performance of the biological fungicidal/bactericidal product S. HOVINGA, Bayer Crop Science, West Sacramento, CA, USA

Growers face many challenges in producing quality fruits and vegetables every year. Pests and disease, abiotic stress, and the demand for sustainable crop protection solutions are just some of the examples of pressures on those in modern agriculture. Biological products offer a complimentary approach to existing crop management programs by demonstrating efficacy while also offering a potential for improvement in yield, quality, and nutritional value of a harvested crop. Serenade ${ }^{\circledR}$, based on a unique fermentation and formulation of the Bacillus subtilis strain QST 713, was originally developed as a foliar fungicide, due to the direct contact activity of lipopeptides present in the final product. Research continues on the bactericidal efficacy of the product focusing on the biological chemistry and induced systemic host response. Most recently, focused research continues to characterize the capabilities of Serenade ${ }^{\circledR}$ in the soil, due to the widespread beneficial effects on plant health observed with product use. Research on modes of action in the soil and the positive effects will be discussed, such as increased chlorophyll fixation, root growth, and in situ production of biological chemistry. Many examples from global research, field trial work, and grower demonstrations will be shown where plant health, yield, and quality are increased due to the benefits that Serenade ${ }^{\circledR}$ treatments provide in root environments. Capitalizing on the effects of biological products like Serenade ${ }^{\circledR}$ in combination with current commercial practices has the potential to increase the value and quality of harvested crops, while meeting sustainability needs of modern farming practices.

Integration of biological control and transgenic insect protection for mitigation of mycotoxins in corn M. A. WEAVER (1), H. K. Abbas (2), M. Brewer (3), L. Pruter (4), N. Little (5), (1) USDA ARS, Stoneville, MS, USA; (2) USDA ARS BCPRU, Stoneville, MS, USA; (3) Texas AgriLife Research and Extension Center-Corpus Christi, Corpus Christ, TX, USA; (4) Texas A\&M AgriLife Research and Department of Entomology, Corpus Christi, TX, USA; (5) Southern Insect Management Research Unit USDA-ARS, Stoneville, MS, USA

Field trials were conducted over two years in two states (MS and TX) to examine the efficacy of biological control of aflatoxin in corn and the interactions with other mycotoxins and transgenic insect protection. Corn hybrid N78N3111, expressing the Cry 1Ab, Cry3A and Vip3Aa20 insecticidal proteins, was nearly 100 percent free from corn earworm damage and generally $\geq 50 \%$ as much fumonisin contamination as compared to N78NGT, a near isogenic corn hybrid without insect protection. This insect protection, however, did not significantly prevent aflatoxin contamination. Soil application of non-aflatoxigenic biocontrol strains of Aspergillus flavus significantly reduced aflatoxin concentrations in corn. Biocontrol strain 21882 of 
A. flavus was especially effective, reducing aflatoxin contamination by about 90 percent over the seven field trials. There was no significant interaction between the insect protection and biocontrol treatments. Although no synergies were detected, the reduction of mycotoxins by both strategies supports application of both strategies in tandem. Economic factors external to the cost of the technologies will be a major determinant if the mycotoxin mitigation attained by use of these technologies will have a positive economic benefit.

Metabolomic-based elucidation of fungicide-microbe interactions in banana and potato fungal pathogens J. Cevallos-Cevallos, M. G. MARIDUENA-ZAVALA, A. Freire-Penaherrera, D. Ochoa, Escuela Superior Politecnica del Litoral, ESPOL, Guayaquil, ECUADOR

The production of bananas and potatoes is continuously being threatened by fungicide-resistant strains of Mycosphaerella fijiensis and Phytophthora infestans, respectively. A deeper understanding of the metabolomic-based fungicide-pathogen interactions would contribute to the development of new control mechanisms. Isolates of $M$. fijiensis and $P$. infestans were submitted to sensitivity tests using the thiabendazole and metalaxyl fungicides, respectively. All isolates were then grown in PDB for one week followed by exposure to 0,1 , or $100 \mathrm{mg} / \mathrm{L}$ of the respective fungicide for 6, 12, 24, and $48 \mathrm{~h}$ in triplicate runs. Mycelial metabolites were extracted by chloroform-methanol-water solutions, identified by GC-MS, and mapped to potential pathways using KEGG Mapper. The response of resistant isolates of $M$. fijiensis to thiabendazole was characterized by a significant upregulation of the metabolites form the fructose and mannose, galactose, and $\mathrm{ABC}$ transporters pathways with low levels of the fungicide observed within the fungal cells. Conversely, exposure of resistant isolates of $P$. infestans to metalaxyl activated the metabolites and pathways associated to membrane rigidity, including the fatty acid biosynthesis and the glycerophospholipid pathways, with no metalaxyl residues detected within the pathogen's cells. The inhibition of the pathways reported in this study could reduce the level of resistance of each pathogen and enhance the control action of the fungicides.

Preserving efficacy of new FRAC group from resistance development in impatiens downy mildew

S. N. SUAREZ (1), P. Lopez (1), A. R. Chase Zemke (2), A. J. Palmateer (3), (1) University of Florida, Homestead, FL, USA; (2) Chase Agricultural Consulting, Cottonwood, AZ, USA; (3) Bayer Environmental Science, Homestead, FL, USA

The re-emergence of impatiens downy mildew (IDM), caused by Plasmopara obducens, in Florida has been the focus of collaborative research efforts for the last five years. While researchers work on understanding the biology and epidemiology of the pathogen, short-term management of IDM is crucial for the industry, particularly growers. Thus far, the only management option for IDM available is chemical control. Based on results from over five years of fungicide efficacy data, the Florida population of $P$. obducens has become resistant to most industry fungicides. Last year, during preliminary field trials in Homestead, FL, oxathiapiprolin provided the longest residual control of IDM in the landscape compared to previous industry standards for downy mildews. Oxathiapiprolin is a novel chemistry in the new FRAC group U15. As a proposed single-site inhibitor, risk for resistance development is assumed to be extremely high. Since $P$. obducens has already exhibited reduced sensitivity to single-site inhibitors (and previous industry standards) mefenoxam (Group 4) and fluopicolide (Group 43), it is assumed resistance to oxathiapiprolin will develop if precautionary measures are not taken. In addition to its excellent residual efficacy, oxathiapiprolin can also be used at low rates and as a seed treatment in other crops the number of applications needed to give adequate control in the landscape is also low. Rotation of or tank mixing with products containing chemistries in different FRAC groups is of utmost importance in order to preserve the efficacy of this new active ingredient.

\section{A Bacillus-based biocontrol system for the mitigation of Aspergillus ear rot and aflatoxin contamination}

S. CHALIVENDRA (1), J. A. Reyes-Pineda (1), C. DeRobertis (1), K. E. Damann (2), (1) Louisiana State UniversityAgCenter, Baton Rouge, LA, USA; (2) Louisiana State University AgCenter, Baton Rouge, LA, USA

The intraspecific aflatoxin (AF) biocontrol requires a physical contact of the biocontrol strain with the toxigenic strain within the first $24 \mathrm{~h}$. This necessitates a continuous deployment of atoxigenic strains every season and may risk the emergence of super toxigenic strains. Biocontrol systems that do not need the presence of another living organism or preclude recombination when physically present will be better alternatives to intraspecific ones. We are developing a biocontrol system using Bacillus isolates commensalistic on the surface of rice leaves. Of the 28 isolates tested on 3 different $A$. flavus lines, $>50 \%$ showed a zone of inhibition, with the isolate 4R being the most fungistatic. The top 5 isolates were field tested for their suppression of $\mathrm{AF}$ in corn and $4 \mathrm{R}$ proved to be the most effective. Since a zone of A. flavus growth inhibition around the Bacillus isolates obviates the need for physical contact and implies the involvement of diffusible antifungal compounds, we tested all isolates on A. flavus growth and AF production. Results showed that the AF-suppressive action is independent of the fungistatic activity and many isolates inhibited AF without affecting $A$. flavus growth. Further, we extracted lipopeptides (LPs) from culture filtrates of isolates 1 and 4R and demonstrated their AF-suppressive activity by infusing them into the medium. Currently, we are purifying the LPs by preparative HPLC and testing them on A. flavus growth and AF synthesis. This approach may prove to be effective on a range of preharvest and postharvest fungal diseases, while avoiding the risk of developing super-toxigenic strains.

Blossom application of novel silver compounds for fire blight (Erwinia amylovora) management in apples

C. NISCHWITZ (1), M. W. Harding (2), E. Petrizzo (1), P. Nadworny (3), (1) Utah State University, Logan, UT, USA; (2) Alberta Agriculture and Forestry, Crop Diversification Center South, Brooks, AB, CANADA; (3) Innovotech, Inc., Edmonton, AB, CANADA

Fire blight, a devastating disease of apple and pear worldwide, causes annual losses of millions of dollars (US). Current primary disease management tools are treatment with streptomycin or kasugamycin, or removal of infected trees. In the US and other countries, antibiotic resistant isolates have appeared, making disease management more difficult. If blossom infections can be decreased/eliminated, yield/tree losses can be greatly reduced. Agress $^{\circledR}$ and AgreGuard ${ }^{\mathrm{TM}}-1$, unique silver compounds for crop protection, could be effective antibiotic replacements. Flower clusters at full-bloom were spray-treated $24 \mathrm{~h}$ post-inoculation with E. amylovora. The Utah State University fire blight risk model showed "extreme" risk for fire blight infection on

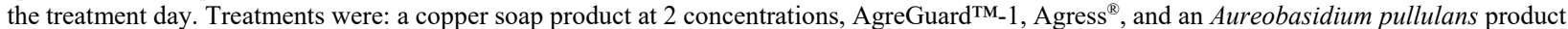
(applied 2-3 days pre-inoculation). Disease levels were compared to untreated controls, and streptomycin and kasugamycin standards in a replicated trial. Agress ${ }^{\circledR}$ outperformed all other products and controls, while AgreGuard ${ }^{\mathrm{TM}}-1$ was equivalent to antibiotic standards for disease incidence reduction. There were few systemic infections; none with silver treatment. No phytotoxicity was observed for any treatment. The results indicate that the silver products could be effective antibiotic alternatives in fire blight management even under conditions of very high disease pressure and infection risk.

\section{Inhibition of Fungal growth by bacterial volatiles}

\section{G. EBADZADSAHRAI, A. Harrison, S. Soby, Midwestern University, Glendale, AZ, USA}

There is increasing evidence that volatile organic compounds (VOCs) play an important role in the interactions between plants, fungi, and bacteria, but little is known about the mechanisms underlying the production of bacterial volatiles or their role in fungal growth inhibition. Sixty-eight bacterial isolates from wild and cultivated cranberry bogs were evaluated for VOC activity against Phytophthora spp., Phomopsis sp., Colletotrichum sp., Coleophoma sp., and Trichodermna sp. Individual bacterial isolates varied in antifungal activity within and between fungi, but Pseudomonas sp. and Chromobacterium sp. showed the strongest antagonistic effect against all fungi. Chromobacterium sp. is a gram-negative bacterium commonly found in soil and water, some strains of which produce the purple pigment violacein. Among 20 different Chromobacterium isolates, wild-type strains were significantly more inhibitory against the five fungi than mutants that are not capable of quorum sensing (QS). C. violaceum inhibited these fungi much better than a QS negative strain. Six new Pseudomonas species isolated from bogs also had VOC activity against fungi. Active VOCs are currently being identified from a range of bacteria using 2D gas chromatography time-of-flight mass spectrometry (GCxGC-TOFMS). 
Spatial pattern of Fusarium wilt (Fusarium oxysporum f. sp. cubense) in banana fields D. W. Heck (1), M. A. Dita Rodriguez (2), E. M. Del Ponte (1), E. S. G. MIZUBUTI (1), (1) Universidade Federal de Vicosa, Vicosa, BRAZIL; (2) Embrapa, Jaguariúna, SP, BRAZIL

Management of Fusarium wilt (FW) or Panamá disease of bananas depends on properly understanding the dynamics of the disease in time and space. Nevertheless, analyses of the spatial pattern of FW are scarce. The objectives of this work were to estimate disease intensity and to investigate the spatial pattern of FW. Thirty fields planted with 'Silk', 'Pome' or 'Cavendish' banana subgroups, totalling 91 ha, were evaluated. In each field, all plants were inspected and symptomatic plants were geo-referenced. Incidence maps were constructed and quadrat-based and point-process distance-based methods were used to investigate the spatial pattern of the disease. FW incidence varied from 0.03 to $45.3 \%$. The average incidence of FW in fields planted to 'Cavendish' (3 fields), 'Pome' (17), and 'Silk' (10) was 2.9\%, 7.7\%, and 20.0\%, respectively. Aggregation of FW was detected by all analytical methods in $50 \%$ of the fields ( 3 of 'Cavendish', 7 of 'Pome' and 5 of 'Silk'). In the other 15 fields ( 10 of 'Pome' and 5 of 'Silk') a total of 135 tests were conducted: 95 revealed aggregation and 40 randomness. In the Vale do Ribeira region, a traditional banana producing area, FW was aggregated in all sampled fields $(\mathrm{N}=4)$. Overall, aggregation was detected in $85 \%$ of the tests. The incidence of $\mathrm{FW}$ in bananas fields in Brazil is high and native populations of $F$. oxysporum $\mathrm{f}$. sp. cubense already present in the fields seem to be driving the spatial distribution of $\mathrm{FW}$.

Fungicides and biological controls for the management of Septoria leaf spot on stevia A. KOEHLER, D. Shew, North Carolina State University, Raleigh, NC, USA

Stevia (Stevia rebaudiana) is a perennial crop harvested for leaves that contain numerous glycosides extracted for use as a nonnutritive sweetening agent Septoria leaf spot was first observed in NC in 2015. Leaf lesions were present throughout the season but spread rapidly and began to coalesce to cause significant defoliation during favorable environmental conditions in September, prior to harvest. There are no fungicides labeled for use on stevia. A pesticide that can reduce end of season lesion expansion and defoliation prior to harvest will be critical for reaching optimum yield potential. Initial efficacy trials for Septoria leaf spot were established in 2016 at two locations in North Carolina, testing seven fungicides and one biological control product. Products were applied twice at the end of the season and plants were rated for percent of leaf area covered with lesions. Trials were combined for analysis and there were significant treatment effects $(P<0.0001)$. All products tested, azoxystrobin + difenoconazole (mean percent leaf lesion area, 9), pyraclostrobin + fluxapyroxad (9.9), benzovindiflupyr + difenoconazole (11.1), benzovindiflupyr + azoxystrobin (11.6), chlorothalonil + azoxystrobin (13.6), tebuconazole + fluopyram (19.2), tebuconazole (20.2), and Serenade (60.5), reduced percent leaf lesion area compared to the untreated control (69.5). Successful management of Septoria leaf spot will impact the long term establishment of stevia in NC.

Temporal distribution pattern of Sclerotinia sclerotiorum apothecia is modulated by row spacing and weather variables in soybean M. L. FALL (1,2), J. F. Willbur (3), D. L. Smith (4), A. Byrne (5), M. Chilvers (5), (1) Agriculture and Agri-Food canada, St-Jean-sur-Richelieu, QC, CANADA; (2) Agriculture and AgriFood Canada, Saint-Jean-sur-Richelieu, QC, CANADA; (3) University of Wisconsin - Madison, Madison, WI, USA; (4) University of Wisconsin, Madison, WI, USA; (5) Michigan State University, East Lansing, MI, USA

Identifying the best fungicide application timing is crucial to maximize Sclerotinia stem rot (SSR; caused by Sclerotinia sclerotiorum) control. In this study, the impact of canopy closure and soil temperature on apothecia development was investigated to optimize fungicide application timing. Replicated soybean plots were established with 0.36- and 0.76-m row spacing's in 2015 and 2016 at the MSU Montcalm Research Center. The number of apothecia, ascospores and SSR were monitored two times per week. In both row spacing experiments, apothecia were observed earlier in 2016 (before R1 growth stage) than in 2015 (R2). The maximum number of apothecia was 50 times higher in the $0.36-\mathrm{m}$ vs. $0.76 \mathrm{~m}$ row spacing in 2015 whereas it was 2.5 times higher in the $0.76-\mathrm{m}$ than in the $0.36-\mathrm{m}$ row spacing in 2016 , though the overall numbers were much lower in 2016 . The apothecia distribution pattern was synchronized with the canopy closure pattern and the soil temperature profile. The peak number of apothecia was observed when the canopy closure reached $60 \%$ and soil temperature in row was between 21.5 and $23.5^{\circ} \mathrm{C}$. In $91 \%$ of the cases, apothecia presence was observed when the shade factor was at $70 \%$ and no apothecia germinated when the shade factor was at $0 \%, 30 \%$ and $100 \%$. In both row spacings, the rate of the canopy closure was two times higher in 2016 than in 2015 and the first diseased plant was observed earlier in 2016 (R2) than in 2015 (R5). These results can explain the inconsistent efficacy of fungicide applications based on soybean growth stage and help fine tune fungicide application strategies.

\section{Management of laurel wilt of avocado, caused by Raffaelea lauricola}

R. C. PLOETZ (1), J. Konkol (2), J. Pérez-Martínez (3), (1) University of Florida - Tropical Research and Education Center, Homestead, FL, USA; (2) University of Florida, Homestead, FL, USA; (3) University of Florida/University of Frankfurt, Homestead, FL, USA

Laurel wilt is caused by Raffaelea lauricola, a nutritional symbiont of an Asian ambrosia beetle, Xyleborus glabratus. In southern Florida, commercial production of an important crop, avocado (Persea americana), has recently been affected by this lethal disease; we summarize studies in which fungicides were tested for its management. Bark applications of Tilt (a propiconazole fungicide for which $18 \mathrm{C}$ emergency registration had been obtained in 2010) in a surfactant, Pentrabark, enabled significant laurel wilt protection on small potted plants in greenhouse studies, but Pentrabark and other surfactants moved little propiconazole into the xylem of fruit-bearing trees in field studies. In field efficacy studies, Propiconazole Pro (an injectable formulation of propiconazole), Tilt, and two experimental formulations of another triazole fungicide, tebuconazole, decreased the development of laurel wilt compared to nontreated control trees when applied as undiluted injections into branches and scaffold limbs (microinjection), or injection of dilute fungicide into tree flare roots (macroinfusion). However, symptoms developed in all treated trees by 10-11 months after inoculation with $R$. lauricola. Regardless of how fungicides were applied, insignificant levels of different active ingredients entered fruit. Management of laurel wilt in commercial avocado production areas will be discussed.

\section{Managing Helminthosporium solani, causal agent of silver scurf in Wisconsin potatoes} S. MACCHIAVELLI GIRÓN, S. A. Jordan, J. M. Crane, A. J. Gevens, University of Wisconsin-Madison, Madison, WI, USA

Silver scurf, caused by the fungus Helminthosporium solani, is a tuber blemish disease of potato which affects periderm quality and appearance, impacting marketability and storability. Cultural management strategies are often impractical, making fungicide applications an important component of integrated management. Helminthosporium solani initially infects tubers in the field, and has secondary infection cycles during storage. Because inoculum can be reduced at multiple times during production, we conducted a full life cycle study during 2015 to 2016 to evaluate the impact of fungicide selection and timing on silver scurf severity in 'Dark Red Norland' potatoes. Fungicides were applied at three times: at planting as seed or infurrow treatments; in-field as a series of 3 foliar sprays; and post-harvest immediately before storage. Non-treated comparisons were included. Results were variable between years. The at-planting treatments gave the greatest reduction in severity in 2015, and the post-harvest treatments were most effective in 2016. Stadium performed the best as a post-harvest treatment in both years. Foliar Phostrol applications provided no significant control in either year. In 2015, the lowest severity resulted from the combined program of a Maxim MZ seed treatment with a Stadium post-harvest treatment. In 2016, the lowest severity resulted from a Stadium post-harvest treatment, regardless of in-field treatments.

Spore production of Phyllosticta spp. in citrus leaf litter associated with citrus black spot in Florida K. ZHANG (1), J. A. Rollins (2), M. M. Dewdney (1), (1) University of Florida, Lake Alfred, FL, USA; (2) University of Florida, Gainesville, FL, USA

The causal agent of citrus black spot (CBS), Phyllosticta citricarpa is a quarantine fungal pathogen in Europe and the US. In the spring of 2010, CBS and the pathogen were found in Florida. Based on previous research, P. cit is heterothallic and due to the clonality of the Floridian P. cit population, 
conidia are presumed to be the primary inoculum in Florida. $P$. capitalensis is a globally distributed endophytic and homothallic species that is widely associated with diverse plant genera including citrus. Ascospores and conidia of $P$. cap are common in Floridian citrus leaf litter. Understanding the dynamics of spore production of these two species can help the development of CBS inoculum management. From May 2014 to May 2017,40 samples of 25 leaves were collected biweekly from leaf litter in a southwest Florida grove. Ascospores and conidia were collected from leaves with Phyllosticta spp. fructifications from each sample. Real-time PCR was performed to determine the presence and quantity of $P$. cit and $P$. cap DNA in leaf tissue, ascospores, and conidia. The results showed from 2014 to 2017, the biomass of $P$. cit in leaf litter tissue increased significantly $(\mathrm{P}<0.01)$ while the biomass of $P$. cap was consistent during 2015-2016 season $(\mathrm{P}=0.19)$ and 2016-2017 season $(\mathrm{P}=0.07)$. $P$. cap DNA is universally present in ascospore suspensions. The increasing ratio of $P$. cit to $P$. cap DNA in conidia suspensions indicated increasing conidia production of $P$. cit in groves.

Cold induced disease resistance may explain unexpected stalling of foliar epidemics of hop powdery mildew (Podosphaera macularis) B. WELDON (1), D. M. Gadoury (1), L. E. Cadle-Davidson (2), (1) Cornell University, Geneva, NY, USA; (2) USDA Grape Genetics Research Unit, Geneva, NY, USA

Epidemics of powdery mildew (Podosphaera macularis) on hop foliage are typified by an initial exponential increase of incidence, while severity, i.e., size of mildew colonies, remains relatively constant, despite daytime temperatures optimal for pathogen growth. A similar pattern reported in grapevine powdery mildew (Erysiphe necator) was associated with cold-induced disease resistance. We investigated the impact of both pre- and post-inoculation cold events on susceptibility of hop to $P$. macularis. Exposing young, ontogenically susceptible hop leaves to a brief $(1-4$ hour $)$ cold event $\left(4^{\circ} \mathrm{C}\right) 24$ hours before inoculation reduced $P$. macularis hyphal density by $26.6 \%$ and colony formation success by $16.5 \%$. A subsequent time course study involving acute cold events suggested that the magnitude of the effect of pre-inoculation cold events was maximized 36 hours after cold exposure. Sequential overnight acute cold events applied post-inoculation extended the latency period by approximately $20 \%$ and, 24 hours after initiation of sporulation, conidia production per colony was reduced by $72.8 \%$. The discovery of a parallel induction of disease resistance by brief overnight cold events in both grapevine and hop powdery mildew pathosystems suggests that host responses to acute nighttime radiational cooling might not only explain stalling of powdery mildew epidemics in grapevine and hop, but also extend to powdery mildews of other crops.

\section{Developing post-harvest fungicides for sugarbeet}

N. GLYNN (1), P. J. Kuhn (2), D. Ruppal (3), (1) Syngenta, Vero Beach, FL, USA; (2) Syngenta Crop Protection, Greensboro, NC, USA; (3) Syngenta Seeds, Akron, MI, USA

Profitability of sugar produced from sugarbeets is influenced profoundly by losses incurred during storage. Such losses vary annually due to a number of factors; storage conditions, in-field crop health, disease pressure. Storage rots caused by fungi such as Botrytis sp., Penicillin sp. and Fusarium sp. contribute to these losses by sucrose degradation and invert sugar formation. Suppression of these pathogens by fungicide application to sugarbeets could play an important role in reducing losses. The utility of three post-harvest fungicide products developed for use in other crops was evaluated for application in sugarbeets. Trials were conducted from 2012 to 2016 in three of the major sugarbeet growing regions of the USA. Harvested sugarbeets grown under commercial conditions were used. Fungicides Scholar, Graduate A+ or Stadium were applied to the roots using a back pack sprayer and an application volume of $0.5 \mathrm{gal} / \mathrm{ton}$. Beets were stored in representative commercial storage conditions. Sugar analysis and estimates of fungal colonization were performed on a representative sample of beets taken before storage, on a sub-sample taken after $\sim 1-3$ months in storage and at the conclusion of the trials after $\sim 3-4$ months in storage. The extent of pathogen colonization, loss of sucrose and increase in invert sugars were all reduced with fungicide application. These experiments highlight the potential of post-harvest applied fungicides towards reducing losses incured during storage of sugarbeets.

\section{Developing the capacity of reflectance spectroscopy for early late blight detection in potato}

K. MOREY GOLD (1), J. Couture (2), P. Townsend (1), A. J. Gevens (1), (1) University of Wisconsin-Madison, Madison, WI, USA; (2) Purdue University, West Lafayette, IN, USA

Late blight of tomato and potato caused by the oomycete pathogen Phytophthora infestans continues to be one of the most challenging diseases to sustainably and proactively manage. Advanced field-based methods of late blight detection that can identify infection before the onset of visual symptoms would improve management by greatly reducing disease potential and spread. The objective of this work was to explore the detection of late blight infection during its latent, or biotrophic phase, using reflectance spectroscopy. We conducted two controlled experiments in growth chambers using inoculated and control, non-inoculated plants to test the ability of reflectance spectroscopy for non-visual detection of infection. We measured continuous visible to shortwave infrared reflectance $(400-2500 \mathrm{~nm})$ on leaves of the plants using a portable spectrometer with contact probe at $8-12 \mathrm{hr}$ intervals. Our results indicated that we could detect late blight infection, caused by clonal lineage US-23, with a $75 \%$ level of accuracy at four days postinoculation and with greater than $50 \%$ accuracy at just 48 hours after inoculation. Shortwave infrared wavelengths ( $>1300 \mathrm{~nm})$ proved to be important to disease detection. Late blight infection was accurately diagnosed in growth chamber and greenhouse settings up to four days before visual symptoms appeared, indicating potential for using spectroscopy as a tool for rapid, early detection of late blight in real-time.

\section{Correlation between fungicide sensitivity of Phakopsora pachyrhizi and Puccinia triticina under greenhouse conditions} C. AVILA-ADAME, C. J. Klittich, G. Gustafson, Dow AgroSciences LLC, Indianapolis, IN, USA

Ten commercial fungicides inhibiting C14-demethylase, cytochrome bc1 ubiquinol oxidase (Qo) or succinate dehydrogenase (SDH) were compared for efficacy against Phakopsora pachyrhizi (Asian soybean rust) and Puccinia triticina (wheat brown rust) under greenhouse conditions. Ten concentrations of each fungicide, ranging from 0.001 to $9.0 \mu \mathrm{g} / \mathrm{ml}$, were applied to wheat and soybean seedlings either three days after inoculation (curative treatment) or one day before inoculation (protective treatment). Percent disease control was evaluated when disease symptoms had fully developed and $\mathrm{ED}_{50}$ values were calculated from dose-response curves. The $\mathrm{ED}_{50} \mathrm{~S}$ ranged from 0.001 to $5.2 \mu \mathrm{g} / \mathrm{ml}$ in the $1 \mathrm{DP}$ treatment and from 0.001 to more than $9 \mu \mathrm{g} / \mathrm{ml} \mathrm{when}$ they were applied as $3 \mathrm{DC}$. Pairwise $\mathrm{ED}_{50}$ values on $P$. pachyrhizi and $P$. triticina were highly correlated when fungicides were applied before inoculation (1DP correlation coefficient of 0.9), but this correlation decreased when fungicides were applied at the mycelia growth stage (3DC correlation coefficient of 0.5 ). The results of this study and additional tests with more than 100 experimental fungicides show that compounds controlling $P$. pachyrhizi are highly likely to deliver strong control of $P$. triticina as well, but compounds with strong control of $P$. triticina may not deliver strong $P$. pachyrhizi control.

Multidrug resistance mechanisms by xenobiotics detoxification, a case story: Sclerotinia homoeocarpa, causal agent of dollar spot G. JUNG (1), H. Sang (2), J. P. Hulvey (1), R. Green (1), T. Chang PhD (3), (1) University of Massachusetts, Amherst, MA, USA; (2) Michigan State University, East Lansing, MI, USA; (3) Kyungpook National University, Sangju, Gyungbuk, KOREA

Dollar spot, caused by the ascomycete fungus Sclerotinia homoeocarpa is the most economically important disease on high amenity turfgrass. $S$. homoeocarpa has developed resistance to multiple fungicide classes through target alteration or overexpression, upregulation of efflux pump overexpression. Multidrug resistance (MDR) conferred by a xenobiotic detoxification system has been a major impediment in treating cancer patients and is an emerging problem in plant and human pathogenic fungi. In cancer cells, a single nuclear receptor, PXR (pregnane X receptor, known as xenobiotic sensing nuclear receptor) has been known to play a pivotal role in concomitantly regulating drug-induced elevated changes in Phase I metabolizing enzymes and Phase III efflux transporters. Using genomics and molecular tools, we also uncovered a mechanistic analogue of PXR, single fungal-specific transcription factor (xenobiotic detoxification regulator 1, ShXDR1) in S. homoeocarpa that coordinately regulates three Phase I cytochrome P450 (CYP450) enzymes in drug metabolism and two Phase III ATP-binding cassette (ABC) transporters in drug secretion, with a 
differential substrate specificity for detoxification of multiple xenobiotics. In addition, glutathione S-transferase was discovered to be involved in the detoxification. This appears to be the first report establishing a molecular mechanism for the regulation of antifungal/xenobiotic detoxification conferring multidrug resistance in filamentous plant pathogenic fungi.

Sensitivity of sugarcane orange rust (Puccinia kuehnii) to fungicides in spore germination and detached leaf bioassays

B. CHAulagaIN (1), M. Hincapie (1), S. Sanjel (2), N. S. Dufault (3), R. N. Raid (4), P. C. Rott PhD (1), (1) University of Florida, Belle Glade, FL, USA; (2) Univeristy of Florida, Belle Glade, FL, USA; (3) Univ of Florida, Gainesville, FL, USA; (4) Univ of Florida, Belle Glade, FL, USA

Orange rust caused by Puccinia kuehnii is a major fungal disease affecting sugarcane production in Florida. Most varieties grown commercially in Florida are susceptible to orange rust; hence, disease management relies on fungicide applications. Using two different in vitro bioassays, we investigated the efficacy of five fungicides used for orange rust control in the field. Capacity to inhibit rust spore germination was determined on agar plates containing increasing concentrations of the active ingredient (a.i.) for each fungicide: at $0,0.01,0.1,1$ and $10 \mu \mathrm{g}$ per ml. Effect on germination of orange rust spores varied among fungicides, from $100 \%$ reduction of germination at $0.01,0.1,1$ and $10 \mu \mathrm{g}$ of a.i. per ml (Priaxor ${ }^{\mathrm{TM}}$ ) to only $50 \%$ reduction at $10 \mu \mathrm{g}$ of a.i. per $\mathrm{ml}\left(\right.$ Sercadis $\left.^{\circledR}\right)$. Capacity to inhibit sugarcane infection and rust sporulation was tested with a detached leaf bioassay. Leaf fragments of the susceptible variety CL85-1040 were first inoculated with spores of $P$. kuehnii and then sprayed with each fungicide at field recommended dose or with surfactant alone as control. Two weeks after inoculation, uredinia formation was inhibited at $99 \%$ in leaf fragments sprayed with all fungicides, except Caramba ${ }^{\mathrm{TM}}$ that showed only $40 \%$ reduction of sporulation. Sercadis ${ }^{\circledR}$, which did not inhibit spore germination, was highly efficient in preventing rust sporulation. Our leaf bioassay appeared to be very useful for rapid screening of fungicides against sugarcane orange rust.

Evaluation of fungicides for control of Ceratocystis fimbriata causing black rot on sweetpotato H. COLLINS, L. M. Quesada, North Carolina State University, Raleigh, NC, USA

Ceratocystis fimbriata, the causal agent of black rot of sweetpotato, is an economically important post-harvest disease. Fungicides that reduce the amount of infective inoculum would provide more commercially acceptable sweetpotatoes and reduce losses for producers. Sweetpotatoes were wounded to create three puncture wounds. Sweetpotatoes were then placed into a bin of water with three petri dish agar plates growing with black rot for twenty minutes and allowed to air dry. Roots were then arranged, wounded side up, and fungicide treatments were applied using a $\mathrm{CO}_{2}$-pressurized backpack sprayer. For dip applications, wounded roots were placed into a perforated metal basket and submerged into the fungicide mixture for 1 minute. After fungicide application, roots were allowed to dry and then placed into plastic containers and stored at $24^{\circ} \mathrm{C}$ and $99 \%$ relative humidity for 29 days. Four replications per treatment were included with 20 roots per replication. Roots were rated for disease incidence at 14, 21 and 29 days after inoculation. After 29 days, disease incidence in the untreated control was high, as estimated by number of infected wounds. Mertect 340F (Dip), TBZ500D, Mertect 340F, Penbotec 400SC, and Bio-Save 10LP, fungicides all consistently provided significant reductions in disease severity. Mertect 340F Dip provided the best control with no disease present.

Fitness, Competitive Ability and Mutation Stability of Strawberry Colletotrichum acutatum Isolates Resistant to Quinone-outside Inhibitor Fungicides

B. B. FORCELINI (1), C. Rebello (2), N. Peres (3), (1) University of Florida, Wimauma, FL, USA; (2) Universidade Estadual Paulista, Registro, BRAZIL; (3) Gulf Coast Research and Education Center; University of Florida, Wimauma, FL, USA

Quinone-outside Inhibitor (QoI) fungicides, are used to manage anthracnose of strawberry, caused by Colletotrichum acutatum. However, selection for C. acutatum resistance to QoI fungicides was first reported in 2013 in Florida, and subsequently in strawberry nurseries and production areas across the USA and Canada. C. acutatum resistance is due to the G143A point mutation in the cytochrome $b$ gene. This mutation is known to cause complete resistance even at high rates of QoIs. In this study, we investigated the relative fitness and competitive ability of QoI-resistant and sensitive C. acutatum isolates. A fitness comparison did not indicate any difference between resistant and sensitive isolates in mycelial growth at different temperatures or on hypertonic media. However, when different ratios of resistant and sensitive isolates were inoculated on detached strawberry fruit, production of conidia by the resistant isolates increased in relation to sensitive isolates. Cultivation of QoI-resistant and QoI-sensitive isolates for four culture cycles in vitro in the absence of azoxystrobin showed that QoI resistance was stable. The observed competitive advantage and stability of the G143A mutation in QoIresistant C. acutatum populations suggest that the interruption and further reintroduction of QoI fungicides might not be an option for strawberry nurseries and fruit production areas.

Variation in sensitivity to DMI fungicides tebuconazole and prothioconazole in Blumeria graminis f.sp. tritici E. A. MEYERS (1), R. Whetten (2), C. Cowger (3), (1) Department of Entomology and Plant Pathology, North Carolina State University, Raleigh, NC, USA; (2) USDA-ARS Plant Science Unit, Raleigh, NC, USA; (3) USDA-ARS, Department of Entomology \& Plant Pathology, North Carolina State University., Raleigh, NC, USA

Blumeria graminis f.sp. tritici (Bgt), the causal agent of wheat powdery mildew, is typically managed through the use of host resistance in the United States (US). However, the pathogen rapidly overcomes widely deployed resistance genes, so effective foliar fungicides must be an available alternative disease control option. Approximately 390 Bgt isolates were collected in 2013 and 2014 from 15 states and evaluated using a detached-leaf method for their sensitivity to two commonly used demethylation inhibitor (DMI) fungicides, tebuconazole and prothioconazole. Populations from 6 states contained isolates with reduced sensitivity to both fungicides; of those, Georgia and New York had the highest proportion of isolates with reduced sensitivity. Genotypic analysis revealed that the presence of a Y136F mutation in the CYP51 gene was usually associated with reduced tebuconazole sensitivity, but not with sensitivity to prothioconazole. Sequencing is underway to detect mutations correlating to reduced prothioconazole sensitivity and any additional genetic components of DMI resistance. Sequencing targets include the CYP51 gene, its upstream region, and candidate CYP51 regulators. Gene expression and copy number of CYP51 will also be determined. Understanding the genetic basis of DMI resistance in US Bgt populations will improve fungicide resistance management and may assist in the identification of new fungicide targets.

Sensitivity and resistant management for the SDHI fungicide fluxapyroxad in the apple scab pathogen Venturia inaequalis K. AYER (1), S. M. Villani (2), K. D. Cox (1), (1) Cornell University, Geneva, NY, USA; (2) North Carolina State University, Mills River, NC, USA

The recent registration of the SDHI fungicide fluxapyroxad for apple scab management prompted investigations into the sensitivity of Venturia inaequalis and fungicide application practices. Baseline isolates of $V$. inaequalis were used to determine the effective concentration of fluxapyroxad at which conidial germination and mycelial growth was inhibited by $50 \%\left(\mathrm{EC}_{50}\right)$. Mean $\mathrm{EC}_{50}$ values for fluxapyroxad conidial and mycelial growth were 0.028 and $0.25 \mu \mathrm{g} \mathrm{ml}^{-1}$, respectively. Using a draft genome of $V$. inaequalis, the $s d h C$ and $s d h D$ genes were identified and characterized to compliment the previously characterized $s d h B$ gene. Translated proteins had $>60 \%$ identity with $s d h C$ and $s d h D$ genes from other fungi. This baseline sensitivity and genetic information will serve as the foundation for phenotyping and genotyping SDHI sensitivity in populations of $V$. inaequalis. To evaluate fungicide application practices for resistance management, field experiments at two research orchards using commercial fluxapyroxad products (Sercadis ${ }^{\circledR}$ and Merivon $^{\circledR}$ ) were performed using both low and high application rates as well as a combination with single-site with multi-site fungicides. In the first year, few significant differences were observed between treatments at both locations. However, plots left untreated and those treated with Merivon had populations with the highest sensitivity to fluxapyroxad. Repeated experiments in subsequent years may lead to an improved evaluation of application practices for resistance management. 
Saponins in chenopods and potential for control of nematodes

K. GWINN (1), E. Batson (2), T. Kouser (2), M. M. Dee (1), (1) University of Tennessee, Department of Entomology and Plant Pathology, Knoxville, TN, USA; (2) University of Tennessee, Knoxville, TN, USA

Exposure of Caenorhabditis elegans to extracts from plants in the Family Chenopodiaceae (chenopods) reduced survival; activity was highest in two chenopods - Blitum bonus-henricus (GKH) and Dysphania ambrosioides (epazote). Our first objective was to evaluate saponin content of chenopod extracts. Aqueous suspensions of dried plant material $(1.5 \mathrm{~g} / 50 \mathrm{ml})$ were autoclaved and filtered; filtrate was autoclaved. Extracts of GKH (hemolytic) had much higher foaming indices than epazote (non-hemolytic). Our second objective was to determine if treatment with commercial Quillaja bark saponin (QBS) affected egg hatch and survival of first stage juveniles (L1) of C. elegans. Three strains (N2, MT7735, ZD500) were cultured using standard procedures. Eggs were collected from 2-day-old cultures and added to QBS, S basal salt, and water mixtures. Experiments were designed as replicated $3 \times 3$ factorials [strain $\times$ QBS $(0,1$, or $10 \mathrm{mg} / \mathrm{ml})$ ] with three trials. Eggs and L1s were counted at $8 \mathrm{~h}$; mobility was monitored at 24 and 120 h. Percentage hatch and mobility were reduced in high QBS treatments $(P<0.001)$. Hatch in ZD500 (defective nose touch response) was higher than either N2 or MT7735 $(P=0.02)$. Effect of QBS on mobility of L1s varied. At $24 \mathrm{~h}$, percentage of mobile L1s was greater in controls than in QBS treatments $(P<0.0001)$; this was reversed at $48 \mathrm{~h}$ for MT7735 (apoptosis mutant). Based on these studies, saponins may play a role in GHK activity against $C$. elegans.

High levels of fludioxonil resistance in Botrytis fragariae and investigation of potential resistance mechanisms M. HU, G. Schnabel, Clemson University, Clemson, SC, USA

Fludioxonil, a phenylpyrrole, is a fungicide commonly used for control of gray mold of strawberry caused by Botrytis spp., including B. cinerea and B. fragariae. In contrast to other site-specific fungicides, resistance frequencies have been comparably low in strawberry fields in the eastern United States. Therefore, the fungicide has been used extensively in rotational programs in recent years. In 2016, B. fragariae isolates were collected from strawberry fields in the east coast. They displayed MIC values greater than $100 \mu \mathrm{g} / \mathrm{ml}$. In contrast, previously reported MIC values of $B$. fragariae from Germany ranged from 0.5 to $3.3 \mu \mathrm{g} / \mathrm{ml}$. Resistance to fludioxonil in $B$. cinerea from eastern strawberry fields is conferred by mutations in the $m r r l$ gene, leading to enhanced expression of the ATP-binding cassette (ABC) transporter gene atrB. Analyses of $m r r l$ sequences indicated no variation in nucleotide sequence compared to sensitive isolates, but real-time gene expression analysis revealed the $\operatorname{atr} B$ gene was constitutional higher expressed in resistant $B$. fragariae isolates compared to sensitive isolates. Relative $\operatorname{atr} B$ copy numbers were identical in resistant and sensitive isolates indicating a lack of gene duplication. Nucleotide sequence analysis of Bos 1 , the osmosensing histidine kinase, did not reveal variations between sensitive and resistant isolates as well. In lab mutants and more recently in field isolates from China, mutations in this gene have been reported to be associated with resistance to dicarboxamides and phenylpyrroles. The high levels of fludioxonil resistance in B. fragariae isolates may pose a selective advantage over $B$. cinerea in commercial strawberry fields.

Assessing fitness of Pythium aphanidermatum isolates with dual resistance to mefenoxam and fenamidone E. LOOKABAUGH (1), B. Shew (2), (1) North Carolina State University, Raleigh, NC, USA; (2) NC State University, Raleigh, NC, USA

Pythium aphanidermatum is the predominant species causing Pythium root rot on commercially grown poinsettias in NC. Mefenoxam (M) and fenamidone (F) are among the fungicides labeled for control of Pythium root rot on poinsettia. Widespread resistance to mefenoxam (MR) has been documented in P. aphanidermatum, but resistance to fenamidone and other QoIs has not been reported. In-vitro sensitivity (S) or insensitivity (R) to mefenoxam $(17.6 \mu \mathrm{la} . \mathrm{i} / \mathrm{ml})$ and fenamidone $(488 \mu \mathrm{l}$ a.i. $/ \mathrm{ml})$ was tested on 96 isolates and the isolates were assigned to four fungicide resistance groups: MSFS, MRFS, MSFR, MRFR. $56 \%$ of isolates were insensitive to one (MRFS $=35 \%$; MSFR $=15 \%$ ) or both fungicides (MRFR $=6 \%$ ). A single point mutation in the cytochrome-b gene (G143A) was identified in fenamidone-insensitive (FR) isolates. Mycelial growth rate, oospore production, and aggressiveness were evaluated to assess fitness of fungicide-resistant isolates. MRFR isolates had reduced growth rates at $30^{\circ} \mathrm{C}$ and were less aggressive on inoculated poinsettias than other isolate groups. MSFS isolates produced significantly more oospores in-vitro than isolates resistant to one or both fungicides. Fungicides failed to prevent disease on plants inoculated with resistant isolates, demonstrating the practical implications of resistance. This is the first report of QoI resistance in P. aphanidermatum from greenhouse floriculture crops and the first report of dual resistance to mefenoxam and QoIs.

Investigating chemical control options for Colletotrichum Dieback of clementine in California

J. S. MAYORQUIN (1), J. D. Carrillo (1), B. B. Peacock (1), K. Moreno (2), L. P. Torres (1), A. Eskalen (1), (1) University of California, Riverside, Riverside, CA, USA; (2) Department of Plant Pathology and Microbiology, UC Riverside, Riverside, CA, USA

Colletotrichum Dieback (CD), caused by Colletotrichum karstii, is a new shoot canker and dieback disease of clementine mandarin and navel citrus. It was first observed in the San Joaquin Valley of California in 2013. Common symptoms of the disease are leaf chlorosis, shoot dieback, crown thinning, and wood necrosis. As an emerging disease, there are no strategies available to growers to manage this canker and dieback disease. In this study, chemical control options for CD were investigated using: i) in vitro fungicide screens of QoI-fungicides, a DMI-fungicide, and a SDHI-fungicide; and ii) a single season field trial using fungicides currently registered for citrus in CA. Preliminary results from in vitro fungicide screens revealed that QoIfungicide compounds azoxystrobin, pyraclostrobin, and trifloxystrobin had 100-fold lower mean $\mathrm{EC}_{50}$ values than the DMI-fungicide compound fenbuconazole, whereas the SDHI-fungicide compound fluopyram exhibited no inhibition. Results from the field trial showed a pre-mix of pyraclostrobin and boscalid was effective in significantly reducing CD incidence one month after application $(\mathrm{P}<0.05)$. Trees treated with pyraclostrobin alone also showed a reduction in incidence both one and three months post-application. These findings suggest that pyraclostrobin formulations may be used to reduce disease incidence of $\mathrm{CD}$ occurring in clementine mandarin and navel orange varieties in California.

In vitro evaluation of efficacy of novel antifungal compounds for control of the rice sheath blight fungus

Z. GUO (1), X. Zhou (1), B. Liu (2), X. Lei (3), (1) Texas A\&M AgriLife Research, Beaumont, TX, USA; (2) Hunan Agricultural University, Changsha, CHINA; (3) Lamar University, Beaumont, TX, USA

Sheath blight, caused by the fungus Rhizoctonia solani AG1-1A, is one of the major diseases of rice worldwide. Fungicides have been the most effective tool for management of this disease in the US. However, recent emergence of resistant populations of the fungus to strobilurin fungicides threatens this management option. The ultimate goal of this study is to develop new chemistries that have a different mode of action and a wide spectrum of fungicide activities against $R$. solani and other pathogens. Toward this goal, an in vitro study was initialized to screen new chemical compounds for the inhibition of growth of $R$. solani on potato dextrose agar (PDA). These chemical compounds were designed and synthesized with a potential of having systemic mode of action. A 6-mm agar disc with $R$. solani was placed onto the center of each of PDA plates amended with different concentrations of chemical compounds. Azoxystrobin served as positive control. Inhibition of the growth of the fungus was measured at $40 \mathrm{~h}$ after inoculation. There were five compounds showing antifungal activities with two having highest levels of inhibition. At $4 \mathrm{ppm}$, compound $78 \mathrm{~B}$ had a significantly higher level of inhibition than azoxystrobin (62\% vs. $49 \%$ ); compound 76 A reduced the growth of $R$. solani to a level similar to azoxystrobin ( $53 \%$ vs. $49 \%$ ). Our results demonstrate that both compound $78 \mathrm{~B}$ and compound $76 \mathrm{~A}$ could be promising candidate fungicides for further investigation.

Predicting ascospore release of Monilinia vaccinii-corymbosi causing mummy berry of blueberry in the Pacific Northwest using machine learning

D. HARTEVELD (1), M. Grant (2), J. W. Pscheidt (3), T. L. Peever (4), (1) Washingtom State University, Mount Vernon, WA, USA; (2) University of Washington, Seattle, WA, USA; (3) Oregon State University, Corvallis, OR, USA; (4) Washington State University, Pullman, WA, USA 
Mummy berry, caused by M. vaccinii-corymbosi, causes economic losses of highbush blueberry in the US Pacific Northwest. Apothecia develop from mummified berries overwintering on soil surfaces and produce ascospores that infect emerging tissue from floral and vegetative buds. Disease control currently relies on calendar-based fungicide sprays rather than on the disease cycle. To establish a prediction model for ascospore release, apothecial development was tracked in three fields, one in northwestern OR and two in northwestern WA in 2015 and 2016. The environmental factors air and soil temperature, precipitation, soil moisture, leaf wetness, relative humidity and solar radiation were monitored using in-field weather stations and WSU's Agweathernet. Four modeling approaches were compared: logistic regression, multivariate adaptive regression splines, artificial neural networks and random forest. A supervised learning approach was used to train the models on two data sets: training (70\%) and testing (30\%). Importance of environmental factors was calculated for each model separately. Soil temperature, soil moisture, and solar radiation were identified as the most important factors influencing ascospore release. Random forest showed the highest accuracy compared to the other models, and was used in the production of an interactive web application aimed at assisting growers in predicting inoculum release in their fields for fungicide optimization and reduced cost.

Determining the Timing of Host Susceptibility to Infection by Fungal Pathogens Associated with Fruit Rot Disease of Winterberry Holly S. LIN (1), M. D. Bellizzi (1), F. Peduto Hand PhD (2), (1) The Ohio State University, Columbus, OH, USA; (2) Ohio State Univ, Dept of Plant Pathology, Columbus, OH, USA

An emergent fruit rot disease is challenging winterberry growers across the U.S. leading in some cases to complete crop loss. Previous studies conducted in Ohio nurseries indicated that a complex of fungi is associated with the disease and that flowers could serve as a point of entry for fruit infections. To test this hypothesis, a container trial of 'Sparkleberry' plants was established in Columbus, $\mathrm{OH}$ in 2016. Alternaria alternata, Colletotrichum acutatum, Diaporthe cf. heveae and Epicoccum nigrum, which are the fungi most frequently recovered from symptomatic fruit, were individually inoculated as a spore suspension of four pooled isolates per species, onto flower or fruit tissues at different stages of development: flower bud, bloom, petal fall, immature fruit, and mature fruit (wounded or unwounded). Following inoculation, plants were arranged in a randomized complete block design and watered by drip irrigation. Fruit rot incidence was assessed weekly mid November through mid December. All pathogens inoculated on mature fruit caused rot symptoms but the presence of a wound deemed necessary for successful infection. Fruit rot also developed on plants inoculated with $D$. $c f$. heveae at full bloom or petal fall. Understanding the timing of host infection is crucial to time appropriate management strategies. Our results indicate that flowers need to be protected to avoid infection by $D$. $c f$. heveae, and that injury to mature fruit can negatively impact berry health.

\section{The influence of water quality on efficacy of fungicides for turf disease control} T. STACY, R. Latin, Purdue University, West Lafayette, IN, USA

Golf course superintendents regularly apply fungicides to control various diseases that cause cosmetic and structural damage to playing surfaces. Although published reports show that water quality (particularly water $\mathrm{pH}$ ) can influence the performance of certain herbicides, evidence of similar effects on fungicide efficacy is weak and largely anecdotal. The objective of this research was to investigate the influence of water $\mathrm{pH}$ on efficacy of fungicides commonly used against dollar spot, a problematic disease on creeping bentgrass. We considered three fungicides (metconazole, thiophanatemethyl, and iprodione) mixed with water stabilized at three $\mathrm{pH}$ levels $(\mathrm{pH}=5.0,7.0$, and 9.0$)$ in in vitro and field experiments. We also considered a time factor, i.e., the time between mixing and application where time $=0$ indicated fungicides were applied immediately after mixing, and time $=24$ indicated that fungicides were applied 24 hours after mixing. Results from field experiments revealed little or no difference in fungicide performance when mixed in acidic $(\mathrm{pH}=5.0$, neutral $(\mathrm{pH}=7.0)$, and alkaline $(\mathrm{pH}=9.0)$ water. Also, the time factor was not significant for all fungicides and water $\mathrm{pH}$ levels. Results from in vitro work supported field observations- few differences in pathogen growth were observed for $\mathrm{pH}$ and time factors.

Calonectria pseudonaviculata microsclerotia viability after exposure to fungicides

J. A. LAMONDIA, K. Maurer, The Connecticut Agricultural Experiment Station, Windsor, CT, USA

The boxwood blight pathogen, Calonectria pseudonaviculata (Cps), can be affected by low rates of fungicides in-vitro, however, management of disease on infected plants can be difficult. We investigated the sensitivity of microsclerotia (MS) to efficacious fungicides. Melanized MS (140-200 $\mu$ diameter) were removed from media, washed for $30 \mathrm{~s}$ in sterile water and soaked in $5 \mathrm{ml}$ of $0,3.16,10.0,31.6,100$, or $316 \mathrm{ppm}$ of kresoxim-methyl, thiophanatemethyl or propiconazole for $24,48,72$ or $96 \mathrm{~h}$. There were 5 replicates and the experiment was conducted twice. After exposure, MS were washed three times with sterile water, blotted and placed on $1 / 2 \mathrm{PDA}$. Viability (germination) and colony diameter were measured after one week, and growth from exposed MS was normalized by the $0 \mathrm{ppm}$ control. Thiophanate-methyl did not affect MS viability or colony diameter after any concentration or time combination. Kresoxim-methyl reduced growth by $50 \%$ only after MS exposure to $316 \mathrm{ppm}$ for $>72 \mathrm{~h}$. Propiconazole affected both survival and subsequent colony diameter. Few MS survived exposure to $100 \mathrm{ppm}$ for $>48 \mathrm{~h}$ or $316 \mathrm{ppm}$ for $>24 \mathrm{~h}$. A multiple regression model predicted $50 \%$ colony diameter reduction after $30 \mathrm{~h}$ in $10 \mathrm{ppm}, 14 \mathrm{~h}$ in $31.6 \mathrm{ppm}$, or any exposure to $>100 \mathrm{ppm}$ propiconazole. The melanized MS of $C p s$ exhibit increased survival after exposure to fungicides toxic to hyphae. However, propiconazole can effectively reduce viability and subsequent colony growth at concentrations within use rates.

How does Botrytis cinerea infect red raspberry?

O. KOZHAR, T. L. Peever, Washington State University, Pullman, WA, USA

Botrytis cinerea, causal agent of gray mold, is the most important pathogen of raspberry in the US Pacific Northwest and worldwide. Despite intensive fungicide application programs used to control the disease, fruit losses often exceed $25 \%$ due to incomplete disease control. Fungicides currently are applied on a calendar basis rather than based on inoculum pressure and infection risk because the disease cycle on red raspberry is poorly understood. The objectives of this study were to determine the dynamics of flower and fruit infection in association with environmental conditions. Recovery of $B$. cinerea from seven developmental stages of raspberry flowers and fruit in northwestern Washington was recorded in 2015-2016 in a field not sprayed with fungicides. Colonization of raspberry flowers by B. cinerea was limited (15\%), increased as fruit developed, and peaked on mature fruit (67\%). In early stages of flower development, the floral part with greatest $B$. cinerea recovery was the carpel $(70 \%)$. As fruit matured, additional floral parts were colonized by $B$. cinerea, which could increase the risk of secondary infections of mature fruit. $B$. cinerea colonization of fruit was significantly associated with air temperature and rainfall. The results suggest that fungicide applications at flowering may be less effective than those at fruit development and maturation, and monitoring environmental conditions may help time fungicide applications effectively to control gray mold.

Deciphering blueberry and cranberry floral involvement in the disease cycle of Colletotrichum fioriniae

T. J. WALLER (1), J. Gager (2), T. Gianfagna (3), P. Oudemans (3), (1) Rutgers University, Chatsworth, NJ, USA; (2) Rutgers University, Bridgeton, NJ, USA; (3) Rutgers University, New Brunswick, NJ, USA

Colletotrichum fioriniae is a hemibiotrophic, fruit-rotting pathogen that limits blueberry (BB) and cranberry (CB) production. Since fungicide applications during bloom are most effective, the link between floral tissue and infection structure formation was investigated. Floral components influence both production of inoculum (secondary conidiation) and formation of infection structures (appressoria). Effects of flower tissue bioactivity on C. fioriniae were tested using two distinct extraction methods: water (W), to simulate mobilization of floral compounds in the field, and chloroform (C), to evaluate nonpolar cuticle compounds. W and C floral extracts significantly increased the rate (200\%) and magnitude (500\%) of appressorium formation and W extracts increased secondary conidiation (500\%) over controls. Extracts of susceptible BB cultivars significantly increased 
appressorial stimulation compared to resistant cultivars, however, this was not observed in CB. Floral rainwater runoff stimulated significantly more secondary conidiation and appressorium formation compared to controls, demonstrating the presence of this phenomenon in the field. Analysis of $\mathrm{CH}$ extract of CB flowers and pure chemical assays showed methyl ester of hexadecanoic acid (HEX) as the most stimulatory compound characterized. Utilizing this, HEX was field-tested with fungicides as a 'trap' spray, synchronizing fungal germination to fungicide applications with moderate early season control.

Efficacy of SDHI fungicide, pydiflumetofen, against Fusarium gramminearum

H. SUN, W. Li, Y. Deng, A. Zhang, H. Chen, Jiangsu Academy of Agricultural Sciences, Nanjing, CHINA

Fusarium head blight (FHB) caused by Fusarium gramminearum is a devastating disease of wheat in China. A new succinate dehydrogenase inhibitor (SDHI) pydiflumetofen was developed recently. The inhibitory activity of pydiflumetofen against FHB and the resistance mechanism of FHB to pydiflumetofen were investigated in this study. Our results showed that isolates of $F$. gramminearum were insensitive (naturally resistant) to thifluzamide, boscalid, flutolanil, penflufen, penflufen and fluopyram on PDA medium. In contrast, these isolates were sensitive to pydiflumetofen. In inoculation trials, control efficacies of pydiflumetofen at 200 g.a.i ha ${ }^{-1}$ was $91.4 \%$ in 2015 . In field trials in 2015 and 2016 , control efficacies of pydiflumetofen at 150 g.a.i ha ${ }^{-1}$ were $84.0 \%$ and $90.3 \%$, and at 200 g.a.i ha ${ }^{-1}$ were $86.7 \%$ and $95.5 \%$. Four pydiflumetofen-resistant (PR) mutants of $F$. gramminearum were generated through UV mutagenesis. The $s d h A, s d h B, s d h C$, and $s d h D$ genes encoding the subunits of succinate dehydrogenase were sequenced, but no mutations were found in these resistant mutants. So, pydiflumetofen should be a promising fungicide for controlling FHB, and its resistance was unrelated to mutation in the genes $s d h A, s d h B, s d h C$, and $s d h D$.

\section{Protection of new cucumber growth from Pseudoperonospora cubensis with oxathiapiprolin-based fungicides}

S. E. SALAS, C. P. Shepherd, H. K. Ngugi, DuPont Crop Protection, Newark, DE, USA

Oxathiapiprolin, is a novel fungicide recently discovered by DuPont with a unique target site in Oomycetes. Growth chamber and greenhouse studies were conducted to evaluate local systemic movement and effectiveness of oxathiapiprolin fungicides at protecting rapidly growing cucumber tissue from downy mildew. In a local systemic movement bioassay, oxathiapiprolin reduced downy mildew severity by 93 to $98 \%$ on cucumber leaf tissue protected from fungicide spray droplets. By comparison, mefenoxam + mancozeb reduced disease severity by $<60 \%$ on similar tissue. Protection of new growth was assessed in two greenhouse experiments. In experiment 1 , an oxathiapiprolin oil dispersion formulation significantly $(P<0.0001)$ reduced disease severity on leaves that had not emerged at the time of fungicide application (new growth) relative to the non-treated plants. Propamocarb + fluopicolide and mefenoxam + mancozeb did not significantly reduce disease on the new growth $(P>0.05)$. In experiment 2 , oxathiapiprolin suspo-emulsion and wettable granules formulations significantly $(P=0.036$ to $<0.0001)$ reduced disease on new growth relative to the non-treated check. By contrast, mefenoxam + mancozeb reduced disease severity on new growth only in one of three experimental runs $(P=0.032)$, and propamocarb + fluopicolide did not reduce disease $(P>0.05)$. These data suggest that oxathiapiprolin is locally systemic and can protect new growth making it a valuable tool for disease control.

Enhancing fruit rot resistance in American cranberry

J. J. POLASHOCK (1), G. Daverdin (2), J. Johnson-Cicalese (2), N. Vorsa (2), (1) USDA ARS, Chatsworth, NJ, USA; (2) Rutgers University, Chatsworth, NJ, USA

Fruit rot is the number one problem facing cranberry growers. Although carefully selected, timed and properly applied fungicides can be partially effective, the loss of some fungicide registrations, stricter MRLs, development of pathogen resistance and other factors underscore the need to breed resistant varieties. To be successful, resistance must be: 1) multigenic, 2) heritable, 3 ) broad spectrum (since cranberry fruit rot is caused by a complex of many different species of fungi), and 4) independent of deleterious traits. We have demonstrated, using DNA fingerprinting, that there are at least four genetically diverse sources of resistance in our germplasm collection. Multiple crosses and extensive evaluation of the progeny demonstrated that progeny segregate for resistance/susceptibility. Resistance was shown to be broad-spectrum since multiple fruit-rotting fungal species were present as latent infections on fruit of resistant accessions. The populations also segregate for a wide range of phenotypic traits independent of resistance. Using GBS, three segregating populations were genotyped. The GBS data were used to generate genetic maps for cranberry and together with the phenotypic data, 60 putative quantitative trait loci (QTL) associated with fruit rot resistance, yield, berry weight and other traits were identified. Selected progeny with good resistance and yield were planted in replicated field trials for further evaluation.

Partitioning and local systemic movement of oxathiapiprolin for protection of potato against Phytophthora infestans H. K. Ngugi, S. E. SALAS, C. P. Shepherd, L. J. Watson, DuPont Crop Protection, Newark, DE, USA

Oxathiapiprolin is a new Oomycete fungicide recently discovered by DuPont. The goal of this study was to evaluate leaf uptake, partitioning and local systemic movement of oxathiapiprolin fungicides for control of Phytophthora infestans in potato. In an experiment comparing different formulations of oxathiapiprolin, $>82 \%$ of the active ingredient (ai) was in the cuticular layer $1 \mathrm{~h}$ after application for both the oil dispersion and suspo-emulsion formulations, $15 \%$ was in a surface water wash, while $3 \%$ had penetrated leaf tissue. By contrast, only $21 \%$ of the ai was recovered in cuticular layer for the wettable granules formulation and $78 \%$ was in the surface water wash $1 \mathrm{~h}$ post application. For both liquid formulations, the amount of the ai that penetrated leaf tissue increased to ca 5\% 3 days post application and remained stable 7 days later. In a rainfastness study, preventive late blight control of oxathiapiprolin fungicides was not affected by $60 \mathrm{~mm}$ of simulated rainfall starting 20 min after application. In a local systemic movement bioassay, oxathiapiprolin fungicides at label rate significantly $(P<0.0001)$ reduced late blight severity by $>82 \%$ on potato leaf tissue protected from fungicide spray droplets. By contrast, all commercial fungicides included for comparison except mefenoxam + mancozeb reduced disease by $<50 \%$ on similar tissue. The results indicate that oxathiapiprolin fungicides are rainfast, locally systemic, and a valuable tool for late blight control.

Investigating the longevity and host range of Cucumber green mottle mosaic virus in the Northern Territory, Australia D. A. LOVELOCK PHD, N. Kurz, S. Mintoff, M. Neilsen, L. T. T. Tran-Nguyen, Department of Primary Industry and Resources, Darwin, AUSTRALIA

Cucumber green mottle mosaic virus (CGMMV) is a tobamovirus that is able to infect a number of economically important cucurbits crops. The first detection of CGMMV in Australia was reported in the Northern Territory (NT) in September 2014; 26 properties in the NT have now been identified as CGMMV positive. As of February 2017, CGMMV has also been detected in Queensland and Western Australia. Initially all infested properties (IP) in the NT were placed under quarantine for a period of two years, where all host plants including cucurbitaceae weeds were destroyed. Previous research had indicated CGMMV was viable for up to 9 months in host free soils. Four IP with varying soil types and climates in the NT were chosen to determine the longevity of the virus starting at 12 months into quarantine, using watermelon and cucumber. At 12,15 and 18 months of the quarantine period, 80 soil samples were collected from each IP, with plant bioassays conducted to determine the viability of any remaining virus. A field trial was also conducted on all four IP at the 12 month soil sampling point. At the initial test period of 12 months, 2 IP from the bioassay and 1 IP from the field trial tested positive for CGMMV, while all 4 IP tested positive in the 15 month bioassays. Because of this discovery, the status of other non-cucurbit commercial crops and selected weeds is being tested to determine the risk of transmission of CGMMV in a wider range of plant host. 
Efficacy of copper hydroxide on four clonal lineages of Phytophthora infestans

T. WU, E. Silva, A. J. Gevens, University of Wisconsin-Madison, Madison, WI, USA

The oomycete Phytophthora infestans causes late blight, one of the most economically important diseases of potato and tomato. Fungicide application is a primary disease management method because few varieties have robust field resistance. In organic systems, copper-based fungicides are considered the most effective means of control. However, concerns with negative environmental effects and potential human exposure associated with copper have made organic farmers hesitant to use regular applications for preventative management. Alternative organic fungicides and biopesticides being developed have not been studied extensively. In a 2014 study, copper did not reduce late blight severity as well as a novel plant-extract-based biopesticide. To investigate this further, resistance to copper-based fungicides was evaluated in four clonal lineages of $P$. infestans, US-1, US-8, US-23, and US-24. Representative isolates were grown on Rye A media amended with varying concentrations $(0,0.0001,0.1,1,10,50,100$ ppm) of an analytical grade of copper (II) hydroxide $\left(\mathrm{CuOH}_{2}\right)$. Preliminary results indicated that growth of isolates of US-1, US-8, and US-24 were completely inhibited at 100 ppm and the isolate of US-23 was completely inhibited at 10, 50, and $100 \mathrm{ppm}$. While copper insensitivity was detected in the US-1 isolate at 1 ppm and in the US-8 isolate at 0.0001 and $1 \mathrm{ppm}$, these rates are negligible when compared to copper label rates applied in field for late blight control.

Detection of cucurbit viruses in Oklahoma combining EDNA with Multiplex RT-PCR coupled with High Resolution Melting L. PENA ZUNIGA (1), A. Espindola (1), H. A. Melouk (2), A. Ali (3), K. F. Cardwell (1), F. Ochoa Corona (1), (1) Oklahoma State University, Stillwater, OK, USA; (2) USDA ARS, Stillwater, OK, USA; (3) Univ of Tulsa, Tulsa, OK, USA

Cultivated acreage of cucurbits in Oklahoma has decreased mainly due to viral diseases. This research combines Electronic probe Diagnostic Nucleic acid Analysis (EDNA) for detection of 17 reported viruses infecting cucurbits with Multiplex RT-PCR and High Resolution Melting (HRM). EDNA is a next generation sequencing (NGS) tool that generates specific electronic probes (e-probes). A mock positive controls database is created in silico with MetaSim using pathogen sequences retrieved from GenBank to mimic single and multiple infections. To avoid false positives, e-probes are made more specific by BLAST alignments of sequences from published databases of near neighbors, to eliminate homologous sequences. Once a sample NGS output is retrieved, the probes and control sequences are used to query the sequence for matches, indicating positive detection. Next, the sample is tested in vitro by a Multiplex RT-PCR+HRM for confirmation and discrimination based on PCR products melting profiles. EDNA probes and multiplex RTPCR+HRM of nine out of 17 cucurbit infecting viruses are presented: Cucurbit green mottle mosaic virus (CGMMV), Cucumber mosaic virus (CMV I and CMV II), Cucurbit aphid-borne yellow virus (CABYV), Cucurbit yellow stunting disorder virus (CYSDV), Melon necrotic spot virus (MNSV), Papaya ringspot virus (PRSV), Squash mosaic virus (SqMV), Watermelon mosaic virus (WMV), and Zucchini yellow mosaic virus (ZYMV). EDNACucurbits can be combined with Multiplex RT-PCR+HRM for virus monitoring in microbial forensics and biosecurity, and for routine diagnostics and epidemiological studies of cucurbit crops.

Field status and dynamics of cassava mosaic disease in Zambia

R. M. MULEnGA (1), P. C. Chikoti (1), J. P. Legg (2), O. J. Alabi (3), (1) Zambia Agriculture Research Institute, Lusaka, ZAMBIA; (2) International Institute of Tropical Agriculture, Dar Es Salaam, TANZANIA; (3) Dept. of Plant Pathology \& Microbiology, Texas A\&M University, Weslaco, TX, USA

The status of cassava mosaic disease in Zambia was determined during a country-wide survey conducted in 2014 in farmers' fields in six major cassavagrowing provinces (Luapula, Lusaka, Northern, Eastern, Western and Northwestern). A symptoms-based assessment of CMD was performed on 30 plants of a predominant variety counted along two diagonals to determine disease incidence and severity. Incidence was calculated as a percentage of symptomatic plants out of 30 counted plants while severity was rated using the standard 1 to 5 scale. Assessment of infection type (cutting- or whiteflyborne) was made and values for whitefly-borne infections were transformed into multiple infection units. The results indicated that mean CMD incidence was lowest in Northern (44\%) and highest in Lusaka province (81\%); averaged 57\% across all six provinces. Mean CMD severity varied significantly $\left(\chi^{2}=249.8, \mathrm{df}=10, \mathrm{P}<0.001\right)$ among provinces ranging from 2.8 in Eastern to 3.0 in Northwestern province. Mean CMD incidence was higher for cassava landraces $(\sim 25$ to $86 \%)$ than improved varieties $(\sim 32$ to $60 \%)$. Disease severity also varied between landraces $(2.2$ to 3.0$)$ and improved varieties (2.7 to 2.8). Cutting-borne infections accounted for $\sim 92 \%$ of CMD-affected plants relative to $8 \%$ for whitefly-borne infections. DNA fingerprinting of field survey samples revealed the occurrence of African cassava mosaic virus, East African cassava mosaic virus and East African cassava mosaic Malawi virus as single or mixed infections of different proportions. The results underscore the importance of clean plant materials for sustainable management of CMD in Zambia.

Developing RNA interference technology to manage whitefly-transmitted begomoviruses D. K. HASEGAWA (1), N. Kaur (2), W. Chen (3), Y. Zheng (3), A. Simmons (1), Z. Fei (3), W. M. Wintermantel (2), K. S. Ling (1), (1) USDA-ARS, Charleston, SC, USA; (2) USDA-ARS, Salinas, CA, USA; (3) Boyce Thompson Institute, Ithaca, NY, USA

Of nearly 300 viruses transmitted by the whitefly (Bemisia tabaci), 90\% of them belong to the genus Begomovirus. Begomoviruses are efficiently transmitted by whiteflies to a range of agricultural crops. This results in billions of dollars lost annually, while jeopardizing food security worldwide. Several begomoviruses have emerged as being especially devastating, including Tomato yellow leaf curl virus (TYLCV). Although the use of pesticides has helped manage whiteflies, there remains an increasing need to develop novel strategies that are effective and sustainable. Recently, our group was the first to sequence the whitefly (B. tabaci MEAM1 or B biotype) genome. In the current study, we were interested in exploring the possibility of using RNA interference (RNAi) technology for whitefly control. Using transcriptome analysis of differentially expressed genes upon TYLCV infection, candidate genes were selected as potential targets for RNAi. Over 20 different double-stranded RNAs (dsRNAs) were synthesized. The effect of RNAi against whitefly survival was evaluated over a course of 10 days through feeding of whiteflies on an artificial diet containing various concentrations of dsRNA. Some RNAi constructs resulted in a dramatic increase in mortality over those whiteflies feeding on the same diet with non-target controls. The potential of using RNAi technology as a non-transgenic topical spray in managing whiteflies and TYLCV transmission will be discussed.

Efficacy of seed treatment fungicides on frequently isolated seedborne fungi of pulse crops in Montana C. PELUOLA, J. Mgbechi-Ezeri, A. Owati, M. E. Burrows, B. Agindotan PhD, Montana State University, Bozeman, MT, USA

The Regional Pulse Diagnostic Laboratory of Montana State University in 2015-2016 isolated high rate of fungal species from chickpea, field pea and lentil seedlots. Prevalent isolates included Alternaria (75-83\%), Botrytis (12-14\%), Stemphylium (16-54\%), Fusarium (17-22\%) and Ascochyta (56$78 \%$ ) species. Seed treatment can help manage these fungi but information on fungicide efficacy is limited. An $\mathrm{EC}_{50}$ value for twelve registered seed treatment fungicides calculated from $0,0.1,1,10,100$ and $1000 \mu \mathrm{g} / \mathrm{ml}$ concentrations of formulated products incorporated into potato dextrose agar. Fungal species included Alternaria, Botrytis, Stemphylium, Fusarium, Sclerotinia, Colletotrichum, Diaporthe and Ascochyta. Fludioxonil had low EC 50 values for most species tested. $\mathrm{EC}_{50}$ values ranged from $0.06-5.6 \mu \mathrm{g} / \mathrm{ml}$ with $<0.2 \mu \mathrm{g} / \mathrm{ml}$ for all fungi except Colletotrichum and Sclerotinia spp. EC E $_{50}$ of thiram ranged from 5.0 - $398.1 \mu \mathrm{g} / \mathrm{ml}$. The $\mathrm{EC}_{50}$ of Quinone outside Inhibitors $(\mathrm{QoI})$-azoxystrobin, trifloxystrobin and pyraclostrobin ranged from $0.32-$ $1000 \mu \mathrm{g} / \mathrm{ml}$. QoI inhibited growth of Stemphylium spp. at $0.32 \mu \mathrm{g} / \mathrm{ml}$ and least effective on Alternaria, Botrytis and Sclerotinia spp. at $1000 \mu \mathrm{g} / \mathrm{ml}$. EC ${ }_{50}$ values ranged from $0.032-501 \mu \mathrm{g} / \mathrm{ml}$ among the Succinate Dehydrogenase Inhibitors-fluopyram, fluxapyroxad, sedaxane and penflufen. Their growth inhibition was strongest on Stemphylium spp. $(0.032 \mu \mathrm{g} / \mathrm{ml})$ and weakest on Colletotrichum and Diaporthe spp. at $501 \mu \mathrm{g} / \mathrm{ml}$. Ethaboxam was least effective at $>1000 \mu \mathrm{g} / \mathrm{ml}$ except on Diaporthe spp. at $25.12 \mu \mathrm{g} / \mathrm{ml}$. This study provides preliminary data for focusing in vivo studies and ultimately providing recommendations to growers for management of seedborne fungi important in pulse crop production. 
Unveiling the population structure of rose rosette virus

A. Katsiani (1), K. H. Lamour (2), I. E. TZANETAKIS (1), (1) University of Arkansas, Fayetteville, AR, USA; (2) University of Tennessee, Knoxville, TN, USA

Rose rosette is one of the most economically important diseases of roses. It is caused by rose rosette virus (RRV), a hepta-segmented, negative-sense RNA virus, member of the genus Emaravirus; transmitted by the eriophyid mite Phyllocoptes fructiphilus. Little is known about the virus and studies on its population structure and evolution are crucial to better understand the epidemiology of virus and disease. We implemented a multiplex amplicon sequencing method to quantify polymorphisms in the RRV U.S. population. On hundred and seven (107) RRV isolates collected from 19 states were used in the study. After reverse transcription, samples were submitted to PCR using virus-specific primers developed to amplify the complete virus genome and sequenced on Illumina platforms. Bioinformatics analysis provided information of more than $95 \%$ of the genome length for more than 90 isolates. The mean genetic distance among isolates was the highest for RNA 5 and the lowest for RNAs 2 and 3 . Phylogenetic trees constructed for all genomic RNAs. Isolates were grouped into distinct clusters with high bootstrap support with geography having minimum impact in virus evolution, possibly because of the movement of infected material across state lines or because the plant genotypes are the driving force shaping virus populations.

Nematicidal effects of absinth wormwood (Artemisia absinthium) against soybean cyst nematode in in-vitro and in-vivo conditions P. BASNET (1), E. Z. Byamukama (2), (1) South Dakota State University, Brookings, SD, USA; (2) South Dakota State Univ, Brookings, SD, USA

Soybean cyst nematode (Heterodera glycines) cause estimated $\$ 1$ billion loss annually in the USA. Breakdown of resistance in the cultivars, complexity of the species identification along with de-registration of some nematicidal compounds challenges the disease management. Discovery of plant-derived nematicidal extract is a benignant, safe and sustainable approach for soybean cyst nematode management. The weed species, absinth wormwood (Artemisia absinthium) is believed to contain compounds such as caeffeic acid, chlorgenic acid, artemisinin, artesunate etc that possess nematotoxic effects. The objective of this study was therefore to evaluate the use of absinth wormwood plant extracts against soybean cyst nematode. Leaves extract from absinth wormwood were prepared at concentrations of $12 \mu \mathrm{g} / \mathrm{ml}, 60 \mu \mathrm{g} / \mathrm{ml}$ and $120 \mu \mathrm{g} / \mathrm{ml}$. In in-vitro study, 20 juveniles were placed in Petridishes with different concentration of plant extract and observed the number of dead juveniles after interval of 24, 48, 72 and 96 hours. In in-vivo study, 1000 eggs were used to infest soybean plant in greenhouse followed by pouring of $100 \mathrm{ml}$ plant extract one week of the post-inoculation and observed cyst on the roots after 35 days. Preliminary result shows that the percentage juvenile mortality in in-vitro condition was $70 \%$ at $120 \mu \mathrm{g} / \mathrm{ml}, 26 \%$ at 60 $\mu \mathrm{g} / \mathrm{ml}$ and $21 \%$ at $12 \mu \mathrm{g} / \mathrm{ml}$. Similarly, cyst formation in soybean roots were reduced by $77 \%$ at $120 \mu \mathrm{g} / \mathrm{ml}, 46 \%$ at $60 \mu \mathrm{g} / \mathrm{ml}$ and $2.5 \%$ at $12 \mu \mathrm{g} / \mathrm{ml}$. Thus, this study suggests that the extracts of $A$. absinthium have nematicidal properties against SCN.

A comparison of ground-based air-blast sprayer and aircraft application of fungicides to manage scab in tall pecan trees C. H. Bock (1), M. W. HOTCHKISS (2), (1) USDA ARS, Southeastern Fruit and Tree Nut Research Laboratory, Byron, GA, USA; (2) USDA ARS, Byron, GA, USA

Scab (caused by Venturia effusa) is the most destructive disease of pecan in the southeastern USA. The most widely used method to apply fungicide is air-blast (AB) sprayers. Aerially (A) applied sprays are also used, but the disease distribution and spray coverage of these two methods has not been compared. Control of scab at 5 heights was compared using AB, A, and AB+A applications using $25 \mathrm{~m}$ 'Schley' pecan in 2013 and 2014 . In control trees scab on fruit was most severe at most heights, except $>12.5 \mathrm{~m}$, where severity in trees receiving the AB spray was most often similar. However, severity at $<12.5 \mathrm{~m}$ was lower in the trees receiving the AB spray compared to the control. The A application often had lower severity at all sample heights compared to the non-treated control, but the difference compared to the $\mathrm{AB}$ treatment was not always significant at 5 to $7.5 \mathrm{~m}$. The combination of $\mathrm{AB}+\mathrm{A}$ treatments had uniformly low severity of scab at all heights. Spray coverage on water sensitive cards indicated a decline in coverage with height using the $\mathrm{AB}$ sprayer, but $\mathrm{A}$ applications resulted in uniformly low spray coverage at all heights. Both $\mathrm{AB}$ and $\mathrm{A}$ sprays resulted in control of scab, but $\mathrm{AB}$ sprays tended to fail at heights $>12.5 \mathrm{~m}$, and A-treated trees tended to show a slight increase in disease severity low in the canopy. The results provide a basis for understanding the relative efficacy and advantages of these methods for fungicide application in tall pecan trees.

Meta-analysis and yield loss analysis of multiple site-year fungicide evaluations for improved Sclerotinia stem rot management in soybean J. F. WILLBUR (1), M. L. Fall (2,3), A. Byrne (4), S. Chapman (1), C. A. Bradley PhD (5), M. Chilvers (4), N. M. Kleczewski (6), D. S. Mueller (7), S. Conley (8), D. L. Smith (1,9), (1) University of Wisconsin - Madison, Madison, WI, USA; (2) Agriculture and Agri-Food canada, St-Jean-sur-Richelieu, QC, CANADA; (3) Agriculture and AgriFood Canada, Saint-Jean-sur-Richelieu, QC, CANADA; (4) Michigan State University, East Lansing, MI, USA; (5) University of Kentucky, Princeton, KY, USA; (6) University of Delaware, Newark, DE, USA; (7) Iowa State University, Ames, IA, USA; (8) University of Wisconsin-Madison, Madison, WI, USA; (9) University of Wisconsin, Madison, WI, USA

Sclerotinia stem rot (SSR) is caused by the fungal pathogen Sclerotinia sclerotiorum and consistently ranks among the top diseases plaguing global soybean crops. As complete resistance in soybean has not been achieved, and SSR continues to be of economic concern, chemical control remains a prevalent disease management strategy. Studies from multiple site-years evaluating the efficacy of fungicide treatments and timings can provide current, regional management recommendations. Fungicide evaluations were conducted in Illinois, Iowa, Michigan, New Jersey, and Wisconsin from 2009 to 2016, for a total of 26 site-years $(\mathrm{n}=2207)$. These studies were used to test eight popular fungicide classes, including 19 active ingredients, and 11 common application timings. Moderator effects of class and active ingredient were found to significantly affect yield $(P<0.0001)$, and application timing was found to significantly affect disease levels $(P=0.0069)$. These studies were also used in nonlinear regression analyses to determine the effect of disease severity index (DSI) on soybean yield. A three parameter logistic model was found to best describe soybean yield loss (pseudo- $\mathrm{R}^{2}=0.24$ ). In modern soybean cultivars, yield loss due to SSR does not appear to occur until 20-25 DSI units and considerable yield loss is observed beginning at 65 DSI (-670 kg/ha). These studies will help growers select cost effective fungicide programs for use in integrated management of SSR in soybean.

Physiological effects and mode of action of $\mathrm{ZnO}$ nanoparticles against postharvest fungal contaminants D. SARDELLA, R. Gatt, V. Valdramidis, University of Malta, Msida, MALTA

Increasing concerns continue to be expressed about health hazards and environmental pollution resulting from the use of conventional fungicides for postharvest disease control. Nanoparticles represent an alternative solution for postharvest disease management. The objective of this work was to assess the physiological effects and the mode of action of $\mathrm{ZnO}$ nanoparticles ( $\mathrm{ZnO} \mathrm{NPs}$ ) against a number of fungal contaminants. The efficacy of ZnO NPs was qualitatively and quantitatively assessed against: Penicillium expansum, Alternaria alternata, Botrytis cinerea and Rhizopus stolonifer. Mycelium growth diameters were measured onto Potato Dextrose Agar (PDA) plates loaded with different ZnO NPs concentrations (from 0 to $15 \mathrm{mM}$ ). Hereafter, the rate of the fungal diameter increase was quantified by linear regression modelling. Microscopic analysis was performed by scanning electron microscopy (SEM) images of agar plugs excised from plates with 0 and $12 \mathrm{mM} \mathrm{ZnO}$. All the fungi were inhibited by ZnO NPs at concentrations higher than $6 \mathrm{mM}$. SEM images showed clear morphological aberrations in the fungal structures of all the isolates grown in presence of $\mathrm{ZnO}$. Additionally, knowing that the chelating agent EDTA sequesters metal ions, it was added to fungal inoculated PDA plates with ZnO to study the NPs' mode of action. Cultures with $\mathrm{ZnO}+$ EDTA had growth trends similar to the controls thus indicating that ions are critical for the antifungal efficacy of the nanoparticles. 
Chemical synthesis of Zinc oxide nanoparticle: Its application for antimicrobial activity and plant health management H. KAUSHIK, P. Dutta, Assam Agricultural University, Jorhat, INDIA

Zinc oxide nanoparticles ( $\mathrm{ZnO} N P s$ ) are cheaper with superior durability, greater selectivity, heat resistance and biocompatible to human cells. ZnO NPs were synthesized chemically and characterized by UV-VIS spectroscopy, zeta sizer, DLS and electron microscopy. Antimicrobial activity of ZnO NPs was tested at seven concentration against Rhizoctonia solani and Sclerotinia sclerotiorum and found to inhibit the mycelial growth at all the tested concentration with highest inhibition $(76.22 \%$ and $70.66 \%$ respectively in poison food technique and $69.44 \%$ and $73.55 \%$ respectively in agar well method) at $225 \mathrm{ppm}$ concentration followed by $100 \mathrm{ppm}$. Similarly, ZnO NPs showed $100 \%$ mortality of $\mathrm{J}_{2}$ of Meloidogyne incognita at $225 \mathrm{ppm}$ concentration after 96 hours of treatment. As a seed treating agent, it also enhanced plant growth parameters, root nodulation, total chlorophyll content, primary and secondary metabolites of french bean and green gram at $100 \mathrm{ppm}$ concentration followed by $50 \mathrm{ppm}$ concentration. This present experiment revealed that $\mathrm{ZnO}$ NPs can be considered as a single bullet for the management of fungal and nematode pathogens, increasing plant growth parameters and strengthening host defense mechanism. More effort will also be given on development of nano-encapsule of ZnO NPs for sustainable plant health management with multiple actions like management of plant pathogens, nutrient supplement, strengthening the host defense mechanism etc.

Fungicide dip treatment of strawberry transplants as management of Botrytis cinerea resistant isolates

M. OLIVEIRA (1), L. Cordova (1), M. Marin (1), N. Peres (2), (1) University of Florida, Wimauma, FL, USA; (2) Gulf Coast Research and Education Center; University of Florida, Wimauma, FL, USA

Botrytis cinerea, causal agent of gray mold, is introduced into Florida strawberry fields through infected transplants. The pathogen can be managed by fungicide sprays throughout the season; however, previous work has demonstrated that several chemical classes are no longer effective due to $B$. cinerea resistance. The objective of this study was to evaluate the effectiveness of pre-plant, fungicide-dip treatments of strawberry transplants to manage $B$. cinerea. Transplants of two cultivars, 'Winterstar' and 'Radiance', were dipped in 11 different fungicides. After planting, overhead irrigation was used for $10 \mathrm{~d}$ to aid plant establishment. Eight leaves per plot were collected before and $14 \mathrm{~d}$ after treatment to evaluate $B$. cinerea incidence. Isolates ( $\mathrm{n}=139)$ obtained from the transplants were tested for fungicide sensitivity. Plant diameter was measured $47 \mathrm{~d}$ after planting. All treatments, including the controls, reduced B. cinerea incidence by at least $77 \%$ compared to pre-dip incidence; however, none of the treatments was $100 \%$ effective. Four treatments significantly reduced plant diameter of 'Radiance' plants, indicating phytotoxicity of some fungicides to this cultivar. Resistant isolates were recovered from all treatments tested. Our results indicate that initial inoculum seems to be naturally reduced after overhead irrigation for plant establishment. Furthermore, other alternatives still need to be tested to reduce initial resistant populations of B. cinerea.

Reduction in the number of fungicide applications for lettuce downy mildew by timing application based on aerial spore load N. DHAR (1), A. Anchieta (2), F. N. Martin (2), S. T. Koike (3), K. V. Subbarao (4), S. Klosterman (2), (1) UC Davis, Salinas, CA, USA; (2) USDA ARS, Salinas, CA, USA; (3) Univ of California, Salinas, CA, USA; (4) University of California at Davis, c/o U.S. Agricultural Research Station, Salinas, CA, USA

The downy mildew pathogen, Bremia lactucae, is an obligate, host-specific oomycete pathogen that infects lettuce. Characteristic symptoms include chlorosis of the infected leaf tissue followed by the appearance of sporulation on the abaxial side of the leaves. Mildew symptoms decrease market value of the produce and the sporulation results in secondary spread. Due to the heterogeneity and quick adaptation of this pathogen in the field, calendar-based fungicide applications have been the most effective means to manage downy mildew. However, repeated applications of chemicals has led to fungicide resistance in the pathogen. The previously developed qPCR assay specific to $B$. lactucae coupled with a solar-powered spore trap system for detection of B. lactucae was deployed in the current work to measure B. lactucae spore load at three commercial fields that each contained experimental plots. Based on the inoculum thresholds detected, fungicides applications were scheduled. Following spray advisories conserved approximately 1.7 sprays on average, relative to the calendar-based sprays. Deployment of this approach in commercial field can reduce grower costs and fungicide use in lettuce production while also decreasing the development of fungicide resistance in B. lactucae.

Chemical options for managing Cytospora canker, a major limiting factor for colorado peach production

S. MILLER (1), K. L. Otto (1), D. Sterle (2), I. Minas (2), J. E. Stewart (1), (1) Colorado State University, Fort Collins, CO, USA; (2) Colorado State University, Grand Junction, CO, USA

Cytospora canker is a major limiting factor of peach production in Colorado, accounting for $15-20 \%$ of production losses annually. Along with pathogen pressure, environmental factors unique to the western slope region of Colorado have exacerbated the problem. Chemical measures are important to growers because few options exist for preventing new infections and management practices have not been standardized. Our specific objectives were to: a) evaluate conventional and organic fungicides efficacy for Cytospora spp., b) test wound sealing with fungicides embedded in paints to develop a preventive approach in existing orchards, and c) monitor spore production and lesion infection size throughout the calendar year. We found Captan and Topsin, combined with 50\% Latex paint, were most effective as preventatives for conventional production practices, and Lime Sulfur was most effective for organic production practices. Further, we observed that 50\% Latex paint alone or mixed with Captan or Topsin, and Surround mixed with Lime Sulfur, were effective at reducing sporulation on existing cankers, thereby reducing the risk of new infections. Trends in spore production and lesion infection size indicate a decrease in inoculum load and pathogen aggressiveness during months of extreme temperatures. Using these chemical measures and pathogen monitoring methods, future infections within existing orchards can be reduced, increasing orchard longevity.

Effect of fungicide seed treatment and temperature on cotton stand establishment in soils with different cropping histories S. YOUNG (1), J. E. Woodward (2), (1) Texas Tech University, Lubbock, TX, USA; (2) Texas A\&M AgriLife Extension Service, Lubbock, TX, USA

New auxin-tolerant herbicide technologies and somewhat improved cotton prices have led to increased interest in production in areas with diverse rotation crops. One concern with this change is the potential for an increased risk in seedling disease. The objective of this research was to determine the efficacy of seed treatment fungicides on stand establishment is soils from different fields. Seed of Phytogen 499WRF were treated with the insecticide imidacloprid, the selective fungicides peflufen or metalaxyl, and the combination of peflufen and metalaxyl. Non-treated seed served as a control. Soils from eight fields from a diverse crop rotation history were collected and used in growth chamber experiments conducted at 18 and $24^{\circ} \mathrm{C}$. Germination and vigor were assessed following the application of treatments. Stands and plant vigor were determined bi-weekly for three weeks. Germination was unaffected by fungicide seed treatment and averaged $98.5 \%$ across the two temperatures. Radical lengths were reduced for seeds treated with metalaxyl $(14.0 \%)$ and metalaxyl and peflufen (17.6\%) compared to the control. Despite this observation, seed treatment fungicides improved stand establishment over the non-treated control under both temperatures. In general, the combination of peflufen and metalaxyl resulted in greater stands and increased vigor compared to the two fungicides alone. Results from these studies indicated that seedling disease pressure from Rhizoctonia solani was more pronounced in fields continually planted to cotton, or previously planted to alfalfa, whereas, fields with a history of peanut production were infested with both $R$. solani and Pythium spp.

The grape powdery mildew conundrum: Fungicide selection and timing B. WARNEKE (1), L. D. Thiessen (2), T. Neill (3), W. Mahaffee (3), (1) Oregon State University, Corvallis, OR, USA; (2) North Carolina State University, Raleigh, NC, USA; (3) USDA, Corvallis, OR, USA 
Grape powdery mildew (GPM, causal agent Erysiphe necator) is the most economically important disease of grapevine in the Western U.S. The inflorescence and early infructescence growth stages are highly susceptible to GPM and its management during these stages is critical to preventing economic losses. Targeting these stages with mobile fungicides could significantly reduce fruit infections. A small-plot experiment was conducted in Corvallis, Oregon during the 2015 and 2016 growing seasons to examine the interaction between fungicide chemistry and application timing on GPM berry infection. Five commonly used fungicides with varying degrees of mobility were applied at three different growth stages around bloom. The controls consisted of a non-treated and sulfur, each applied every 14 days. The proportion of grape berries infected with GPM was significantly influenced by the interaction between fungicide selection and application timing (Drop-in-deviance test, $\chi^{2}=33.1, \mathrm{df}=8, \mathrm{P}<0.01$ ). All treatments significantly reduced fruit infection compared to the non-treated control $(\mathrm{Z}$ test, $\mathrm{P}<0.01)$. The trifloxystrobin, quinoxyfen, and fluopyram applications made at berry set were most effective at reducing the proportion of berries infected, having 4.8, 8.0, and 11.5 times lower odds of berry infection, respectively, compared to the earliest timing $(\mathrm{Z}$ test, $\mathrm{P}<0.01)$. This research indicates that using mobile fungicides during fruit development may improve disease control.

\section{Influence of fungicides on gas exchange of pecan foliage}

B. W. Wood, C. H. BOCK, USDA ARS, Southeastern Fruit and Tree Nut Research Laboratory, Byron, GA, USA

Several fungicide chemistries are used to control disease on pecan (Carya illinoinensis), but there is little knowledge of the side-effects of these chemistries on host physiological processes, including photosynthesis $\left(P_{n}\right)$, stomatal conductance $(C \mathrm{~s})$, transpiration rate $\left(T_{r}\right)$ and water use efficiency $\left(E_{w} ; P_{n} / T_{r}\right.$ ). This study quantifies the impact of several fungicides used to control scab (caused by Venturia effusa) on $P_{n}, C \mathrm{~s} T_{r}$ and $P_{n} / T_{r}$. Multiple field experiments showed that certain fungicide chemistries reduced $P_{n}$, stomatal conductance $(C \mathrm{~s})$, transpiration rate $\left(T_{r}\right)$ and water use efficiency $\left(E_{w} ; P_{n} / T_{r}\right)$, whereas others are benign. Neither triphenyltin hydroxide nor the triazoles tested had a negative impact on gas exchange characteristics. Suppression of $P_{n}$ by certain fungicides was short term, but was long term or permanent for others. Dodine, phosphorous acid, ziram and certain strobilurins were capable of suppressing gas exchange, especially $P_{n}$, up to several weeks after a single treatment. Leaf $P_{n}$ could be suppressed by up to $80 \%$ for at least several weeks after a single exposure. Reductions of such magnitude and duration are potentially problematic in crop species, such as pecan, where low carbohydrate reserves can have major impact on crop quality and return cropping. These results should help provide insight to ensure proper fungicide usage in commercial pecan operations to minimize impacts on the host physiology.

\section{New fungicides for management of avocado root rot in California}

R. J. Belisle, W. Hao, B. McKee, P. Manosalva, J. E. ADASKAVEG, Department of Plant Pathology and Microbiology, University of California, Riverside, CA, USA

Phytophthora root rot (PRR) caused by Phytophthora cinnamomi $(P c)$ is the most serious disease of avocado worldwide. Currently, in the United States only mefenoxam and potassium phosphite (PP) are registered to manage PRR. Therefore, the new oomycete-specific fungicides ethaboxam, fluopicolide, mandipropamid, and oxathiapiprolin (OXA), all with a different mode of action, were evaluated against PRR in greenhouse studies. The soil of potted 8month-old 'Zutano' avocado seedlings was inoculated with $P c$-colonized millet seeds and treated after one week with the four new fungicides as well as mefenoxam and PP. Feeder roots and rhizosphere soil were plated onto a selective medium after 4 months. Treatment efficacy was assessed based on PRR incidence and $P c$ soil population sizes. Most fungicides significantly reduced PRR and $P c$ population size as compared to the control. OXA at the $140 \mathrm{~g} / \mathrm{Ha}-$ rate was most effective, reducing PRR incidence and $P c$ population size by 94 and $92 \%$, respectively. OXA-treated plants also showed significantly increased shoot growth and root dry weight. In another study with the PRR-susceptible 'RO.54' and the -tolerant 'Dusa' avocado rootstocks, OXA was also the most effective treatment. Fluopicolide and mandipropamid also reduced the disease in both studies and had similar efficacy. Based on these studies, OXA, fluopicolide, and mandipropamid should be registered on avocado to develop rotational programs of products with different FRAC codes.

\section{Apple post-harvest infection by Paecilomyces niveus causes spoilage of thermally processed products} M. BIANGO-DANIELS, A. Synder, R. Worobo, K. T. Hodge, Cornell University, Ithaca, NY, USA

Paecilomyces niveus is known in the food industry as an important mycotoxigenic mold that spoils heat-processed fruit products. Though regarded as a soil-borne contaminant, our research shows it can be an apple pathogen like its relative Penicillium expansum. Both fungi synthesize patulin, which acts as a virulence factor in apple infection. Our goals were to assess the ability of $P$. niveus to infect apples and test whether infected apples can be a source of spoilage fungi in the finished apple juice concentrate, a heat-processed product often made from low quality apples. Two varieties of apples were inoculated with $P$. niveus to study pathogenicity and complete Koch's postulates. To determine whether $P$. niveus can survive heat-processing, juice concentrate was made from infected apples. We confirmed $P$. niveus can infect apples through wounds, causing lesions similar to Bull's-Eye Fruit Rot. Apple varieties differed significantly in lesion size $(\mathrm{p}=0.003$ ). Juice concentrate made from infected apples contained viable $P$. niveus propagules, which were reduced by $4-\log$ CFU/L but not eliminated across five stages of processing. We conclude $P$. niveus is an overlooked post-harvest apple pathogen that can survive thermal processing and produce patulin, a mycotoxin limited to $<50 \mathrm{ppb}$ in apple products. The link between post-harvest apple disease and food spoilage is novel and may explain the episodic nature of $P$. niveus food spoilage.

\section{Atoxigenic growth rates and intraspecific aflatoxin inhibition \\ R. R. SWEANY (1), C. DeRobertis (2), K. E. Damann (3), (1) Louisiana State Univ Ag Center, Baton Rouge, LA, USA; (2) Louisiana State UniversityAgCenter, Baton Rouge, LA, USA; (3) Louisiana State University AgCenter, Baton Rouge, LA, USA}

Aflatoxin is an acutely toxic carcinogen produced by Aspergillus flavus. Atoxigenic biocontrol strains reduce aflatoxin production in a thigmoregulated manner. We reasoned increased growth would increase touch (physical interaction) and should theoretically increase toxin inhibition. Toxigenic strains 53 and Af70s were co-inoculated in buffered glucose salts medium in 24-well plates with atoxigenic strains 17, 19, 21, 51, Tx9-8, NRRL 21882 and Af36 to determine intraspecific aflatoxin inhibition and grown alone in petri dishes to determine growth/weight. Growth best fit a quadratic model for $\mathrm{x}=\ln ($ time $)$ and $\mathrm{y}=\ln$ (weight). 21882 and 51 did not significantly reduce aflatoxin and had smallest intercepts and initial growth rates, but highest acceleration in growth. Aflatoxin was reduced by 22, 19 and Tx9-8 which had larger intercepts and initial growth rates but smaller acceleration in growth. Af36 reduced aflatoxin more but intercept and acceleration was similar to 51 and 21882 and an intermediate initial growth rate. The greatest aflatoxin reduction was by 17 which also had the greatest intercept and initial growth rate, but the smallest acceleration. The association of large vs. small initial growth and greatest vs. no inhibition supports earlier observations that touch must occur early during toxigenic germination for best inhibition. Af36 had less initial growth compared to less inhibitory strains, suggesting other factors may lead to inhibition.

Magnesium oxide nanomaterial, a novel bactericide for control of bacterial spot of tomato without accumulating in fruit Y. Y. LIAO (1), A. L. Strayer (1), J. C. White (2), A. Mukherjee (2), R. De La Torre-Roche (2), W. H. Elmer (2), M. Young (3), S. Santra (3), L. Ritchie (4), J. H. Freeman (4), J. B. Jones (5), M. Paret (6), (1) University of Florida, Department of Plant Pathology, Gainesville, FL, USA; (2) Connecticut Agric Exp Station, New Haven, CT, USA; (3) University of Central Florida, Orlando, FL, USA; (4) University of Florida, Quincy, FL, USA; (5) Department of Plant Pathology, University of Florida, Gainesville, FL, USA; (6) North Florida Research and Education Center, University of Florida, Quincy, FL, USA

Bacterial spot of tomato, incited by four Xanthomonas species, can cause major yield losses. There has been no effective chemical control strategy utilizing bactericides. Copper-tolerant strains rendered $\mathrm{Cu}$-based bactericides ineffective. Therefore, finding an efficient alternative has been a long-term 
goal. In this study, magnesium oxide ( $\mathrm{MgO})$ and other metal oxide nanomaterials were evaluated against $\mathrm{Cu}$-tolerant and $\mathrm{Cu}$-sensitive $X$. perforans strains. $\mathrm{MgO}$ had high bactericidal activity against both strains. In greenhouse experiments, disease severity was significantly reduced by $\mathrm{MgO}$ at 200 $\mu \mathrm{g} / \mathrm{mL}$ compared to copper-mancozeb, the grower standard and the untreated control $(\mathrm{p}=0.05)$. In the field, two trials evaluated both nano $(20 \mathrm{~nm})$ and micron $(0.3$ and $0.6 \mu \mathrm{m})$ sized $\mathrm{MgO}$. Only nano-sized $\mathrm{MgO}$ at $200 \mu \mathrm{g} / \mathrm{mL}$ consistently reduced disease severity compared to the untreated control $(\mathrm{p}=$ 0.05), whereas copper-mancozeb was not significantly different from the untreated control. Analysis of fruit from MgO treated plots by Inductively Coupled Plasma Mass Spectrometry analysis revealed no significant accumulation of $\mathrm{Mg}, \mathrm{Cu}, \mathrm{Ca}, \mathrm{K}, \mathrm{Mn}, \mathrm{P}$ and $\mathrm{S}$ compared to untreated fruit. This study demonstrated for the first time the antibacterial potential of $\mathrm{MgO}$ nanomaterials against $X$. perforans and its potential use against bacterial spot of tomato.

Evaluation of new fungicides against Fusarium oxysporum causing wilt in Strawberry A. HANNAN (1,2), S. Atta (3), T. Akhtar (4), I. Ahmad (5), M. Atiq (6), (1) University of Florida, Gainesville, FL, USA; (2) Ghazi University, D G KHAN, PAKISTAN; (3) Ghazi University, Dera Ghazi Khan, PAKISTAN; (4) Univ of Agriculture, Faisalabad, PAKISTAN; (5) University of Agriculture, Faisalabad, PAKISTAN; (6) University of Agriculture, Faislabad, PAKISTAN

Cultivation of strawberry (Fragaria ananassa) is surging among Pakistani famers because of its profit ratio as compared to traditional crops. This crop has been attacked by various pathogens responsible for low yield. The objective of this study was to identify fungicides to control Fusarium wilt caused by Fusarium oxysporum (Schlecht) in the field. Firstly eight fungicides were tested by the poison agar technique against colony growth of $F$. oxysporum under lab conditions. Out of eight chemicals, four were found significantly superior as compared to control in inhibiting pathogen growth. These four were evaluate in the field against the three most common varieties of strawberry (Chandelier, Corona and Douglas) using artificial inoculation and randomized complete block design (RCBD). All the chemicals controlled wilt disease with varying degree of success. The mean results of field trials indicated that Difenconazole 250 SC@350 mL/ha showed 84.6 percent disease reduction over control while Hexaconazole 5\% SC @, 750 ml/ha and Carbendazim 50\% WP @ 612.5 gm/ha gave 80.2 and 76.0\% respectively whereas Tebuconazole+Trifloxystrobim 75 WP @ 150 gm/ha was least effective and expressed $68.4 \%$ disease reduction over control. Therefore Score (Difenconazole 250 SC) is most effective and should be applied for management of Fusarium wilt in strawberry in Pakistan.

Atoxigenic Aspergillus flavus: A hidden mechanism of biocontrol

L. R. L. ARONE (1), P. J. Cotty (2), (1) University of Arizona, Tucson, AZ, USA; (2) USDA-ARS, University of Arizona, Tucson, AZ, USA

Aflatoxins are potent mycotoxins produced by several Aspergillus species. These toxins contaminate foods and feeds worldwide impacting health and trade. Research on managing aflatoxin contamination has focused on breeding, agronomic practices, and biocontrol. Biocontrol with atoxigenic genotypes of A. flavus may result in aflatoxin reductions of over $90 \%$. Applications change the structure of Aspergillus communities so that aflatoxin producers are less common and atoxigenics dominate. In co-infected crop components, contamination is reduced both through competitive exclusion and a second, minor, undefined mechanism. To improve understanding of mechanisms through which atoxigenic $A$. flavus reduce aflatoxins, degradation of aflatoxins by atoxigenic strains of Aspergillus flavus was evaluated. Two commercial biocontrol agents (Aflaguard and AF36) and one biocontrol candidate MZM221-5 were inoculated onto both maize and liquid media containing aflatoxins. The three atoxigenic $A$. flavus genotypes degraded aflatoxins over the 4 week period. The current study reports for the first time the ability of atoxigenic genotypes of $A$. flavus currently used in biocontrol products to degrade aflatoxins in maize. Although all the evaluated atoxigenic genotypes degraded aflatoxin $\mathrm{B}_{1}$, there was variation among the genotypes in the speed of degradation. Optimal selection of atoxigenic biocontrol agents may include assessment of both competitively ability and ability to degrade aflatoxins.

Description of a novel aflatoxin-producing Aspergillus species from a region of the United States with perennial aflatoxin contamination P. SINGH (1), P. J. Cotty (2), (1) University of Arizona, Tucson, AZ, USA; (2) USDA-ARS, University of Arizona, Tucson, AZ, USA

Aflatoxins are potent carcinogens that contaminate a wide range of crops in warm regions, and are produced by several species in Aspergillus section Flavi. To completely describe the etiology of aflatoxin contamination, aflatoxin-producing species must be identified and characterized. During the course of molecular phylogenetic analysis of aflatoxin-producing fungi native to North America, a novel aflatoxin-producing species was identified. The new taxon was assigned the name Aspergillus texensis, P. Singh and P.J. Cotty, species novum. The new taxon has sclerotia production not differentiable from the A. flavus $\mathrm{S}$ strain morphotype. However, A. texensis produces both $\mathrm{B}$ and $\mathrm{G}$ aflatoxins whereas $A$. flavus produces only $\mathrm{B}$ aflatoxins. Total aflatoxins produced by isolates of A. texensis on maize did not differ ( $\mathrm{p}>0.05 ;$ mean $=172 \pm 9.5 \mathrm{mg} / \mathrm{kg}$ ) from quantities produced by the $A$. flavus $\mathrm{S}$ strain. Aspergillus texensis formed a highly supported monophyletic clade in phylogenies based on concatenated partial gene sequences of the betatubulin $(0.5 \mathrm{~kb})$, calmodulin $(1.1 \mathrm{~kb})$, and nitrate reductase $(2.1 \mathrm{~kb})$ genes. Aspergillus texensis is known from eleven isolates, nine from soils cropped to maize in Texas, where aflatoxin contamination of crops is a perennial issue, and one isolate each from maize grown in Arkansas and Louisiana. This is the first report of the occurrence in the United States of an S morphotype Aspergillus with ability to produce both B and G aflatoxins.

Physiological benefits from foliar application of penthiopyrad fungicide in wheat

M. J. MARTIN (1), H. K. Ngugi (2), B. Perotin (3), S. Rossall (4), (1) DuPont Ag \& Nutrition, Columbus, OH, USA; (2) DuPont Crop Protection, Newark, DE, USA; (3) DuPont ERDC, Nambsheim, FRANCE; (4) Univ. of Nottingham, Leicester, UNITED KINGDOM

In greenhouse tests in 2010, penthiopyrad, a succinate dehydrogenase inhibitor (SDHI) fungicide increased root development in wheat seedlings, suggesting it may offer beneficial plant physiological effects. Three greenhouse tests were conducted between 2012 and 2014 to confirm this finding and compare penthiopyrad with SDHI + triazole fungicides commonly used in wheat. Depending on the test, a single application was made at BBCH14 to $\mathrm{BBCH} 31$ and evaluations carried out 4 weeks later. Penthiopyrad at $125-250$ gai/ha significantly increased root and shoot dry weight relative to the untreated check and other SDHI + triazole fungicides. In one of the experiments, penthiopyrad used solo or in tank-mixtures with triazoles increased root dry weight by $>200 \%$ over the untreated check, while the competitive SDHI premixes gave a maximum of $124 \%$. In other laboratory assays, penthiopyrad also increased chlorophyll content of wheat leaves by 110-117\% over the untreated check, while no increase was noted for the other SDHI fungicides tested. In field trials carried out from 2010 to 2011 on winter wheat in absence of disease, penthiopyrad increased nitrogen use efficiency by ca $7 \%$ relative to the untreated check. In laboratory tests, penthiopyrad treatments also increased water use efficiency by $5 \%$ over the untreated check under moisture stress conditions. Together, these results suggest that penthiopyrad may help wheat production during stress events in the growing season.

Colonization, conidiation, and aflatoxin contamination of maize seed by Aspergillus flavus requires oxylipins from both host and pathogen E. J. BORREGO, M. V. Kolomiets, Texas A\&M University, College Station, TX, USA

Oxylipins, generated from the oxygenation of fatty acids, are among the multitude of molecular signals governing the interaction between plant hosts and fungal parasites and are proposed to serve as mediators during the reciprocal signal exchange between organisms. During plant-fungal interactions, this "lipid language" is initiated and maintained by lipoxygenases (LOX) in plants and Psi producing oxygenases (Ppo) in fungi, however the specific genes, enzymes, and products remain to be elucidated in any plant-fungal pathosystem. To date, the best understood oxylipins of non-mammalian origins are the jasmonates of plants and Psi factors (precocious sexual inducers) of fungi. These and other plant and fungal oxylipins display remarkable structural similarity to one another, prompting a hypothesis that oxylipins are reciprocally exchanged and recognized during plant-fungal interactions. To test this hypothesis, we employed a set of oxylipin mutants of both maize and of Aspergillus flavus to determine the role of host- and pathogen-derived oxylipins 
in the ability of the fungus to colonize seed, sporulate, and produce aflatoxin. We found that individual oxylipin biosynthetic enzymes in both host and pathogen determine the outcome of the plant-fungal interaction and regulate specific pathogenicity processes.

Efficacy of a single late-dormant application of mineral oil plus chlorothalonil for control of peach scab, caused by Venturia carpophila T. GLENN (1), P. M. Brannen (2), C. H. Bock (3), M. W. Hotchkiss (4), J. Pitts (5), E. J. Sikora (6), (1) University of Georgia Plant Pathology Department, Athens, GA, USA; (2) University of Georgia, Athens, GA, USA; (3) USDA ARS, Southeastern Fruit and Tree Nut Research Laboratory, Byron, GA, USA; (4) USDA ARS, Byron, GA, USA; (5) Auburn University, Clanton, AL, USA; (6) Auburn University, Department of Plant Pathology, Auburn, AL, USA

Peach scab, caused by Venturia carpophila, is a major disease of peach across the southeastern United States. In multi-year, multi-site field trials, mineral oil plus chlorothalonil late-dormant treatments suppressed both scab incidence and severity, as previously reported for almond scab in California. When applied alone, mineral oil plus chlorothalonil reduced disease incidence and severity by 28.2 and $47.2 \%$, respectively, as compared with an untreated check. A second series of experiments determined whether a late-dormant application of mineral oil plus chlorothanonil, as part of a fullseason fungicide program, would substitute for a missed chlorothalonil application at the shuck split phenology (a critical infection period for scab); this would at times have value (e.g. when rainy conditions make shuck split sprays impossible). Although late-dormant applications of mineral oil plus chlorothalonil did not fully substitute for a missed shuck split chlorothalonil spray, a mineral oil plus chlorothalonil treatment resulted in an average reduction in disease incidence and severity of 28.0 and 33.3\%, respectively, compared with the treatments in which shuck split treatments were removed from a full-season fungicide program. These results are important in that most peach growers already apply oil for scale insect control, so for a relatively small cost, the addition of chlorothalonil in a tank mix with mineral oil could provide better overall scab management.

Fungicide sensitivity and aggressiveness of Phytophthora betacei isolates in Colombia

M. MIDEROS (1), M. Parra (2), N. Guayazan (1), G. Danies (1), S. Restrepo (1), (1) Universidad de los Andes, Bogota, COLOMBIA; (2) Universidad de Los Andes, Bogot, COLOMBIA

In South America, late blight is one of the main threats to several domesticated and semi-domesticated crops. A recently described species, Phytophthora betacei, has been found causing symptoms akin to late blight on tree tomato crops (Solanum betaceum). This pathogen is highly destructive and specialized, causing important economic losses on tree tomato crops. Here, we evaluated the variation in aggressiveness and fungicide sensitivity of a collection of $P$. betacei isolates $(\mathrm{n}=40)$. Five parameters (lesion size, sporulation, latent period, incubation period, and infection efficiency) were measured for each $P$. betacei isolate on five accessions of $S$. betaceum. We tested for differences on each aggressiveness parameter according to the isolate, the host accession, and the interactions between isolate and accession. Data showed marked differences among all aggressiveness parameters determined by the pathogen and host genotype. Fungicide sensitivity was evaluated, under laboratory conditions, for three fungicides (mefenoxam, cymoxanil, and fluopicolide). No mycelial growth and sporulation was detected for either of the $P$. betacei isolates tested on high concentrations of each fungicide. This suggests that all $P$. betacei isolates tested were mostly sensitive to the main fungicides used to control late blight disease in Colombia. This finding is interesting given that isolates of $P$. infestans collected on potato crops neighboring tree tomato crops seem to be highly resistant to these fungicides. We discuss the implications of phenotypic variations in terms of aggressiveness and fungicide sensitivity to the control of this disease in Colombia.

\section{Baseline Sensitivity to Difenoconazole in Penicillium expansum Isolates from Apple} M. E. ALI, D. R. De Angelis, K. A. Mulvaney, L. K. Pandit, A. Amiri, Washington State University, Wenatchee, WA, USA

Blue mold caused by Penicillium expansum is the most prominent postharvest disease of apple and pear. To control this disease, packers mainly rely on the use of the three fungicides registered for postharvest application (thiabendazole (TBZ), fludioxonil, and pyrimethanil). The widespread resistance to $\mathrm{TBZ}$ and the recent emergence of resistance to pyrimethanil have become concerns due to reduced efficacy and the limited options. Thus, there is a need for alternative fungicides with different modes of action to control $P$. expansum and other postharvest pathogens. In this study, we investigated the in vitro and in vivo sensitivities of 130 baseline $P$. expansum isolates to the new demethylation inhibitor (DMI) fungicide difenoconazole. The sensitivity was evaluated using mycelial growth and spore germination inhibition assays to determine effective concentration to inhibit $50 \%$ growth or germination ( $\mathrm{EC}_{50}$ values). The mean $\mathrm{EC}_{50}$ values for difenoconazole were 0.17 and $0.32 \mu \mathrm{g} / \mathrm{ml}$ based on mycelial growth and germination inhibition, respectively. The respective $\mathrm{EC}_{50}$ values ranged from 0.12 to $0.29 \mu \mathrm{g} / \mathrm{ml}$ and from 0.18 to $0.36 \mu \mathrm{g} / \mathrm{ml}$. The minimum inhibitory concentration (MIC) was $1.0 \mu \mathrm{g} / \mathrm{ml}$. Efficacies of difenoconazole to control $P$. expansum isolates were evaluated on detached apple fruit at 0 or $20^{\circ} \mathrm{C}$. Early findings indicate that difenoconazole applied preventively may provide better efficacy against blue mold. For up to 10 days at room temperature and 90 days at $0^{\circ} \mathrm{C}$, no decay was observed on fruit treated with difenoconazole and inoculated with blue mold. When combined with low temperatures and controlled atmosphere, both preventive and curative applications should control blue mold effectively for extended storage periods.

\section{Evaluation of sensitivity of Blumeriella jaapii to SDHI fungicides}

J. C. GLEASON (1), T. J. Proffer (1,2), S. M. Slack (1), C. A. Outwater (1), N. Rothwell (1), G. W. Sundin (1), (1) Michigan State University, East Lansing, MI, USA; (2) Kent State Univ, Salem, OH, USA

Cherry leaf spot (CLS), caused by the fungus Blumeriella jaapii, is a devastating disease of sour cherry (Prunus cerasus 'Montmorency') in the Great Lakes region. Succinate dehydrogenase inhibitor (SDHI) fungicides are widely used for CLS management. We have previously characterized resistance to boscalid in B. jaapii, but have not examined sensitivity to more recently registered SDHI fungicides. In 2016, 36 sour cherry orchards throughout Michigan were sampled, and 883 single conidium isolates were established. The in vitro sensitivity of these isolates to boscalid, fluopyram, and fluxapyroxad was examined using a minimum inhibitory concentration (MIC) test. Our previous analyses have shown that reductions in CLS control with Pristine (boscalid + pyraclostrobin) fungicide occur in orchards with at least $30 \%$ of isolates exhibiting an MIC of $25 \mu \mathrm{g} / \mathrm{ml}$. In this study, $66.6 \%$ and $38.8 \%$ of the 36 sour cherry orchards harbored at least $30 \%$ of isolates with MICs of $25 \mu \mathrm{g} / \mathrm{ml}$ to boscalid and fluxapyroxad, respectively. Overall, a total of $39.1 \%, 72.1 \%$, and $29.1 \%$ of the entire isolate collection exhibited MICs of $25 \mu \mathrm{g} / \mathrm{ml}$ or higher to boscalid, fluopyram, and fluxapyroxad, respectively. We also tested 798 of the more sensitive isolates to four levels of boscalid, and determined MICs of $2.5 \mu \mathrm{g} / \mathrm{ml}(10.5 \%), 5 \mu \mathrm{g} / \mathrm{ml}(9.6 \%), 10$ $\mu \mathrm{g} / \mathrm{ml}(11.2 \%), 25 \mu \mathrm{g} / \mathrm{ml}(33.7 \%)$, and $>25 \mu \mathrm{g} / \mathrm{ml}$ (34.8\%). Further testing with fluopyram and fluxapyroxad is ongoing.

Effects of Fungicide and Fertility on Disease Development and Yield in Winter Wheat M. R. BREUNIG (1), M. Nagelkirk (2), A. Byrne (1), M. Chilvers (1), (1) Michigan State University, East Lansing, MI, USA; (2) Michigan State University Extension- Sanilac County, Sandusky, MI, USA

As growers try to increase yields and profitably, higher rates of nitrogen fertilization are increasingly popular. However, nitrogen can also contribute to disease and lodging. Fungicide and growth regulator products may help mitigate these negative consequences. The objective of this study is to examine the interactions of fungicide, nitrogen, and growth regulators and their effects on disease and ultimately, yield. In 2015, 2016, and 2017, field trials were conducted in East Lansing, MI to evaluate leaf disease, Fusarium head blight (FHB), lodging, deoxynivalenol (DON) production, and yield. The study was a factorial design with different combinations of the fungicides Stratego YLD (Feekes 6) and Prosaro (Feekes 10.5.1) and growth regulator Palisade (Feekes 6), under low (90 lbs N/A at green up) or high (additional $50 \mathrm{lbs}$ N/A at Feekes 6) nitrogen fertilization. The highest yielding treatments and level of disease control varied each year, due to different disease pressures and environmental conditions. In 2015, the highest yielding treatments were low nitrogen treatments containing Prosaro, which were significantly higher than all high fertility treatments, except those with both Stratego YLD and 
Prosaro. Low nitrogen fertility treatments with Prosaro had the lowest DON values, and FHB disease index ratings followed the same trends. In 2016 , there was very little FHB disease development due to dry conditions during flowering. However, stripe rust (Puccinia striiformis) pressure was extremely high. The highest yielding treatment was high nitrogen with both a Stratego YLD and Prosaro application, but this was not significantly different than the five treatments containing either one or both of Stratego YLD or Prosaro.

Fungicide Sensitivity Profiles of Phacidiopycnis piri and Phacidiopycnis washingtonensis Isolates from Pome Fruit in Washington M. E. ALI, L. K. Pandit, D. R. De Angelis, K. A. Mulvaney, A. Amiri, Washington State University, Wenatchee, WA, USA

Phacidiopycnis piri (Teleomorph, Potebniamyces pyri) and Phacidiopycnis washingtonensis are two fungal pathogens reported to cause Phacidiopycnis and Speck rots on pear and apple, respectively. Infections by both pathogens occur in the orchard, remain latent, and only show symptoms after several months of storage. Management relies on orchard sanitation and the use of fungicides pre- and postharvest. In this study, we evaluated the sensitivity of 110 P. washingtonensis and 76 P. piri isolates to the preharvest fungicides pyraclostrobin and boscalid and to the postharvest fungicides thiabendazole (TBZ), fludioxonil, difenoconazole and pyrimethanil. A mycelial growth inhibition test was used to determine the effective concentration sufficient to inhibit $50 \%$ of mycelial growth ( $\mathrm{EC}_{50}$ values). The mean $\mathrm{EC}_{50}$ values in $P$. washingtonensis for fludioxonil, pyrimethanil, TBZ, difenoconazole, pyraclostrobin and boscalid with were $0.1,0.3,0.8,1.8,2.1$ and $4.9 \mu \mathrm{g} / \mathrm{ml}$, respectively. For $P$. piri, the respective mean $\mathrm{EC}_{50}$ values were $0.2,0.6,1.6$, $1.1,0.4$ and $1.8 \mu \mathrm{g} / \mathrm{ml}$. For each pathogen, three isolates with varied sensitivity levels were selected and tested for their in vivo sensitivity to the six aforementioned fungicides. When applied at label rates, fludioxonil, difenoconazole and pyrimethanil effectively controlled both Phacidiopycnis species, regardless of their $\mathrm{EC}_{50}$ values, after 90 days of storage at $0^{\circ} \mathrm{C}$ in a room atmosphere. Although P. piri and P. washingtonensis isolates showed different levels of sensitivity to the six fungicides tested, our results indicate similar sensitivity trends within the same chemical group. The two pathogens should be comparably controlled by fungicides from the same chemical group.

Effect of At-Plant Drench Applications of Actigard on Tomato Seedlings for Bacterial Leaf Spot Control H. ADKISON (1), A. Wen (2), G. E. Vallad (3), (1) Univ of Florida GCREC, Wimauma, FL, USA; (2) University of Florida, Wimauma, FL, USA; (3) Gulf Coast Research and Education Center, University of Florida, Wimauma, FL, USA

Acibenzolar-S-methyl (Actigard) is a plant activator that induces systemic acquired resistance (SAR) in plants to a variety of pathogens. Both soil and foliar applications of Actigard have been evaluated on tomato for the control of bacterial leaf spot caused by Xanthomonas perforans. Replicated field trials were conducted over three growing seasons (spring 2015, fall 2015, and spring 2016) to evaluate the effect of applying Actigard as a drench to tomato seedlings at planting in conjunction with a seasonal program consisting of either weekly foliar applications of Actigard ( $0.5 \mathrm{oz} / \mathrm{A})$, Cuprofix 40D (2.5lbs/A) and Penncozeb 75DF (1.5lbs/A). Four drench application rates of Actigard (0.25, 0.5, 0.75, and 1.0 oz/100 gal) were compared to AgriMycin $(16 \mathrm{oz} / 100 \mathrm{gal})$ and a non-treated control during transplanting. After planting, the tomato plants were inoculated with Xanthomonas perforans at $10^{6}$ $\mathrm{cfu} / \mathrm{mL}$, rated for bacterial spot severity every 10-15 days, and harvested at the end of the season. Overall, the transplant drench applications prior to planting had a significant effect on reducing foliar disease severity over all three seasons. Two seasons (fall 2015 and spring 2016) showed a significant $(P<0.0001)$ combined effect of both drench and foliar applications of Actigard in reducing disease severity. Actigard is commonly applied as a foliar spray one to two weeks after planting. However, treating at transplant may provide better protection during late summer plantings in Florida when weather conditions favor rapid disease development and limit a grower's ability to make foliar applications.

\section{Discovery and characterization of small molecules inhibitory to Erwinia tracheiphila}

C. VRISMAN, L. Deblais, G. Rajashekara, S. A. Miller, The Ohio State University, Wooster, OH, USA

Plant pathogenic bacteria in the genus Erwinia cause destructive diseases, including bacterial wilt of cucurbits caused by E. tracheiphila (Et). Bacterial wilt is a major problem of cucurbit crops in the Midwestern US. Conventional bactericides are limited in availability and insufficient or ineffective in combating bacterial infections. Small, drug-like molecules have shown high efficacy in inhibition of human bacterial pathogens. The objective of this study is to identify and characterize small molecules that are effective in controlling Et. From a high-throughput screening of a library of 4,182 small molecule compounds using a cucumber strain of Et, 231 molecules presented "cidal" activity and 234 showed a "static" effect when tested at a concentration of $100 \mu \mathrm{M}$. When compared to previous tests of this library with other bacteria, both plant- and animal-pathogenic, 16 compounds were specific to Et. These molecules will be tested for their effectiveness in inhibiting bacterial growth of multiple $E t$ strains at lower concentrations and without toxic effects to beneficial bacteria, human cells, cucurbit plants, and pollinators, and likelihood of bacterial resistance development, a major concern in antibiotic development.

\section{Integrated management of stripe rust of winter wheat in Wisconsin}

B. MUELLER (1), S. Chapman (2), S. Conley (1), D. L. Smith (3), (1) University of Wisconsin-Madison, Madison, WI, USA; (2) University of Wisconsin - Madison, Madison, WI, USA; (3) University of Wisconsin, Madison, WI, USA

Wheat stripe rust, caused by Puccinia striiformis f. sp. tritici, has been an increasing problem in areas of the upper Midwest. Over the last four seasons we have observed consistent stripe rust pressure on some varieties of winter wheat throughout the production area of Wisconsin. In an integrated management trial, fungicides Headline and Prosaro were applied at three growth stages: Feekes 6, Feekes 8, and Feekes 10 . These applications were compared to a non-treated control or a full-season fungicide program (positive control). Fungicide applications were applied to winter wheat cultivars varying in resistance to stripe rust: resistant (Pro Seed 380), moderately susceptible (Kaskaskia), and susceptible (Pro Seed 420). Feekes 8 and Feekes 10 fungicide applications led to a significant reduction in stripe rust incidence for the cultivars Kaskaskia and Pro Seed 420 when compared to the nontreated control $(P<0.01)$. Disease incidence scores were not significantly different among all treatments applied to the resistant cultivar Pro Seed 380. Full-season fungicide coverage led to the highest yields across all cultivars. Headline, applied at Feekes 8 and Feekes 10, resulted in comparable yields to full-season fungicide coverage. These results suggest applying a single fungicide spray, at or near Feekes 10, provides nearly optimal control of stripe rust in Wisconsin.

\section{Oxathiapiprolin baseline sensitivity distribution in isolates of Pseudoperonospora cubensis}

G. OLAYA (1), R. Linley (1), K. Edlebeck (1), P. J. Kuhn (2), A. Thomas (3), P. Ojiambo (3), (1) Syngenta Crop Protection, Vero Beach, FL, USA; (2) Syngenta Crop Protection, Greensboro, NC, USA; (3) North Carolina State University, Raleigh, NC, USA

Oxathiapiprolin, the active ingredient in Orondis ${ }^{\mathrm{TM}}$ fungicide, provides outstanding control of diseases caused by Phytophthora species and other oomycete pathogens. It is active against mycelial growth, sporangial germination, and zoospore release and germination. Disease control can be achieved with low fungicide rates applied as a foliar, drench or in drip irrigation. Oxathiapiprolin inhibits the oxysterol binding protein, which is involved in the synthesis or movement of lipids between membranes. Oxathiapiprolin has been grouped in class 49 by FRAC and the risk of resistance development is considered to be medium to high. The baseline sensitivity for Pseudoperonospora cubensis was established using 31 isolates that had never been exposed to oxathiapiprolin. Isolates were obtained from cucumber, zucchini and squash from 12 states in the eastern US (AL, FL, GA, MD, MI, NC, SC, NJ, NY, $\mathrm{OH}, \mathrm{TN}$ and WV). Sensitivity tests were conducted in vivo using 2-3 week-old cucumber plants sprayed with oxathiapiprolin and an untreated control. Disease severity was quantified for oxathiapiprolin at concentrations of: $0,0.00001,0.0001,0.001,0.01,0.1 \mathrm{and} 1 \mathrm{mg}$.ai/L. Baseline sensitivity distributions (ED50 values) of $P$. cubensis ranged from 0.000086 to $0.002868 \mathrm{mg} . \mathrm{ai} / \mathrm{L}$ with a geometric mean of $0.000885 \mathrm{mg} . \mathrm{ai} / \mathrm{L}$ and a range of $33 \mathrm{X}$. Information on the baseline sensitivity is important for implementing resistant monitoring studies focusing on early detection of resistant isolates. 
Identification of two potential small molecule chemical controls for Pierce's Disease of grape S. Zhang, M. JAIN, A. Bernert, L. A. Fleites, D. W. Gabriel, University of Florida, Gainesville, FL, USA

Xylella fastidiosa (Xf) infects a wide range of plant hosts and causes Pierce's Disease (PD) of grapevines. Type 1 multidrug resistance (MDR) efflux system is essential for pathogenicity and survival of Xf in planta. Xf is likely significantly more vulnerable to inhibition by small molecule treatments than most bacterial pathogens that typically carry redundant MDR systems. Single gene mutations in the Xf MDR system are more sensitive to the surfactant Silwet L-77 (SL-77) than wild type Xf. A high throughput cell viability assay for GFP-marked Xf strain Temecula 1 was developed to screen two Prestwick combinatorial small molecule libraries (FDA approved drugs and phytochemicals; 1600 chemicals in total) for cell growth inhibition in the presence and absence of $200 \mathrm{ppm}$ SL-77. The screen identified 215 chemicals resulting in $>50 \%$ inhibition of bacterial growth ( $50 \mu \mathrm{M}$ concentration) in the absence of SL-77. Six chemicals exhibited significantly higher growth inhibition (24-40\%) in the presence of SL-77, indicating that these small molecules possibly target MDR efflux. Furthermore, one Prestwick chemical showed a differential response with SL-77, indicating potential synergism with native plant defenses. Two chemicals identified in this screen showed significant potential for chemical control of PD (when applied as soil drench and/or foliar spray), and significantly reduced progression of disease symptoms (54-59\% compared to untreated controls) over a period of three months.

Early season applications of azoxystrobin with starter fertilizer for Rhizoctonia crown and root rot control in sugar beet G. POON, C. Irwin, Vive Crop Protection, Toronto, ON, CANADA

Rhizoctonia crown and root rot of sugar beets, caused by the fungi Rhizoctonia solani, is a soilborne disease causing significant economic losses worldwide. Early season applications of azoxystrobin can reduce disease severity and increase yield. AZteroid FC is a fungicide containing azoxystrobin that is compatible with all starter fertilizers, giving growers the ability to improve nutrient availability while simultaneously delivering long-lasting disease control. Field trials conducted in 2016 tested the ability of AZteroid to control Rhizoctonia crown and root rot of sugar beets when applied with starter fertilizer. AZteroid was applied in-furrow with 10-34-0 liquid fertilizer with and without a commercial seed treatment for Rhizoctonia control as well as with and without a banded foliar application of AZteroid. We found that the seed treatment alone did not yield statistically better than the untreated control. AZteroid applied in-furrow with and without the seed treatment increased yield by 3.32 and 3.25 tons/acre respectively over the untreated control. AZteroid applied as a banded foliar application following a seed treatment and AZteroid in-furrow increased yield by 6.27 tons/acre. Stand counts 38 DAP in all fungicide treatments were higher than the untreated control, demonstrating good crop safety. Our results show that applying AZteroid FC with starter fertilizer is a safe and efficient way to control Rhizoctonia crown and root rot of sugar beet.

At-bloom Applications of Merivon Provides Season-long Control of Anthracnose on Pomegranate in Florida

A. Nepal (1), K. Xavier (2), G. E. VALLAD (3), (1) Oregan State University, Central Point, OR, USA; (2) UF/IFAS Gulf Coast Research and Education Center, Wimauma, FL, USA; (3) Gulf Coast Research and Education Center, University of Florida, Wimauma, FL, USA

Pomegranate is an emergent fruit crop in the Southeast U.S. However, foliar and fruit diseases are a major constraint to expanded production. In 2016, field trials evaluated Merivon (pyraclostrobin and fluxapyroxad; BASF) for the management of anthracnose fruit rot and leaf spot caused by Colletotrichum spp. Replicated trials were conducted on three separate Florida locations in Dover (var. Vietnam), Plant City (var. Angel Red) and Parrish (vars. Christina and Wonderful). Treatments evaluated the timing of Merivon applications at a 7 and 14 fl. oz./A rate and included a non-treated control. Average disease severity ranged from 12.2 to $28.0 \%$ in Dover, 4.5 to $62.5 \%$ in Plant City, and 5.3 to $30.8 \%$ in Parrish based on the final rating in late August. Based on AUDPC, all treatments that included at-bloom applications of Merivon, statistically reduced disease severity compared to postbloom and the non-treated control across all trial sites regardless of rate. At-bloom applications of Merivon reduced the final disease severity rating compared to the non-treated control by 50.8\%, 82.7\% and 91.6\% in Dover, Parrish and Plant City, respectively. At all trial sites, two at-bloom applications were statistically equivalent to a seasonal Merivon program, consisting of four applications every six weeks beginning at bloom. The integrated use of cultural practices and effective fungicides offers growers a viable means to manage seasonal outbreaks of anthracnose on pomegranate.

Potential persistence of flutriafol in vineyard soils treated for control of cotton root rot, caused by Phymatotrichopsis omnivora S. MCBRIDE (1), D. N. Appel (2), (1) Department of Plant Pathology and MIcrobiology, Texas A\&M University, College Station, TX, USA; (2) Department of Plant Pathology and Microbiology, Texas A\&M University, College Station, TX, USA

The fungicide flutriafol has a section 24(c) special local needs label as Topguard Terra ${ }^{\circledR}$ in Texas for chemigation to control cotton root rot (CRR), caused by Phymatotrichopsis omnivora, in winegrapes. Persistence in vineyard soils is a concern as flutriafol is applied annually to minimize losses to CRR. Testing for CRR control began in March, 2012, when plots in an experimental vineyard (vineyard 1) were treated at rates of $0.26 \mathrm{lbs}$./a.i./acre (1X) and $2.6 \mathrm{lbs}$./a.i./acre (10X) and compared to untreated control plots (0X). Treatments were repeated in vineyard 1 in May and late April of 2013 and 2014, respectively and discontinued thereafter. In late March 2012, similar plots were treated in a commercial vineyard (vineyard 2) at three rates: 1X, $10 \mathrm{X}$ and a $20 \mathrm{X}$ rate of $5.2 \mathrm{lbs}$./a.i./acre. Vineyard 2 plots were treated the following two years. Since then, all vines in vineyard 2 were treated annually at the recommended 1X rate. In 2017, soil samples were collected from both vineyards and analyzed at a commercial pesticide testing lab for residual flutriafol levels. Less than 3 years after the last treatment in vineyard 1, only trace amounts $(0.01-0.02 \mathrm{ppm})$ of flutriafol were found in the soil samples, regardless of rates. In vineyard 2, slightly higher levels were found in plots at average levels $(\mathrm{n}=10)$ of: $0 \mathrm{X}=0.01 \mathrm{ppm}, 1 \mathrm{X}=0.01 \mathrm{ppm}, 10 \mathrm{X}=0.03$ $\mathrm{ppm}$, and $20 \mathrm{X}=0.09 \mathrm{ppm}$. These preliminary results indicate there is little or no residual accumulation of the fungicide in treated vineyards at recommended levels.

Suppression of charcoal rot (Macrophomina phaseolina) in annual strawberry through chemigation treatments J. C. MERTELY (1), R. Martin (1), N. Peres (2), (1) University of Florida-GCREC, Wimauma, FL, USA; (2) Gulf Coast Research and Education Center; University of Florida, Wimauma, FL, USA

Charcoal rot is a destructive emerging disease of strawberry in warm production areas. Macrophomina phaseolina preferentially degrades vascular tissues in strawberry crowns, causing plant collapse and death. To evaluate products for disease control, replicated trials have been conducted in Florida each winter since 2005-06. Inoculum produced on PDA, corn meal sand, or corn cob pet litter is typically placed in planting holes before transplanting. In 2016, colonized toothpicks were inserted into the soil next to the transplants. Test products are injected into plots through dedicated drip irrigation tapes. Over the years, several trials failed to show differences in disease incidence (DI) and/or marketable yield due to unfavorable (cool) weather, poor inoculation response, patchy natural inoculum, sting nematode damage, insufficient replication, etc. Yet, products containing fluopyram, penthiopyrad, thiophanate methyl, Bacillus subtilis, Gliocladium spp., and Trichoderma spp. have shown limited ability to control charcoal rot in multiple trials. Disease pressure was higher in 2016-17 due to the new inoculation method and warm weather early in the season. In this trial, fluopyram, penthiopyrad, and thiophanate methyl, as well as flutriafol, isofetamid, and Gliocladium catenulatum significantly reduced DI. G. catenulatum (Prestop) reduced DI from $41.3 \%$ in the inoculated control to $10.1 \%$ and increased yield $46.7 \%$, promising results that merit further study.

Evaluation of fungicide efficacy and application timing for the management of Glomerella leaf spot and fruit rot in North Carolina S. M. VILLANI, R. Kreis, K. Johnson, North Carolina State University, Mills River, NC, USA

Glomerella leaf spot (GLS) and fruit rot (GFR) caused by members of the Colletotrichum gloeosporioides species complex is the most economically devastating fungal disease in North Carolina. With few management options and a paucity of GLS-related research in the U.S., NC apple growers have been relying on standard summer-disease fungicide programs to manage the disease. To more effectively manage GLS and GFR during the growing 
season and post-harvest, field experiments investigating single-site fungicide efficacy and application timing were conducted in a 'Gala' research orchard in 2016. Single-site fungicides representing different modes of action (Inspire Super, difenoconazole + cyprodinil; Merivon, pyraclostrobin + fluxapyroxad; Sercadis, fluxapyroxad; and Cabrio EG, pyraclostrobin) were applied in non-rotational programs from petal fall through $8^{\text {th }}$ cover. The incidence of GLS, pre-harvest GFR, and post-harvest GFR was significantly lower for programs in which the QoI fungicide, Cabrio EG, and the SDHI/QoI premixed fungicide, Merivon, were applied. The programs of Merivon and Cabrio EG also resulted in a significantly lower incidence of scaffold defoliation due to GLS. In a separate field experiment, the timing of Merivon applications for GFR management was investigated. While no significant differences in GFR incidence prior to harvest were observed between programs with differing Merivon timings, Merivon applications at petal fall and $1^{\text {st }}$ cover resulted in significantly lower fruit rot incidence 14 days post-harvest. Repeated experiments in subsequent years should lead to more refined management recommendations for Glomerella leaf spot and fruit rot in the Southeastern US.

In vitro sensitivity of a vascular wilt pathogen, Ophiostoma novo-ulmi, to paclobutrazol

S. M. ADAMS (1), J. A. Smith (2), (1) The University of Florida \& The Morton Arboretum, Gainesville, FL, USA; (2) University of Florida, Gainesville, FL, USA

Paclobutrazol (PBZ), a gibberellin inhibitor, is a commonly used plant growth regulator in plant production and in arboricultural practices. It also belongs to the DMI-triazoles group of fungicides. Studies in vivo have shown that PBZ can be used to manage fungal diseases, while having limited effect on the host plants. Currently there are no commercially available products to curatively treat Dutch elm disease, which is caused by the fungus $O$. novo-ulmi and responsible for the deaths of thousands of trees in the United States. This in vitro study tested one strain's sensitivity to different concentrations of PBZ-amended potato dextrose agar. Concentrations tested ranged from 2-400 ppm $(0,2,4,6,8,10,20,30,40,50,100,200$, and 400 ppm). O. novo-ulmi was transferred from a ten-day-old unamended PDA culture using a \#3 cork borer $(6 \mathrm{~mm})$. The diameter of isolate growth was measured daily from three different angles and averaged for colony growth. After seven days, growth of $O$. novo-ulmi was inhibited at concentrations higher than $10 \mathrm{ppm}$ and slowed at concentrations below $10 \mathrm{ppm}$. Having a product green industry professionals can use to manage this disease would be valuable in plant health care programs.

\section{Efficacy of fungicide-based management of blueberry rust in southern Georgia}

R. J. INGRAM (1), P. M. Brannen (1), M. Slusher (2), J. Taylor (3), W. Lovett (4), S. Curry (5), (1) University of Georgia, Athens, GA, USA; (2) University of Georgia Extension, Waycross, GA, USA; (3) University of Georgia Extension, Lakeland, GA, USA; (4) University of Georgia Extension, Alma, GA, USA; (5) University of Georgia Extension, Baxley, GA, USA

Fungicides were evaluated for control of blueberry rust in replicated trials in mature southern highbush blueberries (cv. Rebel) in commercial fields in three counties in southern Georgia in 2016. Treatments were assigned using a Randomized Complete Block Design and included an untreated control, Abound (azoxystrobin), Pristine (pyraclostrobin + boscalid), Indar (fenbuconazole), Orbit (propiconazole), Proline (prothioconazole), Quash (metconazole), Aprovia (benzovindiflupyr), Quilt Xcel (azoxystrobin + propiconazole), and a split schedule application of Abound (azoxystrobin) preharvest followed by Bravo (chlorothalonil) until termination. Applications were made bi-weekly on 6 Apr, 21 Apr, 4 May (last prior to harvest), 18 May, 1 Jun, 15 Jun, and 29 Jun. Disease assessments were conducted five times throughout the season. High temperatures over the winter (2015-2016) ensured availability of primary rust inoculum on retained, previous-season leaves. Active urediniospore production was observed on overwintered leaves, and symptoms were observed on new growth as early as 30 Mar 2016. With the exception of Indar, the DMI fungicides (Proline, Quash, Orbit, Quilt Xcel) provided the best control overall and Aprovia, a currently unlabeled SDHI fungicide, provided a level of control equal to the more effective DMI fungicides. Abound and Indar provided similarly moderate levels of control while the previously recommended Pristine provided the least control of all treatments.

Fungicide sensitivity and population structure of Sclerotinia sclerotiorum isolates from Argentina, Brazil, and USA T. J. J. Miorini, A. Pannullo, J. R. Steadman, S. E. EVERHART, University of Nebraska, Lincoln, NE, USA

Sclerotinia sclerotiorum is predicted to have low risk of resistance based on expected population genetic variability, yet intensive use of fungicides may select for resistance. An understanding of fungicide sensitivity and structure of S. sclerotiorum populations is needed. Isolates from soybean and dry bean from Brazil $(\mathrm{n}=100)$, Argentina $(\mathrm{n}=5)$, and the U.S. $(\mathrm{n}=15)$ were selected. For eight fungicides, the average and standard deviation of $\mathrm{EC}_{50}$ values of 23 isolates were used to create a discriminatory dose (DD) for assessment of isolates. For isolates in the $90^{\text {th }}$ percentile of growth, $\mathrm{EC}_{50}$ was determined and converted to resistance factors (RF), where greatest RF were for boscalid (5.39), prothioconazole (3.07), procymidone (2.65), and fluazinam (2.03). As expected, the mixed-mode chemistry resulted in a lowest RF (fluopyram+prothioconazole; 1.41) and one isolate showed reduced sensitivity to both dicarboximide fungicides, procymidone and iprodione. Isolates from soybean $(\mathrm{n}=95)$ were genotyped using 11 microsatellite loci and identified a total of 84 unique multilocus genotypes (MLG). Cluster analysis showed increased partitioning of populations with geographic distance, with significant difference between states $(p>0.05)$. Despite clonal population structures, higher levels of diversity may allow resistance to emerge with intensive fungicide use.

\section{Alternative soil fumigation to control Macrophomina phaseolina, causal agent of charcoal rot of strawberries}

J. BAGGIO (1), E. Zielinski (2), L. Cordova (3), M. Chamorro (4), J. W. Noling (5), N. Peres (6), (1) GCREC - University of Florida, Wimauma, FL, USA; (2) Federal University of Parana (UFPR), Curitiba, BRAZIL; (3) University of Florida, Wimauma, FL, USA; (4) Driscoll's of Europe, Moguer, SPAIN; (5) University of Florida, Lake Alfred, FL, USA; (6) Gulf Coast Research and Education Center; University of Florida, Wimauma, FL, USA

Macrophomina phaseolina, causal agent of charcoal rot in many crops worldwide, has become more prevalent in Florida strawberry fields since methyl bromide was phased out. M. phaseolina is a soil-borne pathogen that infects strawberry crowns, causing wilt, collapse and death of plants. The efficacy of alternative soil fumigation treatments was determined by evaluating the survival of M. phaseolina inoculum on infested corn-cob litter buried in the centers and sides of plastic-covered beds. Different products, concentrations and application methods were tested during two strawberry seasons and compared to methyl bromide treatments and non-treated controls. In the 2015-2016 season, shank applications of 1,3-dichloropropene:chloropicrin 63/35 (T35) and 39/60 (Piclor60) under LDPE (low-density polyethylene) were ineffective against M. phaseolina, whereas drip applications of metam potassium (Kpam) and allyl isothiocyanate (AITC) were as effective as methyl bromide + chloropicrin 67/33 (MeBr67) and 50/50 (MeBr50). In 20162017, shank applications of T35 and 1,3-dichloropropene:chloropicrin 20/80 (Piclor80) under VIF (virtually impermeable film) and drip applications of Kpam and AITC were as effective as MeBr50. However, AITC was not effective in controlling M. phaseolina on the sides of the beds. Dimethyl disulfide (DMDS) applied through shank was less effective in reducing M. phaseolina inoculum than application through drip.

\section{In vitro evaluation of fungicides for the management of rice kernel smut in Texas}

\section{S. S. UPPALA, S. Zhou, Texas A\&M AgriLife Research, Beaumont, TX, USA}

Kernel smut, caused by Tilletia barclayana (Neovossia horrida), was previously considered a minor disease due to its nature of sporadic occurrence. However, this has become an economically important fungal disease of rice in Texas in recent years. This disease has caused significant monetary losses by reducing yield and quality. During the 2015 cropping season, the rice varieties Presidio, Cheniere and XL 753 (hybrid) were severely infected with kernel smut across Texas rice production areas. Isolates of $T$. barclayana were isolated from the infected grain samples and in vitro studies were conducted to evaluate efficacy of various fungicides against these fungal isolates and develop baseline sensitivities to these fungicides. Agar media were amended with propiconazole, azoxystrobin, propiconazole + azoxystrobin, trifloxystrobin plus propiconazole, and fluxapyroxad fungicides at various 
concentrations. Fungicides containing propiconazole as the only active ingredient (Propimax ${ }^{\circledR}$ and Tilt $^{\circledR}$ ) were highly effective and showed complete inhibition of all the isolates evaluated at concentrations as low as $50 \mathrm{ppm}$. Other fungicides showed variable responses among the isolates and were not as effective as Propimax ${ }^{\circledR}$ and Tilt ${ }^{\circledR}$.

\section{Survival of Ceratocystis fagacearum following red oak log fumigation with sulfuryl fluoride}

J. Juzwik (1,2), A. YANG (1), S. Myers (3), M. Furtado (3), A. Taylor (4), (1) University of Minnesota, St. Paul, MN, USA; (2) USDA Forest Service, St. Paul, MN, USA; (3) USDA APHIS PPQ, Buzzards Bay, MA, USA; (4) University of Tennessee, Knoxville, TN, USA

Alternative phytosanitary treatments are needed to replace use of methyl bromide, an ozone depleting chemical, for U.S. oak log exports. Published studies have shown that sulfuryl fluoride (SF) fumigation $\left(280 \mathrm{~g} / \mathrm{m}^{3}\right.$ for $72 \mathrm{hrs}$ ) of Ceratocystis fagacearum (Cf)-colonized red oak logs eradicated the oak wilt pathogen; however, further evaluation is needed. SF treatments of logs from wilted Quercus ellipsoidalis/rubra trees $(20$ to $29 \mathrm{~cm}$ dia at $1.4 \mathrm{~m}$ height) following artificial (AI) or natural inoculation (NI) with Cf were evaluated for ability to kill the fungus in sapwood. Four fumigation chambers (one control; three SF at one of three rates) held three to four logs (combinations of AI and NI). Wood chips from each of eight outer sapwood locations on each of two disks $(7.6 \mathrm{~cm}$ thick) cut at $22 \mathrm{~cm}$ from ends of each $\log (1.8 \mathrm{~m}$ initial length) were plated on semi-selective medium before and after treatment. The experiment was replicated three times. Pre-treatment isolation of Cf was higher from AI ( $83 \%$ of locations; 22 logs) than NI (67\%; 14$)$ $\operatorname{logs}$. Cf was not isolated from any locations on $240 \mathrm{~g} / \mathrm{m}^{3} \operatorname{logs}$ post-treatment. Six and three locations from two AI $\operatorname{logs}$ treated at $280 \mathrm{~g} / \mathrm{m}^{3}$ yielded Cf, as did one location from one AI log and two from one NI log after $320 \mathrm{~g} / \mathrm{m}^{3}$ treatment. All post-treatment controls yielded Cf $(94 \%$ of locations, $5 \mathrm{AI} \operatorname{logs}$; $46 \%, 2 \mathrm{NI}$ ). Studies of SF's movement into sapwood and efficacy of increased exposure time at $</=240 \mathrm{~g} / \mathrm{m}^{3}$ are underway.

\section{Comparison of reflectance meters for evaluation of peanut leaf spot in Georgia} B. S. JORDAN, A. K. Culbreath, University of Georgia, Tifton, GA, USA

The relationships among Normalized Difference Vegetation Index (NDVI) as measured by two crop sensors, Greenseeker and Crop Circle, and visual estimates of percent defoliation caused by late leaf spot (Cercosporidium personatum) of peanut (Arachis hypogaea) were examined across four locations in 2015. Reflectance measurements and visual ratings were all made on the same day for each evaluation. There were linear relationships between defoliation and NDVI calculated with both optical sensors $\left(R^{2}=0.82\right.$ for Greenseeker; $R^{2}=0.91$ for Crop Circle). Likewise, NDVI estimates obtained from the two were linearly correlated $\left(\mathrm{R}^{2}=0.82\right.$. The Greenseeker measurements had more variation than measurements obtained from the Crop Circle, but both devices provided NDVI estimates that predicted percent defoliation well for levels of defoliation of $50 \%$ or greater.

Relationship of yield loss caused by smut in sugarcane and soil properties in Louisiana R. M. Johnson (1), M. P. GRISHAM (2), (1) USDA-ARS, Sugarcane Research Unit, Houma, LA, USA; (2) USDA-ARS, SRU, Houma, LA, USA

Sugarcane cultivar L 01-299, the most widely planted cultivar in Louisiana, is resistant to most economically important diseases; however, it is susceptible to sugarcane smut caused by Sporisorium scitamineum. A study was conducted to determine if the cultivar's susceptibility to smut causes significant yield losses and to determine the relation between soil properties and smut levels in commercial fields of L 01-299. The experiment was initiated in two fields, one a plant-cane and the other a first-ratoon crop in 2013. A grid sampling pattern was established in both fields and soil samples were collected from each point. In addition, smut levels were measured at each point and yield samples were collected in 2013, 2014 and 2015 from each gridded field. Smut levels were highly variable in both fields, while soil properties data and sugarcane yields were less variable. Harvest data showed a negative correlation between cane and sugar yields and smut levels in plant-cane and the first-ratoon crops in 2014. The relationship was inconsistent in older ratoon crops, possibly due to lower levels of smut infection. Smut levels were negatively correlated with soil potassium, magnesium and soil organic matter, possibly indicating that smut could be more serious in lower fertility soils. These combined data suggest that proper management of soil fertility and selecting seed cane from an area with low incidence of smut incidence is important in managing smut in L 01-299.

Incidence-severity relationships for scab on foliage and fruit of pecan

C. H. BOCK, USDA ARS, Southeastern Fruit and Tree Nut Research Laboratory, Byron, GA, USA

The most destructive disease of pecan in the southeastern USA is scab, caused by Venturia effusa. Incidence (I)-severity (S) relationships have not previously been characterized in this pathosystem. Ten scab-susceptible cultivars were assessed for I and S of scab on fruit (1972 trees) and foliage (compound leaves and leaflets, 1129 trees) between 2010 and 2014. Samples were assessed on a tree basis, and sample size ranged from 10 to 100 specimens per tree. The range in mean I and S was different depending on the organ (fruit $\mathrm{I}=0$ to $100 \%, \mathrm{~S}=0$ to $100 \%$; compound leaves and leaflets, $\mathrm{I}$ $=0$ to $100 \%, \mathrm{~S}=0$ to $10.1 \%$, respectively). However, mean I could be $100 \%$ at a mean $\mathrm{S}<2.0$ for fruit, compound leaves and leaflets. Data were transformed by complementary log-log prior to linear regression analysis. A linear regression model described the relationship between I and $\mathrm{S}$ per tree for fruit $\left(\mathrm{P} \leq 0.0001, \mathrm{R}^{2}=0.61\right)$, compound leaves $\left(\mathrm{P} \leq 0.0001, \mathrm{R}^{2}=0.82\right)$ and leaflets $\left(\mathrm{P} \leq 0.0001, \mathrm{R}^{2}=0.91\right)$ for all cultivars. An analysis of covariance showed a significant effect of year and cultivar on intercept and slope, but back-transformed values showed these differences to be very small, numerically. The data indicate that assessing I of scabbed fruit is probably of limited value to estimate severity, although leaves, and more so leaflets may provide a more useful measure for predicting $\mathrm{S}$ on foliage.

\section{Development and validation of risk assessment tools for Verticillium wilt of mint}

D. L. WHEELER (1,2), J. K. S. Dung PhD (3), D. A. Johnson (1), (1) Washington State University, Pullman, WA, USA; (2) Graduate Student Assistant, Pullman, WA, USA; (3) Oregon State University, Madras, OR, USA

The long-lived microsclerotia and wide host range of Verticillium dahliae present management challenges to mint growers because of the limited availability of treatment options. Morphologically identical populations of $V$. dahliae can vary in aggressiveness, interact with plant-parasitic nematodes, and necessitate different management tactics. Detection, differentiation, and quantification of $V$. dahliae populations aggressive to mint is therefore necessary to inform management decisions. Diagnostic tools are being developed to assess the risk of Verticillium wilt of mint. Soil samples were collected from commercial fields from 2014 to present. Verticillium dahliae and nematodes were quantified from soils with soil plating, qPCR and, for nematodes, visual enumeration. Disease and yield were assessed in fields and in mint cultivars grown in the same field soils in a greenhouse. Variation in disease was not fully explained $\left(\mathrm{R}^{2}=0.02, P>0.05\right)$ by $V$. dahliae inoculum and additional predictors were identified with stepwise model selection. Disease observed in fields was explained by interactions between $V$. dahliae and nematodes, interactions among nematodes, and yields from greenhousegrown mint $(P<0.05)$. Inoculum of $V$. dahliae was explained by qPCR data and disease ratings from greenhouse-grown mint $(P<0.05)$. Continued identification and prediction of the factors influencing Verticillium wilt and inoculum density will assist mint stakeholders in disease management.

Comparing the effects of Wheat streak mosaic virus strains on plant growth characteristics and symptom severity in hard red winter wheat D. YABWALO (1), C. Tande (1), R. L. Geppert (2), E. Byamukama (1), M. A. Langham (1), (1) South Dakota State University, Brookings, SD, USA; (2) SDSU Small Grain Pathology, Brookings, SD, USA

Wheat streak mosaic virus (WSMV), infects most cereal crops including wheat and several wild grass weeds. In some agricultural areas, wheat curl mites (the WSMV vector) move from wheat to grassy weeds and from grassy weeds to wheat, hence there is potential of mixing WSMV strains from wheat to grass and vise-versa. An experiment was conducted to compare the impact of WSMV strains from wheat and brome grass on plant height, total seedling biomass and WSMV severity of two winter wheat cultivars, Mace and Millennium. The experiment was maintained in a greenhouse $\left(25-27^{\circ} \mathrm{C}\right)$ 
and growth chamber $\left(19-20^{\circ} \mathrm{C}\right)$ conditions. Both cultivars were inoculated with purified water, WSMV-br (isolated from brome grass) and WSMV-w (isolated from wheat) at Feeke's 2 stage. Both experiments were a randomized complete block design with a split plot arrangement where cultivar was the main plot and inoculum source was a subplot. Results from both the greenhouse and growth chamber demonstrated a similar pattern in disease severity and response of plant growth variables to both WSMV strains. Within cultivar, no significant differences $(P<0.0001)$ in plant height and biomass (dry and wet weight) were observed between the two WSMV strains on Mace while significant differences were observed in Millennium. Plant height and dry weight were reduced by $18 \%$ and $78 \%$ due to WSMV-w, respectively, and $12 \%$ and $28 \%$ due to WSMV-br, in Millennium. These results suggest that different WSMV strains originating from variable hosts differ in aggressiveness and may cause varying yield loss levels.

\section{Ganoderma root and butt rot in California almond}

B. JOHNSON (1), D. M. Rizzo (2), (1) University of Davis-California, Davis, CA, USA; (2) University of California, Davis, CA, USA

Wood decay fungi reduce the structural integrity of trees, leading to limb breakage and wind-driven collapses (windfalls). Of the wood decay types associated with almond, butt rot is the most poorly understood and perhaps the most important. Butt rot fungi decay the tree from the bottom up and from the inside out, often with no obvious external symptoms. Eventually, infection leads to windfall. Attrition from windfall is one of the factors limiting orchard age to 20-25 years. Butt rot fungi are usually considered a problem of older orchards, but our findings challenge these notions. We identified Ganoderma australe and Ganoderma adspersum as the main causes of root and butt rot in California almond. No differences in infection rate were evident among scion varieties, but all identified infections were in orchards grafted on peach rootstocks. We identified G. australe in orchards in the northern San Joaquin Valley, the southern Sacramento Valley, and coastal forests in northern California and southern Oregon. Incidence of G. australe infection in an orchard is often low and associated with old or stressed trees. We isolated G. adspersum, previously unreported in North America, from orchards in five counties in the San Joaquin Valley, and caused extensive tree loss in 9- to 12-year-old orchards. We found that infection rates within orchards tend to exceed 50\%, and result in exponential tree loss over time. Extensive infection appears to be terminal for orchards.

Development of a quantitative assessment method for fungal-soil particle dispersion on glass surfaces during image acquisition A. GAMBE-GILBUENA, A. Coronel, A. A. Eda, M. C. E. Vargas, C. Pangantihon, C. Lopez, M. Chan, N. Tan-Gana, M. R. J. Estuar, Ateneo de Manila University, Quezon City, PHILIPPINES

Rapid image-based detection methods can facilitate monitoring of soil pathogens such as Fusarium sp. especially in large mono-cropping systems. Mobile devices configured for automatic identification are suitable for on-the-spot readouts due to their portability and multi-functionality. Machine learning methods to develop automated systems depend on image properties which rely on specimen preparation quality. Particle dispersion uniformity is important to minimize distortion of parameter estimates. Soil heterogeneity necessitates sucrose-surfactant/dispersant methods to isolate propagules. Behaviour of particles between glass surfaces may thus depend on concentrations of sucrose, surfactant/dispersant and soil debris. To investigate the effects of these factors, definitive screening design experiment (SAS ${ }^{\circledR}$-JMP) was performed and particle dispersion was quantified. $F$. oxysporum (ATCC ${ }^{\circledR}$ MYA1198) propagules were mixed with varying amounts of sucrose, TritonX-100 ${ }^{\mathrm{TM}}$, saponin and soil particules and loaded on glass slides. ImageJ was used to extract Cartesian coordinates of particles from images for all 13 DSD set-ups. Data was imported into MATLAB ${ }^{\circledR}$ for hierarchical clustering using Euclidean, Mahalanobis, Hamming, Jaccard and Minkowski distance metrics. The count, mean and standard deviation of the clusters were computed, with lower values corresponding to better particle dispersion. Least squares regression analyses revealed that concentrations of glucose, soil particles and propagules affect dispersion. These factors will be further investigated to determine optimal conditions. The workflow developed can also be expanded to include more factors.

AG MAPSS: Autonomous Guidance Modular Aerial Plant Survey System

J. RUPP, M. Bruce, Montana State University, Bozeman, MT, USA

The agricultural drone market is reported to generate nearly 100,000 jobs and as much as $\$ 82$ billion dollars in economic activity, demonstrating the critical research and economic impacts of drone use integration for agricultural research. Both the cost to implement and knowledge required can be prohibitive to researchers. AG MAPSS is a custom built, 3-D printed modular drone capable of carrying a variety of components for acquisition of data. Data is stored on MSU's Box and is available to all researchers, free of charge. Our lab focuses on the use of open-source software and instruction for users. This project aims to provide a valuable research dataset to the MSU Research Farm's users, without the onerous requirement they purchase and maintain their own drone platform. AG MAPSS seeks to provide a team model for research and agricultural innovation. As a mobile system, AG MAPSS has potential to serve the horticultural farm and travel to requested sites with operational support within the College of Agriculture and experiment stations. Drone applications represent opportunities for integration and collaboration in many STEM areas, including agricultural sciences, computer science, electrical engineering, mechanical engineering, and natural resource management. The system is designed to be portable, and easy to set up, making it ideal for other events. AG MAPSS can provide real-time demonstrations and an excellent platform for the integration of on-and offcampus learning, outreach and discovery.

Factors affecting emission of AITC and subsequent disease control efficacy of Brassica juncea seed meal soil amendment L. WANG (1), M. Mazzola (2), (1) Washington State University, Wenatchee, WA, USA; (2) USDA ARS, Wenatchee, WA, USA

Soil physical conditions demonstrably affected allyl isothiocyanate (AITC) emitted from Brassica juncea cv Pacific Gold seed meal (SM) amended soil. The AITC concentration detected increased with an increase in temperature from $10^{\circ} \mathrm{C}$ to $30^{\circ} \mathrm{C}$. AITC concentration also increased with an increase in soil moisture, however a significant decrease in AITC generation was observed under the extreme moisture conditions of dry (water potential below $1000 \mathrm{kPa}$ ) and soil saturation. At the same moisture and temperature condition, AITC emission was not significantly affected by soil texture. AITC insensitive fungi Trichoderma spp. and Mortierella spp. were not suppressed in SM treated soil regardless of the soil physical conditions. Incubated for $24 \mathrm{hr}$ in SM treated soil, the AITC sensitive pathogens Pythium ultimum and Rhizoctonia solani AG-5 were effectively controlled when a peak AITC concentration above $0.01 \mu \mathrm{g} / \mathrm{ml}$ was attained. In orchard study, Pratylenchus penetrans root densities were significantly reduced in SM treated soil relative to control and fumigated soil even at 5 months after tree planting. Total fungal density in SM treated soil was significantly higher than control and fumigated soil. In addition, microbial community composition in the rhizosphere of trees planted in SM treated soil was significantly different from the control soil, suggesting that long term pathogen suppression may function through the soil microbiome.

Effect of different Gramineae carbon inputs on efficacy of ASD for control of Macrophomina phaseolina in strawberry S. S. HEWAVITHARANA (1), M. Mazzola (2), (1) Washington State University, Wenatchee, WA, USA; (2) USDA ARS, Wenatchee, WA, USA

Anaerobic soil disinfestation (ASD), using either orchard grass (GR) or wheat shoots (WH) as the carbon input, was examined for the control of $M$. phaseolina in strawberry. The pathogen was artificially infested at a density of 2.5 sclerotia $\mathrm{g}^{-1}$ soil. Treatments included in the study were ASD with orchard grass (GR), wheat leaf residues (WH) or rice bran (RB) at $20 \mathrm{tha}^{-1}$, no treatment control (NTC), and pasteurized control (PC). The effect of soil volatiles produced during the ASD process on M. phaseolina hyphal growth was determined. Post-treatment headspace $\mathrm{O}_{2}$ content, soil $\mathrm{pH}$, fungal growth, and strawberry cv. Albion crown infection were assessed. Soils attained anaerobic state based on the headspace $\mathrm{O}_{2}$ content in response to all ASD treatments by the end of the incubation period irrespective of the carbon source. Soil pH was lowest in ASD-RB soil. Volatiles produced in the GR and WH treatments significantly reduced fungal colony growth compared to RB and NTC, but fungal growth resumed upon transfer to fresh media indicating that the compounds were fungistatic. Among ASD treatments, M. phaseolina crown infection was the lowest in WH. Pathogen DNA detected in crowns was significantly reduced by GR, WH, and PC treatments relative to RB and NTC. 
Deciphering potential mechanisms of anaerobic soil disinfestation (ASD)-mediated control of Pratylenchus penetrans S. S. HEWAVITHARANA (1), M. Mazzola (2), (1) Washington State University, Wenatchee, WA, USA; (2) USDA ARS, Wenatchee, WA, USA

Pratylenchus penetrans is a component of the apple replant disease (ARD) causal pathogen complex. The potential role for biological mechanisms contributing to ASD-mediated suppression of $P$. penetrans was examined in greenhouse study using orchard soil with a history of ARD. Populations of P. penetrans were amplified by cultivating ARD soil with apple seedlings. Subsequently, ASD was applied using orchard grass $\left(20 \mathrm{tha} \mathrm{a}^{-1}\right)$, and half of the treated soil was pasteurized to eliminate biological elements that may have been recruited by ASD. Nematodes extracted from seedling roots were used to infest pasteurized (ASD-P), non-pasteurized (ASD-NP), and no-treatment control (NTC) soil and planted with apple seedlings. Seedlings planted in ASD-NP soil had significantly lower $P$. penetrans root populations than ASD-P indicating a contribution of biology to system resilience against reinfestation. In a separate trial a nylon mesh barrier was placed to separate ASD-P, ASD-NP, NTC, and pasteurized control (PC) soils from P. penetrans infested soil allowing nematode migration into the treated soil but prevented root penetration. Treated soils were planted with apple seedlings. ASD-NP and ASD-P had significantly lower root nematode density compared to NTC and PC inside the mesh barrier. This finding indicates that PC soil was prone to re-infestation in a similar manner to that observed for fumigated soils, whereas ASD treated soil was resistant to re-infestation by $P$. penetrans.

Evaluating the effect of tillage on soil-borne wheat pathogens in the dryland Pacific Northwest D. Kroese (1), K. McLaughlin (2), C. HAGERTY (1), (1) Oregon State University, Pendleton, OR, USA; (2) Oregon State University, Corvallis, OR, USA

No-till seeding is described as planting directly into the crop stubble from the previous season without use of primary tillage. No-till acreage is increasing in the dryland cereal production region of the Pacific Northwest (PNW). A reduction in tillage can result in positive outcomes, including increased soil water holding capacity and reduced fuel costs. However, no-till management can cause shifts in crop disease profiles. There is conflicting evidence in the literature about whether reduced tillage results in increased diversity of plant pathogens. Similarly, some farms have reported a reduction in disease pressure after converting to no-till; but other farms have reported an increase in disease pressure. The goal of this study is to understand how tillage influences disease dynamics. In Fall 2016, 10 soil samples were collected from each of 30 wheat fields representing a wide range of tillage. Fields were scored on a 0-10 scale from inversion tillage (0) to no-tillage (10). Soil samples are currently being evaluated for three soil-borne pathogen groups: Fusarium, Pythium, and Rhizoctonia. Pathogen abundance will be evaluated as a function of tillage. Additional variables including soil $\mathrm{pH}$, organic matter, and field rainfall zone will be investigated. Cultural disease control recommendations were developed mostly under stubble mulch tillage; this study is part of a long-term goal to develop disease management recommendations for no-till producers of the PNW.

The effect of planting date on severity of Fusarium wilt of lettuce in California K. R. PAUGH (1), T. R. Gordon (2), (1) University of California - Davis, Davis, CA, USA; (2) Department of Plant Pathology, University of California Davis, Davis, CA, USA

Development of Fusarium wilt of lettuce, caused by the soilborne fungus Fusarium oxysporum f. sp. lactucae, is strongly influenced by ambient temperature, with more severe disease manifesting at higher temperatures. In principle, lettuce growers can minimize the impact of Fusarium wilt by sowing susceptible cultivars in cooler planting windows and resistant cultivars in warmer planting windows. To evaluate the efficacy of this approach under California conditions, seven lettuce cultivars were transplanted into naturally infested field soil on three planting dates one week apart in August and September of 2014, 2015 and 2016. For moderately susceptible cultivars, one or two weeks delayed planting reduced disease severity by 18 to 33 percent. To determine what stages of lettuce growth are most affected by temperature, cultivars of moderate susceptibility were transplanted into infested soil and maintained in a growth chamber under cool conditions $\left(23 / 18^{\circ} \mathrm{C}\right)$, and a subset of plants was transferred to a warm growth chamber $\left(33 / 23^{\circ} \mathrm{C}\right)$ for one week at either 2, 3, or 4 weeks after transplanting. Preliminary results indicate that plants exposed to high temperatures at 4 weeks posttransplanting have the most severe foliar and vascular symptoms of Fusarium wilt. Adjusting planting dates to avoid high temperatures during a critical stage of growth can contribute to the management of Fusarium wilt of lettuce.

Vacuum steam as a promising alternative to methyl bromide for killing Ceratocystis fagacearum in Quercus rubra logs for global export A. YANG (1), J. Juzwik (1,2), M. White (3), Z. Chen (3), S. Shugrue (4), R. Mack (4), (1) University of Minnesota, St. Paul, MN, USA; (2) USDA Forest Service, St. Paul, MN, USA; (3) Virginia Tech, Blacksburg, VA, USA; (4) USDA APHIS PPQ, Buzzards Bay, MA, USA

Fumigation of oak (Quercus spp.) logs with methyl bromide (MeBr) $\left(240 \mathrm{~g} / \mathrm{m}^{3}\right.$ for $72 \mathrm{hr}$ ) for export from the USA is one of the highest schedules for quarantine and pre-shipment commodity use because of the oak wilt fungus. In spite of the world-wide phase-out of MeBr for most uses, no effective alternative treatment has been found for oak $\log$ exports from states with oak wilt. Vacuum steam treatments $(56 \mathrm{C} / 30 \mathrm{~min} ; 60 \mathrm{C} / 60 \mathrm{~min})$ of $Q$. rubra $\operatorname{logs}$ from wilted trees $(42 \mathrm{~cm}$ to $55 \mathrm{~cm}$ dia at $1.4 \mathrm{~m}$ height) following artificial (AI) or natural inoculation (NI) with Ceratocystis fagacearum (Cf) were evaluated for ability to kill the pathogen in colonized wood. Wood chips from eight inner and eight outer sapwood locations on each of two disks (7.6 cm thick) cut at $22 \mathrm{~cm}$ from ends of each $\log (2.1 \mathrm{~m}$ initial length) were plated on Cf selective agar before and after treatment. Five replicate loads (each with one AI and two NI logs) were tested per schedule. Pathogen isolation rates before treatment were higher for AI than NI logs for both sapwood depths and higher for outer versus inner sapwood within each inoculation type (AI - 45.6\% outer, $29.4 \%$ inner; NI - 24.4\% outer, $12.5 \%$ inner). The fungus was not isolated from any locations assayed in post-treatment logs for either schedule. When coupled with a previously published report of minimal quality defects associated with vacuum steam treatment of oak veneer logs, these results justify further development of this $\mathrm{MeBr}$ alternative.

\section{Design and operation of static and mobile arrays to suppress powdery mildews in greenhouse and tunnel production systems with UV and} visible light

A. Stensvand (1,2), A. Suthaparan (2), P. J. From (2), L. Grimstad (2), N. Bjugstad (2), K. A. Solhaug (2), H. R. Gislerød (2), N. S. Johansen (1), D. M. GADOURY (3), A. Bierman (4), M. Rea (4), (1) Norwegian Institute of Bioeconomy Research, Ås, NORWAY; (2) Norwegian University of Life Sciences, Ås, NORWAY; (3) Cornell University, Geneva, NY, USA; (4) Lighting Research Center, Rensselaer Polytechnic Institute, Troy, NY, USA

To suppress powdery mildews of several crop species, we used static lighting installations, as well as mobile arrays of ultraviolet (UV, at 250 - $280 \mathrm{~nm}$ ) and red lights, adapted to either existing booms or autonomous robotic units. Night applications of UV allowed use of minimal UV doses. Horizontal spray or transportation booms were adapted to apply UV in commercial greenhouses with potted plant production. A light-weight, self-tracking, and battery driven robot developed at the Norwegian University of Life Sciences was equipped with fluorescent UV lamps/UV LEDs and adapted to operate in greenhouses and high tunnels. Employing four drive motors, 4-wheel steering, and adjusted for row width, the robot was used in commercial production of cucumber, tomato, and strawberry. Canopy-detecting ultrasonic sensors equipped with linear actuators facilitated the real-time control of UV applied to the plants. The UV dose was adjusted according to the prevailing Daily Light Integral (DLI) below $500 \mathrm{~nm}$ and the specific sensitivity of each host species to UV, thereby maximizing mildew suppression and minimizing potential phytotoxicity of UV. Treatments with UV every third night provided $80-99 \%$ reduction of powdery mildew compared to untreated plants, without negative effects on yield. Design and operational considerations, including choice of lighting (UVB, UVC, fluorescent, LED, etc.), and potential effects on biocontrol arthopods in diverse applications will be discussed.

Sensing of UV and visible light by powdery mildew pathogens

R. PATHAK (1), A. Sundaram (2), L. E. Cadle-Davidson (3), K. A. Solhaug (1), A. Stensvand (1,4), H. R. Gislerød (1), A. Suthaparan (1), (1)

Norwegian University of Life Sciences, Ås, NORWAY; (2) Norwegian Sequencing Centre, Oslo University Hospital, Oslo, NORWAY; (3) USDA

Grape Genetics Research Unit, Geneva, NY, USA; (4) Norwegian Institute of Bioeconomy Research, Ås, NORWAY 
Optical radiation plays a key role in all forms of life on earth as an environmental sensory cue to anticipate environmental changes and regulate circadian mechanisms. In addition, photosynthetic organisms use optical radiation as a source of energy. Fungi are neither photosynthetic nor capable of observing adjacent objects. Nonetheless, a majority of fungal species exhibit profound responses to light. This is particularly true within the powdery mildews (Erysiphaceae), which sense, interpret and use light to control their growth and development. Using next generation transcriptome sequencing of Erysiphe necator, the grapevine powdery mildew, we recently discovered the presence and expression of putatively photo-responsive genes similar to phytochromes, cryptochromes, white collar, and photolyase. Herein we report draft genome and transcriptome sequencing of Oidium neolycopersici, Podosphaera xanthii, and $P$. aphanis, powdery mildews of cucumber, tomato, and strawberry, respectively. We confirmed the presence of genes similar to all major classes of photoreceptor genes, including photolyase, cryptochrome, white collar, phototropins, phytochrome, and UVR8. Current research on characterization and biological functions of photo-responsive genes will be discussed in the context of disease management via controlled lighting.

\section{Sustainable approaches for soilborne disease management in nursery production} P. Liyanapathiranage, M. N. Kabir, T. Simmons, F. BAYSAL-GUREL, Tennessee State University, McMinnville, TN, USA

Soilborne diseases are the most economically important problem of Southern region nursery producers. The goal of this research was to improve soilborne disease management through the use of biofumigant cover crops, mustard meal and compost. The experiment was established at on-farm with prevalent Rhizoctonia solani pressure, as a randomized complete block design with three replications. Biofumigants (turnip, arugula and amara mustard) were direct seeded into the beds and flowering plants were incorporated $15 \mathrm{~cm}$ deep into the same beds and covered with polythene for 14 days. Compost and mustard meal were incorporated into the beds at the same time of biofumigant plant incorporation. Flowering cherry (Prunus serrulata 'Kwanzan') cuttings were planted two weeks after the incorporation of each treatment. Rooted cuttings were evaluated for disease severity on roots as well as for fresh weight and plant height at the end of the trial. Data were analyzed using Minitab statistical software. Biofumigant cover crops significantly reduced Rhizoctonia root rot compared to the untreated control. Amara mustard cover crop and compost amendment significantly increased plant height compared to the untreated control. Compost amendment also significantly increased plant fresh weight and root weight compared to the untreated control and other treatments. These management approaches will also be evaluated in artificially inoculated field for potential usage in field nursery production.

Effect of Carbon Sources in Anaerobic Soil Disinfestation to Reduce Soilborne Pests in Okra and Brinjal in Nepal

R. B. KHADKA (1), M. Marasini (2), R. Rawal (2), A. L. Testen (3), S. A. Miller (1), (1) The Ohio State University, Wooster, OH, USA; (2) Regional Agricultural Research Station, Nepal Agricultural Research Council, Banke, NEPAL; (3) The Ohio State University OARDC, Wooster, OH, USA

Soilborne diseases, weeds and nematodes are the major yield limiting factors in okra (Abelmoschus esculentus) and brinjal (Solanum melongena). Anaerobic soil disinfestation (ASD) is a promising technique in developed countries to control soilborne pests but it has not been tested in many developing countries. Experiments were conducted in the western Terai region of Nepal in okra and brinjal in 2016 to determine the effectiveness of locally available ASD carbon sources. Experiments were conducted in a randomized complete block design with three replicates of eight carbon source treatments: molasses, rice bran + molasses, rice bran, raw goat manure, mustard cake, lentil husk, berseem clover (Trifolium alexandrinum) leaves, and control (no carbon source). Carbon sources were incorporated into soil followed by irrigation to saturation and plots were covered with white plastic sheeting for 21 days. Significantly lower wilt incidence (brinjal only), nematode index, and weed biomass were observed in all ASD-treated plots than in control plots. Similarly, significantly higher fruit yield and fresh plant biomass were observed in ASD-treated plots than in control plots for both crops. ASD appears to have great potential in Nepal because it has broad spectrum impact on multiple soilborne pests and can be performed using local resources.

\section{Incorporating disease-suppressive rotation crops and organic amendments into improved potato cropping systems}

\section{R. P. LARKIN, USDA ARS, Orono, ME, USA}

Use of longer rotations, disease-suppressive crops, and organic amendments have been shown to increase tuber yield, reduce disease, and improve soil health in previous studies, but need to be implemented in ways that are economically viable for growers. In field trials established in 2013, different 3-yr potato cropping systems focused on specific management goals of soil conservation (SC), soil improvement (SI), and disease-suppression (DS) were evaluated and compared to a 2-yr standard rotation (SQ) and a non-rotation control (PP) for their effects on soil properties, tuber yield, soilborne diseases, and economic viability. SI system resulted in increased potato yield (by $31 \%$ ), higher OM (by $51 \%$ ) and other nutrient contents, and higher microbial activity (by $42 \%$ ), relative to the SQ system. The DS system also increased yield (by $15 \%$ ) and increased microbial activity (by $21 \%$ ) relative to SQ, and reduced black scurf by $25 \%$ and common scab by $21 \%$ relative to PP. Soil microbial community characteristics, as represented by fatty acid methyl ester (FAME) profiles were also distinctly different among the cropping systems. Overall, systems provided comparable or increased net income relative to other 3-yr rotations, but were not quite as profitable as standard 2-yr rotation (SQ). These results indicate that soil health management practices can be incorporated into economically viable cropping systems that may enhance sustainability, productivity, and ecosystem function.

\section{Practical and accurate measurement of ultraviolet radiation (UV) for research and application in plant pathosystems}

A. Bierman (1), L. Radetsky (1), J. S. Patel (1), M. Figueiro (2), T. Plummer (1), M. REA (1), D. M. Gadoury (3), A. Suthaparan (4), A. Stensvand (5), N. Peres (6), R. Borba Onofre (7), (1) Lighting Research Center, Rensselaer Polytechnic Institute, Troy, NY, USA; (2) Lighting Research Center, Troy, NY, USA; (3) Cornell University, Geneva, NY, USA; (4) Norwegian University of Life Sciences, Ås, NORWAY; (5) Norwegian Institute of Bioeconomy Research, Ås, NORWAY; (6) Gulf Coast Research and Education Center; University of Florida, Wimauma, FL, USA; (7) University of Florida - Gulf Coast Research and Education Center, Wimauma, FL, USA

Supplemental ultraviolet radiation (UV) has been repeatedly investigated as a treatment to suppress plant pathogens. Advances in lighting technology, as well as our understanding of pathogen biology have led to several innovations in deployment of UV against pathogens. However, accurate measurement of UV is challenging - much more so than measurement of visible light - and precise quantitative dosing of plants is critical. Under-dosing will be ineffective, while overdosing can stress or even kill plant tissue. Furthermore, diverse plant species differ in their sensitivity to UV, and ability to recover from UV treatments. This presentation explains concepts in radiometry, sensor requirements, and operational procedures that are essential for accurate UV measurements, calculations of dose, and biological activity beyond simple associations with peak wavelengths. This includes detector spectral selectivity, sensitivity and dynamic range, and spatial response considerations. Temporal measurement techniques for dose calculation will be discussed. Practical considerations for choosing instrumentation and converting between units (quantum versus radiometric, dose vs irradiance) will be presented. These concepts will be illustrated with equipment and methods used for in-situ UV dosing of strawberry crops by a tractor-drawn array of UV-C lamps.

\section{Modifying row cover systems to manage bacterial wilt in organic muskmelon in Iowa}

H. M. NELSON, Iowa State University, Ames, IA, USA

Spunbond row covers are used to prevent cucumber beetles from spreading bacterial wilt in organic cucurbits. These row covers must be removed for pollination and to prevent heat stress. Ineffective organic insecticides that are harmful to beneficial insects are sprayed frequently from then until harvest to control beetles. There is need for a row cover system to provide full-season bacterial wilt protection, which would eliminate insecticide use. Four reps of four row cover treatments arranged in a randomized complete block design were compared on muskmelon (cv. Athena): spunbond row covers, partialseason insect netting, full-season insect netting, and a non-covered control. Spunbond row covers were supported by 1.5 -ft. tall wire hoops, and insect netting was supported by 3-ft. tall, 1" conduit hoops. On June 1, 2016, 3-week-old seedlings were transplanted $2 \mathrm{ft}$. apart on 6-ft. row centers. Subplots 
were 3 rows wide by $30 \mathrm{ft}$. long. For pollination, spunbond row covers were removed permanently and partial-season insect netting was removed for two weeks when female flowers appeared. Beehives were placed beneath row covers in full-season insect netting. When not protected by row covers, insecticides were applied to subplots for beetle control. Data on insect and disease pressure as well as number and weight of fruit was recorded. Mean percent bacterial wilt incidence was significantly lower in full-season insect netting compared to non-covered and spunbond. Full-season insect netting had significantly more marketable fruit and required zero insecticide applications. Significantly fewer fruit were culled due to insect damage in fullseason insect netting and the non-covered control.

Impact of cover crop usage on soilborne pathogens in a nursery production system S. Dawadi, K. Addesso, P. A. O’Neal, P. Liyanapathiranage, M. Pandey, F. BAYSAL-GUREL, Tennessee State University, McMinnville, TN, USA

Cover crops are poised to play increasingly important roles on soil conservation, pest and disease management. The goal of this project was to evaluate the effectiveness of a cover crop mix (wheat and crimson clover) for management of wood-boring insects and soilborne pathogens (Phytophthora nicotianae and Rhizoctonia solani) in nursery production. There were four treatments; no insecticide + herbicide, insecticide + herbicide, cover crop + insecticide, cover crop only. The experiment was established as a randomized complete block design with four replications. Soil was sampled from field plots before and after incorporation of the cover crop mix. Greenhouse bioassays were conducted using red maple seeds in 4-in pots. Pots were amended with P. nicotianae or R. solani inoculum. Non-inoculated pots served as controls. Stand and vigor data were recorded four months after seeding. Fluorescent Pseudononads were determined using selective media. The lowest level of damping off was observed in the cover crop treatments for both pathogens. The observed level of damping off was also lowest in cover crop incorporated soils. Plant vigor was greater in the cover crop treatments. There were no significant differences in Pseudononads colony number among treatments, but numerically increased in cover crop incorporated soils. These results indicate that cover crop can provide nursery growers with some quantifiable protection against subsequent outbreaks of soilborne diseases.

UV-C radiation treatment on Andean lupine seed for control anthracnose infection and increase crop production C. E. FALCONI PHD (1), V. Yanez-Mendizábal (2), (1) Univ de Las Fuerzas Armadas - ESPE, Carrera de Ingenieria Agropecuarias, Sangolqui, ECUADOR; (2) Universidad de las Americas, Facultad de Facultad de Ingeniería y Ciencias Agropecuarias, Quito, ECUADOR

The potential of UV-C radiation to control anthracnose seed-borne infections caused by Colletotrichum acutatum and its effect on crop production of Andean lupine (Lupinus mutabilis Sweet) was investigated. First, different UV-C exposure times of 1 to $96 \mathrm{~h}$ giving 7.2 to $691.2 \mathrm{~kJ} / \mathrm{m}^{2}$ were evaluated on anthracnose incidence reduction and seed germination on naturally- and artificially-infected seed. UV-C radiation was effective to reduce or to eradicate the anthracnose infection from $6-7 \%$ to undetectable level $(0 \%)$ compared with untreated controls in all cultivars. The reduction of anthracnose infection and seed germination were dependent on the UV-C doses. Under greenhouse conditions, UV-C doses of 57.6 and $86.4 \mathrm{~kJ} / \mathrm{m}^{2}$ showed seed emergence rates that were similar or better to the fungicide control. Moreover, UV-C doses of $57.6 \mathrm{~kJ} / \mathrm{m}^{2}$ reduced $80 \%$ and $86.4 \mathrm{~kJ} / \mathrm{m}^{2} \mathrm{eradicate}$ transmission of the pathogen from seed to the seedlings. In field experiments, pre-treated seed with UV-C doses of $86.4 \mathrm{~kJ} / \mathrm{m}^{2}$ registered lower disease rate in all lupine cultivars and showed less incidence of anthracnose infection on pods. Crop production from seeds treated with UV-C doses of 57.6 and $86.4 \mathrm{~kJ} / \mathrm{m}^{2}$ was higher than untreated seed and equal than the fungicide-seed treatment. The use of UV-C radiation on seed was effective to control of anthracnose and it is potentially a viable management option for Andean lupine production in high anthracnose risk areas of Ecuador. Research funded by Proyecto "Mejora de la cadena productiva del chocho (Lupinus mutabilis) en Ecuador - SENESCYT/ESPE/UDLA/UTC".

Root response of Huanglongbing-affected citrus trees to steam thermotherapy

K. GERBERICH (1), S. L. Commerford (2), R. Ehsani (3), M. M. Dewdney (3), E. G. Johnson (3), (1) University of Florida Citrus Research and Education Center, Lake Alfred, FL, USA; (2) Univeristy of Florida Citrus Research and Education Center, Lake Alfred, FL, USA; (3) University of Florida, Lake Alfred, FL, USA

Huanglongbing (HLB) is caused by the phloem-limited bacterium, Candidatus Liberibacter asiaticus (Las), transmitted by the Asian Citrus Psyllid. HLB has severely affected the Florida citrus industry in the last 10 years. Steam-based thermotherapy is a possible tool to eliminate infection from the canopy and extend the productive life of the tree. One of the major symptoms of HLB is fibrous root dieback caused by local Las infection and later by carbohydrate starvation. The health of the root system and the effects of thermotherapy on the root system may affect the overall response to thermotherapy. The effects of season ( 3 month intervals) and time/temperature (untreated control, $55^{\circ} \mathrm{C} / 0 \mathrm{~s}, 55^{\circ} \mathrm{C} / 60 \mathrm{~s}, 55^{\circ} \mathrm{C} / 90 \mathrm{~s}, 55^{\circ} \mathrm{C} / 120 \mathrm{~s}$ and $60^{\circ} \mathrm{C} / 30 \mathrm{~s}$ ) of thermotherapy on host response (including roots) were tested in a randomized split plot field trial with 3 replications per season. Soil cores were collected from the pretreatment irrigation zone and after treatment for three months, then every six months to obtain root densities ( $\mathrm{g}$ dry weight/1 of soil). In most cases, trees suffered an initial root dieback after thermotherapy correlated to severity of treatment. The best root recovery within treatments was in the spring when compared to the untreated control. Root recovery could be due to the production of new functional phloem. However, summer displayed the most damage after treatment, with a long root recovery period.

Severity of strawberry powdery mildew in open field vs under plastics that either block or transmit UV light

R. BORBA ONOFRE (1), R. Luis Vieira (2), S. Arupillai (3), A. Stensvand (4), D. M. Gadoury (5), N. Peres (6), (1) University of Florida - Gulf Coast Research and Education Center, Wimauma, FL, USA; (2) Universidade de Passo Fundo, Passo Fundo, BRAZIL; (3) Norwegian University of Life Sciences, Ås, NORWAY; (4) Norwegian Institute of Bioeconomy Research (NIBIO), Ås, NORWAY; (5) Cornell University, Geneva, NY, USA; (6) Gulf Coast Research and Education Center; University of Florida, Wimauma, FL, USA

Strawberry powdery mildew, caused by Podosphaera aphanis, can be particularly destructive in glasshouse and plastic tunnel production systems, which generally are constructed of materials that screen out ultraviolet light (UV). We compared epidemic progress in replicated plots in open fields, and under tunnels constructed of polyethylene, which blocks nearly all solar UVB, or two formulations of ethylene tetrafluoroethylene (ETFE), one of which contained an UV blocker, and another which transmitted nearly $90 \%$ of solar UVB. Disease severity under all plastics was higher than in open field plots, indicating a generally more favorable environment in containment structures. However, the foliar severity of disease within tunnel was directly proportional to their UV transmissibility. Within tunnels, incidence of fruit infection was highest under polyethylene and lowest under UV-transmitting ETFE. These effects likely transcend crop, and the blocking of UV transmission by glass and certain plastics may markedly contribute to the environmental favorability of greenhouse and high tunnel growing systems for powdery mildew.

Heat treatment for management of Botrytis cinerea inoculum on strawberry

A. ZUNIGA (1), N. Peres (2), (1) University of Florida, Wimauma, FL, USA; (2) Gulf Coast Research and Education Center; University of Florida, Wimauma, FL, USA

Chemical control is the primary strategy for managing Botrytis cinerea, the causal agent of gray mold on strawberry. However, the excessive use of fungicides has caused widespread resistance to several fungicide groups, requiring other management strategies. In this study, we evaluated the efficacy of heat treatment on strawberry plants as a possible means to reduce inoculum on naturally infested and artificially inoculated plants. Five different treatments conducted prior to planting included: (1) preheat at $37^{\circ} \mathrm{C}$ for $1 \mathrm{~h} \mathrm{f.b}$. heat treatment at $44^{\circ} \mathrm{C}$ for $2 \mathrm{~h},(2)$ preheat at $37^{\circ} \mathrm{C}$ for $1 \mathrm{~h} \mathrm{f.b}$. heat treatment at $44^{\circ} \mathrm{C}$ for $4 \mathrm{~h},(3)$ heat treatment at $44^{\circ} \mathrm{C}$ for $2 \mathrm{~h},(4) 44^{\circ} \mathrm{C}$ for $4 \mathrm{~h}$, and (5) non-treated control. Leaves were collected before inoculation, after inoculation, and after heat treatment, and evaluated for percentage of inoculum recovery. The natural level of inoculum on the leaves was $42.5 \%$, which following artificial inoculation, increased to $71.9 \%$. For inoculated plants, there was a significant difference $(\mathrm{P}<0.05)$ between treatments 2 and 4 
compared to the control; reducing infection by 100 and $95.9 \%$, respectively. Similar results were obtained for non-inoculated plants, indicating that heat treatments for $4 \mathrm{~h}$ were more effective than for $2 \mathrm{~h}$. Based on these results, heat treatment of strawberry plants is a feasible strategy for reducing inoculum levels and it could be integrated as a strategy to manage fungicide-resistant isolates.

Boron tolerance and clubroot development in Brassica napus

A. McLean (1), B. D. GOSSEN (2), M. R. McDonald (1), (1) University of Guelph, Guelph, ON, CANADA; (2) Agric \& Agri-Food Canada, Saskatoon, SK, CANADA

Plasmodiophora brassicae Wor. causes clubroot in canola (Brassica napus L.) and other brassica crops. Previous studies have shown that application of boron (B) can reduce clubroot severity, but rates $\geq 4 \mathrm{~kg} \mathrm{~B} \mathrm{ha}^{-1}$ can result in phytotoxicity. In 2015, 3-week-old seedlings of B. napus accessions were transplanted on a muck soil ( $70 \%$ organic matter) site naturally infested with P. brassicae. Boron (Solubor, 20.5\% B) was applied at $8 \mathrm{~kg} \mathrm{~B} \mathrm{ha}^{-1}$ in 1500 $\mathrm{L} \mathrm{ha}^{-1}$ of water at 5 days after transplanting. Heavy rainfall and subsequent lack of phytotoxicity prompted a second B application 5 days later. Toxicity was assessed 31 days after seeding. Ten highly B-tolerant lines and nine B-sensitive lines were identified, and clubroot severity (0-3 scale) was assessed in each line at 63-65 days after seeding. Clubroot severity decreased and water content per plant increased in response to added B in the B-tolerant group, but not in the B-sensitive group. When the trial was repeated at an adjacent site in 2016, there were no differences in severity among the lines Warm, wet conditions in spring, favorable for infection and development of clubroot, may have increased disease pressure and overwhelmed the response to $\mathrm{B}$. A reduction in clubbing severity at $8 \mathrm{~kg} \mathrm{~B} \mathrm{ha}^{-1}$ was observed in the B-tolerant lines in two growth room experiments. We conclude that $\mathrm{B}$ reduced clubroot severity, but that disease pressure may affect the consistency of result in the field.

Effective pruning method for twigs showing black shoot blight disease symptoms in apple trees D. H. PARK (1), C. S. Oh (2), (1) Kangwon National University, Chuncheon, KOREA; (2) Kyung Hee Univ, Yongin, KOREA

Black shoot blight disease caused by Erwinia pyrifoliae have damaged economic loss to apple and pear growers until now since it was firstly reported in 1995 in Korea. This study was performed to reduce economic loss by mandatory eradication of all infected trees in case of more $10 \%$ disease incidence per orchard as official control. It also aims to set up effective management protocol for this disease by examining how far bacterial pathogen is present from the border of symptomatic and asymptomatic regions in infected apple twigs. Colony-PCR using isolated bacterial cells instead of genomic DNA was used to identify bacterial pathogen, EpSPF/EpSPR primer designed in ERIC region was selected as specific for E. pyrifoliae. As results of monitoring of this disease during April to October in 2014-2015 by colony-PCR, occurrence of this disease was frequent from mid-May to early-July, when daily average temperature was around $25^{\circ} \mathrm{C}$. Moreover, bacterial cells were continuously detected only in symptomatic regions and also asymptomatic regions of less than $20 \mathrm{~cm}$ from symptomatic regions. Therefore, we concluded that pruning of infected twigs at the region of more than $20 \mathrm{~cm}$ from symptomatic regions might be effective to manage black shoot blight disease in apple trees.

Effects of dry heat treatment on Cucumber green mottle mosaic virus and infection activity C. LIANG $(1,2,3,4)$, L. Luo $(1,2)$, J. Li $(1,2)$, B. Baker $(3,4)$, (1) China Agricultural University, Beijing, CHINA; (2) Beijing Key Laboratory of Seed Disease Testing and Control, Beijing, CHINA; (3) Plant Gene Expression Center USDA-ARS, Albany, CA, USA; (4) Department of Plant and Microbial Biology University of California, Berkeley, Berkeley, CA, USA

Cucumber green mottle mosaic virus (CGMMV; genus Tobamovirus) is one of the most economically important viruses of cucurbit crops worldwide. In order to explore the thermal inactivation efficiency of CGMMV and to characterize the change in infectivity of CGMMV on cucumber seedlings inoculated with virus isolated from contaminated soils, we tested the efficiency of CGMMV inactivation using increasing dry heat treatments (DHTs) $\left(40^{\circ} \mathrm{C}, 80^{\circ} \mathrm{C}, 120^{\circ} \mathrm{C}\right.$ and $\left.160^{\circ} \mathrm{C}\right)$ over increased treatment times $(0 \mathrm{hr}, 1 \mathrm{hr}, 2 \mathrm{hr}, 3 \mathrm{hr}, 5 \mathrm{hr}, 7 \mathrm{hr}, 9 \mathrm{hr}$ and $11 \mathrm{hr})$. The results showed that: 1$)$ Expression levels of CGMMV replicase, movement protein gene and coat protein genes (measure by qRT-PCR) decreased in seedlings inoculated with soil suspensions treated with higher DHTs over longer time periods compared to untreated soil; 2) Contaminated soil treated from 3 hr to 11 hrs at $80^{\circ} \mathrm{C}$ DHT did not abolish CGMMV infection activity and cucumber seedlings inoculated with suspensions showed mottled mosaic symptoms in upper leaves after 20 days; 3 ) Higher DHTs $\left(120^{\circ} \mathrm{C}\right.$ and $\left.160^{\circ} \mathrm{C}\right)$ and longer time periods $(9 \mathrm{hr}-11 \mathrm{hr})$ were required for CGMMV inactivation. These results will provide a scientific basis for prevention and control of CGMMV in the greenhouse.

\section{The Effect of Tree Wound Pastes on callus formation}

S. H. LEE (1), J. H. Park (2), C. H. Jeon (1), (1) National Institute of Forest Science, Jejudo, Korea, Republic of (South); (2) Korea Forest Research Inst, Seoul, Korea, Republic of (South)

Main areas for chestnut production in South Korea used to be the southern part of the country including Jinju and Hadong in Gyungnam province and Gwangyang and Suncheon in Jeonnam province. New chestnut plantations are recently increasing in the central part such as Chungnam and Chungbuk provinces. More than 20 diseases of chestnut (chestnut blight, nut anthracnose, powdery mildew, ink disease, etc.) have been reported from Korea. Pathogens causing the blight or dieback on a main stem and branches include Cryphonectria parasitica, Botryosphaeria dothidea, Pseudovalsa modonia, and Melanconis microspora. Among them, chestnut blight caused by C. parasitica is the most damaging disease, by which infected chestnut trees eventually die. To prevent the pathogen from infecting through wounds, we applied several kinds of wound pastes (auxin, gibberellin, adhesive, and topsin pastes) on artificial stem wounds and evaluated following callus formation. The adhesive paste showed the greatest callus formation (95\%) followed by topsin paste $(93.75 \%)$ and auxin paste $(90 \%)$. The larger the wound size was, the slower the callus formed. With the wound of diameter less than $5 \mathrm{~cm}$, the tree actively formed the callus covering the wound more than $96.7 \%$. Whereas, for the wound of diameter over $10 \mathrm{~cm}$, callus coverage was lower (80 88\%). Treatment with the gibberellin paste has no difference from the control. As a result, applying adhesive, topsin and auxin pastes on the wounds were found to be effective in preventing pathogens or insects from infesting the stems or branches.

Heat treatment as an alternative to fungicide application for control of strawberry anthracnose caused by Colletotrichum acutatum N. Y. WANG (1), W. W. Turechek (2), N. Peres (3), (1) Gulf Coast Research and Education Center, University of Florida, Wimauma, FL, USA; (2) USDA ARS SAA SPP, Fort Pierce, FL, USA; (3) Gulf Coast Research and Education Center; University of Florida, Wimauma, FL, USA

Anthracnose, caused by Colletotrichum acutatum, is a major threat to strawberry production in Florida. The pathogen is commonly spread by contaminated transplants from strawberry nurseries. Due to latent infection of transplants by $C$. acutatum and the emergence of isolates resistant to strobilurin fungicides, management of anthracnose in strawberry nurseries and fruit production fields is challenging. In this study, heat treatment was investigated as an alternative to fungicide applications. In preliminary in vitro tests, spore germination of $C$. acutatum was completely inhibited after incubation at 44,48 , and $52^{\circ} \mathrm{C}$ for 30,5 , and $5 \mathrm{~min}$, respectively. However, a low percentage of $C$. acutatum spores $(<10 \%)$ exposed to $40^{\circ} \mathrm{C}$ for up to $240 \mathrm{~min}$ remained viable. Heat treatment of strawberry transplants at $44^{\circ} \mathrm{C}$ for $4 \mathrm{~h}$, with or without a preheat step $\left(37^{\circ} \mathrm{C}\right.$ for $\left.2 \mathrm{~h}\right)$, via steam in a plant sauna device significantly reduced the colonization of petioles by $C$. acutatum $(<10 \%, P<0.05)$ compared to the non-treated control. Field data from early season harvests revealed that fruit disease incidence in heat-treated plots with or without a pre-heat step was significantly lower $(<1 \%, P<0.05)$ than that in the non-treated control. Our results indicate that heat treatment via steam in a plant sauna device could be an effective alternative for reducing $C$. acutatum inoculum in strawberry nurseries and consequently anthracnose in fruit production fields. 
Evaluation of a nutritional approach for disease control of Brurkholderia glumae and B. gladioli interaction in rice

C. A. Riera-Ruiz (1,2), J. Castro-Lara (3), M. I. Jimenez Feijoo (3), J. CEVALLOS-CEVALLOS (4), (1) Centro de Investigaciones Biotecnológicas del Ecuador, Guayaquil, ECUADOR; (2) CIBE-ESPOL, Guayaquil, ECUADOR; (3) Escuela Superior Politécnica del Litoral, Guayaquil, ECUADOR; (4) Escuela Superior Politecnica del Litoral, ESPOL, Guayaquil, FL, ECUADOR

Burkholderia glumae and B. gladioli are the most important pathogens of rice worldwide but potential synergism among both bacteria is not known. Also, knowing the effects of micronutrients on disease progression would contribute to the development of control strategies. Seeds and plants of four different rice varieties were treated with the micronutrients zinc, boron and a mixture of both micronutrients. Seeds and plants were then inoculated with B. glumae, B. gladioli and a combination of both pathogens and the treatments effect on germination and disease progression (AUDPC) were evaluated in seeds and pants, respectively. Only B. glumae significantly reduced in up to $19 \%$ the percentage of seed germination in all varieties. Both pathogens reduced significantly the coleoptile and root development in up to $83 \%$. In plants, B. glumae yielded the highest AUDPC values in all varieties but no synergism was observed in symptoms development of either seeds or plants. Zinc, boron or the combination did not reduce the effects of the pathogens. Results suggest that nutrition alone is not a significant factor in controlling pathogenic Burkholderia spp.

Broccoli residue and chitin amendments reduce Verticillium wilt in three soil types

K. D. PURI (1), D. P. G. Short (1), P. Inderbitzin (2), D. O. Chellemi (3), K. V. Subbarao (4), (1) University of California, Davis, Salinas, CA, USA; (2) University of California, Davis, CA, USA; (3) Agricultural Solutions, Fernandina Beach, FL, USA; (4) University of California at Davis, c/o U.S. Agricultural Research Station, Salinas, CA, USA

To develop economically feasible Verticillium wilt management practices in the absence of fumigation, the effects of soil amendments on Verticillium wilt and associated microbiome dynamics were studied. Experiments were conducted using soil with three distinct land use histories- intense production agriculture, transition from pasture to agriculture, and native, undisturbed soil. The tested amendments were: broccoli residue applied at $10 \% \mathrm{w} / \mathrm{w}$, a chitin-based blend of crustacean shell applied at $0.2 \% \mathrm{w} / \mathrm{w}$, and a combination of broccoli and crustacean shell. Soil chemical and physical properties were evaluated at the start and end of the experiments. Disease severity on eggplant, microsclerotia (MS) density, chitinase enzyme activity, and soil microbiome community structure were analyzed over time. Broccoli and broccoli+chitin resulted in significant reductions in disease severity and MS density relative to the unamended control. A higher MS reduction was observed in non-agricultural than in agricultural or transition soils. Soil microbiome dynamics, enzyme activity and soil properties associated with disease suppressiveness will be presented. Broccoli residue alone or in combination with crustacean shell reduced Verticillium wilt in all soil types, and thus represents a cultural management approach for soilborne pathogens in the absence of fumigation.

Canopy and Root Response of HLB-affected Citrus trees to Steam-generated Thermotherapy N. THAPA (1), S. L. Commerford (2), R. Ehsani (1), E. G. Johnson (1), M. M. Dewdney (1), (1) University of Florida, Lake Alfred, FL, USA; (2) Univeristy of Florida Citrus Research and Education Center, Lake Alfred, FL, USA

Citrus greening (HLB) is threatening the viability of Florida citrus industry. The phloem-limited bacterium, Candidatus Liberibacter asiaticus (Las), is the causal agent of HLB and infects both canopy and root system. This study explores steam as a heat source for rapid treatment of HLB-affected citrus trees, with the hypothesis that thermotherapy can reduce Las titer and slow tree decline. Canopy and root responses were studied, along with the bacterial movement in tree. A randomized split-plot design field trial was laid out with the four seasons as main plots to determine optimum time-of-year for tree response and subplots at 6 temperature-time combinations $\left(55^{\circ} \mathrm{C} / 0 \mathrm{~s} ; 55^{\circ} \mathrm{C} / 60 \mathrm{~s} ; 55^{\circ} \mathrm{C} / 90 \mathrm{~s} ; 55^{\circ} \mathrm{C} / 120 \mathrm{~s} ; 60^{\circ} \mathrm{C} / 30 \mathrm{~s}\right.$ and untreated control) with 3 replications/treatment. Four leaves/tree from 10 trees/rep were collected monthly and tested for Las titer via qPCR. Roots, the untreated reservoir, were also assessed for Las titer. The greatest decrease in Las titer was in winter for both midribs and roots. Overall analysis showed that month, season and the interaction of month*season were significant $(p$-value $\leq 0.0001)$ for midribs. For roots, month, month*season and treatment*month*season were significant $(p$-value $\leq 0.0001)$. Season and month post treatment are important for suppression of Las. Changes in Las titer in canopy and roots may be due to change of phloem flow of Las along with the stored carbohydrates from root to the treated canopy due to damage from thermotherapy.

Switchgrass extractives reduce bacterial diseases of tomato foliage and populations of foodborne pathogens on tomato fruit A. I. Bruce (1), B. H. OWNLEY (1), J. Tao (2), N. Labbé (2), K. Gwinn (1), D. D’Souza (3), N. Moustaid-Moussa (4), (1) University of Tennessee, Department of Entomology and Plant Pathology, Knoxville, TN, USA; (2) University of Tennessee, Center for Renewable Carbon, Knoxville, TN, USA (3) University of Tennessee, Food Science Department, Knoxville, TN, USA; (4) Texas Tech University, Department of Nutritional Sciences, Lubbock, TX, USA

Panicum virgatum (switchgrass) is a prominent feedstock for production of lignocellulosic biofuel. Non-structural, phenolic components (extractives) of switchgrass can hinder enzymes and microorganisms in the conversion process and reduce the overall yield of biofuel. Extractives have potential uses as value-added coproducts. Development of switchgrass extractives as biopesticides or food decontamination surface washes could make switchgrass a more cost-effective feedstock. Previously, we described the in vitro antimicrobial activity of switchgrass extractives against fungal and bacterial plant pathogens and foodborne bacterial pathogens. The objectives of this study were to evaluate switchgrass extractives to control bacterial diseases of tomato plants, caused by Xanthomonas perforans, Clavibacter michiganensis subsp. michiganensis, and Pseudomonas syringae pv. tomato, and to eliminate the foodborne pathogens, Salmonella enterica serovars Typhimurium and Enteritidis, when applied as a fruit wash. In plant assays, extractives were tested as pre- and post-inoculation treatments against the bacterial diseases. Switchgrass extractives reduced disease caused by the plant pathogens. The degree of control varied depending on pre- or post-inoculation treatment and pathogen. Populations of Salmonella enterica serovars were reduced significantly in all food wash assays. Switchgrass extractives have valuable potential for development as a biopesticide or decontamination wash.

The use of eco-mesh bags for environmentally friendly controlling Pine Wilt

S. H. LEE (1), J. H. Park (2), C. H. Jeon (1), K. S. Kang (3), C. S. Kim (4), (1) National Institute of Forest Science, Jejudo, Korea, Republic of (South);

(2) Korea Forest Research Inst, Seoul, Korea, Republic of (South); (3) National institute of Forest science, seogwipo, jejudo, Korea, Republic of (South); (4) National Institute of Forest Science, Seogwipo, jejudo, Korea, Republic of (South)

Pine wilt is the most damaging forest disease by which pine trees are killed within 1 3 years in Korea. It is caused by pine wilt nematode which is carried by pine sawyer beetles (Monochamus alternatus) or pine sawyer beetles (M. saltuarius). The disease has been also reported from Asian countries including China and Japan and European countries including Spain and Portugal. In Korea, various chemical control means have been introduced to control the disease such as aerial spray, ground spray, fumigation, and tree injection, but the damage has been increasing. In addition, the wide use of such chemical control means was controversial due to its negative effects on environment. To control pine wilt in an environmentally friendly way, we sealed logs obtained from pine wilt infected trees using mesh bags made of eco-mesh cloth, polyester, and mesh bags in field. For eco-mesh bags, control efficacy was highest $(100 \%)$ followed by polyester mesh bags (97\%), and mesh bags $(78 \%)$. No Japanese pine sawyer beetles, which emerged from the infected logs, were able to come out of the eco-mesh bags and most of the insects stayed killed on the logs or on the bottom of the bags within 4 to 7 days. The sealing of infected logs by eco-mesh bags are environmentally friendly without using any chemicals and have a high control efficacy, thus being expected useful to manage such large areas affected by pine wilt in South Korea. 
Pre-inoculation treatment of basil plants with ultraviolet-B radiation induces resistance to downy mildew

J. S. PATEL (1), L. Radetsky (1), T. Plummer (1), A. Bierman (1), D. M. Gadoury (2), M. Rea (1), (1) Lighting Research Center, Rensselaer Polytechnic Institute, Troy, NY, USA; (2) Cornell University, Geneva, NY, USA

Chemical control of downy mildew on basil, caused by Peronospora belbahrii, is problematic, as leaves are consumed as the final product and the preharvest intervals of many fungicides preclude their use. We have explored use of ultraviolet-B (UVB) radiation as an alternative method of control. Forty 8-day-old basil plants were exposed to UVB light emitting diodes (LEDs) for 6, 3 and 0 days before inoculation with sporangia of $P$. belbahrii. The 292 $\mathrm{nm}$ UVB LEDs (FWHM=13 nm) were positioned 1.5 inches in front of the pots, providing an average dose of $1368 \mathrm{~J} / \mathrm{m}^{2}$ over a period of $120 \mathrm{~min}$. The percent sporulation and number of sporangia were assessed for each treatment at $8,9,10$, and 11 days post inoculation. The basil plants exposed to UVB for 3 and 6 days exhibited reduced percent sporulation per plant compared to non-exposed plants. Fewer numbers of sporangia were recorded from plants exposed to UVB for 6 days than those exposed for 3 days or 0 days. The basil plants exposed to UVB for 3 days also had lower sporangia counts than the non-exposed basil. While some changes in leaf appearance were observed on the UVB-treated plants, UVB treatments did not significantly $(\mathrm{P}<0.05)$ reduce fresh or dry weight. The results suggest that an optimum pre-inoculation dose of UVB can suppress basil downy mildew.

Utility of planting variable-sized whole tubers simulating a European belt-style planter to reduce seed piece decay in seed potato production K. M. DUELLLMAN KINZER (1), M. C. Bertram (1), M. A. Lent (1), J. C. Randall (1), S. A. Clark (2), (1) University of Idaho, Idaho Falls, ID, USA; (2) Brigham Young University - Idaho, Rexburg, ID, USA

Seed potato growers in Idaho use cut tubers or single-drop tubers (small tubers $42.5 \mathrm{~g}$ to $85.0 \mathrm{~g}$ ) for seed potato production, in part because cup-type planters that predominate in the region limit seed piece or tuber size. Both approaches pose challenges. European-style belted planters allow whole tubers of different sizes, including large ones, to be placed in the planter hopper at once, without cutting or pre-sorting. An experiment was established at two locations in Idaho in 2016 with treatments consisting of cut seed potato pieces with 9-inch within-row spacing, single drop whole tubers with 9-inch within-row spacing, variable sized whole tubers ( $42.5 \mathrm{~g}$ to $226.8 \mathrm{~g}$ ) with 9-inch within-row spacing, or variable-sized whole tubers (42.5 g to $226.8 \mathrm{~g}$ ) with variable within-row spacing simulating a European belt-style planter. These were protected with fungicides or left non-protected prior to planting. Use of fungicide led to improved quality for nearly all parameters measured, regardless of seed type used. Use of variable-sized tubers at variable spacing led to significantly increased vigor, number of stems, and yield per plant, and reduced yield of culls for both locations $(\mathrm{P}<0.05)$. Seed piece decay was not consistently lower in the variable-sized/variable-spaced treatment compared to cut seed. When considering all measures, European beltstyle planters may improve seed potato quality, and fungicide seed piece treatment is beneficial regardless of planting strategy.

Survey of viruses infecting sweet cherry (Prunus avium) in Oregon

L. A. LUTES, J. W. Pscheidt, Oregon State University, Corvallis, OR, USA

Sweet cherry (Prunus avium) is a major fresh market fruit crop in Oregon, which ranks third among U.S. states for this commodity. Though extensive virus surveys have been conducted in the top-ranked sweet cherry-producing state, Washington, much less is currently known about the occurrence of sweet cherry viruses in Oregon. To gain perspective on virus diversity and distribution, a survey was conducted in Oregon sweet cherry orchards. Guided by local cooperators, orchards with suspected virus disease symptoms were sampled. Symptomatic and asymptomatic leaves were collected in summer 2016 from three main production areas: The Dalles, Hood River, Willamette Valley, as well as, Umpqua Valley. Virus-specific ELISA were used to test 64 symptomatic and 42 asymptomatic samples for the presence of common sweet cherry viruses: Cherry leaf roll (CLRV), Cherry rasp leaf (CRLV), Plum pox (PPV), Prune dwarf (PDV), Prunus necrotic ringspot (PNRSV), Tomato bushy stunt (TBSV), Tobacco ringspot (TRSV), and Tomato ringspot viruses (ToRSV). Isothermal AmplifyRP ${ }^{\circledR}$ (Agdia, Inc., Elkhart, IN) was used for detection of Little cherry virus 2 (LChV2). CLRV, a new virus on sweet cherry in Oregon, was found associated with dieback symptoms in The Dalles. Sweet cherry viruses reported in new regions, included: Hood River (PDV, PNRSV, ToRSV) and Umpqua Valley (PDV, PNRSV). Determining the prevalence of sweet cherry viruses throughout the state will allow for more effective disease management.

A real-time PCR assay for the differentiation of Pantoea stewartii subsp. stewartii from Pantoea stewartii subsp. indologenes in corn seed N. Pal (1), C. C. BLOCK (2), (1) USDA-ARS, Ames, IA, USA; (2) Iowa State University, Ames, IA, USA

Stewart's wilt of corn caused by the bacterium Pantoea stewartii subsp. stewartii is a seedborne disease of major phytosanitary importance. Over 60 countries have restrictions on seed imports to prevent introduction of the pathogen. Traditional methods for detection of $P$. stewartii include field inspection of parent plants and an ELISA seed assay. Current lab methods (ELISA and PCR) cannot readily distinguish P. stewartii subsp. stewartii from the closely-related but non-pathogenic (on corn) subspecies $P$. stewartii subsp. indologenes. We developed a SYBR-Green real-time PCR assay targeting the intergenic region of the cps gene cluster for the specific detection of $P$. stewartii subsp. stewartii. The assay detected all 27 P. stewartii subsp. stewartii reference strains while 13 Pantoea agglomerans, 11 P. ananatis, seven P. stewartii subsp. indologenes, and four distantly-related Erwinia species were negative. The detection limits for purified genomic DNA and cells were $0.1 \mathrm{pg}$ and $11 \mathrm{CFU}$ per PCR reaction, respectively. The assay successfully detected $P$. stewartii subsp. stewartii from 16 naturally-infected seed lots. Several seed lots that had tested positive by ELISA and published PCR methods were negative for $P$. stewartii subsp. stewartii and were later confirmed by sequencing to be subspecies indologenes. By distinguishing the two subspecies, the assay may help prevent unnecessary restrictions on the international movement of corn seed.

\section{Development and validation of two rapid isothermal detection assays for root-infecting fungi detection on turfgrass} B. KARAKKAT, M. Franchett, M. Olson, C. Mullenberg, P. L. Koch, University of Wisconsin, Madison, WI, USA

Necrotic ring spot, summer patch, and take-all patch are important diseases of turfgrass caused by root-infecting fungi. These diseases can ruin the aesthetic and functional value of cool-season turfgrasses on golf courses, home lawns and athletic fields from late spring to early summer. These pathogens are notoriously difficult to diagnose through traditional microscopic and culture-based diagnostic procedures, and many turfgrass diagnostic facilities don't have the equipment, expertise, or time to conduct routine molecular assays such as polymerase chain reaction (PCR). We developed two assays that can quickly and accurately detect these fungi on turfgrass samples using standard diagnostic laboratory equipment in an hour or less. The two assays we developed are a loop mediated isothermal amplification (LAMP) assay and recombinase polymerase amplification (RPA) assay. Both LAMP and RPA do not require pure genomic DNA but only a solution of crushed infected root sample. Both LAMP and RPA rely on DNA strand displacement activity for its rapid amplification and can be completed at $65^{\circ} \mathrm{C}$ in an hour and $39^{\circ} \mathrm{C}$ in 20 minutes incubation, respectively. New England Biolab's color change reaction assay was used to identify the specific fungal DNA amplified for the LAMP assay and Agdia's Immunostrip was used for the same purpose in the RPA assay. Both assays produced consistent and specific amplification for all three fungi. Validation and applicability of the assays will be performed on samples submitted to the University of Wisconsin's Turfgrass Diagnostic Lab in 2017.

Development and Validation of a Loop-Mediated Isothermal Amplification (LAMP) Assay for Detection of Citrus Leprosis Cytoplasmic Type 2 in Plants B. ADDUCCI, G. Wei, A. Roy, V. A. Mavrodieva, M. K. Nakhla, USDA-APHIS-PPQ-S\&T-CPHST, Beltsville, MD, USA

Cytoplasmic Citrus leprosis virus C2 (CiLV-C2) is currently reported only from Colombia, where it causes a substantial loss of citrus production. Even though CiLV is not present in the US, the emergence of CiLV is possible because the host, environmental conditions and mite vectors are present. New methods are needed to rapidly and accurately detect CiLV in the field, ports of entry and inspection stations. Loop-mediated isothermal amplification 
(LAMP) is a portable, fast, and specific detection system that has been applied to various pathogens, including CiLV-C. The specific objectives of this work are to design, optimize and validate an assay for the detection of CiLV-C2. Six (6) primer sets were designed, screened and an optimal primer set was selected based on performance. A closely related hibiscus infecting virus (CiLV-C2H) and CiLV-C tested negative with the optimal primer set. Commercially available master mixes from 2 companies [Optigene and New England Biolabs (NEB)] were compared. The NEB master mix produced a greater level of fluorescence, while Optigene was able to achieve a lower limit of detection (LOD). Temperature, primer concentrations, and time for optimal amplification were also optimized. Initial studies suggest that this CiLV-C2 LAMP assay is comparable in sensitivity to the currently used CiLV-C2 real-time RT-PCR. Further validation for the CiLV-C2 LAMP assay, including selectivity testing using common citrus pathogens, is in progress.

Validation of a Quadruplex Real-Time RT-PCR assay for Simultaneous Detection of Three Citrus leprosis Viruses in Plants B. ADDUCCI (1), G. Wei (1), A. Roy (1), V. A. Mavrodieva (1), G. Dennis (2), W. Schneider (3), R. H. Brlansky (4), M. K. Nakhla (1), (1) USDAAPHIS-PPQ-S\&T-CPHST, Beltsville, MD, USA; (2) USDA-APHIS-PPQ-S\&T-CPHST, Raleigh, NC, USA; (3) USDA ARS FDWSRU, Fort Detrick, MD, USA; (4) University of Florida, Lake Alfred, FL, USA

Citrus leprosis is one of the most destructive viral diseases of citrus in the Americas, associated with two distinct groups of viruses; a cytoplasmic type and a nuclear type. Currently, Citrus leprosis is not present in the US. However, to prevent the re-emergence of Citrus leprosis viruses (CiLVs) and to save the multi-billion dollar citrus industry, a specific and sensitive detection method is needed. To reduce testing time, cost, and labor, a quadruplex one-step real-time RT-PCR assay was developed and validated for simultaneous detection of cytoplasmic CiLV-C, CiLV-C2, and nuclear CiLV-N with a Nad5 plant RNA internal control. The results of repeated experiments performed in triplicate yielded a linear standard curve for each virus that was as efficient and linear when compared to the currently used species-specific real-time RT-PCR assay. Other common citrus pathogens, including Hibiscus infecting Cilevirus (CiLV-C2H), were tested negative in this multiplex assay. Healthy citrus RNA was spiked with in vitro transcripts for CiLV-C, -C2, and $-\mathrm{N}$ to determine the limits of detection (LOD) for each virus. The LOD was determined to be 30, 13, and 20 copies for CiLV-C, $-\mathrm{C} 2$, and $-\mathrm{N}$, respectively. With the availability of the described multiplex real-time RT-PCR assay, sensitive and specific detection of CiLV-C, $-\mathrm{C} 2$, and $-\mathrm{N}$ can be achieved in a timely and less labor intensive manner.

Development of two multiplex RT-PCRs for simultaneous detection of five cytoplasmic and three nuclear viruses associated with citrus leprosis complex

A. ROY (1), A. L. Stone (2), G. Leon Martinez (3), G. Otero-Colina (4), M. J. Melzer (5), J. S. Hartung (6), G. Wei (1), V. A. Mavrodieva (1), R. H. Brlansky (7), W. Schneider (2), M. K. Nakhla (1), (1) USDA-APHIS-PPQ-S\&T-CPHST, Beltsville, MD, USA; (2) USDA ARS FDWSRU, Fort Detrick, MD, USA; (3) CORPOICA, Villavicencio, COLOMBIA; (4) Colegio de Postgraduados, Texcoco, MEXICO; (5) University of Hawaii, Honolulu, HI, USA; (6) USDA ARS MPPL, Beltsville, MD, USA; (7) University of Florida, Lake Alfred, FL, USA

Two conventional multiplex reverse transcription-polymerase chain reactions (mRT-PCR) were developed for the simultaneous detection of 5 cytoplasmic $(\mathrm{C})$ and 3 nuclear $(\mathrm{N})$ leprosis viruses. The C-type mRT-PCR was optimized for Citrus leprosis virus -C (CiLV-C), CiLV-C2, the SJRP isolate of CiLV-C, hibiscus infecting Cilevirus (CiLV-C2H) and Hibiscus green spot higrevirus 2 (HGSV-2), whereas N-type mRT-PCR was optimized for 3 Dichorhaviruses (CiLV-N, CiLV-N2 and CiLV-N0) with nad5 plant gene as internal control. Genus and species specific primers were designed using leprosis associated virus sequences and validated using simplex- and mRT-PCR assays. In single infections, C type mRT-PCR produced a generic Cilevirus amplicon ranging from 887 to $905 \mathrm{nt}$, a nad5 amplicon (183 nt) and specific amplicons for CiLV-C (140 nt), CiLV-C-SJRP (371 nt), CiLV-C2 (784nt), CiLV-C2H (590nt) or HGSV-2 (480nt). The N type mRT-PCR amplified a 254 nt generic amplicon for Dichorhaviruses, the nad5 amplicon as internal control and specific amplicons for CiLV-N0 (691nt), CiLV-N (1143nt), or CiLV-N2 (1023 nt). One hundred CiLV suspected citrus and hibiscus samples were collected from Belize, Colombia, Costa Rica, Florida, Hawaii, Mexico and Panama and tested for CiLVs by mRT-PCR. The consistent results of the mRT-PCR were confirmed by sequencing analysis. Identification of multiple leprosis viruses by mRT-PCR will aid in quarantine and production of virus-free citrus and hibiscus plants.

Development of a rapid, specific and sensitive isothermal assay for the detection of Verticillium alfalfae

A. J. Reyes Gaige (1), J. K. S. Dung PhD (2), J. E. WEILAND (3), (1) Oregon State University, Corvallis, OR, USA; (2) Oregon State University, Madras, OR, USA; (3) USDA ARS, Corvallis, OR, USA

The genus Verticillium contains 10 plant pathogenic species that are responsible for billions of dollars of damage annually to $>400$ plant hosts worldwide. In alfalfa, Verticillium alfalfae causes Verticillium wilt, a serious disease of susceptible cultivars with the potential to reduce yields by the second harvest and to limit the productive stand life to $<3$ years. Susceptible alfalfa cultivars exhibit disease symptoms which include v-shaped chlorosis in leaflets, discoloration, stunting and wilting of shoots. New regulations by the Chinese government prohibit the entry of alfalfa samples contaminated with the Verticillium pathogen. In this investigation, we developed a recombinase polymerase amplification (RPA) assay coupled with a lateral flow device for the specific and sensitive detection of $V$. alfalfae. The primers targeted the TEF-1 $\alpha$ gene and incorporated mismatches to increase the specificity of the assay. This assay detected all tested isolates of $V$. alfalfae to at least $800 \mathrm{fg}$. Moreover, there was no nonspecific amplification of closely related $V$. nonalfalfae or other Verticillium species. Inoculation experiments using alfalfa cultivars with high resistance (55V50) and low resistance (La Jolla) further demonstrated the specificity and sensitivity of the assay. The assay is easy-to-use and will allow growers, diagnostic labs, and regulatory agencies to determine whether $V$. alfalfae is present in alfalfa products.

Development of digital PCR assays for plant pathogen diagnosis

Q. XIANG (1), J. Rascoe (1), S. Costanzo (1), Z. Liu (2), M. K. Nakhla (1), (1) USDA-APHIS-PPQ-S\&T-CPHST, Beltsville, MD, USA; (2) USDA APHIS PPQ, Riverdale, MD, USA

Digital PCR is a sensitive and precise analytical tool in absolute DNA/RNA quantification. We are applying the RainDance droplet digital PCR (ddPCR) platform to develop assays for plant pathogen diagnosis, using Huanglongbing (HLB) as model. HLB is caused by obligate bacterial phytopathogens of the Candidatus Liberibacter species. Early detection is very important for disease management and control, but is challenging due to low titer and irregular distribution within infected trees. Its diagnosis relies heavily upon DNA-based molecular diagnostic approaches. In this study, singleplex or duplex ddPCR detection assays targeting HLB 16S rDNA or/and nrdB gene are developed. The singleplex assays can precisely and reproducibly quantify the copy numbers of linearized plasmid DNA templates. The assays also can reproducibly quantify gene copy numbers in diseased HLB samples, and we are determining the precision of this quantification. The sensitivity of the singleplex nrdB assay was investigated and its Limit of Detection is $\sim 5$ gene copies (one bacterium), comparable to the sensitivity of real time PCR. We are optimizing the $16 \mathrm{~S}$ rDNA-nrdB duplex assay in an attempt to develop it into a more sensitive tool than the singleplex assays.

Genomics-informed molecular detection of Xanthomonas vasicola causing bacterial leaf streak of corn in the United States M. J. STULBERG (1), B. Kasiborski (1), D. Studholme (2), G. P. Munkvold (3), C. C. Block (3), S. Arias (3), J. Rascoe (1), M. K. Nakhla (1), (1) USDA-APHIS-PPQ-S\&T-CPHST, Beltsville, MD, USA; (2) University of Exeter, Exeter, UNITED KINGDOM; (3) Iowa State University, Ames, IA, USA

Multiple U.S. states recently reported the presence of an emerging disease, bacterial leaf streak of corn, caused by Xanthomonas vasicola (Xv). Bacterial leaf streak of corn often looks similar to gray leaf spot, which is caused by a fungal pathogen. Disease management, however, differs substantially 
depending on the pathogen, meaning a detection tool that quickly identifies $X v$ could be useful for making management decisions for growers. Four bacterial genomes, including three U.S. isolates from Minnesota, Kansas, and Texas and a historical Xanthomonas campestris pv. zeae (Xcz), were sequenced and used for whole-genome phylogenetic analysis. This analysis placed all four isolates among $X v$ pv. vasculorum strains. $X v$ strains are typically sorghum and sugarcane pathogens with weaker pathogenicity than $X c z$ strains on maize, so we used comparative genomics to identify a genomic region shared among the U.S. and $X c z$ strains that is not found in other $X v$ strains. This region was used to develop TaqMan-based real-time PCR methods and isothermal methods for in-field detection. Additionally, we adapted the propidium monoazide live-dead cell assay into a seed-wash protocol followed by qPCR to detect the presence of live cells on seeds.

Optimizing the molecular tools for Phytophthora cinnamomi detection in environmental samples

S. CRANDALL (1), P. M. Manosalva PhD (2), T. Miles (1), (1) California State University Monterey Bay, Seaside, CA, USA; (2) Department of Plant Pathology and Microbiology, University of California, Riverside, CA, USA

Phytophthora cinnamomi $(P c)$ is a devastating root pathogen on a wide variety of hosts including avocado and blueberry. Approaches based on pathogen isolation, immunological strip tests, conventional polymerase chain reaction (PCR), and real-time PCR (qPCR) have been traditionally used for detecting P. cinnamomi. Recently, new tools based on isothermal technologies including loop mediated isothermal amplification (LAMP) and recombinase polymerase amplification (RPA) have been developed for rapid $P c$ detection. By optimizing the amount of initial plant material (avocado roots) and extraction buffer composition, we successfully optimized the extraction protocol to maximize DNA yields for a successful molecular detection. Furthermore, we compared three molecular techniques targeting two different mitochondrial loci (trnM-trnP-trnM and atp9-nad9). In all cases qPCR was the most sensitive technique detecting down to $100 \mathrm{fg}$ of $P c$ DNA, but LAMP and RPA were able to detect down to 1 pg $P c$ DNA. These differences can be attributed to: i) different enzyme chemistries and ii) the high polysaccharides within avocado samples that could inhibited some of the amplifications. By optimizing these tools, P. cinnamomi detection techniques could be developed for diagnosis and efficient control of Phytophthora root rot disease in the field.

Development of a sensitive real-time PCR detection method for Citrus tatter leaf virus M. KUNTA, J. W. Park, M. Gonzalez, P. Vedasharan, J. V. da Graça, Texas A\&M University Kingsville Citrus Center, Weslaco, TX, USA

Citrus tatter leaf virus (CTLV), taxonomically regarded as a strain of Apple stem grooving virus in the Capilloviridae family, is a graft-transmissible capillovirus that causes the tatter leaf disease of citrus and tissue incompatibility between scion and some trifoliate hybrid rootstocks resulting in serious economic losses to citrus worldwide. Although several CTLV detection protocols have been previously described, there is a need for a highly sensitive high throughput detection assay that can be used in routine diagnostic tests for virus-free budwood certification programs. To develop a new CTLV detection method, we analyzed nucleotide sequence information of six CTLV strains of which full genome sequences are available at NCBI GeneBank by multiple sequence alignment. The sequence analysis revealed that the region near the 3 ' end of the viral genome is more conserved among different CTLV strains than the rest of the viral genome, from which we designed a new set of primers and TaqMan ${ }^{\otimes}$ probe for the detection of CTLV by real time reverse transcription-polymerase chain reaction (real time RT-PCR). The newly designed real time RT-PCR primer set for CTLV detection has $\sim 98.6 \%$ amplification efficiency and is highly specific for CTLV as no amplification was observed in RNA templates from Citrus tristeza virus (CTV), Citrus leprosis virus (CiLV), Citrus psorosis virus (CPsV), Citrus exocortis viroid (CEVd), Hopstunt viroid (HSVd), and Citrus viroid IIa (CVdIIa). This new TaqMan-based method confirmed the presence of CTLV in some field trees and also in previously known CTLV-positive trees maintained in the greenhouse. The new real-time RT-PCR will be a valuable tool for CTLV diagnosis in citrus.

A new diagnostic real-time PCR method for huanglongbing detection in citrus root tissue

J. W. Park (1), W. E. Braswell (2), P. Stansly (3), J. Rascoe (4), E. Louzada (1), G. Mccollum (5), J. V. da Graça (1), M. KUNTA (1), (1) Texas A\&M University Kingsville Citrus Center, Weslaco, TX, USA; (2) USDA APHIS PPQ CPHST, Edinburg, TX, USA; (3) University of Florida/IFAS/SWFREC, Immokalee, FL, USA; (4) USDA-APHIS-PPQ-S\&T-CPHST, Beltsville, MD, USA; (5) USDA, ARS, USHRL, Fort Pierce, FL, USA

Pre-symptomatic detection of Candidatus Liberibacter asiaticus (CLas), the presumed causal bacterium of huanglongbing (HLB), and subsequent removal of the infected trees as early as possible is recommended to slow spread by reducing sources of the disease. However, HLB symptom development is a slow process and takes place unevenly in the aerial parts of an infected tree, making HLB early diagnosis challenging. The current study evaluated the root system as a source material for HLB early detection in the absence of visible symptoms. We optimized the root DNA preparation and developed a new TaqMan real-time PCR method for CLas detection in the root tissue. This method can detect all three species of HLB causing bacteria. The newly developed TaqMan method was evaluated with 76 HLB positive mature trees that were previously determined as CLas positive with HLBaspr real-time PCR tests. All of them were tested positive for CLas, suggesting that the new method for root tissue is comparable to the one developed for leaf tissue. In addition, an experiment was conducted over 12 months assessing young citrus trees with minor or no HLB symptoms. The number of CLas positive trees detected in root tissue drastically increased during the $2^{\text {nd }}$ quarter of the experiment, while no such change was observed with leaf tissue samples. Conventional PCR followed by amplicon sequencing re-confirmed the test results, which indicated that HLB screening of root tissue can be a more reliable alternative to sampling leaf tissue, especially when no visible HLB symptoms are present.

Measurement of complex permittivity of plant cells and detection of cellular state alteration by Waveguide Penetration and Coaxial Probe T. FURUKAWA (1), A. Kazamaki (2), A. Kik (2), Y. Suzuki (2), (1) Dept Biol. Sci., Tokyo metropolitan University, Hachioji-shi, JAPAN; (2) Electr. \& Electron. Eng., Tokyo Metropolitan University, Tokyo, JAPAN

To prevent extinction of a great number of endemic species in isolated island such as the Ogasawara Islands in Japan, their propagation at mainland and transfer to the islands are now being attempted. A possible problem of the system is invasion of diseases which originally do not exist in the islands. Plant diseases are sometimes observed only after they are widely spread in plants. Even if no symptoms are found before transferring, diseases develop by the stress after planting to the original places. Therefore, establishment of non-destructive, fast, and simple disease detecting method is strongly demanded. We tried to develop a diagnosis method of plants by measuring their complex permittivity. To get fundamental information, we used two kinds of cultured cells (rice and tobacco) and tried two methods. The complex permittivity of both cells were successfully measured at the range of 8.2$12.4 \mathrm{GHz}$ and $2.6-3.95 \mathrm{GHz}$ by waveguide penetration method and of $1-50 \mathrm{GHz}$ by coaxial probe method. The values of both cells were almost identical, though the cell shape and the size are rather different each other. Furthermore, we could detect the slight difference of water content, less than $1 \%$, of the cells clearly by coaxial probe method.

Rapid detection of Hop stunt viroid, Potato spindle tuber viroid and Tomato chlorotic dwarf viroid using isothermal AmplifyRP S. ZHANG (1), P. Russell (1), B. Davenport (1), R. Li (1), D. Groth-Helms (1), R. W. Hammond (2), (1) Agdia, Inc., Elkhart, IN, USA; (2) USDA ARS Molecular Plant Pathology Laboratory, Beltsville, MD, USA

Viroids, the smallest known pathogens, are naked, circular, single-stranded RNA molecules that do not encode any protein but are able to replicate in host plants. Since the discovery of the first viroid, Potato spindle tuber viroid (PSTVd) in 1971, several dozen viroids have been reported and most of them are classified into the family of Pospiviroidae within which Hop stunt viroid (HSVd) is a member of the genus Hostuviroid, while PSTVd and Tomato chlorotic dwarf viroid (TCDVd) are members of the genus Pospiviroid. These three viroids each cause a serious disease in numerous economically important crops such as tomato, potato or hop and impose a great threat on crop production. Thus, rapid diagnostic assays are needed for 
these viroids, particularly due to the lack of any conventional antibody-based tests. Based on recombinase polymerase amplification, one of the leading isothermal amplification tools for detection of nucleic acids, Agdia has developed its own isothermal platform, AmplifyRP ${ }^{\circledR}$, for rapid detection of plant pathogens. Currently available at Agdia are three formats of AmplifyRP ${ }^{\circledR}$ : Acceler8 ${ }^{\circledR}$, XRT and XRT ${ }^{+}$. Here we report development and application of AmplifyRP ${ }^{\circledR}$ assays that utilize reverse transcription-recombinase polymerase amplification in one of these different formats for rapid diagnostic detection of HSVd, PSTVd or TCDVd in plants. The assays are specific and as sensitive as PCR. In addition, they are rapid and field deployable.

Use of LAMP for in-field early detection of Raffaelea lauricola, the causal agent of laurel wilt disease

J. N. Workman (1), S. W. Fraedrich (2), C. VILLARI (1), (1) D.B. Warnell School of Forestry and Natural Resources, University of Georgia, Athens, GA, USA; (2) USDA Forest Service, Athens, GA, USA

Laurel wilt is a devastating disease caused by Raffaelea lauricola, an invasive fungus vectored by the redbay ambrosia beetle. The pathogen is decimating redbay and threatening other lauraceous species native to Central and North America, including sassafras and avocado. Early detection and rapid removal of infected plants are crucial steps to slow disease spread, and are critical components of an integrated pest management program. The goal of this project is to provide forest health monitoring agencies with a loop-mediated isothermal amplification (LAMP)-based assay that would allow them to perform early detection of $R$. lauricola in the field on their own, without having to rely on external laboratories. The first step is to develop and validate the LAMP-based assay. Two pathogen-specific primer sets were designed to the $\beta$-tubulin gene and to a taxon-specific simple sequence repeat locus currently used for R. lauricola molecular identification, respectively. Reactions are being optimized for use with crude DNA extracts on portable devices and tested for specificity and sensitivity on pure fungal cultures, symptomatic and asymptomatic infected plant tissues, and insect vectors. The final steps will involve evaluation of the assay under field conditions, and training of forest health monitoring personnel. The implementation of this assay would strongly enhance the effectiveness of early detection, allowing for more successful management of laurel wilt disease.

First detection of Phytophthora species of concern at the USA: The role of the USDA-APHIS-PPQ-CPHST-Beltsville Laboratory Z. G. ABAD, J. C. Bienapfl, L. M. Knight, D. D. Picton, A. Barth, M. K. Nakhla, USDA-APHIS-PPQ-S\&T-CPHST, Beltsville, MD, USA

Phytophthora with 166 species contains plant pathogens of high economic impact particularly in crops, ornamentals, and forest ecosystems. Species identification is many times challenging due to misidentified sequences in public databases including the NCBI. In addition, many of the ex-types lack sequences for multiple genes and rely primarily only on ITS sequences. Recently we have acquired from the "World Phytophthora Collection - CA" a vast collection of specimens of the ex-types and selected specimens for the development of the online "Phytophthora ID", and the implementation of molecular diagnostic and identification tools for species of concern for the USA listed in the Cooperative Agriculture Pest Survey, Plant Epidemiology and Risk Analysis Laboratory, and Prioritized Offshore Pest List. Reference sequences for five nuclear and two mitochondrial genes are used for the "confirmation" of first detections at the US. Based on this method using sequences of the Ex-types, we confirmed identification for suspect samples including $P$. tentaculata (2012), P. hedraiandra (2013), P. parvispora, P. quercina (2016) and P. chrysanthemi (2016). Suspect of $P$. fallax (2013) was confirmed to be $P$. aff. fallax and suspect of $P$. boehmeriae (2016) was confirmed to be $P$. aff. boehmeriae. The laboratory performed additional confirmations for new hosts and locations for $P$. tentaculata after the first determination. Aspects of the robust system for identification will be presented.

Detection of Papaya ringspot virus using an ultra-sensitive single-tube nested PCR

I. HAMIM (1), J. S. Hu (2), (1) University of Hawaii at Manoa, Honolulu, HI, USA; (2) Univ of Hawaii At Manoa, Honolulu, HI, USA

Papaya ringspot virus (PRSV) is the most dominant disease threat to the commercial production of papaya worldwide. Specific ultrasensitive assays are important for the early detection of PRSV infection in the field. In this study, a single tube nested PCR (STNP) was developed for the detection of PRSV. Two nested PCR primer sets (inner and outer) were designed to target the P3 gene of PRSV. The annealing temperatures and concentrations of both primer pairs were optimized to reduce the potential competition between primer sets in PCR. The assay shows much more sensitivity when compared with regular RT-PCR in serial dilutions of cDNAs and RNAs from infected plants. The STNP could detect PRSV targets at the dilutions of sample extract up to 1:81,920, while PRSV targets could only be detected at the dilutions up to 1:10,240 and 1:320 by RT-PCR and ELISA. STNP allowed detection of PRSV infections at 7 days post inoculations from PRSV inoculated plants, whereas regular PCR and ELISA could only detect PRSV two weeks more after inoculations. This new assay could detect virus from healthy-looking plants without cross-contamination. This system could facilitate studies PRSV epidemiology in the field and in quarantine systems of governments by providing early detection of very low infection rates of PRSV in field and imported plant samples.

Exploring the use of NGS technology for citrus HLB diagnosis and microbiome research

Z. Zheng (1), F. Wu (1), M. Xu (1), X. Deng (1), M. J. Stulberg (2), J. Rascoe (2), X. Sun (3), A. Jeyaprakash (3), J. CHEN (4), (1) South China Agricultural University, Guangzhou, CHINA; (2) USDA-APHIS-PPQ-S\&T-CPHST, Beltsville, MD, USA; (3) Florida Dept of Agric \& Consumer Service, Gainesville, FL, USA; (4) USDA-ARS-PWA, Parlier, CA, USA

Citrus Huanglongbing (HLB) is currently threatening citrus production around the world. HLB is most prevalently associated with "Candidatus Liberibacter asiaticus" (CLas), an unculturable alfa-proteobacterium. Accurate diagnosis of HLB exclusively depends on PCR detection of CLas, which is determined by the presence of a targeted DNA band (standard PCR) or/and a Ct value below the defined threshold (TaqMan real-time PCR). In the case of a weak DNA band or high Ct value, it is difficult to distinguish low CLas titer from non-specific DNA amplification. In this study, next generation sequencing (NGS) technology was used in HLB diagnoses and microbiome research. Fourteen NGS (10 MiSeq and 4 HiSeq) data sets from whole genome sequencing of CLas samples, which varied in Ct values measured by the primer set HLBas/HLBr, were subject to sequence read count assisted by BLASTn search. The results showed that: 1) Increase of HLBas reads was correlated to the decrease of Ct values; 2) At high Ct values (e.g. $\mathrm{Ct}>35$ ), a sample could have either low titer of CLas, or no CLas; 3) NGS detection sensitivity was related to sequencing throughput (HiSeq of 40M reads $>$ MiSeq 20M reads); 4) Detection on nrdB gene (5 copies) was more sensitive than that on 16S rRNA gene (3 copies); 5) Target (e.g. 16S rRNA gene) sequencing was more cost-effective than whole genome sequencing for CLas detection; and 6) Other non-CLas bacteria, fungi and DNA viruses in HLB samples were also detected.

Molecular detection and quantification of root-lesion nematode, Pratylenchus penetrans from soil using real-time PCR R. Baidoo (1), G. YAN (2), (1) North Dakota State University, Department of Plant Pathology, Fargo, ND, USA; (2) Department of Plant Pathology, North Dakota State University, Fargo, ND, USA

The root-lesion nematode, Pratylenchus penetrans, is a major pathogen of potato world-wide. Yield losses may be exacerbated by interaction with other soil-borne pathogens. Distinction between P. penetrans and other closely related Pratylenchus spp. based on morphology is a tedious task. A SYBR Green I-based real-time PCR assay was developed to detect, discriminate and quantify $P$. penetrans directly from field soil. $P$. penetrans-specific realtime PCR primers were designed from the D2-D3 region of the 28S rRNA. The specificity of the primers was evaluated using eight isolates of $P$. penetrans and 31 isolates of closely related Pratylenchus spp. and other plant-parasitic nematode species. A standard curve relating threshold cycles and $\log$ values of nematode numbers was generated from artificially infested soils and used to determine nematode numbers from naturally infested field soils. The $P$. penetrans numbers in both artificially and naturally infested field soils estimated by conventional methods strongly correlated with the realtime PCR estimates. Grinding the field soil prior to DNA extraction improved P. penetrans detection from soil. The real-time PCR assay will be useful 
for detection, discrimination and quantification of $P$. penetrans directly from field soil with mixed populations of Pratylenchus spp. without the need for conventional nematode extraction. The assay requires no expertise in nematode taxonomy and morphology, and may serve as a useful diagnostic tool in research, diagnostic labs and extension services for pest management.

Development of a CANARY (Cellular Analysis and Notification of Antigen Risk and Yield) assay for detection of Ralstonia solanacearum R3B2 W. CAI (1), F. Nargi (2), J. Elphinstone (3), S. Costanzo (1), J. Rascoe (1), Z. Liu (4), M. K. Nakhla (1), (1) USDA-APHIS-PPQ-S\&T-CPHST, Beltsville, MD, USA; (2) MIT Lincoln Laboratory, Lexington, MA, USA; (3) Central Science Laboratory, York, UNITED KINGDOM; (4) USDA APHIS PPQ, Riverdale, MD, USA

CANARY is a fast, specific and sensitive pathogen biosensor developed by scientists at MIT Lincoln Laboratory. Ralstonia solanacearum race3, biovar2 (R3B2), is a plant pathogenic bacterium and a select agent with significant quarantine implications. The strain is considered a serious threat to the US potato industry. The R3B2 strain also causes southern wilt of geranium and the US imports millions of geranium cuttings each year. Therefore, rapid detection of the pathogen is essential to prevent the introduction of the disease into the US. During validation, the original CANARY assay developed as a rapid screening tool for $R$. solanacearum, was found to have a higher detection sensitivity for $R$. solanacearum R1B1 (strain already established in the US), than R3B2. A hybridoma from the Food and Environmental Research Agency (FERA), UK was used in this study, which produces a monoclonal antibody $(\mathrm{mAb})$ more specifically targeting the R3B2 strain. We first developed CANARY biosensor clones based on the FERA mAb. We then tested these biosensors for assay feasibility, specificity and sensitivity against target bacteria. Preliminary results showed that these new FERA antibody-based CANARY biosensors are more specific and sensitive to the R3B2 strain than R1B1 strain. Two of the clones were selected for insertion of a longevity gene to extend shelf-life and will be used for final stage assay development for detection of $R$. solanacearum R3B2.

Evaluation of immunoreagents for development of a diagnostic assay specific for Rathayibacter toxicus

D. LUSTER (1), M. B. McMahon (1), A. Sechler (1), E. E. Rogers (1), W. Schneider (1), B. Schroeder (2), T. D. Murray (3), (1) USDA ARS FDWSRU, Fort Detrick, MD, USA; (2) University of Idaho, Moscow, ID, USA; (3) Washington State University, Pullman, WA, USA

Rathayibacter toxicus is gram-positive bacterium causing gummosis of forage grasses, and produces a corynetoxin responsible for recurring livestock poisonings in Australia. Because R. toxicus is an APHIS-listed Plant Pathogen Select Agent, threatening grass seed and livestock production areas in the Pacific Northwest and elsewhere, we are developing diagnostic assays to prepare for the possible introduction of the pathogen into the U.S. Using proteomic, transcriptomic and comparative genomics data, we identified proteins expressed in R. toxicus but not in other Rathayibacter spp. Potential candidate antigens were selected based upon predicted extracellular localization, antigenicity, and solubility. Recombinant proteins corresponding to extracellular domains were used as immunogens to raise polyclonal and monoclonal antibodies against the two top candidate protein targets. Antibodies were evaluated for sensitivity and specificity in western blot and ELISA format immunoassays against a panel of Rathayibacter spp. and near neighbor genera. One polyclonal and multiple monoclonal antibodies exhibited high sensitivity and specificity for $R$. toxicus in assays with cultured bacterial cells The highest-performing immunoreagents were identified for further testing with field samples collected in Australia and for eventual development of an immunoassay for bacterial detection in grass samples.

Rollout of a Simplified DNA Isolation Method and LAMP for On-Site Detection of Citrus Black Spot G. MARRERO (1), K. A. Zeller (1), K. Levin (1), T. D. Riley (2), H. D. Gomez (3), Z. G. Abad (1), (1) USDA-APHIS-PPQ-S\&T-CPHST, Beltsville, MD, USA; (2) USDA APHIS PPQ, Orlando, FL, USA; (3) USDA APHIS PPQ, Davie, FL, USA

Citrus Black Spot (CBS) disease, caused by the fungus Guignardia citricarpa, was discovered in South Florida during 2010. The disease has resulted in restrictions on the movement and transport of symptomatic fruit, and disease presence can prohibit shipping and sales to EU markets. Currently, if CBS symptoms are discovered in a packing house on even a single fruit, an entire lot of perishable fruit can be put on hold. This can cost the growers and packing house valuable time and limited storage space while waiting for a diagnostic decision to be reached. Methods for the confirmation of the CBS pathogen require off-site sampling, a time-consuming DNA isolation procedure, and conventional and/or real-time PCR to provide a definitive answer. Since the diagnostic process can take 1-2 days to complete the objective of this study was to establish a simple, accurate and sensitive diagnostic assay that can detect $G$. citricarpa from symptomatic fruit. We have identified and verified a simplified DNA isolation method, in conjunction with Loopmediated Isothermal Amplification (LAMP) conducted on portable equipment that can provide diagnostic resolution at a packing house in one hour or less for CBS suspect samples. Untreated fruit, fruit treated with wax and morpholine, and fruit treated with wax only were tested to determine the efficacy of LAMP on various stages of fruit in a packing house. Pilot testing of the procedure was conducted during February 2017.

Development and evaluation of molecular methods for species-specific detection of Phytophthora quercina

J. C. BIENAPFL (1), K. Jennings (1), L. Schena (2), J. Juzwik (3), Z. G. Abad (1), (1) USDA-APHIS-PPQ-S\&T-CPHST, Beltsville, MD, USA; (2) Universita' Mediterranea Di Reggio Calabria, Reggio Calabria, ITALY; (3) USDA Forest Service, St. Paul, MN, USA

Phytophthora quercina is considered a priority pest in the commodity-based survey listing for the USDA Cooperative Agriculture Pest Survey. It is also ranked \#1 out of 29 Phytophthora species exotic to the USA by PPQ as a species of concern due to the potential damage that may be caused if introduced into the country. P. quercina has been reported as a prevalent root rot pathogen of multiple oak species in several European countries. In 2005 and 2007 , isolates from declining oak trees in Minnesota and Missouri were identified as $P$. quercina, respectively. However, as part of efforts to enhance systems in pest detection of Phytophthora species of concern, these isolates were re-examined and compared with the ex-type of $P$. quercina, as well as other Phytophthora species. Through extensive analysis using eight target genes these isolates were determined to be $P$. aff. quercina. In addition, the availability of these isolates aided in the development of screening and confirmatory diagnostic assays for detection of $P$. quercina. Furthermore, the sequence information from these isolates and the development of diagnostic assays for detection of $P$. quercina allowed for the first confirmation of this species in the USA during 2016. Information regarding the importance of using the ex-holotypes, as well as sequence analysis, and the verification process for the diagnostic assays developed for detection of $P$. quercina will be presented.

Comparison of next generation sequencing platforms and library preparation for the analysis of metagenomic diversity D. Sherman (1), A. L. Stone (1), J. King (2), A. Bronzato (2), W. SCHNEIDER (1), (1) USDA ARS FDWSRU, Fort Detrick, MD, USA; (2) Mississippi State University, Mississippi State, MS, USA

Next-generation sequencing (NGS) has become a common method for metagenomic studies with potential for use in pathogen detection. Sample analysis may be affected by the library preparation and/or the sequencing platform used. Library and platform comparisons were performed on metagenomes generated from plant and insect samples infected with two RNA viruses, polyadenylated (Plum Pox Virus), non-polyadenylated (Soybean Dwarf Virus) or bacterial pathogen Candidatus Liberibacter asiaticus (Huanglongbing). We evaluated multiple library protocols on Illumina MiSeq, Roche 454Jr and Nanopore MinIon. Alignments were completed using BLASTn or GraphMap, followed by analysis with MEGAN6 for taxonomic content and Geneious R9 16S Biodiversity for rRNA diversity of prokaryotes. Read lengths range from 50 to thousands of bases, while number of reads per run range from thousands to millions, depending on sequencing platform used. The ability to detect low titer organisms was correlated to the number of total reads. Read length had minimal impact provided that reads were greater than 50 bases. The advantage of using RNA libraries is the ability to detect RNA based viruses. These results may be a useful guide for choosing the appropriate library and platform protocols for optimal metagenomic analysis. 
A Novel Molecular Toolkit for Rapid Detection of the Pathogen and Primary Vector of Thousand Cankers Disease

E. Oren (1), W. Klingeman (1), R. O. Gazis (1), J. Moulton (1), P. Lambdin (1), M. Coggeshall (2), J. Hulcr (3), S. J. Seybold (4), D. HADZIABDIC (1), (1) University of Tennessee, Knoxville, TN, USA; (2) USDA Forest Service, Northern Research Station, West Lafayette, IN, USA; (3) University of Florida, Gainesville, FL, USA; (4) USDA Forest Service, Pacific Southwest Research Station, Davis, CA, USA

Thousand Cankers Disease (TCD) involves a tree host (Juglans or Pterocarya spp.), the fungal pathogen Geosmithia morbida, and the primary insect vector, Pityophthorus juglandis. TCD was described originally from cases of dying J. nigra in the western United States (USA), but was subsequently detected in the eastern USA and northern Italy. Often difficult to diagnose, symptoms of TCD can be confused with those caused by other biotic and abiotic agents. Using species-specific microsatellite loci, we developed a molecular protocol to rapidly detect G. morbida and P. juglandis. We demonstrated the utility of the method for delineating TCD quarantine zones by testing whether geographical incidence of disease symptoms correlated with the presence of G. morbida and/or P. juglandis. A total of 1600 drill cores were taken from trees surveyed in three regions ( $n=40$ trees for each location): California-J. hindsii (heavy disease symptoms), Tennessee-J. nigra (mild disease symptoms) and Missouri-J. nigra (no symptoms). The latter is outside of the known distribution of TCD. California samples had the highest incidence of the TCD organisms $(85 \%, 34 / 40)$ when compared to Tennessee $(37.5 \%, 15 / 40)$. Neither organism was detected in samples from Missouri. The molecular protocol developed here has a high degree of sensitivity and specificity, and it significantly reduces sample-processing time, making the protocol a powerful tool for TCD detection.

Development of a recombinase-polymerase amplification assay for Clavibacter michiganensis subsp. sepedonicus

R. Li (1), P. Russell (1), D. GROTH-HELMS (1), S. Zhang (1), B. Davenport (1), K. Schuetz (2), C. A. Ishimaru (3), (1) Agdia, Inc., Elkhart, IN, USA; (2) Agdia, Inc, Elkhart, IN, USA; (3) University of Minnesota, St. Paul, MN, USA

Clavibacter michiganensis is a Gram-positive plant pathogenic species. Nine subspecies are now recognized based on host specificity and other bacterial characteristics. C. michiganensis subsp. sepedonicus ( $\mathrm{Cms}$ ) is highly infectious and lives in the vascular system of potato (Solanum tuberosum) causing ring rot disease of tubers. The disease is controlled primarily through a "zero tolerance" policy for Cms in production of certified potato seed. The detection and quantification of $\mathrm{Cms}$ from infected plants have previously been achieved using indirect fluorescent antibody staining (IFAS), ELISA, PCR, and real-time PCR. Agdia has developed a fast and user-friendly AmplifyRP XRT+ assay for testing the presence of $\mathrm{Cms}$ in potato tuber and stem through recombinase-polymerase amplification technology. The assay performs as both a real-time and an endpoint test using a single reaction tube incubated at $39^{\circ} \mathrm{C}$ for 20 minutes. Detection was confirmed in over $30 \mathrm{Cms}$ cultures. No cross-reaction was observed to 24 non- $\mathrm{Cms}$ cultures. Testing DNA purified from Cms cultures showed that this XRT+ assay had equal sensitivity to PCR and real-time PCR. The limit of detection was determined to be 10-20 cells per reaction by spiking cells into potato tuber core-soaking water. Cms cells in potato tuber core-soaking water can be tested directly without the need of DNA purification. This assay adds a new tool for users to monitor Cms movement between regions or countries.

A reverse transcription-loop mediated isothermal amplification assay for the detection of pospiviroids in tomato seeds N. A. GONZALEZ, G. P. Munkvold, Iowa State University, Ames, IA, USA

Pospiviroids such as Potato spindle tuber viroid and Tomato apical stunt viroid are considered important seedborne pathogens in tomato because of their devastating effects on fruit production. Most detection assays are based on RT-PCR, which is not suitable for on-site applications outside of a laboratory. Testing for pospiviroids at ports of entry or production facilities requires an effective detection assay that is faster and easier to perform while maintaining its specificity and reliability. We have developed a reverse transcription loop-mediated isothermal amplification (RT-LAMP) assay to detect pospiviroids based on a consensus sequence obtained from an alignment of more than 300 published sequences of the most common pospiviroids found in tomato. RT-LAMP primers were designed using the Primer Explorer V5 software. A total of 630 primer sets were generated, of which the ten most stable sets were selected for further testing. RNA was extracted from contaminated seeds and tissue with the EZNA RNA extraction kit. The RT-LAMP reactions were performed in single tubes in $27 \mu \mathrm{l}$ total reaction volume with $5 \mathrm{U}$ of AMV reverse transcriptase per reaction and incubated at $66^{\circ} \mathrm{C}$ for 40 minutes. The best RT-LAMP primer set was chosen based on its ability to produce a repeatable banding pattern in agarose gels with all samples tested. This RT-LAMP assay provides a new, fast and reliable method to assess tomato seeds for the presence of economically important pospiviroids.

Development of Multiplex-PCR for simultaneous detection of phytoplasmas causing Lethal Yellowing and Date Palm Lethal Decline in palms A. RAVINDRAN, J. Ueckert, K. Ong, Texas A\&M AgriLife Extension Service, College Station, TX, USA

Lethal Yellowing (LY) and Date Palm Lethal Decline (DPLD) are two important diseases of palms caused by phytoplasmas which are identified as Candidatus Phytoplasma palmae 16SrIV-A and 16SrIV-D, respectively. In Texas, there are plant quarantine regulations on these two palm diseases. To date, DPLD has been found sporadically in parts of South Texas and the greater Houston area, but LY has never been detected in Texas. Nested PCR followed by Restriction Fragment Length Polymorphism (RFLP) analysis is currently used for the detection and differentiation of the DPLD and LY strains. In order to improve sample diagnostic turnaround time, we developed a multiplex-PCR with simultaneous detection of LY and DPLD phytoplasma in palms. The strain-specific primers were designed based on groEL gene that target to identify the variable sequence to differentiate the phytoplasmas strains of lethal yellowing (16SrIV-A) and date palm lethal decline (16SrIV-D) in palms. This multiplex-PCR was able to detect LY and DPLD strains in infected palm samples based on the different amplification size of LY (310bp) and DPLD (489bp). The two target regions were amplified simultaneously in a multiplex reaction, suggesting that this method would allow us to differentiate at the strain level and also determine the presence and absence of one or both of the phytoplasmas of lethal yellowing (16SrIV-A) and date palm lethal decline (16SrIV-D) in palms. Preliminary test results indicate that this multiplex-PCR system is sensitive, reliable, and cost-effective method for rapid strain identification in infected palms samples, especially co-infected plants.

Coupling Spore Traps and Quantitative PCR Assays for Detection of Cercospora sojina, the Causal Agent of Soybean Frogeye Leaf Spot B. LIN (1), H. M. Kelly (1), H. Yu (2), A. Mengistu (3), (1) University of Tennessee, Jackson, TN, USA; (2) Tusculum College, Greeneville, TN, USA; (3) USDA ARS, Jackson, TN, USA

Frogeye leaf spot (FLS), caused by Cercospora sojina Hara, is a common disease of soybean reported to cause yield losses from $10-60 \%$. We present a novel trapping approach using petroleum jelly-coated slides placed at a $45^{\circ}$ angle within a passive, wind-vane spore trap used in combination with realtime PCR to detect the presence of wind-blown inoculum. In 2016, multiple spore traps were deployed at 3 areas: 1) at Jackson, TN sampled weekly and twice a week; 2) within non-treated tilled and no-till research plots sampled once weekly at Milan, TN; and 3) on the edge of large soybean plots in 4 different counties in TN sampled once weekly. DNA was extracted from petroleum jelly-coated slides and qPCR was conducted to estimate the number of spores based on a standard curve developed from known C. sojina DNA. FLS severity was recorded at each reproductive growth stage using percent leaf area affected at areas 2 and 3. Comparing data from spore traps at area 1, there was no weekly significant difference between sampling twice or once per week. There was no significant difference between spore amounts from non-treated tilled and no-till plots. Spore trap data collected from different counties varied in trends and resulted in several major peaks of inoculum during the season most likely influenced by variety susceptibility to FLS, field history, and weather conditions. These results indicate this trapping approach has the potential to benefit FLS management decisions.

Detection, quantification, and seasonal movement of Xylella fastidiosa in grafted and own-root Meadowlark blueberries D. BAYO (1), R. Darnell (2), J. Williamson (2), P. Harmon (1), (1) Department of Plant Pathology, University of Florida, Gainesville, FL, USA; (2) Horticultural Sciences Department, University of Florida, Gainesville, FL, USA 
Southern highbush blueberries (SHB) (interspecific hybrid of Vaccinium corymbosum) are a lucrative crop in Florida, where growers receive the highest prices per pound in the nation due to advantageous harvest times. Blueberry leaf scorch (BLS), a disease caused by the bacterium Xylella fastidiosa, reduces yields and kills several commercial cultivars including the University of Florida (UF) variety 'Meadowlark'. Currently, rootstocks are not used in commercial blueberry production; however, the native sparkleberry (Vaccinium arboreum) is being evaluated in SHB production for improved root function and structure in ongoing UF research. Grafted 'Meadowlark' plants were observed to have less severe disease symptoms than own-rooted plants. In an initial sampling, the BLS pathogen was detected in both plant types with a diagnostic qPCR assay. The objective of this research was to quantify differences in disease severity and determine the presence and quantity of $X$. fastidiosa in stems, crowns, and roots of own-root and grafted 'Meadowlark' plants. No grafted plants were symptomatic at the time of the January 2017 sampling, and samples all tested negative. An average of $44 \%$ of crown samples from symptomatic own-root plants were positive, compared to only $11 \%$ of stem samples. Inconsistent positive results likely reflect low bacterial titers associated with the winter period. When sampling in the winter, these results suggest crown material is the most reliable.

\section{Detection of Spiroplasma citri by droplet digital PCR}

Y. Maheshwari (1), V. Selvaraj (2), S. Hajeri (3), R. K. YOKOMI (4), (1) USDA, Agricultural Research Service, Parlier, CA, USA; (2) ARS-USDA, parlier, CA, USA; (3) Citrus Pest Detection Program (CCTEA), Tulare, CA, USA; (4) USDA ARS PWA, Parlier, CA, USA

Spiroplasmas are motile, helical bacteria belonging to the Class Mollicutes, a group of prokaryotics with no cell wall and phylogenetically related to Gram-positive bacteria. Spiroplasma citri is the first-cultured spiroplasma and causal agent of citrus stubborn disease (CSD). Detection of CSD is difficult due to low and erratic titer and distribution of the pathogen in infected tissues and isolation by culturing is technically demanding and time consuming. This study evaluated the applicability of droplet digital PCR (ddPCR) as an improved new tool with higher accuracy and precision for detection of $S$. citri at low titer and, presumably, at an early stage of infection using two sets of primers SP1 and ORF1 for spiralin (housekeeping) and prophage (multi-copy) genes, respectively. Standard curve analyses on tenfold dilution series of $S$. citri cells showed that ddPCR assay had good linearity and PCR efficiency. The sensitivity of ddPCR assay for SP1 and ORF1 plasmids were 1 copy/20 $\mu 1$ reaction and 3.4 copies/20 $\mu 1$, respectively. The sensitivity of ddPCR assay with $S$. citri DNA was $0.00001 \mathrm{ng}$ using SP1 and ORF1. Leaves and fruit samples were collected from different quadrant branches from fifty trees in a citrus orchard near Ducor, Tulare County, CA. The ddPCR assay showed that $S$. citri titer was higher in fruit columella compared to leaf petioles. The ddPCR assay was $100 \%$ accurate in detecting $S$. citri-infected versus -negative trees and exhibited reproducible quantitation of the pathogen without need of an external curve regardless of low target concentration in field samples.

\section{Dickeya Black Leg - A New Challenge for Florida's Potato Farmers and a New Pathogen Detection Task for University of Florida Plant Diagnostic Center \\ S. BEC (1,2), C. Lapaire Harmon (3), (1) UF-Plant Diagnostic Center, Gainesville, FL, USA; (2) University of Florida, Plant Diagnostic Center,} Gainesville, FL, USA; (3) Univ of Florida PDC, Gainesville, FL, USA

The value of Florida's potato production in 2016 was estimated at $\$ 86,650,000$. A majority of Florida's potato production occurs in the northeastern part of the state, in the tricounty area (St. Johns, Flagler and Putnam counties). Black leg and bacterial soft rot are among the most important diseases affecting seed quality and potato yields in Florida. Pectobacterium carotovorum subsp. carotovorum and Dickeya dianthicola have been the main causal agents of black leg and bacterial soft rot in the northeast region of the United States. Disease detection is critical for potato seed management as well as determining offseason management strategies for field infections. In order to provide rapid and accurate diagnostics and pathogen species identification to the Florida potato farmers, we have adapted the USDA protocols for Dickeya black leg diagnostics and molecular assays for studying the bacterial soft rot pathogen population in Florida's potato growing area. The University of Florida Plant Diagnostic Center now offers Dickeya dianthicola detection in potato tubers, plant tissue, and bacterial culture identification using a targeted gene sequencing approach.

Utilizing mitochondrial markers and T-RFLP data to identify Phytophthora species in mixed environmental samples

T. MILES (1), K. P. Coats (2), G. A. Chastagner (2), F. N. Martin (3), (1) California State University Monterey Bay, Seaside, CA, USA; (2) Washington State University, Puyallup, WA, USA; (3) USDA ARS, Salinas, CA, USA

Multiple Phytophthora species can often be present in environmental samples such as plant tissue, soil and streams. When using molecular markers to detect Phytophthora, a genus specific marker would be helpful to identify species in unknown samples. However, when using these techniques, multiple Phytophthora spp. can be amplified and conventional amplicon Sanger-based sequencing can fail or multiple peaks can be observed making it difficult to interpret the results. For this project we chose to adapt a previously developed mitochondrial marker (atp9-nad9) in conjunction with a terminal restriction fragment length polymorphism (T-RFLP) system. This technique terminally labels the forward and reverse primers with different fluorescent dyes and the amplicon is digested with two restriction endonucleases (AseI and DraI). In silico predictions were made for both of these restriction enzymes from 145 Phytophthora taxa and an interactive Java based program was developed that allows users to sort fragments in order to identify Phytophthora species in unknown samples. These predictions were validated using purified DNA samples from a diverse group of Phytophthora spp. and compared with predicted fragment sizes. We are currently in the process of validating these techniques with environmental samples and comparing these results with purified DNA and in silico predictions. If effective, this technique has the potential to lower the cost of identifying Phytophthora spp. in mixed environmental samples.

EDNA-Rose a novel approach for detecting rose viruses combining next generation sequencing and bioinformatics

L. PENA ZUNIGA (1), A. Espindola (1), P. Klein (2), T. Debener (3), J. Rees (4), D. Byrne (2), K. F. Cardwell (1), F. Ochoa Corona (1), (1) Oklahoma State University, Stillwater, OK, USA; (2) Texas A\&M University, College Station, TX, USA; (3) Leibniz Universität Hannover. Institute of plant genetics, Hannover, GERMANY; (4) Agricultural Research Council-Biotechnology Platform, Pretoria, SOUTH AFRICA

Garden rose production is threatened by viral infections in the US. Twenty-four viruses have been reported to infect rose worldwide and many of these are reported in the U.S. Early viral infections in rose are difficult to diagnose until symptoms develop. Currently single-virus serological and molecular methods are used for diagnosis of most of these pathogens. Next-generation sequencing (NGS) can be used as a diagnostic tool because unique DNA signatures of plant viruses can be detected in the sequences of rose tissue samples. Electronic probe Diagnostic Nucleic acid Analysis (EDNA) was used to detect rose viruses in simulated metagenomics runs (mock sequencing databases, MSDs) containing reference positive controls and the host genome. MSDs were generated using MetaSim and configurations that included Illumina average read length and error rates. Electronic probes (e-probes) with 20-40 nucleotides length were designed comparing each target viral genome versus all potential co-infecting viral genomes and rose metagenomic sequences. To avoid false positives, e-probes were distinguished and specified by BLAST alignments of sequences from published public databases (nt $\mathrm{NCBI}$ ) to eliminate homology. Positive MSDs containing only the intended target viral genome were considered positive controls. MSDs mimicking single and multiple infections were also generated. EDNA is a relatively rapid diagnostic method when next generation sequencing is available and affordable.

Detection and Differentiation of Arthropod-Transmitted Viruses of Cereal Crops Using Multiplex PCR and High Resolution Melting P. RYDZAK (1), F. Ochoa Corona (1), A. E. Whitfield (2), A. C. Wayadande (1), (1) Oklahoma State University, Stillwater, OK, USA; (2) Kansas State University, Manhattan, KS, USA

The high plains region of the United States is comprised of approximately 45 million hectares of grasslands where several economically important cereal crops are grown. Cereal-infecting pathogens vary in incidence from year to year and are often difficult to detect in large acreages. Nucleic acid based 
diagnostic tools are among the most sensitive and flexible available for the detection of plant pathogens. To facilitate detection of multiple RNA and DNA plant viruses in wheat, barley, and maize, we developed a multiplex PCR platform assay that can be used in combination with high resolution melting (HRM) to detect and differentiate between five arthropod transmitted plant viruses; Wheat streak mosaic virus (WSMV), Maize mosaic virus (MMV), Maize fine streak virus (MFSV), Barley yellow dwarf virus (BYDV), Maize streak virus (MSV). We report a successful multiplex PCR detection of WSMV, MMV, and BYDV, as well as HRM differentiation of two strains of BYDV, BYDV-PAS and BYDV-PAV. The goal of this project will be to successfully detect additional cereal-infecting viruses, MSV and MFSV in multiplex PCR, as well as differentiate two more strains of BYDV, BYDV-SGV and BYDV-MAV, using HRM in cereal crops and insects.

\section{Introducing PrimerCHECKER, a PCR primer performance graphic display generator}

K. V. Salinas Villacís (1), H. Espitia-Navarro (2), J. Garcia-Suarez (3), F. OCHOA CORONA (4), (1) Freelance Consultant, Quito, ECUADOR; (2) Georgia Institute of Technology, Atlanta, GA, USA; (3) The Army University - ESPE, Sangolquí, ECUADOR; (4) Oklahoma State University, Stillwater, OK, USA

PrimerCHECKER is a bioinformatic tool developed to provide visual post primer design assessment of the thermodynamic values of primers. The application allows a comprehensive visual confirmation of predictable primer performance in PCR runs. Even though primer thermodynamics can be obtained from literature, or extracted from applications outputs, for example from web interface applications like Primer-BLAST, the assembly of data and further analysis is still needed. Once these values are submitted, PrimerCHECKER graphically displays the melting temperature (Tm) difference between two reacting primers, the percent of Guanine-Cytosine (GC \%), and the Delta G ( $\triangle \mathrm{G})$. Additional graphic output includes Primer3's ANY score for self-complementarity, as well as Primer3's 3' score for self-complementarity of the 3' termini of the primers of interest. Stringent thresholds are set seeking optimal performance. We are presenting plots of optimal and suboptimal primers tested in silico by PrimerCHECKER and the in vitro validation of sensitivity. The example primer sets illustrate amplification of targeted plant viruses commonly infecting ornamental crops. PrimerCHECKER facilitates decision making through visual aids that allow the assay designer to visualize strengths and weaknesses of oligos in real time as a means for improving reaction efficiencies during assay development.

\section{Two Genomospecies in the Culture Collection Strains of Pseudomonas syringae pv. atrofaciens} D. SHIN (1), G. I. Heo (2), H. I. Lee (3), J. S. Cha (4), (1) Chungbuk National Univ, cheungju-si, Korea, Republic of (South); (2) Chungbuk National Univ, Cheongjoo-si, KOREA; (3) Animal and Plant Quarantine Agency, Gimcheon, KOREA; (4) Chungbuk National Univ, Cheongju Chungbuk, KOREA

'Pseudomonas syringae complex' is a heterogeneous and contains more than 60 pathovars. A great deal of effort has been put into study for better classification of this group of bacteria. From DNA relatedness analysis, 48 pathovars were allocated into 9 genomospecies. In addition, MLST has proved its usefulness for the rapid genomospecies identification of a new isolate. In order to develop P. syringae pv. atrofaciens (Psa) - specific DNA marker, genetic diversity of the 11 strains of Psa at ICMP and LMG were analyzed with MLST and rep-PCR. Nucleotide sequences of the 4 loci (gapA, gltA, gyrB, rpoD) of the Psa strains were determined by PCR amplification and sequencing. The sequences for 38 stains ( 32 pathovars) of $P$. syringae were obtained from the PAMDB (Plant Associated and Environmental Microbe Database). MLST indicated that 9 Psa strains were grouped into genomospecies 1 clade, while 2 Psa strains were grouped into genomospecies 4 clade. Amplification band patterns by the rep-PCR also yielded the same grouping pattern: the 9 Psa strains formed a clade with genomospecies 1 strains and the 2 Psa strains formed a clade closest to genomospecies 4 strains. These results suggest that either the 2 Psa strains grouped into genomospecies 4 may have been mis-identified in the pathovar or Psa contains two different genomospecies. In the case where the latter is true, phenotypic identification (pathovar assignment) has not coincided with genotypic grouping in the genomospecies level of the Pseudomonas syringae pv. atrofaciens.

\section{Development of a sequence-specific PCR assay for burrowing nematode (Radopholus similis) detection}

C. S. CHANG (1), J. I. Yang (2), (1) Department of Plant Pathology and Microbiology, National Taiwan University, Taipei, TAIWAN; (2) National Taiwan University, Taipei, TAIWAN

Burrowing nematode is a damaging plant parasitic pathogen of over 350 hosts in subtropical and tropical regions. The purpose of this study is to construct a species-specific PCR detection assay targeting the highly species-conserved region of mitocondria DNA (mtDNA) of burrowing nematode, Radopholus similis. A sequence-specific primer pair was designed to target $R$. similis mtDNA for a 224bp PCR product amplification. The specification of the assay was examined against other common plant parasitic nematodes including Bursaphelenchus xylophilus, Aphelenchoides besseyi, Meloidogyne incognita, Ditylenchus destructor, Tylenchorhynchus sp., Pratylenchus sp. and Rhadinaphelenchus cocophilus. No amplification was detected on nontargeted samples. The detection limits of the PCR assay was tested with series of different concentrations of cloned target gene DNA plasmids. As low as ten copies of cloned plasmid DNA could be successfully detected with this assay. In addition, the PCR assay was examined with environmental samples that contain mixed DNA of the target nematode and the host plants Anubias bateri and Anthurium andraeanum. The detection was successful and no false positive was shown in the samples containing only plant DNA. The assay provides a specific and sensitive detection option for burrowing nematode detection in environmental samples that contain host plant tissues.

\section{Validation of viability/enrichment (ve) PCR-based detection of viable Acidovorax citrulli in melon and watermelon seed samples} R. WILLMANN, A. Beerepoot, B. P. Woudt, Syngenta Seeds, Enkhuizen, NETHERLANDS

Viability/enrichment (ve) PCR allows the quick and exclusively laboratory-based detection on seeds of viable Acidovorax citrulli (Willmann et al. 2015), the causative agent of bacterial fruit blotch (Schaad et al. 1978). This provides a resource-efficient alternative to sweatbox grow-outs, being the industry standard for detecting viable $A$. citrulli (NSHS 2014). vePCR exploits cellular integrity and propagation of viable $A$. citrulli using Taqman real-time PCR as read-out. Here we present performance characteristics of vePCR. Sensitivity was determined to be $<10 \mathrm{CFU}$ of viable $A$. citrulli per ml seed extract with all three $A$. citrulli lineages behaving equally. Single naturally infected seeds were detected in subsamples of 5000 seeds. Possible competition of other microbes with $A$. citrulli by was studied by adding microflora harvested from seed wash suspensions to sample extracts. Excess microflora did not interfere with $A$. citrulli detection. During extraction an early spike of the related Acidovorax cattleya is added as process control. Its detection verifies suitable conditions which was confirmed in recently produced seed lots. Naturally infected commercial seed lots containing different loads of $A$. citrulli were tested positive whilst seed lots harboring dead A. citrulli did not yield false positives. vePCR detecting $A$. citrulli is highly sensitive, specific, robust and comparable to grow-outs and thus fit for purpose as an alternative for conventional testing.

\section{Detection and genome characterization of a mixed infection of Crinum mosaic, Nerine latent and Nerine yellow stripe viruses in ornamental} Crinum

R. L. JORDAN (1), M. Wingert (2), C. Louden (2), M. A. Guaragna (1), (1) USDA-ARS, USNA, Floral \& Nursery Plants Research, Beltsville, MD, USA; (2) Biotechnology Academy, Applications and Research Lab (Howard County), Ellicott City, MD, USA

Ornamental flower bulbs (including true bulbs, bulbils, corms, tubers and rhizomes) are increasingly important floriculture crops. Crinum is a large genus of herbaceous perennial flowering bulbs in the family Amaryllidaceae and most species have large, showy, fragrant flowers. Only a few viruses have been reported to infect cultivated Crinum. These include three potyviruses: Nerine yellow stripe virus (NeYSV; India), Hippeastrum mosaic virus (HiMV; UK), and Crinum mosaic virus (CriMV; Australia, FL and HI) and a tospovirus. Total RNA from leaves of individual Crinum plants growing at the US National Arboretum exhibiting mosaic symptoms were purified and cDNA libraries prepared for paired-end Illumina MiSeq next-generation 
sequencing. De novo assembly of contigs (from 8.67M 250bp reads) and their analysis was done using Geneious Pro. Contigs were subjected to BLAST analysis against GenBank databases. Complete or near-complete genome sequences were obtained for the two potyviruses CriMV and NeYSV and the carlavirus Nerine latent virus (NeLV) from a single plant. This work extends our previous results for CriMV [KX911718]. The NeLV has 78-96\% NT and $90-99 \%$ AA coat protein identity with other NeLVs. The NeYSV has $79-96 \%$ NT and $88-95 \%$ AA identity with other isolates; the highest (96\% NT, $95 \%$ AA) with a Stenomesson isolate and only $87 \%$ NT and $92 \%$ AA with the other Crinum isolates. To our knowledge, this is the first report of NeYSV in the US and of NeLV in Crinum.

Development of a CANARY ${ }^{\circledR}$ multiplex testing platform for rapid identification of Ralstonia solanacearum A. A. AHMAD (1,2), D. N. Kalkofen (3), R. Rana (3), J. J. Lehett (3), A. R. Flannery (3), Q. Huang (1), (1) FNPRU, US National Arboretum, USDA/ARS, Beltsville, MD, USA; (2) MINIA University, EL-MINIA, EGYPT; (3) PathSensors, Inc., Baltimore, MD, USA

Ralstonia solanacearum (Rs) race 3 biovar $2(\mathrm{r} 3 \mathrm{~b} 2)$ causes devastating potato brown rot disease and is a select agent pathogen in the United States. The commercial movement of latently infected plant material such as geranium cuttings is the primary route by which this pathogen can spread. Fast and accurate early detection diagnostics are therefore needed for the efficient quarantine and management of r3b2. CANARY ${ }^{\circledR}$ (Cellular Analysis and Notification of Antigen Risks and Yields) technology, developed by the MIT Lincoln Laboratory, is a fast, sensitive, and easy to use biosensor technology that has been successfully utilized for the detection of microorganisms and toxins. In collaboration with USDA-APHIS and USDA-ARS, PathSensors, Inc. has developed a CANARY ${ }^{\circledR}$-based 96-well high-throughput diagnostic platform capable of detecting $R s$ including r3b2 strains. Our in vitro testing demonstrated that the $R s$ assay had an analytical detection level down to $100 \mathrm{CFU}$ and detected both $R s \mathrm{r} 3 \mathrm{~b} 2$ and non-r $3 \mathrm{~b} 2$ strains, but none of the out group bacteria. Our in planta testing showed that the assay had high specificity and sensitivity for Rs r $3 \mathrm{~b} 2$ with very low false positive/negative rates in r3b2-inoculated asymptomatic and symptomatic zonal geraniums. Sample preparation time is less than 10 minutes per sample, with a time to result at 1 minute per well. The combination of CANARY ${ }^{\circledR}$ technology with the high-throughput format makes this an attractive platform for the rapid and accurate screening of plant material for $R s$ before specific identification can be made for $\mathrm{r} 3 \mathrm{~b} 2$.

Multiplex assay for the quantitative assessment of Rhizoctonia solani AG2-2, AG4 and $R$. zeae from the soil T. ALBRECHT (1), K. M. Webb (2), R. M. Harveson (3), K. D. Broders (1), (1) Colorado State University, Fort Collins, CO, USA; (2) USDA-ARS, Soil Management and Sugar Beet Research Unit, Fort Collins, CO, USA; (3) Univ of Nebraska - Panhandle Research \& Extension Center, Scottsbluff, NE, USA

Rhizoctonia solani causes damping off and root and crown rot in sugar beets resulting in substantial losses in the field and during storage. Root rot is a difficult fungal disease to diagnose and manage, as the pathogen is usually not detected until after damage has occurred. The objective of this study was to develop a multiplex quantitative PCR assay to detect pathogenic and beneficial Rhizoctonia spp. in the soil. The assay was designed to simultaneously detect AG4 and two ISGs of AG2-2 known to cause disease in sugar beets. The assay also detects isolates of a potential antagonistic fungus, $R$. zeae, which has been found to provide some protection against pathogenic $R$. solani. Upon optimization, this assay provides early detection of pathogenic $R$. solani in the soil allowing for field treatment before significant damage occurs. This assay will be used for quantification of these Rhizoctonia species in multiple production fields in order to determine the role cropping history and soil characteristics play in the abundance of pathogenic and beneficial species. This information will help us determine if specific farming practices or soil types enhance the abundance of $R$. zeae and suppress $R$. solani. This will add valuable evidence to the feasibility of using $R$. zeae as a biological control agent for root rot disease in sugar beet production.

\section{Detection and quantification of Septoria glycines in soybeans with Real-time PCR}

H. A. LIN (1), S. X. Mideros Mora (2), (1) University of Illinois At Urbana-Champaign, Urbana, IL, USA; (2) University of Illinois at UrbanaChampaign, Urbana, IL, USA

Brown spot of soybean, caused by Septoria glycines is an extremely common late season foliar disease. The pathogen overwinters in the plant debris and becomes the primary inoculum in the next growing season. The disease remains at the lower canopy until maturity and might lead to premature defoliation under warm temperature and high humidity. In average, the yield losses caused by $S$. glycines are estimated between 3 to $8 \%$ per year. To accurately track the development of the pathogen, quantitative real-time PCR (qPCR) primers and hydrolysis probes were designed from polymorphic regions of the actin, $\beta$-tubulin and calmodulin genes. Twenty-six $S$. glycines isolates and four isolates of other foliar pathogens were collected from soybean leaves. The DNA were extracted by the CTAB method and used to sequence the ITS region to confirm the species identification and to test the specificity and sensitivity of the designed primers. Two of the designed primers and probe combinations were specific for detecting $S$. glycines, and no fluorescent signals were detected $\left(\mathrm{C}_{\mathrm{t}}>38\right)$ from other tested pathogens. The qPCR technique developed in this research will be applied to developing a yield model in the further studies, which is indispensable to define an economic threshold for the application of control measures against chronic soybean diseases.

Improved media for isolation of Pseudomoans savastanoi pv savastanoi from olive knots and development of an in vitro pathogenicity bioassay M. Salman (1), D. A. KLUEPFEL (2), (1) Palestine Technical University-Kadoorie, Tulkarm, PALESTINE; (2) USDA ARS, Crops Pathology \& Genetics Research Unit, Davis, CA, USA

Pseudomonas savastanoi pv. savastanoi (Psav) is the causal agent of olive knot (OK) on olive trees in many parts of the world where olive is grown. Understanding the mechanism of disease development by Psav requires proper isolation and purification methods that facilitate rapid recovery of Psav from the knots as well as reliable and reproducible in vitro pathogenicity bioassay. We developed an enhanced selective medium for the isolation and purification of Psav from plant tissue which consisted of KB medium amended with Vancomycin, Cephalexin and Cyclohexamide at 150,50 and $50 \mu \mathrm{g}$ $\mathrm{ml}^{-1}$, respectively. To isolate Psav, internal OK tissue was extracted from surface sterilized knots and placed in $100 \mu 1$ sterile distilled water for 30 min. A loopful of the suspension was then streaked onto the enhanced $\mathrm{KB}$ medium and incubated at $25^{\circ} \mathrm{C}$ for $24 \mathrm{hrs}$. Eighty Psav isolates were obtained from 15 olive genotypes grown in the National Clonal Germplasm Repository in Davis, California. The genetic diversity of these isolates was resolved using BOX, ERIC and REP PCR profiles. A highly virulent Psav strain was used to develop a quick and non-destructive pathogenicity bioassay using detached leaf segments of the olive cultivar "Manzanillo". Symptom development was obvious under $20 \mathrm{X}$ magnification after 10 days of incubation at $25^{\circ} \mathrm{C}$. This bioassay will facilitate the rapid screening of the olive germplasm collection for resistance to OK disease.

Comparison of primers for the detection of North American strains of Dickeya species in seed potato stems and tubers

A. S. B. NASARUDDIN (1), B. N. Babler (2), N. T. Perna (3), J. D. Glasner PhD (4), A. O. Charkowski (1), (1) Colorado State University, Fort Collins, CO, USA; (2) University of Wisconsin, Madison, WI, USA; (3) UW-Madison, Madison, WI, USA; (4) University of Wisconsin Madison, Madison, WI, USA

The 2015 Dickeya outbreak in the eastern US prompted the molecular testing of seed potatoes prior to planting to detect the presence of Dickeya dianthicola, an aggressive species of Dickeya. PCR-based detection assays are available for the soft rot bacteria Pectobacterium and Dickeya, however, they were not yet validated with the North American strains. We tested five different PCR-based detection assays: a) multiplex (Pcc, Pba, Pwa and Dsp primers); b) ECH; c) DIA-A; d) DIA-C; and e) PelADE assays on at least 115 stem and 240 tuber samples obtained from multiple locations in the United States. We found no evidence of false positives with the multiplex assay, but, six samples gave false negative results when compared with the DIA-C results. The ECH primer set has a high rate of false positive results. The DIA-A primer set, which is specific for Dickeya dianthicola, failed to detect all 
D. dianthicola strains. Genome sequence showed that one $D$. dianthicola strain is missing the target operon of the DIA-A primer set. The DIA-C primer set was sensitive and capable of detecting $D$. dianthicola in all samples tested. The PelADE primer set was the most consistent and is capable of detecting almost all Dickeya species. However, the published thermal cycler reaction profile needed to be altered to improve assay sensitivity. Both the PelADE and DIA-C primers are currently being used by the potato industry for Dickeya detection.

High-Throughput Molecular Screening for the presence of Citrus Pathogens in the Citrus Germplasm Collection of Punjab, Pakistan R. Haq (1), S. BODAGHI (2), T. Dang (2), I. Lavagi (2), S. H. Tan (2), S. Naz (1), G. Vidalakis (2), (1) Lahore College for Women University, Lahore, Pakistan, Lahore, PAKISTAN; (2) University of California, Riverside, Riverside, CA, USA

In 2013, a survey of the citrus germplasm collection at the Citrus Research Institute in Punjab, Pakistan, was initiated for the detection of grafttransmissible pathogens with a view to create a clean citrus germplasm program and to provide citrus growers in Pakistan with pathogen-tested propagative materials. Trees of 7 grapefruit, 15 lemon/lime, 15 mandarin, 30 sweet orange, 1 tangelo, 3 kumquat, and 7 rootstock varieties were selected and tested for the presence of viruses: Citrus tristeza virus (CTV), Citrus psorosis virus (CPsV), Citrus leaf blotch virus (CLBV), citrus tatter leaf virus (CTLV), citrus vein enation virus (CVEV); viroids: Citrus bent leaf viroid (CBLVd), Hop stunt viroid, Citrus dwarfing viroid (CDVd), Citrus bark cracking viroid (CBCVd) and Citrus exocortis viroid (CEVd); bacteria: Candidatus Liberibacter asiaticus (CLas), Spiroplasma citri, and Xylella fastidiosa. A combination of PCR-based techniques was used to screen 400 samples form the selected trees. Of the tested samples, $7 \%$ were positive for CTV (25/78 varieties, of which 22 from sweet orange), $22 \%$ were positive for different viroids ( $31 / 78$ varieties, of which 20 from sweet orange), and $65 \%$ were positive for CLas (47/78 varieties, of which 25 from sweet orange), whereas CPsV, CLBV, X. fastidiosa and S. citri were not detected. This screening was the first step towards the establishment of a clean citrus germplasm program in Pakistan.

\section{Molecular diagnostics for exotic cotton leaf curl begomovirus-betasatellites undergoing diversification and expansion} A. S. AVELAR, J. K. Brown, M. Ilyas, School of Plant Sciences, University of Arizona, Tucson, AZ, USA

Recently, begomoviruses that cause leaf curl disease of cotton and vegetable crops have emerged and spread throughout Asian and/or Africa, and spread to adjacent locales has been realized, further increasing the risks to unaffected areas, particularly along international plant trade routes. To accomplish begomoviral surveillance in plants and the whitefly vector, the molecular diagnostics development is imperative. PCR primers were designed to detect two most prevalent emergent leaf curl pathogens: Cotton leaf curl Kokhran virus-Burewala and Cotton leaf curl Gezira virus, and their associated betasatellite. Ongoing and extensive genomic diversification was evident from nucleotide sequence alignment and single nucleotide polymorphism analysis, both features that created challenges for primer design. The extensive number of closely related species and strains in the "core leaf curl virus complex' poses further constraints to designing broad-spectrum and species/strain-specific primers. Here, PCR primers were strategically designed and validated using cloned viral genomes and betasatellites for representative 'core leaf curl' variants, and virus-infected plant DNA extracts. A strategy involving 'community shared use' of standardized diagnostics for begomovirus-satellite detection in plants/whitefly vector prior to export/import of internationally-transported plants would reduce the risk of exotic virus spread in food and fiber crops globally.

Validation of species specific primers for detection and discrimination of Pythium aphanidermatum and P. deliense S. Wallace (1), M. F. Proano (1), A. Espindola (1), M. Arif (2), M. L. Daughtrey (3), C. D. GARZON PHD (1), (1) Oklahoma State University, Stillwater, OK, USA; (2) University of Hawaii at Manoa, Honolulu, HI, USA; (3) Cornell University, Long Island, NY, USA

Pythium aphanidermatum is an important pathogen affecting the greenhouses industry. This oomycete attacks seeds and plants at any age causing damping off, root rot and death. The pathogen is aggressive and quick identification is needed to assist growers in management decisions. Morphologica identification of $P$. aphanidermatum can be challenging due to overlapping similarities with other Pythium species, particularly from its sister species $P$. deliense. Two species-specific end-point PCR primer sets previously developed to detect and differentiate between $P$. aphanidermatum and $P$. deliense were validated on 47 isolates collected from floricultural greenhouses on Long Island, New York, that had been tentatively identified as Pythium aphanidermatum. The primer sets were species specific. Of the 47 samples, 41 produced amplicons with $P$. aphanidermatum primers. The six samples that did not produce amplicons were sequenced with ITS universal primers and identified as Pythium myriotylum $(\mathrm{n}=3)$, Phytopythium helicoides $(\mathrm{n}=1)$, Pythium irregulare $(\mathrm{n}=1)$ and Pythium deliense $(\mathrm{n}=1)$. Independent, blind validation of the $P$. deliense primers produced amplicons only with the positive control and the $P$. deliense sample assayed. The validated PCR assay can be used for quick and reliable discrimination between $P$. aphanidermatum and P. deliense.

Seed extract TaqMan PCR for the detection of Clavibacter michiganensis subsp. michiganensis in seeds of tomato H. KOENRAADT, Naktuinbouw, Roelofarendsveen, NETHERLANDS

Clavibacter michiganensis subsp. michiganensis $(\mathrm{Cmm})$ is a destructive seed transmittable pathogen and the causal organism of bacterial canker of tomato. Dilution plating (DP) of concentrated seed extract on semi-selective media is the reference method for detection of Cmm. After DP the identity of putative Cmm suspected colonies can be determined by TaqMan PCR. Finally, the identity of suspected colonies is verified by a pathogenicity assay. A high throughput seed extract TaqMan PCR was developed and validated as a pre-screening method for detection of Cmm in seeds of tomato. After stomaching, the seed extract is spiked with Clavibacter michiganensis subsp. tesselarius (Cmt) as an internal isolation control. Then bacteria are concentrated by centrifugation to increase the sensitivity of the assay. After bead beating, the bacterial DNA is purified using a KingFisher system. A triplex TaqMan PCR with two complementary Cmm specific and one Cmt specific primers-probe set is performed to determine whether Cmm DNA is present in the sample. A minimal recovery of the Cmt spike is used to evaluate the validity of the test result for each sub sample. The seed extract TaqMan PCR allows the rapid screening (within 2 days) of many samples. An advantage over the DP method is that Cmm is easily detected irrespective of the saprophyte load. Unlike the DP method the seed extract cannot discriminate between viable and dead Cmm cells. Validation data and experiences with the seed extract PCR in a routine laboratory will be presented.

\section{Identification and distribution of pathogens associated with field pea diseases in Montana State} J. MGBECHI-EZERI, A. Owati, C. Peluola, M. E. Burrows, B. Agindotan PhD, Montana State University, Bozeman, MT, USA

Surveys of pea fields were conducted in 2016 to determine diseases of economic importance in Montana State. A total of 17 fields were surveyed in June for foliar, root and soil-borne pathogens while 11 fields were resampled in July for pod and foliar diseases in 3 pea production zones (Gallatin Valley, Golden Triangle and Northeast). Fungi and bacteria were isolated on potato dextrose agar and Kings B medium, respectively. Identification of fungal species was based on cultural and morphological characteristics, and confirmed using species specific primers, sequences of the internal transcribed spacer (ITS) region and translation elongation factor (EF1- $\alpha$ ) gene (for Fusarium spp.) and 16S sequence for bacterial isolates. Ten fungal species and 135 pathogenic bacterial strains were obtained from 541 plants with necrotic symptoms. Alternaria (30\%) and Fusarium (25\%) were the most frequent and widespread species, followed by Ascochyta (13\%) and Stemphylium (7\%) from foliar, stem and pod tissues. Fusarium (69\%) was the most common fungal species recovered from pea root infection. Root rot disease was prevalent in the Northeast zone: observed in 7 of the 8 fields surveyed. Among the Fusarium species, F. avenaceum was predominant and widely distributed in all the production zones. Pseudomonas syringae pv. syringae and Pseudomonas syringae pv. pisi were the bacterial agents detected causing bacterial blight of pea on both foliar and pod. The preliminary results present the disease agents of field peas and their distribution in the different production zones in Montana, a knowledge needed for the management of field pea diseases. 
Rapid Detection of Pathogen in Complex Metagenomic Data by EDNA M. SHARP, Oklahoma State University, Stillwater, OK, USA

E-probe Diagnostic Nucleic-acid Analysis (EDNA) is a probe-based bioinformatic approach that has the potential to quickly detect any pathogen present in a single complex sample through metagenomic data mining. The objectives of this study are: 1) to evaluate the impact of sequencing platform and probe length on the detection of pathogen (Salmonella) by EDNA in metagenomic data, and 2) to assess the performance consistency of EDNA. Two sequencing platforms (454 and Illumina) were used to sequence metagenomic DNA extracted from the surface washes of commercial tomatoes that were spiked with Salmonella enterica at $10^{6}$ cell/tomato (S1), $10^{3}$ cells/tomato (S2), or un-spiked control (T). Salmonella enterica probes were created using the EDNA program and then curated. The probes were run against the raw sequence data and matches were retrieved having $97 \%$ identity and an e-value $10^{-9}$ or above. This process was repeated 100x for consistency testing. EDNA allows Salmonella enterica to be identified in spiked samples in less than 5 minutes from raw metagenomic DNA data. Probe lengths had limited impact on the detection of the pathogen; whereas, metagenomic data generated by Illumina provided higher numbers of matches (89-92 matches) and increased detection ( $10^{3}$ cells of pathogen/tomato or higher) compared to 454 (22-23 matches) at $\left(10^{6}\right.$ cells of pathogen/tomato). The E-probes were run against the three metagenomic data sets $100 \mathrm{x}$ with no identifiable change in detection (matches) indicating that the method is consistent. Rapid detection of human and plant pathogens in complex metagenomic data sets has important applications in the field of agriculture and phytopathology in the next generation sequencing era.

\title{
Detection and Absolute Quantification of Sugarcane Yellow Leaf Virus -SCYLV by Real-Time PCR for Successive Vegetative Cycles in Sugarcane in Colombia \\ C. Cardozo (1,2), Y. C. Acosta Vega (2,3), M. Cadavid (2), H. A. Chica (2), J. C. Angel S. (2), J. I. Victoria (2), C. A. ANGEL (2,4), (1) Universidad Nacional de Colombia campus Palmira, Palmira, COLOMBIA; (2) Cenicaña, Colombia, Cali, COLOMBIA; (3) private, Bogota, COLOMBIA; (4) Cenicafe - Colombia, Manizales, COLOMBIA
}

Sugarcane yellow leaf virus (SCYLV) is an important sugarcane pathogen worldwide detected in Colombia in 1998 by CENICAÑA in variety SP 716163 from Brazil. SCYLV infects most varieties used in Colombia (Cauca's river valley), and spreads efficiently by aphid transmission and infected cane seed setts. SCYLV is detected by tissue blot immuno-assay and reverse transcription PCR; however, low virus titers and reduced sensitivity of some techniques hamper detection resulting in false negatives, favoring virus dissemination. A reverse transcription quantitative PCR was validated to detect SCYLV in sugarcane plants used for seed production. Absolute quantification and detection limits were determined. A 147bp diagnostic fragment of SCYLV from ORF 0-1 was amplified, cloned, and in vitro transcribed to perform serial dilutions from $1 \times 10^{-3} \mu \mathrm{g}$ to $1 \times 10^{-11} \mu \mathrm{g}$ in DEPC water. Healthy plant total RNA samples were spiked to simulate routine detection assays. SCYLV was consistently detected in leaves from plants at different ages (2 to 9 months old) propagated from individual stem buds confirming SCYLV seed transmission during three successive vegetative cycles. Spiked samples required 2 to 3 additional PCR amplification cycles to overcome presence of plant inhibitors. Detection limit was 6 copies in samples diluted in DEPC water. A consistent quantification was estimated in 658 copies $(0.1 \mathrm{fg})$. A linear model $\left(\mathrm{R}^{2}=0.97\right)$ was developed to predict SCYLV copies in infected leaf samples in four varieties at different ages, determining virus accumulation curves in a time-course 12 months experiment to assess plant resistance to SCYLV, and virus effects on the plant performance.

Mitochondrial markers to detect Pythium at a genus and species-specific level using TaqMan and recombinase polymerase amplification T. MILES (1), F. N. Martin (2), (1) California State University Monterey Bay, Seaside, CA, USA; (2) USDA ARS, Salinas, CA, USA

Mitochondrial marker systems have been utilized in other oomycete systems to develop novel molecular diagnostic tools in both laboratory and field settings. Multiple gene order differences were identified using comparative genomics that allowed for genus specific amplification of more than 75 Pythium species across a wide range of clades within the genus. Furthermore, single nucleotide polymorphisms allowed for the development of some species-specific assays, particularly for the inflated filamentous Pythium species (clades A-D). PCR results for this locus tested negative on 145 Phytophthora taxa and several Phytopythium spp. Genus and species specific TaqMan assays were developed using labeled probes and validated with purified DNA and environmental samples from over 40 host plants (nursery and field samples). In addition to the TaqMan assay a genus-specific recombinase polymerase amplification (RPA) assay was developed for this locus. This isothermal assay was validated using the same purified and field samples and the RPA technique was effective at detecting Pythium species in less than 20 minutes, with a sensitivity around $1 \mathrm{pg}$ without a laborious DNA extraction. The use of these genus and species specific marker systems will not only help confirm Pythium infection in plants, it may also be useful in environmental samples and it will complement the other marker system developed for Phytophthora. Due to the amplicon size, this locus may also be useful in future metagenomic studies of Pythium diversity.

\begin{abstract}
Analysis of Amylostereum areolatum (Russulales: Amylostereacea) symbiont from two populations of Sirex nigricornis Fabricius (Hymenoptera: Siricidae)

R. O. OLATINWO (1), T. Schowalter (2), (1) USDA Forest Service, Pineville, LA, USA; (2) Louisiana State University Agricultural Center, Baton Rouge, LA, USA

The wood-decay fungus Amylostereum areolatum (Chaillet ex Fr.) is a symbiont of the native North American woodwasp Sirex nigricornis Fabricius (Hymenoptera: Siricidae) and the non-native Sirex noctilio Fabricius. However, the association between A. areolatum and S. noctilio is particularly damaging to pines in regions around the world. Our objective was to investigate the genetic differences between the A. areolatum strain from a population of native $S$. nigricornis in Louisiana, USA (where S. noctilio currently does not exist) and the A. areolatum strain from another population in Ontario, Canada where both Sirex species currently co-exist. We analyzed the intergenic Spacer (IGS) ribosomal DNA (rDNA) and the mitochondrial small subunit (mtSSU) rDNA of isolates and sequences from Louisiana and Canada, respectively. Results showed that $A$. areolatum sequences from native $S$. nigricornis in Louisiana were different from those found in both native S. nigricornis and non-native S. noctilio in Canada. Analysis indicated reciprocal exchange of $A$. areolatum (alleles not found in Louisiana) between the native $S$. nigricornis and non-native $S$. noctilio in Canada. The genetic information identified could be useful for tracking A. areolatum strains across regions.
\end{abstract}

\section{Detection of mefenoxam-insensitive populations of Pseudoperonospora humuli in Wisconsin hop yards} M. E. MARKS, A. J. Gevens, University of Wisconsin-Madison, Madison, WI, USA

Hop downy mildew caused by the oomycete Pseudoperonospora humuli is primarily managed with fungicides as commercial varietal resistance is unavailable. Mefenoxam, of the phenylamide fungicide class, is highly effective for systemic disease control but is at high risk for the development of pathogen resistance. In 2015 and 2016, 35 P. humuli isolates collected from 5 commercial hop yards in 4 Wisconsin counties were screened for mefenoxam insensitivity using a leaf disk assay. Leaf disks $(\mathrm{n}=14)$ from cleanly grown 'Nugget' variety hop plants were placed onto either $1 \%$ water agar or $1 \%$ water agar amended with $25 \mu \mathrm{g} / \mathrm{ml}$ of mefenoxam (Ridomil Gold SL, Syngenta). For each isolate, disks were inoculated at 3 sites with sporangial suspensions and the incidence of sporulation at each site was assessed after 5-7 days. Isolates were considered insensitive if the incidence of sporulation on amended media was at least $50 \%$ of that on non-amended media. Twelve (34.3\%) isolates tested as insensitive to mefenoxam, while the remaining $23(65.7 \%)$ were sensitive. Insensitive isolates were detected in all counties sampled. These data suggested that mefenoxam may be less effective in many WI hop yards. Growers are advised to follow resistance management guidelines to best steward the use of this potentially effective fungicide. Continued monitoring of mefenoxam sensitivity in the $P$. humuli population of Wisconsin will further support the downy mildew management decisions of hop growers. 
Occurrence of fungicide resistance in Pseudoperonospora cubensis populations causing cucurbit downy mildew in commercial and wild hosts K. D'ARCANGELO (1,2), T. Miles (3), L. Quesada-Ocampo (4), (1) North Carolina State University, Raleigh, NC, USA; (2) North Carolina State University Department of Entomology and Plant Pathology, Raleigh, NC, USA; (3) California State University-Monterey Bay, Seaside, CA, USA; (4) Department of Plant Pathology North Carolina State University, Raleigh, NC, USA

Pseudoperonospora cubensis, the causal agent of cucurbit downy mildew (CDM), is a broad host range oomycete pathogen of one of largest and most important horticultural food crops, the Cucurbitaceae family. P. cubensis has recently re-emerged post 2004 due to a break down in host resistance. After 2004, many different fungicides have been used to control the disease but only few are still effective due to the development of fungicide resistant populations of $P$. cubensis. Carboxylic Acid Amide (CAA) fungicides and Quinone outside Inhibitor (QoI) fungicides have known target site mutations that result in fungicide resistance in some oomycetes, G1105W and G143A, respectively. Samples were collected from commercial and wild cucurbit hosts from Eastern, Central and Western North Carolina at four different time points from 2013 to 2014. G1105W and G143A fungicide resistance alleles were detected and amplified via PCR. Results revealed that isolates from commercial hosts such as cucumber which have an intensive spray program for downy mildew control, show a higher occurrence of fungicide resistance mutations than hosts with less intensive spray programs or wild hosts. By better understanding the impact that fungicide programs have on pathogen populations and the occurrence of fungicide resistance, management practices could be modified to more sustainably control CDM in the United States.

Fungicide Resistance in B. fragariae and Species Prevalence in the United States East Coas M. DOWLING, M. Hu, G. Schnabel, Clemson University, Clemson, SC, USA

Botrytis fragariae was recently found causing gray mold of strawberry in Germany and the USA. It is unknown how widespread the new species is in commercial strawberry fields and whether fungicide resistance has developed. 208 Botrytis isolates were collected from the states of GA (28), SC (46), $\mathrm{NC}$ (51), VA (47), and MD (36), and B. fragariae made up 34.1\% of the entire collection. The two species coexisted in 12 fields, while other field samples consisted of only $B$. fragariae (5) or $B$. cinerea (13) isolates. Isolates with fungicide resistance were found, and resistance profiles differed from those of $B$. cinerea. No resistance to cyprodinil, boscalid, or penthiopyrad was observed, but $B$. fragariae isolates were more frequently resistant to thiophanate-methyl and fludioxonil and reduced sensitive to polyoxin $\mathrm{D}$ than $B$. cinerea. This is the first report of $B$. fragariae resistance to iprodione, fludioxonil, and thiophanate-methyl in the USA, and to fenhexamid worldwide. We also detected $B$. fragariae isolates with simultaneous resistance to multiple fungicide chemical classes. $B$. cinerea isolates with 3 or more chemical class resistances were more common in fields where no $B$. fragariae was observed $(p=0.0184)$. This indicates that these $B$. cinerea isolates had a selective advantage in commercial fields with routine fungicide use. In conclusion, $B$. fragariae is a common pathogen of strawberry and must be considered when designing gray mold management practices.

\section{Characterizing spatial and temporal variation in fungicide sensitivity of Venturia effusa within a pecan orchard}

\section{J. R. STANDISH, T. B. Brenneman, K. L. Stevenson, University of Georgia, Tifton, GA, USA}

Management of pecan scab, caused by Venturia effusa, has been significantly impacted by fungicide resistance. Results of recent monitoring efforts revealed unexpected variation in fungicide sensitivity among samples collected from the same orchard. In 2015 and 2016, a study was conducted to further investigate this variation. A pecan orchard block covering approximately 18 hectares was divided into $64(8 \times 8)$ quadrats, each containing nine trees. Infected leaflets were collected from trees in each quadrat and tested for sensitivity to propiconazole (PROP; $1 \mu \mathrm{g} / \mathrm{ml}$ ), thiophanate-methyl (TPM; $5 \mu \mathrm{g} / \mathrm{ml}$ ), and fentin hydroxide (TPTH; $30 \mu \mathrm{g} / \mathrm{ml}$ ). Sensitivity to all three fungicides varied significantly among the quadrats within years. Mean and standard deviation of percent relative growth for PROP were 28 and $75 \%$ in 2015; and 47 and 28\% in 2016. For TPM, mean and standard deviation of percent relative germination values were 45 and $24 \%$ in 2015 ; and 57 and $21 \%$ in 2016 . For TPTH, these values were 7 and $12 \%$ in 2015 ; and 13 and $7 \%$ in 2016. Sensitivity to all three fungicides was significantly lower in 2016 than in 2015. Significant spatial autocorrelation in sensitivity to TPM (Geary's $c=0.9481, p=0.0402$ ), suggesting clustering, was observed in 2016, but not for any other fungicide or year. The spatial and temporal variation in fungicide sensitivity within an orchard will be important to consider in designing future sampling protocols for fungicide sensitivity monitoring.

\section{Botrytis cinerea isolates lacking the Mrr1 R632I mutation are multi-resistant to postharvest fungicides which impacts apple grey mold} management

W. M. Jurick II PhD (1), O. Macarisin (2), V. L. Gaskins (1), I. Vico (3), J. Yu (2), K. A. Peter (4), W. J. Janisiewicz (5), K. D. COX (6), (1) USDAARS Food Quality Laboratory, Beltsville, MD, USA; (2) USDA ARS, Beltsville, MD, USA; (3) University of Belgrade, Faculty of Agriculture, Belgrade, SERBIA AND MONTENEGRO; (4) Penn State University, Biglerville, PA, USA; (5) USDA-ARS AFRS, Kearneysville, WV, USA; (6) Cornell University, Geneva, NY, USA

Botrytis cinerea causes grey mold of fruits and vegetables in the field and during storage. It is one of the most economically important pathogens worldwide and is controlled by fungicides and cultural practices. $B$. cinerea populations can quickly develop multiple fungicide resistance in the field resulting in severe losses. The concept of multi-fungicide resistance development in a postharvest environment was studied using $B$. cinerea isolates collected from commercial apple storage. Eight isolates were resistant to pyrimethanil (PYR) and thiabendazole (TBZ), but sensitive to fludioxonil (FLU). Resistance to FLU was generated in vitro by growing eight PYR and TBZ resistant $B$. cinerea isolates on a sub-lethal dose of FLU. Three vigorously growing multi-fungicide resistant isolates were obtained and characterized in vitro. A representative multi-fungicide resistant isolate was also evaluated on apples treated with labeled rates of three postharvest fungicides $\left(\right.$ Scholar $^{\circledR}$, Mertect ${ }^{\circledR}$, and Penbotec $\left.{ }^{\circledR}\right)$ and caused decay. The R632I mutation in the Mrr1 gene, associated with fludioxonil resistance in $B$. cinerea, was not detected in multi-fungicide resistant $B$. cinerea isolates. Results from this study indicate that the fungus can rapidly develop resistance to fludioxonil and may use additional mechanism(s) to mediate resistance. These findings reinforce the value of rotating postharvest fungicides with differing modes of action to limit development of multi-resistant isolates.

Effect of SDHI-fungicide sprays and pistachio hedging on Alternaria alternata sensitivity

P. D. S. F. LICHTEMBERG (1), W. Zeviani (2), R. Puckett (1), T. J. Michailides (1), (1) University of California - Davis, Parlier, CA, USA; (2) Universidade Federal do Paraná, Curitiba, BRAZIL

Alternaria late blight (ALB) of pistachio caused by Alternaria alternata, among two other species, continues to be a major disease in California pistachio orchards. Alternaria spp. is subject to selection of resistance to succinate dehydrogenase inhibitors. In 2015, resistance build-up to fluxapyroxad and penthiopyrad fungicides were observed, thus we decided to investigate which of the two products would result in faster selection of resistance in an $A$. alternata population in a commercial pistachio orchard with conditions conducive for high ALB infection. Our objectives were, (i) to evaluate different spray programs regarding their capacity to change SDHI fungicide sensitivity, (ii) to determine the effects of fungicide treatment and hedging on ALB disease severity components. In 2015 and 2016 less resistance build-up was observed in plots treated with spray programs that included the penthiopyrad fungicide. In 2015, the heavy hedging of trees resulted in less defoliation and lower ALB incidence. However, this effect was not observed in 2016. The 2015 and 2016 results obtained in pistachio orchards with conditions conducive for ALB disease suggest that fungicide spray programs that include penthiopyrad slow down the resistance build-up in comparison to similar spray programs that include fluxapyroxad. The heavy hedging of trees promotes less disease severity, but this practice needs to be conducted prior to low yield ("off year") production.

QoI resistance among Colletotrichum species found in Virginia vineyards

D. McHenry, A. Bly, C. L. Oliver, M. NITA, Virginia Polytechnic Institute and State University, Winchester, VA, USA 
In this study, one of the common mutations for QoI fungicide resistance among many fungal species, the G143A mutation in the cytochrome $b$ gene, was investigated using a newly developed real-time PCR (qPCR), and the result was compared with that of a fungicide-amended media assay. Various species of Colletotrichum are known to cause ripe rot in grapes, and our primary results exhibited evidence of resistance to QoI fungicides, in particular to an active ingredient azoxystrobin (e.g., Abound ${ }^{\mathrm{TM}}$ ). A total of 274 single-spore isolates, cultured from specimens collected in 30 vineyards across eight Virginia wine regions, were tested for QoI resistance. A combination of $100 \mathrm{ppm}$ azoxystrobin and 1,000 ppm SHAM was used in the amended media assay, and fungal growth was measured after 6 days. Probes were developed for the qPCR to target a 17-bp region around the G143A mutation, and the mutation (or lack of it) was assessed based on Ct values. Statewide prevalence of QoI-resistance was $29 \%$ based on the amended media assay and $24 \%$ according to the qPCR. A matched pairs analysis showed $66.7 \%$ correlation with a significantly higher number of QoI resistant isolates identified with the amended media assay $(P=0.03)$. Also, results from logistic regressions showed that the prevalence of QoI resistance was significantly affected by Colletotrichum species $(P<0.01)$ as well as the region effect $(P<0.01)$.

Resistance of Botrytis cinerea from California strawberries to ten fungicides

S. D. Cosseboom (1), G. SCHNABEL (2), K. L. Ivors (1), G. J. Holmes (1), (1) Strawberry Center, California Polytechnic State University, San Luis Obispo, CA, USA; (2) Clemson University, Clemson, SC, USA

Gray mold of strawberry, caused primarily by Botrytis cinerea, is a very destructive pre- and post-harvest fruit rot and is routinely managed by foliar fungicide applications. Outside of California, resistance of $B$. cinerea has been reported to every single-site active ingredient currently labelled for use against gray mold. We characterized the resistance profiles of $702 \mathrm{~B}$. cinerea isolates to ten active ingredients. Isolates were collected from $37 \mathrm{California}$ strawberry fields during the early-season ( 0 to 8 fungicide applications) and late-season (16 to 26 fungicide applications) of 2016. Sensitivity of each isolate was determined for each fungicide using the following discriminatory dosages $(\mu \mathrm{g} / \mathrm{ml})$ : boscalid (75), cyprodinil (4), fenhexamid (50), fludioxonil (0.5), fluopyram (10), iprodione (10), isofetamid (5), penthiopyrad (5), pyraclostrobin (10), and thiophanate-methyl (100). The percentage of isolates (early-season/late-season) resistant to each active ingredient was: boscalid (12/35), cyprodinil (12/46), fenhexamid (53/91), fludioxonil (1/4), fluopyram (2/7), iprodione (24/8), isofetamid (0/1), penthiopyrad (8/25), pyraclostrobin (77/98), and thiophanate-methyl (81/96). Resistance between early-season and late-season increased for all active ingredients except for iprodione, likely due to its restricted use. The observed shift in resistance to most fungicides within one season demonstrates the rapid, within-season selection of resistant subpopulations.

Occurrence of prochloraz resistance in Colletotrichum higginsianum in South China

L. YU, G. Lan, X. She, Y. Tang, M. Deng, Z. He, Plant Protection Research Institute, Guangdong Academy of Agricultural Sciences, Guangzhou, CHINA

Colletotrichum higginsianum Sacc. is a destructive pathogenic fungus causing anthracnose disease on crucifer plants. Brassica rapa var. parachinensis (Baily) Hanelt, also known as Choi sum, is one of the most popular crucifer vegetables in China. Prochloraz is a sterol demethylation inhibitor fungicide which is commonly used to control crucifer anthracnose disease. One-hundred-twenty field isolates of $C$. higginsianum were collected from Choi sum in 15 cities of Guangdong province in South China from 2010 to 2015. $\mathrm{EC}_{50}$ values of 100 isolates to prochloraz ranged from 0.0032 to $54.3101 \mathrm{mg} / \mathrm{L}$, indicating that resistance in $C$. higginsianum to prochloraz is beginning to emerge in South China, but its molecular basis is unknown. Two $14 \alpha-$ demethylase genes, including ChCYP51A and ChCYP51B, were investigated in three resistant and three sensitive isolates. Several point mutations leading to amino acid changes were found. Two resistant isolates BL1-14 and MX4-13 both exhibited a D161E substitution of ChCYP51A protein and an F508I substitution of ChCYP51B protein. The resistant isolate BL9-14 exhibited D161E and F508L substitutions of the ChCYP51A protein. Analysis of upstream nucleotide sequences of two CYP51 genes revealed an 1879-bp element, namely ChTE1, at base pair position -470 from the translational start site of ChCYP51A in isolate BL9-14 but not in the other two resistant isolates or sensitive isolates. ChTE1 might be a transposable element with inverted repeat sequences of 52-bp in length at both ends and three transposase domains, including DDE, CENPB and HTH_psq. ChTE1 also contains six predicted promoters. To our knowledge, this is the first report of occurrence of prochloraz resistance in C. higginsianum.

\section{Alternaria leaf spot in Michigan and fungicide sensitivity issues}

L. E. HANSON (1), N. Rosenzweig (2), Q. Jiang (3), P. Samohano (4), (1) USDA ARS, East Lansing, MI, USA; (2) Michigan State Univ, East Lansing, MI, USA; (3) MSU, East Lansing, MI, USA; (4) Michigan State University, East Lansing, MI, USA

Since 2010 there has been an increase in identification of Alternaria leaf spot on sugar beet in Michigan and other growing regions in the US and Canada. In 2016, the disease was severe enough to cause economic losses in the Michigan growing region. Michigan isolates from sugar beet were examined for species identification and tested for their response to fungicides commonly used on sugar beet to control foliar diseases. All isolates collected belonged to the Alternaria alternata species complex by both morphological and DNA sequence analysis. From 48 isolates collected in 2016 and 38 isolates collected in prior years, over $50 \%$ were identified as having resistance to one or more of the major fungicides currently used on sugar beet, including quinone outside inhibitor, demethylase inhibitor, and organo-tin fungicides. In addition to fungicide sensitivity, isolates were screened for pathogenicity on an alternate host, tomato, and for virulence on sugar beet germplasm. All isolates tested infected tomato fruit. Varietal differences in response to the isolates were observed in sugar beet germplasm. Further research on management options is needed for this disease.

\section{Effect of mefenoxam-acquired resistance on sporulation and dry weight in Phytophthora infestans} M. REGNIER, J. Gonzalez Tobon, M. Mideros, S. Restrepo, G. Danies, Universidad de los Andes, Bogota, COLOMBIA

Phytophthora infestans is an oomycete pathogen that causes potato and tomato late blight disease. This pathogen represents a worldwide concern for farmers given its explosive epidemic potential. Control of the disease is mainly achieved by the use of fungicides. Studies have reported that different strains of $P$. infestans acquire resistance to mefenoxam in vitro, after being exposed to sub-lethal concentrations of this fungicide. So far, this phenomenon has only been assessed in terms of mycelial radial growth. The aim of this study was to evaluate if there is an effect on the sporangia production and mycelial dry weight in isolates of $P$. infestans that have acquired resistance to mefenoxam. Originally sensitive isolates of $P$. infestans and those same isolate after acquisition of resistance, were exposed to different concentrations of fungicide-amended medium. When mycelia in the control plate $\left(0 \mu \mathrm{g} \mathrm{ml}^{-1}\right.$ of mefenoxam $)$ reached $80 \%$ of the petri plate, sporangia production and mycelial dry weight were assessed. The consequences of an acquisition of resistance to mefenoxam on sporangia production and on mycelial dry weight are discussed.

Is the phenomenon of acquired resistance in Phytophthora infestans universal?

J. GONZALEZ TOBON (1), R. Childers (2), M. Regnier (1), M. Mideros (1), S. Restrepo (1), G. Danies (1), (1) Universidad de los Andes, Bogota, COLOMBIA; (2) Harvard University, Cambridge, MA, USA

Phytophthora infestans, a plant pathogenic oomycete, is the causal agent of late blight disease of potatoes and tomatoes. This disease causes devastating economical losses each year and control is mainly achieved by the use of fungicides. Previous studies have reported that sensitive isolates to the phenylamide fungicide, mefenoxam, become tolerant after a single passage on mefenoxam-containing medium. The acquired resistance of originally sensitive isolates appeared to have a cost on the rate of mycelial growth. The aim of this study was to investigate whether the same phenomenon is observed when exposing sensitive isolates of $P$. infestans to sub-lethal concentrations of fluopicolide and cymoxanil. These two systemic fungicides, used to control late blight, present modes of action different to that of mefenoxam. Sensitive isolates of $P$. infestans were exposed to a range of concentrations of fungicide-amended medium. To assess if the isolates had acquired resistance, mycelium growing at the two lowest concentrations were 
transferred once again to media amended with the highest concentrations of the fungicide. Mefenoxam was also included in our assays as a positive control for the acquisition of resistance. This study provides further insights into the in vitro adaptability of $P$. infestans against antifungal stress.

Sclerotinia homoeocarpa Populations and Their Resistance to Fungicides

C. STEPHENS, J. E. Kaminski III, Penn State University, University Park, PA, USA

Sclerotinia homoeocarpa is an economically important pathogen that causes dollar spot in turfgrass. Turfgrass managers often apply fungicides throughout the growing season in order to control the disease. With an increase in documented fungicide resistance across multiple chemical classes, it is pertinent for turfgrass managers to gain a better understanding of the $S$. homoeocarpa populations present at their golf course. A total of 101 isolates were collected from different sites throughout PA, OH, MI, NY, and MA and screened in vitro across four different fungicides. Fungicides evaluated included thiophanate-methyl at $1,000 \mathrm{ug}$ a.i $\mathrm{mL}^{-2}$, iprodione at $10,1,0.1,0.01 \mathrm{ug} \mathrm{a.i} \mathrm{mL} \mathrm{m}^{-2}$, propiconazole at $1,0.1,0.01,0.001 \mathrm{ug}$ a.i $\mathrm{mL}^{-2}$, and boscalid

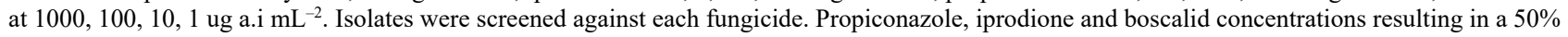
reduction in growth (EC50) were determined. Resistance to thiophanate-methyl was determined qualitatively. Isolates from the Martha's Vineyard Golf Club (MVGC), which has never been sprayed with a synthetic pesticide, were served as a baseline for sensitivity. Mean EC50 values of isolates from MVGC to iprodione, propiconazole, and boscalid were $0.34,0.01$, and $141.48 \mathrm{ug} \mathrm{a.i} \mathrm{m}^{-2}$, respectively. All other isolates had a mean (range) of 0.52 ( 0.12 to 2.16 ug a.i mL $\mathrm{mL}^{-2}$ ), 0.13 (0.01 to $0.917 \mathrm{ug}$ a.i $\mathrm{mL}^{-2}$ ), and $371 \mathrm{ug} \mathrm{a.i} \mathrm{mL}^{-2}$ (38 to $990 \mathrm{ug} \mathrm{a.i} \mathrm{mL}$ ) for iprodione, propiconazole, and boscalid, respectively. A total of 63 of 101 isolates were resistant to thiophanate-methyl. Based on these results there is a wide range of $S$. homoeocarpa sensitivity to thiophanate-methyl, iprodione, propiconazole, and boscalid in turfgrass.

Sensitivity to azoxystrobin in selected Kentucky populations of Cercospora nicotianae, the frogeye leaf spot pathogen of tobacco W. Barlow, E. W. Dixon, R. Pearce, E. E. PFEUFER, University of Kentucky, Lexington, KY, USA

Resistance to azoxystrobin fungicide has been documented in several Cercospora pathogens, including C. sojina and C. beticola. In 2016, burley tobacco in south-central Kentucky was affected by atypically severe frogeye leaf spot, caused by $C$. nicotianae. Azoxystrobin has been used to manage tobacco leaf spot diseases in this region for eight seasons or more, often without rotation due to few alternative modes of action labeled for tobacco. In August 2016, tobacco leaves with frogeye were collected from five fields, at ten sites spaced at least $50 \mathrm{~m}$ apart. Single-spore cultures from each site were isolated on 1/4-strength PDA, then transferred to V8 agar to induce sporulation. Conidial suspensions were plated on PDA amended with SHAM and azoxystrobin at concentrations of $0,0.001,0.01,0.1,1$, or $10 \mu \mathrm{g} / \mathrm{mL}$. After 18 to 24 hours, germination of 100 spores per plate was determined at each concentration, in triplicate. The effective concentration to inhibit $50 \%$ of germination $\left(\mathrm{EC}_{50}\right)$ was calculated based on each isolate's dose response curve. $\mathrm{EC}_{50}$ values ranged from 0.03 to $2.80 \mu \mathrm{g} / \mathrm{mL}$ azoxystrobin; most were $0.30 \pm 0.04 \mu \mathrm{g} / \mathrm{mL}$. Two isolates' $\mathrm{EC}_{50} \mathrm{~s}$ exceeded $2.50 \mu \mathrm{g} / \mathrm{mL}$ azoxystrobin, nearly 100 -fold greater than isolates collected from certified organic tobacco. This is the first record of shifted sensitivity to azoxystrobin in $C$. nicotianae, and future work will focus on understanding how disease dynamics may differ in field populations containing such individuals.

Occurrence of fungicide resistance in Alternaria populations to commonly used fungicides in Idaho K. FAIRCHILD (1), A. Malek (1), P. S. Wharton (2), (1) University of Idaho, Aberdeen, ID, USA; (2) University of Idaho, Aberdeen Research and Extension Center, Aberdeen, ID, USA

Early blight (caused by Alternaria solani) and brown leaf spot (caused by Alternaria alternata), are important diseases of potatoes in Idaho. Previous research documented the widespread occurrence of resistance to SDHI and QoI fungicides in Idaho. Fungicide sensitivity testing was done using spiral plate dilution gradients. Fungicide solutions were applied to PDA in a $2.5-\log$ dilution in a continuous radial concentration gradient using a spiral plater. Fungal inoculum was placed in radial lines across the gradient. Fungicide sensitivity was expressed as an EC50, the fungicide concentration at which a fungal isolate's radial growth was equal to $50 \%$ of the average growth of the isolate on non-amended PDA. A three year study from 2010 to 2013 showed that resistance to the commonly used SDHI fungicide boscalid had risen from under $50 \%$ to over $80 \%$ by 2013 and resistance to the QoI fungicides (e.g. azoxystrobin) was over 90\%. This study was expanded between 2014-2016 during which a total of 75 isolates were collected and screened for resistance to a larger group of fungicides which have shown potential for the control of both $A$. solani and A. alternata. This study will give and update on the status of fungicide resistance in Alternaria populations in Idaho.

Response of sorghum stalk pathogens to near isogenic brown midrib plants and their soluble extracts D. L. FUNNELL-HARRIS (1), P. O’Neill (1), S. Sattler (1), T. Gries (1), M. Berhow (2), (1) USDA-ARS, Lincoln, NE, USA; (2) USDA-ARS, Peoria, IL, USA

Sorghum [Sorghum bicolor (L.) Moench] has great potential for lignocellulosic feedstock for biofuels production. Reducing lignin content can increase conversion efficiency. Brown midrib (Bmr) 6 and 12 genes both encode enzymes in the monolignol pathway, which is responsible for synthesizing the subunits of lignin. Near-isogenic lines containing recessive loss of function mutations in Bmr6, Bmr12, or both genes were previously developed and shown to have significantly reduced lignin content and increased levels of soluble phenolics. In the current study, response of these lines to Fusarium stalk rot pathogens, Fusarium verticillioides and Fusarium proliferatum, and the charcoal rot pathogen, Macrophomina phaseolina, was assessed. The bmr6, bmr12, and bmr6 bmr12 lines in two backgrounds were shown to not be more susceptible to $F$. verticillioides, $F$. proliferatum and $M$. phaseolina inoculations. Some bmr lines exhibited increased resistance to F. proliferatum and M. phaseolina, compared to wild-type lines. When another stalk rot pathogen, Fusarium thapsinum, was grown on methanol-soluble stalk extracts from bmr6 and wild-type plants, it grew significantly faster on medium with bmr6 extract than on wild-type extract or controls. This result suggested that factors other than soluble phenolics from the extract, such as cell wall bound phenolics compounds or inducible defense compounds, may be contributing to increased resistance observed in bmr6 plants.

Evaluation of Cucurbita pepo breeding lines with reduced susceptibility to root and crown rot caused by Phytophthora capsici

G. VOGEL (1), K. LaPlant (1), M. Mazourek (1), M. Gore (1), C. D. Smart (2), (1) Plant Breeding and Genetics Section, Cornell University, Ithaca, NY, USA; (2) Plant Pathology and Plant Microbe Biology Section, Cornell University, Geneva, NY, USA

The squash species Cucurbita pepo is highly susceptible to crown rot caused by the broad-host range oomycete pathogen Phytophthora capsici. No source of complete resistance to $P$. capsici has been found in C. pepo, but an accession, PI 615089, with reduced susceptibility compared to commercial cultivars was previously identified. Evaluation of progeny from crosses with PI 615089 suggests the tolerance is quantitatively inherited. The aim of this project was to evaluate advanced breeding lines derived from PI 615089 to identify the most suitable parent of a biparental mapping population. Eleven breeding lines and a susceptible zucchini variety used as a control were evaluated as seedlings for crown rot tolerance over 15 days in a replicated greenhouse trial. Over $50 \%$ of susceptible control plants were symptomatic 3 days after inoculation, compared to an average of 8.4 days for the breeding lines, and 11 days for the most tolerant line identified. Plants were further evaluated for their response to eight different isolates of Phytophthora capsici to characterize the isolate specificity of the tolerance, no evidence of which was found. The most tolerant line was crossed with the highly susceptible zucchini variety 'Dunja' to develop a large F2 mapping population. Sequencing of pooled DNA samples from extreme susceptible and tolerant tails of the F2 population will enable the identification of genomic regions contributing to variation for susceptibility to the pathogen.

Molecular mapping and comparison of $\operatorname{Yr} \operatorname{Tr} 1$ with other genes on chromosome $1 \mathrm{BS}$ for resistance to wheat stripe rust

J. Feng (1,2), M. Wang PhD (3), X. CHEN (4), (1) Washington State University, Pullman, WA, USA; (2) Biotechnology and Nuclear Technology Research Institute, Chengdu, CHINA; (3) Washington Sate University, Pullman, WA, USA; (4) USDA ARS, Pullman, WA, USA 
Stripe rust, caused by Puccinia striiformis f. sp. tritici (Pst), is one of the most destructive diseases of wheat worldwide. Races of the pathogen are differentiated with a set of $Y r$ single-gene lines. Among the $18 \mathrm{Yr}$ genes in the current differentials, the chromosomal location of $Y r \operatorname{Tr} 1$ is unknown. To map the gene, the near-isogenic line (AvSYrTr1NIL) carrying $\operatorname{YrTr} 1$ was backcrossed to the susceptible recurrent parent 'Avocet S' (AvS), and seedlings of $\mathrm{BC}_{7} \mathrm{~F}_{2}$ and $\mathrm{BC}_{7} \mathrm{~F}_{2: 3}$ were phenotyped with an avirulent race of $P s t$ and genotyped with simple sequence repeat (SSR) and single-nucleotide polymorphism (SNP) markers. Genetic analysis of the phenotypic data of $177 \mathrm{BC}_{7} \mathrm{~F}_{2}$ plants and their $\mathrm{BC}_{7} \mathrm{~F}_{2: 3}$ lines identified a single partially dominant gene. A linkage map was constructed for $Y r T r 1$ with four SSR and seven SNP markers, and the gene was flanked by SNP markers IWA2583 (1.8 cM distal) and IWA7480 (1.3 cM proximal). The four SSR markers mapped $\operatorname{YrTr} 1$ to the short arm of chromosome 1B. Amplification of the complete set of 21 Chinese Spring (CS) nulli-tetrasomic lines and seven CS deletion lines with three of the SSR markers confirmed the chromosomal arm and further mapped the gene in the chromosomal bin 1BS $18(0.5)$ close to the telomere. Based on race spectra and chromosomal locations, $\operatorname{YrTr} 1$ is different from all other permanently named stripe rust resistance genes in chromosome 1BS. This information is useful for identifying Pst races and understanding wheat-Pst interactions.

\section{Adaptation of Phytophthora nicotianae to partial resistance in tobacco}

J. JIN, I. Carbone, D. Shew, North Carolina State University, Raleigh, NC, USA

Phytophthora nicotianae causes tobacco black shank worldwide. The ability of $P$. nicotianae to rapidly overcome single-gene resistance was documented following the widespread deployment of the Php gene in tobacco cultivars in the 1990s. Increased levels of aggressiveness in $P$. nicotianae have been documented following exposure to tobacco varieties with high levels of partial resistance. In a previous study, isolates of $P$. nicotianae were adapted on multiple sources and levels of partial resistance by inoculating and reisolating isolates from a given resistance type and level for six consecutive host generations. A single isolate was obtained from each host after each generation of adaptation and these six isolates were characterized for their level of aggressiveness. Our objective was to characterize the adaptation to partial resistance using phenotypic and molecular approaches. We used in vivo inoculum production and rate of lesion expansion to quantify the adaptation by $P$. nicotianae. The adapted isolates also were subjected to double digest restriction site associated DNA sequencing to identify genetic changes during adaptation. Understanding how $P$. nicotianae adapts to partial resistance in tobacco will inform better resistance deployment strategies and increase the durability of partial resistance.

\section{Host resistance of flue-cured tobacco (Nicotiana tabacum L.) for the management of tobacco black shank (Phytopthora nicotianae)} L. D. THIESSEN, North Carolina State University, Raleigh, NC, USA

Black shank of tobacco, caused by Phytophthora nicotianae, causes significant losses to cultivated tobacco production. For management, producers rely heavily on host resistance and soil-applied fungicide applications to reduce the severity of disease epidemics. The assessment of varietal resistance to $P$. nicotianae has been conducted cooperatively for over 30 years in numerous states throughout the southeast U.S. More recently, a race shift in $P$. nicotianae populations has occurred from the prolonged use of the $P h$ gene from the cultivar Coker 371-Gold, which has affected breeding and disease management strategies. Newly developed cultivars are incorporating multiple genes for resistance to $P$. nicotianae to increase survival under high disease pressure. This study assesses host resistance over time with relation to disease dynamics, regional differences, and environmental changes.

\section{Use of FT-IR for rapid phenotyping of European ash resistance levels to ash dieback}

C. VILlaRI (1), A. Dowkiw (2), R. Enderle (3), M. Ghasemkhani (4), T. Kirisits (5), E. Kjaer (6), D. Marčiulynienè (7), L. McKinney (6), B. Metzler (3), F. Munoz (2), L. Rostgaard Nielsen (6), A. Pliūra (7), L. G. Stener (8), V. Suchockas (7), L. Rodriguez-Saona (9), P. Bonello (10), M. Cleary (4), (1) D.B. Warnell School of Forestry and Natural Resources, University of Georgia, Athens, GA, USA; (2) INRA, Ardon, Orléans Cedex 2, FRANCE; (3) Forest Research Institute Baden-Wurttemberg, Department Forest Protection, Freiburg, GERMANY; (4) Swedish University of Agricultural Sciences (SLU), Southern Swedish Forest Research Centre, Alnarp, SWEDEN; (5) Univ Fur Bodenkultur (BOKU), Vienna, AUSTRIA; (6) Department of Geosciences and Natural Resource Management, University of Copenhagen, Copenhagen, DENMARK; (7) Lithuanian Research Centre for Agriculture and Forestry, Institute of Forestry, Girionys, Kaunas district, LITHUANIA; (8) The Forestry Research Institute of Sweden (SKOGFORSK), Svalöv, SWEDEN; (9) Department of Food Science and Technology, The Ohio State University, Columbus, OH, USA; (10) Department of Plant Pathology, The Ohio State University, Columbus, OH, USA

Ash dieback is a devastating disease caused by the invasive fungus Hymenoscyphus fraxineus, and is currently decimating European ash (Fraxinus excelsior) in much of its distribution range in Europe. Despite the host population being highly susceptible to the pathogen, a small proportion of trees $(1-5 \%)$ show low susceptibility, which appears to be genetically controlled, suggesting that selection and breeding could be a successful management strategy. The lack of viable tools for rapid resistance phenotyping, however, impedes significant advances in this direction. The objective of this study was to test if Fourier-transform infrared (FT-IR) spectroscopy, a vibrational-based chemical fingerprinting technique, can be applied for rapid phenotyping of disease resistance in ash. We sampled non-infected leaves and stems from known susceptible and resistant $F$. excelsior genotypes across six European countries. We analysed purified phenolic extracts via FT-IR, and used chemometric models to discriminate between resistant and susceptible trees. While the model built with leaves had no differentiating power, the one built using stems strongly discriminated between resistant and susceptible phenotypes. These findings, further strengthened by the validation of the model using a separate set of random blind samples, show that FTIR holds real promise as a fast, efficient, and reliable screening tool for the selection of genetically resistant trees.

Genetic characterization of resistance to Sclerotinia in lettuce cultivar Eruption

B. E. MAMO PHD (1), R. J. Hayes (2,3), M. J. Truco (4), R. W. Michelmore (4), K. V. Subbarao (1), I. Simko (5), (1) University of California at Davis, c/o U.S. Agricultural Research Station, Salinas, CA, USA; (2) USDA, ARS, Forage Seed and Cereal Research Unit, Corvallis, OR, USA; (3) USDA, ARS, Corvallis, OR, USA; (4) University of California at Davis, Genome Center and Department of Plant Sciences, Davis, CA, USA; (5) USDA, ARS, Crop Improvement and Protection Unit, Salinas, CA, USA

Lettuce drop caused by the fungal pathogens Sclerotinia minor and S. sclerotiorum is a serious disease of lettuce. The use of genetic resistance as part of an integrated lettuce drop management strategy should have a significant economic advantage in mitigating yield loss. Sclerotinia resistance is often confounded with morphological traits, primarily premature bolting. As such, previous attempts of identifying Sclerotinia resistance in lettuce were largely unsuccessful. Recently, high level of resistance to $S$. minor and $S$. sclerotiorum independent of plant morphology has been identified in the slowbolting red Latin type cultivar Eruption (ERN). To characterize the inheritance of resistance to Sclerotinia in Eruption, a cross was made to the susceptible lettuce $\mathrm{cv}$. Batavia Reine des Glaces (BRG). One hundred sixty $\mathrm{F}_{6: 7}$ recombinant inbred lines (RILs) were evaluated for lettuce drop resistance and bolting in a field infested with S. minor; genotyped by sequencing (GBS) identified 840 single nucleotide polymorphism (SNP) markers. The variation in the phenotypic data indicates the presence of quantitative resistance in Eruption. QTL analysis identified one resistance QTL on each of lettuce chromosomes 1 and 5, explaining $12 \%$ and $12-25 \%$ of the variation, respectively. These genomic locations are not associated with bolting in Eruption. The SNP markers identified may be useful in marker-assisted introgression of lettuce drop resistance into breeding materials.

Two new late blight resistant home garden varieties of tomato with tolerance to Septoria leaf spot R. MAHFUZ, M. E. Gallegly Jr., West Virginia University, Morgantown, WV, USA

Late blight resistant West Virginia '63 tomato is a favorite to organic and small growers in West Virginia. It remains susceptible to Septoria leaf spot (SLS) incited by Septoria lycopersici. Cornell University released a $\mathrm{F}_{1}$ hybrid, Iron Lady, with resistance to SLS in addition to late blight resistance 
partly obtained from WV ' 63 . We crossed WV '63 with Iron Lady and selected in $F_{2}$ field-grown plants for plant and fruit type and resistance to SLS. The SLS resistance was low. We selected for this tolerance by inoculating 6-week old plants showing nutrient deficiency. WV ' 63 plants were killed whereas the hybrid selections survived. Two of the selections, tentatively named WV' $17 \mathrm{~A}$ and ' $17 \mathrm{~B}$ are being released in 2017 in honor of the $150^{\text {th }}$ anniversary of the founding of WVU Davis College of Agriculture. Both selections carry single-gene and multiple-gene resistance to late blight, and the dominant Ve-gene and I-gene for resistance to Verticillium and Fusarium wilt, respectively. The vine type of both selections is indeterminant. The fruit carry the uniform ripening gene and high color. Fruit set is higher in WV '17A and fruit is more firm than that of WV '63. Fruit of WV '17B tend toward a beefsteak type and is sweeter with significantly $(P<0.003)$ higher brix content compared with WV'63. Limited seed is available. Final release and name is dependent upon feedback from 2017 growers.

Using allele-specific PCR for genotyping different mutations selected by the use of SDHI in populations of Alternaria alternata in California P. D. S. F. LICHTEMBERG, Y. Luo, T. J. Michailides, University of California - Davis, Parlier, CA, USA

Among the fungicide groups used to control the Alternaria late blight (ALB) of pistachio are the succinate dehydrogenase inhibitors (SDHI). In California, four SDHI active ingredients (a.i.) are currently registered for pistachio (boscalid, fluxapyroxad, penthiopyrad, and fluopyram). However, in a relatively short time after registration, resistance to these a.i. were found to be associated with mutations on the genes $A a S d h B^{\mathrm{H} 277 \mathrm{R} / \mathrm{YL}}, A a S d h C^{\mathrm{H} 134 \mathrm{R} \& \mathrm{~S} 135 \mathrm{R}}$, and $A a S d h D^{\mathrm{D} 123 \mathrm{E} \& H 133 \mathrm{R}}$. To monitor and quickly identify SDHI-mutant genotypes of Alternaria species, an allele-specific PCR (AS-PCR) assay was developed. For that, the reverse primer was designed to match each putative point mutation at the 3' end of the primer. In total, six primer pairs were designed to specifically target the mutations mentioned above except for the H133R. The primer specificity was tested with real-time PCR to successfully amplified 460-bp (H134R) and 350-bp (H277R), with 30 cycles, and 90-bp (H277Y), 179-bp (H277L), 398-bp (S135R), and 255-bp (D123E) with 35 cycles. The full PCR conditions included initial-heat $95^{\circ} \mathrm{C}$ for $3 \mathrm{~m}$, denaturation $94^{\circ} \mathrm{C}$ for $40 \mathrm{~s}$, annealing $66^{\circ} \mathrm{C}$ for $40 \mathrm{~s}$, extension $72^{\circ} \mathrm{C}$ for $1 \mathrm{~m}$ and final extension $72^{\circ} \mathrm{C}$ for $5 \mathrm{~m}$. In conclusion, the AS-PCR will allow a very fast and costless genotyping assay, and several other laboratory applications, such as determination of genotype competition, assessment of mutation frequency for large-area surveys, and the development of a multiplex PCR assay.

QTL mapping for Meloidogyne incognita and M. hapla resistance in one recombinant inbred line population of soybean C. Li (1), J. Wang (1), J. Abe (2), F. Kong (1), C. WANG (1), (1) Northeast Institute of Geography and Agroecology, Chinese Academy of Sciences, Harbin, CHINA; (2) Laboratory of Plant Genetics and Evolution, Hokkaido University, Sapporo, JAPAN

Host-plant resistance is the most economically effective strategy for root-knot nematode (Meloidogyne spp.) control in soybean (Glycine max). A recombinant inbred line (RIL) population was originally developed for flowering gene mapping from a cross between a Japanese breeding line G. max cv. TK780 and a wild G. soja accession line H4. We identified TK780 as resistant and H4 as susceptible to both M. incognita and M. hapla. To identify the quantitative trait loci (QTLs) determining the response to the two nematodes, the RIL population was phenotyped in the greenhouse for root response (galling index, GI) and nematode reproduction (egg masses, EM and eggs per gram root, EGR). QTL analysis indicated that one major QTL in TK780, accounting for 39\% of phenotypic variance (PVE) in M. incognita GI, was identified on Chromosome 10 (Chr10), previously reported in other resistant genotypes. Two new major QTLs accounting for $26 \%$ and $21 \%$ of PVE in $M$. incognita EGR were mapped on Chr14 and Chr20, respectively. One major QTL linked to the marker Sat_389 was detected on Chr7, explaining 21\% and 26\% of PVE in M. incognita EM and EGR, and 37\%, 48\% and 45\% in M. hapla GI, EM and EGR, respectively. This is the first report for $M$. hapla resistance mapping in soybean. The results suggest genetic independence for root response and egg production. These newly identified QTLs with flanking markers will be helpful in marker-assisted breeding for nematode resistance in soybean.

Population dynamics of blast fungus leads to breakdown of resistance to rice blast

Y. KIM, J. Goh, I. J. Kang, H. K. Shim, S. Heu, J. H. Roh, National Institute of Crop Science, RDA, Suwon, Korea, Republic of (South)

Rice blast caused by Magnaporthe oryzae is one of the most serious diseases that affect the quantity and quality of rice production. The use of resistant rice varieties would be the most effective way to control the rice blast. Pathogenic diversity of $M$. oryzae isolates collected in the rice field from 2004 to 2015 in rice field in Korea was analyzed using rice blast monogenic lines, each harboring a single blast resistance gene. Compatibility of blast isolates against rice blast monogenic lines carrying the resistance genes Pi5, Pi9, Pib, and Piz showed dynamic changes by year. It indicates that pathogen has high evolutionary potential adapted host resistances to increase fitness and would lead to rice blast resistance bred into the cultivar becoming ineffective eventually. Major rice blast resistant genes were determined in 120 rice cultivars of Korea by molecular screening. Rice cultivars, Hoban, Younghae and Taebong carrying Pi5, Pi , Pib, Pita and Piz and showed high resistant reaction to rice blast in nursery test. On the other hand, rice cultivars carrying a single resistance gene showed susceptibility against rice blast fungus. These results support that resistant gene combination enhances durable blast resistance in rice.

Tomato functional genomic screen to find novel resources for development of resistant cultivars against Sclerotinia minor A. HATTORI (1), Y. Ishiga (2), (1) Graduate School of Life and Environmental Sciences, University of Tsukuba, Tsukuba, JAPAN; (2) University of Tsukuba, Tsukuba, JAPAN

Sclerotinia minor (S. minor) is the plant pathogen known to cause white mold disease (WMD) in various crop plants. The resistant cultivars against WMD have not been developed. Micro-Tom, a model tomato has been utilized to develop mutant resources for the functional genomics of tomato. To identify novel resources that confer resistance against WMD, we developed a forward-genomics screen with Micro-Tom ethyl methanesulfonate (EMS) mutants. From this screen, we identified a white mold resistancel (wmrl) mutant that showed high resistance against WMD. To determine resistance of $w m r l$, we made artificial S. minor mixed soil for germination test. In the result, wmrl showed higher germination rate than wild-type in artificial $S$. minor mixed soil. Commonly, oxalic acid is known as a significant virulence factor to infect host plant and transformed oxalate oxidase confers resistance to Sclerotinia spp. (Marciano P. et al., 1982; Livingstone D. M. et al., 2005; Kyoung S. K. et al., 2008; Walz A. et al., 2008). We carried out oxalic acid assay to investigate sensitivity of wild-type and wmrl to oxalic acid. wmrl showed sensitivity to oxalic acid same as wild-type. Therefore, this result suggests that resistant mechanism of wmrl is independent of oxalate oxidase like activity. Further studies will be needed to reveal the molecular function of WMR1 in the tomato and S. minor interactions.

Development of an RAPD-based SCAR marker linked with smut disease resistance in commercial sugarcane cultivars Y. B. PAN (1), M. Khan (2), M. P. Grisham (1), J. Iqbal (2), (1) USDA-ARS, SRU, Houma, LA, USA; (2) School of Biological Sciences, Lahore, PAKISTAN

Sugarcane smut has been a major disease worldwide that can cause a significant yield loss if not controlled. A 300-bp RAPD-derived Sequence Characterized Amplified Region (SCAR) marker linked with smut disease resistance was developed. Bulked segregant analysis and RAPD were conducted using 480 random decamers in the initial screening of 12 sugarcane cultivars (two completely smut resistant, two completely smut susceptible, four moderately smut resistant, and four moderately smut susceptible). Eighty-four of these primers proved to be polymorphic while only one (B17) produced a reproducible polymorphic fragment, which appeared to co-segregate in repulsion with sugarcane smut resistance. A 300-bp B1SM-F/B1SMR SCAR marker was designed based on the sequence alignment of the B17 products. Testing of this SCAR marker on these 12 cultivars confirmed its specificity on two completely and four partially resistant cultivars. Further screening of this SCAR marker on seven additional sugarcane cultivars of Pakistan verified the earlier findings. Therefore, it was deduced that the B1SM-F/B1SM-R SCAR marker is linked with smut resistance in sugarcane. 
NCBI blast of the SCAR marker sequence showed no homology to any of the earlier identified sequences in GenBank. The newly discovered B1SMF/B1SM-R SCAR marker for sugarcane smut resistance will facilitate sugarcane breeding programs since it can be used for marker-assisted selection in sugarcane.

\section{Identification of factors involved in foliar resistance to bacteria in potato}

D. A. HALTERMAN (1), P. Basnal (2), (1) USDA ARS, Madison, WI, USA; (2) Madison Area Technical College, Madison, WI, USA

Foliar bacterial pathogens are a constant problem of many Solanaceous crops including tomato, pepper, and eggplant. However, cultivated potato has benefitted from low infection rates from most pathogenic Pseudomonas and Xanthomonas species. The purpose of this project is to understand the mechanisms involved in limiting infection of bacteria in the foliage of potato. Using P. syringae pv. tomato DC3000, we have screened many popular potato cultivars and found them all to be immune. Using DC3000, we expanded our screen to over 100 wild species accessions of potato. The majority of these species were susceptible or tolerant, suggesting that resistance to foliar pathogens may have been selected during cultivation of potato as a food crop. A population between wild and cultivated potato was developed and is segregating for immunity. This population has been used for SNP marker analysis in order to identify loci involved in immunity to foliar bacterial pathogens in potato.

Using decoys to expand the recognition specificity of a plant disease resistance protein M. HELM, R. W. Innes, Indiana University, Bloomington, IN, USA

Genetic-based disease resistance is the most effective and environmentally sustainable approach to protecting crops from disease. Although significant progress has been made with respect to understanding the mechanistic basis of plant-pathogen interactions, a remaining challenge is to expand the recognition specificity of plant resistance $(R)$ proteins to confer entirely new specificities. We recently reported a novel approach for expanding the recognition specificity of an Arabidopsis $R$ protein by generating 'decoy' proteins that function as substrates for pathogen proteases. This strategy is based on the observation that modifying the protease cleavage site within Arabidopsis PBS1, a substrate of the AvrPphB cysteine protease, expands the recognition specificity of the Arabidopsis RPS5 immune response pathway. Thus, the specificity of RPS5 can be changed simply by altering the protease cleavage sequence within PBS1. We are now using this technology to engineer resistance in soybean (Glycine max) to Soybean Mosaic Virus (SMV) based on the recognition of the viral protease by a PBS1 'decoy' protein. Soybean encodes three co-orthologs of PBS1, and all three can be cleaved by AvrPphB. Furthermore, we show soybean recognizes AvrPphB, thus soybean likely contains an $R$ protein that detects PBS1 cleavage. We hypothesize that we will be able to engineer resistance to SMV by introducing a cleavage site recognized by the SMV protease into a soybean PBS1 ortholog.

Identification of major-gene resistance to Leptosphaeria maculans in winter canola cultivars and breeding lines F. CEVALLOS (1), M. Stamm (2), J. P. Damicone (1), (1) Oklahoma State University, Stillwater, OK, USA; (2) Kansas State University, Manhattan, KS, USA

Black leg caused by Leptosphaeria maculans, is the most important disease of winter canola in Oklahoma and worldwide. Genetic resistance is currently the most effective and economic strategy to reduce the impact of this disease. Identifying major-resistance genes in winter canola is important to make decisions on which cultivars to plant and what breeding lines to develop. Cultivars and breeding lines from the Kansas State University canola breeding program were screened for seedling resistance to $L$. maculans using isolates with different avirulence genotypes (races). Local races (AvrLm6,7 and AvrLm 1,6,7), and one Australian race ( $A v L m 1,2,4,7, S)$ were used. Seedling responses were resistant if $>80 \%$ of plants showed a resistance response, susceptible if $>80 \%$ of plants showed a susceptible response, and heterogeneous when the cultivar-isolate interaction showed that $20 \%$ to $80 \%$ of the plants were resistant. Cultivars resistant to local races were found to carry genes $R \operatorname{lm} 1, R \operatorname{lm} 6$, and/or $R \operatorname{lm} 7$. Some breeding lines were susceptible to all three races and the lack of specific resistance genes was inferred. However, most breeding lines showed heterogeneous resistance to all three races, which means a possible presence of the genes $R \operatorname{lm} 1, R \operatorname{lm} 2, R \operatorname{lm} 6$ and/or $R \operatorname{lm} 7$ and a high probability of harboring $R \operatorname{lm} 4$. Planting cultivars with major gene resistance could reduce the impact of black leg disease in winter canola in the Southern Great Plains.

\section{Understanding the genetic control of quantitative resistance to yellow spot in wheat}

E. DINGLASAN (1), D. Singh (1), I. Godwin (2), L. Hickey (1), (1) Queensland Alliance for Agriculture and Food Innovation, Brisbane, AUSTRALIA; (2) The University of Queensland, Brisbane, AUSTRALIA

Yellow spot or tan spot caused by Pyrenophora tritici-repentis (Ptr) is a serious disease of wheat crops globally. Ptr can produce necrotrophic effectors (ToxA, ToxB, ToxC) that interact with specific sensitivity genes in wheat (Tsn1, Tsc2, Tsc1, respectively). Although the absence of effector-host-gene interactions is correlated with resistance there is considerable phenotypic variation in response to Ptr. This suggests the role of additional genetic factors that may contribute quantitative forms of resistance like in other cereal-pathosystems. Here, we report a rapid phenotyping protocol utilising accelerated growth conditions (constant light and controlled temperature) suitable for evaluating adult plant response to Ptr. The phenotyping technique and ToxA bioassay was used to screen 300 diverse wheat accessions originally sourced from the Vavilov Institute. Genome-wide association studies using DArTseq markers identified novel genomic regions conferring resistance. Surprisingly, some accessions carried Tsn1 yet displayed a high degree of resistance to ToxA-producing races. To further examine these genetic factors, a donor accession was crossed to Banks, a susceptible Australian cultivar, to develop a recombinant inbred line (RIL) mapping population, which was fixed for Tsn1. The population was genotyped using DArT-seq and evaluated for response to Ptr and ToxA. Thus, current efforts are focussed on understanding the genetics and mechanisms of resistance to Ptr in the presence of Tsn1. We propose manipulation of host loci that do not interact with effectors could deliver an alternative solution for wheat breeders instead of removing major sensitivity loci that are widespread in elite germplasm.

\section{Quantitative analysis of wilt using time lapse videography}

E. LARSON (1), Q. Jiang (2), M. K. Clayton (3), A. O. Charkowski (4), (1) University of Wisconsin-Madison, Madison, WI, USA; (2) Citi Bank, Madison, WI, USA; (3) University of Wisconsin, Madison, WI, USA; (4) Colorado State University, Fort Collins, CO, USA

Pectobacterium caratovorum $(\mathrm{Pc}$ ) causes soft rot, stem wilt, and blackleg in potato (Solanum tuberosum). Genetic resistance presents the best potential for controlling Pc, but there are currently no resistant cultivars available. Phenotyping potato responses to Pc is difficult because environmental factors play a large role in symptom development. In addition, most potato cultivars are tetraploid, making mapping of complex traits, such as Pc resistance, difficult. Previously, we used a petiole assay to identify highly resistant and highly susceptible clones in an F2 population derived from a cross between the Pc susceptible doubled monoploid S. tuberosum clone DM1-3 and the diploid S. chacoense clone M6, which is more resistant to wilt symptoms when inoculated with Pc than DM1-3. To further study this system, we stab inoculated whole plants of DM1-3, M6, and select F2 lines and recorded their response using time lapse videography. To analyze plant response, we used a greenness ratio video assay (GRVA) designed to compare the change in the ratio of green color between two treatments over time using a functional concurrent linear model for time series data, developed in R. We found plant age was associated with wilting. This novel time lapse video assay provides a quantitative analysis of a complex trait and its behavior over time and facilitates phenotyping for future genetic analysis of potato.

\section{Genotypic and phenotypic evaluation of Pyrenophora tritici-repentis isolates for their race structure in South Dakota} S. ABDULLAH (1), S. Sehgal (1), K. D. Glover (1), S. Ali (2), (1) South Dakota State University, Brookings, SD, USA; (2) South Dakota State Univ, Brookings, SD, USA 
The fungus Pyrenophora tritici-repentis (PTR), causes tan spot an important foliar disease of durum and common wheat in the US Northern Great Plains. Eight races of $P T R$ has been identified and resistance in the host to individual race is controlled by independent gene (s). Knowledge of virulence variation in PTR in the region is an important step in the development of durable tan spot resistant cultivars. We characterized 569 PTR isolates for the presence of PTR ToxA and Ptr ToxB genes using Ptr ToxA and Ptr ToxB specific primers. Of 569, 510, and 2 isolates amplified Ptr ToxA, and Ptr ToxB gene, whereas, 52 isolates amplified neither of the two toxins, respectively. Further, we race typed randomly selected 134 isolates (from 569) on tan spot wheat differential set. Of 134 isolates, $74.6 \%(100), 0.4 \%(1), 18.8 \%(25)$, and $1.2 \%$ (2) were identified as race 1, 2, 4, and 5, respectively. Six (4.4\%) of the isolates lacked Ptr ToxA gene but behaved like race 2. Our results indicate though race 1 is most common but diversity in PTR population exists in South Dakota and need to periodically monitor for any potential new races. The isolates lacking Ptr ToxA are under investigation to study potential novel toxin (s) and their association with necrosis symptoms.

Response of wheat cultivars to tan spot against race 1,5 , and unknown race isolates in South Dakota

S. ABDULLAH (1), S. Sehgal (1), K. D. Glover (1), S. Ali (2), (1) South Dakota State University, Brookings, SD, USA; (2) South Dakota State Univ, Brookings, SD, USA

Tan spot (TS) is one of the important leaf spotting diseases of wheat in South Dakota and its neighboring states. TS is caused by an ascomycete fungus Pyrenophora tritici-repentis (PTR). Eight races have been identified in the fungal population based on an isolate ability to produce necrosis and chlorosis symptoms on the wheat differential set. The fungus is known to produces three host-selective Ptr ToxA, Ptr ToxB, and Ptr ToxC toxins, which are associated with necrosis and chlorosis symptoms. In South Dakota, we have observed Race 1 and race 5, and some PTR isolates similar to race 2 but lacking Ptr ToxA gene. The response of wheat cultivars widely grown in the region to these commonly observed PTR races/isolates is important to avoid any potential disease epidemic. In this study, 45 cultivars/lines were screened against race 1 and race 5, a new isolate RO5-23 (similar to race 2 but lacked Ptr ToxA gene), and Ptr ToxA at seedling stage in a greenhouse. Wheat cultivars react differentially to both races, RO5-23 isolate, and Ptr ToxA. Seven wheat cultivars showed susceptibility (15\%) to races 1 and 5 . Only three cultivars react differentially to race 1 and RO-5-23 isolate. Wheat cultivars susceptible to both races 1 and 5 could play a role in their establishment and development of new races. Further investigation of wheat genotypes reacting differentially to RO-5-23 and race 1 would help us in understanding the genetic basis of host-parasite interaction in RO-5-23.

High resolution genetic dissection of two disease resistance genes in common bean cultivar Ouro Negro G. Valentini (1), O. P. Hurtado-Gonzales (2), S. de Lima Castro (1), M. Gonçalves Vidigal (1), Q. Song (2), M. PASTOR-CORRALES (2), (1) Universidade Estadual de Maringá, Maringa, PR, BRAZIL; (2) Soybean Genomics \& Improvement Lab, BARC-West, ARS-USDA, Beltsville, MD, USA

Gene pyramiding of disease resistance genes is a cost-effective strategy to manage common bean diseases. The Ouro Negro common bean cultivar carries three closely linked disease resistance genes that confer resistance to the highly variable pathogens causing the rust, anthracnose, and angular leaf spot diseases, respectively. The objective of this study was to perform a high resolution genetic dissection of the rust and anthracnose resistance genes in Ouro Negro. These studies were conducted using $\mathrm{F}_{2: 3}$ families derived from the Rudá (susceptible) $\times$ Ouro Negro (resistant) cross. To investigate the cosegregation between the rust and anthracnose genes, $\mathrm{F}_{2: 3}$ families were phenotyped separately with races of the rust and anthracnose pathogens. These studies established that these two genes were closely linked. To develop markers tightly linked to these genes, we performed bulk segregant analysis combined with SNP genotyping of $179 \mathrm{~F}_{2: 3}$ families, comprising 4,293 $\mathrm{F}_{2: 3}$ plants, using the BARCBEAN6K_3 SNP chip containing 5,398 SNPs. We then used SSR and KASP markers specifically designed for the candidate region containing the rust and anthracnose loci to create a high-resolution linkage map. This map revealed that the rust and anthracnose loci were located in chromosome 4 of common bean and were positioned at $2.2 \mathrm{cM}$ from each other. Several KASP and SSR markers were tightly linked to these two loci. These markers are being validated in a wide range of common bean cultivars for their utility in marker-assisted selection. In addition, both resistance loci contained a cluster of five to seven disease resistance NB-ARCLRR genes.

\section{Identification of markers associated with race-specific resistance to Aphanomyces root rot in alfalfa}

D. A. SAMAC (1), B. Bucciarelli (1), M. Dornbusch (2), S. Miller (3), L. X. Yu (4), (1) USDA ARS, St Paul, MN, USA; (2) USDA-ARS, St Paul, MN, USA; (3) USDA-ARS, St. Paul, MN, USA; (4) USDA-ARS, Prosser, WA, USA

Aphanomyces root rot, caused by Aphanomyces euteiches, is one of the most important diseases of alfalfa in the United States. Two races of the pathogen are recognized and although most cultivars are resistant to race 1, fewer have resistance to race 2, the predominant race in North America. Molecular markers are needed to facilitate breeding for resistance and to clarify race/resistance gene structure. Resistant and susceptible seedlings were identified from three resistant cultivars, WAPH1, WAPH5 and 53V52, and used as parents to produce F1 populations. Severity of symptoms corresponded with amount of pathogen DNA and oospore density in roots. Race-specific resistance involves a hypersensitive response of individual epidermal or cortical cells upon pathogen attack followed by suberization of cells surrounding the stele and strong autofluorescence in cortical cells, indicating the presence of phenolic compounds. Segregation ratios of F1 populations suggested that resistance to race 1 in WAPH1 is conditioned by a single gene but resistance to race 1 is multigenic in WAPH5 and 53V52, and resistance to race 2 is multigenic in all three cultivars. Segregation for resistance to seven strains of $A$. euteiches in $70 \mathrm{~F} 1$ full-sib plants derived from $53 \mathrm{~V} 52$ suggested the presence of clustered resistance genes and multiple race types. Identification of resistance gene loci is in progress using genotyping by sequencing and genetic mapping of F1 populations.

Development and evaluation of four molecular markers tightly linked to the Potato virus $\boldsymbol{Y}$ resistance gene $\boldsymbol{R} \boldsymbol{y}_{\text {chc }}$ in diploid potato populations A. C. FULLADOLSA (1), S. H. Jansky (2), D. R. Smith (1), C. M. Abramczak (1), A. O. Charkowski (3), (1) University of Wisconsin-Madison, Madison, WI, USA; (2) USDA-ARS, University of Wisconsin-Madison, Madison, WI, USA; (3) Colorado State University, Fort Collins, CO, USA

In the last 15 years, Potato virus $Y$ (PVY) has been the main pathogen causing seed potato lot rejections in North America. The most efficient and environmentally sound method of limiting incidence and spread of PVY is the use of virus resistant potato cultivars. Several genes for extreme resistance to PVY have been found in wild potato relatives. We identified a PVY resistant, diploid clone of Solanum chacoense (CHC 39-7) carrying the resistance gene $R y_{\text {chc }}$, previously mapped to the distal end of potato chromosome IX. CHC 39-7 was crossed with the susceptible, diploid clone of S. tuberosum, US-W4, and a resistant F1 progeny, XD3, was selfed to produce a segregating population. The genome of CHC 39-7 was sequenced and used to develop two TaqMan ${ }^{\circledR}$ SNP markers and two SCAR markers linked to $R y_{c h c}$. Phenotypic and genotypic data obtained from the XD3 selfed population did not show Mendelian segregation for resistance but suggested that there was a lethal factor associated with susceptibility. All four markers were tightly linked to $R y_{c h c}$ and were efficient for selecting resistant individuals, with less than $5.2 \%$ recombination. The resistant germplasm, as well as the molecular tools generated and evaluated through this work, will better equip breeders to introduce a new source of PVY resistance into North American potatoes and develop virus resistant potato cultivars.

Development of KASP markers for the identification of Pea seed-borne mosaic virus pathotype P-1 resistant alleles of eIF4E in Pisum sativum K. D. Swisher (1), L. D. PORTER (2), (1) USDA/ARS, Prosser, WA, USA; (2) USDA-ARS, Prosser, WA, USA

Pea seed-borne mosaic virus (PSbMV) is an economically important potyvirus of legumes, specifically of pea (Pisum sativum) where it causes leaf curling, mosaic, and vein discoloration, and can reduce seed quality and quantity. Resistance of $P$. sativum to the P-1 pathotype of PSbMV has been mapped to the eIF4E translation initiation factor, a common resistance gene effective against different members of the family Potyviridae. Two 
kompetitive allele-specific PCR (KASP) markers were designed to target allelic differences previously identified in the eIF4E gene of $P$. sativum. The first marker targeted a single nucleotide polymorphism that generated the W62L amino acid mutation in the resistant eIF4E allele, and the second targeted an alternate resistance allele with a three-nucleotide deletion that generated the S78 amino acid deletion. These two KASP markers were effective in differentiating known susceptible $P$. sativum accessions from those known to have one of these two modes of eIF4E resistance to PSbMV pathotype P-1. The endpoint genotyping analysis used with the KASP markers provides a quick and effective way to identify $P$. sativum plants with PSbMV resistance, and provides an effective tool for plant breeders, who routinely screen for resistance to PSbMV by large, time-consuming greenhouse trials that require phenotypic and serological identification of virus resistant accessions.

Managing bacterial wilt disease of tomato in open field conditions by improving host resistance through transgenic approach S. Kunwar (1), E. da Silva (2), F. B. Iriarte (3), L. Ritchie (2), D. Clark (2), J. H. Freeman (2), R. E. Stall (1), J. B. Jones (4), G. V. Minsavage Jr. (4), C. Zipfel (5), D. M. Horvath (6), M. Paret (7), Q. FAN (8), (1) University of Florida, Gainesville, FL, USA; (2) University of Florida, Quincy, FL, USA; (3) Univ of Florida - IFAS NFREC, Quincy, FL, USA; (4) Department of Plant Pathology, University of Florida, Gainesville, FL, USA; (5) The Sainsbury Laboratory, Norwich, UNITED KINGDOM; (6) 2Blades Foundation, Evanston, IL, USA; (7) North Florida Research and Education Center, University of Florida, Quincy, FL, USA; (8) University of florida, Quincy, FL, USA

Bacterial wilt of tomato, caused by Ralstonia solanacearum, is one of the major constraints for tomato growers worldwide. Two field trials were conducted in the fall season of 2015 and 2016 to investigate whether transgenic tomato lines expressing $E F R$ (Elongation Factor Tu-Receptor) from Arabidopsis thaliana or $B s 2$ gene from pepper or both provides improved resistance to bacterial wilt. The transgenic lines containing EFR and $B s 2$ genes or EFR gene alone, on the highly susceptible FL 8000 background, significantly reduced disease incidence by $34.7-66.5 \%$ in 2015 and by $22.9-33.3 \%$ in 2016 compared to $B s 2$ line (carrying only $B s 2$ ) or non-transformed control. Although, the yields of marketable fruit from $E F R$ or $E F R / B s 2$ lines were statistically not different than those from $B s 2$ lines or non-transformed control, the total yield was significantly improved by $57.2-111.3 \%$ in 2015 and by $57.3-114.2 \%$ in 2016 compared to Bs 2 line or non-transformed control. Following harvest, the basal and middle stem sections of the remaining living plants in the field were assessed for bacterial colonization by dilution plating on modified SMSA media. The results revealed no significant differences in the population at the basal stem. Interestingly, in the middle stem, no bacteria could be recovered from $E F R$ lines or EFR/Bs2 lines in both the field trials but viable bacterial populations were recovered from $B s 2$ and non-transformed control lines at $10^{2}-10^{4} \mathrm{CFU} / \mathrm{g}$ tissue, indicating that upward movement of bacteria is affected in the transgenic lines. This finding indicates the potential of $E F R$ gene for field management of tomato bacterial wilt.

Genomic approaches to dissecting Phytophthora crown and root rot resistnace and applicatons to strawberry improvement S. LEE (1), Y. Noh (2), J. A. Mangandi (3), S. Verma (2), V. Whitaker (4), (1) University of Florida, WIMAUMA, FL, USA; (2) University of Florida, Wimauma, FL, USA; (3) Gulf Coast Research \& Education Center, Wimauma, FL, USA; (4) Univ of Florida, Winauma, FL, USA

Phytophthora crown rot $(\mathrm{PhCR})$ is the most common and a destructive disease in strawberry production in Florida. At the moment, all major strawberry varieties grown in Florida are susceptible to PhCR. Also, soil fumigation before planting is ineffective in controlling this disease because the main inoculum source is infected nursery stock. It is critical to develop new strawberry varieties for PhCR resistance. In our previous study, we identified a major quantitative trait loci (QTL) for PhCR resistance, FaRPc2, located on the distal end of linkage group 7D, using whole-genome SNP genotyping (IStraw90 Axiom ${ }^{\circledR}$ SNP Array) and marker-trait association (FlexQTL ${ }^{\text {TM}}$ ) in a complex, multi-family population. Four major SNP haplotypes (H1, H2, $\mathrm{H} 3$ and $\mathrm{H} 4$ ) were identified at the FaRPc2 locus, and especially the SNP haplotype $\mathrm{H} 3$ was found to be highly associated with the FaRPc2-mediated resistance. SNP-based high-throughput DNA-test such as endpoint-genotyping, high resolution melting (HRM), and kompetitive allele specific (KASP) assay has been developed for the FaRPc2 resistance allele. For allele mining and candidate gene discovery for PhCR resistance, next-generation sequencing technology has been conducted. In this presentation, it will be discussed how integrated genomic approaches are able to identify specific genes and genetic markers associated with PhCR resistance for new cultivar development. We also provide select examples of contemporary work involving various genomic strategies to identify the resistance genes, and further applications for durable and sustainable PhCR resistance breeding in strawberry.

\section{QTL mapping and candidate gene discovery in potato for resistance to the Verticillium wilt pathogen Verticillium dahliae}

A. KUMAR (1), S. H. Jansky (2), J. Endelman (3), D. A. Halterman (4), (1) University of Wisconsin-Madison, Madison, WI, USA; (2) USDA-ARS, University of Wisconsin-Madison, Madison, WI, USA; (3) University of Wisconsin-Madison, Madison, WI, Madison, WI, USA; (4) USDA ARS, Madison, WI, USA

Verticillium wilt (VW) of potato (Solanum tuberosum), caused by fungal pathogens Verticillium dahliae and V. albo-atrum, is a disease of major significance throughout the potato growing regions in the world. In the past, researchers have focused on the Ve gene, which is a major dominant gene that confers resistance to VW in tomato. Using $V e$ sequence information from tomato, orthologues in potato were sequenced to develop functional molecular markers. However, the use of such markers was complicated by gene duplication and the association between phenotypic and marker data was unreliable. These studies suggested that there might be other genetic determinants of VW resistance in potato. Difficulties in disease phenotyping along with the complicated genetics of tetraploid potato cultivars have limited the progress in this direction and no major resistance gene from potato has been reported so far. We developed an F2 mapping population by selfing an F1 individual, which was derived from a cross between two homozygous diploid parents, S. tuberosum DM1-3 (susceptible to VW) and S. chacoense M6 (resistant to VW). This population was SNP genotyped and phenotyped using a rooted cutting protocol developed in our lab. Using R/qtl software, a QTL in chromosome 1 was identified, which explains $31 \%$ of phenotypic variation. Using SNP marker data, 22 genes were found in the QTL region, among which, based on bioinformatic analysis, two genes have been selected for further functional validation studies.

Characterization of Verticillium dahliae Disease Reactions in Lettuce Differential Cultivars D. P. G. SHORT (1), K. D. Puri (1), G. V. Sandoya (2), I. Simko (3), R. J. Hayes (4,5), K. V. Subbarao (6), (1) University of California, Davis, Salinas, CA, USA; (2) University of Florida, Gainseville, Belle Glade, FL, USA; (3) USDA, ARS, Crop Improvement and Protection Unit, Salinas, CA, USA; (4) USDA, ARS, Corvallis, OR, USA; (5) USDA, ARS, Forage Seed and Cereal Research Unit, Corvallis, OR, USA; (6) University of California at Davis, c/o U.S. Agricultural Research Station, Salinas, CA, USA

Soilborne diseases significantly affect high value crops in coastal California, including lettuce and strawberry. Breeding crops for pathogen resistance is a strategy for the sustainable management of crop disease. Verticillium dahliae is a soilborne fungus that causes wilt and economic losses in a broad range of hosts. Homologs of a conserved plant locus that confers resistance to Verticillium infection were first identified in tomato and are now known to occur widely in plants. In lettuce, a dominant allele of the locus $V r l$ confers resistance to race 1 isolates of $V$. dahliae, which are defined by the fungal avirulence gene $A v r 1$. Currently, only partial resistance in lettuce to $V$. dahliae race 2 is available in a few plant introductions (PIs). Pathogenicity tests involving rootstocks of tomato differentials have led to the identification of race 3 among isolates that previously belonged to race 2 . Experiments involving several lettuce cultivars and $V$. dahliae isolates and cultivars revealed diverse reactions that point to the potential of putative race 3 differentials among lettuce cultivars. Results of greenhouse experiments confirming the pathogenicity and virulence of a diverse panel of $V$. dahliae tested with race 1-resistant and race 2-partially resistant PIs will be presented, as well as the status of allelism tests to determine the nature of putative race 2 resistance in lettuce. 
Interactions between diverse Clavibacter michiganensis subsp. nebraskensis isolates and maize inbreds and hybrids influence severity of Goss's wilt

R. R. MCNALLY, B. T. Webster, R. Curland, C. A. Ishimaru, D. K. Malvick, University of Minnesota, St. Paul, MN, USA

Clavibacter michiganensis subsp. nebraskensis $(\mathrm{Cmn})$ is the causal agent of Goss's wilt, a bacterial disease of maize. Although first observed in Nebraska in 1969, this disease reemerged in the past 10 years when it became important in multiple Midwestern states and distribution expanded to 18 states and two Canadian provinces. To investigate the reemergence of Goss's wilt and virulence diversity in Cmn, leaves of four maize inbreds and four hybrids with different levels of resistance to Goss's wilt were inoculated with $\mathrm{Cmn}$ isolates in field and greenhouse studies. The isolates (n=45) were collected from multiple states between 1969 and 2014. All hybrids and inbreds were susceptible to Goss's wilt based on lesion development, and no complete resistance was observed with any isolate or host combination. While resistance ratings affected disease severity and yield, inbreds were not more susceptible to contemporary isolates of $\mathrm{Cmn}$ than to older isolates. Our results also show that $\mathrm{Cmn}$ exhibits diverse virulence phenotypes ranging from avirulent to different levels of virulence within and among hybrids and inbreds. Most isolates displayed decreased virulence on resistant maize, but some isolates were equally virulent on both resistant and susceptible hybrids and inbreds. The results support that resistance to Goss's wilt is quantitative, virulence varies widely among isolates of $\mathrm{Cmn}$, and resistance mechanisms are not equally effective against all isolates.

Development of a sweet cherry pepper line with resistance to the southern root-knot nematode Meloidogyne incognita W. B. RUTTER, S. Buckner, M. Farnham, R. Fery, C. Kousik, U.S. Vegetable Laboratory, USDA, ARS, Charleston, SC, USA

The southern root-knot nematode (Meloidogyne incognita) is a major pathogen of pepper (Capsicum spp.), causing significant yield losses in heavily infected plants. The N-gene confers resistance to $M$. incognita, and has been successfully used to mitigate nematode damage in specific pepper varieties for over 50 years. Unfortunately, the $\mathrm{N}$-gene is only available in a limited number of commercial pepper varieties. With consumer demand for a selection of pepper varieties, and the removal of many traditional fumigant nematicides from the market, there is an increased need for a wider variety of peppers with resistance to $M$. incognita. To help fill this need, we have used a recurrent backcross strategy to develop a variety of sweet cherry pepper that carries the N-gene. This new sweet cherry variety shows a high level of resistance to $M$. incognita, equal to that of the widely-used nematode resistant variety Charleston Belle. Under field conditions it produces fruit yields, shape and color comparable to its recurrent commercial parent 'Sweet Cherry'. The release of this sweet cherry pepper variety will provide useful options for pepper growers and breeders looking to introduce nematode resistance in their fields and breeding programs.

GBS-SNP-based linkage mapping and QTL associated with resistance to race 1 Fusarium wilt in Cucumis melo W. P. WECHTER (1), S. Branham (2), A. Levi (2), (1) USDA-ARS, Charleston, SC, USA; (2) USDA, ARS, Charleston, SC, USA

With the loss of inexpensive soil fumigants due to environmental and human health concerns, soil-borne pathogens are becoming a greater concern to vegetable growers. The use of host resistance to combat these pathogens is a method of choice for many growers and consumers of these crops. Marker assisted selection (MAS) can speed the introgression of resistance from lesser quality resistant lines into a high-quality, but susceptible cultivars. Identification and development of markers linked to the genes responsible for resistance is essential for development of MAS systems. Utilizing a recombinant inbred line derived from the multi-pathogen resistant Cucumis melo breeding line MR-1 by the susceptible cultivar Ananas Yokneum, QTL associated with resistance to Fusarium oxysporum f. sp. melonis race 1 were identified. Genotyping-by-sequencing was performed on 190 RILs in association with screening for resistance to this pathogen. Mapping and QTL data will be presented.

Gene expression profiling of resistant vs susceptible tomato (Solanum lycopersicum) cultivar after challenging with Ralstonia solanacearum M. DASGUPTA (1), M. R. Sahoo (1), S. V. Ngachan (2), (1) University of Tennessee, Knoxville, TN, USA; (2) ICAR Research Complex for NEH Region, Meghalaya, INDIA

Tomato production is threatened by bacterial wilt caused by a gram-negative bacterium Ralstonia solanacearum (Rs). During bacterial wilt pathogenesis, $R s$ invades the host through wounded roots; effectively colonizes the host xylem tissue and cause wilting in the whole plant. Because of its soil-borne nature, the protective measures turn out to be ineffective. Identification and characterization of host gene changes those are potentially involved during $R s$-tomato interaction, is important to understand the molecular basis of resistance. We have conducted whole genome transcriptome analysis on resistant (Megha Tomato-3; MT3) and susceptible (Pusa Ruby; PR) tomato cultivars challenged with either R. solanacearum or mock inoculations. At the onset of wilt symptom appearance ( 5 days post inoculation), about 374 genes differentially expressed (DEGs) in resistant cultivar; 151 being induced and 223 being repressed and only 11 genes were differentially expressed in susceptible cultivar; out of which 9 were up and 2 were down regulated. The Mapman analysis revealed that the up regulated DEGs in the resistant cultivar were involved in various metabolic process (lipid and carbohydrate), signal transduction, transcriptional regulation, cell wall organisation and response to various stresses (biotic and abiotic). Among the up regulated DEGs, one third represent defense associated genes in resistant cultivar MT-3. The most highly up regulated gene was a tomato oleosin-1-like protein, 13-fold induced. The present study provides insights into various reactions underlying the successful establishment of resistance to Rs in MT-3 and helps in the identification of important defense related genes in tomato for wilt management.

Quantitative Trait Loci (QTL) conferring resistance in soybean to root rot caused by Pythium oopapillum E. LERCH (1), N. Arritt (2), A. E. Dorrance (3), A. E. Robertson (4), (1) Iowa State University, Ames, IA, USA; (2) Iowa State University, Ames, IA, USA; (3) The Ohio State University, Wooster, OH, USA; (4) Iowa State University, Department of Plant Pathology, Ames, IA, USA

Pythium species are causal agents of soybean seedling disease, specifically pre- and post-emergence damping-off and root rot. One of the most prevalent species recovered from damped-off soybeans in a seedling disease survey done in the Midwest was $P$. oopapillum. Resistance is often an effective tool to manage disease. Screening the parents of the SoyNAM population for resistance to damping-off caused by $P$. oopapillum identified PI 398.881 as a source of potential resistance. Consequently, the objective of this research was to identify quantitative trait loci (QTL) contributing to resistance to root rot caused by $P$. oopapillum in the SoyNAM population, PI $398.881 \times$ IA3023. Three traits were assessed as a measure of disease; root rot severity (RRS), adjusted germination (GERM), and adjusted wet root weight (WRW). A significant correlation ( $\mathrm{p}<0.0001)$ between traits was found for RRS/GERM and RRS/WRW. Inclusive composite interval mapping identified two QTL, one each on chromosomes 3 and 4, each with a logarithm of the odds value above 3. Together the two QTL explain, 21.07\% of the variance seen in RRS. These QTL were located in regions with known resistance to other root pathogens. Incorporation of these QTL into adapted soybean cultivars will provide a useful tool to manage damping-off and protect early season plant stands.

Physiologic specialization of Puccinia hordei on barley in the United States from 2012 to 2016 M. ROUSE, USDA-ARS Cereal Disease Laboratory, Saint Paul, MN, USA

Collections of Puccinia hordei, the barley leaf rust pathogen, were received from collaborators around the United States from 2012 through 2016. Annually 21-54 single pustule isolates were derived from 20-41 collections received. The single pustule isolates were increased on susceptible barley cultivars Robust or Moore. $P$. hordei urediniospores were inoculated onto a differential set of 15 barley single-gene lines for barley leaf rust resistance genes. The $P$. hordei population in the United States was fixed or nearly fixed for virulence to resistance genes $R p h 1, R p h 2$, $R p h 4, R p h 6$, and $R p h 8$. The population was fixed for avirulence to $R p h 9, R p h 12$, and $R p h 15$. The isolates varied in response to $R p h 3, R p h 5, R p h 7, R p h 10, R p h 13$, and $R p h 14$. 
Isolates from the western United States were more diverse in virulence profile compared to isolates from the eastern United States. These data suggest the efficacy of barley leaf rust resistance genes $R p h 9, R p h 12$, and $R p h 15$ for protecting barley from leaf rust in the United States.

The Colombian strategy against devastating coffee diseases: Preparation for Coffee Berry Disease (Colletotrichum kahawae subsp. kahawae) C. E. MALDONADO, L. Angel, C. P. Florez, C. A. Angel, A. L. Gaitan, CENICAFE, Colombia, Manizales, COLOMBIA

Colombia is the third largest world coffee producer with 14.5 million $60 \mathrm{~kg}$ bags, being the most important crop in the country planted by 555,000 families in 932,000 hectares. Coffee Leaf Rust (CLR, Hemileia vastatrix) and Coffee Berry Disease (CBD, Colletotrichum kahawae subsp. kahawae) are two of the most devastating diseases worldwide. In absence of them, CENICAFÉ started a breeding program since 1960s, focused first on CLR, crossing the susceptible C. arabica cv. Caturra and the resistant Timor Hybrid (TH). TH is also source for resistance to CBD and bacterial blight (Pseudomonas syringae pv. garcae), still absent in Colombia, and to Root Knot Nematode (Meloidogyne exigua). Before and after CLR was found in Colombia in 1983, a complete strategy based on regulations, biology and epidemiology studies, chemical control, varieties, and extension was established for integrated management. Multiline resistant varieties were released: "Colombia" in 1982, "Tabi" in 2002 and "Castillo" in 2005, these three currently planted in $74 \%$ of the area, and "Cenicafé 1" in 2016. Based on CLR, a similar strategy is being prepared for CBD. Here, by using bioinformatics, mapping, and comparative genomics, this study determined the distribution of CBD resistance gene $C k$-1, derived from TH. Sat207 and Sat 235 microsatellites were linked to resistance, and candidate CBD and CLR resistance genes were found. $97.1 \%$ of the lines of "Colombia" variety, 83.7\% of "Castillo ${ }^{\mathbb{B}}$ ", $76 \%$ of "Tabi", and $100 \%$ of "Cenicafe 1" showed evidence of $C k-1$ associated markers, and an introgressed block from TH contains six candidate CBD and CLR resistance gene analogs.

Genetics behind avirulence: The identification of avr-factors in Magnaporthe oryzae D. TATE (1), T. K. Mitchell (2), (1) The Ohio State University, Columbus, OH, USA; (2) Ohio State University, Columbus, OH, USA

Rice blast disease, caused by Magnaporthe oryzae, accounts for 10-30\% of yearly rice loss which is estimated to represent enough food to feed 60 million people. The study of interactions between M. oryzae and rice can be used to help mitigate one of the most devastating diseases plaguing a primary food source and knowledge of this pathosystem can be used to manage related fungal diseases. The objective of this study is to identify avirulence factors related to the Pi-2 resistance (R)-gene. In previous studies, we identified five candidate genes by Agrobacterium tumefaciens-mediated transformation; infection assays; and TAIL-PCR with the incompatible strain KJ201. These genes were cloned into the compatible strain CHNOS. To determine the genes' ability to regulate virulence, Pi-2 R plants were infected with the CHNOS transformants and disease assessed. Punch inoculations demonstrated that transformed line CHNOS4842 produced a phenotype similar to KJ201 with a significance level of 0.05 . Further, the transformants distribution of virulence stages was evaluated through leaf sheath assays and CHNOS4842's distribution was found to be similar to KJ201 with a significance level of 0.001 . Sequencing results between KJ201 and CHNOS show two polymorphisms in regions thought to regulate translation. These findings indicate that MGG_04842 plays a role in the virulence of the rice pathogen M. oryzae and that this phenotype is potentially translationally regulated.

Identification of markers associated with resistance to Leptosphaeria maculans in a collection of Brassica napus plant introductions S. M. MANSOURIPOUR (1), M. Rahman (1), L. E. del Rio Mendoza (2), (1) North Dakota State University, Fargo, ND, USA; (2) North Dakota State Univ, Fargo, ND, USA

Blackleg, caused by the fungus Leptosphaeria maculans (Desm.) Ces. et de Not. has become the most important disease affecting canola production in North Dakota, state that contributes $>85 \%$ of the canola production in the United States. The objective of this study was to identify markers associated with resistance to blackleg. Seedlings of 229 Brassica napus accessions were inoculated with a mixture of five isolates from pathogenicity group 4 of $L$. maculans in replicated greenhouse trials. Disease reaction was recorded 12 days after inoculation. Genomic DNA samples were extracted from all accessions and sequenced. Single nucleotide markers were used for association mapping analysis. One major marker (A04 15177269) associated with resistance was identified in chromosome A04 with $P<0.000142$ and 31 other markers distributed in A04 and 10 other chromosomes were identified with $P<0.00106$. These markers could be used for marker-assisted breeding in future.

\section{Reaction of potato genotypes to infection with Tomato chlorosis virus (ToCV)}

L. R. PINTO, D. Bampi, J. A. M. Rezende, A. Bergamin Filho, University of São Paulo - ESALQ, Piracicaba, BRAZIL

Tomato chlorosis virus (ToCV) is a member of the genus Crinivirus (family Closteroviridae), phloem limited and transmitted by whitefly in a semi persistent manner. In Brazil, ToCV has been found affecting mainly tomato and potato crops. Therefore, the objective of this work was to evaluate the reaction of potato genotypes to infection with ToCV. Potato tubers of 21 genotypes (five tubers each) were randomly planted on polypropylene trays and kept on an insect-proof cage. Two weeks after germination, plants were inoculated with ToCV with Bemisia tabaci biotype B (MEAM1). Approximately 1000 viruliferous insects per day were released into the cage, during five consecutive days. After that, plants were sprayed with insecticide, individually transplanted to $2 \mathrm{~L}$ pots for further evaluations based on symptoms expression and quantification of virus titer by qRT-PCR. Evaluations were carried out 45 days after inoculation. None of the potato genotypes was resistant to infection with ToCV. Virus titer varied among genotypes. Variety Camila was the only one symptomless and with the lowest virus titer. Such characteristic might affect virus acquisition/transmission and consequently the epidemiology of the disease under field conditions. Studies to evaluate the effect of virus titer in different potato genotypes on virus acquisition by $B$. tabaci are under way.

Reaction of sweet potato genotypes to the root-knot nematode (Meloidogyne javanica)

A. FURTADO SILVEIRA MELLO, R. Souza, A. Macedo, D. Biscaia, A. Moita, R. Lemos, L. Fonseca, J. Pìnheiro, EMBRAPA, Brasilia, BRAZIL

One of the main obstacles to food production in many developing countries is the damage caused by nematodes, especially those forming galls, belonging to the genus Meloidogyne. Symptoms are associated to reduction of fibrous roots, plant growth and yield loss. The objective of this work was to characterize the resistance level of one Embrapa pre-commercial genotype of sweet potato to the root-knot nematode Meloidogyne javanica. In addition to the pre-commercial clone four different sweet potato commercial cultivars, one resistant and one susceptible tomato controls, Nemadoro and Rutgers, respectively, were used. The experiment was installed in $0.3 \mathrm{~L}$ plastic pots and conducted under greenhouse conditions on a completely randomized design, with six replications. Plants were evaluated 47 days after soil infestation. The classification of resistance levels was performed according to the Reproduction Factor (RF), gall index (GI), egg mass index (IMO) and number of eggs per gram of root (NEGR). M. javanica presented low aggressiveness in the evaluated genotypes but the pattern of the control results were consistent with previous evaluations. The pre-commercial clone was statistically different from the susceptible tomato cultivar in all parameters evaluated and was not statistically different to the resistant tomato control. In addition, this new sweet potato clone also was more resistant than its direct agronomic control, the sweet potato Beauregard.

\section{Tomato Spotted Wilt Virus (TSWV) on Pepper Seedlings (Capsicum annuum) in a Breeding Nursery and Difference in Susceptibility among} Breeding Lines

B. S. KIM, S. H. Lee, Kyungpook National University, Daegu, Korea, Republic of (South)

Severe mosaic and wilting symptoms occurred on greenhouse-grown seedlings of breeding lines of pepper (Capsicum annuum L.) in spring, 2016. In electron microscopic and RT-PCR examination, the causal agent was identified as Tomato Spotted Wilt Virus (TSWV). The disease fast spread to all the 
pepper plants in the greenhouse causing necrotic spots on the leaves and stem tips curving down. Most of the breeding materials in the greenhouse were susceptible but variation in susceptibility was observed among the materials. When the plants were transplanted to greenhouse, the plants developed diverse symptoms on foliage and fruits ranging from concentric ring spots, downward curving of shoots, and colored mosaic spots on maturing fruits. A few materials including lines developed by incorporation of resistance to Phytophthora capsici into Subicho, a land race in Yeongyang, Gyeongbuk province, Korea, and a few other lines showed some tolerance to the disease to bear fruits to mature. In another greenhouse where only a few infected plants were mixed in planting, those tolerant lines picked up the disease in less frequency and later than susceptible lines.

\section{Assessment of soybean breeding lines for resistance to Phomopsis seed decay from field trials in Stoneville, Mississippi} S. LI (1), J. R. Smith (2), (1) USDA ARS CGRU, Stoneville, MS, USA; (2) USDA-ARS, Stoneville, MS, USA

Phomopsis seed decay (PSD) is one of the most important seed diseases in soybean. A fungal pathogen, Phomopsis longicolla (syn. Diaporthe longicolla), is the primary causal agent of PSD. Planting PSD-resistant soybean cultivars is the most effective strategy to manage this disease. However, few commercial cultivars have resistance to PSD. For this study, breeding lines were developed from three crosses between PSD-resistant and high germination parents (DS25-1 × PI 424324B, DS25-1 × PI 417274, DS30-1 × PI 424324B) and from one cross between two PSD-resistant parents (PI $80837 \times$ SS93-6181). Based on seed plating assays of 122 breeding lines from field trails in 2014 and 2015 , the range of percent seed infection by $P$. longicolla among all lines tested was from 0 to $52 \%$, with an overall line mean of $1.9 \%$. There were significant differences in the reaction to PSD among lines derived from DS25-1 $\times$ PI 424324B and PI $80837 \times$ SS93-6181. However, there was no PSD incidence in lines derived from DS25-1 $\times$ PI 417274 and DS30-1 $\times$ PI 424324B. Breeding lines with no Phomopsis seed infection in both years will be tested again in 2017 . Resistant lines will be released for future use in developing PSD-resistant soybean cultivars.

\section{Characterization of black walnut genotypes for resistance to Thousand Cankers disease} E. LAURITZEN, C. Nischwitz, Utah State University, Logan, UT, USA

Black walnut, Juglans nigra, is an important nut and timber crop in the United States. Shortly after the beginning of the $21^{\text {st }}$ century, a decline in the health and, later, death of black walnut trees was observed in several western states and the pathogen-vector complex now known as Thousand Cankers Disease (TCD) was discovered. TCD, caused by Geosmithia morbida, is vectored by the walnut twig beetle (WTB), Pityophthorus juglandis. TCD has been confirmed in nine western states and seven eastern states with quarantines issued in 18 states. Cankers develop around the entry holes of the WTB and quickly expand, coalesce and kill the branch or stem. There are currently no management practices for TCD. The research and development of resistant cultivars is important to maintain sensitive ecosystems and livelihoods of forest managers, timber producers and growers. The purpose of this project was to evaluate Juglans nigra, Juglans regia, and Juglans hybrid trees for resistance to TCD through direct inoculation of the pathogen Geosmithia morbida. Inoculation of limbs took place in early summer at two Utah State University research orchards. Inoculated limbs were removed from the tree after senescence and canker size measured. Of the nearly 400 trees inoculated in 2015 and 2016 over ten percent showed resistance to TCD indicated by no or small canker size. The results of this project indicate that breading for resistance to TCD could be a management option for the disease.

\section{Host resistance to Botrytis bunch rot in Vitis spp. and its correlation with Botrytis leaf spot} R. P. NAEGELE, USDA ARS, Parlier, CA, USA

Botrytis cinerea, the causal agent of Botrytis bunch rot, is the number one postharvest disease of fresh grapes in the United States. Fungicide applications are used to manage the disease, but resistant isolates are common and postharvest losses occur annually. Host resistance is needed for long-term management of the disease as fungicide resistance becomes more prevalent. Sources of resistance in grape have been identified, but often have poor fruit quality. In this study, twenty-five grape genotypes (cultivars, breeding lines, and species) were evaluated for fruit and leaf susceptibility to two isolates of Botrytis cinerea. Significant differences in virulence and disease incidence were detected between the two isolates and among lines, respectively. Isolate 16-23 from apricot was more virulent than isolate 449 collected from grape, but no differences in pathogenicity were observed. Most of the Vitis vinifera cultivars had high disease severity in fruit and leaves, while the complex hybrids, $V$. aestivalus, and $V$. arizonica had low to moderate disease severity. Three $V$. vinifera lines had moderate resistance ( $<50 \%$ diseased) to Botrytis bunch rot when inoculated with either isolate. Only two genotypes, both with V. labrusca in their background, had little to no detectable disease across isolates. Correlations were examined among brix, leaf susceptibility, and fruit susceptibility for each of the cultivars. No correlations between brix and disease susceptibility were detected. Moderate resistance to Botrytis bunch rot and leaf spot were detected in $V$. vinifera breeding lines, suggesting these may be potential sources for selecting grapes with resistance to $B$. cinerea and high fruit quality.

Egyptian Blumeria graminis f. sp. tritici virulence frequencies and powdery mildew resistance of Egyptian wheat cultivars A. S. G. ABDELRHIM (1,2), H. Abd-Allah (2), M. Ismail (2), C. Cowger (3), (1) North Carolina State University, Raleigh, NC, USA; (2) Minia University, El Minia, EGYPT; (3) USDA-ARS, Department of Entomology \& Plant Pathology, North Carolina State University., Raleigh, NC, USA

Powdery mildew caused by Blumeria graminis (DC.) Speer f. sp. tritici (Em. Marchal) is a serious disease of wheat that can cause a severe reduction in yield. In Egypt, little information is available about the virulence of the Egyptian $B$. graminis f. sp. tritici population, and the response of Egyptian wheat cultivars to powdery mildew. Virulence frequencies of a representative sample of the Egyptian $B$. graminis f. sp. tritici population were studied. Seven provinces were chosen to represent the country: Qena, Sohag, El Minia, Alexandria, Kafrelsheikh, Dakahlia and Sharqia. Ten isolates from each province, or 70 isolates total, were derived from single ascospores and used for this study. They were inoculated individually on 19 powdery mildew differential lines, each bearing a single resistance $(P m)$ gene. Also, the responses of 20 recent commercial Egyptian wheat cultivars to each of the 70 isolates were evaluated individually. Only five Pm genes (Pm1b, Pm2, Pm 34, Pm37 and Pm53) were effective against at least 70\% of B. graminis f. sp. tritici isolates from each province. Several other genes were effective against most or all isolates from a majority of provinces. Two durum wheat cultivars, BeniSweaf 5 and BeniSweaf 6 , had intermediate responses to a large percentage of the isolates, but all tested bread wheat cultivars showed full susceptibility to all isolates. To enhance mildew resistance in Egyptian wheat cultivars, it is recommended to use combinations of genes that are nationally effective or effective against multiple provincial $B$. graminis $\mathrm{f}$. sp. tritici populations.

\section{Resistance in species of Cucurbitaceae to gummy stem blight caused by Stagonosporopsis citrulli under field conditions} G. RENNBERGER, A. P. Keinath, Coastal Research and Education Center, Clemson University, Charleston, SC, USA

Gummy stem blight, caused by the Stagonosporopsis species complex, is one of the most important diseases of cucurbits. High levels of resistance are rare, and no resistant cultivars are currently available for any commercial cucurbit crop. In spring 2015 and spring and fall 2016,13 previously untested species of Cucurbitaceae and Cucumis melo 'Athena' (a susceptible control) were inoculated in the field. Treatments were arranged in four randomized complete blocks with seven plants per plot. The severity of foliar blight was rated seven, nine and five times, and the incidence of crown cankers was rated three, four and two times, respectively, in each season. There was a species-by-season interaction for both symptoms $(P<0.0001)$. Coccinia grandis and Zehneria pallidinervia were highly resistant to foliar blight in all three seasons and had significantly less severe symptoms than all other species except Sicana odorifera and Zehneria scabra. In the fall of 2016, severity on these two species was as low as on C. grandis and Z. pallidinervia. Coccinia grandis and $S$. odorifera were among the four species with the lowest number of crowns with cankers in each season. Ecballium elaterium was consistently at least as susceptible as 'Athena'. There were significant differences between the tribes and geographic origins represented by the species included. The resistant species identified in this study could be used to identify resistance genes for future breeding efforts. 
Breeding of high quality Korean carrot lines resistant to root-knot nematodes

Y. H. KIM (1), E. Kim (2), Y. Seo (2), Y. S. Kim (3), Y. Park (3), (1) Seoul National University, Seoul, Korea, Republic of (South); (2) Department of Agricultural Biotechnology, Seoul National University, Seoul, Korea, Republic of (South); (3) KC Carrot Breeding Institute, Co. Ltd., Daegu, Korea, Republic of (South)

A total of 56 Korean carrot lines were inoculated with second-stage juveniles $\left(\mathrm{J}_{2}\right)$ of Meloidogyne incognita and M. hapla to examine their responses to the nematode infection determined by the root-knot gall formation \{using gall index (GI)\} at 6 weeks after inoculation. Among these carrot lines, 10 (3 parental +7 inbreed lines) were determined to be resistant (with GI $\leq 1.0)$ to $M$. incognita; 20 (6 parental +14 inbreed), resistant to $M$. hapla; and 9 (3 parental +6 inbreed lines) to both nematodes; but no maternal line was resistant to either nematode. This suggests more Korean carrot lines may be resistant to M. hapla than M. incognita. The histopathological responses of various resistant and susceptible lines were examined after nematode infection. The carrot lines susceptible to the nematodes showed well-developed giant cells in the stele around which xylem vessels were extensively formed regardless of nematode species. However, histopathological responses of the carrot lines resistant to the nematodes differed apparently from those susceptible to the nematodes, showing the formation of under-developed giant cells (or modified cells), confirming their true resistance to the nematodes, which were accompanied by the surrounding necrotic layers in $M$. incognita and cellular degeneration and cytoplasmic vacuolation in $M$. hapla, indicating different resistance mechanisms from each other. Among the carrots resistant to either or both of the nematodes, 8 lines were selected as potential carrot lines to be developed for commercialization because they are all Western cultivar of Nantes type with orange-colored surface and with higher quality (higher carotene and/or sugar contents) than current commercial carrot cultivars.

Foliar and tuber reactions of five fresh market potato cultivars with three Potato virus $\boldsymbol{Y}$ strains

D. A. INGLIS (1), B. Gundersen (1), A. Beissinger (2), A. V. Karasev (3), (1) Washington State University-Mount Vernon NWREC, Mount Vernon, WA, USA; (2) University of Connecticut, Storrs, CT, USA; (3) University of Idaho, Moscow, ID, USA

Potatoes grown in western WA are primarily for fresh market and seed potato sales. Until recently, $\mathrm{PVY}$ was the only known strain of PVY in the area, but now PVY ${ }^{\mathrm{N}-\mathrm{Wi}}$ and $\mathrm{PVY} \mathrm{NTN}^{\mathrm{NT}}$ also are present. To clarify symptoms on 5 common cultivars, greenhouse plants from nuclear seed of All Blue (AB), Chieftain (CH), French Fingerling (FF), Russet Burbank (RB), and Yukon Gold (YG) were inoculated with the 3 strains or buffer in 2016. Pots were arranged in a RCB design ( 4 reps $\times 3$ plants per strain $\times$ cultivar). PVY foliar symptoms across cultivars presented on $51 / 60$ plants $(85 \%)$ for PVY ${ }^{O}$, $59 / 60(98 \%)$ for PVY ${ }^{\mathrm{NTN}}$ and PVY ${ }^{\mathrm{N}-\mathrm{Wi}}$, and $0 / 60(0 \%)$ for buffer; $85 \%$ of symptomatic plants were PVY positive by ELISA. Mosaic displayed on all cultivars $\times$ strains. Veinal necrosis on $>50 \%$ plants occurred for PVY $\times A B, R B$ and $\mathrm{YG}$, and PVY $\mathrm{NTN}^{\mathrm{TTN}} \times$ YG. Leaf drop on $>50 \%$ plants was noted for all reactions except $\mathrm{PVY} \times \mathrm{CH}, \mathrm{PVY}^{\mathrm{NTN}} \times \mathrm{FF}$ and $\mathrm{RB}$, and $\mathrm{PVY} \mathrm{N}^{\mathrm{N}-\mathrm{Wi}} \times \mathrm{RB}$. Over-reliance of leaf drop as a key PVY indicator may have resulted in past missed detections of PVY ${ }^{\mathrm{NTN}}$ and PVY ${ }^{\mathrm{N}-\mathrm{Wi}}$ on RB in the field. There were significant $(P<0.0001)$ strain $\times$ cultivar interactions for yield. Potato tuber necrotic ring spot disease (59.8\% by wt.) only was observed for PVY $\mathrm{Y}^{\mathrm{NTN}} \times \mathrm{YG}_{\text {. PVY }}$ infections resulted in shallow, suberized canoe-shaped cracks on tubers of all cultivars (3.1-9.3\% by wt.) and most PVY ${ }^{\mathrm{NTN}}$ and PVY ${ }^{\mathrm{N}-\mathrm{Wi}}$ tubers had cracks, but at low levels (0.7-3.3\% by wt.). Tuber cracking did not result in storage weight loss.

Seasonal and potato cultivar effects on pathogenic Streptomyces spp.

K. NAHAR (1), C. Goyer (1), B. Zebarth (1), D. Burton (2), S. Whitney (1), (1) Agriculture and Agri-Food Canada, Fredericton, NB, CANADA; (2) Dalhousie University, Truro, NS, CANADA

Potato common scab is caused by pathogenic Streptomyces spp. Potato cultivar can influence the abundance and diversity of microbial communities; however the effect of cultivar on the abundance of pathogenic Streptomyces spp. is unclear. The objective of this study was to measure changes in the abundance of pathogenic Streptomyces spp. in potato cultivars over the growing period. The experiment was conducted in a naturally common scab infested field located at Fredericton, NB, Canada. Two tolerant (Gold Rush, Hindenberg) and two susceptible cultivars (Green Mountain, Agria) were tested. Soil sampling was done in bulk (BS), rhizosphere (RS) and tuber surface (TS) soils over the growing season. When averaged over time, abundance of pathogenic Streptomyces spp., measured using quantitative polymerase chain reaction, was significantly greater in TS for susceptible than for tolerant cultivars. In general, the abundance of pathogenic Streptomyces spp. was increased in BS, RS and TS for susceptible cultivars at 90 days after planting compared with other dates, whereas no changes were observed for tolerant cultivars. These results suggest that growth of pathogenic Streptomyces spp. was stimulated in soil associated with the plant roots and tuber surface of susceptible cultivars, perhaps due to organic compounds in the root and tuber exudates.

Interaction of onion cultivar and growth stages on incidence of Pantoea ananatis bulb infection

S. STUMPF (1), R. D. Gitaitis (1), T. W. Coolong (1), C. Riner (2), B. Dutta (1), (1) University of Georgia, Tifton, GA, USA; (2) University of Georgia, Lyons, GA, USA

The effect of onion growth stages on susceptibility to Pantoea ananatis (causal agent of center rot) infection was investigated. In addition, differences in onion varietal susceptibility to bulb infection was also investigated. Five cultivars of sweet onion (Pirate, Sweet Harvest, 1518, Granex YPRR, and 1407) were inoculated at three growth stages (first leaf senescence, bulb initiation, and bulb swelling). Total center rot incidence in bulb was significantly higher for Granex YPRR (84\%), compared to other cultivars. Also, cultivars $1518(49 \%)$ and $1407(33 \%)$ had significantly lower incidence of bulb infection compared to other tested cultivars. Onions were significantly more susceptible to bulb infection when inoculated during first leaf senescence $(62 \%)$ as compared to bulb initiation (37\%) and bulb swelling (31\%) stages when assessed prior to curing $(P=0.041)$. When total bulb incidences were calculated, significantly higher incidence of center rot was observed for bulbs whose foliage were inoculated at first leaf senescence stage (64\%) compared to bulb initiation (55\%) and bulb swelling $(52 \%)$ stages $(P=0.048)$. Interactions between onion cultivar and inoculation stage on center rot bulb incidence was not significant $(P \geq 0.218)$. However, different cultivars displayed significant variability in susceptibility to bulb infection. The outcomes of this study may have implications in devising management strategies against $P$. ananatis.

Evaluating host resistance to Macrophomina crown rot in strawberry

J. WINSLOW (1), M. Mazzola (2), G. J. Holmes (3), K. L. Ivors (3), (1) Strawberry Center, Cal Poly State University, San Luis Obispo, CA, USA; (2) USDA ARS, Wenatchee, WA, USA; (3) Strawberry Center, California Polytechnic State University, San Luis Obispo, CA, USA

Macrophomina crown rot, caused by the soilborne fungus Macrophomina phaseolina, is an emerging pathogen in California strawberry production. When established, the pathogen can cause extensive plant decline and mortality. Host resistance will be a critical tool for managing this disease and guiding breeding programs in this post methyl bromide era. In order to evaluate strawberry germplasm for resistance to M. phaseolina, a total of ninety cultivars and elite selections chosen from both public and private breeding programs were planted in an artificially inoculated field during the fall of 2016 at the California Polytechnic State University in San Luis Obispo. Crown rot severity and plant mortality were assessed over time in both inoculated and non-inoculated plots. Pathogen colonization of strawberry crown and root tissues of four resistant cultivars (Fronteras, Grenada, Petaluma and Radiance) and four susceptible cultivars (Albion, Festival, San Andreas and Monterey) was quantified using a previously described qPCR protocol. The primary objectives of this research were to correlate host symptom expression with the extent of pathogen colonization in different strawberry tissues, and to determine if resistant germplasm can contribute to secondary inoculum production in the field. 
Resistance of almond cultivars to Aspergillus flavus and A. parasiticus

J. Moral (1), R. Puckett (2), K. Tomari (3), A. Ortega-Beltran (4), T. M. Gradziel (5), T. J. MICHAILIDES (2), (1) Univ De Cordoba / Univ Davis, Parlier, CA, USA; (2) University of California - Davis, Parlier, CA, USA; (3) Kearney Agricultural and Extension Center, PARLIER, CA, USA; (4) International Institute of Tropical Agriculture, Ibadan, NIGERIA; (5) Dept. of Plant Sciences, University of California Davis, California, CA, USA

Aflatoxins (AF), produced by Aspergillus flavus and A. parasiticus, are among the most potent liver carcinogens that can occasionally contaminate California almonds. The risk of almond contamination by AF depends on weather conditions, levels of damage by navel orange worm (Amyelois transitella), and cultivar resistance but it is unkown. Here, we evaluate the resistance of almond cultivars to Aspergillus spp. The effects of 29 almond cultivars, shell, seed coat, and Aspergillus species were studied through artificial inoculations in several experiments. The dependent variables were: colonization, sporulation, and AF production that was evaluated by TLC and HPLC. The cultivars showed a wide-range of response to AF contamination, from moderately to highly susceptible. The cvs. Sonora and Tarragona had significantly less concentration of AF than the other cultivars. In our experiments, none of the cultivars were immune to AF contamination. The concentration of AF from kernels was correlated with the extent of colonization by the pathogen. The pathogen produced AF solely in the kernel albeit, when fruits with shell were inoculated, the fungus could overcome the shell barrier and arrive at the kernel. Even so, the presence of intact shell on the fruit reduced AF contamination by more than 100-fold. The use of almond cultivars with some tolerance to the pathogen may be a useful strategy for integrated management of AF.

Kiwifruit susceptibility to Botrytis cinerea and Botrytis prunorum during fruit development in Chile D. RIQUELME, H. Valdés, J. P. Zoffoli, Pontificia Universidad Católica de Chile, Santiago, CHILE

Gray mold, the main postharvest disease of kiwifruit (Actinidia deliciosa (A. Chev.) C.F. Liang et A. R. Ferguson 'Hayward') is caused by Botrytis cinerea and Botrytis prunorum. Symptoms appears in colstorage but infections produced in the field. Fruit tissue susceptibility to $B$. cinerea (isolate Kw_F58) and B. prunorum (isolate Kw_6) was studied since $20 \mathrm{~d}$ after flowering (fruit immature) until near harvest of Hayward kiwifruit. Fruits were injured and inoculated on the cheek, in the picking wound, and at the base of the berry, in the insertion of the sepal with the receptacle with a mycelial plug $(3 \mathrm{~mm})$ or a conidial suspension $\left(10^{6}\right.$ conidia $\left.\mathrm{mL}-1\right)$ and evaluated after 5,7 or $12 \mathrm{~d}$ at $20^{\circ} \mathrm{C}$ and $100 \%$ humidity. Results showed that the fruits were susceptible to $B$. cinerea and $B$. prunorum during all the season. Fruits collected early in the season were more susceptible than those collected at harvest, varying according to the wounded tissue and pathogen. Kiwifruits inoculated on cheeks with conidial suspension were susceptible during all the season. Inoculation on sepal insertion was more susceptible early, while picking wound was susceptible only $20 \mathrm{~d}$ after flowering. The isolate of $B$. cinerea was significantly $(\mathrm{P}<0.001)$ more virulent than $B$. prunorum in all fruit inoculated tissues. Differences in fruit susceptibility throughout the season could be attributed to host defence mechanisms that vary within the season. These results suggest that early fruit protection could reduce stem end rot in kiwifruit in postharvest period.

Susceptibility of dry bean varieties to species of Fusarium and Rhizoctonia associated with root rot disease A. O. ADESEMOYE (1), S. Kodati (1), M. Eskelson (1), R. M. Harveson (2), (1) University of Nebraska Lincoln, North Platte, NE, USA; (2) Univ of Nebraska - Panhandle Research \& Extension Center, Scottsbluff, NE, USA

Root rot diseases in dry bean are caused mainly by species of Fusarium and Rhizoctonia. However, the virulence of these diverse pathogen groups on dry bean market classes in Nebraska (NE) is unclear. The objectives of this study were (1) to understand the nascent genetic diversity of root rot pathogens of dry bean in NE and (2) the susceptibility of common varieties of dry bean in NE to different root rot pathogens. Over a two-year period, infected dry bean samples were collected from 8 field locations in western and west central NE. Field locations were selected based on a history of disease or current disease. Isolates were recovered and identified using molecular techniques with ITS and $\beta$-tubulin primers. Among the 117 isolates collected, 16 were selected as representatives and used to challenge five commonly grown dry bean varieties - Draco (Great Northern), La Paz (Pinto), Marquis (Great Northern), Medalist (Navy), and Pink Panther (Kidney) - in a greenhouse assay. The species and anastomosis groups recovered included $R$. solani AG-K, $R$. solani AG4, $R$. solani AG-2-2, $R$. solani AG-5, F. oxysporum, F. equiseti, F. redolens, $F$. solani, $F$. cuneirostrum, and $F$. sporotrichioides Pathogenicity results indicated that among Fusarium, F. sporotrichioides was the most virulent on dry bean with a rating of 5.26 on a 9-point rating scale. Also, AG4 was the most virulent anastomosis group of $R$. solani. Marquis was the most susceptible variety for both pathogen groups.

Resistance to Cereal Cyst Nematode in Spring Wheat in Southeastern Idaho

P. ENSAFI (1), M. T. Moll (2), J. M. Marshall (2), (1) University of Idaho, Aberdeen, ID, USA; (2) University of Idaho, Idaho Falls, ID, USA

Heterodera avenae is the only documented Cereal Cyst Nematode $(\mathrm{CCN})$ species in Idaho. The widespread occurrence and high populations throughout southern Idaho are responsible for consistent yield losses. The diversity of CCN, susceptibility of all Poaceae, variable resistance responses, and lack of effective registered nematicides make control strategies limited. In 2016, an experiment conducted as a randomized complete design with 6 reps on a heavily infested, irrigated field in St. Anthony was established to test the response of seven spring wheat cultivars. Plant roots from six plants at flowering (Feekes GS 10.5.1) were collected from each plot, washed, and stained to evaluate the number of cysts per root system. Soil was sampled before planting and after harvest, and changes in abundance of cyst and eggs were quantified for the CCN multiplication rate assessment. WB-Rockland and Expresso ranked moderately resistant ( 3 to 6 cysts per plant) and moderately susceptible (6 to 12), respectively. WB9668 and Patwin515 were found to be susceptible (12 to 25), whereas Snow Crest, WB9411 and Westbed936 ranked very susceptible with more than 25 cysts per plant. Susceptibility to $\mathrm{CCN}$ and stripe rust, and the fact that resistance to both pathogens are regulated by closely linked genes, demonstrates that targeting the transfer of $\mathrm{Cre}$ genes from resistant cultivars to locally adapted ones would be an optimal approach in integrated management of CCN in wheat. Considering the long survival of cysts and small number of resistant cultivars, introducing new resistant cultivars with reasonable tolerance is the most effective management strategy.

Rose rosette disease resistance field trials in north Texas

M. SHIRES (1), K. Ong (2), D. Byrne (1), (1) Texas A\&M University, College Station, TX, USA; (2) Texas A\&M AgriLife Extension Service, College Station, TX, USA

Rose Rosette Disease (RRD) is a viral disease caused by the Rose Rosette Virus (RRV) that is greatly affecting the United States rose industry. Currently, there are no rose cultivars that are known to be resistant to this disease. There are efforts by several researchers, including us at Texas A\&M, to try to identify potential sources of resistance or tolerance to RRD. In 2016, 10 cultivars of roses were selected for resistance trials. Those cultivars were Double Red Knockout, Belinda's Dream, Pink Homerun, Homerun, Shreveport, Hot Cocoa, Nearly Wild, Caldwell Pink, Chuckles, and Basye's Purple. These cultivars were selected on the basis of: (1) susceptible plant to serve as positive control; (2) potentially susceptible plant; or (3) popular cultivar that is planted in Texas. Two trial locations were established in 2016 - Dallas and Wichita Falls, Texas. To date, no symptoms of RRD have been observed at the Dallas plot. At the Wichita Falls site, disease symptoms were observed in 30 out of 51 plants, five months after trial initiation. Samples were collected from each of the 51 plants in the plot and tested for RRV via PCR. Of the 51 samples collected, 23 were found to be infected with RRV. Three of the 10 cultivars did not test positive for RRV. Those three cultivars were Basye's Purple, Caldwell Pink, and Chuckles, and were carried over for further resistance testing in spring 2017. 
Potential sources of resistance to Phytophthora crown rot in Cucurbita maxima and Cucurbita moschata

K. MANTOOTH (1), J. Ikerd (2), M. K. Mandal PhD (3), S. Kousik (2), (1) Clemson University, Coastal Research and Education Center, Charleston, SC, USA; (2) U.S. Vegetable Laboratory, USDA, ARS, Charleston, SC, USA; (3) ORISE participant, US Vegetable Laboratory, USDA, ARS, Charleston, SC, USA

Phytophthora capsici is an economically important oomycete pathogen, infecting 27 plant families across the globe, and causing several plant diseases including seedling death, crown rot, fruit rot, root rot, and foliar blight. The popularity of grafting watermelons for disease management has risen over the past few years. Interspecific hybrids of Cucurbita (C. maxima $\times$ C. moschata) are commonly used as rootstocks, primarily due to their non-host resistance to Fusarium wilt of watermelon. However, they are susceptible to P. capsici, which is of concern in the southeastern U.S. The goal of this research was to identify and develop new sources of resistance to $P$. capsici in C. maxima and C. moschata. All available U. S. Plant Introductions (PIs) of C. maxima (510 PIs) and C. moschata (319 PIs) were evaluated for tolerance to $P$. capsici by inoculating 4-week-old plants with $10^{4}$ zoospores at their crowns. Fourteen days after inoculation they were rated for disease development using a 0-5 scale. Four PIs of C. maxima rated 2 or less (e.g. PI 508462) and five PIs of $C$. moschata rated less than 1 (e.g. PI 442265) were considered resistant. New sources of crown rot resistance in both $C$. maxima and $C$. moschata were identified. These sources of resistance will be used to develop improved resistant germplasm lines and hybrids to be utilized as rootstocks for grafted watermelon. These sources can also be used to develop crown rot-resistant squash cultivars for human consumption.

Assessing spring wheat for Fusarium head blight susceptibility in Idaho

S. Arcibal (1), J. M. MARSHALL (2), (1) University of Idaho, Aberdeen, ID, USA; (2) University of Idaho, Idaho Falls, ID, USA

Fusarium head blight (FHB), caused by Fusarium graminearum, has been increasingly damaging to spring wheat in southern Idaho. FHB can cause significant yield losses, Fusarium-damaged kernels (FDK) and mycotoxin contamination from deoxynivalenol (DON) in high-desert production areas under irrigated conditions. To evaluate FHB susceptibility of spring wheat, field screening nurseries were established at Aberdeen, ID in mid-April of 2016. Fifty-three spring wheat varieties were planted using a randomized complete block design with two replications. Corn inoculum was produced in corn spawn inoculated with local $F$. graminearum isolates. Plots were inoculated at a rate of $30 \mathrm{~g} / \mathrm{m}^{2}$ two to three weeks before heading. Disease severity and incidence were evaluated 21 days after anthesis (Feekes 10.5.1) to determine FHB index. Despite a low FHB pressure, FHB index (P=0.0384), FDK $(\mathrm{P}=0.0103)$ and $\mathrm{DON}(\mathrm{P}=0.0034)$ were significantly different among wheat varieties. Louise (soft white) had the highest FHB index (10.7\%). However, hard red wheat varieties Jefferson and Kelse had the highest FDK of 6.5 to $6.8 \%$, respectively. DON accumulation ranged up to 9.8 ppm in Klasic (hard white). Although not statistically different, yield ranged from $52.3 \mathrm{bu} / \mathrm{A}$ (Snow Crest) to $117.6 \mathrm{bu} / \mathrm{A}$ (Seahawk). Since the majority of spring wheat varieties available are susceptible to FHB, it is crucial to continue screening for FHB susceptibility to develop integrated management strategies for local growers in Idaho.

Factors influencing the occurrence of foliar pathogens in commercial watermelon fields in South Carolina in 2015 and 2016 G. RENNBERGER (1), A. P. Keinath (1), P. Gerard (2), (1) Coastal Research and Education Center, Clemson University, Charleston, SC, USA; (2) Department of Mathematical Sciences, Clemson University, Clemson, SC, USA

Survey data from three seasons were analyzed to identify how environmental and agronomic factors influence the occurrence of foliar pathogens of watermelon in commercial fields. In spring 2015 and spring and fall 2016, 100 leaves with symptoms were collected in each of 56 fields in seven counties in South Carolina. After microscopic identification, all pathogens were recorded on each leaf. Survey procedures for logistic regression and frequencies were used for analysis (SAS). There were significant differences among counties in all seasons. Plant age had no consistent effect on pathogen occurrence. Stagonosporopsis spp. were more likely to occur in fields that were cropped to a cucurbit the previous season $(P \leq 0.0001)$. Pathogens were less likely to occur in fields aligned west or southwest compared to other directions $(P \leq 0.0003)$. If Stagonosporopsis spp. were present, Cercospora citrullina and Podosphaera xanthii were more likely $(P \leq 0.0002)$ to be present and Colletotrichum orbiculare was less likely $(P=0.0360)$ to be present on the same leaf. Systemic fungicides decreased the occurrence of Stagonosporopsis spp. and $P$. xanthii compared to no fungicide and protectants. Pathogen-specific fungicides decreased the occurrence of both pathogens compared to non-specific fungicides and no fungicides. Results support the importance of crop rotation and systemic, pathogen-specific fungicides for integrated disease management.

Evaluation of $\beta$-nicotinamide adenine dinucleotide for the potential to manage fungal and bacterial diseases on vegetable crops S. ZHANG (1), S. Bibi (2), Z. Mou (3), (1) University of Florida, Homestead, FL, USA; (2) PMAS Arid Agriculture University, Rawalpindi, PAKISTAN; (3) Univniversity of Florida, Gainesville, FL, USA

$\beta$-nicotinamide adenine dinucleotide (NAD) is a ubiquitous electron carrier involved in many metabolic pathways. It has been reported that NAD induces systemic acquired resistance (SAR)-like defense responses in Arabidopsis. In the greenhouse, NAD was evaluated for its potential to reduce powdery mildew (Podosphaera xanthii) on squash, halo blight (Pseudomonas syringae pv. phaseolicola) on snapbean, and black rot (Xanthomonas campestris pv. campestris) on cabbage. NAD was applied at $5 \mathrm{mM}$ as leaf infiltration, a foliar spay, or a soil drench at 1,3, or 7 days, respectively, prior to pathogen inoculation. Disease severity was rated $7-10$ days after inoculation. NAD applied by all the methods significantly $(P<0.05)$ reduced disease severity of powdery mildew on squash compared to the untreated control. The best efficacy (up to $86.2 \%$ reduction) was achieved by leaf infiltration or a foliar spray, followed by a soil drench. Disease severity of halo blight on snapbean and black rot on cabbage was significantly lower on plants treated with NAD as a foliar spray or leaf infiltration than the untreated plants. NAD applied as a soil drench failed to reduce severity of these bacterial diseases. Preliminary results from this study indicated that NAD has the potential to reduce fungal and bacterial diseases on vegetable crops. Exploration of the efficacy of NAD in disease suppression will substantially promote integrated disease management for vegetables and other crops.

Seed treatments to eradicate Pyrenopeziza brassicae from infected mustard (Brassica juncea) seed

S. CARMODY, L. du Toit, Washington State University, Mount Vernon, WA, USA

Light leaf spot of brassicas is caused by Pyrenopeziza brassicae, a new disease to the USA. The fungus can be seedborne and seed transmitted. A seed lot of 'Caliente 199' mustard (B. juncea) infected with $P$. brassicae was used to assess the efficacy of chlorine $(1.2 \% \mathrm{NaOCl}$ for $10,20,30$, and 40 minutes), hot water $\left(50^{\circ} \mathrm{C}\right.$ for 15 and 30 minutes), steam $\left(62.8,65.6,68.3\right.$, and $\left.71.1^{\circ} \mathrm{C}\right)$, and 10 fungicide treatments to manage seedborne $P$. brassicae. Each seed treatment was compared to non-treated seed, and fungicide treatments were also compared to seed treated with a polymer colorant (seed coating) added to each product. All treatments reduced the incidence of seed infected with $P$. brassicae, from an average of $13.5 \%$ for non-treated seed to 0 to $4.3 \%$, based on seed health assays. Likewise, all treatments, including the seed colorant control treatment, reduced seed transmission of $P$. brassicae from an average of $3.4 \%$ for non-treated seed to 0 to $0.4 \%$. Seed transmission was not observed for the hot water, steam, and six of the fungicide treatments (azoxystrobin, fludioxonil, iprodione, thiabendazole, pyracostrobin + boscalid, and difenoconazole + fludioxonil + mefenoxam + sedaxane + thiamethoxam). The hottest steam treatment reduced seed germination from $98.0 \%$ for non-treated seed to 90.0 and $93.8 \%$ in Trials 1 and 2 , respectively. The results demonstrate there are effective organic and conventional seed treatments for management of $P$. brassicae.

Non-target effects of horticultural and pest management practices in fall and winter on development of Exobasidium leaf and fruit spot of blueberry

H. SCHERM (1), R. M. Allen (2), R. J. Ingram (1), P. M. Brannen (1), (1) University of Georgia, Athens, GA, USA; (2) UGA Extension, Alma, GA, USA 
Exobasidium maculosum causes Exobasidium leaf and fruit spot (ELFS), an early-season disease of blueberry in the southern United States. Population monitoring studies suggest that the pathogen oversummers and overwinters epiphytically on the surface of blueberry bark and buds, most likely as yeast. This is supported by the finding that a single application of lime sulfur at the late dormant stage affords substantial control of ELFS the following spring. As a surface resident for much of the year, E. maculosum is thus exposed to various horticultural and pest management practices which may affect its epiphytic population dynamics and impact subsequent disease development. In multi-year field trials on susceptible 'Premier' rabbiteye blueberry, two fall applications of captan (timed to manage other foliar diseases) did not suppress ELFS the following spring, whereas two fall applications of lime sulfur reduced the disease significantly. A single late dormant spray of the growth regulator hydrogen cyanamide (a caustic compound) reduced subsequent ELFS incidence significantly, whereas a single dormant oil application increased the disease compared with the untreated check. The significant non-target effects associated with these off-season applications could explain variations in ELFS risk among blueberry sites and years. The results will be discussed in the context of treatment effects on E. maculosum epiphytic population densities during the winter and early spring.

Modelling Daytime Light Integral and light quality effects to optimize suppression of powdery mildews by nighttime applications of UV and red light

A. Suthaparan (1), K. A. Solhaug (1), H. R. Gislerød (1), A. STENSVAND (1,2), D. M. Gadoury (3), A. Bierman (4), M. Rea (4), (1) Norwegian University of Life Sciences, Ås, NORWAY; (2) Norwegian Institute of Bioeconomy Research, Ås, NORWAY; (3) Cornell University, Geneva, NY, USA; (4) Lighting Research Center, Rensselaer Polytechnic Institute, Troy, NY, USA

Nighttime use of ultraviolet (UV) light suppresses diverse powdery mildews; especially when supplemented with red light. However, the Daily Light Integral (DLI) preceding and following UV treatments, particularly quanta of blue and red light, as well as proximal timing of blue and red quanta relative to nighttime UV treatments can profoundly alter the efficacy. In the Podosphaera xanthii/cucumber system, plant growth increased, and efficacy of brief nighttime UV treatments against $P$. xanthiidecreased with increasing DLI. At a constant DLI, efficacy of UV decreased if day length increased from 16 to $20 \mathrm{~h}$. Increasing the dose of blue light during daytime reduced the efficacy of UV in controlling the disease, whereas growth light lacking blue wavelengths caused some UV mediated curling of young leaves, illustrating the importance of maintaining a quantity of blue light before UV treatment to increase resistance to phytotoxic effects. Efficacy of UV increased if red light was applied at the end of the day, and more so if applied immediately after UV treatment. Thus, red light can be strategically used during periods of extended day length to circumvent the tendency of increased daylength and increased DLI to reduce the efficacy of nightime UV treatments against powdery mildews. Pairing a simple rule structure with global databases of DLI can provide guidance for optimal deployment of nighttime UV and red light for suppression of powdery mildews.

Effect of UV-C/dark treatment on bacterial and fungal microbiome of strawberry fruit

W. J. JANISIEWICZ (1), B. Evans (1), F. Takeda (1), W. M. Jurick II PhD (2), P. Ramachandran (3), E. Reed (3), A. Ottesen (3), (1) USDA-ARS AFRS, Kearneysville, WV, USA; (2) USDA-ARS Food Quality Laboratory, Beltsville, MD, USA; (3) FDA, College Park, MD, USA

Treatment of strawberry plants with UV-C light followed by a 3-4 hour dark period has been shown to kill several important pathogens of fruit and leaves. This promising practice is close to commercialization for strawberry production in protective culture. There is an important knowledge gap concerning the broader impact of this UV-C treatment on the total microbiome of fruit and leaves. Therefore, we determined the composition of the bacterial and fungal microbiome of fruit and leaves after repeated application of the UV-C/dark treatment at two harvest times. Total DNA was isolated from washes of leaf and fruit samples and amplified using primers for 16S rRNA gene and ITS (18S intergenic). Sequencing was performed using the Illumina MiSeq platform and amplicons were analyzed with QIIME. A slight increase (not statistically significant) in OTUs was observed in UV-C/dark treated samples for both fruit and leaves, but it was not detectable using the Whole Phylogenetic Diversity (pd_whole) or Chao1 diversity indices. Principal component analyses of UV-C vs. non-UV-C treated fruits and leaves showed no clear separation by treatment. Several Enterobacteriaceae OTU's were enriched in UV-C/dark samples. They could not be identified at genus level; however, further phylogenetic analysis showed close relation to Enterobacter or Erwiniaspp. Our results indicate that UV-C/dark treatment does not appear to increases the risk of foodborne pathogens.

Nighttime treatments of ultraviolet (UV) light targeting powdery mildews also suppress the two-spotted spider mite (Tetranychus urticae) N. S. Johansen (1), B. A. Tadesse (2), A. Suthaparan (3), A. STENSVAND (1), P. J. From (3), D. M. Gadoury (4), (1) Norwegian Institute of Bioeconomy Research, Ås, NORWAY; (2) Norwegian Institute for Bioeconomy Research (NIBIO), Aas, NORWAY; (3) Norwegian University of Life Sciences, Ås, NORWAY; (4) Cornell University, Geneva, NY, USA

We have used brief periodic nighttime applications of ultraviolet (UV) at 50 to $300 \mathrm{~J} / \mathrm{m}^{2} /$ day to suppress powdery mildews on diverse crops in greenhouse and tunnel production systems. During these studies, we noted effects of the UV treatments on populations of the two-spotted spider mite (Tetranychus urticae) on strawberries. Subsequent experiments focused upon direct effects of the UV treatments upon various life stages of the mites. Fluorescent UVB lamps (Q-PANEL UVB-313EL) were used nightly to apply $1.6 \mathrm{~W} / \mathrm{m}^{2}$ over a period of $3 \mathrm{~min}\left(288 \mathrm{~J} / \mathrm{m}^{2} /\right.$ day). To maintain a Daily Light Integral sufficient for optimal plant growth and treatment efficacy, supplemental light was provided with HPS-lamps when natural solar insolation was below $200 \mathrm{~W} / \mathrm{m}^{2}$. Effects of the UV treatments on survival and egg production of adult females, as well effects on survival and hatching rates of mite eggs were assessed. UV treatments had a negligible effect on adult female survival and egg production, but killed more than $99 \%$ of immature mites and $100 \%$ of eggs. The magnitude of the treatment effects was similar both in a laboratory strain of $T$. urticae and a wild-type strain collected shortly before experiments were conducted. No phytotoxic effects were observed from the UV treatments. The ovocidal and juvenile effects observed may provide useful suppression of phytophageous mite populations if instituted early in the development of pest populations.

\section{Characterizing Colletotrichum orbiculare, the causal agent of cucurbit anthracnose, for fungicide efficacy and host susceptibility in North Carolina} N. NOEL, L. M. Quesada, North Carolina State University, Raleigh, NC, USA

Colletotrichum orbiculare syn. lagenarium, a hemibiotrophic ascomycete, is responsible for yield losses across the United States due to foliar, stem, and fruit lesions when it causes anthracnose in cucurbits. Favorable environmental conditions, diverse and available hosts, and long growing seasons offer ample opportunity for disease development in North Carolina (NC). Current control measures include application of effective fungicides and planting resistant cultivars, along with cultural practices. Despite these efforts, new emerging races and fungicide resistance have greatly hampered control strategies. Our objectives were to characterize C. orbiculare populations infecting NC cucurbit crops, determine their sensitivity to fungicides, and characterize their virulence in watermelon cultivars. Diverse isolates were obtained from cucurbit fields in North Carolina and elsewhere for fungicide sensitivity evaluations in vitro with 10 fungicides at 6 concentrations. North Carolina isolates of different races were evaluated for virulence in 36 commercial cultivars of watermelon (Citrullus lanatus) in both field and greenhouse trials. Results suggest that control of cucurbit anthracnose requires an integrated disease management strategy that combines effective fungicides and host tolerance.

Control of root rot diseases of woody ornamentals using biorational products and fungicides M. N. Kabir, P. Liyanapathiranage, T. Simmons, F. BAYSAL-GUREL, Tennessee State University, McMinnville, TN, USA

Phytophthora and Rhizoctonia root rots are two destructive diseases with a wide host range among nursery-grown ornamentals. The efficacy of biorational products and fungicides against Phytophthora root rot of hydrangea and Rhizoctonia root rot of viburnum were assessed in separate field trials. Treatments were arranged in randomized complete block design with four replications in each trial. Plots were inoculated with Rhizoctonia solani agar slurry or Phytophthora nicotianae grown on rice grains. Mutual treatments were RootShield PLUS, MBI110, IT-5103, TerraClean 5.0 + TerraGrow 
and Empress. SoilGard, Mural and Paegant were also used only in the Rhizoctonia trial; OxiPhos, Segovis and Subdue Maxx were used only in the Phytophthora trial. TerraClean 5.0 was drenched into the soil $24 \mathrm{~h}$ prior to transplanting in dedicated plots. Dedicated rooted cuttings were dipped in a TerraGrow ( $1 \mathrm{oz} / 10 \mathrm{gal})$ solution prior to planting and same plants received TerraGrow $(0.4 \mathrm{oz} / 10 \mathrm{gal})$ as drench application after transplanting. The other treatments were applied as drench starting after transplanting. All of the treatments significantly reduced both root rot severities compared to the untreated, inoculated controls. The treatments most effective in reducing Phytophthora root rot severity were Segovis, Empress, Subdue Maxx and MBI110 and in reducing Rhizoctonia root rot severity were Mural, Empress, Paegant and TerraClean 5.0 + TerraGrow. Both trials will be repeated in controlled environments.

\section{Management of Rhizoctonia diseases in sugar beet using an integrated approach}

A. K. CHANDA (1), J. R. Brantner (2), (1) Department of Plant Pathology, University of Minnesota, Crookston, MN, USA; (2) University of Minnesota, Crookston, MN, USA

Damping-off and crown and root rot (RCRR) caused by Rhizoctonia solani AG 2-2 have been the most common root diseases on sugar beet in Minnesota and North Dakota. In 2016, a field trial was established to evaluate an integrated management strategy consisting of a resistant and moderately susceptible variety with seed treatments (penthiopyrad, sedaxane, and fluxapyroxad) in combination with a postemergence azoxystrobin application at the 4- or 8-leaf stage for 1) control of early-season damping-off and RCRR and 2) effect on yield and quality. There were no significant $(P$ $=0.05$ ) two or three way interactions for stand or harvest data. All three seed treatments had higher stands compared to the untreated control from 5 to 8 weeks after planting, but did not affect root rot rating or harvest parameters. The moderately susceptible variety had higher yield but also higher sugar loss to molasses compared to the resistant variety. Root rot severity was significantly higher in the treatments without postemergence azoxystrobin application, intermediate in 4-leaf, and lowest in the 8-leaf azoxystrobin application. Yield and recoverable sugar was higher for 8-leaf azoxystrobin application compared to no azoxystrobin because of favorable conditions for development of disease later in the season. Results suggest an integrated approach utilizing a seed treatment to protect seedlings followed by a postemergence application for mid- to late-season disease pressure.

Fungicides and cultivars can limit hop downy mildew (Pseudoperonospora humuli) in Michigan

D. S. HIGGINS (1), M. K. Hausbeck (2), (1) Michigan State University Department of Plant, Soil and Microbial Sciences, East Lansing, MI, USA; (2) Michigan State University, East Lansing, MI, USA

Michigan's hop industry is valued up to $\$ 16.4$ million (2016) with 800 harvested acres in 2016. Downy mildew (DM) incited by Pseudoperonospora humuli limits production and quality. Separate fungicide and cultivar trials were conducted in 2016 on first-year, nontrellised hop yards at two locations. Eight foliar Oomycete-specific fungicides were sprayed every 10 days and compared to an untreated control; treatments were initiated before inoculation. For the cultivar trial, twelve cultivars selected by Michigan growers and not treated with fungicides were compared for susceptibility. Each trial was arranged in a randomized complete block design with four replications. Plots were inoculated with zoospores via mist irrigation. Disease density (infected shoots) and severity (infected shoots and foliage) were assessed weekly from 23 Jul to 7 Oct. 'Newport' and 'Tahoma' were least susceptible to DM based on infected shoots and foliage; 'Centennial' and 'Cascade', among the most popular cultivars grown in Michigan, were highly susceptible. All fungicides significantly reduced DM symptoms compared to the control. Under high (Site 1) and low (Site 2) DM pressure, the fungicide oxathiapiprolin was most effective. Ametoctradin + dimethomorph, fluopicolide, cyazofamid and mandipropamid were significantly more effective than cymoxanil and dimethomorph (Site 1). Fosetyl-Al was the least effective fungicide (Site 1), but limited shoot infection under low disease pressure (Site 2).

Options for control of Fusarium wilt Tropical race 4 in bananas

A. DRENTH, J. Henderson, University of Queensland, Brisbane, AUSTRALIA

Fusarium oxysporum f.sp. cubense Tropical race 4 (TR4) is one of the most important pathogens of banana which severely affects many varieties, including Cavendish types which account for almost $50 \%$ of worldwide production. Disease symptoms progress from yellowing of the leaf margins to collapse of the leaves at the lower end of the leaf stalks and splitting of the leaf sheaths at the base of the pseudostem. Internal symptoms include dark brown to black discolouration of the water conducting tissues. Plants affected by Fusarium wilt seldom produce a marketable bunch. The recent intercontinental spread of TR4 and the rapid development of disease epidemics in many locations after arrival raises questions concering diagnostics, containment and ongoing management of this disease. Management options are few, and data on their effectiveness is often lacking. Our integrated research program has resulted in several outcomes which include improved molecular diagnostics for TR4, testing and improving containment treatments in the field, and screening banana germplasm for resistance in multiple locations containing different races of Fusarium wilt.

Progress in managing bacterial spot of cucurbits, caused by Xanthomonas cucurbitae M. BABADOOST (1), S. Thapa (2), X. Zhang (1), S. Sulley (1), (1) University of Illinois, Urbana, IL, USA; (2) Univ of Illinois, Urbana, IL, USA

Bacterial spot, caused by Xanthomonas cucurbitae, is an emerging disease in pumpkin and squash. Yield losses up to $96 \%$ in pumpkin fields, caused by $X$. cucurbitae, have been documented. The minimum, maximum, and optimum temperatures for cell multiplication of isolates of $X$. cucurbitae from the Midwestern states were 4-6, 34-36, and $24-30^{\circ} \mathrm{C}$, respectively. $X$. cucurbitae is a seedborne pathogen and survived longer than 18 months in naturallyinfected and artificially-inoculated pumpkin seeds stored at 4 and $22^{\circ} \mathrm{C}$. The pathogen also survived in the field for more than 24 months. Hot-water treatment at $55^{\circ} \mathrm{C}$ for $15 \mathrm{~min}$ and $\mathrm{HCl}$ treatment at $0.5 \%$ concentration for 40 min eradicated $X$. cucurbitae in both naturally-infected and artificiallyinoculated pumpkin seeds without any significant adverse effect on either seed germination or seedling vigor. More than 25 chemical compounds and biocontrol agents were tested for their efficacy for management of $X$. cucurbitae in pumpkin and found that mancozeb (Dithane M45), cupper hydroxide (Kocide 3000), copper octanoate (Cueva SC), and an extract from Reynoutria sachalinensis (Regalia) were the most effective compounds in reducing incidence and severity of bacterial spot in leaves and fruits of pumpkin. Effects of crop rotations and using antagonistic bacteria for management of bacterial spot are being studies. In addition, investigation is underway to determine resistance in pumpkin and squash germplasms to $X$. cucurbitae .

\section{Novel phytosanitary treatment of Xylella fastidiosa-infected pecan scions using carbon nanotubes}

A. HILTON (1), M. Handiseni (1), W. Choi (1), X. Wang (2), L. J. Grauke (2), C. Yu (1), Y. K. Jo (1), (1) Texas A\&M University, College Station, TX, USA; (2) USDA-ARS Pecan Breeding and Germplasm Repository, Somerville, TX, USA

Pecan bacterial leaf scorch (PBLS) is an emerging issue for the U.S. and international pecan industry and was recently reported in CA, AZ, NM, and TX Caused by Xylella fastidiosa, PBLS is a chronic disease that causes yield losses in susceptible cultivars. Hot-water treatment of pecan scions is the sole method to minimize disease transmission. The establishment of novel, effective remediation methods is imperative to prevent dissemination of PBLSinfected pecan. The goal of this study is to develop a disinfection method for X. fastidiosa using microwave-absorbing carbon nanotubes (CNT). In preliminary tests, bacterial model Burkholderia glumae was injected into pecan scions. A $1 \times 10^{2-3} \mathrm{CNT}$ solution was dispersed through the xylem with a Scholander-type pressure chamber and exposed to microwave for 5-10 sec. Dissected scions were inoculated onto LB media. B. glumae viability was reduced when amended with CNT and treated with microwave, compared with the nonamended control. Comparable methods of CNT treatment will be performed with pecan isolates of $X$. fastidiosa. Following treatment, concentration of $X$. fastidios $a$ will be determined, and scions will be monitored for viability in hydroponic culture. Hot-water treatments will be used as a comparison and serve as the conventional disinfection method for $X$. fastidiosa. We will determine the optimal threshold thermal energy levels of CNT that affects the viability of $X$. fastidiosa with low adverse impact on plant tissue. 
Integration of biopesticides, a SAR inducer and copper for managing bacterial spot of tomato; a long-term evaluation in production areas in Florida

M. PARET (1,2), P. D. Roberts (3), G. E. Vallad (4), J. H. Freeman (5), S. M. Olson (6), L. Ritchie (5), D. Clark (5), (1) North Florida Research and Education Center, University of Florida, Quincy, FL, USA; (2) University of Florida, Department of Plant Pathology, Gainesville, FL, USA; (3) University of Florida, Immokalee, FL, USA; (4) Gulf Coast Research and Education Center, University of Florida, Wimauma, FL, USA; (5) University of Florida, Quincy, FL, USA; (6) Univ of Florida NFREC, Quincy, FL, USA

Tomato growers in Florida use a SAR inducer and biopesticides against tomato bacterial spot caused by Xanthomonas perforans. However, the efficacy of these materials in integrated practice remains a question. Identification of alternatives and integrated programs is critical, but consistency in performance and an economic return is important for increased adoption by growers. Streptomyces lydicus, Bacillus subtilis, acibenzolar-S-methyl (ASM), copper hydroxide and copper hydroxide + mancozeb were experimented in 12 field trials from 2011-2016 in Quincy, Immokalee, and Wimauma; three tomato production areas in Florida. Foliar applications of ASM in 9/10 trials, S. lydicus in 8/11 trials, B. subtilis in 4/5 trials, copper hydroxide + mancozeb in $6 / 12$ trials, and copper hydroxide in $1 / 3$ trials significantly reduced bacterial spot disease severity than the untreated $(\mathrm{P}=0.05$, LSD). The most effective integration program was ASM + S. lydicus which reduced disease severity in $6 / 9$ trials compared to the untreated ( $\mathrm{P}=0.05$, LSD). These trials demonstrate and indicate that ASM, S. lydicus, and B. subtilis by itself or integration of ASM $+S$. lydicus, are viable programs against bacterial spot of tomato; while use of copper should be avoided by all tomato producers in Florida.

Survey and field efficacy trials to manage bacterial spot of fresh market tomatoes in North Carolina

F. LOUWS (1), P. Adhikari (2), T. B. Adhikari (3), D. Panthee (4), I. Meadows (5), (1) North Carolina State University, Raleigh, NC, USA; (2) NC State University, Raleigh, NC, USA; (3) Sakata Seed America Inc, Salinas, CA, USA; (4) NC State University, Mills River, NC, USA; (5) North Carolina State University, Mills River, NC, USA

Bacterial spot (Bsp), caused by Xanthomonas perforans, is one of the most devastating diseases of tomato in North Carolina. Current IPM practices offer insufficient control due to inoculum prevalence, conducive weather and lack of host resistance. An area-wide survey of over 300 isolates in 2015 to 2016 documented over $90 \%$ of strains were resistant to copper and $25 \%$ to $45 \%$ resistant to streptomycin. Analysis of genetic diversity and race-typing is underway to inform the tomato breeding program. A field study that tested 12 treatments including bactericides, biological control agents (BCAs) or biorational products, and their combinations, was implemented both years using a RCBD with 4 replications and weekly applications of product until fruit harvest. Commercially grown 'Picus' transplants arrived with Bsp symptoms and disease progress was rapid both years. Severity ranged from 20 to $45 \%$ defoliation in 2015. AUDPC values differed among treatments with the water-only treated (UTC) plots exhibiting the greatest levels of disease whereas several treatments suppressed severity. In 2016, severity values ranged from $59 \%$ to $76 \%$; the UTC had the highest AUDPC values with some suppression associated with selected treatments. Bsp was unacceptably high both years, which highlights the critical need to develop durable host resistance and/or efficacious products to emerge into the market. Both goals remain elusive.

Cloning of a chitinase gene from Trichoderma asperellum and its efficacy against Alternaria leaf spot R. T. P. Pandian, M. Raja, A. Kumar, P. SHARMA, ICAR- Indian Agricultural Research Institute, New Delhi, INDIA

Products from Trichoderma spp. are versatile bio-fungicides used in agriculture. Chitinases produced by Trichoderma spp. are highly effective against fungal pathogens as chitin is a major component of fungal exoskeleton. In this study, we cloned the endochitinase 42 (Chit42) gene from T. asperellum strain Ta13 into the Arabidopsis thaliana ecotype Dijon-G and validated its efficacy against the Alternaria leaf spot pathogen, A. brassicicola. Cloning and characterization of chit 42 gene from Ta13 revealed a 1275 bp amplicon. The Chit 42 gene was then overexpressed in A. thaliana ecotype Dijon-G using Agrobacterium tumefaciens strain GV3101 harbouring the binary vector, pBI121 with in-built GUS reporter gene. Eleven putative transformants (T1, T2, T3, T4, T5, T6, T7, T8, T9, T10, and T11) were obtained in $\mathrm{T}_{1}$ generation and confirmed by PCR at the genomic DNA and transcript level. A 41 - 817-fold higher expression of Chit 42 was observed in transformed plants depending on the tranformant used relative to non-transformed plants. Three transformants, T4 (0), T6 (1), and T7 (0) had low Chit42 expression. Transgene presence was confirmed using PCR and GUS expression in the $\mathrm{T}_{2}$ generation plants. Bioassays on A. thaliana plants over expressing Chit 42 against $A$. brassicicola at $\mathrm{T}_{2}$ generation showed no disease development compared with the high susceptibility of non-transgenic control plants.

The interaction between the Lon protease and other major regulatory systems in Erwinia amylovora J. H. LEE, Y. Zhao, University of Illinois at Urbana-Champaign, Urbana, IL, USA

The ATP-dependent protease Lon, belonging to the $\mathrm{AAA}^{+}$protein superfamily, is a major player of post-translational control system in bacteria. In this study, we examined the Lon-mediated virulence regulation in Erwinia amylovora, the causal agent of fire blight disease in apple and pear. The type III secretion system (T3SS) and the exopolysaccharide (EPS) amylovoran are two pathogenicity factors in E. amylovora. Lon negatively regulates both traits by directly targeting two T3SS components, HrpS (bacterial enhancer binding protein) and HrpA (pilin subunit), and RcsA (auxiliary protein of the Rcs system). The lon mutant exhibited increased levels of RcsA/B proteins, which altered $h r p S$ and $\operatorname{csr} B$ sRNA expression. Accumulation of RcsA/B also suppressed $f l h D$ expression, rendering the lon mutant non-motile. In addition, this study provided evidence of possible feedback regulation between Lon and the Gac-Csr system by showing that mutation in $\operatorname{csr}$, encoding an RNA-binding protein, affected lon expression at both transcriptional and post-transcriptional levels. Our previous studies showed that CsrA is essential for both T3SS and EPS amylovoran production in E. amylovora. However, the lon/csrA double deletion mutant exhibited restored amylovoran production, but not T3SS. These suggest that CsrA positively regulates E. amylovora virulence in part by repressing Lon activity and that the regulatory role of CsrA in T3SS activation is mediated by unknown targets.

\section{Macrophomina phaseolina promotes charcoal rot susceptibility in sorghum through induced host cell wall degrading enzymes (CWDEs)} A. Y. BANDARA, D. K. Weerasooriya, S. Liu, C. R. Little, Kansas State University, Manhattan, KS, USA

Cell wall degrading enzymes (CWDEs) are virulence factors secreted by necrotrophs. Macrophomina phaseolina (MP) is an important necrotrophic fungus causing charcoal rot disease in Sorghum bicolor (L.) Moench. An RNASeq experiment revealed the MP's ability to up-regulate CWDEs encoding genes of a charcoal rot susceptible sorghum genotype (Tx7000), but not in a resistant genotype (SC599) at 7 days post-inoculation. These CWDEs included cellulase, endoglucanase, glycosyl hydrolases involved in cellulose degradation, and homogalacturonan degrading pectinesterase and polygalacturonase. For functional validation, crude enzymes mixtures (CEM) were extracted from MP- and mock-inoculated resistant (SC599, SC35) and susceptible (Tx7000, BTx3042) sorghum genotypes. The fluorimetric determination of glucose in carboxymethylcellulose (substrate), 2cynoacetamide, and CEM reaction mixture revealed the significantly increased cellulose degrading enzyme activity in two MP-inoculated susceptible genotypes. The absorbance determination of remaining ruthenium red (RR) in polygalacturonic acid (substrate), RR, and CEM reaction mixture showed the significantly increased polygalacturonase activity in MP-inoculated Tx7000 and BTx3042. A gel diffusion assay (pectin substrate) demonstrated significantly increased pectinesterase activity in MP-inoculated Tx7000 and BTx3042. These results confirmed MP's ability to promote charcoal rot susceptibility in sorghum via induced host CWDEs.

Dynamics of host glutathione and glutathione-related enzymes in the Macrophomina phaseolina-sorghum bicolor interaction A. Y. BANDARA, D. K. Weerasooriya, S. Liu, C. R. Little, Kansas State University, Manhattan, KS, USA 
Glutathione plays a key role in detoxification and redox buffering. The dynamics of glutathione and related enzymes in sorghum bicolor (L.) Moench after Macrophomina phaseolina (MP) infection were investigated using an RNASeq experiment. Upon infection, glutathione synthetase, glutamate cysteine ligase (involved in glutathione biosynthesis), glutathione s-transferase (GST), glutathione peroxidase (GPX), and glutathione reductase (GR) genes were up-regulated in a charcoal rot susceptible sorghum genotype (Tx7000), but not in a resistant genotype (SC599) at 7 days post- inoculation. The net $\log 2$ fold up-regulation of the aforesaid genes in MP-inoculated Tx7000 was 1.9, 0.9, 120.0, 9.0, and 4.5, respectively. For functional validation, cell extracts were obtained from MP- and mock-inoculated resistant (SC599, SC35) and susceptible (Tx7000, BTx3042) sorghum genotypes. Reduced (GSH), oxidized (GSSG) glutathione, and GST, GPX, and GR activities were measured using specified reagents and absorbance measurements. A significantly reduced GSH/GSSG ratio was observed in Tx7000 and BTx3042 indicating strong oxidative stress generated under MP infection. MP significantly increased the GST, GPX, and GR activities of Tx7000 and BTx3042. While increased GR activity helps to regenerate GSH, increased GST activity depletes GSH through vacuolar sequestration of GSH-S-conjugates at the expense of relaxing oxidative stress experienced by susceptible genotypes under MP infection.

The necrotrophic fungus Macrophomina phaseolina induces strong oxidative stress in charcoal rot susceptible sorghum genotypes A. Y. BANDARA, D. K. Weerasooriya, S. Liu, C. R. Little, Kansas State University, Manhattan, KS, USA

The induction of cytochrome P450 (CP450) results in increased conversion of endogenous compounds into reactive metabolites and is a source of oxidative stress. Increased P450 expression, therefore, is a direct indication of enhanced oxidative stress. Through an RNASeq experiment, we found that Macrophomina phaseolina (MP) is capable of significantly up-regulating CP450 encoding genes (net $\log 2$ fold up-regulation $=42$ ) in a charcoal rot susceptible sorghum genotype (Tx7000), but not in a resistant genotype (SC599) at seven days post inoculation. Moreover, peroxidase genes showed a net $\log 2$ fold up-regulation of 19.8 in MP-inoculated Tx7000. To confirm the MP-inoculation associated oxidative stress, reactive oxygen species (ROS) content of cell extracts of charcoal rot resistant (SC599, SC35) and susceptible (Tx7000, BTx3042) sorghum genotypes was assayed using dichlorodihydrofluorescein while peroxidase activity was measured using amplex red reagent. Both assays were based on fluorimetric determinations. Malondialdehyde (MDA) content was measured to confirm the ROS-mediated lipid peroxidation. MP significantly increased ROS content and peroxidase activity in the susceptible genotypes. Significantly increased MDA content confirmed the strong oxidative stress experienced by MPinoculated susceptible genotypes. Findings revealed the MP's ability to promote strong oxidative stress in Tx7000 and BTx3042 which leads to induced cell death-associated disease susceptibility.

Development of antisense antimicrobials for fire blight management Q. ZENG, Department of Plant Pathology \& Ecology, The Connecticut Agricultural Experiment Station, New Haven, CT, USA

Erwinia amylovora is the causal agent of fire blight, a devastating disease of apple and pear. Fire blight is traditionally managed by the application of the antibiotic streptomycin during bloom, but this strategy has been challenged by the development and spread of streptomycin resistance. Thus, there is an urgent need for effective, specific, and sustainable control alternatives for fire blight. Antisense antimicrobials are oligomers of nucleic acid homologs with antisense sequence of essential genes in bacteria. The binding of these molecules to the mRNA of essential genes can result in translational repression and antimicrobial effect. Here, we explored the possibility of developing antisense antimicrobials against E. amylovora and using these compounds in fire blight control. We determined that a 10-nucleotide oligomer of peptide nucleic acid (PNA) targeting the start codon region of an essential gene acpP is able to cause complete growth inhibition of E. amylovora. We found that conjugation of cell penetrating peptide (CPP) to PNA is essential for the antimicrobial effect, with $\mathrm{CPP} 1[(\mathrm{KFF}) 3 \mathrm{~K}]$ being the most effective against E. amylovora. Examination of the antimicrobial mechanisms demonstrated that anti-acpP-CPP1 caused dose-dependent reduction of acpP mRNA in E. amylovora upon treatment and resulted in cell death (bactericidal effect). Anti-acpP-CPP1 $(100 \mu \mathrm{M})$ is able to effectively limit the pathogen growth on stigmas of apple flowers. Finally, unlike streptomycin that does not display any specificity in inhibiting pathogen growth, anti-acpP-CPP1 has more specific antimicrobial effect against E. amylovora.

\section{A multi-resistance plasmid transfers at high frequencies among Pseudomonas syringae}

L. BARDAJI, M. Echeverría, J. Murillo, Universidad Pública de Navarra, Pamplona, SPAIN

Chemical control of bacterial diseases often results in the selection of resistant pathogen populations containing mobile genetic elements (MGEs) that encode resistance to antimicrobials. The bean pathogen Pseudomonas syringae pv. syringae UPN800 carries the chromosomal MGEs Tn5393, conferring resistance to streptomycin, and the ICE GI16, coding for copper resistance. In liquid media and in planta, UPN800 transferred resistance to both compounds to $P$. syringae pv. phaseolicola $1448 \mathrm{~A}$ at a very high frequency of up to $6 \cdot 10^{-1}$. However, the resulting transconjugants did not contain the copper resistance copABCD-RS genes from ICE GI16, and also showed increased MIC values for arsenic and mercury, suggesting they inherited a different MGE from strain UPN800. Comparative genomics of UPN800, 1448A and a multi-resistant 1448A transconjugant, identified $c a$. $272 \mathrm{~kb}$ that were collectively transferred to 1448A. This sequence was annotated and functionally characterized, including transference assays, qPCR, hybridization, phylogenetic analyses, and mutagenesis. Altogether, our data indicate that this 272-kb sequence constitutes a single-copy, mobilizable plasmid, designated pE800, that harbors a second copy of Tn5393 and four operons for resistance to diverse heavy metals. pE800 shows extensive conservation and synteny with chromosomal and plasmid sequences from species of Pseudomonas isolated from clinical environments, waste water and contaminated soils. Thus, plant pathogenic pseudomonads can acquire readily transferable multi-resistant MGEs circulating in different ecological niches that contribute to their resistance to phytosanitary products and survival in polluted environments.

\section{Direct evidence of $C S L O B 1$ being the susceptibility gene of citrus canker caused by Xanthomonas citri} S. DUAN, Univerisity of Florida, Lake Alfred, FL, USA

Xanthomonas citri ssp. citri $(\mathrm{Xcc})$ is an important plant pathogenic bacteria and causes citrus canker disease worldwide. PthA, a type III secretion system (T3SS) effector, is a critical pathogenicity determinant of Xcc. PthA and its variants are transcriptional activator like (TAL) effectors, which direct the expression of specific disease susceptibility genes during infection. In citrus, it has been suggested that PthA4 targets the CsLOB1 to induce its expression and downstream genes to cause pustule formation. However, our previous evidence linking CsLOBI induction to canker disease symptom development is correlative. In this study, we used the dexamethasone(DEX) induced nuclear targeting of reporter construct containing glucocorticoid receptor (GR) and CsLOB1. Transgenic Duncan grapefruit (Citrus paradisi Macf.) plants expressing 35S:CsLOB1-GR were generated. DEX (100 $\mu \mathrm{M}$ ) treated leaves of the $C S L O B 1$-transgenic plants exhibited a pustule formation, which was not observed in DEX treated wild type plants and in non-treated $C s L O B 1$ - transgenic plants. Importantly, no water soaking was oberved with those pustule symptoms in DEX-treated CsLOB1-transgenic plants. When CsLOB1-transgenic Duncan grapefruit leaves were inoculated with Xcc306 $\Delta$ pthA4, then treated with DEX solution and mock, water-soaking symptoms were observed on DEX-treated transgenic plant leaves, but not on mock treated plants at 7 days post inoculation. We have selected 12 genes which were induced by PthA4 were also induced by CsLOB1. In summary, we have provided the direct evidence of CsLOBI being the citrus susceptibility gene for Xcc.

Does Salmonella enterica translocate type III effectors into plant cells?

L. CHALUPOWICZ (1), G. Nissan (2), M. T. Brandl (3), M. McClelland (4), G. Sessa (5), I. Barash (2), S. Manulis-Sasson (6), (1) ARO, The Volcani Center, Rishon LeZion, ISRAEL; (2) Tel Aviv University, Tel Aviv, ISRAEL; (3) Produce Safety and Microbiology Research Unit, Albany, CA, USA; (4) University of California, Irvine, CA, USA; (5) Tel Aviv University, Tel-Aviv, ISRAEL; (6) ARO The Volcani Center, Rishon LeZion, ISRAEL 
Salmonella enterica, a human enteric pathogen, can multiply and survive endophytically in plants. This colonization has been associated with an induction and subversion of pattern-triggered immunity (PTI). PTI suppression can be achieved by type III effectors (T3Es) injected into the plant cells through the type III secretion system (T3SS). Based on previous reports, it remains unclear whether the T3Es of Salmonella contribute to plant colonization. We employed two genetically engineered plasmids based on the HR domains of avirulence proteins from the Pantoea agglomerans/beet and Xanthomonas euvesicatoria/pepper pathosystems to explore whether the T3SS of Salmonella can translocate T3Es into plants. T3Es of $S$. Typhimurium (14028), sipB (SPI-1 T3SS) and sifA (SPI-2 T3SS), were not translocated by Salmonella into beet cubes or pepper leaves. In contrast, these effectors were translocated into plant cells by the phytopathogenic bacteria P. agglomerans pv. betae, Erwinia amylovora and X. euvesicatoria. No difference was observed in endophytic colonization of lettuce leaves by Salmonella strains mutated in the T3SS structural or effectors genes invA, spaS, sipB and sifA compared to the wild type. Our results support the conclusion that the Salmonella T3SS system, which is phylogenetically different from the T3SS of plant pathogens, cannot translocate T3Es into plant cells.

Contribution of $d s p E$ and eop1 to Erwinia tracheiphila virulence

O. I. OLAKUNLE, M. L. Gleason, G. A. Beattie, Iowa State University, Ames, IA, USA

Erwinia tracheiphila is a xylem-inhabiting bacterium causing wilt of cucurbits in the Northeastern and Midwestern USA. Previous studies have shown that E. tracheiphila strains fall into groups exhibiting differences in host-specific virulence, as illustrated by the ability of individual strains to induce rapid wilting on Cucumis melo or Cucurbita pepo hosts, but not both. DspE and Eop1 are plant bacterial effectors that belong to the AvrE and YopJ/AvrRxv family of type III secreted proteins, respectively. Although these effectors have been examined for their contribution to the virulence and host-specificity of other plant pathogens, nothing is known about their roles in the xylem pathogen E. tracheiphila. Here we report the first characterization of the roles of $d s p E$ and eop 1 in E. tracheiphila virulence. We constructed deletion mutants lacking $d s p E$, $d s p E F$ (the effector and its chaperone), or eop 1 in representative strains isolated from Cucumis or Cucurbita hosts using the Lambda Red Recombineering system. Mutants of a C. melo isolate lacking $d s p E$ and $d s p E F$ were reduced, but were not deficient, in their ability to wilt plants, indicating that $d s p E$ contributes to, but is not required for, pathogenicity. This is contrary to a requirement for DspE for pathogenicity in closely related species like E. amylovora and Pantoea stewartii. Mutants of two C. melo isolates lacking eopl were not altered in virulence when inoculated into C. melo plants, but this does not address whether eop 1 functions to limit virulence on a heterologous host. We are currently exploring the impact of eop 1 in host-specific virulence by examining the impact of heterologous eopl expression on E. tracheiphila virulence in Cucumis and Cucurbita hosts.

Identification and characterization of plasmids from Xylella fastidiosa using next generation sequencing analyses C. VAN HORN (1), F. Wu (2), Z. Zheng (2), J. Chen (1), (1) USDA-ARS-PWA, Parlier, CA, USA; (2) South China Agricultural University, Guangzhou, CHINA

Xylella fastidiosa causes diseases in many economically important crops, e.g. Pierce's disease (PD) of grapevines and blueberry bacterial leaf scorch (BBLS) disease in U.S. Biological research on X. fastidiosa has been difficult due to its nutritionally fastidiousness. Genomic research provides a new venue to study Xylella biology. Next generation sequencing (NGS) has recently become a common tool in bacterial biology studies due to the increasing affordability and abundance of data produced. In this study, MiSeq sequencing, an NGS technology, was used to study plasmids in Stag's leap, a PD strain (X. fastidiosa subsp. fastidiosa) and BB01, a BBLS strain (X. fastidiosa subsp. multiplex). Plasmids are extrachromosomal mobile genetic elements often related to bacterial survival, environmental adaptation, and virulence. Using DNA extracted from in vitro pure culture, MiSeq sequencing generated data sets of $6.59 \times 10^{6}$ sequence reads $(301 \mathrm{bp} / \mathrm{read})$ for Stag's leap, and $1.45 \times 10^{7}$ sequence reads $(250 \mathrm{bp} / \mathrm{read})$ for BB01. Analyses of de novo assemblies identified two plasmids: pXFSL01 (20,542 bp) from Stag's leap carrying a predicted acriflavin resistance gene, and pXFBB01 (31,648 bp) from BB01 carrying a predicted NAD-specific glutamate dehydrogenase gene. The two plasmids likely exist as single copies and are capable of integration into their host chromosomes. These results set forward a base for further studies on Xylella-host interactions.

Role of cytosine-5 DNA methyltransferases in morphogenesis and virulence of phytopathogen Cryphonectria parasitica J. Bal, Y. H. Ko, K. K. So, D. H. KIM, Chonbuk National University, Jeonju, Korea, Republic of (South)

Cytosine-5 (C5) DNA methylation, mediated by enzymes called DNA methyltransferases (DMTase), is a well characterized epigenetic mechanism associated with a variety of functions such as gene regulation or control of genomic stability. Silencing of genes may account for degeneration of phenotype or attenuation of virulence. Cloning and characterization of C5- DMTases from Cryphonectria parasitica was performed. Two putative C5DMTase genes, $C p D m t 1$ and $C p D m t 2$, consisting of highly conserved DNA cytosine methyltransferase domain were identified from the genome database. Phylogenetic analysis put $C p D m t 1$ closer to Neurospora crassa RID whereas $C p D m t 2$ closer to $N$. crassa Dim2. CpDmt1-and $C p D m t 2$-null mutants were constructed and confirmed through PCR and Southern blot analysis. In complex medium PDAmb, the CpDmt1-null mutant displayed retarded growth, but as the culture proceeded, the mutant colony showed sporadic fluffy mycelial growth at the margin. However, the $C p D m t 2$-null mutant showed less fluffy growth than the wild type with outer concentric circular pigmentation. The robust mycelial growth of the $C p D m t 1$-null mutant was stably inherited without reverting to the parental morphology. Virulence assay through inoculation into excised chestnut tree bark suggested that the $C p D m t 1$ - and $C p D m t 2$-null mutants were less virulent. This study will help us to understand the role of epigenetics in the pathogenicity of $C$. parasitica.

Characterization of a putative tunicamycin gene cluster in Rathayibacter toxicus M. TANCOS, A. Sechler, M. B. McMahon, D. Luster, W. Schneider, E. E. Rogers, USDA ARS FDWSRU, Fort Detrick, MD, USA

The APHIS-listed select agent Rathayibacter toxicus, causal agent of annual ryegrass toxicity, is a Gram-positive bacterium that infects a variety of forage grasses through its close association with seed gall nematodes belonging to the genus Anguina. R. toxicus produces a tunicamycin-related toxin under undefined field conditions, resulting in sporadic disease outbreaks that cause morbidity and mortality among grazing livestock. At present, little is known about this toxigenic bacterium and the regulatory mechanisms involved in toxin production. Sequencing of several $R$. toxicus strains has demonstrated the conservation of a putative tunicamycin-like biosynthetic gene cluster that spans 14 genes, in which 12 of these genes (tunA-tunL) are homologous to the tunicamycin gene cluster (TGC) of Streptomyces chartreusis. All the genes necessary for tunicamycin biosynthesis are present, however their order and orientation differ in $R$. toxicus relative to $S$. chartreusis. Similar to $S$. chartreusis, no apparent regulatory genes are present Functionality of the $R$. toxicus TGC was evaluated with gene-specific disruptions. In order to characterize environmental triggers and potential forms of regulation for tunicamycin biosynthesis, the $R$. toxicus TGC was investigated at the transcriptional and translational level under a variety of stress conditions. Characterization of the TGC will help determine the regulatory mechanisms that induce and modulate toxin production in $R$. toxicus.

Characterization of effectors from Fusarium graminearum

G. HAO, M. Vaughan, S. Mccormick, A. Kelly, T. J. Ward, USDA/ARS/NCAUR, Peoria, IL, USA

Fusarium graminearum is the causal agent of Fusarium head blight (FHB), which reduces crop yield and quality by producing various mycotoxins. Effectors play an important role in the pathogenesis of many bacterial and fungal pathogens. In this study, twenty six effector candidates were selected for investigation. The expression of these effectors was evaluated during pathogenesis in wheat. Our analysis revealed that some effectors were highly up-regulated at the early or late infection stage. Expression of the effector gene located at the FGSG_03598 locus was strongly upregulated $36 \mathrm{~h}$ post inoculation but was then reduced at $7 \mathrm{~d}$ post inoculation. FGSG_03598 encodes a 309-aa protein that shares homology to sialidases from other organisms. Sialidases play important roles in diverse biological processes such as biofilm formation and microbial pathogenesis. The FGSG_03598 protein contains a signal peptide but no transmembrane domain. Further examination of the protein sequence suggests that the protein is secreted from 
the fungus and targeted to the plant chloroplast. Knock-out mutant of FGSG_03598 exhibited reduced FHB development. The role of FGSG_03598 in pathogen virulence and its localization in plant are under investigation.

TsnRNA-IIIb induces citrus dwarfing and alters the hormone profile of the trees

I. LAVAGI, G. Greer, T. Dang, S. H. Tan, S. Bodaghi, P. E. Rolshausen, C. Lovatt, G. Vidalakis, University of California, Riverside, Riverside, CA, USA

'Transmissible small nuclear Ribonucleic acids' (TsnRNAs) are well-characterized viroid RNA species that act as regulatory genetic elements modifying tree performance in specific citrus hosts without inducing disease. TsnRNA-IIIb (syn. citrus dwarfing viroid) can reduce canopy volume of navel orange trees on trifoliate rootstock (approx. 50\%), increase yield per canopy volume, and concentrate fruit in the optimum canopy zone for harvest without affecting fruit quality. These findings raised questions regarding the mechanism of this plant-TsnRNA interaction. To understand the cellular and physiological mechanisms modulated by TsnRNAs, we initiated a physiological study of navel orange on trifoliate rootstock with and without TsnRNA planted in 1997. Initial measurements showed that the TsnRNA-IIIb-induced decrease in canopy volume resulted from reduced apical shoot growth. The involvement of phytohormones, key regulators of growth and development, was considered and a phytohormone profiling study was undertaken. The results will be discussed in relation to observed TsnRNA-IIIb-induced dwarfing versus control trees. The importance of elucidating the TsnRNA dwarfing mechanism lies in the potential to develop commercial applications that do not require a transmissible agent. Dwarfed trees are fundamental for high-density plantings that will be critical for meeting challenges posed by water shortages, disease spread, farmland reduction, and increasing labor costs.

Influence of Xylella fastidiosa cold shock proteins on pathogenesis in grapevine L. BURBANK, USDA-ARS, Parlier, CA, USA

Cold shock proteins (CSPs), a family of nucleic acid binding proteins are an essential part of microbial adaptation to temperature changes. Bacterial CSPs are often expressed in a temperature-dependent manner, and act as chaperones, facilitating translation at low temperature by stabilizing mRNA. In addition, CSPs are important for response to osmotic and oxidative stress and for stationary phase survival, highlighting the importance of these proteins for bacterial adaptation to changing environments such as during pathogenesis. Recent evidence also suggests that CSPs can be present at the surface of bacterial membranes, influencing interactions with host such as elicitation of plant defense responses. Xylella fastidiosa (Xf), the causal agent of Pierce's disease in grapes, has two CSP homologs, at least one of which $(X f \mathrm{Csp} 1)$ is important for virulence in grapevine. A knockout mutant in Csp1 also was less able to survive cold temperatures and osmotic stress. A knockout mutant in Csp2 was unstable, suggesting it may be essential for viability during in vitro growth. Interestingly, expression of $X f \mathrm{Csp} 1$ and $\mathrm{Csp} 2$ appear to be temperature-independent, rather than cold-inducible. Further investigation of the role of $X f$ CSPs in bacterial adaptation to environmental stress, and its interactions with the host plant will lead to a better understanding of persistence and long term survival of this pathogen.

Analysis of a virulence gene family of Pyricularia from cereal and grass hosts D. J. EBBOLE (1), Y. Han (1,2), M. L. Chen (1,2), W. Zheng (1,2), X. Wu (1,2), G. Lu (2), Z. H. Wang (2), (1) Texas A\&M University, College Station, TX, USA; (2) Fujian Agriculture and Forestry University, Fuzhou, CHINA

A 21 member gene family of the rice blast fungus (Pyricularia oryzae) is expressed primarily during infection and colonization of rice. At least one of the gene family members is delivered into rice cells. This gene is able to suppress programmed cell death in Nicotiana benthamiana. No allelic variation leading to amino acid substitutions were found in analysis of rice isolate genome sequences, however, presence/absence polymorphism and other null alleles were noted. In populations of $P$. oryzae infecting other hosts, amino acid substitutions relative to the rice isolate sequences were common. Some gene family member alleles are unique and diagnostic of rice-adapted isolates. The number of sequences of isolates from non-rice hosts is limited, however, the gene family haplotypes within host-specific populations have shown relatively little variation so far, except for isolates from wheat. Several of the haplotypes in the wheat-infecting population resemble those from other grass hosts, suggesting that genetically distinct grass-infecting populations may have contributed individuals to the wheat-infecting population. $P$. grisea is a pathogen of digitaria grasses that has diverged from $P$. oryzae. The gene family has diverged much faster than expected compared to other genes. We propose adaptation to a host species involves selection operating on gene family members.

In silico predictive studies translate into an understanding of complex molecular interactions between pospiviroid pathogens and their tomato host

R. W. HAMMOND, N. Kovalskaya, N. Kreger, K. Avina-Padilla, USDA ARS Molecular Plant Pathology Laboratory, Beltsville, MD, USA

Viroids are unencapsidated, single-stranded, covalently closed circular, highly structured, noncoding RNAs of 239 - 401 nucleotides that cause disease in several economically important crop plants. In tomato, symptoms of pospiviroid infection include stunting, reduced vigor, flower abortion, and reduced size and number of fruits, resulting in significant crop losses. These dramatic alterations in plant development that are triggered by viroid infection are the result of differential gene expression patterns where many genes are up-regulated and others are down-regulated; evidence is accumulating that short RNA fragments derived from the viroid molecule (vd-sRNAs) target host gene transcripts for cleavage and result in downregulation. Alternatively, the mechanisms by which viroids increase gene transcription are poorly understood. In our studies, in silico predictions of viroid sRNA targets in plant genes and experimental validation of predicted targets was performed, including genes that are down-regulated, such as WD-40 (growth and pollen viability) and the tomato homolog of OVA6 (fertility), and those that are up-regulated, including the tomato homolog of BIGPETAL1 (petal morphology) and PKV protein kinase (phospholipid signaling). We report the identification of unexpected interactions between viroid RNAs and their plant host, contributing fundamental knowledge to our present understanding of viroid pathogenesis.

A five amino acid motif in the potato leafroll virus minor capsid protein regulates local virus movement

Y. XU (1), S. Gray (2), (1) Section of Plant Pathology and Plant-Microbe Biology, Cornell University, Ithaca, NY, USA; (2) Cornell University, Ithaca, NY, USA

The minor structural protein of potato leafroll virus, known as the readthrough protein (RTP) is required for efficient virus movement in plant hosts. Analysis of numerous deletion mutations in the RTP identified a five amino acid motif in the C-terminal domain that is required for RTP function. Deletion of the motif severely reduced virus accumulation and symptom development in hairy nightshade (Solanum sarrachoides) and Nicotiana benthamiana. Although the mutant virus was able to move long distance in inoculated plants, there were limited infection foci in the systemically infected leaves. The five amino acid deletion did not alter the efficiency of RTP translation, nor impair RTP interaction with P17, the virus movement protein, however it did alter the subcellular localization of RTP. Normally in the presence of P17, RTP is localized to the cell membrane and plasmodesmata. The mutant RTP is localized mainly to aggregates in the cytoplasm. Unexpectedly, 2 of 30 plants infected with the mutant developed a wild type virus infection phenotype 10 weeks post-inoculation due to compensatory mutations that restored normal RTP function. The five amino acid motif is crucial for normal RTP function and is under strong selection pressure.

TAL effector PthA4 is required for the hypersensitive response by Xanthomonas citri in kumquat D. TEPER, N. Wang, University of Florida, Lake Alfred, FL, USA 
Citrus canker disease, caused by Xanthomonas citri sp. citri (Xcc), has a significant economic impact on the citrus industry. We characterized the responses of Meiwa kumquat (Fortunella crassifolia) to Xcc infection. Kumquat is closely related to commercial citrus cultivars and displays high resistance to $X c c$. Upon inoculation with $X c c$ kumquat plants display defense features such as up-regulation of ethylene biosynthesis and PR genes, production of hydrogen peroxide, localized hypersensitive response-like cell death and early leaf defoliation when compared to susceptible sweet orange. Deletion of the virulence associated TAL effector $p t h A 4$ in Xcc significantly affected kumquat responses to the bacteria including abolishing early leaf defoliation, hypersensitive response and significantly reducing the expression of ethylene biosynthesis marker genes and PR1. The effect of $p t h A 4$ mutant on kumquat was complemented with pthA4 and designed TALE constructs directed to the previously identified PthA4 target gene CsLOB1, indicating that kumquat response to PthA4 is mediated through ectopic overexpression of CsLOB1. Our study suggest a novel avirulence recognition system against a TAL effector based on the expression of its putative target gene.

Generating canker-resistant citrus varieties by modification of the citrus canker susceptibility gene CsLOB1 via Cas9/sgRNA technology H. JIA, Y. Zhang, V. Orbovic, J. Xu, N. Wang, CREC, University of Florida, Lake Alfred, FL, USA

Citrus bacterial canker is one of biotic challenges for citrus production. Traditional breeding of canker-resistant citrus varieties is challenging due to multiple limitations, including polyploidy, polyembryony, extended juvenility, and long crossing cycles. Targeted genome editing technology has the potential to shorten varietal development for many critical traits, including disease resistance. Here, we used CRISPR/Cas9/sgRNA technology to modify the canker susceptibility gene $C S L O B 1$ in Duncan grapefruit. Six independent lines, $\mathrm{D}_{\mathrm{LOB}} 2, \mathrm{D}_{\mathrm{LOB}} 3, \mathrm{D}_{\mathrm{LOB}} 9, \mathrm{D}_{\mathrm{LOB}} 10, \mathrm{D}_{\mathrm{LOB}} 11$ and $\mathrm{D}_{\mathrm{LOB}} 12$, were generated. Targeted next-generation sequencing of the six lines showed the mutation rate was $31.58 \%, 23.80 \%, 89.36 \%, 88.79 \%, 46.91 \%$ and $51.12 \%$ for $\mathrm{D}_{\mathrm{LOB}} 2$, $\mathrm{D}_{\mathrm{LOB}} 3, \mathrm{D}_{\mathrm{LOB}} 9, \mathrm{D}_{\mathrm{LOB}} 10, \mathrm{D}_{\mathrm{LOB}} 11$, and $\mathrm{D}_{\mathrm{LOB}} 12$, respectively, of the cells in each line. $\mathrm{D}_{\mathrm{LOB}} 2$ and $\mathrm{D}_{\mathrm{LOB}} 3$ showed canker symptoms similar to wild type grapefruit, when inoculated with the pathogen Xanthomonas citri subsp. citri (Xcc). No canker symptoms were observed on $D_{\mathrm{LOB}} 9, \mathrm{D}_{\mathrm{LOB}} 10, \mathrm{D}_{\mathrm{LOB}} 11$ and $\mathrm{D}_{\mathrm{LOB}} 12$ at 4 days post inoculation (DPI) with Xcc. Pustules caused by Xcc were observed on $\mathrm{D}_{\mathrm{LOB}} 9, \mathrm{D}_{\mathrm{LOB}} 10, \mathrm{D}_{\mathrm{LOB}} 11$ and $\mathrm{D}_{\mathrm{LOB}} 12$ in later stages, which were much reduced compared to that on wild type grapefruit. The pustules on $\mathrm{D}_{\mathrm{LOB}} 9$ and $\mathrm{D}_{\mathrm{LOB}} 10$ did not develop into typical canker symptoms. No side effects and off-target mutations were detected in the mutated plants. This study indicates that genome editing using CRISPR technology will provide a promising pathway to generate disease resistant citrus varieties.

Characterization of a 21 member effector gene family from the rice blast fungus M. L. CHEN (1,2), Y. Han (1,2), W. Zheng (1,2), X. Wu (1,2), G. Lu (2), Z. Wang (2), D. J. Ebbole (1), (1) Texas A\&M University, College Station, TX, USA; (2) Fujian Agriculture and Forestry University, Fuzhou, CHINA

A 21-member effector gene family of the rice blast fungus (Pyricularia oryzae) is expressed primarily during infection and colonization of rice. We are characterizing the role of gene family members in suppression of plant defense responses. To assess the timing of expression and localization, gene family members were expressed as fluorescent protein fusions. The length of promoter required for proper expression and protein localization was explored. We also assessed the role of the proteins in suppression of plant resistance. A gene that induces programmed cell death when expressed in Nicotiana benthamiana, BAX, was used as a marker for plant defense activation. Some, but not all, gene family members were able to suppress BAXmediated cell death. Additional efforts to assess the role of the effectors in suppression of defense and identify the interacting host target proteins are underway.

Two citrus viruses encode silencing suppressors to suppress posttranscriptional gene silencing S. H. TAN, S. Bodaghi, S. Abuhajar, G. Vidalakis, University of California, Riverside, Riverside, CA, USA

Viral proteins have been known to have multiple functions in virus-host interactions. In this study, we identified the coat protein (CP) and movement protein (MP) of Citrus tatter leaf virus (CTLV) and P0 of Citrus vein enation virus (CVEV) as suppressors of host antiviral RNA silencing. CTLV CP acts as a local suppressor of RNA silencing while the MP as a systemic suppressor and CVEV uses the P0 as a local and systemic silencing suppressor. When the Potato virus $\times$ infectious vector harboring CTLV CP, MP, or CVEV P0 was inoculated on Nicotiana benthamiana, all three constructs promoted viral infection and symptom development, likely through their RNA silencing suppression activities. Mass spectrometry-based immunoprecipitation proteomics, identified both CTLV CP and MP interacting with components of the host ubiquitin-proteasome pathway and probably hijacking it to degrade host silencing pathway proteins. CVEV P0, which possessed an F-box motif, interacted with the Skp and Cullin to form SCF complex. This complex is a muti-protein E3 ubiquitin ligase complex catalyzing the ubiquitination of proteins destined for proteasomal degradation. We also observed that the P0 triggered robust hypersensitive response-like cell death in $N$. benthamiana probably through gene-for-gene interaction. P0 deletion assay showed that the first 140 amino acids at the $\mathrm{N}$ terminal and the last 40 amino acids at the $\mathrm{C}$ terminal have important role in host recognition leading to cell death.

Pseudomonas syringae pv. tomato oxidative stress transcription factor OxyR and sigma factor AlgU play an important role for virulence Y. ISHIGA (1), T. Ishiga (1), Y. Ichinose (2), (1) University of Tsukuba, Tsukuba, JAPAN; (2) Okayama University, Okayama, JAPAN

Reactive oxygen species (ROS) have a crucial role in plant defense responses and signaling pathways. In addition, ROS also have direct toxicity against invading pathogens. Alginate is known to protect the bacterium from oxidative stress. However, the molecular mechanisms of plant ROS in the direct effects against pathogens is still unclear. To investigate the function of plant ROS in the interactions of plant and bacterial pathogens, we focused on the oxidative stress-regulated transcription factor OxyR and sigma factor AlgU, a key regulator involved in alginate biosynthesis, and generated $\Delta o x y R$ and $\triangle a l g U$ mutants in Pseudomonas syringae pv. tomato DC3000 (Pto DC3000). The Pto DC3000 $\Delta$ oxyR mutant showed high sensitivity to oxidative stress in comparison to the wild-type. The host plants of Pto DC3000 including tomato and Arabidopsis inoculated with the $\Delta$ oxy $R$ and $\Delta a l g U$ mutants showed reduced disease symptoms as well as reduced bacterial populations. Expression profiles of Pto DC3000 genes revealed that OxyR regulates the expression of genes related to oxidative stress including catalases. We also demonstrated that AlgU regulates not only genes involved in alginate biosynthesis but also genes related to type III secretion system. These results suggest that OxyR and AlgU have an important role in the virulence of Pto DC3000.

AefR transcription factor negatively regulates the virulence of Pseudomonas syringae pv. tomato DC3000 T. ISHIGA (1), Y. Ishiga (1), T. Kiyokawa (1), N. Maruyama (1), S. Betsuyaku (1), Y. Ichinose (2), N. Nomura (1), (1) University of Tsukuba, Tsukuba, JAPAN; (2) Okayama University, Okayama, JAPAN

The mechanism by which bacteria take intercellular communication and regulate gene expression according to bacterial density is called Quorum sensing (QS). To date, it has been reported that expression of virulence-related genes is controlled by QS in phytopathogenic bacteria. However, there are still many unclear points about the regulatory mechanism of virulence-related genes by QS. In this study, we investigated the molecular function of a transcription factor AefR, which is suggested to be involved in QS in P. syringae pv. tomato DC3000 (Pto DC3000). The $\triangle a e f R$ mutant showed enhanced virulence in host plants including tomato and Arabidopsis thaliana. Furthermore, gene expression profiles of Pto DC3000 during infection revealed that AefR controls not only $p s y R$ and $p s y I$ related to QS, but also the expression of genes involved in multidrug efflux pumps. These results suggest that AefR functions as a key molecule that controls QS and multidrug efflux pumps and plays an important role in the virulence of Pto DC3000. 
FRN1, a gene encoding putative GPCR protein, is critical for ear rot and fumonisin biosynthesis in maize pathogen Fusarium verticillioides H. YAN (1), M. Kim PhD (1), J. Huang (2), W. B. Shim (1), (1) Texas A\&M University, College Station, TX, USA; (2) Plant Protection College, Fujian Agriculture \& Forestry University, Fuzhou, CHINA

Fusarium verticillioides is one of the major fungal pathogens causing ear rot and fumonisin contamination in maize, posing significant food safety and security risks. Here, we studied a large-scale $F$. verticillioides RNA-seq datasets by a systematic network-based computational analyses to identify potential genetic modules involved with virulence and fumonisin regulation. Our aim was to characterize subnetwork modules in F. verticillioides coexpression network that are significantly deferentially expressed in two different maize lines (moderately resistant vs susceptible), then to predict hub genes in these functional subnetwork modules. We identified a putative hub gene (FRNI), encoding a putative GPCR with 7 transmembrane regions, in a subnetwork module exhibiting a strong discriminative power. We hypothesized that $F R N 1$ operates collectively with other linked genes in subnetwork to regulate fumonisin biosynthesis and pathogenesis. Knockout mutant frnl showed little effect on morphology and fertility but failed to produce fumonisin and pigmentation. Furthermore, frn 1 mutant showed significant alteration in the expression of FUM1, FUM8 and select PKS genes, which are associated with fumonisin biosynthesis and secondary metabolism. Notably, FRN1 demonstrated polymorphism in different Texas field isolates. Protein alignment revealed significant similarity within Fusarium species, while predominantly absent in other fungal species.

Characterization of two major facilitator superfamily (MFS) transporters in multi-drug resistance in the citrus fungal pathogen Alternaria alternata

K. R. CHUNG, National Chung-Hsing Univ, Taichung Taiwan, TAIWAN

Two major facilitator superfamily (MFS)-coding genes (designated MFS19 and MFS54) were originally identified from the citrus fungal pathogen Alternaria alternata cDNA library after subtracting with that of a YAP1 mutant defective for oxidative stress resistance. MFS19 and MFS54 showed similarity, respectively, to the drug resistance transporters Bcr/CflA subfamily and EmrB/QacA subfamily. Mutational inactivation of MFS19 or MFS54 yielded fungi that displayed increased sensitivity to a broad range of compounds, such as 2,3,5-triiodobenzoic acid (an auxin transport inhibitor), 2chloro-5-hydroxypyridine (a heteroaromatic compound) and hematoporphyrin (a potent singlet oxygen-generating compound), iprodione and vinclozolin (dicarboximide fungicides), fludioxonil (a phenylpyrrole fungicide), clotrimazole, copper chloride, and copper sulfate. Fungal strains defective in MFS54 but not MFS19 also displayed elevated resistance to copper fungicide, cumyl $\mathrm{H}_{2} \mathrm{O}_{2}$, and eosin Y; however, neither MFS19 nor MFS54 mutant displayed hypersensitivity to $\mathrm{H}_{2} \mathrm{O}_{2}$. Semi quantitative RT-PCR and Northern blotting revealed that the expression of the MFS19 and MFS54 genes was downregulated in fungal strains carrying defective YAP1 or "two-component" histidine kinase (HSK1). The expression of the MFS19 but not the MFS54 gene also was regulated by the HOG1 and FUS3 MAP kinases. Infection assays on detached citrus leaves revealed MFS19 and MFS54 were not required for fungal virulence.

\section{Chromosomally encoded peroxiredoxin CLIBASIA_00485 of $\mathrm{Ca}$. Liberibacter asiaticus is a virulence factor needed for survival and colonization of citrus}

M. JAIN, A. Munoz Bodnar, S. Zhang, D. W. Gabriel, University of Florida, Gainesville, FL, USA

We previously reported that a prophage-encoded peroxidase (SC2_gp095) in Ca. Liberibacter asiaticus (Las) is an important lysogenic conversion gene required for bacterial fitness and delayed symptom development. Some Las strains appear to lack prophage, prompting us to review alternative $\mathrm{H}_{2} \mathrm{O}_{2}-$ detoxification mechanisms in Las. Two putative peroxiredoxin genes (CLIBASIA_00980 and CLIBASIA_00485) are conserved among all sequenced Las strains. CLIBASIA 00980 appears to be a housekeeping gene with similar transcript abundance in both Las infected psyllids and citrus. However, CLIBASIA_00485 expression was nearly undetectable in psyllids, similar to SC2 peroxidase. Both CLIBASIA_00980 and CLIBASIA_00485, when expressed in the cultured Las proxy, Liberibacter crescens (Lcr), conferred significant resistance against oxidative damage, despite Lcr carrying native orthologs of both genes. Lcr cells expressing CLIBASIA_00980 were 65-fold more resistant to $\mathrm{H}_{2} \mathrm{O}_{2}$. Similarly, Lcr with CLIBASIA_00485 were 36fold more resistant to $\mathrm{H}_{2} \mathrm{O}_{2}$ but additionally, a striking $21 \overline{4}$-fold higher level of resistance to tert-butyl hydroperoxide (an organic peroxide). Transient over-expression of CLIBASIA_00485 in tobacco suppressed $\mathrm{H}_{2} \mathrm{O}_{2}$-induced activation of $R b o h B$, the key gatekeeper of plant defense signaling cascade. As with SC2 peroxidase, CLIBASIA_00485 is secreted, suppresses defense signaling, and also provides defense against a chain reaction-like spread of oxidative damage to membrane lipids.

Verticillium dahliae LHS1 is required for virulence and expression of extracellular enzymes involved in cell-wall degradation W. Cui, X. Dai, W. GUO, Institute of Food Science and Technology, Chinese Academy of Agricultural Sciences, Beijing, CHINA

Verticillium dahliae is a devastating soilborne fungus causing vascular wilt disease in a wide range of economically important crops. Cell wall-degrading enzymes (CWDE) secreted by $V$. dahliae have been considered to play important roles in pathogenesis. However, little is known about the secretion mechanisms of CWDE used by this pathogen. In this study, we investigated the role of the $V$. dahliae LHSI (VdLHSI), the homolog of which in Magnaporthe oryzae is an endoplasmic reticulum (ER) chaperone protein that was previously implicated in pathogenicity and expression of extracellular enzymes. We showed that VdLHS1 colocalized with ER-tracker dye Blue-White DPX in ER. The $\triangle V d L H S 1$ mutant showed defects in radial growth and asexual conidiospores. The disease index of cotton inoculated with $\triangle V d L H S 1$ mutant was significantly lower than that of wildtype strain throughout the assay up to 30 days postinoculation. On complete medium (CM) plates supplemented with hydrogen peroxide, sodium chloride, sorbitol and SDS, the growth rates of the $\triangle V d L H S 1$ mutant and the wildtype were not significantly different from those on CM. Furthermore, the activities of several extracellular enzymes in culture filtrates from the $\triangle V d L H S 1$ mutant and the wildtype were compared using different test plate assay and enzyme linked immunosorbent assay. The activities of celluase, ligninase, laccase, pectinase and xylanase were significantly reduced in the $\triangle V d L H S 1$ mutant, and those of amylase and beta-glucosidase did not change markedly in the mutant. However, activities of protease increased remarkably in the $\triangle V d L H S 1$ mutant. Together, our results suggest that the $V d L H S 1$ is requisite for pathogenicity, conidiation and secretion of extracellular enzymes.

The involvement of LsGRP1 in salicylic acid-related disease resistance and photosynthesis C. A. Chen, C. H. Lin, C. Y. CHEN, National Taiwan University, Taipei, TAIWAN

The defense-related gene $L s G R P 1$ encoding a plant class II glycine-rich protein exhibits an increased level of expression in Lilium spp. after Botrytis elliptica infection, the fungal pathogen of lily leaf blight. A number of putative LsGRP1-interacting proteins were isolated from lily leaves via coimmunoprecipitation and identified as proteins taking a part in electron transport chain and Calvin cycle. A test of lily plants with part of the leaves enveloped in black paper bags demonstrated that a dark treatment conducted a decrease of LsGRP1 content whereas a complement of light exposure enabled a recruit of LsGRP1 accumulation. In addition, fluorescence observation indicated that LsGRP1 accumulated in the chloroplasts of lily protoplasts, revealing that lily defense mediated by LsGRP1 might be photosynthesis-related. Furthermore, virus-induced gene silencing system was used to decrease the expression levels of LsGRP1 and a putative LsGRP1-interacting protein, Rubisco small subunit (RbcS). Inoculation test revealed that VIGS-LsGRPI and VIGS-RbcS lily plants were more susceptible to B. elliptica infection, the decrease of photosynthesis rate caused by $B$. elliptica significantly occurred as conducted by a pretreatment of salicylic acid, and the silencing of LsGRPI and RbcS affected gene expression of Photosystem I reaction center subunit $N$ and Photosystem II subunit $O-2$. Presumably, LsGRP1 may interact with chloroplast proteins to regulate the physiological and biochemical reactions of lily, and increase the resistance against $B$. elliptica infection.

Stringent response regulator DksA positively regulates virulence traits of Xanthomonas citri

Y. ZHANG (1), N. Wang (2), (1) University of Florida, Lake alfred, FL, USA; (2) University of Florida, Lake Alfred, FL, USA 
The stringent response is a pleiotropic bacterial global regulatory response responding to various nutritional and metabolic stresses. Small signal molecule ppGpp and transcription factor DksA are involved in stringent response. Plant pathogenic bacteria encounter many stresses during the infection process. Here, we investigate the relationship between bacterial stringent response and virulence in Xanthomonas citri subsp. citri (Xcc), the agent of citrus canker disease. A $d k s A$ mutant was made by double crossover recombination. The mutant was unable to cause canker symptoms and HR after inoculation into its host Valencia sweet orange and non-host Nicotiana benthamiana respectively. Growth of the $d k s A$ mutant was significantly reduced in minimal medium M9 and in planta. To elucidate the role of DksA in virulence, qRT-PCR is used to test the expression of T3SS genes, important pathogenicity factors in gram-negative bacteria. Expression of the T3SS genes was significantly reduced in the $d k s A$ mutant compared to wild type, which is further confirmed by measuring promoter activity via Gus assay. DksA is also involved in biofilm formation and motility. We will discuss our findings in the context of how Xcc coordinates its stringent response and virulence traits in planta.

\section{Genetic analysis of motility and phytohormone biosynthesis of an endohyphal bacterium from Rhizoctonia solani} P. ZHANG, J. C. Huguet-Tapia, F. F. White, University of Florida, Gainesville, FL, USA

Fungal-bacterial interactions are pervasive in the rhizosphere, while the nature of the symbioses are relatively understudied. An Enterobacter sp. strain, named En-Cren, was isolated from the hyphae of the soil pathogen fungus Rhizoctonia solani AG2-2IIIB, the causal agent of brown patch disease. The free-living bacteria grow in close association with hyphae, and, upon contact, can rapidly colonize the entire fungal culture. Apart from hyphal colonization, we have focused on the production of phenylacetic acid (PAA) and indole acetic acid (IAA), which have been hypothesized to play a role in the interaction between $R$. solani and infected plants. PAA and IAA production were observed in En-Cren cultured in minimal media supplemented with precursor compounds phenylalanine or tryptophan, respectively. We will present a genetic analysis of Enterobacter sp. En-Cren with respect to motility and phytohormone biosynthesis. The $4.6 \mathrm{Mb}$ genome of En-Cren was sequenced to facilitate the genetic analysis. Initial mutations were created in the flagellar biosynthesis gene $f l g E$ and the $i p d C$ gene encoding indole-3-pyruvate decarboxylase, a key enzyme for indole-3-acetic acid (IAA) biosynthesis in Enterobacter. Loss of flagellar motility did not restrict movement along the fungal hyphae, while loss of ipdC did reduce apparent IAA production. The results of further genetic and biochemical characterization of motility and phytohormone production will be presented.

\section{Screening of Secreted in Xylem (SIX) Genes in Fusarium Wilt Pathogens of Palms}

S. PONUKUMATI (1), M. L. Elliott (1), J. A. Rollins (2), B. Desjardin (1), (1) University of Florida, Fort Lauderdale, FL, USA; (2) University of Florida, Gainesville, FL, USA

Fusarium wilt on ornamental palms in Florida is a lethal disease caused by Fusarium oxysporum f. sp. canariensis (FOC) and F. oxysporum f. sp. palmarum (FOP). While FOC and FOP share many similarities, including pathogenicity on Phoenix canariensis, FOP has a wider palm host range, causes a more aggressive disease and is primarily localized to Florida, whereas FOC is found world-wide and restricted primarily to one palm species. Secreted in Xylem (SIX) genes produce small secretory proteins, which may play a role in virulence and in the infection process of Fusarium oxysporum formae speciales. Currently, fourteen proteins have been identified in F. oxysporum f. sp. lycopersici, of which four have been characterized as effectors. Whole genome sequencing of FOC and FOP indicates the presence of SIX7, SIXIO and SIXI2 in FOC and SIX8 and SIX9 in FOP. A PCR based approach, using previously identified $S I X$ primers, were used to detect $S I X$ genes in these two pathogens. Twenty isolates each of FOC and FOP, obtained from different palm hosts and geographical locations, were used in this study. Results thus far confirm the presence of SIX7 and SIXI0 genes in all FOC isolates. An additional $S I X$ gene, $S I X 1$, was detected in most, but not all, FOC isolates. SIX 8 and $S I X 9$ genes are present in all FOP isolates. The different SIX gene profiles observed thus far suggest FOC and FOP utilize distinct complements of effectors for pathogenicity of palms.

\section{Identification and characterization of Fusarium graminearum pathogenesis genes}

M. SALAZAR (1), F. Kolb (2), S. X. Mideros Mora (1), (1) University of Illinois at Urbana-Champaign, Urbana, IL, USA; (2) University of Illinois, Urbana, IL, USA

Fusarium graminearum, the causal agent of Fusarium Head Blight (FHB) of wheat, produces trichothecene secondary metabolites such as deoxynivalenol (DON). Apart from DON, little is known about other pathogenesis compounds used by $F$. graminearum. To identify essential fungal pathogenesis genes, a paired strategy of isolates and transcriptome characterization of naturally infected lines was implemented. To collect a variety of $F$. graminearum strains, research sites were established at five Illinois locations using the University of Illinois soft red winter wheat improvement program plots. Wheat lines included the following: a resistant line, a moderately resistant line, a moderately susceptible line, and two susceptible lines. Ten naturally infected heads were identified for each line and two spikelets were collected. One spikelet was kept on ice for fungal isolation and the other was immediately submerged in RNAlater. A total of 197 Fusarium isolates were recovered. RNA extraction of selected samples yielded high-quality RNA and have been sequenced and produced $391 \mathrm{Mbp}$ of raw data. RNAseq analysis to compare the transcriptomes of resistant, moderately susceptible, and susceptible interactions and to identify pathogenesis genes that are required for infection on wheat is underway. To determine functional pathogen aggressiveness, pathogen strains will be inoculated onto wheat cultivars with varying levels of resistance in field and greenhouse experiments.

Hormone cross-talk in the interaction between 'Candidatus Liberibacter asiaticus' and citrus rootstock J. WU (1), F. Alferez (2), E. G. Johnson (1), J. H. Graham (1), (1) University of Florida, Lake Alfred, FL, USA; (2) University of Florida, Immokalee, FL, USA

Huanglongbing (HLB), caused by 'Candidatus Liberibacter asiaticus' (Las), is devastating citrus trees in Florida. To understand hormone's role in host response to Las infection, hormone metabolism status were evaluated in two Las-infected citrus rootstocks, Cleopatra mandarin (Citrus reticulata Blanco) and Swingle citrumelo (C. paradisi Macfad. $\times$ Poncirus trifoliata [L.] Raf.). A significantly higher number of Las copies was measured in roots of Cleopatra mandarin compared to Swingle citrumelo at 5 and 8 weeks post root trimming (wpt). In fibrous roots of Cleopatra mandarin, genes associated with salicylic acid (SA), ethylene (ET), and abscisic acid (ABA) synthesis and signaling, phospholipases D (PLD), and phospholipase A2 (PLA2) were activated by Las infection at 5 wpt. Expression of downstream effectors of SA, i.e., NPR1, WRKY 70 and PR1, did not change in Cleopatra mandarin, suggesting inhibition of the response to SA by the elevation of ABA, ET, PLD. In contrast, the up-regulation of PR1, and downregulation of biosynthesis of phytohormones indicated that Swingle citrumelo responds to Las infection via a more effective way against this biotrophic pathogen than the defense activated in Cleopatra mandarin.

\section{Investigating sRNA regulation of carbohydrate utilization in Erwinia amylovora} E. SWEENEY, Michigan State University, East Lansing, MI, USA

Fire blight, caused by the gram-negative bacterium Erwinia amylovora, is a destructive disease of apple and pear trees worldwide. A unique aspect of flower infection by E. amylovora is the progression of the organism through three different carbohydrate zones: from the glucose-containing stigma surface, to the high sucrose environment of the nectary, then to leaves and shoots where sorbitol is most abundant. It is not yet known how the sugarutilization genes of E. amylovora are regulated in response to these host changes. However, in Escherichia coli, small RNAs (sRNAs) mediated by the chaperone protein, Hfq, have been implicated in the metabolic regulation of non-preferred sugars such as sucrose and sorbitol. These sRNAs act by basepairing with target mRNAs to affect translation or stability. One such sRNA is called Spot 42 and is known to target a sorbitol uptake gene (srlA) in $E$. coli; this sRNA has recently been identified in E. amylovora. In this study, we hypothesized that the Spot 42 sRNA is involved in regulating $E$. amylovora carbohydrate utilization. To test this hypothesis, we generated knock-out mutants of $h f q$, spot 42 , and srlA. Using these strains, we conducted 
growth analyses, RNAseq, and additional gene expression studies in vitro and in planta to determine the role of Hfq-dependent sRNAs in regulating transitions between the carbohydrate zones as E. amylovora infects susceptible hosts.

\section{A mass spectrometry-based approach to quantitatively measure translocation of multiple Type III Secretion System effectors}

D. GILLIS, University of Georgia - Department of Plant Pathology, Athens, GA, USA

Many bacterial pathogens rely on a Type III Secretion System (T3SS) to deliver Type III Effectors (T3E) into plant cells to cause disease. Pseudomonas syringae pv. tomato DC3000 (Pto), a pathogen of tomato and brassicas, delivers a repertoire of 29 T3Es into plant cells. Current methods to quantify effector delivery do not allow for an absolute quantification nor simultaneous measurement of multiple T3Es. We have developed a method that involves a barcoded glycogen synthase kinase (GSK) motif fused to target T3Es to quantify T3E delivery. Our GSK tag consists of a 13-residue epitope from human GSK-3 beta with a unique four amino acid barcode fused to a target effector using Gibson Assembly. The GSK motif is phosphorylated at serine 9 in eukaryotic cells providing the signature of translocation into plant cells while the unique barcodes facilitate quantifiable measurement by mass spectrometry. Using transient expression, we have validated that barcoded GSK is phosphorlylated in plant cells. Eight peptides were synthesized and validated via LC-MS/MS for use in the system. A subset of validated tags were cloned into Pto for analysis of T3E delivery. Standard translocation assays confirmed delivery of tagged effectors. Use of this method would allow for an absolute quantification of multiple tagged T3E during a Pto infection. This will facilitate a systems-level understanding of effector coordination by pathogens during their interaction with plants.

Differential gene expression within Meloidogyne incognita: Response to exposure to sub-lethal doses of post-plant nematicides C. WRAM (1,2), A. Peetz (3), I. A. Zasada (4), (1) Oregon State University, Corvallis, OR, USA; (2) USDA ARS Horticultural Crops Research Unit, Corvallis, OR, USA; (3) USDA ARS HCRU, Corvallis, OR, USA; (4) USDA ARS, Corvallis, OR, USA

Meloidogyne species, root-knot nematodes, are one of the most important groups of plant-parasitic nematodes globally due to their ability to infect and damage almost all cultivated plants. Estimated crop loss by Meloidogyne spp. worldwide is 5\% annually. Despite the importance of these plant-parasitic nematodes, there are few control measures to prevent infection of plants by these nematodes. This is exemplified by the lack of robust post-plant nematicides to combat this economically-important group of nematodes. To better understand the mechanisms of these limited controls and facilitate the discovery of new control methods for plant-parasitic nematodes, new technologies can be employed to gain a deeper understanding of how to better target this pest. In this study, the lethal dose which resulted in 50\% morality $\left(\mathrm{LD}_{50}\right)$ for each of four post-plant nematicides was determined for Meloidogyne incognita second-stage juveniles (J2) using a microwell assay. Post-plant nematicides included in the study included both new and old nematicide chemistries, encompassed various modes of action, and represented a range of $\mathrm{LD}_{50}$ concentrations. Twenty-four hour $\mathrm{LD}_{50}$ concentrations for oxamyl, fluopyram, fluensulfone, and Q8U80 were 100 ppm, 1 ppm, 95 ppm, and 230 ppm, respectively. Meloidogyne incoginta J2 were exposed to $\mathrm{LD}_{50}$ concentrations of each nematicide for 24 hours, after which RNA was extracted and gene expression determined using RNAseq. RNAseq revealed genes potentially involved in metabolizing and overcoming these nematicides.

Evolutionary and biological basis of Xanthomonas systemic pathogenesis of plants J. M. JACOBS (1), T. Vancheva (2), A. Cerutti (3), J. M. Lang (1), C. Pesce (4), L. Noel (3), C. Allen (5), J. E. Leach (1), B. Szurek (6), S. Cunnac (6), C. G. Bragard (2), R. Koebnik (6), (1) Colorado State University, Fort Collins, CO, USA; (2) Universite Catholique de Louvain, Louvain-la-Neuve, BELGIUM; (3) LIPM Laboratoire des Interactions Plantes Micro organismes, UMR CNRS / INRA 2594/441, Castanet-Tolosan, FRANCE; (4) University of New Hampshire, Durham, NH, USA; (5) Univ of Wisconsin, Madison, WI, USA; (6) Institut de Recherche pour le Developpement, Montpellier, FRANCE

Pathogenic microbes cause systemic and non-systemic diseases of plant and animal hosts. Systemic diseases are particularly destructive because the pathogen moves through the host vasculature causing widespread infection; meanwhile non-systemic pathogens remain restricted to the non-vascular tissue near the site of infection. The basis of systemic and non-systemic pathogenesis is unclear. Here we describe the role of a cell wall-degrading enzyme in the evolution and biology in the Gram-negative phytobacterial genus Xanthomonas. Xanthomonas comprises a diverse group of vascular and non-vascular pathogens of over 200 plant species. We demonstrate that a single, vascular pathogen-unique cell wall degrading enzyme called CelA contributes to systemic pathogenesis in multiple pathogenic lineages in this diverse genus. We determined that CelA was conserved only in systemic pathogenic bacteria in the genera Xanthomonas, Xylella and Ralstonia but absent in non-systemic plant pathogenic bacteria. Most notably expression of this cell wall-degrading enzyme in two distinct non-systemic pathogen species, barley-infecting Xanthomonas translucens and rice-infecting Xanthomonas oryzae, permitted systemic spread in the vascular tissues of their respective host plants. From furhter genomic analysis, we determined that non-systemic Xanthomonas pathogens inactivated this trait through different mechanisms suggesting they arose from related vascular subgroups upon adapting to the non-vascular plant environment. Overall this work provides a framework to describe pathogen emergence based on symptom development and tissue-specificity in an important pathogen genus.

Live visualization of Type III effector activity in stomata and mesophyll cells by Xanthomonas translucens during leaf infection J. M. JACOBS (1), C. Pesce (2), T. Vancheva (3), F. Bini (4), J. Butchacas (5), G. Hensel (4), I. Otto (4), B. Szurek (5), J. Kumlehn (4), C. G. Bragard (3), R. Koebnik (5), (1) Colorado State University, Fort Collins, CO, USA; (2) University of New Hampshire, Durham, NH, USA; (3) Universite Catholique de Louvain, Louvain-la-Neuve, BELGIUM; (4) Inst for Plant Genetics \& Crop Plant Res, Gatersleben, GERMANY; (5) Institut de Recherche pour le Developpement, Montpellier, FRANCE

Many plant and animal pathogenic Gram-negative bacteria require a Type III secretion system and associated virulence effector proteins to cause disease. Although Type III effector activity has been largely studied, we still lack a strong understanding about the host cells targeted by effectors during infection. Type III effectors are typically difficult to detect because they are translocated into host cells at low concentrations. We developed a novel method to detect plant cells targeted by the plant bacterium Xanthomonas translucens, arguably the most important bacterial cereal and forage crop pathogen. A class of Type III effectors called Transcriptional activator-like (TAL) effectors found in many Xanthomonas spp. including X. translucens induce plant gene expression by binding promoters of host genes. We created transgenic barely plants that express GFP upon infection by $X$. transclucens expressing TAL effectors that target and activate the promoter of the GFP gene. We confirmed TAL effector-dependent GFP expression with quantitative PCR and visualization with fluorescence confocal microscopy. We overall provide evidence that major barley cell targets for $X$. translucens include both mesophyll cells and the stomata, the entry point for most major plant pathogenic foliar pathogens.

Evolution of avirulence genes in the wheat blast pathogen Pyricularia graminis-tritici

V. L. CASTROAGUDIN (1), S. Sousa Moreira (1), P. C. Ceresini (2), (1) University of Sao Paulo State, Ilha Solteira, SP, BRAZIL; (2) University of Sao Paulo State, Ilha Solteira, SP., BRAZIL

Races have been identified in Pyricularia graminis-tritici (Pgt), yet the genetics of their virulence spectra remains unknown. We studied presence/absence and sequence polymorphisms, and type of selection acting on 12 avirulence genes (Avrs) in 64 Brazilian isolates of Pgt representing 25 races and obtained from wheat and other Poaceae invasive of wheat fields. For comparison, we examined 16 isolates of Pyricularia oryzae (Po) representing 13 rice blast races from Brazil. A multilocus genotype (MLGN) was also determined for each isolate. Avr-Pi-a, $A v r-P i-t a^{2}, A v r-P i-k$ and PWL1 were absent in Pgt and Po. Avr-Pi-k and Avr-Pi-ta were absent in Pgt, and Avrl-CO39 and PWL4 were absent in Po. PWL2 and PWL3 prevailed in Pgt isolates from other Poaceae. For all loci, Pgt haplotypes (and encoded proteins) were different from those of Po. In Pgt, Tajima's D test showed Acel-avr and Avr1-CO39 were under neutral selection, and Avr-Piz- $t$ was under balancing selection. However, Ka/Ks ratio indicated Acel-avr and Avrl- 
CO39 were under directional selection. In Po, both Tajima's D and Ka/Ks ratio showed Avr-Pi-i, Avr-Pita, Avr-Piz-t and PWL4 were under neutral selection, and $A v r-P i-k$ under balancing selection. Pgt and Po did not share MLGNs. In Po, but not in Pgt, a direct correlation was observed between race and Avr-MLGN. This indicates that in Pgt a higher number of Avrs are needed to discriminate among races or Pgt has a strong recombination component in its reproductive cycle.

AM-Toxin and virulence in Alternaria tenuissima isolated from apple moldy core K. ELFAR (1), B. A. Latorre (1), J. P. Zoffoli (2), (1) Pontificia Universidad Católica de Chile, SANTIAGO, CHILE; (2) Pontificia Universidad Católica de Chile, Santiago, CHILE

Moldy core (MC) is an important disease affecting 'Red Chief', 'Red King Oregon' and 'Scarlet' apples, characterized by fruits with open sinus until harvest. Alternaria tenuissima and Alternaria spp. have been associated to MC in Chile. The presence of AM-toxin gen and virulence in six isolates of $A$. tenuissima were studied. Isolates of A. alternata, A. arborescens, and A. infectoria, previously isolated from MC of apple, were included as reference isolates. Pure cultures were obtained by single hypal tip transfer to PDA, and the presence of AM-toxin gen was examined with the specific primers LINF1/ LINR and AM-f/AM-r. The presence of AM-toxin gen was determined in four out of six A. tenuissima isolates, and in two A. infectoria isolates. No evidence of the presence of AM-toxin gen were found in isolates of $A$. alternata and A. arborescens. Virulence was study on detached wounded leaves $(\mathrm{n}=4)$ of 'Red Chief' apples, inoculated with $15 \mu 1$ of conidial suspensions $\left(10^{6}\right.$ conidia/ml $)$ and incubated for 7 days at $25^{\circ} \mathrm{C}$. All isolates of Alternaria studied induced grey brown necrotic lesions, but differences in virulence were obtained. Based on our results, AM-toxin producing isolates of A. tenuissima were more virulent than AM-toxin producing isolates of A. infectoria and non AM-toxin producing isolates of $A$. alternata and $A$. arborescens.

Small RNA-mediated gene regulation of pathogen and host during wheat stripe rust infection

N. A. MUETH (1), S. H. Hulbert (2), (1) Washington State University, Pullman, WA, USA; (2) Dept. of Plant Pathology, Washington State University, Pullman, WA, USA

The fungus Puccinia striiformis f. sp. tritici causes wheat stripe rust, a costly and widespread disease of cereal crops. This biotrophic pathogen carries genes encoding effector proteins that are capable of suppressing plant defenses. Recently, it was discovered that many fungi also possess highlyexpressed small RNA molecules, including microRNA-like and tRNA-derived sequences, yet the role of most pathogen-derived sRNA is unknown. We hypothesize that post-transcriptional gene silencing controls endogenous fungal development, as well as cross-kingdom RNA exchange to facilitate pathogenicity. In this study, we combined high-throughput small RNA sequencing with parallel analysis of RNA ends (PARE), a technique that reveals genes cleaved by the action of RNAi. Target genes corresponding to observed fungal small RNA sequences were discovered in both stripe rust and wheat. Select targets are being verified using RNA ligase-mediated 5' rapid amplification of cDNA ends. This work lends credence to the idea that small RNAs may function as effectors, and proposes intriguing new gene targets for the development of novel fungal pathogen control strategies.

Stop and smell the fungi: Uncovering overlooked roles of volatile metabolites emitted by Verticillium species in plant growth and development N. LI (1), S. Kang (2), (1) the Pennsylvania State University, University Park, PA, USA; (2) the Pennsylvania state University, University Park, PA, USA

Members of the genus Verticillium cause Verticillium wilt in diverse crop plants around the world. However, due to their soil habitat, long-term persistence in the infested soil, and capability of infecting a broad spectrum of plants, as well as the scarcity of resistant host germplasm, effective strategies for controlling Verticillium wilt have been very limited. Enhanced understanding of how Verticillium spp. interact with plants is vital to facilitate the development and deployment of novel disease management strategies. Using Verticillium, we investigated the role of fungal volatile metabolites, largely overlooked signals that mediate diverse organismal interactions, in plant growth and development. Strains representing ten Verticillium spp. significantly promoted growth of Arabidopsis thaliana and tobacco via volatile production. Using $V$. dahliae and A. thaliana, we investigated the mechanism underpinning Verticillium volatile-mediated growth enhancement in plants. Growth responses of $A$. thaliana auxin signaling mutants suggested the involvement of several components of the auxin signaling pathway in processing Verticillium volatile signals, with TIR3 playing a key role. Other components, including AUX1, TIR1 and AXR1, were also implicated but appeared to play lesser roles. Studies on auxin-responsive gene expression using a reporter system and growth response to the inhibition of polar auxin transport further supported the involvement of auxin signaling in Verticillium volatile-mediated growth promotion. This research helped uncover a novel mechanism that soil-borne fungal pathogens utilize to manipulate host plants.

Multiple roles of Wheat streak mosaic virus coat protein in wheat curl mite transmission, disease modulation and host range extension S. TATINENI (1), A. J. McMechan (2), G. L. Hein (3), (1) USDA ARS, Univ of Nebraska, Lincoln, NE, USA; (2) University of Nebraska-Lincoln, Lincoln, NE, USA; (3) University of Nebraska, Lincoln, NE, USA

Wheat streak mosaic virus (WSMV; genus Tritimovirus, family Potyviridae) is an economically important wheat virus transmitted by the wheat curl mite (Aceria tosichella Keifer). The coat protein (CP) of WSMV is unusually tolerant of extensive deletions for systemic infection of wheat. In this study, we found multiple functions for CP amino acids (aa) 58 to 100 in WSMV biology. Deletion of CP aa 58 to 84 dramatically increased WSMV symptom phenotype in wheat, maize, barley and rye. Additionally, deletion of CP aa 58 to 100 completely abolished WSMV transmission by wheat curl mites and failed to infect oat by specifically blocking long-distance transport but not cell-to-cell movement. The amino-proximal (aa 6 to 27 and 36 to 57 ) or carboxy-terminal (14 aa) regions of CP were expendable for mite transmission. Mutation of aspartic acid at aa position 289 or $326\left(D_{289} A\right.$ or $\left.D_{326} A\right)$ at the carboxy-proximal region of CP significantly affected mite transmission. Interestingly, mite-transmitted progeny virus but not wheat infected with in vitro transcripts of mutants $\mathrm{D}_{289} \mathrm{~A}$ and $\mathrm{D}_{326} \mathrm{~A}$ contained a second-site mutation of $\mathrm{R}_{131} \mathrm{C}$ and $\mathrm{N}_{275} \mathrm{H}$, respectively. Collectively, these data demonstrated that $\mathrm{CP}$ aa 58 to 84 are involved in symptom modulation in multiple cereal hosts, and aa 58 to 100 are required for host range extension and wheat curl mite transmission.

FvLcp1, a novel LysM/Chitin-binding protein, is important for ear rot virulence and fumonisin biosynthesis in Fusarium verticillioides H. Zhang (1), M. Kim PhD (1), J. Huang (2), W. B. SHIM (1), (1) Texas A\&M University, College Station, TX, USA; (2) Plant Protection College, Fujian Agriculture \& Forestry University, Fuzhou, CHINA

Fusarium verticillioides is one of the key maize ear rot pathogens and produces fumonisins. In this study, we performed a systematic network-based analysis of large-scale $F$. verticillioides RNA-seq datasets to identify potential gene subnetwork modules that are associated with virulence and fumonisin regulation. In one of the highly discriminative subnetwork modules, we identified a putative hub gene $F v L C P 1$. The predicted FvLcp1 protein contains a signal peptide, three LysM domains in the $\mathrm{N}$-terminus, and two chitin binding domains in the $\mathrm{C}$-terminus, indicating that FvLcp1 is a putative type-D fungal LysM protein. FvLcp1 sequence alignment against fungal protein database revealed a unique domain composition with both LysM and ChtBD1 domains, suggesting that FvLcp1 is quite different from previously characterized fungal LysM proteins. In addition, $F v L C P 1$ showed polymorphism in Texas $F$. veriticillioides isolates. Localization experiment revealed that FvLcp 1 is a cytoplasmic protein, but our study also showed that FvLcp1 is a secreted protein important for fumonisin production with LysM domain playing a critical role. Currently we are performing carbohydrate binding experiments to further understand the virulence mechanisms of FvLcp1. In addition, we are testing whether FvLcp1 can suppress program cell death triggered by mouse BAX protein in Nicotiana benthamiana through Agrobacterium tumefaciens-based transient assays. 
Development of a Virulence Model System to Assay Virulence in Chromobacterium vaccinii

E. WIESNER, J. Park, G. Ebadzadsahrai, A. Harrison, S. Soby, Midwestern University, Glendale, AZ, USA

Chromobacterium vaccinii (Betaproteobacteria) was isolated from wetlands and irrigation ponds in Southeastern Massachusetts. C. vaccinii has insecticidal activity against Aedes sp. larvae, but little is known about its virulence factors. Two isolates of $C$. vaccinii and two other species of Chromobacterium were assayed for virulence in brine shrimp (Artemia salina). C. vaccinii were more virulent than the other species. To make genetic manipulation of $\mathrm{C}$. vaccinii possible, a transformation method was developed using electroporation with a derivative of the broad host range plasmid RSF 1010. Transformation efficiency averaged $6.7 \times 10^{5}$ transformants $\mathrm{mg}^{-1}$ plasmid DNA. A $T n 5$ mutant library is being constructed and to evaluate virulence genes in the brine shrimp model system.

Virulence factors regulated by Quorum Sensing in Chromobacterium vaccinii

J. PARK, E. Wiesner, A. Harrison, G. Ebadzadsahrai, S. Soby, Midwestern University, Glendale, AZ, USA

Chromobacterium vaccinii is a newly discovered species from wild and cultivated cranberry bogs that has antifungal and insecticidal activity, but little is known about the mechanisms involved in virulence or in the regulation of virulence genes. Virulence factors are regulated by Quorum Sensing (QS) in other bacteria, suggesting that a mutation in the QS signaling molecule synthesis pathway gene (cvil) would allow analysis of the regulation of likely virulence factors such as hydrogen cyanide, chitinases, sideropores, and violacein. The cviI gene was targeted for site-specific mutagenesis, and candidate $C$. vaccinii virulence factors were assayed and compared to expression in wild type and cvil C. violaceum.

\section{The type III effector AvrBst enhances Xanthomonas perforans fitness in tomato}

P. ABRAHAMIAN (1,2), S. Timilsina (1,2), G. V. Minsavage Jr. (2), N. Potnis (3), E. M. Goss (2), J. B. Jones (2), G. E. Vallad (1,4), (1) Gulf Coast Research and Education Center, University of Florida, Wimauma, FL, USA; (2) Department of Plant Pathology, University of Florida, Gainesville, FL, USA; (3) Department of Entomology and Plant Pathology, Auburn University, Auburn, AL, USA; (4) University of Florida, Department of Plant Pathology, Gainesville, FL, USA

Bacterial leaf spot (BLS) is a major disease affecting tomato production. BLS in Florida is caused by two phylogenomic groups of Xanthomonas perforans $(X p)$. Recent surveys showed an increasing prevalence of the type III secretion effector, avrBst, among $X p$ strains in Florida. We evaluated the contribution of avrBst to fitness on tomato based on disease severity and the recovery of wild-type (WT) and avrBst mutant (MT) strains from inoculated plants in greenhouse and field studies. Strains representing the two phylogenomic groups, GEV872 and GEV1001, were selected for generating avrBst mutants. In planta growth of WT and MT strains did not differ following leaf infiltration of greenhouse grown tomato plants. Replicated field trials with 15 plant plots were conducted during fall 2015 and spring 2016. The center plant of each plot was inoculated with WT and MT strains of either GEV872 or GEV1001, and monitored over an eight-week period. Disease severity did not differ between GEV872 and GEV1001 in either trial. Differences in strain recovery were significant, with WT being recovered two to ten times more than MT for GEV872 or GEV1001. The spread of WT and MT strains, based on the recovery beyond the inoculated center plant within each field plot only statistically differed for strain GEV1001. Findings suggest that AvrBst affects the fitness of $X p$ strains under field conditions, making it an ideal candidate for BLS resistance breeding efforts in tomato.

\section{Panicum mosaic virus modulates reactive oxygen species homeostasis in Brachypodium distachyon}

S. PANT (1), S. Irigoyen (2), S. Christensen (3), J. Sedbrook (4), K. B. G. Scholthof (5), K. K. Mandadi (6), (1) Texas A\&M AgriLife Research \& Extension Center, Texas A\&M University System, Weslaco, TX, USA; (2) Texas A\&M AgriLife Research and Extension Center, Texas A\&M University System, Weslaco, TX, USA; (3) Chemistry Unit, Center of Medical, Agricultural, and Veterinary Entomology, USDA, Gainesville, TX, USA; (4) School of Biological Sciences, Illinois State University, Normal, IL, USA; (5) Department of Plant Pathology and Microbiology, Texas A\&M University, College Station, TX, USA; (6) Texas A\&M AgriLife Research, Weslaco, TX, USA

Panicum mosaic virus (PMV) is an important pathogen of bioenergy and food crops in the Poaceae, including switchgrass, maize and millets. We recently reported genome-wide transcriptomic and alternative splicing changes in Brachypodium distachyon (Brachypodium) during compatible virus infections of PMV. Here, we analyzed Brachypodium reactive oxygen species (ROS) homeostasis modulated by PMV. In contrast to the typical accumulation of $\mathrm{H}_{2} \mathrm{O}_{2}$ during plant-pathogen interactions, $\mathrm{H}_{2} \mathrm{O}_{2}$ was suppressed in PMV-infected Brachypodium leaves at 7, 14 and 21 days post infection, as determined by in situ histochemical staining using 3,3'-diaminobenzidine and luminol-based chemiluminescence assays. Metabolic profiling of PMV-infected Brachypodium, using LC-MS/MS tools, revealed increased levels of cinnamate (CA) and salicylic acid (SA), but lowered levels of jasmonic acid (JA) and fatty-acid precursors linoleic acid (18:2) and $\alpha$-linolenic acid (18:3). The modulated ROS and metabolite profiles correlated with changes in gene expression of corresponding genes in the respective signaling pathways. Furthermore, genetic studies by RNAi-mediated knockdown of PHENYLALANINE LYASE (PAL), a key enzyme in CA and SA biosynthesis, revealed that Bd-PAL activity is required for basal resistance to PMV and for modulation of ROS in Brachypodium during PMV infection. Our results provide new insights into ROS homeostasis and signaling during grass-virus interactions.

\section{A novel type III Xop effector in Xanthomonas cynarae associated with rapid cell death}

S. Kara (1), S. TIMILSINA (2), M. A. Jacques (3), N. Potnis (4), G. E. Vallad (5), M. Fischer-Le Saux (3), J. C. Hulbert (6), G. V. Minsavage Jr. (2), J. B. Jones (2), (1) university of florida, gainesville, FL, USA; (2) Department of Plant Pathology, University of Florida, Gainesville, FL, USA; (3) INRA UMR PAVE, Beaucouze Cedex, FRANCE; (4) Department of Entomology and Plant Pathology, Auburn University, Auburn, AL, USA; (5) Gulf Coast Research and Education Center, University of Florida, Wimauma, FL, USA; (6) Department of Chemistry, Physics and Geology, Winthrop University, Rock Hill, SC, USA

Xanthomonas gardneri and X. cynarae causal agents of bacterial spot of tomato and pepper and bacterial bract spot of artichoke, respectively, have greater than $99 \%$ average nucleotide identity indicating they could be members of the same species. $X$. cynarae was shown to be pathogenic on pepper but elicits a hypersensitive reaction (HR) on tomato. We identified a gene in X. cynarae that when expressed in X. gardneri and infiltrated at high bacterial concentrations into tomato leaflets resulted in rapid cell death. Using an avrBs 2 reporter system in which the N-terminus of the suspect type III effector gene was fused onto the C-terminus of $a v r B s 2$ and was confirmed to be a novel type III effector, which we are proposing to place in a new Xop family as XopAZ. The gene has high homology to FKBP-type peptidyl-prolyl cis-trans isomerases. When bacterial suspensions of wild-type (WT) $X$. gardneri alone or carrying the type III effector were infiltrated into tomto leaflets, bacterial populations reached similar levels indicating the rapid cell death was not an HR. When this gene was mutated in X. cynarae, electrolyte leakage was significantly reduced indicating that this gene contributes to rapid cell death; however, bacterial populations for mutant strain were similar to the WT strain indicating an additional factor(s) is associated with inability to grow in tomato. We are in the process of identifying the other factor(s) associated with tomato incompatibility in X. cynarae.

\section{Pump up the virulence: RND efflux pumps in Pseudomonas syringae} N. ECKSHTAIN-LEVI (1), B. Kvitko (2), B. A. Vinatzer (1), (1) PPWS Department, Virginia Tech, Blacksburg, VA, USA; (2) University of Georgia, Athens, GA, USA

Perception of pathogen-associated molecular patterns (PAMPs) constitutes the first layer of plant innate immunity. Failure to recognize PAMPs may leads to enhanced disease susceptibility. Ralstonia solanacearum is a soil-borne bacterium that causes bacterial wilt disease in many food crops. To date, elf-18 (an epitope of elongation factor Tu) is the only known recognized PAMP of this pathogen. In order to identify new PAMP candidates, we used a 
comparative genomics approach to search for genes that are under strong selective pressure. First, the core genome of 37 sequenced $R$. solanacearum strains was identified to comprise 1935 genes. In this list, genes not evolving neutrally were flagged based on the statistical test Tajima's D. Numerous multidrug efflux pumps (MDRs) were at the top of the list, along with EF-Tu. When searching the Pseudomonas syringae pv. tomato (Pto) DC3000 genome for homologues of these MDRs, one of them was found to be more frequently present in $P$. syringae crop pathogen genomes rather than in environmental isolates, thus suggesting an important role for MDRs in Pto. Several MDR mutants were constructed in Pto DC3000 and characterized, revealing some expected results, such as increase in sensitivity to antibiotics, and unexpected results: one mutant strain reaches a significantly higher population size in tomato compared to the wild type. Additional characterization is underway to unravel the role of MDRs in pathogen-plant interactions.

Transcriptomic profiling of watermelon-powdery mildew (Podosphaera xanthii) interactions

M. K. MANDAL PHD (1), S. Haktan (2), C. Kousik (3), (1) ORISE participant, US Vegetable Laboratory, USDA, ARS, Charleston, SC, USA; (2) Virginia Tech, Blacksburg, VA, USA; (3) U.S. Vegetable Laboratory, USDA, ARS, Charleston, SC, USA

Watermelon is an important vegetable crop and is widely cultivated in USA with an approximate global production of $>100$ million tons. Powdery mildew (PM) caused by Podosphaera xanthii is a major production limiting factor on watermelon and other cucurbits. Numerous powdery mildew resistant (PMR) watermelon germplasm lines have been developed by the USDA in Charleston, SC. To gain a better understanding of the innate and activated molecular defense mechanisms involved during compatible and incompatible powdery mildew-watermelon interactions, we inoculated powdery mildew susceptible and PMR watermelon plants with $10^{5}$ conidia $^{-\mathrm{ml}}$ of $P$. xanthii. RNAseq profiling was done on leaf samples collected at 0,1 , 3 , and 8 days post inoculation (DPI). The compatible interactions resulted in distinct plant gene activation ( $>2$ fold unique transcripts, 2121:1942:2670:: 1:3:8 DPI) as compared to incompatible interaction ( $>2$ fold unique transcripts, 1590:1946:1932 :: 1:3:8 DPI). Compatible interactions mostly involved pathogenesis events including carbohydrate metabolism, ethylene signaling and activation of stress responsive transcripts. Incompatible interaction results in activation of both TIR and CC-NBS-LRR mediated signaling events including induced transcripts of shikimate kinase, receptor kinase, ankyrin repeat containing protein and RIN4 like protein within $24 \mathrm{hr}$ of PM infection. A detailed study on NBS-LRR genes activated during watermelon-PM interaction is under progress.

\section{Influence of the P6 Effector Protein of Cauliflower mosaic virus (CaMV) on the Expression and Subcellular Localization of the CaMV Movement Protein}

J. E. Schoelz (1), M. ADHAB (2), (1) University of Missouri, Columbia, MO, USA; (2) University of Missouri - Columbia, Columbia, MO, USA

The P6 protein of Cauliflower mosaic virus (CaMV) is a multifunctional, $66 \mathrm{kDa}$ protein that has key roles in translation of viral proteins, intracellular movement, elicitation of plant defenses in hypersensitive hosts, and modulation of plant defenses in susceptible hosts. The P6 protein forms the matrix for the electron dense, amorphous inclusion bodies (IBs) that accumulate in the cytoplasm and has been shown to physically interact with all other CaMV proteins, including the CaMV movement protein (P1). In this study, we have investigated the influence of the P6 protein on the expression and subcellular localization of the CaMV P1 protein in transient expression assays in Nicotiana benthamiana. Previous studies involving the agroinfiltration of P1-GFP with a silencing suppressor such as the CaMV P6 protein or the P19 protein of Tomato bushy stunt virus had shown that P1-GFP was localized to punctate spots associated with plasmodesmata. However, agroinfiltration of P1-GFP in the absence of any silencing suppressor revealed a different pattern of expression, in which only a few individual cells expressed P1-GFP. Furthermore, the P1-GFP protein was distributed throughout the cytoplasm instead of within punctate spots associated with the cell wall. We propose that the silencing suppressor function of CaMV P6 may facilitate the expression of P1-GFP in a broader cross section of cells and alter the localization of the P1 protein from the cytoplasm to the cell wall.

Cofactor engineering as a pathogenesis strategy: A Xanthomonas secreted effector modifies NAD in planta

T. Shidore (1), J. Long (2), C. Broeckling (2), J. Kirkwood (2), J. E. Leach (2), L. R. TRIPLETT (1), (1) Connecticut Agricultural Experiment Station, New Haven, CT, USA; (2) Colorado State University, Fort Collins, CO, USA

Bacterial pathogens secrete type III secreted effectors to suppress host immunity. While effectors are generally understood to modify proteins or activate gene transcription, a few have recently been reported to degrade small molecules. The effector AvrRxo1 from Xanthomonas, Burkholderia, and Acidovorax spp. is a virulence factor that also triggers activation of the maize resistance gene Rxo1. Because AvrRxo1 has structural similarities to a small-molecule kinase, a global metabolomics approach was used to identify metabolic changes resulting from AvrRxo1 expression in yeast and bacteria. The NADP analog 3'-NADP was identified as the likely product of AvrRxo1 activity, and an in vitro assay confirmed that AvrRxo1 phosphorylates NAD at the 3' position. 3'-NADP accumulated in an AvrRxo1-dependent manner during transient expression and pathogen infection experiments in tobacco and rice. Mutation of key kinase residues abolished AvrRxo1 kinase activity as well as the phenotypes of toxicity, suppression of PAMP-triggered immunity (PTI), and activation of Rxo1-mediated immunity. These findings demonstrate that a secreted effector modifies the central redox carrier NAD in plants, and that this catalytic activity is required both for suppression of PTI and resistance gene activation.

\section{Identifying the host targets of rice blast effector proteins using proximity-dependent biotin labelling} K. HAYDON, A. Rogers, M. Egan, University of Arkansas, Fayetteville, AR, USA

The filamentous ascomycete Magnaporthe oryzae causes rice blast disease on Oryza sativa, resulting in global losses of up to $30 \%$ every year. To establish disease, $M$. oryzae secretes a large repertoire of effector proteins into rice tissue, which are thought to suppress plant defenses and modulate host biology to promote fungal colonization. Importantly, the host targets and cellular activities of many putative rice blast effectors remain unknown. Interactions between fungal effector proteins and their host target proteins are potentially transient in nature, or of low affinity, making them difficult to identify by traditional methods. To overcome these limitations, we are exploiting proximity-dependent biotin labelling approaches to probe effector-host protein interactions. Using this strategy, putative and known effector proteins are genetically tagged with the small promiscuous biotin ligase, BioID2. Upon secretion into rice cells, and in the presence of biotin, these BioID2-tagged effectors irreversibly biotinylate host proteins within a limited labeling radius, therein generating a history of potential effector-host protein interactions as they occur over time. Here we report our recent progress in exploiting this approach as an effector-host target discovery tool during the hemi-biotrophic infection of rice by M. oryzae.

\section{Identification of phloem specific translatome alterations in response to tobacco mosaic virus infection}

T. COLLUM (1), J. N. Culver (1,2), (1) Institute for Bioscience and Biotechnology Research, College Park, MD, USA; (2) Department of Plant Science and Landscape Architecture University of Maryland College Park, College Park, MD, USA

For a plant virus, gaining access to phloem vascular tissue is often a key factor in the ability to establish a systemic infection and cause disease. To gain insight into how this important tissue is altered during viral infection we used vascular specific promoters and a translating ribosome affinity purification strategy to identify phloem-associated translatome responses to infection by tobacco mosaic virus (TMV) in Arabidopsis thaliana ecotype Shahdara and Nicotiana benthamiana. Results demonstrate that within inoculated leaves phloem specific alterations account for over $80 \%$ of the tissue specific changes observed in both hosts. Even in comparison to gene changes that occurred in all leaf tissues, phloem specific alterations made up the majority of observed changes ( $>2$ fold alteration) in Arabidopsis and in highly altered ( $>10$ fold alteration) $N$. benthamiana genes. Additional comparisons of phloem alterations between the two hosts identified sets of genes that were either similarly or differentially regulated. Similarities between host phloem responses included genes involved in systemic acquired resistance and metabolic processes that likely reflect conserved host responses to TMV infection 
Genes differentially affected in the two hosts included those involved in the production of reactive oxygen species that conceivably contribute to differences in symptom responses. Combined these results indicate phloem tissue plays a disproportion role in the mediation and control of host responses to virus infection.

Transcriptional regulation in Geminiviruses

J. GUERRERO (1), G. Sunter (2), (1) University of Texas at San Antonio, San Antonio, TX, USA; (2) UT San Antonio, San Antonio, TX, USA

The Geminiviridae are a family of ssDNA plant infecting viruses that are transmitted by insects. Geminivirus diseases are responsible for billions of dollars in annual losses of agricultural crops worldwide; development of disease resistance strategies is crucial. A single mRNA (AL1629) transcribed by Tomato golden mosaic virus (TGMV) directs expression of two viral genes, AL2 and AL3. AL2 suppresses host defenses and regulates later promoters. $A L 3$ encodes a replication enhancement protein. Previous work showed that binding of AL1, required for replication, to its cognate binding site is required for transcription of the downstream AL1629 mRNA. Spinach curly top virus (SCTV) transcribes a similar mRNA (C2045) encoding the C2 and C3 proteins. We have demonstrated that the promoter sequences for both AL1629 and C2045 are conserved, active in the context of transient expression assays, and exhibit a significant-fold change above background. For TGMV, addition of AL1 in the absence of replication results in a 2-fold increase in AL2 expression. The presence of AL1 and replication results in a 10-fold increase in AL2 expression. For SCTV, no significant change was observed when AL1 is added. Thus, the TGMV and SCTV promoters (AL1629 and C2045) seem to be regulated differently. Our work suggests that in TGMV, AL1 seems to have a direct influence on downstream promoter activity, likely through binding to its cognate binding site and an indirect effect through replication.

\section{Controlling Sclerotinia stem rot using gene silencing M. MCCAGHEY, A. Ranjan, J. Kurcezewski, M. Kabbage, D. L. Smith, University of Wisconsin, Madison, WI, USA}

Sclerotinia sclerotiorum, the causal agent of Sclerotinia stem rot (SSR), is a yield limiting fungal pathogen in soybean (Glycine max). RNA interference (RNAi) has emerged as a technology to control pests within agricultural systems. Virus induced gene silencing (VIGS) was used as a proof-of-concept for controlling S. sclerotiorum in soybean using RNAi. Target sequences of 300-400 bp, in sense and antisense directions, were cloned into a Bean pod mottle virus (BPMV) vector. Targets include important genes related to fungal structure and oxalic acid synthesis. Soybeans were inoculated with BPMV vectors using particle bombardment, and silencing was evaluated by lesion size, fungal biomass, and mRNA levels of target sequences of $S$. sclerotiorum. Silencing of fungal sequences will serve as proof-of-concept for host induced gene silencing (HIGS) as a viable strategy to control SSR without chemicals. Our study also evaluated exogenous applications of dsRNA and sRNA to limit fungal growth in vitro and in planta. Expression level of target genes, growth inhibition, and disease development using exogenous RNA was evaluated on G. max. Additionally, oxalic acid production was quantified using a colorimetric assay. These RNAi strategies will provide new tools for resistance to S. sclerotiorum in soybeans. Targets include important genes related to fungal structure and oxalic acid synthesis.

\section{Functional characterization of potential Sclerotinia sclerotiorum candidate virulence genes on canola} K. CHITTEM (1), L. E. del Rio Mendoza (2), (1) North Dakota State University, Fargo, ND, USA; (2) North Dakota State Univ, Fargo, ND, USA

The necrotrophic fungus Sclerotinia sclerotiorum has a wide host range that includes canola and many other broadleaf crops and is considered a devastating plant pathogen capable of causing huge economic losses worldwide. Several potential S. sclerotiorum candidate pathogenicity/virulence genes were identified from a transcriptome sequencing study conducted to understand canola $-S$. sclerotiorum interaction. Four of these candidate genes, cytochrome P450 (SS1G_05491), Glycosyltransferase SsBGT1 (SS1G_09997), Glycosylhydrolase (SS1G_14184) and a Serine/Threonine kinase (SS1G 11866), were selected for functional characterization. Single-gene knockout mutants were generated by targeted gene replacement following split marker approach. The effect of deletion of these genes on mycelial growth characteristics on PDA and pathogenicity/virulence on canola were evaluated. Deletion of cytochrome P450 and SsBGT1 affected growth characteristics on PDA, resulting in significant reduction in colony diameter and mycelial density. However, deletion of the other two genes had no effect on fungal growth. In addition, virulence assays on detached canola leaves showed reduced lesion sizes in case of cytochrome P450, SsBGT1, and Serine/Threonine kinase deletion mutants, indicating their possible role in virulence of $S$. sclerotiorum. Results of this study suggest these genes could be potential targets for developing novel disease management strategies.

Interplay of ascorbate glutathione cycle and redox signaling in host resistance of Taro-Phytophthora interaction M. R. SAHOO (1), M. Dasgupta (1), N. Prakash (2), S. V. Ngachan (3), (1) University of Tennessee, Knoxville, TN, USA; (2) ICAR Research Complex for NEH Region, IMPHAL, INDIA; (3) ICAR Research Complex for NEH Region, Meghalaya, INDIA

Ascorbate-glutathione cycle (AsA-GSH) is an efficient metabolic pathway deployed in detoxification of $\mathrm{H}_{2} \mathrm{O}_{2}$. It involves four enzymes viz., ascorbate peroxidase (APX), glutathione reductase (GR), dehydroascorbate reductase (DHAR) and monodehydroascobate reductase (MDAR). A study was undertaken in taro genotypes to assess the changes in $\mathrm{H}_{2} \mathrm{O}_{2}$ content, lipid peroxidation, total antioxidative enzymes, ascorbate and reduced glutathione content to understand how the activity of AsA-GSH cycle plays an important role in taro-Phytophthora colocasiae interaction. We have performed in situ detection of $\mathrm{H}_{2} \mathrm{O}_{2}$ in resistant and susceptible taro leaves owing to Phytophthora colocasiae infection using 3,3-diaminobenzidine (DAB) staining. The elevated levels of $\mathrm{H}_{2} \mathrm{O}_{2}$ content (by spectrophotometric method) and increased malondialdehyde (MDA content), marker of lipid peroxidation, showed positive correlation with DAB results. About 1.5-2-fold increase in APX, GR, DHAR and MDAR was also recorded in resistant genotype (RCMC-5, Duradim) as compared to susceptible ones (Telia). A significant and negative correlations was observed between $\mathrm{H}_{2} \mathrm{O}_{2}$ and antioxidative enzymes. Results of these experiments clearly indicate that enhanced activities of all the enzymes of ascorbate-glutathione cycle, signifying a potential role of these enzymes in providing antioxidative defense under Taro-Phytophthora interaction.

\section{Methodology for profiling of phloem translatomes in Prunus domestica $\mathrm{L}$ in response to virus infection}

T. COLLUM (1), E. Lutton (2), A. L. Stone (3), D. Sherman (3), W. Schneider (3), C. D. Dardick (2), J. N. Culver (4), (1) Institute for Bioscience and Biotechnology Research, College Park, MD, USA; (2) Appalachian Fruit Research Station, USDA, Agricultural Research Service, Kearneysville, WV, USA; (3) USDA ARS FDWSRU, Fort Detrick, MD, USA; (4) Department of Plant Science and Landscape Architecture University of Maryland College Park, College Park, MD, USA

In woody perennials, virus interactions within the vasculature are essential to the establishment of disease. Unfortunately, little is known regarding this aspect of virus - host interactions. In this study a translatome - RNAseq profiling method that incorporates phloem specific promoters to express FLAGtagged ribosomal proteins for immuno-capture of cell-specific mRNAs was adapted for use in Prunus domestica $\mathrm{L}$. This method allows for the identification of host phloem specific transcripts that are altered in response to virus infection as well as the viral RNA that is actively being translated. Plum lines expressing a FLAG-tagged ribosomal protein, RPL18, from phloem specific promoters pSUC2 and pSULTR2;2 as well as a control p35S promoter were created. Anti-FLAG conjugated magnetic bead methodologies were developed and used to immunopurify tagged ribosome complexes from plum leaves, stems and buds. Translatome RNA with high RNA integrity was recovered from plants expressing FLAG-RPL18 but not from nontransformed plants. Subsequently, these plum lines were infected with plum pox virus (PPV) and efforts are underway to identify phloem specific transcripts that are altered in response to PPV infection. The adaptation of translatome - RNAseq profiling methods for perennials allows for the characterization of tissue specific responses to viral infection during dormant and growth periods, providing a unique means to examine host responses to viral infection. 
Tomato yellow leaf curl virus $\mathrm{C} 4$ protein is a determinant of disease phenotype in tomato C. PADMANABHAN (1), Y. Zheng (2), M. Shamimuzzaman (1), Z. Fei (2), K. S. Ling (1), (1) USDA-ARS, Charleston, SC, USA; (2) Boyce Thompson Institute, Ithaca, NY, USA

Tomato yellow leaf curl virus (TYLCV) is a monopartite begomovirus. Its genome contains six open reading frames, with V1 and V2 in sense, and C1 to $\mathrm{C} 4$ in complementary orientation. The functions of $\mathrm{V} 1$ and $\mathrm{V} 2$ are for coat protein and pre-coat, respectively. $\mathrm{C} 1$ is for virus replication, $\mathrm{C} 2$ for transactivation, and $\mathrm{C} 3$ for replication enhancer. The function of $\mathrm{C} 4$ protein, however, has not been determined in TYLCV, although studies in other geminiviruses suggest its role in pathogenicity and disease phenotype. To evaluate the function of TYLCV- encoded C4 protein, we generated transgenic tomato plants via Agrobacterium-mediated transformation. Surprisingly, transgenic tomato plants displayed an upward leaf curling phenotype, which is similar to the symptoms of tomato plants infected by wild TYLCV. To investigate any host genes and pathways that might be altered by the transgene C4 expression, we conducted genome-wide transcriptome profiling using RNA sequencing and identified a total of 241 differentially expressed genes (DEGs), including two microRNA target genes, in the transgenic C4 plants in comparison to those from the transgenic GFP control plants. Interestingly, expressions of several leaf developmental genes were altered, which might play a role leading to the leaf curl phenotype in the transgenic $\mathrm{C} 4$ plants. These results provide direct evidence that C4 of TYLCV is a unique virus-encoded protein that causes leaf curling phenotype upon virus infection in tomato.

Exploring the trans-acting short interfering RNAs (ta-siRNAs) technology for virus control in plants C. PADMANABHAN (1), Y. Zheng (2), M. Shamimuzzaman (1), Z. Fei (2), K. S. Ling (1), (1) USDA-ARS, Charleston, SC, USA; (2) Boyce Thompson Institute, Ithaca, NY, USA

Small RNAs ( 20-24nt) processed from double-stranded RNA in plants can trigger degradation of the target mRNAs in cytoplasm or de novo DNA methylation in nucleus leading to gene silencing. Trans-acting short-interfering RNAs (ta-siRNAs) have been shown to enhance the target mRNA degradation and maintenance of host gene expression. In this study, we were interested in exploring ta-siRNAs technology for virus control on tomato. It was expected that by targeting the viral genomic RNA for degradation, these transgenic plants might interfere or suppress virus replication, thus confer disease resistance in the transgenic plants. Using $22 \mathrm{nt}$ miRNAs targeting the viral RNA genomes of Pepino mosaic virus (PepMV) or Tomato spotted wilt virus (TSWV) for insertion into tomato pre-miRNA sequences, we were able to generate gene constructs with multiple virus-specific ta-siRNAs (21 nt). Through Agrobacterium-mediated transformation, transgenic tomato plants containing the ta-siRNAs against PepMV or TSWV were generated and the transgene integration was confirmed by PCR. In screening transgenic plants for virus resistance through mechanical inoculation, although no immunity to the respective virus infection was observed, a number of transgenic plants exhibited delayed or milder symptoms over control tomato plants under the same treatments. The ta-siRNA-mediated resistance may be a novel strategy in controlling viruses in plants.

Functional characterization of the triple gene block 1 (TGB1) gene of Pepino mosaic virus in tomato C. PADMANABHAN (1), Y. Zheng (2), M. Shamimuzzaman (1), Z. Fei (2), K. S. Ling (1), (1) USDA-ARS, Charleston, SC, USA; (2) Boyce Thompson Institute, Ithaca, NY, USA

Pepino mosaic virus (PepMV) has caused serious economic losses to many greenhouse tomato productions around the world. This potexvirus genome contains five major open reading frames (ORFs) encoding for a 164-kDa RNA-dependent RNA polymerase (RdRp), three triple gene block (TGB) proteins of 26, 14 and $9 \mathrm{kDa}$, and a 25-kDa coat protein, respectively. Although there is some knowledge about the functions for TGB proteins in other potexviruses, the function of the PepMV TGB gene is currently unknown. Using the TGB1 gene from a severe strain of PepMV CH2 genotype, we constructed a plant transformation vector for Agrobacterium-mediated transformation on tomato (cv. Moneymaker). In screening transgenic plants for resistance to PepMV infection, through mechanical inoculation, a number of transgenic tomato plants exhibited resistance to PepMV infection, with a delay in symptom expression over that of GFP- control tomato plants. To investigate the effect of differential gene expression through RNA sequencing between the TGB1 transgenic plants and control GFP-plants upon PepMV infection, we identified a total of 217 differentially expressed genes (DEGs) through transcriptome analysis. Those DEGs and their roles in host gene regulation and virus resistance will be discussed. Understanding the PepMVencoded TGB1 function might guide us to better utilize the TGB1 gene to manage PepMV infection in greenhouse tomato productions.

Towards the control of $\mathrm{Ca}$. Liberibacter asiaticus (Las) by interfering with lytic phage repressors A. MUNOZ BODNAR, L. A. Fleites, M. Jain, A. Bernert, D. W. Gabriel, University of Florida, Gainesville, FL, USA

Most but not all Las genomes sequenced to date harbor SC1 and SC2 bacteriophages. SC1 is maintained as a stable lysogen in psyllids but in periwinkle and citrus its lytic phase is activated. Las strains carry either full length or truncated versions of a C1-like phage repressor, as well as a $\mathrm{C} 2$ repressor on the $\mathrm{SC} 1$ bacteriophage. The mechanism behind the activation of the lytic cycle in planta and its implied repression in psyllids is unknown. We hypothesize that both LC1 and/or LC2 could play an important role in the repression of the SC1 lytic cycle. To test this hypothesis, predicted lytic gene promoters were fused with a GFP reporter and these constructs were transformed into Liberibacter crescens (Lcr) and Escherichia coli. The LC2-like and LC1-like promoters were bidirectionally functional in Lcr, but only the LC2-like repressor was functional in E. coli. LC2-like promoter activity was significantly higher than any other Lcr promoters tested in Lcr, and also higher than the strong E. coli lacZ promoter. Both the truncated and full length versions of chromosomal LC1 functionally bind and repress the C2-like repressor promoter (late gene direction). The SC1 phage C2-like repressor selfrepresses its own promoter. Both repressors are potential molecular targets for chemical control of Las.

C-di-GMP regulates pectate lyase activity through the H-NS-rsmB-RsmA pathway in the soft-rot bacterial pathogen Dickeya dadantii X. YUAN (1,2), F. Tian (2,3), G. Severin (4), C. Waters (4), F. Liu (1), C. H. Yang (2), (1) Institute of Plant Protection, Jiangsu Academy of Agricultural Sciences, Nanjing, CHINA; (2) Department of Biological Sciences, University of Wisconsin-Milwaukee, Milwaukee, WI, USA; (3) Institute of Plant Protection, Chinese Academy of Agricultural Sciences, Beijing, CHINA; (4) Department of Microbiology and Molecular Genetics, Michigan State University, East Lansing, MI, USA

Pectate lyases (Pels), involved in plant cell wall degradation, is the major virulence factor in Dickeya dadantii. We have shown previously that the bacterial second messenger c-di-GMP regulated Pel production. However, the regulatory mechanism remains unclear. Here, we examined Pel production in eighteen mutants that are defective in genes related to c-di-GMP metabolism. Our results showed that a GGDEF-domain protein, GcpA, negatively regulated Pel production. Using UPLC mass spectrometry, we confirmed that GcpA is a diguanylate cyclase (DGC). Flow cytometry and qRT-PCR were performed to determine promoter activities and RNA levels of pelD and pelE, Interestingly, only pelD expression was significantly enhanced in the $g c p A^{D 418 A}$ mutant. The mutant showed increased expression of Pel activators RsmB and H-NS (heat-stable nucleoid-structuring protein), but reduced expression of the Pel repressor, RsmA. A hnsgcp A ${ }^{D 418 A}$ double mutant restored $r s m B$ RNA levels, pelD promoter activity and Pel production in the $g c p A^{D 418 A}$ mutant, suggesting that GcpA represses pelD via the H-NS-rsm $B$-RsmA pathway. Deletion of phosphodiesterase (PDE) encoding genes, egcpB and $е с p C$, decreased $r s m B$ RNA levels and $p e l D$ promoter activity. Interestingly, only the egcpB mutant exhibited reduced hns RNA levels. These results reveal a novel and sophisticated regulatory pathway involving the ability of individual DGC and PDE to differentially control the Pel regulators.

Transcriptome changes in the whitefly $B$. tabaci in response to feeding on Cucurbit yellow stunting disorder virus infected melon N. Kaur (1), W. Chen (2), Z. Fei (2), W. M. WINTERMANTEL (1), (1) USDA-ARS, Salinas, CA, USA; (2) Boyce Thompson Institute, Ithaca, NY, USA 
Cucurbit yellow stunting disorder virus (CYSDV), a crinivirus transmitted by the whitefly, B. tabaci, causes widespread losses in melon and other cucurbits. The virus emerged in the southwestern United States in 2006. It established in crops and weeds, and is transmitted to cucurbits by the resident population of B. tabaci MEAM1 each year. CYSDV has a semipersistent mode of transmission and can be retained for seven to nine days in the whitefly vector. In order to understand the specific interactions between B. tabaci and CYSDV, RNA-Seq was performed on whiteflies following acquisition feeding on CYSDV-infected melon leaves at three different time points, $24 \mathrm{~h}, 72 \mathrm{~h}$, and 7 days. Of the 15,664 genes present in the whitefly, B. tabaci MEAM1, 275 genes were differentially expressed in the whiteflies in response to feeding on CYSDV-infected melon host plants over the three time points. Transcriptome analysis of the whiteflies identified a temporal shift in gene expression, with only 3 down-regulated genes at $24 \mathrm{~h}$, followed by higher numbers of genes differentially expressed at 72 h (221 DEGs: 82 up-regulated and 139 down-regulated), and 7 days (51 DEGs: 49 up-regulated and 2 down-regulated). Several distinct gene categories were represented among the DEGs in the whiteflies. Interestingly, 59 DEGs were common between whiteflies fed on CYSDV-infected melon with those fed on ToCV-infected tomato plants including several cathepsins, $\alpha$-glucosidase, and a glucose transporter, as well as many unknown genes.

\section{Transcriptional Profiling And Phenotypic Analysis To Identify Genes Involved In Stress Response And Conidiogenesis In Fusarium graminearum \\ C. Blaschke, Z. O. Bilton, K. Lambert, M. Rothrock, R. Manspeaker, J. FLAHERTY, Coker College, Hartsville, SC, USA}

Fusarium graminearum is an important plant pathogen that causes major yield $\neg$ limiting diseases like head blight of wheat and ear rot of maize. $F$. graminearum persists in the soil in the absence of a host and reproduces asexually through the production of conidia (asexual spores) to initiate repeating cycles of infection. Our project involves undergraduate students on both gene discovery in $F$. graminearum using bioinformatics approaches and phenotyping analysis of mutant strains. Candidate genes putatively involved in stress responses/conidiogenesis have been identified through two gene expression projects. RNAseq data obtained from F. graminearum PH-1 and two mutant strains, all grown in conditions either conducive or nonconducive for conidiation, was processed using cluster analysis (based on unbiased measures of similarity rather than sorting objects into predefined categories). Attributes for clustering genes from each strain were locus and change in expression. Analysis of this data provides a robust way of examining gene expression patterns to identify candidate genes and gene clusters for future investigation. Towards characterizing specific candidate genes, a series of mutant strains were confirmed as targeted disruption mutants and four of these, found to be closely linked $F$. graminearum genes FGSG_12704,_12705,_12744, and_05476, were interrogated for phenotypes related to growth and development. The characterization of candidate genes identified in this study will help to fill gaps in our understanding of fungal development and facilitate building models depicting developmental processes required for the survival and dissemination of $F$. graminearum.

\section{Taxonomic reorganization of the family Endornaviridae}

S. SABANADZOVIC (1), R. A. Valverde (2), M. Khalifa (3), M. N. Pearson (4), R. Okada (5), N. Aboughanem-Sabanadzovic (6), (1) Dept of Biochem, Mol Biology, Entomology and Plant Pathology, Mississippi State University, Mississippi State, MS, USA; (2) Dept of Plant Pathology and Crop Physiology, Louisiana State University Agricultural Center, Baton Rouge, LA, USA; (3) School of Biological Sciences, The University of Auckland, Auckland, NEW ZEALAND; (4) Univ of Auckland, Auckland, NEW ZEALAND; (5) Faculty of Agriculture, Tokyo University of Agriculture and Technology, Tokyo, JAPAN; (6) Institute for Genomics, Biocomputing and Biotechnology, Mississippi State University, Mississippi State, MS, USA

Endornaviruses are persistent and capsidless viruses that infect plants, fungi, and oomycetes. They have monocistronic RNA genomes that code for large polyproteins containing several functional domains. According to the present classification, all 12 currently recognized species belong to a single genus Endornavirus in the family Endornaviridae. However, recent studies on these viruses resulted in identification of several new putative endornaviruses with distinct molecular traits and evolutionary history. Therefore, in July 2016 we proposed a taxonomic reorganization of the family to mirror the progress in the research and knowledge of these viruses. Proposed taxonomic changes included renaming the extant genus Endornavirus to Alphaendornavirus (to contain 11 out of 12 recognized species at that time) and the creation of a new genus named Betaendornavirus typified by Sclerotinina sclerotiorum endornavirus 1. In addition, we proposed recognition of 10 new species and their assignment to these two genera. Finally, we proposed renaming recognized species by adopting an updated non-Latinized binomial nomenclature to reflect names of new higher taxa (genera). All proposals were recently discussed by the Executive Committee of the International Committee on Taxonomy of Viruses (EC-ICTV) and currently have been submitted to ratification vote.

\section{Influence of reactive oxygen species on the Res phosphorelay and its relationship to a large RTX toxin in Pantoea stewartii} P. VIRAVATHANA, M. C. Roper, University of California, Riverside, Riverside, CA, USA

Pantoea stewartii subsp. stewartii causes Stewart's wilt in sweet corn and maize. This disease is characterized by water soaked lesion formation, and synthesis of exopolysaccharide (EPS) as a part of biofilm formation that wilts seedlings. Water soaked lesions contain high levels of the reactive oxygen species (ROS), $\mathrm{H}_{2} \mathrm{O}_{2}$ and superoxides. The transcription factor OxyR is essential in protecting P. stewartii against $\mathrm{H}_{2} \mathrm{O}_{2}$. Its absence abrogates EPS production and increases sensitivity to $\mathrm{H}_{2} \mathrm{O}_{2}$. In the genome of $P$. stewartii, a conserved binding site for OxyR is upstream of an operon containing genes encoding components of the Rcs pathway along with two RTX proteins. Deletion of the gene encoding the larger RTX toxin $(r t x 2)$ decreases plant colonization, negates water soaked lesion formation, and reduces EPS production. Sublethal in-vitro, exposure to paraquat and $\mathrm{H}_{2} \mathrm{O}_{2}$ increased induction of expression of components of the Rcs phosphorelay via OxyR. This research will determine if ROS species can act as an external stimuli to induce the process of biofilm formation in P. stewartii via the Rcs phosphorelay by OxyR. In addition to forming water soaked lesions, the multifunctional Rtx 2 appears to play a role in the transitioning between the apoplastic and xylem phases of Stewart wilt.

A role for Erwinia amylovora elongation factor $P$ in fire blight disease development

S. Klee (1), I. Mostafa (2), S. Chen (3), C. Dufresne (4), B. L. Lehman (5), J. P. Sinn (6), K. A. Peter (5), T. W. MCNELLIS (6), (1) Pennsylvania State University, University Park, PA, USA; (2) Zagazig University, Zagazig, EGYPT; (3) University of Florida, Gainesville, FL, USA; (4) Thermo Fisher Scientific, West Palm Beach, FL, USA; (5) Penn State University, Biglerville, PA, USA; (6) Penn State University, University Park, PA, USA

Elongation Factor P (EFP) is a highly conserved bacterial protein that is associated with the 70S ribosome and is thought to be involved in translation initiation and the alleviation of translational stalling at polyproline sequences. EFP undergoes an essential post-translational beta-lysylation through the activities of enzymes YjeA and YjeK. Despite several decades of study, relatively little is known about EFP biological function. Recently, EFP has been associated with virulence in several different bacterial species, including Salmonella enterica, E. coli, and Agrobacterium tumefaciens. In a forward genetics screen for loss of virulence mutants in the fire blight pathogen Erwinia amylovora, we discovered Tn5 transposon mutations in yjeA and yjeK. In addition, we created a deletion mutant of the E. amylovora efp gene. E. amylovora efp, yjeA, and yjeK mutants all had a similar phenotype of greatly reduced pathogenicity in apple and increased chemical sensitivity, particularly to hydrogen peroxide. The increased chemical sensitivity of the $e f p, y j e A$, and $y j e K$ mutants likely contributes to their reduced pathogenicity in apple, especially considering that high levels of reactive oxygen species are produced at E. amylovora infection sites. Quantitative proteomics analysis of the $y j e K$ mutant detected numerous protein expression differences from the wild-type, including changes in the abundance of proteins associated with virulence, stress tolerance, and motility. However, the EFP-dependence of most proteins was contingent on environmental conditions. These results suggest that EFP might play a regulatory role in protein expression, rather than a purely mechanical one. 
Two Class I Hydrophobins, TvHyd1 and TvHyd2, from Trichoderma virens

J. TAYLOR (1), F. Crutcher (2), B. A. Horwitz (3), C. M. Kenerley (1), (1) Texas A\&M University, College Station, TX, USA; (2) Montana State University, Sidney, MT, USA; (3) Israel Inst of Technology, Haifa, ISRAEL

Trichoderma virens is a plant symbiotic fungus that contributes multiple beneficial effects upon colonization of plant roots. Defining the elements that drive these interactions is pivotal to our understanding of induced systemic resistance (ISR). Based upon microarray and secretomic analyses of $T$. virens grown in the presence of maize and/or tomato roots, two class I hydrophobins (TVHYD1 and TVHYD2) were identified. Colonization of maize roots was shown to be significantly reduced in knockout mutants of $T v H y d l$. These mutants were also impaired in their ability to contribute to ISR in maize against infection by Colletotrichum graminicola and hydrophobicity of hyphae as measured by a contact angle assay. Transcriptomic analysis demonstrated that both $T v H y d 1$ and $T v H y d 2$ are expressed only after three days post inoculation in potato dextrose broth (PDB), and between 30 and 54 hours in the presence of maize roots. $T v H y d 2$ shares significant homology with $T v H y d l$, however, promoter and terminator sequences for each are unique to the genome. $T v H y d 2$ is expressed in the $\Delta$ TvHyd 1 mutants at approximately the same level as wild type at 72 hours in PDB via RT-PCR, suggesting that there is no compensation. Hydrophobins appear to play a multifaceted role in $T$. virens-plant interactions, ranging from attachment to induction of plant defense. Identifying fungal genes involved in these $T$. virens-plant interactions will assist in strain selection for enhanced crop production.

\section{A Coin with Two Sides: Probing Non-Pathogenic versus Pathogenic interactions using the Fusarium oxysporum - Arabidopsis thaliana pathosystem \\ K. VESCIO, L. Guo, L. J. Ma, University of Massachusetts, Amherst, MA, USA}

Fusarium oxysporum $(F o)$ is a soil-dwelling fungal pathogen that results in vascular wilt diseases of a broad range of plants, including agricultural crops as well as the model plant Arabidopsis thaliana ${ }^{1}$. There are also members of the $F$. oxysporum species complex that are non-pathogenic, and confer various defense benefits against other pathogens to the host plant ${ }^{2}$. Through RNA-seq analysis of Arabidopsis thaliana infected by two Fo strains Fo5176 (pathogenic) and Fo47 (nonpathogenic), we have identified shifts in plant gene expression specific to treatment with Fo5176 and Fo47, respectively. Annotating protein-protein interaction networks highlighted an enrichment of genes and pathways related to nutrient acquisition and assimilation, and Salicylic acid mediated defense responses in Fo47 infected A. thaliana roots. In the contrast, in responding to pathogen infection, genes and pathways related to secondary metabolite biosynthesis, protein homeostasis, and Jasmonic acid and systemic defense responses are differentially induced. Phenotypic characterization of $A$. thaliana plants co-inoculated with Fo47 and Fo5176 revealed a significant reduction in disease progression and outcome when compared to plants inoculated with Fo5176. With insights from RNA-seq and phenotypic data, we propose that infection with Fo47 improves plant immunity, and mechanisms taking place on the molecular and cellular level will be discussed.

\section{Functional Characterization of ubiquitin carboxyl-terminal hydrolase gene Moubp1 in Maganaporthe oryzae}

D. CHEN, X. Yang, Z. Zhao, G. Lu, Fujian Agriculture and Forestry University, Fuzhou, CHINA

Deubiquitination is an important regulatory step in the Ub-dependent pathway. Deubiquitinases are cystering proteases that specifically remove Ub from substrate proteins, which are the largest family of enzymes in Ub-dependent pathway. DUBs are involved in lots of regulatory mechanisms including growth and oncogenesis, differentiation and development, chromosome structure and transcriptional regulation, memory and neurodegenerative diseases We here studied the function of Ub C-Terminal Hydrolase in Magnaporthe oryzae. MGG 03527 in Magnaporthe oryzae which has conservated domain including Zn-finger domain and UCH domain, which is highly homologous to the Ubp 8 in Saccharomyces prombe and Saccharomyces cerevisiae. Through functional analysis of knockout mutant, we found the conidiophore and conidia formation of Moubpl knockout mutant reduced, and it produced more abnormal conidia with less septum than wild type. And the defect phenotype also include the delay of germination and appressorium formation. Besides, we all found the dramatic decrease of pathogenicity of mutant through inoculating on onion epidermis, barley and rice.

Further characterization of OsSULTR3;6 the first susceptibility gene identified for bacterial leaf streak of rice A. C. READ (1), J. Belanto (2), A. Cernadas (3), L. Wang (1), M. Willmann (4), D. Voytas (2), A. J. Bogdanove (1), (1) Cornell University, Ithaca, NY, USA; (2) University of Minnesota, St. Paul, MN, USA; (3) University of Buenos Aires, Buenos Aires, ARGENTINA; (4) Cornell University Plant Transformation Facility, Ithaca, NY, USA

Xanthomonas oryzae pv. oryzicola (Xoc), the causal agent of Bacterial leaf streak of rice, encodes as many as 28 type III secreted transcription activatorlike (TAL) effectors, each of which may target a specific host DNA sequence and upregulates expression of nearby host genes. A target of a TAL effector that leads to increased susceptibility is considered an S-gene. The only known S-gene in the Xoc-rice pathosystem encodes a putative sulfate transporter, SULTR3;6, targeted by the highly conserved Xoc TAL effector Tal2g. Studies of the related pathogen Xo pv. oryzae have identified members of a sugar transporter gene family as S-genes and shown that paralogs can function as substitute S-genes when activated with designer TAL effectors (dTALEs). In our study dTALEs have been constructed targeting SULTR3;6 paralogs to ascertain which, if any, can act as substitute S-genes So far, no paralog is able to restore full susceptibility. In fact, activation of a closely related paralog results in greatly reduced susceptibility. Functional studies are underway to confirm SULTR3;6's role as a sulfate transporter and gain insight into the mechanism of susceptibility. We report preliminary sub-cellular localization findings as well as characterization of CRISPR generated OsSULTR3;6 knock-out and Tal2g target disruption rice lines. Understanding the mechanism and spectrum of susceptibility will empower breeding and engineered resistance strategies.

\section{Effector repertoire of the citrus fungal pathogen Colletotrichum acutatum}

V. Candian (1), L. CANO (2), D. Oppelaar (3), (1) University of Torino, Dep of Agricultural Forestry and Food Science, Grugliasco, ITALY; (2) University of Florida, IFAS, Dep Plant Pathology, Indian River Research and Education Center, Fort Pierce, FL, USA; (3) Wageningen University, Wageningen, NETHERLANDS

Postbloom fruit drop caused by the fungal pathogen Colletotrichum acutatum is a damaging disease affecting citrus production in Florida mainly sweet oranges since 2014. Filamentous plant pathogens secrete molecules that manipulate host physiology to achieve colonization. We describe here the identification of $C$. acutatum secreted effector candidates from a recently generated reference PacBio genome assembly of $53.4 \mathrm{Mb}$ for an isolate obtained from sweet orange Valencia in 2016. We also generated RNAseq libraries for 15 C. acutatum strains collected pre and post 2014 epidemics and from diverse locations that will serve as transcript evidence sequencing data for the annotated effectors. Identified effector candidates can be employed in high throughput functional genomics screens, commonly referred to as effectoromics, to test citrus germplasm for specific pathogen recognition by resistance $R$ proteins.

\section{Effect of Phymatotrichopsis root rot disease on alfalfa root morphology and forage quality} C. MATTUPALLI, S. Kwon, J. K. Rogers, C. A. Young, The Samuel Roberts Noble Foundation, Ardmore, OK, USA

Phymatotrichopsis root rot (PRR) caused by Phymatotrichopsis omnivora severely reduces crop stand causing huge economic losses to alfalfa growers in the southwest USA. To gain insight into the effects of PRR on alfalfa, a field study was conducted during 2015-16 on a PRR-infested 50.4 hectare alfalfa hay production field at the Noble Foundation's Red River Farm, Burneyville, OK. Sampling was performed from five (2015) and three (2016) PRR disease sites at regular time intervals along random transects that spanned both sides of the disease front (survivor and asymptomatic zones). Three alfalfa plants were sampled from ten quadrats at each disease site and were assessed for shoot, root characteristics, and forage quality. Data was analyzed in $\mathrm{R} / \mathrm{lmerTest}$ package using a mixed model with sampling period and quadrat as fixed effects and disease site as a random effect. Disease site and 
quadrat significantly affected tap root diameter, stem length, and all forage quality parameters tested. Interestingly, plants sampled from quadrats in the survivor zone had severely diseased tap roots but strong lateral roots. Within the survivor zone, older surviving plants at $3.5 \mathrm{~m}$ away from the disease front were more likely to have strong lateral roots (41\% in 2015 and $13 \%$ in 2016) compared to plants at $0.5 \mathrm{~m}$ (3\% in 2015 and $5 \%$ in 2016 ) away from the disease front. These results suggest that alfalfa might change its root architecture to overcome PRR disease stress.

\section{Infection rate by Fusarium proliferatum in aerial garlic bulbils is reduced compared to rate in seed cloves when both originate from infected bulbs}

F. M. DUGAN (1), S. Lupien (1), B. Hellier (2), (1) USDA ARS WRPIS, Pullman, WA, USA; (2) USDA-ARS WRPIS, Pullman, WA, USA

Fusarium proliferatum, a fungal pathogen of garlic (Allium sativum) world-wide, infects bulbs and infests soil. Chemical management is not consistently cost-effective. Garlic is typically propagated via seed cloves, but planting of aerial bulbils is an alternative means of propagation for cultivars that produce them. Bulbs were harvested at the USDA-ARS Plant Introduction Station near Pullman WA in fall 2015. From a sample of 18 bulbil-producing accessions whose bulbs were previously documented as infected, a mean of $64 \%$ of cloves from such bulbs contained the pathogen. In umbels resulting from planting of cloves from bulbs of the 18 accessions, a mean of only $11 \%$ contained the pathogen. The mean infection rate in bulbils in infected umbels was $42 \%$. Given that a bulb was infected, the probability of a bulbil in the resulting umbel being infected was approximately $5 \%$. In bulbs harvested in fall 2016 from 9 accessions whose bulbs were previously documented as infected, a mean of $35 \%$ of the cloves in such bulbs contained the pathogen, but to date the pathogen has not been detected in either umbels or bulbils. Bulbils take at least a year longer to mature into full size bulbs than do seed cloves, presenting a longer window for infection by several pathogens, but if planted to pathogen-free soil might represent a cost-effective means to strongly reduce infection of propagation material by $F$. proliferatum.

Disease interaction of late leaf spot and white mold on peanut

M. MUNIR (1), D. J. Anco (2), (1) Clemson University, Clemson, SC, USA; (2) Clemson University, Blackville, SC, USA

Under standard production conditions in the field, peanuts are commonly exposed to multiple diseases. The occurrence or severity of one disease can potentially affect the development of another disease through such mechanisms as microclimate modification. In 2016, field experiments were initiated to study the potential interaction of late leaf spot and white mold in peanut. To manipulate levels of late leaf spot defoliation, three programs of late leaf spot management (5, 4 and 3 chlorothalonil applications) were applied across 4 peanut varieties (Georgia 12Y, TUFRunner 511, Georgia 13M, and Georgia $06 \mathrm{G}$ ) via a split-plot design in three fields. Within canopy and soil temperature were measured from 56 days after planting until inversion. Fungicide management program, variety and the interaction thereof significantly affected late leaf spot severity. However, among these factors only variety significantly affected white mold severity. Georgia $13 \mathrm{M}$ showed the most severe late leaf spot defoliation followed by TUFRunner 511 . Within canopy maximum daily temperatures were not significantly different among treatments, despite significant differences in canopy defoliation. Data from 2016 did not suggest the presence of a significant interaction between late leaf spot and white mold under the conditions experienced in the trial. A lack of an interaction between the two diseases benefits growers and peanut production in the sense that it simplifies management recommendations.

\section{Multivariate analysis of fungal communities associated with diseased winter squash in western Oregon} H. RIVEDAL, A. G. Stone, P. Severns, K. B. Johnson, Oregon State University, Corvallis, OR, USA

Winter squash is produced on 2500 ha in Oregon's Willamette Valley for edible seeds, processing and fresh market. Growers of the prominent cultivar, Cucurbita maxima cv. 'Golden Delicious', are experiencing significant yield losses due to a soilborne disease. Symptoms include stunting, root and crown rot, vascular discoloration, and late-season vine collapse. To identify potential causes, a total of 64 fields were surveyed in the 2014-2016 seasons to characterize the community of fungi associated with roots and crowns of diseased and healthy squash. Over 2500 fungal isolates from sampled plants were subjected to ITS and EF1 $\alpha$ sequencing to identify to species. Isolated fungal communities were analyzed with multivariate statistical techniques in PC-ORD software (multi-response permutation, indicator species analysis and nonmetric multidimensional scaling). From these analyses, three species were consistently associated with disease symptoms: Fusarium oxysporum, F. solani and Plectosphaerella cucumerina. These species were also identified as indicator species of crop rotation history, cucurbit species sampled, and plant tissue sampled. Ordinations of these results showed the fungal community structure associated with winter squash and highlighted the influence of these three fungi on diseased plants. Results of community analyses provided insight into potential causal agents and will allow for more focused pathogenicity trials to further understand this problem.

\section{Armillaria altimontana is not associated with damage to western white pine (Pinus monticola) planted in northern Idaho}

J. W. Hanna (1), M. V. Warwell (1), G. I. McDonald (1), M. S. Kim (2), B. M. Lalande (3), J. E. STEWART (3), N. B. Klopfenstein (1), (1) Rocky Mountain Research Station, USDA Forest Service, Moscow, ID, USA; (2) Kookmin University, Seoul, Korea, Republic of (South); (3) Colorado State University, Fort Collins, CO, USA

Armillaria solidipes (A. ostoyae) is a virulent primary pathogen on diverse conifers in regions of interior western North America, where A. altimontana also frequently co-occurs. However, little is known about impacts of $A$. altimontana on tree health. In 1971, a provenance planting of western white pine (Pinus monticola) was established on a 0.8-ha plot at the Priest River Experimental Forest in northern Idaho, USA to examine growth and survival. In 1987, at 16 years of age, 1215 living/recently dead trees were measured and inspected/sampled for Armillaria to determine potential influences of Armillaria on growth and survival. Armillaria was associated with ca. $48 \%$ of the trees. Based on somatic pairing and elongation factor 1- $\alpha$ sequencing, ca. $83 \%$ of the Armillaria isolates comprised two genets of A. altimontana, and ca. $17 \%$ of the isolates comprised five genets of $A$. solidipes. A. solidipes was associated with decreased tree growth and survival. In contrast, $A$. altimontana was not associated with increased tree mortality or decreased tree growth, suggesting that $A$. altimontana was not damaging to western white pine in northern Idaho. Furthermore, $A$. solidipes was uncommon in areas dominated by A. altimontana. The wide coverage of individual Armillaria genets suggest that both Armillaria spp. have occupied this site for $>250$ years. Understanding the interactions of these two Armillaria species appears key to understanding Armillaria root disease in this region.

Influence of temperature and moisture on germination of Cercospora kaki, causal agent of persimmon angular leaf spot R. FERNANDES ALVES (1), M. Bellato Spósito (2), (1) University of São Paulo, Piracicaba, BRAZIL; (2) University of Sao Paulo, Piracicaba, BRAZIL

Persimmon angular leaf spot, caused by Cercospora kaki, is one of the main foliar diseases on persimmon. The determination of favorable environmental conditions on conidia germination is important to understand the pathosystem epidemiology. In order to determine the favorable conditions for conidia germination, the effect of temperature and moisture were evaluated. Three $70 \mu 1$ aliquots of $10^{4}$ conidia $\mathrm{mL}^{-1}$ suspensions were placed on each of four plastic Petri dishes and incubated in BODs at $10,15,20,25,30,35,37$ and $40^{\circ} \mathrm{C}$ for $6,8,10,12,18,24,30,36$ and 48 hours. Germination percentage was calculated through observation of 100 uredospores per aliquot. After 8 hours, $12 \%$ of germination was observed for conidia placed between 25 and $35^{\circ} \mathrm{C}$. The minimum temperature for conidia germination, estimated by beta-monomolecular function, was at $12.4^{\circ} \mathrm{C}$ and the maximum at $37.1^{\circ} \mathrm{C}$, with an optimal at $28.3^{\circ} \mathrm{C}$. The best condition for conidia germination, more than $90 \%$, occurred between 25 and $30^{\circ} \mathrm{C}$ and moisture longer than 48 hours.

Phylogeny of Northern Hemisphere Armillaria and Desarmillaria: Neighbor-net and Bayesian analyses of translation elongation factor 1- $\alpha$ gene sequences

M. S. KIM (1), J. E. Stewart (2), J. W. Hanna (3), N. B. Klopfenstein (3), (1) Kookmin University, Seoul, Korea, Republic of (South); (2) Colorado State University, Fort Collins, CO, USA; (3) Rocky Mountain Research Station, USDA Forest Service, Moscow, ID, USA 
Armillaria and Desarmillaria species are common in many forests. This study provides further insights into the phylogenetic relationships among Northern Hemisphere Armillaria and Desarmillaria species, based on elongation factor 1- $\alpha$ (tefl) sequences from well-characterized isolates. Bayesian analysis showed Armillaria and Desarmillaria species from the Northern Hemisphere as generally comprised within the following four superclades: 1) Socialis/Tabescens (exannulate) superclade including Eurasian D. ectypa and two separate clades of D. socialis (D. tabescens) from North America/Europe; 2) Mellea superclade including undescribed annulate Armillaria sp. (Mexico) and four separate clades of A. mellea (Europe and Iran, eastern Asia, and two groups - North America); 3) Gallica superclade including Armillaria Nag E (Japan), multiple clades of $A$. gallica (Asia/Europe), A calvescens (eastern North America), A. cepistipes (North America), A. altimontana (western USA), A. nabsnona (North America/Japan), and at least two A. gallica clades (North America); and 4) Solidipes/Ostoyae superclade including two A. solidipes/ostoyae clades (North America), A. gemina (eastern USA), A. solidipes/ostoyae (Eurasia), A. cepistipes (Europe/Japan), A. sinapina (North America/Japan), and A. borealis (Eurasia) clade 2. Although these analyses are based solely on a set of partial tefl sequences, several potentially important phylogenetic relationships are revealed that warrant further study.

\section{Microfungi associated with diseases on Theobroma cacao $\mathrm{L}$. in Merida state, Venezuela}

S. MOHALI-CASTILLO (1), J. E. Stewart (2), (1) Universidad de Los Andes. Facultad de Ciencias Forestales y Ambientales., Merida, VENEZUELA; (2) Colorado State University, Fort Collins, CO, USA

Venezuela has been home to Cacao trees for over 400 years, much earlier than when Spaniards arrived. In the 1630s, diseases were reported on Cacao, though little is known about the fungal species involved in these diseases in the small plantations in Merida state, Venezuela. The goal of this work is to identify fungi associated with diseases in cacao plantations such as die-back, sudden death, and anthracnose on fruits. Samples were collected and transported to the phytopathology laboratory of the School of Forestry Engineering, University of Los Andes (ULA) Venezuela. Samples were cut (5 $\mathrm{mm}^{2}$ ) from the margin of healthy and diseased tissues. Tissues were surface-disinfected for $30 \mathrm{~s}$ in $70 \%$ ethanol followed by $60 \mathrm{~s}$ in $0.5 \%$ sodium hypochlorite, washed 3 times with sterilized water for $60 \mathrm{~s}$, dried with sterilized filter paper, plated onto $2 \%$ malt extract agar and incubated at $25^{\circ} \mathrm{C}$ for 7 days. Cacao fruits with anthracnose were checked for the presence of fruiting bodies. Fungal mycelia of pure cultures were cultured onto malt extract agar. Through morphological descriptions, five fungal genera have been identified associated with these diseases, such as: Lasiodiplodia spp., Neofusicoccum spp., Cylindrocladium and Fusarium spp. isolated from stems and branches; Colletotrichum spp., Neofusicoccum spp. and Fusarium spp. from fruit. Future work will include molecular characterization of these isolates to determine fungal species associated with diseases on cacao.

\section{Effects of soil conditions on root rot of soybean caused by Fusarium graminearum}

D. R. CRUZ, D. A. Mayfield, G. P. Munkvold, L. F. S. Leandro, Iowa State University, Ames, IA, USA

Fusarium graminearum (Fg) is an important soybean pathogen that causes seedling disease, root rot, pre- and post-emergence damping-off. However, effects of soil conditions on the disease are not well understood. Our objective in this greenhouse study was to observe the impacts of soil texture, $\mathrm{pH}$, and soil moisture on seedling root rot symptoms and detrimental effects on seedling development caused by Fg. Fg-infested millet was added (10\% v/v) to soil with four different textures (sand, loamy sand, sandy loam, and loam). Soil moisture was maintained at saturation, field capacity or permanent wilting point at soil $\mathrm{pH}$ levels of 6 or 8 . Seedlings were evaluated 4 weeks after planting for root rot, root length, root and shoot dry weights, and leaf area. There was a significant interaction between soil moisture and soil texture for root rot assessed visually $(P<0.0001)$. Highest severity $(\sim 70 \%)$ was observed at pH6 and permanent wilting point in sandy loam soils. In contrast, pot saturation resulted in the lowest levels of disease in sandy loam and loam soils (11.6 and 10.8\%, respectively). Foliar area was reduced in sandy loam and loam soils at permanent wilting point. Root length and root and shoot dry weights were reduced in sandy loam and loam soils at saturation. In contrast, sandy soils did not present reductions in plant variables. According to our results, levels of root rot increased in water-stressed plants, producing detrimental effects on plant development.

\section{The effect of temperature and wounding on resistance of ornamental grasses to Sclerotinia sclerotiorum}

M. A. GRABOWSKI (1), D. K. Malvick (2), (1) University of Minnesota Extension, Andover, MN, USA; (2) University of Minnesota, St. Paul, MN, USA

Sclerotinia sclerotiorum causes stem rot and death of many flowering annual plants. Significant plant loss occurs in infested landscape beds. Identification of plants with resistance to this disease will help in management. Six genera of annual ornamental graminoids from four families were evaluated to determine the effects of temperature and wounding on resistance to $S$. sclerotiorum. To evaluate the effect of temperature, graminoids were inoculated by securing an $8 \mathrm{~mm}$ square of agar media containing S. sclerotiorum to the base of the stem with a sterile toothpick. Plants were maintained in a growth chamber at $13,16,19$, or $22^{\circ} \mathrm{C}$. Lesion length was measured 28 days after inoculation. Lesion length of inoculated Acorus gramineus, Pennistetum glaucum, and Setaria italica was significantly greater than uninoculated controls and other graminoids. Lesion length on inoculated $P$. glaucum and S. italica increased exponentially with decreasing temperature. Juncus effuses, Carex flagellifera, and Scirpus cernus were highly resistant and lesion length on inoculated plants did not differ from uninoculated controls at any temperature. To evaluate the effect of wounding, $P$. glaucum and A. gramineus were inoculated as described above, with or without wounding, and maintained at $13^{\circ} \mathrm{C}$. Acorus gramineus became infected both with and without wounding. External stem discoloration was observed on unwounded P. glaucum plants but internal stem discoloration occurred only with wounding.

\section{Distribution of AM fungi in Coal mine soils associated with four forest tree Species of North Telangana region} S. PODETI, Department of Biotechnology, Warangal, INDIA

The aim of present study was to investigate the abundance of AM fungal spores in Coal mine overburden soils from India. Rhizosphere soil samples were collected from four different Coal mine locations (Bhupalpally, Kothagudem, Godavarikhani, and Mancherial) of North Telangana region, and were examined to analyze the distribution and diversity pattern of AMF. The root colonization and spore population was influenced by a wide range of environmental factors, which have influence on host plants and AM fungal distribution. Due to mining activities the soil ultimately becomes unproductive and lost its fertility, which leads to decreasing the percentage of AM colonization and lowest spore population. Rhizosphere soil samples were collected along with young tender roots of Albizia lebbeck, Acacia nilotica, Azadiracta indica, and Tamarindus indica from four different coal mine sites. The mycorrhizal roots were stained and percentage of root colonization was assessed. AM fungal spores in the rhizosphere soils were extracted. The results showed the association of AM fungi in all the investigated plants. Among these four plants, percentage of root colonization and spore incidence was highest in Kothagudem rhizosphere soils of Albizia lebbeck and least in Bhupalpally soils of Tamarindus indica. Among all extracted AM fungal spores, Glomus species was dominant in all samples; Glomus and Gigaspora were recorded more in Kothadugem soils when compared to other AM fungal species. Percentage of root colonization was recorded lowest in Godavarikhani and Mancheriyal. The Sclerocystis, Scutellospora and Acaulospora were less abundant in the soils of Godavarikhani and Mancheriyal.

\section{Alternaria species causing moldy core of apple in Chile}

K. ELFAR (1), B. A. Latorre (1), J. P. Zoffoli (2), (1) Pontificia Universidad Católica de Chile, SANTIAGO, CHILE; (2) Pontificia Universidad Católica de Chile, Santiago, CHILE

Moldy core (MC) of apple is an important disease in Chile, reducing yields and quality. Infected fruits ripe and drop prematurely, and show a slight deformation, a black fungal mycelia colonize the carpels and eventually a core rot occur. Disease prevalence of 12 to $60 \%$ were estimated in 'Fuji', 'Red 
Chief', 'Red King Oregon' and 'Scarlet' apples in 2015-2016. The etiology of MC was studied. Apple samples (n=100) were collected from ten orchards in Central Chile two weeks before harvest. A total of 319 of Alternaria isolates were obtained on PDA, and selected small-spore Alternaria isolates $(\mathrm{n}=14)$ were examined molecularly with the primer of plasma membrane ATPase, calmodulin and Alternaria major allergen sequence analysis Based on a concatenated phylogenetic analysis, A. alternata, A. arborescens, A. infectoria and A. tenuissima were identified. All Alternaria spp. were pathogenic in inoculated mature apples and seeds and detached leaves of 'Red Chief' apples. A gray to dark olive green mycelium colonized the carpel cavity after 10 days at $25^{\circ} \mathrm{C}$. Inoculated seeds were superficially colonized. Leaf symptoms consisted on round grayish brown necrotic lesions after 7 days at $25^{\circ} \mathrm{C}$. Alternaria spp. were re-isolated from inoculated fruits, seeds and leaves. In conclusion, MC of apple was associated to an Alternaria complex, being A. alternata, A. arborescens, A. infectoria and A. tenuissima identified in this study.

Transmission of mycoviruses of $R$. solani and effects on fungal growth T. J. STETINA, I. E. Tzanetakis, University of Arkansas, Fayetteville, AR, USA

Rhizoctonia solani Kühn consists of at least 14 genetically distinct anastomosis groups (AG). The goals of this research are to understand Rhizoctonia mycovirus epidemiology in Arkansas and their effects on fungal biology. A virus discovery survey was conducted using 190 geotagged isolates from five different fields in Arkansas. More than 20 new viruses, belonging to at least three different virus families have been discovered. Six viruses were selected for their potential use for biological control. Mycovirus transmission is accomplished laterally via hyphal anastomosis, but transmission may be limited by somatic incompatibility. Experiments were conducted to achieve horizontal transmission from a virus-infected donor isolate to a virus-tested recipient, and to observe the effects of mycovirus infection on fungal growth. Horizontal virus transmission between two pairs of isolates (AG-4 and AG11) was confirmed with RT-PCR using virus specific detection primers. Isolate identity was confirmed by observation of hyphal anastomosis. To our knowledge, this is the first report of transmission of a mycovirus between two non-clonal $R$. solani isolates in vitro. Future experiments will determine the effects of mycovirus infection on fungal virulence.

\section{Isolation of Diaporthe longicolla from asymptomatic sunflower root}

A. ADHIKARI, T. Olson, B. Kontz, F. M. Mathew, South Dakota State University, Brookings, SD, USA

In 2016, Diaporthe longicolla (Hobbs) Santos, Vrandecic and Phillip was recovered from asymptomatic roots of sunflower (Helianthus annuus L). plants in Brookings County, South Dakota. The pathogen was identified using colony morphology characteristics on potato dextrose agar and sequencing of the ITS gene region. To test the competency of $D$. longicolla as a root pathogen in sunflower, an experiment was set up in a completely randomized design under greenhouse conditions at $22^{\circ} \mathrm{C}$ and 12 - h photoperiod. The study included three treatments $[$ D. longicolla 303 (Brookings, SD), D. longicolla 204 (Brookings, SD) and D. helianthi 006 (causal pathogen of Phomopsis stem canker of sunflower, Sully, SD)] and 6 plants (replications) per treatment. Sunflower cv. 'HA 288' was inoculated 2 weeks and 4 weeks after planting using 4 inoculation methods which includes two wounding and two nonwounding methods. At 49 days after inoculation, plants were rated for vascular discoloration on the roots using a $0-5$ scale. No vascular discoloration was observed on roots inoculated with $D$. longicolla at 2 weeks and 4 weeks after planting with any of the four inoculation methods. However, visible discoloration was observed on roots inoculated with $D$. helianthi at 4 weeks after planting with two of the inoculation methods. Preliminary research results suggest that $D$. longicolla is not a root pathogen of sunflower.

\section{Interceptions of fungal pathogens from grass seed imported to Oregon}

J. J. QIU, USDA, Animal and Plant Health Inspection Service, Plant Protection and Quarantine, Portland, OR, USA

Oregon annually produces over 280 million $\mathrm{kg}$ of grass seed or more than $90 \%$ of the US crop. It also imports about three to four million kg of such seed each year. The majority of grass seeds are ryegrass (Lolium spp.), fescue grass (Festuca spp.), Kentucky bluegrass (Poa pratensis), orchard grass (Dactylis glomerata), and bentgrass (Agrostis spp.). From 2014 to 2016, there were 1187 total pest, weed and disease interceptions from the agricultural quarantine inspection of import seed at the port entry. Among the pests intercepted, fungal pathogens comprised $54.5 \%$, split among nine reportable and 18 non-reportable taxa. More frequently intercepted fungi are Phaeoseptoria sp., Mycosphaerella sp., Cladosporium sp., Claviceps purpurea, Phaeosphaeria sp. and Ascochyta sp., having 183, 154, 83, 81, 28 and 21 interceptions, respectively. Other intercepted pathogenic fungi are Glomerella sp., Phoma sp., Pleospora sp., Puccinia graminis ssp. graminis, Puccinia striiformis, Septoria sp., and Stagonospora sp. Due to concerns with regulated pathogens and diseases, eight shipments of seeds were rejected. The quarantine status of some pathogens is discussed.

\section{Species and population densities of stubby root nematodes from multiple states in the United States}

G. YAN (1), D. Huang (2), A. Plaisance (2), N. C. Gudmestad (2), J. L. Whitworth (3), K. Frost (4), C. R. Brown (5), W. Ye (6), P. A. Agudelo (7), W. T. Crow (8), S. L. Hafez (9), (1) Department of Plant Pathology, North Dakota State University, Fargo, ND, USA; (2) North Dakota State University, Department of Plant Pathology, Fargo, ND, USA; (3) USDA-ARS, Aberdeen, ID, USA; (4) Oregon State University, Hermiston Agricultural Research \& Extension Center, Hermiston, OR, USA; (5) USDA-ARS, Prosser, WA, USA; (6) North Carolina Department of Agriculture \& Consumer Services, Raleigh, NC, USA; (7) Clemson University, Plant and Environmental Sciences Department, Clemson, SC, USA; (8) University of Florida, Entomology and Nematology Department, Gainesville, FL, USA; (9) University of Idaho, Parma Research and Extension Center, Parma, ID, USA

Stubby root nematodes (SRN) are an important group of pests on many crops, particularly on potato due to its ability to transmit Tobacco rattle virus causing potato corky ringspot disease. To determine the species and densities of SRN present in the USA, a total of 242 soil samples and 16 nematode suspensions were collected from eight states in 2015 and 2016. Nematodes were extracted from soil using decanting and sugar centrifugal flotation method. SRN were found in 73 out of 194 samples from North Dakota with the densities of 10-250/100 $\mathrm{cm}^{3}$ of soil, seven samples from Minnesota (15$200 / 100 \mathrm{~cm}^{3}$ soil), 19 of 21 samples from Idaho (10-240/100 $\mathrm{cm}^{3}$ soil), five of 13 samples from Oregon (20-300/100 $\mathrm{cm}^{3}$ soil), two samples from Washington $\left(20-35 / 100 \mathrm{~cm}^{3}\right.$ soil), three samples from Florida $\left(40-60 / 100 \mathrm{~cm}^{3}\right.$ soil), two samples from South Carolina $\left(170-320 / 100 \mathrm{~cm}^{3}\right.$ soil), and eight out of 16 nematode suspensions from North Carolina. The species of SRN were identified by sequencing of three genomic regions (28S rRNA, ITS rDNA, 18S rRNA), species-specific PCR, and/or morphological measurements. Results suggested the presence of four SRN species. Paratrichodorus allius was detected from North Dakota, Minnesota, Idaho, Oregon, and Washington; P. minor from North Carolina; P. porosus from South Carolina and North Carolina; and Trichodorus obtusus from Florida and South Carolina. Grid sampling in North Dakota demonstrated an uneven distribution pattern of $P$. allius in a potato field. Identification of SRN species and awareness of population density in affected fields are essential for designing effective control measures.

\section{Changes in Vertical Distribution of Pratylenchus crenatus Associated with Corn in Ohio}

A. C. M. SIMON (1), T. L. Niblack (2), R. Lewandowski (3), P. A. Paul (4), (1) The Ohio State University, Columbus, OH, USA; (2) The Ohio State University, Columbus, OH, USA; (3) Ohio Sate University Extension, Athens, OH, USA; (4) Ohio State Univ, Ohio Agricultural Research and Development Center, Dept of Plant Pathology, Wooster, OH, USA

The vertical distribution of the economically important plant-parasitic nematode, Pratylenchus crenatus, was monitored in a commercial corn field with a history of lesion nematode infestation. Soil samples were collected between growth stages VE and V7 from five blocks. Each block was divided into subplots from which 15 cores were taken to a depth of $70 \mathrm{~cm}$ and then divided into seven segments of $10 \mathrm{~cm}$ each. Nematodes were extracted from the soil mineral fraction (SMF) and root fraction (RF) of each of the seven segments with the sucrose centrifugation and Baermann funnel techniques. $P$. crenatus was present in all soil layers at all growth stages, but mean population density averaged across SMF and RF increased over time. Between V3 and V7, counts were highest in the 10-20 and $20-30 \mathrm{~cm}$ segments (approximately 35 to 50 nematodes $/ 100 \mathrm{~cm}^{3}$ soil). At these depths, mean population 
densities were higher in SMF than in RF at earlier growth stages, but reached comparable or higher levels in RF between V6 and V7. These results suggest that $P$. crenatus population density may be underestimated if either the soil mineral fraction or the root fraction alone is used for extraction. This is consistent with earlier reports on $P$. penetrans and $P$. scribneri. The observed patterns of increase in nematode counts over time suggest reproduction and movement of nematodes based on root resource availability.

Response of cotton cultivars with varying levels of resistance to Meloidogyne incognita to irrigation

J. E. WOODWARD (1), R. Roper (2), T. Wheeler (3), (1) Texas A\&M AgriLife Extension Service, Lubbock, TX, USA; (2) Texas Tech University, Lubbock, TX, USA; (3) Texas A\&M AgiLife Research, Lubbock, TX, USA

Irrigation is important in maximizing cotton yield in west Texas, where the southern root-knot nematode (Meloidogyne incognita) is an important pest. Field trials were conducted from 2014-2016 to evaluate the response of cotton cultivars to three irrigation levels in a field naturally infested with $M$. incognita. Overall, nematode densities increased with higher irrigation levels; however, damage was more pronounced under low irrigation. Nematode reproduction varied by year, but differences among cultivars were observed. Cultivars that contain two $M$. incognita resistance genes supported the lowest level of reproduction, followed by cultivars with a single resistance gene. Irrigation affected yield in all years of the study. Yield for partially resistant (PR), resistant (R) and susceptible (S) cultivars were not different in 2014 under the three irrigation levels. In 2015, yields of R cultivars were similar to yields of S cultivars within an irrigation level. When averaged across irrigation levels, yields for the PR cultivars increased by $14 \%$ over R and $\mathrm{S}$ cultivars. Yield increases over S cultivars were observed for PR and R cultivars under all irrigation levels in 2016. Yield increases for R over $\mathrm{S}$ cultivars averaged 13, 15 and 3\% for the low, base and high irrigation levels; whereas, yields for PR cultivars increased by 28,48 and $42 \%$ for the same irrigation levels. While high levels of resistance to M. incognita exist in R cultivars, higher yields where achieved with PR cultivars. Furthermore, yield responses of PR cultivars to irrigation were greater than those for $\mathrm{R}$ cultivars.

Evaluating fertilizer effect on the interaction between Fusarium proliferatum and soybean cyst nematode on soybean P. N. OKELLO (1), S. Osborne (2), J. Kleinjan (1), F. M. Mathew (1), (1) South Dakota State University, Brookings, SD, USA; (2) USDA Agricultural Research Service, Brookings, SD, USA

In this study, we hypothesized that fertilizer levels can impact root rot severity caused by Fusarium proliferatum in the presence of SCN. To test this hypothesis, a greenhouse and field study were conducted using two soybean cultivars (SCN susceptible and SCN resistant). Two N-P-K fertilizer ratesstarter (15:15:15) and high (50:80:110) were applied to each of the treatments ( $F$. proliferatum alone, SCN alone, combination of SCN and $F$. proliferatum and a control). In the greenhouse, both cultivars were inoculated at planting with either $F$. proliferatum-colonized millet seeds or 2000 SCN eggs/ mL. For field experiment, the two cultivars were planted in a naturally infested SCN field plots and inoculated with F. proliferatum-colonized millet seeds at rate of $80 \mathrm{~g}$ per plot. For the two studies, root rot severity and SCN population data were recorded after 35 days. Our greenhouse results suggest that the fertilizers rates significantly $(P<0.001)$ increased root rot severity caused by $F$. proliferatum in the presence and absence of SCN. However, SCN reproduction was reduced by $F$. proliferatum under both fertilizer treatments. In the field study, root rot severity was not impacted by either fertilizer treatments. Fusarium proliferatum and N-P-K rates did not significantly reduce SCN reproduction. Our study suggests that a balanced N$\mathrm{P}-\mathrm{K}$ rates and $\mathrm{SCN}$ resistant cultivar are important to manage F. proliferatum and $\mathrm{SCN}$ in affected soybean fields.

Association Mapping of Resistance to Heterodera glycines within the USDA Core Collection of Common Bean

S. JAIN (1), S. Poromarto (2), J. Osorno (3), B. D. Nelson (4), (1) North Dakota State University, Fargo, ND, USA; (2) Agrotechnology, Sebelas Maret University, Surakarta, INDONESIA; (3) Plant Sciences 7670, NDSU, Fargo, ND, USA; (4) Plant Pathology 7660, North Dakota State University, Fargo, ND, USA

Common bean (Phaseolus vulgaris L.) is an important high protein crop grown worldwide. North Dakota is the largest producer of dry beans in USA, but the crop is threatened by soybean cyst nematode (SCN; Heterodera glycines) as most current cultivars are susceptible. Seed yield losses of 30-50\% were observed in different bean classes due to SCN infection. The USDA common bean core collection is a diverse collection of bean germplasm and a valuable genetic resource for resistance to different diseases. The data from a recent greenhouse screening of 353 accessions from the core collection for resistance to SCN HG type 0 was used to conduct a genome wide association study to find genomic regions associated with SCN resistance. Phenotypic results showed that accessions could be classified as highly resistant (22\%), moderately resistant (52\%), moderately susceptible (24\%) and highly susceptible (2\%) based on the SCN female index. Four genetic subpopulations as well as four principal components (PCs) were defined for the association analysis using 4575 SNP markers evenly distributed across all 11 chromosomes. Two SNPs on chromosome 8 and one SNP on chromosome 11 were found to be associated with SCN resistance. A gene cluster analog of $r h g l$ locus which confers resistance to SCN in soybean was identified in close proximity to associated SNPs on chromosome 8 of dry bean. These genomic regions will be examined to identify candidate genes associated with resistance in dry bean.

\section{Characterization of a new tymovirus from Ecuador}

K. GREEN (1), D. S. Mollov (2), D. F. Quito-Avila (3), A. V. Karasev (1), (1) University of Idaho, Moscow, ID, USA; (2) USDA, Beltsville, MD, USA; (3) PROMETEO, CIBE-ESPOL, Guayaquil, ECUADOR

Naranjilla (Solanum quitoense) is a solanaceous shrub cultivated in Ecuador for fresh fruit and juice production. A naranjilla plant exhibiting stunting and chlorotic spots was sampled near Quito, Ecuador. An infectious agent was transferred to indicator plants by mechanical inoculation, and subsequently characterized at biological and molecular levels. The pathogen from naranjilla systemically infected eggplant producing mild mosaic symptoms, and naranjilla producing chlorotic spots, mosaic, and stunting. However, it did not infect potato or tobacco. An RNA-Seq analysis conducted on inoculated symptomatic Nicotiana benthamiana plants produced a single sequence contig sharing the closest relationship with several tymoviruses. The entire genome of the virus was amplified using RT-PCR and sequenced by the Sanger methodology. The genome was found to encode a set of open reading frames typical of tymoviruses, with the highest nucleotide sequence identity to the closest tymovirus, Eggplant mosaic virus, below $70 \%$. The data suggest that this is a new tymovirus infecting naranjilla, for which the tentative name Naranjilla chlorotic mosaic virus (NarCMV) is proposed.

Complete nucleotide sequence and genome organization of a novel filamentous virus from alfalfa (Medicago sativa) L. G. NEMCHINOV (1), S. Grinstead (2), D. S. Mollov (3), (1) USDA ARS MPPL, Beltsville, MD, USA; (2) USDA-ARS-NGRL, Beltsville, MD, USA; (3) USDA, Beltsville, MD, USA

A new species of the family Alphaflexiviridae provisionally named Alfalfa virus S (AVS) was diagnosed in alfalfa samples originating from Sudan. A complete nucleotide sequence of the viral genome consisting of 8,349 nucleotides excluding the 3' poly(A) tail was determined by Illumina NGS technology and 5' RACE system (Thermo Fisher Scientific) using total RNA extracted from infected plants. BLAST search revealed that the virus shares the greatest degree of sequence identity with members of the family Alphaflexiviridae, genus Allexivirus. The AVS genome contains six computationally predicted tentative open reading frames (ORF): viral replication protein, triple gene block protein 1 (TGB1), TGB2, putative TGB 3-like protein, an unknown $38.4 \mathrm{kDa}$ protein resembling serine-rich $40 \mathrm{kDa}$ protein characteristic for allexiviruses, and coat protein (CP). However, it appears that AVS lacks a clear 3' proximal ORF that is typical for allexiviruses and encodes a nucleic acid-binding protein. On the nucleotide level, the next closest species to the AVS are not yet classified Arachis pintoi virus A (54.6\% identities); unassigned species within the family Alphaflexiviridae, Blackberry virus E (50.6\%); and the type member of the genus Allexivirus, Shallot virus X (49.7\%) (PASC tool, Bao et al., 2014). DIG-labeled RNA probes generated from 
cloned AVS P38.4 and CP cDNAs successfully hybridized to the total RNA extracted from infected alfalfa tissues in dot blot assays thus confirming identity of the virus. To the best of our knowledge, this is the first report on the presence of a putative allexivirus in alfalfa (Medicago sativa).

A new virus complex in babaco (Carica pentagona) shows evolutionary relationships with two viruses involved in meleira disease of papaya $(C$. papaya)

J. F. Cornejo (1), R. A. Alvarez-Quinto (2,3), D. QUITO-AVILA (4,5), (1) Escuela Superior Politecnica del Litoral, Guayaquil, ECUADOR; (2) CIBEESPOL, Guayaquil, ECUADOR; (3) Centro de Investigaciones Biotecnologicas del Ecuador, Guayaquil, ECUADOR; (4) Escuela Superior Politecnica del Litoral, Facultad Ciencias de la Vida, Guayaquil, ECUADOR; (5) Escuela Superior Politecnica del Litoral, CIBE-ESPOL, Guayaquil, ECUADOR

In recent years, the presence of a new umbra-like virus was reported in papaya plantings of Ecuador. Thus far, the virus, tentatively named Papaya virus $\mathrm{Q}(\mathrm{PpVQ})$, is symptomless and found quite frequently in commercial plantings. A recent study conducted in Brazil reported the presence of a virus with $75 \%$ nt identity to PpVQ. The brazilian virus was shown to act synergistically with Papaya meleira virus (PMeV) to induce a devastating disorder known as papaya meleira. This prompted us to investigate whether PpVQ was associated with meleira-like symptoms in Ecuador. Virus surveys showed that PpVQ was present in both symptomatic and asymptomatic plants; whereas PMeV was not detected in any of the tested plants, suggesting that either $\mathrm{PMeV}$ is not present in Ecuador or - similarly to the umbra-like PpVQ - the virus is highly divergent and escaped detection. Considering the importance of implementing a surveillance scheme for meleira disease in Ecuador, symptomatic babaco (Carica pentagona), grown to a lesser extent in papaya producing areas, was collected and subjected to dsRNA extractions followed by DOP-RT-PCR. Sequencing revealed the presence of two viruses with homology to PpVQ and $\mathrm{PMeV}$, respectively. Detection primers developed to partial sequences obtained for both viruses were used to determine their presence in papaya showing meleira-like symptoms. Results from virus surveys and phylogenetic analysis will be presented and discussed in detail.

Five Fusarium species associated with root rot and sudden death of industrial hemp in Nevada

J. Schoener, R. Wilhelm, R. Rawson, P. Schmitz, S. WANG, Nevada Department of Agriculture, Sparks, NV, USA

Industrial hemp (Cannabis sativa L.) is a regulated emerging economic crop in the United States. In the summer of 2016, high mortality was observed in an industrial hemp field in Nevada, with up to $70 \%$ of plants killed. In three varieties planted, the "Canda" variety was most affected followed by the "Futura-75" and the "Joey". Affected plants exhibited rapid death of foliage and extensive root rot with little lateral root growth. Selected root tissue was surface sterilized, placed onto potato dextrose agar amended with streptomycin sulfate, and incubated at $22^{\circ} \mathrm{C}$ in the dark. Diverse Fusarium species were isolated from root samples collected from the three varieties. Thirty representative Fusarium isolates were obtained and grouped into five distinct species based on colony and sporulation characters. Sequence analysis of the ITS region of rDNA classified these 30 isolates into five species, namely, F. oxysporium, F. solani, F. redolens, F. tricinctum, and F. equiseti, consistent with morphological grouping. F. oxysporium, the most prevalent species, was recovered from $82 \%$ of sampled plants; and $F$. solani, F. equiseti, F. tricinctum, and F. redolens were isolated from $29 \%, 29 \%, 18 \%$, and $6 \%$ of sampled plants, respectively. While the role of each species in the disease remains to be investigated, the data suggest that $F$. oxysporium, $F$. solani, and $F$. redolens are the primary pathogens causing root rot, while $F$. tricinctum and $F$. equiseti may be secondary pathogens.

First detection for Fusarium oxysporium and $F$. solani causing wilt of medical marijuana plants in Nevada J. Schoener, R. Wilhelm, R. Rawson, P. Schmitz, S. WANG, Nevada Department of Agriculture, Sparks, NV, USA

Medical marijuana plants (Cannabis sativa L.) are produced in registered facilities in Nevada for medical use. Since the production is highly regulated, information on diseases associated with marijuana plants is scarce. In 2016, a systemic problem was observed in seedlings, cuttings, and mother plants in one production facility. Affected plants exhibited leaf chlorosis, wilting, and failure of nutrient absorption from the soil. Reddish-brown streaks were observed in the vascular tissue of examined stems. To isolate the causative agent, stem tissue was surface sterilized, placed onto potato dextrose agar amended with streptomycin sulfate, and incubated at $22^{\circ} \mathrm{C}$ in the dark. Two Fusarium species, morphologically resembling F. oxysporium and $F$. solani, were isolated from the vascular tissue of two Cannabis varieties, "Fire OG", and "Remedy CBD", respectively. Sequence analysis of the ITS region of rDNA confirmed that these two species belong to $F$. oxysporium and $F$. solani, consistent with morphological identification. Inoculation tests on four industrial hemp (Cannabis sativa L.) varieties showed that both $F$. oxysporium and $F$. solani caused stunting, root rot, and occasionally vascular discoloration at the basal stem. Both Fusarium species were recovered from roots or stems of inoculated plants. The data suggest that both $F$. oxysporium and $F$. solani cause severe vascular wilt on marijuana plants but only cause root rot and mild vascular diseases on hemp plants.

\section{The blueberry stem and twig blight complex in New Jersey} J. J. POLASHOCK, USDA ARS, Chatsworth, NJ, USA

Stem blight, caused by Botryosphaeria dothidea, is a growing problem in blueberry production in New Jersey. Stem blight will kill whole canes on mature bushes, but crown infections can kill the entire bush. Twig blight, caused by Phomopsis vaccinii, is another disease of growing importance. Twig blight is typically limited to the tips of the canes. Only twig blight can be controlled with fungicides. It is unclear why incidence of both diseases seems to be increasing. We explored two possibilities: 1) shifts in the pathogen species complex to more virulent isolates and/or species, and 2) fungicide resistance development. Samples with typical symptoms (dying canes with necrotic lesions) were collected in 2014 and 2016. Fungi were isolated and characterized to determine their species, virulence on 'Duke' blueberry plants, and fungicide resistance. In 2014, about half the pathogens (75 of 140) were $B$. dothidea and the rest (65) were $P$. vaccinii. In 2016, there was a species shift wherein most were $P$. vaccinii (67 of 96), only 2 were $B$. dothidea and the remaining (27) were found to be Neofusicoccum spp. In virulence tests, all isolates caused disease, but $P$. vaccinii and Neofusicoccum spp. were more virulent than $B$. dothidea. All isolates were susceptible to the fungicides tested in vitro. Thus, the increasing incidence of these diseases is not related to fungicide resistance development. The species complex causing these diseases needs further exploration.

New virus from the family Tombusviridae infecting sugarcane

M. N. Tahir (1), S. Grinstead (2), D. S. MOLLOV (3), (1) USDA ARS, Beltsville, MD, USA; (2) USDA-ARS-NGRL, Beltsville, MD, USA; (3) USDA, Beltsville, MD, USA

Sugarcane is an economically important crop cultivated in tropical and sub-tropical regions worldwide. Viral diseases are one of the major constraints for sugarcane production. Total RNA was extracted from leaves exhibiting mosaic symptoms on a sugarcane quarantine accession. A cDNA library was constructed from the genomic RNA for whole-transcriptome analysis by high-throughput sequencing (HTS). HTS reads were assembled using CLC Workbench. BLAST analyses of the assembled contigs revealed the presence of Sugarcane mosaic virus (family Potyviridae), Sugarcane yellow leaf virus (family Luteoviridae), and contig of 2869 nt. This additional contig showed identity to umbraviruses and umbra-like viruses belonging to the family Tombusviridae. RT-PCR, cloning and Sanger sequencing were used to verify the new virus-like contig. Open reading frame (ORFs) analysis revealed two proteins, which encode a putative replicase and a hypothetical protein with unknown function. BLAST analyses of both ORFs show 44\% and 60\% identity, respectively, with Citrus yellow vein-associated virus (JX101610). The genome organization, sequence identity, and phylogenetic analysis provide sufficient evidence to conclude that this is a new virus in the family Tombusviridae.

First Report of Armillaria spp. infecting citrus trees on sour orange rootstock in the Lower Rio Grande Valley, Texas M. KUNTA, P. Vedasharan, J. W. Park, E. Louzada, Texas A\&M University Kingsville Citrus Center, Weslaco, TX, USA 
During December 2015, clusters of light brown colored fruiting bodies were observed around the base of the citrus tree trunk in a commercial orchard in the Lower Rio Grande Valley, TX. Citrus trees located both in residential and commercial areas have been affected. Trees showed initial leaf and twig dieback, premature leaf drop, dried and dead branches, sometimes the fruit still hanging on the tree which eventually dried up, and later death of the whole tree or in some trees sudden wilt and total collapse of the tree was observed. Although it appears as sudden death since initially no apparent symptoms were seen above the ground, the disease is well established in the larger roots and crown, destroying the whole root system. White mycelial mats were observed underneath the bark of infected roots and crown area, and spreading into the wood. The roots were rotten, wet, spongy, with brown to black bark which peels off easily, and with strong mushroom odor, all of which are typical symptoms of root rot caused by Armillaria spp. PCR using Armillaria mellea specific primers on DNA extracted from fungal mycelial mats produced positive results. Currently, fungal isolations are under progress.

A dieback disease affecting blackberry in North Carolina

J. Mercier, P. WIESE, K. Cobaugh, Driscoll Strawberry Associates, Watsonville, CA, USA

A dieback disease of blackberry was found in Eastern North Carolina during the summer of 2016. The main symptoms were shallow lesions and purplish discoloration on floricanes, accompanied by necrotic foliage. The lesions often coalesced and killed the canes. The disease affected single plants and was apparently randomly distributed in the field. It was usually present on all canes of each affected plant. The disease was found in three ranches and affected only the proprietary cultivar BJ110.2. Other cultivars at these locations were not affected. Fungi isolated from the lesions were identified as Phomopsis spp., Colletotrichum fructicola and Pestalotia sp. through morphological characteristics and molecular analyses of the internal transcribed spacer (ITS) region. Further DNA sequencing work is being done to identify the Phomopsis species. All three fungi occurred together on the same canes. These pathogens caused lesions within two to three weeks when each isolate was inoculated singly to detached BJ110.2 cane segments held in humid plastic boxes and all three were successfully re-isolated. The weather data for Wilmington N.C. shows significant frost events $\left(-6^{\circ} \mathrm{C}\right)$ in two weeks with a high above $19^{\circ} \mathrm{C}$ in both January and February 2016 . We hypothesize that BJ110.2 early onset of growth might make it susceptible to damage from weather events such as early frosts alternating with warm temperatures, creating conditions for infection by opportunistic pathogens.

Pseudomonas viridiflava: A potential emerging pathogen associated with bacterial canker of plum trees in the Western Cape, South Africa K. BOPHELA (1), Y. Petersen (2), J. Roux (1), T. Coutinho (1), (1) University of Pretoria, Pretoria, SOUTH AFRICA; (2) Agricultural Research Council, Stellenbosch, SOUTH AFRICA

Pseudomonas viridiflava is a common pathogen of naturally occurring Arabidopsis thaliana, but is also known to infect other crop plants such as tomato, cabbage, cauliflower and lettuce. Pseudomonad strains were isolated from the twigs of diseased plum trees cultivated in spatially separated orchards in the Western Cape. A multilocus sequence typing approach of four housekeeping loci, namely, cts, gapA, gyrB and $r p o D$, was used to identify and delineate these isolates to their respective phylogenetic groups within the Pseudomonas syringae species complex. The pseudomonad strains from diseased plum twigs clustered into two phylogenetic groups, i.e. phylogroup 2a (non-pathogenic or non-plant derived group) and 7a ( $P$. viridiflava group). Since phylogroup 2a strains are non-pathogenic, the pseudomonad strains that clustered in this group were not tested for pathogenicity but the role of $P$. viridiflava in the etiology of bacterial canker of plum trees remained to be elucidated. Upon infection of bean pods, three of five plum phylogroup 7a strains were able to induce soft-rotting of tissue, possibly a result of a pectate lyase activity which is a known characteristic of pathogenic $P$. viridiflava strains. The same strains were able to induce water-soaked lesions, which later became necrotic, when inoculated into 18 day-old bean seedlings. Gummosis was evident around the site of inoculaton of an artificially wounded stem of a plum seedling. The isolation of pathogenic $P$. viridiflava strains from plum trees showing symptoms of bacterial canker suggests that a complex of bacteria may be responsible for tree death in South Africa.

First detection of two new, unnamed root-lesion nematodes Pratylenchus spp. on soybean in North Dakota

G. YAN (1), D. Huang (1), A. Plaisance (1), Z. A. Handoo (2), (1) North Dakota State University, Department of Plant Pathology, Fargo, ND, USA; (2) USDA-ARS, Mycology and Nematology Genetic Diversity and Biology Laboratory, Beltsville, MD, USA

Root-lesion nematodes (RLN; Pratylenchus spp.) are important nematode pests on soybean. In 2015 and 2016, 17 soil samples were collected from two different soybean fields in Richland County, ND. All six samples from Field-1 contained RLN ranging from 125 to 2,000/kg of soil. Ten of the 11 samples collected from Field-2 had RLN from 150 to $875 / \mathrm{kg}$ of soil. Infested soil samples from these two fields were used to inoculate a soybean cultivar, Barnes, in four replicates. After 15 weeks of growth at $22^{\circ} \mathrm{C}$ in a greenhouse room, nematodes were extracted from soil using the sugar centrifugal flotation method and from roots using the Whitehead tray method. The final RLN populations were found to have increased greatly. The reproduction factor of RLN was 5.02 for Field-1 and 3.76 for Field-2, indicating that these nematodes infect and reproduce well on this soybean cultivar. Individual RLN were hand-picked and examined morphologically and molecularly for species identification. The Pratylenchus sp. in Field-1 differs from four morphologically closely related Pratylenchus spp. including P. convallariae, P. pratensis, P. fallax, and P. flakkensis. The Pratylenchus sp. in Field2 differs from $P$. flakkensis and three other morphologically closely related Pratylenchus spp. including $P$. alleni, P. hexincisus, and $P$. gibbicaudatus. The root-lesion nematode species in Field-1 is distinct from that in Field-2. According to both morphological and molecular observations, these two North Dakota Pratylenchus spp. populations represent new species able to parasitize soybean and new records of the two Pratylenchus spp. in the literature.

First detection of the spiral nematode Helicotylenchus microlobus on soybean in North Dakota

G. YAN (1), D. Huang (2), A. Plaisance (2), Z. A. Handoo (3), (1) Department of Plant Pathology, North Dakota State University, Fargo, ND, USA; (2) North Dakota State University, Department of Plant Pathology, Fargo, ND, USA; (3) USDA-ARS, Mycology and Nematology Genetic Diversity and Biology Laboratory, Beltsville, MD, USA

Spiral nematodes (Helicotylenchus spp.) are common plant-parasitic nematodes in fields of many crops. In 2015 and 2016,12 soil samples were collected from a soybean field in Richland County, ND. Nematodes were extracted from soil using a sugar centrifugal flotation method. Spiral nematodes were found in 11 of the samples, ranging from 125 to 3,300 per $\mathrm{kg}$ of soil. One soil sample with 1,500 spiral nematodes per kg was planted with two soybean cultivars, Sheyenne and Barnes, each in four replicates. After 15 weeks of growth at $22^{\circ} \mathrm{C}$ in a greenhouse room, the population of spiral nematodes increased substantially. The final density was $9,300 \pm 1,701$ spiral nematodes per $\mathrm{kg}$ of soil for Sheyenne and 9,451 $\pm 2,751$ for Barnes. The reproductive factor in Sheyenne and Barnes was 6.2 and 6.3, respectively, demonstrating that this spiral nematode reproduces well on these two soybean cultivars. Individual spiral nematodes were examined morphologically and molecularly for species identification. The spiral nematode was identified as Helicotylenchus microlobus according to morphological and morphometric characteristics. The species identity was further confirmed by the ITS rDNA sequence. This nematode had only $91 \%$ sequence similarity to seven isolates of $H$. pseudorobustus, a spiral nematode species very closely related to H. microlobus in morphology. The H. microlobus nematode was reported as one of the most commonly observed spiral nematodes in soil samples in Minnesota and all 13 soybean cultivars tested except Hawkeye were rated as hosts. This finding represents the first occurrence of $H$. microlobus in North Dakota.

Neofusicoccum australe, $N$. luteum and $N$. mediterraneum are new blight and canker pathogens of Coast Redwood (Sequoia sempervirens) in California

S. G. AĆIMOVIĆ (1,2), S. N. Rooney-Latham (3), D. M. Grosman (2), J. J. Doccola (2), (1) Cornell University, Hudson Valley Research Laboratory, Highland, NY, USA; (2) Research and Development Laboratory, Arborjet Inc., Woburn, MA, USA; (3) California Department of Food \& Agriculture, Sacramento, CA, USA 
Coast redwood is among the most planted landscape trees in California. Outside its natural range, coast redwood is in decline due to factors including climate change and the movement of plant pathogens. We investigated the fungi associated with leaf blight and canker in declining urban stands of coast redwood. Symptomatic leaves, shoots and branch cankers were collected from 11 coastal and inland locations of CA between 2010-2014. Fifty-nine Botryosphaeria-like fungal strains were isolated and 18 were selected for identification. PCR sequencing of the ITS and EF-1 $\alpha$ regions revealed at least 5 different Botryosphaeriaceae fungi. In addition to Botryosphaeria dothidea, which is known on redwood in CA, Neofusicoccum australe, $N$. luteum, $N$. mediterraneum and a $N$. sp. closely related to $N$. parvum were detected. Pathogenicity tests on healthy saplings showed that these fungi had different levels of virulence. Average lesions lengths were longest on saplings inoculated with $N$. australe, followed by $N$. luteum, $N$. sp., $N$. mediterraneum and B. dothidea. Fungi were reisolated from all inoculated trees, completing Koch's postulates. Our results support the increasing incidence of Botryosphaeriaceae pathogens in California's landscape and agriculture trees, which may be due to prolonged drought. Accurate diagnosis of fungal pathogens of coast redwood is important for disease management and the improvement of horticultural practices in urban stands of this tree species.

Identification of the Constricta yellow dwarf virus strain of Potato yellow dwarf virus in field grown peppers and tomatoes in Maryland J. HAMMOND (1), J. Stommel (2), M. Reinsel (3), C. Gulbronson (4), M. M. Goodin (5), (1) USDA ARS Floral and Nursery Plant Research Laboratory, Beltsville, MD, USA; (2) USDA ARS, Genetic Improvement of Fruits \& Vegetables Laboratory, Beltsville, MD, USA; (3) USDA ARS, Floral and Nursery Plants Research Unit, Beltsville, MD, USA; (4) USDA ARS Electron and Confocal Microscopy Unit, Beltsville, MD, USA; (5) University of Kentucky, Department of Plant Pathology, Lexington, KY, USA

In 2006, several pepper (Capsicum annuиm) plants at Beltsville, MD were observed with virus-like symptoms of vein clearing, leaf curling, and uneven chlorosis. Transmission electron microscopy (TEM) revealed rhabdovirus-like particles, also observed in Nicotiana benthamiana, N. edwardsonii, and $N$. glutinosa mechanically inoculated with pepper leaf extracts. Tomato (Solanum lycopersicum) grown in the same area in 2010 showed fruit symptoms of yellowish speckling, with some irregular rings; TEM revealed typical rhabdovirus particles. RT-PCR using generic rhabdovirus primers amplified a c. 1 $\mathrm{kb}$ product, with a sequence most closely related to, but distinct from the Sanguinolenta yellow dwarf virus strain of Potato yellow dwarf virus (PYDVSYDV; 73\% nt identity; 94\% coverage). Pepper plants showing fruit distortion, uneven coloration, and sunken lesions with a necrotic center were also observed in 2016; TEM of thin sections revealed large groups of rhabdovirus particles in the nucleus of infected cells. RT-PCR from pepper, and from a nightshade plant growing at the field margin, yielded a rhabdovirus PCR product with c. $99 \%$ identity to the earlier tomato PCR product. Comparison to the newly completed genome of the Constricta yellow dwarf virus strain of PYDV (PYDV-CYDV) also revealed c. 99\% nt identity. We therefore identify the rhabdovirus isolates from pepper, tomato, and nightshade as PYDV-CYDV; nightshade may function as an overwintering host in Maryland.

Zucchini tigré mosaic virus, an emerging potyvirus in cucurbit vegetables in South Asian countries B. Bagewadi (1), K. Nagendran (2), R. B. Khadka (3), G. Karthikeyan (4), M. S. Hossain (5), L. Shah (6), A. C. Fayad (7), R. NAIDU (8), (1) Washington State University, Prosser, Prosser, WA, USA; (2) ICAR-Indian Institute of Vegetable Research, Varanasi, INDIA; (3) Khajura Regional Agriculture Research Station, Khajura, Banke, NEPAL; (4) Tamil Nadu Agricultural Univ, Madurai, INDIA; (5) Horticulture Research Center, Gazipur, BANGLADESH; (6) iDE, Kathmandu, NEPAL; (7) Virginia Polytechnic Institute and State Univ, Blacksburg, VA, USA; (8) Washington State University-Prosser, Prosser, WA, USA

Zucchini tigré mosaic virus (ZTMV, Potyviridae: Potyvirus) has been reported in cucurbit crops from several countries, including Guadeloupe (French West Indies), Myanmar, China, and the USA. As part of improving the production of cucurbit vegetables in subsistence agriculture in South Asian countries, a survey of cucurbit vegetable crops grown in farmers' fields was conducted in South India, Bangladesh and Nepal. Leaf samples showing virus-like symptoms were tested in the field using immunostrip diagnostic kits. Symptomatic samples were pressed onto FTA ${ }^{\mathrm{TM}}$ PlantSaver Cards and air-dried. Total nucleic acids captured on the FTA cards were eluted and tested by RT-PCR using primers specific to a portion of the cylindrical inclusion body protein of potyviruses, followed by sequence analysis of the amplicons. In addition, total RNA extracted from field samples was subjected to high-throughput sequencing using Illumina's HiSeq 2500 system. The quality filtered 125-basepair paired-end reads were assembled de novo into contigs using the CLC Genomics workbench 8.0 software and annotated against the viral RefSeq database in GenBank by BLASTN program to identify viral sequences present in symptomatic samples. The results indicated the presence of ZTMV in different types of gourds belonging to the genus Lagenaria. Molecular characterization of ZTMV isolates revealed the presence of genetic variants of the virus in cucurbit vegetable crops in South Asian countries.

\section{First report of bacterial leaf streak of corn caused by Xanthomonas vasicola in Oklahoma} J. P. DAMICONE, F. Cevallos, J. D. Olson, Oklahoma State University, Stillwater, OK, USA

Following reports of a new bacterial disease of corn in nearby states, irrigated corn fields in Oklahoma were surveyed for bacterial leaf streak caused by Xanthomonas vasicola in August 2016. Symptoms consisting of elongated necrotic lesions with irregular margins often surrounded by yellow borders were observed in Texas Co., although disease severity was low (less than 5\%). Bacterial streaming was observed from excised leaf sections and yellow mucoid colonies formed on nutrient agar. The species-specific primers Xvv3 and Xvv5 were used in PCR assays to identify the pathogen. Four isolates were PCR positive for both primer pairs, confirming the pathogen in 2 of the 19 fields surveyed. Other bacteria were PCR negative. Pathogenicity tests were performed on 21-d old plants grown in the greenhouse. Bacterial suspensions $\left(10^{7} \mathrm{cfu} / \mathrm{ml}\right)$ were used to spray-inoculate plants incubated for $48 \mathrm{hr}$ pre and post-inoculation in humidity chambers, or were infiltrated into leaves of non-incubated plants. Sterile water served as the non-inoculated controls. Typical bacterial leaf streak lesions developed within 7-d post inoculation with both inoculation methods. Other bacteria and the water control did not cause any symptoms. The pathogen was re-isolated from diseased leaves. This is the first report of bacterial leaf streak of corn caused by $X$. vasicola in Oklahoma and expands the current reported geographic range of this new disease in the United States.

Current assessment of Xanthomonas citri subsp. malvacearum race designation in Texas cotton

J. E. WOODWARD (1), T. Wheeler (2), T. S. Isakeit (3), K. Ong (4), R. Bart (5), A. Phillips (6), (1) Texas A\&M AgriLife Extension Service, Lubbock, TX, USA; (2) Texas A\&M AgiLife Research, Lubbock, TX, USA; (3) Texas A\&M University, College Station, TX, USA; (4) Texas A\&M AgriLife Extension Service, College Station, TX, USA; (5) Donald Danforth Plant Science Center, St Louis, MO, USA; (6) Danforth Plant Science Center, St Loius, MO, USA

The resurgence of Bacterial blight, caused by Xanthomonas citri subsp. malvacearum $(\mathrm{Xcm})$, throughout cotton production areas in the U.S. has led to renewed interest in the disease. Overall, race 18 is the most prevalent race in the U.S. Observations from fields in 2015 and 2016 revealed leaves displaying small, water-soaked lesions that were restricted by leaf veins occurring on both susceptible and immune cultivars. As the disease progressed, premature defoliation occurred. Symptoms were easily observed on susceptible cultivars; however, the manifestation of symptoms on immune cultivars occurred more so in the lower canopy and appeared to be less severe. Incidence in immune cultivars was low and generally occurred in small isolated pockets, whereas, symptoms were uniformly distributed across susceptible cultivars. Isolations from numerous leaf samples yielded approximately 200 yellow, mucoid colonies that were tested for pathogenicity using immune and susceptible cultivars. All reactions were consistent with Xcm race 18 reactions. Additional isolations from field samples produced colonies of a white, non-Xanthomonas sp. Sequences from four isolates were identified as Pseudomonas syringae $(P s)$ based on the $16 \mathrm{~S}$ and $23 \mathrm{~S}$ r-DNA. When inoculated with both $X c m$ and $P s$, weak Bacterial blight symptoms occured on an immune cultivar, where $X \mathrm{~cm}$ alone caused no symptoms. These data suggest that $\mathrm{Xcm}$ populations identified to date conform to race 18; however, the presence of $P S$ may predispose immune cultivars to infections by $X \mathrm{~cm}$. 
Identification of a novel bipartite begomovirus isolated from Lycianthes biflora in China

Z. HE, Y. Tang, Plant Protection Research Institute, Guangdong Academy of Agricultural Sciences, Guangzhou, CHINA

In 2015, we found Lycianthes biflora (Lour. Bitter) (Solanaceae) plants exhibiting yellow mosaic symptoms in Nanxiong County, Guangdong Province, China. The five leaf samples were collected from the symptomatic plants and the total DNA was extracted from them using the CTAB method, respectively. PCR with degenerate primers AV494/CoPR indicated a begomovirus presence in the samples. The apparently full-length DNA-A and DNA-B viral components were cloned by rolling circle amplification (RCA) and restriction digestion with BamH I and Pst I endonucleases. The DNA-A component (2752nt, KT582302) shares the highest nucleotide sequence identity at $80.2 \%$ with tomato leaf curl Sulawesi virus (FJ237618), causing yellow leaf curl disease of chilli pepper in Indonesia. The DNA-B component (2704nt, KT582303) shares its highest nucleotide sequence identity at 76.3\% with pepper yellow leaf curl Indonesia virus (AB213599), associated with yellow leaf curl disease of tomato in Indonesia. According to the ICTV guidelines for begomoviral species demarcation, the virus is a novel, previously undescribed bipartite begomovirus species, for which the name Lycianthes yellow mosaic virus (LyYMV) is proposed.

A new Potyvirus found in Dioscorea bulbifera in Florida

K. DEY (1), M. J. Melzer (2), C. Kerr (1), C. Li (1), X. Sun (3), S. T. Adkins (4), (1) Florida Department of Agriculture and Consumer Services, Gainesville, FL, USA; (2) University of Hawaii, Honolulu, HI, USA; (3) Florida Dept of Agric \& Consumer Service, Gainesville, FL, USA; (4) USDA ARS USHRL, Fort Pierce, FL, USA

Air potato, Dioscorea bulbifera, an herbaceous twining vine, native to tropical Asia, was introduced to Florida in 1905. Due to its ability to displace native species and disrupt natural processes such as fire and water flow, air potato has been listed as one of Florida's most invasive plant species since 1993, and was placed on the Florida Noxious Weed List by the Florida Department of Agriculture and Consumer Services in 1999. In March 2016, air potato specimens displaying mosaic symptoms were collected in Alachua County, FL and submitted to the Division of Plant Industry, Gainesville, FL for diagnosis. Symptomatic leaf tissue tested positive for potyvirus infection using both a lateral flow assay (Agdia) and antigen coated plate (ACP) ELISA. Total RNA that was extracted from symptomatic tissue tested positive for the presence of a potyvirus by RT-PCR using universal potyvirus primers. The 3'-teminal $1.62 \mathrm{~kb}$ region spanning the partial NIb gene, coat protein gene, and the 3' untranslated region (UTR) was sequenced and compared to the GenBank database using a BLAST search. This sequence was found to be most similar to Commelina mild mosaic virus (71\% nucleotide identity) and Sweet potato latent virus ( $61 \%$ nucleotide identity). The virus infecting air potato appears to be a new potyvirus species and is tentatively named Dioscorea mosaic virus. The complete genomic sequencing and inoculation of healthy Dioscorea bulbifera and closely related species is in progress.

Report and characterization of bacterial diseases caused by Xanthomonas oryzae in Senegal

H. Tall (1), K. Noba (2), B. Szurek (3), S. Cunnac (3), C. Tekete (4), V. M. VERDIER (5), (1) ISRA, Dakar, SENEGAL; (2) UCAD, Dakar, SENEGAL; (3) Institut de Recherche pour le Developpement, Montpellier, FRANCE; (4) LBMA, USTTB, Bamako, Mali, Bamako, MALI; (5) IRD, Cirad, Univ Montpellier, IPME, Montpellier, FRANCE

Over the last decades rice cultivation has been increasing drastically in Africa. Rice is becoming the number one crop produced for human consumption feeding more than half of the population in Africa. Among factors that reduce rice yield in Africa and impact food security, are bacterial diseases. Xanthomonas oryzae cause two important diseases of rice. Both bacterial blight (BB) and bacterial leaf steak (BLS) are reported in various countries in Africa. Xanthomonas oryzae pv. oryzae (Xoo) was reported in Senegal in the eighties. The use of rice cultivars with introgressed disease resistance (R) genes is currently the best way to control BB disease. Resistance breeding depends on harnessing genetic diversity of pathogen populations. So far no strains of $X o O$ and $X o c$ were collected and characterized in Senegal consequently no strategy has been pursued to control the diseases. Over the last two years presence and prevalence of BB and BLS was monitored in different regions of Senegal. Leaf samples were collected and analyze. A PCR multiplex and a LAMP assay were used to confirm the presence of pv. oryzae and oryzicola in different sites. A set of IRBB lines each carrying a single R gene was used to identifying the race of Xoo in Senegal. We assessed the virulence of Xoc strains on a susceptible rice cultivar. The results during the last two year's survey in Senegal will be presented.

\section{Survey of spinach leaf spot diseases in Texas}

B. LIU (1), C. Feng (2), A. Manley (2), R. Garcia Rodriguez (2), L. Stein (3), K. Cochran (4), L. du Toit (5), (1) Univ of Arkansas, Fayetteville, AR, USA; (2) University of Arkansas, Fayetteville, AR, USA; (3) Dept. of Horticulture, Uvalde, TX, USA; (4) Texas A\&M University, Uvalde, TX, USA; (5) Washington State University, Mount Vernon, WA, USA

Leaf spot diseases of spinach, which reduce yield and quality, have become a major concern in spinach production in Texas. Determining the causal agents of the various leaf spot diseases on spinach, and their relative frequency, is vital for effective disease management. Spinach leaves with leaf spot symptoms were collected from 44 locations in the Winter garden area of Texas between November 2016 and March 2017. Leaf samples were incubated in a moist chamber at $22^{\circ} \mathrm{C}$ for $1-5$ days. In addition, isolations were performed on water agar and PDA. Fifty lesions were evaluated for each of the 44 samples. Fungal and bacterial colonies were recovered to pure cultures. Fungal isolates were identified to species based on morphology and molecular analysis, including DNA sequencing of the ITS rDNA and use of pathogen-specific PCR primers. The survey demonstrated that anthracnose (Colletotrichum dematium) and Stemphylium leaf spot (Stemphylium botryosum) were the predominant diseases, with each pathogen recovered from 33 and 16 of the 44 samples, respectively. Several additional fungi were recovered from leaf spot lesions at limited frequency, and were demonstrated to be pathogenic to spinach, including a Cercospora sp., an Alternaria sp., a Cladosporium sp., Colletotrichum gloeosporioides, a Nigrospora sp., and a Myrothecium sp. None of the bacterial isolates recovered was pathogenic on spinach and none was of a known pathogenic genus on spinach.

\section{A hunt for novel pathogens in cranberries}

Z. ZALEWSKI (1), P. S. McManus (2), (1) University of Wisconsin Madison, Madison, WI, USA; (2) University of Wisconsin-Madison, Madison, WI, USA

Cranberry (Vaccinium macrocarpon) is important to the economies of several states and Canadian provinces. Currently the most economically damaging disease is cranberry fruit rot. This disease occurs pre- and post-harvest and is attributed primarily to a complex of filamentous fungi. Even with proper fungicide applications, however, growers still experience losses from fruit rot. Consequently, we tested the hypothesis that bacterial pathogens contribute to fruit rot. Cranberries treated with prothioconazole (Proline) or left untreated were harvested at the end of the season from two locations. Individual sound or rotten berries were placed on media amended with streptomycin or cycloheximide, to differentially select fungi and bacteria, respectively. An average of $97 \%$ of berries yielded pathogenic fungi while $12 \%$ yielded $>50$ CFU's of bacteria or yeast. Upon inoculation into sound berries, none of the bacterial isolates consistently caused rot. However, two morphologically distinct yeasts were identified by ITS sequencing as Hanseniaspora uvarum and Candida railenensis, and were proven through Koch's postulates to cause rot. With a new focus on yeast, culturing in the presence of cycloheximide is problematic, but without it filamentous fungi dominate. To prevent the inherent bias of culturing, culture independent methods such as Illumina MiSeq will be used to compare microbial populations between rotten and sound berries.

\section{A New Virus Disease of Sunflower in Nebraska}

R. M. HARVESON (1), M. Al Rwahnih PhD (2), T. Tian (3), A. V. Karasev (4), T. Gulya (5), (1) Univ of Nebraska - Panhandle Research \& Extension Center, Scottsbluff, NE, USA; (2) Department of Plant Pathology, University of California-Davis, Davis, Davis, CA, USA; (3) California Dept of Food \& Agric, Sacramento, CA, USA; (4) University of Idaho, Moscow, ID, USA; (5) USDA-ARS, Northern Crop Science Laboratory, Fargo, ND, USA 
During the 2010 and 2011 growing seasons, sunflower plants exhibiting stunting, mottling, and chlorotic ringspots were observed from commercial fields in Box Butte County, Nebraska. Field symptoms were reproduced in seedlings in the greenhouse on multiple occasions confirming the presence of a sap-transmissible virus. Symptomatic greenhouse-inoculated plants from both seasons tested negative for sunflower mosaic virus (SuMV) and sunflower chlorotic mottle virus (SuCMoV) by ELISA and RT-PCR. Similar viral-like symptoms were observed in a commercial sunflower field in Kimball County in September 2014, were reproduced on greenhouse sunflower seedlings after mechanical transmission. Symptom expression began 1012 days after inoculation as a mild chlorotic mottle followed by a mosaic with line patterns and/or chlorotic ringspots. Transmission electron microscopy assays of greenhouse-infected plants from 2014 revealed distinct, polyhedral-shaped virus particles. To characterize the virome of this sample, total nucleic acid was extracted and analyzed by high throughput sequencing (HTS). HTS analysis identified five contigs (363-1239 bp) of varying coverage $(41-99 \%)$ and identity (49-71\%) to known members of the family Tombusviridae, thus pointing to a distinct and novel member of this family. Several viral contigs were also identified in the families Endornaviridae, and Partitiviridae. Additional diagnostic and characterization efforts are ongoing.

Bacterial wilt outbreaks of multiple sequevars on blueberries in Florida

D. J. NORMAN (1), A. M. Bocsanczy (2), P. Harmon (3), C. Lapaire Harmon (4), A. Khan (5), (1) Univ of Florida MREC, Apopka, FL, USA; (2) University of Florida MREC, Apopka, FL, USA; (3) Department of Plant Pathology, University of Florida, Gainesville, FL, USA; (4) Univ of Florida PDC, Gainesville, FL, USA; (5) University of Florida, Orlando, FL, USA

In the fall of 2016 Ralstonia solanacearum was isolated from blueberry hosts at five farms in three counties in Florida. In all cases, strains were isolated from the Southern highbush blueberry cultivar 'Arcadia'. Symptoms were bronzing of leaves, leaf necrosis, and bacterial streaming. Symptoms resembled those of common blueberry Xylella fastidiosa infections. Strains were characterized into populations by sequencing the endoglucanase genes and profiling metabolic biovars. Three distinct populations were identified infecting blueberry: a) phylotype IIa, sequevar 38, biovar 1, b) phylotype IIa, sequevar 7, biovar 1, and c) phylotype I, sequevar 13, biovar 3. Whole genome sequencing of selected strains has also revealed unique profiles differing from those commonly found in vegetable and ornamental plant production in Florida. It is very unusual for a bacterial wilt outbreak in commercial crops confined to a limited geographic distribution not to be clonal. The blueberry industry is expanding rapidly in Florida and may be now moving into areas with different endemic $R$. solanacearum populations making it susceptible to multiple bacterial wilt outbreaks. It is important to characterize those populations and their structure to assess the risk of outbreaks.

Status of Huanglongbing (HLB) Multi Agency Coordination (MAC) projects on thermal treatment of citrus trees with citrus greening (2017) D. A. KOMM (1), R. Ehsani (2), P. Ravenna (3), (1) USDA, Raleigh, NC, USA; (2) University of Florida, Lake Alfred, FL, USA; (3) Ag Harvester, Au Gres, MN, USA

Citrus trees infected with 'Candidatus Liberibacter asiaticus' (Las), subjected to heat treatments, have shown an improvement in citrus yields and vigor. Thermal treatment equipment is being developed and improved for all sizes of citrus trees infected with this pathogen. Two MAC projects are contributing to the development and improvement of this thermal treatment equipment. Researchers involved in the first project have developed a mobile physical system to prolong the productive life of citrus trees up to nine feet tall. In this system, the trees are covered with a tent canopy and subjected to a steam treatment, optimally conducted at $131^{\circ} \mathrm{F}$ for 30 seconds. For each season, researchers have observed a significant interaction between the treatments and time after thermotherapy for the roots. Furthermore, seasonality and physiological state of the tree seem to play a critical role for Las distribution and movement within a particular tree. Heat treatment of the HLB infected trees significantly reduced Las bacteria, initiating an improvement of the physiological conditions of the heat-treated trees. The second project involved the development of a mobile thermotherapy system for citrus trees up to fifteen feet tall. The entire system was designed to continuously apply steam as the unit moves down a row of trees. Excessive heat loss with open canopy ends resulted in closed ends during treatment. Additional design changes are ongoing to improve the ability of the apparatus to supply thermal therapy to large trees. Both projects have resulted in the development of equipment that has successfully heat-treated grove trees infected with HLB.

An assessment of putative foliar pathogens on different accessions of tea grown in Florida

J. ORROCK, K. Kemfert, K. Sandsted, R. Healy, B. Spakes Richter, B. Rathinasabapathi, University of Florida, Gainesville, FL, USA

Tea [Camellia sinensis (L.) O. Kuntze] is an economically important beverage crop, under investigation as a new specialty crop in the US. Florida agriculture, long dominated by citrus, is in flux due to Huanglongbing (HLB; syn. citrus greening). Tea may present an alternative crop. We examined the impact of two fungal foliar diseases of tea on eight genetic accessions. Plants were grown in a mulched, drip-irrigated field in North Central Florida since March 2016 in a completely randomized block design and rated every two months for growth and disease incidence. We characterized two types of leaf spots and a tip die-back. While no field resistance was noted, different accessions showed varying levels of incidence and severity, with the anthracnose-like leaf spot incidence ranging from $35 \%$ in 'MS-SL' to $90 \%$ in 'MS-GEO.' Severity ratings were similar, with 'MS-SL' having the lowest rating and 'MS-GEO' the highest. Incidence of the smaller leaf spot ranged from $44 \%$ in 'MS-BL' to $91 \%$ in 'CF-CH,' with similar severity ratings, highest in 'CF-CH' and lowest in 'MS-BL.' Different accessions showed significant differences in incidence and severity ratings using a Tukey's HSD. Potential pathogens were isolated, cultured and sequenced using the ITS region. Koch's Postulate experiments are pending. By examining disease impacts, this work can help shape future directions of tea research, breeding, as well as recommendations for growers in establishing a novel industry.

\section{Molecular characterization of a new member of the genus Waikavirus}

T. Thekke Veetil (1), T. Ho (2), J. D. Postman (3), I. E. TZANETAKIS (1), (1) University of Arkansas, Fayetteville, AR, USA; (2) Driscoll's, Watsonville, CA, USA; (3) USDA ARS, Corvallis, OR, USA

As a part of an effort to ensure the health status of the black currant collection maintained at the USDA National Clonal Germplasm Repository in Corvallis, Oregon, accessions exhibiting virus-like symptoms were subjected to large scale sequencing (LSS). A new virus, putative member of the family Sequiviridae, was identified in one of the symptomatic plant. The genome was obtained by a combination of LSS and Sanger sequencing. Insilico analysis showed that the virus code for a single polyprotein of $390 \mathrm{kDa}$ showing the highest amino acid identity with its counterpart of bellflower vein chlorosis virus. Phylogenetic analysis and molecular features at the genome termini indicated that the virus is a putative new member of the genus Waikavirus. The new virus is tentatively named as black currant waikavirus A (BCWVA) and a detection method has been developed. In addition to BCWVA, four additional new viruses were discovered in some of the plants analyzed which underscored the importance of sensitive molecular screening techniques in certification and quarantine programs for black currant.

Phylogenetic relationship of Grapevine vein clearing virus isolates in wild plant species and in cultivated grapevines S. PETERSEN, C. Keith, K. Austin, W. Qiu, Missouri State University, Springfield, MO, USA

Grapevine vein clearing virus (GVCV) is a badnavirus that contains a circular, double-stranded DNA genome in a range of 7,725 base pairs (bp) to $7,765 \mathrm{bp}$. Three open reading frames (ORFs) are predicted on the plus-strand genome. To understand the genetic evolution of GVCV populations, the entire genomes of seven GVCV isolates were analyzed. These viral genomes were obtained from cultivated and wild grapevines and native Ampelopsis cordata in the Vitaceae family. Comparative analysis showed that the most pronounced sequence diversity is found in ORF II of GVCV in a range of $83 \%$ to $100 \%$, including a 9-bp indel. The ORF II sequences were acquired from 48 isolates and used to build a phylogenetic tree to unveil the relationships of GVCV isolates among plant populations in the two genera Vitis and Ampelopsis growing in seven regions. Phylogeny segregated the 
isolates based on the 9-bp indel in ORF II, with 26 isolates branching from a common ancestor which do not contain the 9-bp insert, in comparison to the 22 isolates stemming from the trunk which contain these nucleotides. One instance of $100 \%$ identical nucleotide sequence was found in ORF II between an A. cordata isolate and a Vitis isolate which were found adjacent to each other in the field. Phylogenetic analysis suggested that GVCV isolates are related more closely according to geographical proximity rather than host species. The results infer local spread of GVCV across plant species and provide crucial clues on epidemics of GVCV.

\section{A species of Pestalotiopsis identified infecting red mangrove in The Bahamas}

R. E. ROSSI (1), C. A. Layman (2), J. B. Ristaino (3), (1) North Carolina State, Raleigh, NC, USA; (2) Dept of Applied Ecology, NC State University, Raleigh, NC, USA; (3) NC State University, Raleigh, NC, USA

Mangroves are foundation species in coastal ecosystems providing an estimated US \$1.6 billion in ecosystem services worldwide. These services range from providing essential nursey habitat for marine organisms to land accretion and carbon sequestration. Unfortunately, mangrove forests are declining because of myriad factors, many related to human activity. Here, we present a case study from Abaco, The Bahamas in which dwarf red mangroves (Rhizophora mangle) are dying in a $5 \mathrm{~km}$ area. Initial data suggest that these dwarf $R$. mangle are stressed by multiple factors, including a fungal pathogen, herbivory and high salinity. Disease incidence surveys were completed in the die-off area and elsewhere along the island. Simultaneously, we engaged the public to create awareness about plant disease and developed curriculum for Bahamian high school students to learn isolation techniques and Koch's postulates. We found disease present across the Island and disease incidence varied from 1 to $46 \%$. Infected leaf samples were collected and fungi were isolated. DNA was sequenced using internal transcribed spacer, elongation factor 1-alpha, and beta-tubulin genes. Pestalotiopsis sp. were the most commonly identified fungi from tissue samples. Koch's postulate trials are ongoing but initial trials indicate the pathogen caused disease. Although Pestalotiopsis sp. infects banana and coconut, this is the first report documenting Pestalotiopsis sp. infecting mangroves in The Bahamas.

Identification of a caulimo-like virus in pistachio via high-throughput sequencing A. DIAZ LARA (1), K. Stevens (2), N. Westrick (1), D. A. Golino (3), M. Al Rwahnih PhD (4), (1) Department of Plant Pathology, University of California-Davis, Davis, CA, USA; (2) Foundation Plant Services, University of California-Davis, Davis, CA, USA; (3) University of California, Davis, CA, USA; (4) Department of Plant Pathology, University of California-Davis, Davis, Davis, CA, USA

Pistachio (Pistacia vera L.) has seemingly been free of viral diseases, but this may be misleading due to limited studies. To increase the knowledge about viruses in pistachio, symptomatic and asymptomatic trees from different regions in California were analyzed by high-throughput sequencing (HTS). Contigs generated from HTS reads were compared to the NCBI database. Many contigs across multiple samples showed homology to species in the family Caulimoviridae. Viral contigs were aligned with draft genome assemblies of $P$. vera, $P$. atlantica, and $P$. integerrima using GMAP which resulted in the identification of a subset of integrated or partially integrated copies. Thirteen contigs (527-6,889 bp) not exhibiting extensive integration were analyzed further. Specific PCR primers confirmed the presence of viral contigs in the original sample. A complement sequence of the detection primers was used to obtain the missing region from the contigs. One set of outward facing primers amplified a fragment of around $6 \mathrm{kbp}$, with initial similitude to Rudbeckia flower distortion virus (Identities: 63/122=52\%). Additionally, RNaseH and CP domains from Caulimoviridae were identified. These results suggest a novel virus in the family Caulimoviridae infecting pistachio. A PCR assay will be designed to investigate the field occurrence and potential effects on pistachio trees. Further work will determine the extent to which caulimo-like DNA is integrated into the pistachio genome.

Characterizing Sclerotinia sclerotiorum as the cause of white rot on short-day strawberry cultivars in the Mid-Atlantic region E. HELLMAN (1), E. Koivunen (2), C. L. Swett (1), (1) University of California, Davis, Davis, CA, USA; (2) Plant Sciences, Inc., Watsonville, CA, USA

With over 1,000 producers in the Mid-Atlantic states, short-day strawberry (Fragaria $\times$ ananassa) cultivars represent an important, multi-million dollar industry. In spring 2016, a white rot of unknown etiology was observed on strawberry crowns and fruit at two farms in the Mid-Atlantic; preliminary surveys indicated that cultivars Chandler and Flavorfest might be particularly susceptible. Five isolates were identified as the white rot pathogen Sclerotina sclerotiorum, based on ITS sequence analysis (533 bp, 99-100\% homology, GenBank). In crown rot assays with two isolates, both isolates produced lesions at 3 days post inoculation (DPI) $(5.8 \pm 0.2 \mathrm{~mm}$ in length); at $6 \mathrm{DPI}$, all plants had developed symptoms and many lesions expanded to the petiole base ( $42.1 \pm 4.8 \mathrm{~mm}$ lesion length). In fruit rot assays, 41 to $53 \%$ of inoculated fruit developed white rot symptoms within 6 DPI. Comparing susceptibility of four popular short day cultivars, petiole rot was significantly more severe on cv. Flavorfest $(P=0.0001)$. This is the first report of white rot on strawberries in Maryland; our results indicate that cv. Flavorfest, a new anthracnose-resistant cultivar with rapidly expanding acreage, has unusually high susceptibility to white rot. The rapid increase in cv. Flavorfest acreage may explain, in part, why this disease is being observed for the first time in the region.

Characterization of Tomato leaf curl purple vein virus, a new monopartite New World begomovirus infecting tomato in Northeast Brazil M. MACEDO (1), L. Albuquerque (2), M. Maliano (1), J. Souza (1), M. Rojas (1), R. L. Gilbertson (1), A. K. Inoue-Nagata (3,4), (1) University of California Davis, DAVIS, CA, USA; (2) Goiano Federal Institute, Morrinhos, BRAZIL; (3) EMBRAPA, Brasilia, BRAZIL; (4) Embrapa Vegetables, Gama, BRAZIL

A new begomovirus was identified from tomato (Solanum lycopersicum) plants with leaf curl and purple vein symptoms in Piaú state, Brazil, in 2014. PCR with begomovirus degenerate DNA-A and DNA-B primers and restriction enzyme digestion of rolling circle amplification products revealed a single DNA-A-like component of $\sim 2.6 \mathrm{~kb}$ and no DNA-B. The genomic DNA was cloned and sequenced, and has a genome organization typical of DNA-A components of New World bipartite begomoviruses. Sequence comparisons revealed the highest identity ( $80 \%)$ with the DNA-A component of Macroptilium yellow spot virus (MaYSV). A phylogenetic analysis placed this virus into a distinct clade, with the closest relative MaYSV, including a $334 \mathrm{nt}$ recombination event. Tomato plants agroinoculated with a multimeric clone developed leaf curling and purple vein symptoms, indistinguishable from those observed in the field. This fulfilled Koch's Postulates, and established the monopartite nature of the virus. This virus has a narrow host range with tomato, Nicotiana benthamiana and N. glutinosa developing symptoms and $N$. tabacum and Phaseolus vulgaris (cv. Topcrop) having symptomless infections. Co-infection experiments with Tomato severe rugose virus and Tomato mottle leaf curl virus, two predominant begomoviruses infecting tomato in Brazil, revealed a synergistic interaction among these begomoviruses. This new monopartite New World begomovirus species is tentatively named Tomato leaf curl purple vein virus.

Sugarcane (Saccharum spp.) and itch grass (Rottboellia cochinchinensis): New hosts to maize yellow mosaic virus (genus Polerovirus) A. YAHAYA (1), M. Al Rwahnih PhD (2), D. B. Dangora (1), L. Gregg (3), M. D. Alegbejo (1), P. L. Kumar (4), O. J. Alabi (5), (1) Ahmadu Bello University, Zaria, NIGERIA; (2) Department of Plant Pathology, University of California-Davis, Davis, Davis, CA, USA; (3) Dept. of Plant Pathology and Microbiology, Texas A\&M AgriLife Research and Extension Center, Weslaco, TX, USA; (4) International Institute of Tropical Agriculture, Ibadan, NIGERIA; (5) Dept. of Plant Pathology \& Microbiology, Texas A\&M University, Weslaco, TX, USA

The virome of sugarcane and itch grass plants encountered during routine surveys in the northern guinea savannah region of Nigeria in 2015 and showing yellow mosaic symptoms were explored using highthroughput sequencing (HTS). Symptomatic leaf tissue samples from five randomly selected plants (sugarcane $=4$; itch grass $=1$ ) were dried and stored under $\mathrm{CaCl}_{2}$ at room temperature then used for total RNA isolations. cDNA library constructed from ribosomal RNA-depleted RNA aliquots from the pooled sample was sequenced on the Illumina NextSeq 500 platform. Analysis of the $\sim 43.5$ million raw Illumina reads (76 nucleotides each) resulted in 31,486 reads mapped to maize yellow mosaic virus (MaYMV) along with reads 
mapped to Sugarcane mosaic virus. Assembly of the reads yielded 5,566 nucleotides (nt) long near complete genome of MaYMV that was 95-97\% identical and phylogenetically related to corresponding sequences of MaYMV isolates in GenBank. The HTS results were confirmed via amplification and Sanger sequencing of $\sim 1100$ bp DNA band specific to a region encompassing partial P1-P2 fusion protein and coat protein genes of luteovirids from two sugarcane and one itch grass sample using published primers. Sequences from both plants shared $99-100 \%$ nt identity with each other and $97-99 \%$ identity with the corresponding MaYMV sequences derived by HTS in this study, and those retrieved from GenBank. The results showed the occurrence of MaYMV, a recently characterized maize-infecting tentative member of the genus Polerovirus (family Luteoviridae), in sugarcane and itch grass worldwide and the first report of the virus in an African country.

Survival of the Goss's wilt pathogen, Clavibacter michiganensis subsp. nebraskensis on maize leaf residue in Central and South Texas T. S. ISAKEIT (1), G. Steele (2), S. Halfmann (3), (1) Texas A\&M University, College Station, TX, USA; (2) Monsanto, Hillsboro, TX, USA; (3) Monsanto, College Station, TX, USA

Goss's bacterial wilt and leaf blight, long restricted to portions of the Corn Belt, was more recently confirmed in central Texas in 2014, and again in 2016. To devise management recommendations for central and south Texas, it is necessary to determine how well the pathogen survives in this climate. The objective of this study was to determine whether the pathogen could overwinter in maize leaf residue. Symptomatic leaves were collected from a field in central Texas and dried flat. In September, 2016, leaf pieces were placed in plastic mesh bags and either left on the soil surface, or buried $10 \mathrm{~cm}$ deep, at three locations. Samples were removed every 5 weeks and leaf tissue and/or adhering soil was suspended in buffer and aliquots were injected into the stems of 12-day-old corn seedlings. Aliquots from most of the surface samples from all locations, collected 5 weeks later, caused symptoms in the seedlings, but the buried samples, which were noticeably decomposed, did not. Recovery of the pathogen during the winter months from surface samples declined during the winter, but was still detectable after 20 weeks. One buried sample from this sampling time also caused disease. Average soil temperatures during the first 7 weeks ranged from $23^{\circ} \mathrm{C}$ to $30^{\circ} \mathrm{C}$, with $5 \mathrm{~cm}$ rain in 4 events. The results suggest that the inoculum in residue is greatly diminished over the winter, but not completed eliminated, and may survive to the following season.

Differential accumulation and transmission of traditional and emergent Beet curly top virus strains from the western United States W. M. WINTERMANTEL (1), L. Hladky (1), A. Cortez (1), L. F. Chen (2), R. L. Gilbertson (2), (1) USDA-ARS, Salinas, CA, USA; (2) University of California, Davis, CA, USA

Curly top disease, caused by Beet curly top virus (BCTV), causes significant economic losses to several crops throughout the western US. Recent surveys in California and Idaho identified a striking shift in the predominant strains of BCTV, with strains related to BCTV-CO and -LH71 emerging in both regions and becoming the most prevalent forms of the virus. These emergent strains are supplanting BCTV-Svr (CFH) in Idaho, and both BCTVSvr and -Wor in California. The latter two strains had been the most common for the past 20 years throughout the western US. To identify factors leading to emergence of new strains, studies were conducted in which crop and weed host plants were inoculated with emerging and traditional BCTV strains alone or in combination and delivered by agroinoculation of cloned viral DNA. After four weeks, agroinoculated plants were evaluated for virus accumulation by qPCR, and used as acquisition hosts for leafhopper transmission to healthy plants, followed by quantification of virus accumulation of each strain in these leafhopper-inoculated plants. Results demonstrated that some BCTV strains are better adapted to certain host plants than others, suggesting that host plants may have a substantial influence on emergence and predominance of new BCTV strains. These results contribute to our knowledge of factors influencing emergence of new BCTV strains as well as toward long-term virus management and disease prediction efforts.

\section{Rapid research response to emerging wheat stem rust pathogen races in Ethiopia}

B. Girma (1), W. Denbel (2), E. Hailu (3), P. D. Olivera Firpo (4), M. S. Newcomb (5), Y. Jin (6), D. Hodson (7), A. Badebo (7), B. Abeyo (7), G. Wolderufael (3), B. Hundie (8), G. Cisar (9), M. ROUSE (6), (1) Cornell University, Kulumsa, ETHIOPIA; (2) Ethiopian Institute of Agricultural Research, Debre Zeit, ETHIOPIA; (3) Ethiopian Institute of Agricultural Research, Ambo, ETHIOPIA; (4) University of Minnesota, St. Paul, MN, USA; (5) University of Arizona, Maricopa, AZ, USA; (6) USDA-ARS Cereal Disease Laboratory, Saint Paul, MN, USA; (7) CIMMYT, Addis Ababa, ETHIOPIA; (8) Ethiopian Institute of Agricultural Research, Kulumsa, ETHIOPIA; (9) Cornell University, Loveland, CO, USA

Wheat stem rust, caused by Puccinia graminis f. sp. tritici (Pgt), is a significant disease limiting wheat yield in Ethiopia. Epidemics occurred in the southern wheat production regions of Bale and Arsi in Ethiopia in the 2013-2014 and 2014-2015 crop seasons caused by race TKTTF that is virulent to the widely grown Ug99-resistant wheat variety 'Digalu'. These epidemics underscored the need for wheat varieties with resistance to multiple Pgt races, including Ug99. In response to these epidemics, Pgt races TKTTF, TTKSK, TRTTF and JRCQC were isolated from field populations and multiplied in isolation in a greenhouse starting in May 2014. In order to facilitate germplasm screening in the field, a field nursery was inoculated at Debre Zeit, Ethiopia in 2014 and 2015. Out of a total of 1,433 wheat lines assessed, 822 were resistant to race TKTTF with resistant infection responses or disease severities less than 30\%. In addition, we evaluated advanced breeding lines in separate single race nurseries at Kulumsa, Ethiopia 2014-2015. The data indicated that 21 out of 111 wheat lines tested displayed resistant infection responses or disease severities less than $30 \%$ in response to the four races across both years. Variety 'Kingbird' was released in Ethiopia in 2015 and was confirmed as resistant to the four Pgt races in the field. This effort demonstrated that rapid identification, characterization, and increase of $P g t$ races in Ethiopia is possible to facilitate field screening of breeding lines to select for cultivars with resistance to multiple virulent races of Pgt.

\section{Viral metagenomics unravels the etiology of lethal necrosis of St. Augustinegrass 'Floratam'}

\section{R. I. ALCALÁ-BRISEÑO, P. Harmon, J. Polston, Department of Plant Pathology, University of Florida, Gainesville, FL, USA}

Lethal necrosis of St. Augustinegrass in Stenotaphrum secundatum (Walt.) Kuntze 'Floratam' was first reported in West Palm Beach Co. (Belle Glade), Florida in 2007 but remerged in Pinellas Co. in 2011, West Palm Beach Co. (West Palm Beach) in 2013 and Dade and Monroe counties in 2016. The disease is characterized by necrosis and death of 'Floratam' plants at temperatures below $25^{\circ} \mathrm{C}$ and a mosaic at higher temperatures. 'Floratam' has been the most widely planted cultivar of St. Augustinegrass in Florida for several decades. While Sugarcane mosaic virus (SCMV) has been associated with the disease, SCMV has been reported to cause only mosaic symptoms in St. Augustinegrass since the 1960's, therefore the presence of a second causal agent is suspected. A metagenomic survey was conducted using dsRNA extracted from symptomatic St. Augustinegrass plants collected from Belle Glade, Pinellas and West Palm Beach, Florida. The samples were multiplexed and sequenced by Illumina HiSeq 2000. The RNA-seq was assembled de novo with Spades 3.8. As expected BLASTx search showed many contigs with homology to SCMV and a full genome of SCMV was recovered from each location. In addition, there were contigs from each location that had homology to all three species in the genus Panicovirus (Tombusviridae). These were assembled into a partial genome (70\%) of a novel Panicovirus with an overall similarity to panicoviruses of $63 \%$. Studies are in progress to determine the role of this new panicovirus in the disease.

Identifying the causal agent of the rotten rhizome syndrome in Achira (Canna edulis)

P. URIBE, M. C. Ortega, Corporación Colombiana de Investigación Agropecuaria, Pasto, COLOMBIA

The rhizome of achira is important in the household economy of Colombian Andean farmers. The starchy content of this organ can be used as food source and as an input in agro industrial processes. Nonetheless, this crop is being limited by the presence of several phytosanitary problems. One of them is the rotten rhizome syndrome, which affects the rhizome, and reduces yields. With the purpose of identifying and characterizing this problem, we visited achira producing areas in Colombia where we collected symptomatic plants with signs of wilting and lack of turgor. For the most part, collected rhizomes had typical disease signs such oxidized, brown or blackened tissues, some of them soft. Microbiological cultures of affected areas yielded fungi 
of the Fusarium, Rhizoctonia and Sclerotinia genera. Bacterial isolates yielded some Gram negative organisms which. Among all the collected isolates, Fusarium sp. was the most abundant organism isolated. Morphological and molecular analysis of the Fusarium isolates indicated the presence of at least five different species, $F$. oxisporum, $F$. equiseti, $F$. circinatum, $F$. fujikuroi and $F$. verticillioides. Of these, $F$. oxysporum was isolated most often and more importantly from almost all the areas scouted. Accordingly with the results, we have started to pursue Koch's postulates working on the hypothesis that $F$. oxisporum is the causal agent of the rotten rhizome syndrome in achira.

Charles Darwin and the Irish Potato Famine: "A Painfully Interesting Subject"

J. B. RISTAINO (1), D. Pfister (2), (1) NC State University, Raleigh, NC, USA; (2) Harvard University, Cambridge, MA, USA

Charles Darwin's famous voyage on the HMS Beagle led him around the world on a collecting journey that culminated in his theory of evolution. In 1835, the Beagle traveled to the island of Chiloé, and there, Darwin discovered and sent potatoes back to England. During the Irish famine, late blight appeared on Darwin's farm at Down House and the Chilean potatoes proved useful. Darwin's interest in the potato and potato late blight spanned four decades. He used the potato to investigate questions of what a species is, understand its ravages by a plant pathogen, and investigate ideas on clonal versus sexual reproduction on species fitness. Darwin's letters reveal his thoughts on free trade, population growth and food security during the Irish famine. Darwin was involved in the first research to find resistance to late blight and personally funded the breeding program of Torbitt in Ireland. Darwin's studies on potato late blight are relevant today for scientists studying global migrations of the pathogen and development of durable resistance.

Assessing the potential for Phytophthora to move between native plant nurseries and interstate shipping nurseries T. Miles (1), W. Schweigkofler (2), S. Sharma (2), N. Luecke (3), K. L. Kosta (4), K. G. SUSLOW (5), (1) California State University Monterey Bay, Seaside, CA, USA; (2) Dominican Univ of California, San Rafael, CA, USA; (3) California State Univ Monterey Bay, Seaside, CA, USA; (4) California Dept of Food \& Agric, Sacramento, CA, USA; (5) Dominican University of Califo, San Rafael, CA, USA

The California nursery and floriculture industry has a farm-gate value greater than $\$ 3.5$ billion. Commercial, interstate shipping nurseries purchase native plants to supplement their drought-tolerant inventory; this practice creates a likely pathway for Phytophthora species to enter the mainstream nursery shipping channels and move into new regions. The bulk of native plants produced in CA are grown for local habitat restoration projects and remain within the state; however, the buy-in practices of interstate shipping producers have underscored the concern for Phytophthora species to be introduced into their operations. Longitudinal sampling for root-rotting Phytophthora species was conducted over two seasonal periods, Fall 2016 through Spring 2017, among 15 participating nurseries. The 15 nurseries were divided into three categories: 5 interstate shipping nurseries that do not buy-in native plants, 5 interstate shipping nurseries that do, and 5 native plant nurseries that sell product to interstate shipping nurseries. Root samples were taken from 20 symptomatic plants at each nursery. Symptomatic samples were tested using ImmunoStrip ${ }^{\circledR}$ Phytophthora assays (Agdia Inc., Elkhart, IN) and DNA was extracted from the homogenized tissue of the positive samples. Using a genus specific qPCR marker and subsequent sequencing, 14 different Phytophthora species were identified, the most abundant being P. niederhauserii. In addition, a unique Phytophthoraspecies was found in two nurseries and four of the same species were identified in all three categories. These findings demonstrate the need to characterize the Phytophthora species that are present in order to implement the most appropriate management practices.

Distribution of root rot on pea and lentil crops on the Canadian prairies, 2016

B. D. GOSSEN (1), S. G. Chatterton (2), D. L. McLaren (3), (1) Agric \& Agri-Food Canada, Saskatoon, SK, CANADA; (2) Agriculture and Agri-Food Canada, Lethbridge, AB, CANADA; (3) Agriculture and Agri-Food Canada, Brandon, MB, CANADA

Wetter-than-normal spring seasons across large portions of the Canadian prairies in recent years have been associated with the emergence of root rot as a major constraint to production of field pea and lentil. Also, use of molecular diagnostic tools indicate that Aphanomyces euteiches Drechs. may be a more important component of the root rot complex across the northern Great Plains than previously thought. Therefore, a survey was conducted to assess root rot etiology, incidence and severity on pea and lentil across the Canadian prairies in 2016. Root samples were collected at 5-10 sites from 187 pea and 94 lentil fields across the region during flowering and sent to a central laboratory for assessment of visible symptoms $(1-7$ scale, $7=$ dead $)$ and DNA tests to determine the causal agent. On field pea, root rot symptoms were present in almost $90 \%$ of fields, with mean severity per field (3.2) slightly below yieldlimiting levels. On lentil, incidence was $>90 \%$ but mean severity was slightly lower (2.9). Differences among soil zones were generally small. Fusarium spp. and Rhizoctonia solani Kühn were detected in about $90 \%$ of fields, A. euteiches in $>60 \%$ and Pythium spp. in about $50 \%$ of fields. A. euteiches was present at high levels in fields in every soil zone and was commonly associated with the most severe root rot symptoms. This study demonstrated that $A$. euteiches is a widespread and important root pathogen of field pea and lentil across the region.

Viability of resting spores of Plasmodiophora brassicae throughout the soil profile

F. Al-Daoud (1), B. D. Gossen (2), M. R. MCDONALD (1), (1) University of Guelph, Guelph, ON, CANADA; (2) Agric \& Agri-Food Canada, Saskatoon, SK, CANADA

Resting spores of Plasmodiophora brassicae, the causal agent of clubroot in Brassica crops, can remain viable in soil for many years. Resting spores have been detected throughout the soil profile $(0-100 \mathrm{~cm})$ of infested fields but it is not known how many of these are viable. The objective of this study was to quantify the viable resting spores of $P$. brassicae throughout the soil profile of a naturally-infested field. Two sites in a field in Alberta were sampled 0-45 cm deep at $7.5 \mathrm{~cm}$ increments. Two cores were analyzed per site. Propidium monoazide-assisted quantitative PCR (PMA-PCR) was used to estimate the number of viable resting spores. Treatment with PMA inhibits amplification of DNA from non-viable resting spores in a subsequent qPCR assay. The proportion of viable spores was estimated by comparing the PMA-PCR estimates to the total number of spores detected by qPCR with no PMA treatment. Resting spores were detected at all soil depths. The proportion of viable spores in the top layer varied by core from $19 \%$ to $66 \%$ and the number of viable spores ranged from $7.4 \times 10^{5}$ to $3.3 \times 10^{6} \mathrm{~g}^{-1}$. The rest of the samples varied from $0 \%$ to $100 \%$ viable spores throughout the soil profile. The highest concentrations of viable spores were found at $30-45 \mathrm{~cm}$ depth and ranged from $1.8-6.2 \times 10^{6} \mathrm{~g}^{-1}$. The presence of viable resting spores throughout the soil profile may reduce the efficacy of clubroot management strategies that target resting spores only in the top soil layers.

\section{Phytophthora species present in two Missouri Ozark valleys with white oak mortality}

\section{S. E. REED, J. T. English, R. M. Muzika, University of Missouri, Columbia, MO, USA}

Rapid white oak mortality (RWOM) was first observed in the Missouri Ozarks circa 2011. Affected tree crowns wilt and die within a few weeks. A survey was conducted to determine the distribution of dead and dying trees attributed to RWOM in two valleys and to identify associated Phytophthora species. One valley was located in Sunklands Conservation Area (SCA) and the other valley was next to Harmon Springs Campground (HSC). Crown vigor was assessed for white oaks in 42 plots ( 0.08 ha) at SCA and in 43 plots at HSC during 2014. Plots were categorized as high or low vigor based on the average crown vigor score. Low vigor white oaks were most often located on the lower-half of hillsides. Soil samples were collected from three trees in each high and low vigor plot and the soil was baited for Phytophthora. Low vigor plots without detectable Phytophthora in 2014 were resampled in 2015. Phytophthora species were identified based on analysis of ITS and COX-2 gene sequences. Phytophthora cinnamomi was the most frequently detected species and was detected in $36 \%$ of plots at SCA and $76 \%$ of plots at HSC. P. cinnamomi was associated with high and low vigor plots at HSC but not at SCA. Phytophthora cactorum, P. europaea, and P. pini were infrequently detected, with percentage recoveries of $8 \%$ or less. Phytophthora cinnamomi is known to cause fine root damage in white oak species. Its potential role in the decline of white oak trees should be further investigated. 
The risk of seedling disease of corn caused by Pythium increases with proximity of the corn seedling to terminated winter rye cover crop plants J. N. ACHARYA (1), T. Kaspar (2), A. Lenssen (3), A. E. Robertson (1), (1) Iowa State University, Department of Plant Pathology, Ames, IA, USA; (2) USDA ARS Natl Soil Tilth Lab, Ames, IA, USA; (3) Iowa State University, Department of Agronomy, Ames, IA, USA

Yield loss of corn following a winter rye cover crop discourages farmers in Iowa from adopting cover crops to manage soil and water quality. The addition of cover crops into the cropping system alters microbial community within the rhizosphere of both winter rye and corn. For example, Pythium species that may cause seedling disease are more prevalent in corn seedlings following winter rye. We used growth chamber experiments to determine the susceptibility of corn to seedling disease caused by Pythium Clade B and Clade F members as a function of corn seedling proximity to winter rye plants. Corn seedlings were planted at three distances, $0 \mathrm{~cm}, 2-3 \mathrm{~cm}$, and $8-10 \mathrm{~cm}$, from terminated rye plants. Root rot severity was assessed at crop development stage V2. Quantitative PCR was used to estimate the abundance of Pythium Clade B and Clade F members present in corn roots. Radicle and seminal root rot severity was greater the closer the seedling was to the rye plants $(P=0.01)$. Moreover, a greater abundance of $P y t h i u m$ Clade $\mathrm{B}$ was detected in corn grown within the terminated rye compared to corn planted further away $(P=0.01)$. No effect of distance between corn and rye was detected for Pythium clade F. This study will inform management practices for farmers to reduce occasional yield loss of corn following a winter rye cover crop.

\section{Incidence of Phytophthora in Maryland nurseries}

R. R. POKHAREL, Maryland Dept of Agric, Annapolis, MD, USA

Leaf spots, blight, and plant death, caused by both biotic and abiotic factors, are common symptoms observed in nursery, landscape and forest plants each year in Maryland. Phytophthora spp. are among the causes of such symptoms. One species, $P$. ramorum, which is under federal quarantine, can cause rapid plant death. To rule out the association of Phytophthora with such symptoms, nursery surveys were conducted of Azalea, Camellia, Kalmia, Pieris, Rhododendron and Viburnum at 36 different nurseries in 2014, 2015, and 2016 inspecting 115,327, 34,206, and 15,032 plants, respectively. Symptomatic plants observed, less than 1\% in 2014 and 2015, and 4.8\% in 2016, were collected and tested for Phytophthora spp by ELISA kit. An increasing trend of Phytophthora spp. incidence in nurseries from 2014 to 2016 was found. Numbers of symptomatic plants and percentage of infection varied with plant species and year. Camellia, followed by Kalmia, had more symptomatic plants in 2014 and 2016, but not 2015. No Phytophthora infection was found in Kalmia in 2014 and 2015, but had more than others, except Rhododendron in 2016. All Phytophthora positive samples were tested for P. ramorum. For the first time, in 2016 , P. ramorum was detected at a nursery where $33 \%$ of symptomatic plants were positive for Phytophthora spp. P. ramorum was observed in Rhododendron 'Cunningham White' and in two mountain laurels -- Kalmia 'Olympic Wedding'. No possible infection source was detected.

Survival of Peronospora belbahrii sporangia isolated from host plant tissue K. S. ALLEN (1), G. Higgins (1), L. J. Ma (2), R. L. Wick (1), (1) University of Massachusetts Amherst, Amherst, MA, USA; (2) University of Massachusetts, Amherst, MA, USA

Basil downy mildew is a devastating disease of sweet basil caused by the obligate parasitic oomycete Peronospora belbahrii. Survival of the pathogen is limited in the absence of a host as no oospores or chlamydospores have been reported in North America; thus destruction of the affected crop following a disease outbreak should be an effective means of preventing subsequent disease development in successive basil crops. However, it is not known how long the sporangia remain viable in the absence of host tissue following release. This study uses two approaches to evaluate the long-term viability of $P$. belbahrii sporangia: an in vitro assay to assess germination potential following release, and a plant infection bioassay to determine infection potential. Sporangia were isolated from sporulating plants and stored for increasing time intervals at different temperature and light intensity treatments. At each interval, stored sporangia were collected in water and plated onto $1.5 \%$ water agar plates, incubated at $12^{\circ} \mathrm{C}$ for 8 hours, and then assessed for germination percentage. After just 2 hours of storage on the greenhouse bench or growth chamber and rapid rehydration, sporangia were unable to germinate. The experiment will be replicated with earlier time-points to assess the limiting time after drying and slow or rapid rehydration that sporangia can germinate, and then supported with plant infection bioassays. This data can be utilized by growers who are trying to maximize their greenhouse space and find a safe re-entry interval to re-start basil production in a greenhouse following an epidemic.

Susceptibility to infection of soybean by $P$. sylvaticum depends on germination stage R. L. MATTHIESEN (1), A. E. Robertson (2), (1) Iowa State University, Ames, IA, USA; (2) Iowa State University, Department of Plant Pathology, Ames, IA, USA

Pre- and post- emergence damping-off of soybean in Iowa can be caused by several Pythium species, including P. sylvaticum. Little is known about which germination stages are affected by Pythium species infection. The objective of this study was to determine if susceptibility to damping-off of soybean caused by $P$. sylvaticum depended on the germination stage at which infection occurred. Soybean seeds or roots were inoculated with $P$. sylvaticum at $0,2,4$, or 7 days after planting (DAP) and incubated at $23^{\circ} \mathrm{C}$. A slurry of $P$. sylvaticum was placed on the seed or $1.5 \mathrm{~cm}$ below the seed on the root. Seeds and roots were evaluated for disease incidence (percent of symptomatic seeds or roots) and disease severity (percent of seed or root tissue that was rotted) at 2, 4, 7, and 10 days after inoculation (DAI). Disease incidence was greatest when inoculation occurred at 2 DAP, increasing from $75.4 \%$ to $100 \%$ by 7 DAI. Roots inoculated at 2 DAP showed greater disease incidence and disease severity compared to 4 DAP when assessed at 2, 4 , and 7 DAI. Disease severity was greatest at 0 and 2 DAP, increasing over time from $83.7 \%$ to $100 \%$ and $69.4 \%$ to $100 \%$, respectively, by 7 DAI. No disease developed on roots inoculated at 7 DAP. An improved understanding of when Pythium species infect soybean could advance development of effective management strategies, including targeted fungicide seed treatments, which will reduce damping-off and stand loss in soybean.

\section{Historical pattern of Phytophthora species associated with Abies root rot in Pennsylvania}

J. E. Kim (1), E. V. NIKOLAEVA (2), T. N. Olson (3), S. Kang (4), S. H. Kim (5), (1) Penn State Univ., University Park, PA, USA; (2) Pennsylvania Department of Agriculture, Harrisburg, PA, USA; (3) Pennsylvania Dept of Agric, Harrisburg, PA, USA; (4) the Pennsylvania state University, University Park, PA, USA; (5) PA Department of Agriculture, Harrisburg, PA, USA

The PA Department of Agriculture (PDA) monitors a diverse array of specialty crops, nurseries and surrounding environments to detect diseases of regulatory concern through plant disease inspection and diagnostic service. Samples of diseased plants, mainly collected by PDA plant inspectors, are processed at the PDA Plant Diagnostic Laboratory (PDAPDL) for diagnosis, Among several groups of pathogens isolated and preserved since as early as 1980s, the largest group corresponds to Phytophthora. True fir trees (Abies spp.) are common hosts of Phytophthora species. Christmas tree root rot associated with Phytophthora species has been accountable for heavy losses in seedling beds and plantations for many years in Pennsylvania. We identified Phytophthora species isolated from root rot samples of Abies spp. using their sequences to understand their historical pattern of occurrence. Among 225 samples of Abies spp. diagnosed as Phytophthora root rot from 1988 to 2013, A. fraseri, A. concolor and A. balsamea were the most commonly infected hosts, and $P$. sp. kelmania, P. cactorum, P. cinnamomi and $P$. sansomeana were most commonly isolated species, while $P$. chlamydospora, $P$. europaea and $P$. colocasiae have been isolated only once. This information will help guide Phytophthora root rot inspection on true firs in Pennsylvania.

\section{Exciting changes for the Journal of Environmental Horticulture}

J. CALABRO (1), J. Derr (2), (1) AmericanHort/HRI, Washington, DC, USA; (2) Weed Science Society of America, Virginia Beach, VA, USA

The Journal of Environmental Horticulture (JEH) was created in 1983 to publish sound research and communicate valuable information to nurserymen. JEH is the only peer-reviewed journal with a focus on nursery and landscape research and directly functions as the outlet for research pertinent to our 
industry. The Horticultural Research Institute (HRI) believes that JEH should enhance the image of environmental horticulture as a profession and thereby attract more people to our discipline. Maintaining visibility in academia is key to continue attracting young students to our industry. JEH needs to be updated to continue serving its intended purpose and has struggled in recent years to remain relevant in the scientific community. One of the greatest obstacles is a general lack of awareness among researchers. HRI is undertook major revisions to JEH to better reach this goal and be relevant. For example, JEH is now hosted on a new platform, one that ensures that articles will be cataloged. Furthermore, the scope of JEH was broadened to include all aspects of the green industry, including but not limited to floriculture, herb and vegetable production in controlled environments, turf, cut flower production, container and field nursery production, and landscape maintenance. Within these areas, research articles are encouraged that address breeding, carbon sequestration, economics and marketing, growth regulator use, IPM, irrigation, nutrition, propagation, pest biology and control, and other issues of interest to the green industry, JEH will continue to publish information on new cultivars. The subscription service was discontinued, and JEH became open source.

Survey and awareness plan for identification and management of clubroot on canola in North Dakota V. CHAPARA (1), N. Kalwar (1), L. Lubenow (1), A. Chirumamilla (2), (1) NDSU, Langdon, ND, USA; (2) 901 3rd Street, Langdon, ND, USA

Clubroot (caused by Plasmodiophora brassicae) is a serious yield robbing disease of brassica crops such as canola, cauliflower, cabbage, rutabaga, radish, turnip, brussel sprouts, kale etc. P. brassicae infects roots causing clubs or galls thereby restricting the flow of water and nutrients to the plant Yield losses of $50-80 \%$ have been reported at $100 \%$ plant infection in Europe and Sweden. P. brassicae is a soil-borne pathogen that moves from field to field primarily through farm equipment. Once in the soil, the pathogen can live as resting spores for up to 20 years. $P$. brassicae prefers acidic soils (pH below 6.5), however, it is found in soils of $\mathrm{pH}$ up to 7.2. The disease clubroot was identified in Cavalier County, ND in 2013, and has been found in 2014, 2015 and 2016. Canola growers are facing challenges to manage this disease with no known management options at their disposal. A clubroot survey group has been formed at the Langdon Research Extension Center with one plant pathologist, two extension specialists and seven county extension agents to create awareness of clubroot and its management for growers in North Dakota. Out of 119 fields surveyed in 2016, only one field was found positive for clubroot. In 2016, twelve awareness meetings have been conducted in seven counties to educate about clubroot identification and management options available to growers to practice. Surveying to monitor the prevalence and spread of clubroot along with awareness meetings, field days, and field visits will be continued in the 2017 cropping season.

A model for sustainable IPM technology transfer in Nepal

A. C. FAYAD (1), R. N. Muniappan (2), L. Shah (3), (1) Virginia Polytechnic Institute and State Univ., Blacksburg, VA, USA; (2) Virginia Polytechnic Institute and State Univ, Blacksburg, VA, USA; (3) International Development Enterprises iDE, Lalitpur, Kathmandu, NEPAL

The Feed the Future Innovation Lab for Integrated Pest Management (IPM IL), a USAID funded program managed by Virginia Tech, improves the livelihoods of smallholder farmers around the world by increasing crop production, nutrition, health, income, and food security. Based on two decades of experience in the developing world, the IPM Innovation Lab has developed IPM packages for high value vegetable crops and transferred them to farmers in collaboration with local scientists, extension services, NGOs, value chain projects, and other organizations, using all available paths to reach farmers in a given country. Farmer access to the recommended products, such as Trichoderma spp. or other biopesticides, remains a major constraint to the widespread adoption of IPM in developing countries. In Nepal, we worked with agrovets to promote knowledge about the benefits and use of bioproducts and access and distribution of IPM components; we also collaborated with private entrepreneurs by training them on production and quality control of biopesticides and Trichoderma spp. to encourage the production of these products. This approach led to privatization of IPM technologies, development of a market for these IPM products, and a sustainable technology transfer in Nepal.

An Abridged Technique to Determine Phyllocoptes fructiphilus Populations on Roses Endangered by Rose Rosette Disease S. COLLINS (1), K. Solo (1), A. S. Windham (2), F. Hale (2), Q. Cheng (1), M. T. Windham (1), (1) University of Tennessee, Knoxville, TN, USA; (2) Soil, Plant, and Pest Center, Nashville, TN, USA

Rose rosette disease, also known as witches broom, is caused by Rose Rosette Virus. Vectored by an eriophyid mite, Phyllocoptes fructiphilus, rose rosette disease is difficult to control in wholesale and retail nurseries and public and private gardens. The inability to determine when eriophyid mite populations increase on plantings, makes timing of miticide application difficult, and therefore increases the chance of Rose Rosette Virus transmission. Although methods of eriophyid mite extraction and counting have been published for laboratory settings, it is often costly (estimates in excess of $\$ 9,000$ (U.S. dollars)), thus prohibiting nursery and landscape professionals and homeowners from determining P. fructiphilus populations on their roses. The objective of this study was to develop a method for counting eriophyid mites with readily available materials for horticultural professionals and private individuals, with a combined cost of less than \$100. A Phyllocoptes fructiphilus Rapid Detection Kit has been developed and includes a handbook that describes the kit components and protocol for eriophyid mite extraction and population determination. The eriophyid mite counting kit achieves its economic goal $(<\$ 100)$ and allows users to photograph mites via cellphone. Twelve kits were distributed to nursery owners, rose garden professionals, and private garden owners. Participants were asked to fill out a survey on kit operation, ease-of-use, and satisfaction of results. Survey results were used to amend and improve the mite detection kit for future users.

Engaging non-traditional and online students in Ohio State's Master in Plant Health Management: A SWOT analysis M. M. LEWANDOWSKI (1), A. E. Dorrance (2), L. Canas (3), C. Welty (4), F. Peduto Hand PhD (1), D. Shetlar (4), P. A. Paul (5), A. Londo (6), S. D Ellis-Williams (1), W. Klooster (4), (1) Ohio State Univ, Dept of Plant Pathology, Columbus, OH, USA; (2) The Ohio State University, Wooster, OH, USA; (3) Ohio State Univ, Ohio Agricultural Research and Development Center, Dept of Entomology, Wooster, OH, USA; (4) Ohio State Univ, Dept of Entomology, Columbus, OH, USA; (5) Ohio State Univ, Ohio Agricultural Research and Development Center, Dept of Plant Pathology, Wooster, OH, USA; (6) Ohio State Univ Extension, Columbus, OH, USA

Ohio State's Master in Plant Health Management (MPHM) program has attracted a diverse population of students, including non-traditional and online students. The MPHM program, administered by the Departments of Plant Pathology and Entomology, has an enrollment of over 20 students and recently graduated its $10^{\text {th }}$ student. This marked an opportune time to evaluate the program with a Strengths, Weaknesses, Opportunities and Threats (SWOT) analysis. SWOT feedback from alumni was discussed with the MPHM Graduate Studies Committee and an advisory board comprised of representatives from industry and other key organizations. From the SWOT's alumni responses, the program's strength is the interdisciplinary curriculum, including coursework in plant pathology, entomology, weed science, soil science, statistics, education and leadership. As an area for improvement, we are working to engage online and part-time students professionally and academically by expanding the scope of the independent projects that are required for the degree, and we hold weekly webinars with participation from members of industry to discuss professional development and plant health topics. To engage distance students, we are developing ways to foster an online student community and connect students to professional events, job shadowing and experiential learning in their locales. We continually evaluate feedback to improve and expand the opportunities for graduate education in plant health.

News and 2016 Activities of the Clemson University Plant Problem Clinic M. WILLIAMSON, Clemson University Department of Plant Industry, Pendleton, SC, USA

The Clemson University Plant Problem Clinic (PPC) will be changing its name to the Plant and Pest Diagnostic Clinic (PPDC) in January, 2018. However, its mission will remain the same. The Clinic is both an Extension Clinic and the state lab for the Division of Plant Industry in the Regulatory Services Department. As such, samples to detect pathogens, insects and weeds of regulatory concern are routinely submitted by Plant Industry 
Inspectors. The lab also assists with CAPS surveys. This close cooperation facilitates communication with Regulatory Officers and allows the State of South Carolina to react quickly to prevent the spread of any pests of concern that are found. In 2016, boxwood blight, caused by Calonectria pseudovaniculata, was detected for the first time in a nursery and a landscape. For a fun promotional activity, PPC staff worked with the CAPS Coordinator to design a scarecrow display promoting the "Don't Move Firewood" pest introduction prevention program. This was placed in the annual Scarecrow Contest in the town of Pendleton, SC.

\section{The Sunflower Pathology Working Group}

S. G. MARKELL (1), R. M. Harveson (2), C. C. Block (3), T. Gulya (4), F. M. Mathew (5), S. Thompson (6), (1) North Dakota State University, Fargo, ND, USA; (2) Univ of Nebraska - Panhandle Research \& Extension Center, Scottsbluff, NE, USA; (3) Iowa State University, Ames, IA, USA; (4) USDA-ARS, Northern Crop Science Laboratory, Fargo, ND, USA; (5) South Dakota State University, Brookings, SD, USA; (6) Univ of Southern Queensland, Toowoomba, AUSTRALIA

Approximately $85-90 \%$ of the $2.0 \mathrm{M}$ acres of sunflower in the U.S. are planted in the North Central States. According the National Sunflower Association, diseases are the most significant biological yield-limiting factor for sunflower production, yet few pathologists work on sunflower and until recently, limited reference and Extension materials on sunflower diseases were available. As a consequence, disease identification and management consistently have been challenging and growers may resort to a 'spray and pray' approach to disease management. To address this situation, a small group of pathologists formed a Sunflower Pathology Working Group (SPWG) in 2013 with funding support from the North Central IPM Center. The mission of the SPWG is to develop academic reference and Extension materials that will help growers better identify diseases and facilitate their ability to use IPM. Contributions to SPWG-initiated projects have come from scientists in over a dozen countries and U.S. states, most notably to the first APS Compendium of Sunflower Diseases and Pests (2016). Outputs also include a 20-disease field diagnostic card set (2015), two disease chapters in monographs/books $(2015,2016)$, a diagnostic playing-card deck (2016), and other funded grants, Extension and research articles. Currently, the SPWG is composing a Plant Disease feature article, several Plant Health Progress - Diagnostic Guides and is in the formative phase of an interactive 'ibook'.

Volunteerism in International Development: Disease Notes from Abroad

J. C. HIMMELSTEIN, ACDIVOCA, Washington, DC, USA

A large tonnage of agricultural crops die every year in the developing world due to lack of resources, skills, and infrastructure. Plant pathologists have a depth of knowledge that, when shared with relevant organizations and people, can have a significant impact on the food security of developing communities. There are a range of programs that offer opportunities for plant pathologists to share their expertise, but many of these scientists aren't aware of these programs, how they work, or the potential positive effect they could engender by participating. This presentation covers several case studies of a few such volunteer programs in Kyrgyzstan, Bangladesh, and Ghana, which illustrate measurable results and demonstrate the various means in which plant pathologists can contribute to the developing world. This includes strategies for finding the right volunteerism program, methods for collecting data for potential publication outcomes, and the value such activities can add to your day-to-day work.

Relative abundance of Potato virus $Y$ strains in commercial potato fields of the Columbia Basin, 2011 to 2015

C. FUNKE (1), O. V. Nikolaeva (2), K. Green (1), L. Tran (1), A. V. Karasev (1), (1) University of Idaho, Moscow, ID, USA; (2) Univ of Idaho, Moscow, ID, USA

Potato virus $Y(\mathrm{PVY})$ exists as a complex of strains, including multiple recombinants often associated with potato tuber necrotic ringspot disease (PTNRD). These recombinant strains have been spreading in the U.S. for the past several years, although this spread was not documented in a systematic way. To document and assess this spread between 2011 and 2015, strain composition of PVY isolates circulating in the Columbia Basin potato production area was determined from hundreds of seed lots of various cultivars. The proportion of non-recombinant $\mathrm{PVY}^{\mathrm{O}}$ isolates circulating in Columbia Basin potato dropped nine-fold during this period, from 63\% of all PVY-positives in 2011 to less than 7\% in 2015. This drop in PVY ${ }^{\mathrm{O}}$ was concomitant with the rise of the recombinant $\mathrm{PVY}{ }^{\mathrm{N}-\mathrm{Wi}_{\mathrm{i}}}$ strain incidence, from less than $27 \%$ of all PVY-positives in 2011 to $53 \%$ in 2015 . The proportion of the PVY ${ }^{\text {NTN }}$ recombinant strain, associated with PTNRD symptoms in susceptible cultivars, increased from $7 \%$ in 2011 to ca. $24 \%$ in 2015.

Spread of bacterial leaf spot (Xanthomonas perforans) among tomato seedlings in the greenhouse K. Hernandez, S. Sharpe, I. MEADOWS, North Carolina State University, Mills River, NC, USA

Bacterial leaf spot (BLS) of tomato, caused by four species of Xanthomonas, is a devastating disease that impacts tomato production in North Carolina and worldwide. This seedborne disease reduces yield by causing defoliation and unmarketable fruit. If properly maintained, seedlings produced in the greenhouse may appear healthy at the time of transplant. However, if symptoms are present on a few plants, how many other plants are infected but asymptomatic? We examined the spread of $X$. perforans among tomato transplants in the greenhouse and assessed the number of symptomatic and asymptomatic plants. Disinfested tomato seeds were grown in the greenhouse and one plant in each of 340 -cell trays was inoculated with a suspension of $X$. perforans; 3 trays were treated similarly with water and the experiment was repeated. Young leaves were systematically harvested weekly for 3 weeks or one time at 6 weeks; leaves were assayed to isolate $X$. perforans in culture and quantify the number of colony-forming units using qPCR. Xanthomonas perforans was regularly detected in asymptomatic plants and throughout the trays by qPCR, although the pattern of detection was not consistent among trays. The frequency of detection of $X$. perforans was greater by qPCR than by culturing and was isolated from asymptomatic plants. Results suggest that when symptomatic plants are present, asymptomatic plants may be present in the remainder of the tray and, possibly, in adjacent trays.

Transmission of Monilina fructicola genotypes from blossoms to twigs

M. DOWLING (1), B. Cox (1), T. Sroka (1), J. Wilson (2), G. Schnabel (1), (1) Clemson University, Clemson, SC, USA; (2) Section of Plant Pathology and Plant-Microbe Biology, Cornell University, Ithaca, NY, USA

Monilinia fructicola, causal agent of brown rot on peach, causes blossom blight and subsequently twig cankers in the spring. Conidia asexually produced on blossoms and cankers are believed to cause secondary infections that largely remain quiescent until fruit matures. It is unknown whether this pathogen's transition from blossom to canker affects genetic diversity, but such information would help in our understanding of its epidemiology. Fungal spores from blossoms and, two months later, from their corresponding cankers were collected from a conventional and an unsprayed orchard in 2015 and 2016. Simple sequence repeat markers were used to genotype 10 to 20 single spores from each of four blossom/canker pairs per orchard. The average number of genotypes in blossoms and corresponding cankers were not significantly different for both years $(p=0.4788, p=0.8612)$ and for both farms $(p=0.6573, p=0.2688)$, indicating that genetic diversity remained the same through the transition. The average number of genotypes unique to blossom or canker was not significantly different in either year $(p=0.1202, p=0.8704)$ or farm $(p=0.7311, p=0.2663)$, although blossoms had a numerically greater number of unique genotypes. In conclusion, a single blossom may be infected by one or more genotypes of $M$. fructicola, and this diversity is not lost during canker formation. This information will help assess the pathogen's ability to asexually maintain and spread diverse genotypes in an orchard.

Investigating the dispersal efficiency of pathogens causing potato early blight and brown spot

S. DING, D. Rouse, K. Meinholz, A. J. Gevens, University of Wisconsin-Madison, Madison, WI, USA

The combined diseases of early blight caused by Alternaria solani and brown spot caused by A. alternata have major impacts on potato production worldwide. Both pathogens are windblown, but differ in conidial morphology, which can significantly influence their dispersal and subsequent 
epidemiology and management. The dispersal pattern of Alternaria conidia was studied in 2016 using a 10-m tower to collect environmental data and capture conidia in the center of a naturally-infected potato field in Wisconsin. The aerial concentration of the conidia was monitored with Rotorod samplers mounted on the tower and deployed at $0,1,2$, and $3 \mathrm{~m}$ above the potato canopy. Another $2-\mathrm{m}$ tower mounted with samplers was set up at the edge of the field in the upwind direction to monitor the source of conidia captured on the $10-\mathrm{m}$ tower. Aerial concentrations of both conidia decreased rapidly with height on the $10-\mathrm{m}$ tower, and the mean daily vertical flux and escape fraction of small $A$. alternata conidia $\left(1.8 \times 10^{5} \mathrm{conidia} / \mathrm{m}^{2}, 0.08\right)$ were higher than that of large $A$. solani conidia $\left(4.0 \times 10^{4}\right.$ conidia $\left./ \mathrm{m}^{2}, 0.01\right)$. Numbers of both conidia were reduced on the upwind edge compared with the center of the field, with $A$. solani reduced even more, suggesting that $A$. solani captured on the $10-\mathrm{m}$ tower most likely escaped from the field in which it was deployed, while A. alternata could be from other sources. Our results indicated that conidia of $A$. alternata have a higher dispersal potential than A. solani.

\section{Incidence of Silverleaf disease caused by Chondrostereum purpureum in apple nursery plants} A. FRANCE, D. Grinbergs, J. Chilian, INIA Quilamapu, Chillan, CHILE

Silverleaf, caused by the Basidiomycete Chondrostereum purpureum, is an important disease in Chile affecting trees, fruit yield and quality. The disease affects adult trees, because of basidiospores require well-developed xylem tissue to grow. However, the new methods of tree production, such as Knipboom, are releasing well developed plants with strong stems. Therefore, the objective was to determine if C. purpureum is infecting apples trees at the nursery, when visual symptoms are absent. Sixty-five 2-year-old, non-symptomatic nursery plants (cv. Gala Brookfield) were randomly selected. Ten mature leaves were collected from each plant and the endopolygalacturonase (endoPG) concentration was measured through DAS-ELISA. Healthy plants were used as negative controls and purified endoPG as positive ones. The presence of C. purpureum from wood was confirmed through fungal isolation on PDA and by amplification of the large intergenic spacer region. Results indicate $100 \%$ of the plants were infected by the pathogen through high concentration of endoPG, which was confirmed by PCR. However, the fungus was only isolated from $26.5 \%$ of the plants, indicating the ELISA method was more sensitive. In addition, $94.3 \%$ of the ELISA-positive and $89.8 \%$ of the isolation-positive samples did not show internal necrosis symptoms. Therefore, visual symptoms are insufficient to determine healthy plants and the amount of silverleaf plants provided by nursery are by far more than is expected.

Transmission efficiency of Cucumber green mottle mosaic virus via Soil C. LIANG $(1,2,3,4)$, X. A Bie $(1,2)$, L. Luo $(1,2)$, J. Li $(1,2)$, B. Baker $(3,4)$, (1) China Agricultural University, Beijing, CHINA; (2) Beijing Key Laboratory of Seed Disease Testing and Control, Beijing, CHINA; (3) Department of Plant and Microbial Biology University of California, Berkeley, Berkeley, CA, USA; (4) Plant Gene Expression Center USDA-ARS, Albany, CA, USA

Cucumber green mottle mosaic virus (CGMMV), which belongs to the genus Tobamovirus, is one of the most important quarantine pests in the world. It is well known that CGMMV is transmitted by seed, pollen, farm operation and plant debris. Cucumber green mottle mosaic virus disease poses a threat to the growth of cucumber and other plants of the family Cucurbitaceae and causes great economic losses. Soil transmission of CGMMV has been reported, however, this mode of transmission has not been confirmed. This study was carried out to test for CGMMV infection rates of cucumber and zucchini seedlings grown in virus-contaminated soil with and without plant debris. CGMMV infection of seedlings was tested 10 to 60 days after sowing using RT-PCR (reverse transcription-polymerase chain reaction) analysis of RNA collected from leaves. The results indicated that transmission of CGMMV to seedlings grown in contaminated soil with plant debris was $21.4 \%$; whereas transmission of CGMMV in plants grown in contaminated soil without plant debris was $5.9 \%$. Mechanical inoculation of CGMMV on control seedlings grown in virus-free soil without debris was $42.9 \%$ measured in leaves collected 25 to 60 days post inoculation.

Epidemic network analysis for mitigation of invasive pathogens in seed systems: Potato in Ecuador C. BUDDENHAGEN (1,2,3), J. F. Hernandez Nopsa (1,2,3), K. F. Andersen (1,2,3), J. L. Andrade-Piedra (4), P. Kromann (5), G. A. Forbes (6), S. Thomas-Sharma (7), K. A. Garrett (2,3,8), (1) Plant Pathology Department, Gainesville, FL, USA; (2) Institute for Sustainable Food Systems, Gainesville, FL, USA; (3) Emerging Pathogens Institute, Gainesville, FL, USA; (4) International Potato Center (CIP), Lima, PERU; (5) International Potato Center, Quito, ECUADOR; (6) International Potato Center, Servas, FRANCE; (7) Plant Pathology Department, Manhattan, KS, USA; (8) Plant Pathology Department, University of Florida, Gainesville, FL, USA

Seed system structure defines pathways for the spread of pathogens involved in seed degeneration and influences their ability to supply high quality seed of disease resistant varieties to farmers. We evaluated seed system networks defined by a regional potato farmer consortium (CONPAPA) in Tungurahua, Ecuador. The structure of networks of farmer seed and potato transactions, and the linked network of information about pest and disease management, were estimated based on surveys. We performed a scenario analysis of disease spread in this multilayer network to identify key nodes for sampling and mitigation. CONPAPA's centrality in the network means that disease management interventions, such as training, monitoring and variety dissemination, should target CONPAPA staff and facilities. A market in the largest nearby town, Ambato, was the next most important node. Farmers reported receiving advice about disease and pest management through trusted CONPAPA technical staff. Advice from agrochemical stores was common but viewed as significantly less reliable. Farmer access to information (number and quality of sources) was similar for both genders. Women had a smaller amount of the market share, however. Understanding seed system networks provides a window into options for system improvement that include environmental and societal concerns.

Pollen can spread Colletotrichum acutatum among citrus flowers

M. C. DE GODOY GASPAROTO (1), A. B. Gama (2), S. de Afonseca Lourenço (2), G. J. Silva Junior (3), L. Amorim (2), (1) UNESP, Registro, BRAZIL; (2) USP, Piracicaba, BRAZIL; (3) FUNDECITRUS, Araraquara, BRAZIL

Colletotrichum acutatum, causal agent of postbloom fruit drop (PFD), can infect citrus pollen. The hypothesis is that pollen carried by honeybees can spread the fungus from diseased to healthy flowers when the insects are visiting citrus plants. The experiment was carried out in controlled conditions and it was performed 3 times. Flowers from potted sweet orange plants were inoculated by spraying a suspension of C. acutatum spores $\left(1.25 \times 10^{5}\right.$ conidia. $\mathrm{ml}^{-1}$ ). Controls had the same number of flowers as inoculated plants and they were sprayed with sterile water. In total, 138 flowers were used in the experiment. The plants were kept for 24 hours under moist chamber conditions at $24^{\circ} \mathrm{C}$. Anthers were transferred from inoculated and control flowers to healthy flowers. PFD symptoms were evaluated for 3 days in order to isolate the pathogen on potato dextrose agar $\left(25^{\circ} \mathrm{C}, 12 \mathrm{~h} / 12 \mathrm{~h} \mathrm{for} 7\right.$ days $)$. DNA was extracted from all isolates and Polymerase Chain Reaction (PCR) assays confirmed the fungal species. The specific primer CaInt2/ITS4 was used for PCR detection. All flowers that received inoculated anthers showed PFD symptoms. All isolates were identified as C. acutatum by PCR. None of the flowers that received control anthers exhibited PFD symptoms. Images of scanning electron microscope showed citrus pollen surrounded by conidia of C. acutatum on petals 48 hours after the anther transference. This study confirmed the hypothesis that citrus pollen is able to disperse C. acutatum.

Assessment of the presence of Plasmodiophora brassicae viable inoculum in the irrigation sediments in the Savannah of Bogota J. S. Navas Martínez, A. BOTERO RAMIREZ, D. J. Urbano Muñoz, J. S. Urquijo Ruiz, C. García, Universidad Nacional de Colombia, Bogotá, COLOMBIA

The presence of viable resting spores of Plasmodiophora brassicae in the irrigation sediments has been stated as an important source of the pathogen dissemination. Based on that affirmation, this research aimed to evaluate the potential of the pathogen dissemination through the irrigation water in the Savannah of Bogota by the evaluation of the presence of P. brassicae's viable inoculum in the irrigation sediments. For this purpose, four samples of 
irrigation sediments were collected in three points at the irrigation canal of the experimental field of the Universidad Nacional de Colombia and one at the reservoir of one commercial field. The evaluation was done using a hydroponic system in a growth chamber, where broccoli var. calabrense was grown in a suspension with the irrigation sediments and a positive control with $10^{6}$ resting spores $\cdot \mathrm{mL}^{-1}$ for a period of three days. Afterwards, the suspension was replaced for a 1:10 Hoagland solution where plants were kept for 21 days. The presence of viable inoculum was assessed by the observation of primary infection in radical hairs; by this method disease incidence and the percentage of infected hairs were estimated. Positive control had an incidence of $100 \%$ while other samples showed incidence values between $20 \%$ and $46.9 \%$. The highest percentage of infected radical hairs was observed in the positive control (43.9\%), all other treatments showed values between 2.4 and $7.1 \%$. From this trial, it can be concluded that irrigation systems can be a source of $P$. brassicae inoculum in the Savanah of Bogota fields.

Assessment of the vertical and horizontal distribution of Plasmodiophora brassicae in soil L. Tarazona, A. BOTERO RAMIREZ, C. García, Universidad Nacional de Colombia, Bogotá, COLOMBIA

Knowledge of spatial patterns of pathogens can provide important insights about their biology and ecology. This work aimed at describing spatial patterns of inoculum density (ID) for resting spores of Plasmodiophora brassicae the pathogen of clubroot of crucifers, in a 3D approach and relate them to disease development. Soil sampling was done in a commercial field of broccoli in a grid of $30 \times 20 \mathrm{~m}$, with 30 georeferenced points; at each point, samples were collected at $15 \mathrm{~cm}$ and $30 \mathrm{~cm}$ depth. ID quantification was done according to Takahashi and Yamaguchi (1987). Finally, a bio-assay in greenhouse conditions was done on hybrid 'Delus' cabbage plants; incidence and severity were measured 80 days after planting. Average ID per gram of soil at $15 \mathrm{~cm}$ was $1 \cdot 10^{6}$ resting spores while at $30 \mathrm{~cm}$ it was $7 \cdot 10^{5}$ resting spores. Incidence and severity did not show differences among depths. The spatial structure analysis using variograms, showed an anisotropic trend at $45^{\circ}$. Structural variance was between $95 \%$ and $100 \%$ that confirmed that ID and severity variance are almost completely explained by the spatial variation. All estimated semivariograms were adjusted to the spherical model. Range value, which estimates the patch size, at $15 \mathrm{~cm}$ was $77.14 \mathrm{~m}$ for ID and severity, while at $30 \mathrm{~cm}$ it was $34.96 \mathrm{~m}$ for ID and $24.58 \mathrm{~m}$ for severity. In summary, $P$. brassicae shows a patchy distribution in the horizontal axis, while in the vertical axis, the deeper the lesser inoculum density and the smaller the patch size

Environmental Factors Impact Temporal Passalora sequoiae Conidia Counts from Leyland Cypress

J. L. Williams-Woodward (1), W. E. COPES (2), (1) University of Georgia - Department of Plant Pathology, Athens, GA, USA; (2) USDA ARS, Poplarville, MS, USA

Needle blight disease, caused by Passalora sequoiae, results in a progressive loss of leaf tissue on Leyland cypress $(\times$ Cupressocyparis leylandii) within container and field tree nurseries, in the landscape, and on Christmas tree farms. Fungicide schedules have been developed in response to seasonal symptom progression and conidia dispersion without clarification of the influence of weather conditions on disease. Conidia count data of $P$. sequoiae were collected approximately weekly from June to December in 2001 in Watkinsville, GA and in 2002 in Dearing, GA. Peak number of conidia were collected in September and October. Daily weather data summaries were obtained from a weather station at each location. Deterministic models were developed individually for summer (June 1 to September 30) and fall (October 1 to December 21) seasons. A 3-day lag of average daily temperature (T) had a positive quadratic relationship with conidia counts in summer. A 10-day lag of average daily T, vapor pressure deficit (VPd) and total solar radiation had a positive quadratic, a negative linear and positive linear relationship, respectively, with conidia counts in fall. The models show that the seasonal fall shift to cooler temperatures, along with a reduction in VPd and an increase in solar radiation, favor production and dispersal of $P$. sequoiae conidia.

Germination of oospores of the Impatiens downy mildew at different temperatures N. SHISHKOFF, USDA ARS FDWSRU, Frederick, MD, USA

Plasmopara obducens causes downy mildew of Impatiens walleriana, threatening a crop valued annually at more than \$91 million wholesale in 2013. The pathogen is thought to overwinter vegetatively in warmer regions of the US but it also produces oospores. We examined the germination of oospores from 4 isolates (collected from NY, MD, and NJ) after infected stem segments were incubated for up to 7 months at $-10,0,10$ or $20 \mathrm{C}$. Every month four stems per treatment were dissected in water and incubated at $10 \mathrm{C}$, examined for signs of oospores germination every 2-6 days for 3 weeks. Germination was commonly seen for oospores incubated at $0 \mathrm{C}$ for at least 1 month and occasionally for those incubated at $10 \mathrm{C}$. Oospore germination was also observed in infected stems moved from 0 to $10 \mathrm{C}$ every few days. When infected stems incubated for 7 months at $-10 \mathrm{C}$ were moved to $0 \mathrm{C}$, germination was not seen at one and two months subsequently, but when infected stems at $0 \mathrm{C}$ were moved to $-10 \mathrm{C}$, oospores continued to germinate after one or two months. These results should eventually contribute to our understanding of the overwintering of the pathogen.

\section{Lack of pheromone reduces nematode dispersal}

A. PERRET-GENTIL (1), A. Mirti (1), J. Giurintano (1), E. Sampson (1), X. Gao (1), D. I. Shapiro-Ilan (2), F. Kaplan (1), (1) Pheronym, Inc., Gaineville, FL, USA; (2) USDA/ARS, Southeastern Fruit and Tree Nut Research Laboratory, Byron, GA, USA

Nematode dispersal is important for host encounter and regulated by pheromones. A mixture of ascaroside pheromones disperses Caenorhabditis elegans dauer/dispersal larvae. The same mix of pheromones was recognized by plant parasitic and entomopathogenic nematode (EPN) dispersal larvae/infective juveniles (IJs). Furthermore, in the absence of pheromones, Steinernema feltiae, an EPN, IJs become sluggish and clump (decondition) within 2 days. However, it is not known whether deconditioning happens to all EPNs in the absence of pheromones or whether deconditioning times vary among nematode species. To determine whether pheromone deconditioning periods are variable, 4 to 5 day old $S$. feltiae IJs from 3 different sources were washed with water to remove pheromone and monitored for dispersal for 6 days at $22^{\circ} \mathrm{C}$. IJs were placed on agar for $15 \mathrm{~min}$ and then a $1.3 \mathrm{~cm}$ diameter cylinder separated the dispersed IJs from deconditioned IJs for quantification. S. feltiae IJ deconditioning time varied depending on the EPN sources. To determine whether this was specific to $S$. feltiae, we tested two other EPN species, S. carpocapsae and Heterorhabditis bacteriophora, for deconditioning in the absence of pheromones. Currently, deconditioned IJs are being quantified. Understanding how pheromones regulate dispersal has two important practical applications for plant biotic stress: 1- to improve EPN biological control efficacy 2- to control plant parasitic nematodes.

\section{Elevated temperature reduces seed-to-seedling transmission of bacterial fruit blotch of cucurbits caused by Acidovorax citrulli M. ZHAO, R. R. Walcott PhD, The University of Georgia, Athens, GA, USA}

Bacterial fruit blotch (BFB) caused by Acidovorax citrulli can be transmitted by seeds. Though seeds are the most important primary inoculum source, little is known about BFB seed-to-seedling transmission. We hypothesized that elevated temperature negatively affects the ability of $A$. citrulli to switch from saprophytic to pathogenic growth on germinating seeds, hence, the aim of the study was to investigate the effect of temperature on BFB seedling transmission. We conducted seedling grow out (SGO) assays, in which watermelon seeds were vacuum-infiltrated with $10^{6} \mathrm{CFU} / \mathrm{mL}$ A. citrulli cell suspensions, germinated at $28^{\circ} \mathrm{C}$, and transferred to $40^{\circ} \mathrm{C} 3,5$ or 7 days after planting (dap). Control treatment included seeds incubated at $28^{\circ} \mathrm{C}$. The SGO assays were conducted for 14 days and BFB incidence was assessed visually. We observed lower mean BFB incidence (54\%) for seeds transferred to $40^{\circ} \mathrm{C}$ at 3 dap compared to seeds incubated at $28^{\circ} \mathrm{C}(89 \%)$ or transferred to $40^{\circ} \mathrm{C}$ at $5(69 \%)$ or 7 dap $(78 \%)$. In addition, seeds inoculated with $10^{2}$ $\mathrm{CFU} /$ seed showed reduction in BFB transmission when transferred from $28^{\circ} \mathrm{C}$ to $40^{\circ} \mathrm{C}$ at 3 dap, while seeds inoculated with $10^{6}$ and $10^{4} \mathrm{CFU} /$ seed did not. We concluded that the negative effect of elevated temperature on BFB seedling transmission only occurs when seedlings are transferred to $40^{\circ} \mathrm{C}$ at 3 dap and with inoculum of $10^{2} \mathrm{CFU} /$ seed. These results suggest $A$. citrulli is vulnerable to environmental conditions during initial stages of seed germination. 
Analyzing Key Nodes and Epidemic Risk in Seed Networks: Sweetpotato in Northern Uganda

K. F. ANDERSEN (1,2,3), C. Buddenhagen (1,2,3), P. Rachkara (4), R. Gibson (5), S. Kalule (4), D. Phillips (5), K. A. Garrett (2,3,6), (1) Plant Pathology Department, Gainesville, FL, USA; (2) Institute for Sustainable Food Systems, Gainesville, FL, USA; (3) Emerging Pathogens Institute, Gainesville, FL, USA; (4) Department of Rural Development and Agribusiness, Gulu, UGANDA; (5) Natural Resource Institute, Greenwich, UNITED KINGDOM; (6) University of Florida, Gainesville, FL, USA

Seed systems, both formal and informal, are critical to global food security. Even so, efforts to implement seed systems that work better for smallholder farmers in low-income countries have too often been unsuccessful. The structure of seed system networks provides important information about epidemic risk within the network and can be used to implement management strategies. Sweetpotato is a major staple food crop in many African countries, and Uganda is the second largest producer in Africa. Planting material from sweetpotato is vegetatively propagated and largely sold by informal sellers. In this study, we evaluated the structure of a sweetpotato seed system in Northern Uganda in terms of its utility for distributing improved varieties and its vulnerability to the spread of potential seed-borne pathogens. Sellers in this study were surveyed in the Gulu Region of Northern Uganda. Weekly vine sales transactions were tracked through the growing season (April-October) creating a robust dataset of planting material sales over time, including price, village sold to, volume, and information about the buyer and seller. From this dataset of known transactions and the distance between villages, a network of vine movement was constructed. Results indicate that varieties are not equally distributed throughout the network. In silico simulations of the introduction of a novel virus into the systems indicated the potential for rapid spread. Through simulation of multiple epidemic starting points, nodes to be targeted for pathogen sampling and mitigation were identified. We present a method that can serve as an example, with potential to be used across a wide variety of seed systems.

A network model to predict spread and mesoscale level development of hop powdery mildew

D. H. GENT (1), S. Bhattacharyya (2), T. Ruiz (2), M. Twomey (3), S. Wolfenbarger (3), (1) US Department of Agriculture, Agricultural Research Service, Corvallis, OR, USA; (2) Oregon State University, Department of Statistics, Corvallis, OR, USA; (3) Oregon State University, Department of Botany and Plant Pathology, Corvallis, OR, USA

For aerially dispersed pathogens, the probability of disease transmission between individuals, fields, and other scales is influenced by connectivity of the host and the pathogen in space and time. These relationships can be expressed in a model that explicitly considers network structure to better represent disease spread through a finite set of connections. In this research, we formulated and fit a network model to describe the month-to-month spread of powdery mildew on hop (caused by Podosphaera macularis) among yards at the mesoscale level. An individual hop yard was considered a node in the model, whose disease status in a given month was modeled to be a function of its disease status in the preceding month, and disease spread from other nodes as influenced by their disease incidence, distance away, and susceptibility to two races of the fungus, as well as wind run and direction in the preceding month. Parameter estimates for edge weights representing these variables were estimated by penalized regression. Evaluation of predicted versus actual incidence of diseased plants during 2014, 2015, and 2016 yielded coefficients of determination ranging from 0 to 0.77 during May, whereas predictions for June and July ranged from 0.51 to 0.91 . Given that $P$. macularis overwinters in less than $5 \%$ of hop yards in western Oregon, the network model provides a useful description of risk factors that underlie the spread of powdery mildew at the mesoscale.

A low-cost spore trap allows collection of Fusarium circinatum airborne spores for real-time PCR quantification T. QUESADA (1), J. Hughes (1), K. Smith (2), P. James (1), K. Shin (1), J. A. Smith (1), (1) University of Florida, Gainesville, FL, USA; (2) USDA Forest Service, Gainesville, FL, USA

A variety of commercial instruments are available for sampling and quantifying microscopic airborne organisms from the environment. Most utilize air suction mechanisms to draw particles into a matrix, where they are trapped and processed for quantification. Such samplers are highly sensitive, but also expensive, costing thousands of dollars per unit and often requiring additional post-sampling services. For initial tests, low-cost methods are preferred before investing in more sophisticated equipment. We developed a simple, low-cost spore trap that, for under $\$ 50.00$ per unit allows surveying spore abundance in outdoor environments. The trap consists of a rotating motor that holds a metal rod and two petroleum jelly-coated microscope slides. As the motor rotates, the slides collect airborne particles. Its low cost allows placing multiple traps per site, increasing statistical power for analysis. To test whether the traps allowed detection of Fusarium circinatum spores, we placed six traps on three sites: an actively-managed commercial stand located in Lake Butler, FL, a semi-managed stand near Gainesville, FL, and a site with little perturbance at Goethe State Forest, FL. The slides were replaced weekly and $F$. circinatum was detected by quantitative PCR using species-specific primers. Results show detection of low levels $(\mathrm{AVG}=4.8-22.7 \mathrm{pg} \pm$ $\mathrm{SE}=0.9-5.6$ ) of the pathogen with high reproducibility. Further adaptations can be customized for sampling other organisms.

Biological relevance of the detection of pospiviroids on pepper and tomato seeds H. KOENRAADT, Naktuinbouw, Roelofarendsveen, NETHERLANDS

Pepper and tomato crops may become infected by the pospiviroids Citrus exocortis viroid (CEVd), Columnea latent viroid (CLVd), Pepper chat fruit viroid (PCFVd), Potato spindle tuber viroid (PSTVd), Tomato apical stunt viroid (TASVd), Tomato chlorotic dwarf viroid (TCDVd) and Tomato planta macho viroid (TPMVd). To study the role of seed transmission in spreading these pospiviroids, a high-throughput test was used for the detection of pospiviroids on pepper and tomato seeds. This test consists of four TaqMan RT-PCR reactions to detect all seven pospiviroids. To monitor test performance, Dahlia latent viroid (DLVd) is used as an internal isolation control. RNA is extracted from pepper and tomato seeds with a bagmixer or genogrinder and purified using a KingFisher system. To examine whether pospiviroid contaminated tomato and pepper seeds lead to infection of seedlings, grow-out experiments were performed with contaminated seeds. Two to three weeks after sowing, 1,000 - 1,200 seedlings were transplanted. Plants were grown at a constant temperature of $25^{\circ} \mathrm{C}$ with supplemental daily illumination for $14 \mathrm{~h}$. Five to six weeks after transplantation, all plants were tested for viroid presence. Results of the transmission studies will be presented and discussed.

Is the begomovirus, Sweet potato leaf curl virus, really seed transmitted in sweetpotato?

K. S. LING, P. Wadl, L. Williams, A. Simmons, M. Jackson, USDA-ARS, Charleston, SC, USA

Sweetpotato is one of the major root crops in the world and is also widely grown in the southern United States. Sweet potato leaf curl virus (SPLCV) is a begomovirus posing a serious threat to sweetpotato production worldwide and is primarily transmitted by whitefly (Bemisia tabaci) or through vegetative propagated materials (cuttings or storage roots). A recent publication on seed transmission of SPLCV in sweetpotato, the first report of seed transmission by any Geminivirus, prompted us to investigate our sweetpotato breeding seed stocks for possible seed transmission. Through symptom observation and real-time PCR on seedlings, experiments were conducted in three separate years (2012, 2016 and 2017). In the first experiment conducted in 2012 using a smaller number of seedlings $(\sim 100)$, no seed transmission of SPLCV on the germinated seedlings was detected. The second experiment conducted in 2016, used 3,500 seedlings from seeds collected in 2015 from 20 genotypes in Charleston, SC; none of these seedlings tested positive for SPLCV. In the third experiment conducted in 2017, nearly 14,000 seedlings were germinated in insect-free conditions in greenhouses and a growth chamber. Again, no seed transmission of SPLCV was detected in the test. In conclusion, our large scale seedling grow-out tests conducted in multiple years did not support seed transmission of SPLCV in sweetpotato. 
Presence and prevalence of Raffaelea lauricola, cause of laurel wilt, in different species of ambrosia beetle in Florida

R. C. PLOETZ (1), J. Konkol (2), T. Navarez (2), R. Duncan (2), J. R. Saucedo Carabez (2), A. Campbell (3), J. Mantilla (2), D. Carrillo (1), P. Kendra (3), (1) University of Florida - Tropical Research and Education Center, Homestead, FL, USA; (2) University of Florida, Homestead, FL, USA; (3) USDA-ARS, Miami, FL, USA

Laurel wilt, a lethal disease of American members of the Lauraceae plant family, is caused by Raffaelea lauricola, the primary nutritional symbiont of an Asian ambrosia beetle, Xyleborus glabratus, which serves of the pathogen's primary vector in native lauraceous hosts. After the US introduction of $X$. glabratus (early 2000s?), R. lauricola moved into other species of ambrosia beetle that were present in the US prior to the introduction of $X$. glabratus. We summarize what is known about the lateral transfer of this pathogen to new, potential vectors. Extracted mycangia, heads (taxa with pre-oral mycangia), or intact bodies (taxa with mycangia in other locations) of 14 species of Ambrosiodmus, Euwallacea, Premnobius, Xyleborus, Xyleborinus and Xylosandrus were assayed from six collections from native species in the southeastern USA (Persea borbonia, $P$. palustris and $P$. humilis) and four from avocado (P. americana). Raffaelea lauricola was identified based on its cultural phenotype, and the identity of a random subset of isolates was confirmed with taxon-specific microsatellite markers. The pathogen was recovered from $34 \%$ (246 of 726 ) of the individuals that were associated with the native Persea spp., but only 6\% (58 of 931) of those that were associated with avocado. Over all studies, R. lauricola was recovered from 10 of the ambrosia beetle species, but was most prevalent in Xyleborus congeners. The potential impacts of $R$. lauricola's promiscuity are discussed.

Species diversity of Leptographium and Grossmannia blue-stain fungi associated with root-feeding beetles in loblolly pine stands M. BULAND (1), B. Barnes (1), K. Klepzig (2), K. Gandhi (1), C. Villari (3), (1) D.B. Warnell School of Forestry, University of Georgia, Athens, GA, USA; (2) USDA Forest Service, Asheville, NC, USA; (3) D.B. Warnell School of Forestry and Natural Resources, University of Georgia, Athens, GA, USA

Loblolly pine (Pinus taeda L.), the most commercially important tree species in the southeastern United States, is experiencing higher levels of mortality around the Fall-line and Upper coastal plain regions in Alabama and Georgia. Root-feeding beetles (e.g., Hylastes and Hylobius spp.) and their Ophiostomatoid fungal obligates (e.g., Leptographium and Grosmannia species) have been associated with areas of dieback; however, the actual roles of these beetles and their fungal obligates in loblolly pine mortality is yet to be established. Our research objectives are as follows: 1) to assess the diversity and composition of Ophiostomatoid fungal complex associated with these root-feeding taxa in loblolly pine stands; and 2) to analyze whether pathogen pressure and species composition varies in stands with various management practices and environmental conditions. Root-feeding beetles will be collected in loblolly pine stands with differing management histories, such as thinning regimens, and across multiple seasons. DNA will be extracted directly from the adult beetles and species-specific PCR primers will be developed to determine the incidence of each fungal species. Results from this study will assist in better understanding of the role of Ophiostomatoid fungi in the dieback and mortality of loblolly pine, and their implications for sustainable forest management practices in the southeastern U.S. forests.

Two novel fungal symbionts of invasive Kuroshio shot hole borer (Euwallacea sp. nr. fornicatus) causing Fusarium Dieback on woody hosts in California

J. D. CARRILLO (1), J. S. Mayorquin (2), F. Na (2), J. Stajich (3), A. Eskalen (1), (1) Department of Plant Pathology and Microbiology, UC Riverside, Riverside, CA, USA; (2) UC Riverside, Riverside, CA, USA; (3) University of California Riverside, Riverside, CA, USA

Fusarium Dieback (FD) is a new disease complex affecting hardwood trees in California and is vectored by two distinct, but related ambrosia beetles (Euwallacea sp. nr. fornicatus) called Polyphagous shot hole borer (PSHB) and Kuroshio shot hole borer (KSHB). This pest-disease complex causes branch dieback symptoms which affect several native plant species, landscape trees, as well as the agricultural commodity, avocado. The discovery of two different vectors of FD in California initiated an investigation to compare the fungal symbionts of PSHB and KSHB. Eight isolates of Fusarium sp. and Graphium sp., respectively, were recovered from the mycangia of adult KSHB females captured in three different locations (Bonsall, El Cajon, Escondido) in San Diego County and compared to the known symbiotic fungi of PSHB. Multi-gene phylogenetic analysis and morphological comparison revealed the Fusarium sp. and Graphium sp. obtained from KSHB were related to, but distinct from the fungal symbionts, Fusarium euwallaceae and Graphium euwallaceae associated with PSHB. Pathogenicity tests on healthy, young avocado plants revealed Fusarium sp. and Graphium sp. to be pathogenic. The novel fungal species associated with KSHB are now described as Fusarium kuroshium sp. nov. and Graphium kuroshium sp. nov. These findings highlight the pest disease complexes of KSHB-FD and PSHB-FD as distinct, but collective threats adversely impacting woody hosts throughout California.

Genetic diversity and spatial structure of the Walnut Twig Beetle, Pityophthorus juglandis, the vector of Thousand Cankers in USA and Europe E. Oren (1), W. Klingeman (1), J. Moulton (1), P. Lambdin (1), M. Faccoli (2), D. HADZIABDIC (1), (1) University of Tennessee, Knoxville, TN, USA; (2) University of Padua, Legnaro, ITALY

Thousand Cankers Disease (TCD) is caused by the interactions between the fungal pathogen Geosmithia morbida, an insect vector Pityophthorus juglandis, and the plant hosts, Juglans spp. and Pterocarya spp. Disease is manifested by canopy thinning, which results from numerous cankers initiated during aggregated attacks on hosts and consequent excavation and tunneling by the insect vector. Although the TCD has caused significant mortality among native and non-native walnut tree populations, we have limited knowledge regarding genetic diversity of the insect vector. The study objective was to evaluate genetic diversity and population structure of $P$. juglandis collected across a broadly representative geographical area where TCD occurs. Pityophthorus juglandis specimens from 40 subpopulations across U.S. and Italy were genotyped using 12 polymorphic microsatellite loci. Results demonstrated a high genetic diversity, presence of population structure, and limited gene flow that has occurred between these groups. High levels of genetic diversity present across all groups is likely to best be explained by human mediated movement of infested plant material from multiple source locations on multiple occasions. These findings further substantiate previous hypotheses that infer thousand cankers disease has been established in these different source locations for a longer period of time than was originally proposed.

Occurrence of Xanthomonas vasicola on maize seeds in the United States

S. ARIAS (1), C. C. Block (1), D. A. Mayfield (1), T. A. Jackson-Ziems (2), K. D. Broders (3), G. P. Munkvold (1), (1) Iowa State University, Ames, IA, USA; (2) University of Nebraska-Lincoln, Lincoln, NE, USA; (3) Colorado State University, Fort Collins, CO, USA

Xanthomonas vasicola, the causal agent of bacterial leaf streak (BLS) of maize, was officially reported in the U.S. in 2016. The disease has reached epidemic proportions in areas of Colorado, Kansas and Nebraska with disease incidence levels greater than $90 \%$ in several fields and disease severities of more than $50 \%$ leaf area infected. It is not known if seed is a pathway in the spread of disease. Therefore, the objective of this study was to determine the incidence of $X$. vasicola on maize harvested from fields with BLS. To evaluate the role of seed contamination, we obtained seed samples from 30 fields in Colorado, Iowa, and Nebraska, all with moderate to severe BLS symptoms. Seed testing involved shaking 400-seed subsamples in phosphate-buffered saline with $0.05 \%$ Tween- 20 and $20 \mathrm{mg} / \mathrm{L}$ nystatin for 4 or 20 hours, followed by centrifugation, DNA extraction and qPCR amplification. Serial dilutions of seed washes were spread onto CS20-ABN semi-selective agar medium. In initial assays, samples from four fields were identified as likelypositives by TaqMan PCR with Ct values under 30; eight samples were weakly positive (Ct values of 30-32); 18 samples had $\mathrm{Ct}$ values $\geq 33$ and are likely negative. However, live bacteria have been recovered from only one of the 30 fields. Testing of additional seed samples from all 30 fields continues, with particular emphasis on the recovery of bacteria from the likely-positive fields, but it is clear that seed contamination is not extensive. 
Preliminary assessment of insect-associated Geosmithia species in Tennessee

K. CHAHAL, R. O. Gazis, J. Grant, D. Hadziabdic, P. Lambdin, W. Klingeman, M. T. Windham, University of Tennessee, Knoxville, TN, USA

Geosmithia sp. 41 and G. morbida are insect vectored and are the causal agents of Foamy Bark Canker disease on California live oak, and Thousand Cankers Disease of black walnut, respectively. To explore Geosmithia species associations with forest insects in Tennessee, USA, bark and ambrosia beetles were collected using ethanol lured traps. To isolate fungi, beetle 'wash offs' were plated on nutrient media. Fungi were re-isolated onto clean plates until axenic cultures were obtained. Cultures morphologically similar to Geosmithia spp. were selected for molecular identification using the Internal Transcribed Spacer (ITS) and the Elongation Factor alpha regions (TEF-1) for species identification. The 30 obtained strains were grouped within the "G. pallida" species complex and corresponded to previously reported, but not formally described Geosmithia species. Another 8 strains were identified as G. obscura, Gesomithia sp. 10, and Geosmithia sp. 21. We did not find signs of vector specificity. Vectors associated with the recovered Geosmithia spp. are: Cnestus mutilatus, Hylocurus spadix, Madoniella dislocatus, Stenomimus pallidus, Xyleborinus saxesenii, Xylobiops basilaris, and Xylosandrus crassiusculus. Our preliminary results indicated a close genetic relationship between some of the strains discovered in Tennessee and the lineage Geosmithia sp. 41. This report raises awareness of a potential emergent threat to the native forests, especially the native oak species.

Potential alternative vectors of Geosmithia morbida (thousand cankers disease) in east Tennessee K. CHAHAL, R. O. Gazis, J. Grant, D. Hadziabdic, P. Lambdin, W. Klingeman, D. Paulsen, M. T. Windham, University of Tennessee, Knoxville, TN, USA

Fungal pathogen, Geosmithia morbida along with its vector, Pityophthorus juglandis are the causal agents of Thousand Cankers Disease (TCD) in black walnut. In the last two decades, TCD outbreaks have been reported in the eastern and western U.S. In the eastern U.S., G. morbida has also been detected on other insect species. To better access the potential for alternative beetles to vector the fungal pathogen, wood boring beetles were collected using ethanol-lured traps. Traps were placed on TCD-symptomatic trees at several locations in east Tennessee across different time periods in 2016. Morphological characters and the mitochondrial cox1 gene were used to identify beetle species. Geosmithia morbida presence was validated using a molecular-based method that involved the amplification of the species-specific microsatellite marker GS004. To date, nine beetle species have been found to carry G. morbida including Cnestus mutilatus, Dryoxylon onoharaense, Monarthrum fasciatum, M. mali, Xyleborinus saxesenii, and Xylosandrus crassiusculus (Coleoptera: Curculionidae: Scolytinae), Stenomimus pallidus (Coleoptera: Curculionidae: Cossoninae), Xylobiops basilaris (Coleoptera: Bostrichidae), and Melanophthalma spp. (Coleoptera: Latridiidae). These findings raise the awareness of the potential threat imposed by alternative vectors that may contribute to the spread of G. morbida within the native range of black walnut.

Impact of late season vine-kill on incidence of ' $C$ andidatus Liberibacter solanacearum' in potato tubers F. WORKNEH (1), L. Paetzold (1), C. M. Rush (2), (1) Texas A\&M AgriLife Research, Bushland, TX, USA; (2) Texas A\&M AgriLife Research, Amarillo, TX, USA

Late season potato infection by 'Candidatus Liberibacter solanacearum' (Lso) has become a growing concern since it was discovered that Lso continues to develop in storage. It is a routine practice for growers to kill potato vines weeks prior to harvest to promote skin set for reducing the likelihood of bruise and rot in storage. However, the effect of vine-kill on development of Lso in tubers from plants infected late in the season is currently unknown. To address this question, caged plants were infested with bacteriliferous psyllids 7 and 2 days before, and 2 and 7 days after vine-kill in replicated trials. The vines were killed with chemicals possessing slow (reglone) and fast (sulfuric acid) vine-kill activities, and tubers were dug about 4 weeks later and evaluated for Lso and zebra chip symptoms. Over $85 \%$ of tubers from plants infested 7 days before vine-kill tested positive for Lso in both chemical treatments. Plants infested 2 days before vine-kill had nearly 30 and $50 \%$ of tubers testing positive, in the slow and fast chemicals, respectively, with no significant difference between the two $(P>0.05)$. However, none of the tubers from plants infested 2 days or 7 days after vine-kill were positive. The study showed that Lso can reach tubers if psyllid infestation occurs as late as 2 days before vine-kill and that the pathogen continues to develop in tubers and cause symptoms in the soil. These results have significant implications for psyllid management.

Pathogenicity of Verticillium dahliae Klebahn in rootstock rose cv. Manetti

R. GARCIA-VELASCO, G. Dominguez-Arizmendi, E. A. Chavarro-Carrero, M. E. Mora-Herrera, Universidad Autónoma del Estado de México, Tenancingo, MEXICO

Verticillium dahliae is the causal agent of wilt in rose cv. Manetti. In Mexico the disease has not been reported, however its presence may represent a risk for the 1,385.5 hectares of cut rose crops. The aim of this research was to determine the pathogenicity of $V$. dahliae in rootstock rose cv. Manetti. The experiment was arranged in a completely randomized design with two treatments and 17 replicates in greenhouse. Treatments consisted of rootstocks with and without $V$. dahliae inoculum $\left(1 \times 10^{6}\right.$ conidia $\left.\mathrm{mL}^{-1}\right)$. Incidence, fresh root weight and length, fresh and dry weight of foliage were evaluated, in addition root tissue was placed in PDA medium to recover the pathogen. The variables evaluated were analyzed with t-Student test $(\mathrm{P}<0.05)$. The symptoms were manifested 18 days after inoculation (dai) in all inoculated plants, and death of some of these occurred 56 dai. The initial symptomatology was wilting leaves at the tips of young canes, followed by yellowing of the lower leaves, defoliation and finally death of entire plant. No significant differences were found in fresh root weight and length; However, fresh and dry weight of foliage were lower when the plants were inoculated with the pathogen, reducing their biomass by $28 \%$. The microorganism was recovered in $60 \%$ of the inoculated plants. The results suggest that $V$. dahliae is the pathogen responsible of wilt disease in the rootstock rose cv. Manetti, causing biomass decreasing and even the death of the plant.

\section{Investigations on the lily bulb- and root rot disease complex involving multiple pathogenic fungi and the root lesion nematode Pratylenchus} penetrans

D. LAKSHMAN (1), K. Kamo (2), P. Vieira (3), R. Pandey (4), (1) USDA-ARS, beltsville, MD, USA; (2) USDA-ARS, FNPRU, National Arboretum, Beltsville, MD, USA; (3) Virginia Polytechnic Institute and State University, Blacksburg, VA, USA; (4) Sustainable Agricultural System Lab, beltsville, MD, USA

Lilies are popularly grown for flowers in the garden and commercially as a cutflower. A survey was conducted to diagnose and assess pathogenic fungi from rotted bulbs and roots from Easter lilies, Lilium longiflorum cv. Nellie White grown in the U.S. Pacific Northwest. Randomly-selected fungal isolates were identified by morphological characteristics, microscopy, and molecular analyses using ITS rDNA sequences. The fungal isolates were identified as Fusarium oxysporum (5 isolates), F. tricinctum (2 isolates), and Ceartobasidium sp. AG-I isolates (2 isolates). In addition, several Trichoderma isolates $(\mathrm{n}=15)$ were obtained from diseased bulbs and roots. For the pathogenicity assay, pure cultures of each isolate were used to infect the roots of Easter lily plants growing in vitro. Moreover, Easter lily plants were inoculated with fungal isolates either with or without prior inoculation with Pratylenchus penetrans, a root lesion nematode species commonly found parasitizing Easter Lily roots. All the characterized fungal species, including four Trichoderma isolates, caused necrosis and root rots when inoculated individually. However, symptoms developed more rapidly with prior exposure to $P$. penetrans. Fungi with identical morphologies to the source inocula were re-isolated from root lesions, thus proving Koch's postulates. Combined inoculation of lily seedlings with all the fungal pathogens with or without $P$. penetrans further accelerated disease development and severity; demonstrating the complex nature of this disease. 
Host preferences of Colletotrichum species isolated from mango and tree tomato in Colombia

L. Cabrera Villamizar (1), C. P. Rojas (1), C. J. Pardo De la hoz (1), S. Rojas (1), M. Mideros (1), L. Lopez (2), P. Jimenez (3), S. RESTREPO (1), (1)

Universidad de los Andes, Bogota, COLOMBIA; (2) Universidad Nacional, Bogota, COLOMBIA; (3) Univ Militar Nueva Granada, Bogota, COLOMBIA

The high incidence of anthracnose symptoms due to Colletotrichum species is one of the main problems in crop production in Colombia. However, there is a lack of studies dealing with the relationship that different fungal species have with their hosts, whether they present any host preference, or even host specificity or not. In Colombia, diseases caused by species in this genus are particularly severe in mango (Mangifera indica) and tree tomato (Solanum betaceum) crops. In a previous study, we characterized the Colletotrichum species attacking these crops. Now, this study aims to determine whether isolates collected from tree tomato and mango show any host preference. Isolates presenting Colletotrichum morphologies were identified using their macroscopic and microscopic morphology, and molecular tools. We performed inoculation essays in both entire plant and detached leaves with strains corresponding to the species C. asianum, C. gloeosporioides, C. tamarilloi and C. theobromicola. Latency period, aggressiveness, sporulation and fitness were recorded to evaluate the pathogenicity of each Colletrotrichum strain on the different hosts. The results found, in whole plants as well as in detached leaves, agreed. Our results did not show strong evidence for host specificity, but several strains showed different degree of host preference. This result is relevant due to the fact that both crops are cultivated in the same geographical areas.

Pathogenicity of Stemphylium vesicarium on asparagus and onion

J. Foster (1), C. S. Tayviah (1), B. D. Gossen (2), M. R. MCDONALD (1), (1) University of Guelph, Guelph, ON, CANADA; (2) Agric \& Agri-Food Canada, Saskatoon, SK, CANADA

Stemphylium vesicarium (Wallr.) E. Simmons (teleomorphs Pleospora herbarum or Pleospora alli) has a diverse host range including crops such as asparagus, Allium spp. and pear. The pathogen has recently become a problem in asparagus and onion in Ontario, Canada. In both crops, small elliptical lesions develop on infected tissue, which may coalesce, resulting in elongated lesions in asparagus and leaf dieback in onion. Cultivars of asparagus and onion were inoculated with isolates from onion and asparagus under controlled conditions, to determine cultivar susceptibility and aggressiveness of isolates. Field trials were conducted to determine the susceptibility of cultivars to the mix of naturally-occurring isolates. For the controlled environment trials, the asparagus spears were wounded prior to inoculation and onion leaves were wiped with cheesecloth to remove the wax, which probably caused microwounds. All isolates were pathogenic on asparagus and onion. Differences in aggressiveness of the isolates were found. Source host was not a factor for onions, but some onion isolates were less aggressive on asparagus spears. There were differences in cultivar susceptibility among onions; cv.'s Pontiac, Hendrix and Milestone had less leaf dieback than cv. Highlander in the field and controlled environment studies. There was no correlation between the number of lesions on young onion plants and the extent of leaf dieback. Asparagus cultivars differed in susceptibility to purple spot (spear infection) in both field trials and one detached spear trial. However, results varied with field site and inoculation method. There was no correlation between spear infection and the severity of foliar disease in asparagus in the field.

\section{Identification of Colletotrichum Species Causing Anthracnose of Pomegranate in Florida}

K. V. XAVIER (1), A. Nepal (2), N. Peres (1), G. E. Vallad (3), (1) Gulf Coast Research and Education Center; University of Florida, Wimauma, FL, USA; (2) Oregan State University, Central Point, OR, USA; (3) Gulf Coast Research and Education Center, University of Florida, Wimauma, FL, USA

Colletotrichum sp. has been identified as the major pathogen of pomegranate (Punica granatum) in Florida. Correct identification of the causal agent is important in developing the correct management strategies. Species-specific primers for Colletotrichum acutatum and C. gloeosporioides showed that more than $75 \%$ of the isolates recovered from pomegranate are C. gloeosporioides. Multigene sequence analyses confirmed that these isolates grouped within C. acutatum and C. gloeosporioides complexes. The pathogenicity of representative isolates from each complex was tested on detached pomegranate fruits. Results showed that isolates from the C. acutatum species-complex were more aggressive than those from the C. gloeosporioidesspecies complex. Fungicide sensitivity tests and cross-infection assays on strawberries, blueberries, citrus, and mango are in progress. Crosspathogenicity of Colletotrichum species with other crops could complicate disease management and breeding efforts.

Genome wide association mapping of resistance to tan spot in durum wheat N. N. Galagedara (1), X. Li (1), S. Chao (2), S. Xu (2), J. D. Faris (2), Z. LIU (1), (1) North Dakota State University, Fargo, ND, USA; (2) USDA-ARS Northern Crop Science Laboratory, Fargo, ND, USA

Tan spot, caused by Pyrenophora tritici-repentis, is a major foliar disease on all cultivated wheat crops. The disease is known to involve race-specific susceptibility determined by three necrotrophic effector and host sensitivity gene interactions, including Ptr ToxA-Tsn1, Ptr ToxB-Tsc2 and Ptr ToxCTsc1. Recently, QTL for race-nonspecific resistance have also been identified. Compared to hexaploid wheat, sources of resistance in durum are limited, and genetics of resistance in durum background are not well understood. The objectives of this study are to identify sources of resistance and to locate QTL for tan spot resistance in a core durum collection using a genome wide association study (GWAS). Disease evaluations showed that seventeen lines were highly resistant to all races tested. GWAS employed over 12,000 mapped genotype-by-sequencing SNP markers. Sensitivity to Ptr ToxA, which is conferred by Tsn1, was mapped to the chromosome 5B as expected, but it did not significantly associate with susceptibility to the disease caused by races 1 and 2. In contrast, genomic regions on $1 \mathrm{AS}$ and 2BS, likely corresponding to $T s c 1$ and $T s c 2$ locus, respectively, were identified as major QTL. The genomic region on 3BL conferring race-nonspecific resistance was also identified for all races in GWAS. This work indicates race-specific interactions of Ptr ToxC-Tsc1 and Ptr ToxB-Tsc2 as well as race-nonspecific interaction play significant roles in tan spot development in durum.

\section{Pathogenicity of Rhizoctonia solani and Phytophthora nicotianae to Brassicaceae Biofumigant Cover Crops} P. Liyanapathiranage, T. Simmons, F. BAYSAL-GUREL, Tennessee State University, McMinnville, TN, USA

Soilborne diseases reduce plant performance, increase costs to the grower and cause potential ecological damage to the natural environment. Some soilborne pathogens may have broad host ranges and crop species may be susceptible to several different pathogens. The use of biofumigant cover crops has been explored most extensively in vegetable, fruit and flower production. However, their use and value have not been documented in woody ornamental nursery production. The objective of this study was to evaluate 15 biofumigant cover crops in the Brassicaceae family for susceptibility to Rhizoctonia solani and Phytophthora nicotianae. Sterilized soil was inoculated with $R$. solani agar slurry and P. nicotianae infested rice grains. Control pots received only sterilized rice grains or pathogen free agar slurry. Cover crops were sown into the infested soil and disease incidence was recorded. Data was analyzed using MINITAB statistical software. Rhizoctonia and Phytophthora disease incidences were significantly lower in Oilseed radish (Raphanus sativus), mustard (Sinapis alba), Amara mustard (Brassica carinata) and Oriental mustard (B. juncea) than the other biofumigant cover crops. Sylvetta green arugula (Diplotaxis tenuifolia) and Dragon's tongue arugula (D. tenuifolia) were the most susceptible cover crops to both pathogens. Future research will be conducted using selected cover crops to evaluate their ability in controlling soilborne diseases in field nursery production.

Host specificity and survival of Colletotrichum species on celery and common weeds S. Reynolds (1), M. J. Celetti (2), K. S. Jordan (1), M. R. MCDONALD (1), (1) University of Guelph, Guelph, ON, CANADA; (2) OMAFRA, Guelph, ON, CANADA

Multiple species within the Colletotrichum acutatum species complex cause anthracnose on many crops, and can survive asymptomatically on other plants. The objective was to determine the pathogenicity of isolates of $C$. fioriniae and C. nymphaeae, and determine if they survive on some common 
weed species. Conidia of C. fioriniae from celery and apples and C. nymphaeae from strawberry were inoculated onto the foliage of celery (cv. TZ 9779) and five weed species: redroot pigweed (Amaranthus retroflexus), lamb's quarters (Chenopodium album), oak-leaved goosefoot (Chenopodium glaucum), yellow nutsedge (Cyperus esculentus), and common groundsel (Solanum sarachoides). Leaf tissue segments were excised at 10 and 20 days post inoculation, and plated directly or surface-sterilized and plated on semi-selective agar to determine percent survival. All isolates caused typical leaf curl symptoms on celery. Data for 10 and 20 days post inoculation were pooled. Weeds remained asymptomatic; however, plated leaf excisions from all weeds and celery showed Colletotrichum growth (25-85\% of non-sterilized segments). Survival of the two Colletotrichum species was high on all weeds except yellow nutsedge (3-44\% survival) and was highest on lamb's quarters (50-90\% survival). Endophytic colonization (growth from surface sterilized leaves) was low for non-celery isolates on celery. These five weeds support growth of Colletotrichum species and may serve as a source of inoculum for celery.

\section{Cross pathogenicity of Diaporthe gulyae on sunflower and weed hosts}

B. KONTZ (1), A. Adhikari (1), T. Olson (1), P. O. Johnson (1), S. G. Markell (2), F. M. Mathew (1), (1) South Dakota State University, Brookings, SD, USA; (2) North Dakota State University, Fargo, ND, USA

Diaporthe gulyae is one of the fungal pathogens causing Phomopsis stem canker of sunflower (Helianthus annuus L.) in the United States, in addition to Diaporthe helianthi and Diaporthe stewartii. In 2016 D. gulyae was isolated from asymptomatic kochia (Kochia scoparia L.) and lambsquarters (Chenopodium album) plants sampled from a commercial sunflower field in Hyde County, South Dakota. The identity of $D$. gulyae was confirmed using morphology and phylogenetic analyses of the internal transcribed spacer (ITS) and translation elongation factor 1-alpha (EF1- $\alpha$ ) gene regions. In order to perform Koch's postulates, greenhouse experiments are being setup to test the cross-pathogenicity of pure D. gulyae isolates recovered from kochia, lambsquarters and sunflower. Briefly, the stem-wound method developed by Mathew et al. (2015) will be used to inoculate 18 plants of sunflower cv. 'HA 288' (susceptible check), kochia and lambsquarters using a D. gulyae-infested agar plug. At 14 days after inoculation, all plants will be rated for disease severity using a 0-5 rating scale. Koch's postulates will be completed by recovering the pathogen from the inoculated plants and confirming its identity via phylogenetic analysis. This research will possibly confirm the role of kochia and lambsquarters as alternative hosts for $D$. gulyae, especially in commercial, no-tillage systems involving sunflower.

Determination of rose black spot (Diplocarpon rosae) race diversity in Canada to facilitate targeted disease resistance breeding A. M. POLEATEWICH (1), I. Perez-Valdes (2), P. Sandhu (2), T. Banks (2), D. Somers (2), (1) University of New Hampshire, Durham, NH, USA; (2) Vineland Research and Innovation Centre, Vineland Station, ON, CANADA

Black spot caused by Diplocarpon rosae is a serious disease of roses worldwide and is best controlled using resistant cultivars. Breeding for race-specific resistance relies on knowing which pathogenic races occur in target geographical regions. To date, eleven pathogenic races have been documented based on a set of nine host differentials. There is limited knowledge of the geographic distribution of these races worldwide and in Canada. The objective of this study was to examine the distribution of rose black spot genetic diversity in Canada using host differentials and to incorporate this information into the Canadian Hardy Rose breeding program. Over 600 D. rosae single spore isolates were collected from six provinces in Canada from $2014-2016$. Isolates were characterized on the nine differential cultivars in a detached leaf assay. Each host/isolate combination was evaluated and scored after 7 and 14 days. Each lesion was scored on a 0-5 scale based on the presence of acervuli and conidia. A host/isolate combination was considered incompatible when symptoms were scored 0,1 or 2 . A compatible interaction was between 3 and 5 . Race 10 was the most predominant race found in all six provinces. Races 7, 8, 9 and 11 were found in lower frequency. Several isolates resulted in infection patterns that did not match any of the published patterns suggesting the presence of new races in Canada. Among these new patterns, six isolates exhibited the ability to infect all nine differential cultivars which has not previously been reported in the literature.

Genetic Structure, Geography and Host Specialization within Populations of Pseudoperonospora cubensis in the United States A. THOMAS, I. Carbone, P. Ojiambo, North Carolina State University, Raleigh, NC, USA

Pseudoperonospora cubensis, the causal agent of cucurbit downy mildew (CDM), re-emerged in the US in 2004 overcoming host resistance that was effective for over 40 years. Host compatibility assays conducted using 22 P. cubensis isolates and 15 host differentials revealed the presence of five pathotypes in the US including pathotypes 1 and 3 that were previously known to exist only in Asia. The role of different cucurbit host types and geography in shaping the genetic structure of $P$. cubensis was examined using 93 isolates collected from diverse cucurbits with the double digest Restriction Associated DNA Sequencing technology. Results obtained from phylogenetic and STRUCTURE analyses revealed two distinct host specialized lineages and significant structuring between lineage I and II isolates of $P$. cubensis $\left(F_{\mathrm{ST}}=0.72\right)$. Lineage II was specialized on Cucumis sativus and lineage I was specialized on Cucurbita spp. and Citrullus lanatus. A distinct genetic cluster within lineage I was associated mainly with $C$. lanatus. There was no evidence of geographical structuring of $P$. cubensis populations. The association of lineage II with new pathotypes and a lack of genetic differentiation among lineage II isolates suggest that lineage II may be associated with the resurgence of CDM in the US. Information generated here may aid in the development of diagnostic tools and more precise management strategies for CDM.

\section{Survey of fungi from symptomatic leaves of cornelian cherry dogwood (Cornus mas) in eastern Tennessee}

T. COLLINS (1), M. M. Dee (2), D. D. Hensley (2), B. H. Ownley (2), (1) University of Tennesse, Department of Entomology and Plant Pathology, Knoxville, TN, USA; (2) University of Tennessee, Department of Entomology and Plant Pathology, Knoxville, TN, USA

Cornelian cherry dogwood (Cornus mas) is an ornamental, fruit-bearing tree, with potential as a specialty crop in Tennessee. Cornus mas is native to southwestern Asia and southern Europe, where few pathogens have been reported. The aim of this project was to identify potential plant pathogens of $C$. mas when grown for the ornamental market in Tennessee. Field trials were established in Oak Ridge and Crossville, TN. Symptomatic leaves of $C$. mas were collected from the field trial plantings and nearby landscape trees, and more than twenty fungi were isolated, many of which are known pathogens of other hosts. However, only Fusarium sporotrichioides caused moderate to severe disease symptoms in pathogenicity assays. Isolates of $F$. sporotrichioides were grown on various media to determine fungal growth rate and morphological characteristics. Identification of $F$. sporotrichioides was based on morphology and sequences of the internal transcribed spacer region of 18S ribosomal DNA and of translation elongation factor 1-alpha. DNA sequences were compared with the nucleotide database sequences of Fusarium-ID and the National Center for Biotechnology Information. Sequence identity was 95 to $99 \%$. Pathogenicity of $F$. sporotrichioides was confirmed with whole plant assays. Identification and management of pathogens of cornelian cherry dogwood could increase its potential for the ornamental market in Tennessee, and enhance its aesthetic value as a landscape tree.

Thousand cankers disease severity is not determined by Geosmithia morbida genetic grouping nor a synergistic relationship with Fusarium solani

R. A. SITZ (1,2), E. K. Luna (1), J. R. Ibarra Caballero (1), N. A. Tisserat (1), W. S. Cranshaw (1), J. E. Stewart (1), (1) Colorado State University, Fort Collins, CO, USA; (2) United States Department of Agriculture Forest Service, Moscow, ID, USA

Geosmithia morbida is the widely recognized fungal causal agent of thousand cankers disease of black walnut trees. Little is known about whether phenotypic differences occur among recently identified genetically distinct isolates of G. morbida, or if isolates of Fusarium solani (which are commonly found co-occurring with G. morbida) alter the aggressiveness of G. morbida strains. Historically, F. solani has been identified as a pathogen of black walnut trees, but the impact this pathogen has on thousand cankers disease is not known. Pathogenicity assays on walnut were conducted with 
genetically distinct isolates of $G$. morbida with and without $F$. solani (phylogenetic lineage 6). Genetic grouping did not explain variation in aggressiveness, as large differences in canker areas were observed from isolates in the same genetic grouping. Co-inoculation experiments with $G$. morbida and $F$. solani suggest that this species does not interact to increase or decrease aggressiveness on black walnuts.

Long term monitoring of cucurbit powdery mildew (Podosphaera xanthii) races in Charleston, South Carolina

C. KOUSIK (1), J. Ikerd (1), M. K. Mandal PhD (2), (1) U.S. Vegetable Laboratory, USDA, ARS, Charleston, SC, USA; (2) ORISE participant, US Vegetable Laboratory, USDA, ARS, Charleston, SC, USA

Cucurbit powdery mildew (PM, Podosphaera xanthii) can be a severe production limiting factor in greenhouses and fields. Presently seven races of $P$. xanthii have been classified using melon (Cucumis melo) differentials. We have been monitoring the PM pathogen races on melon differentials (Iran $\mathrm{H}$, Hales Best Jumbo, Vedrantais, PMR 5, PMR 45, WMR 29, Edisto 47, PI 414723 and MR1) and various other cucurbits since 2008 in an open field in Charleston, SC. Every year since 2008, severe powdery mildew was mainly observed on the melon differentials Iran H, Hales Best Jumbo and Vedrantais indicating that race 1 was the predominant race. We did not observe powdery mildew during the summer months on other melon differentials In two years mild PM symptoms were observed on PMR 45 primarily in the fall indicating the potential presence of race 2, but the symptoms were not severe. Other cucurbit cultivars including cucumber (Straight 8), watermelon (Mickey Lee), Squash (EPSN), Pumpkin (Baby Boo), bottle gourd, and butternut squash (Waltham) were also susceptible to PM. However cultivars of smooth gourd (Luffa, Harita), ridge gourd (Surekha) and bitter Gourd (green long and white bitter) were very resistant. Resistance to PM race 1 and 2 currently available in numerous melon cultivars should prove useful for managing this disease in South Carolina. Similar routine monitoring of PM pathogen across the U.S can prove useful for appropriate deployment of resistant cultivars.

\section{Comparing inoculation methods to study the aggressiveness of Diaporthe aspalathi causing southern stem canker of soybean}

K. GHIMIRE (1), B. Kontz (1), D. L. Smith (2), C. A. Bradley PhD (3), D. S. Mueller (4), K. A. Wise (5), N. Braun (1), F. M. Mathew (1), (1) South Dakota State University, Brookings, SD, USA; (2) University of Wisconsin, Madison, WI, USA; (3) University of Kentucky, Princeton, KY, USA; (4) Iowa State University, Ames, IA, USA; (5) Purdue University, West Lafayette, IN, USA

Stem canker of soybean (Glycine max L.) is divided into two geographically distinct diseases - southern stem canker (Diaporthe aspalathi Janse van Rensburg, Castlebury and Crous) and northern stem canker (Diaporthe caulivora (Athow and Caldwell) Santos, Vrandecic and Phillips). Collectively, the two pathogens caused soybean yield losses of an estimated 326,600 metric tons in the United States in 2014. In this study, we are comparing inoculation methods to determine differences in aggressiveness of $D$. aspalathi isolates. Four inoculation methods including the cut seedling assay, toothpick method, mycelium contact, and spore injection method were tested on soybean cv. Williams 82 under greenhouse conditions $\left(25-27^{\circ} \mathrm{C}\right.$ and 12 $\mathrm{h}$ daylight). For each of the methods, five isolates representing each of five Kentucky counties (Ballard, Breckinridge, Cumberland, Daviess, and Hopkins) were used to inoculate 10 plants per isolate-inoculation method in each of the two experimental repeats. At 14 days post-inoculation, disease severity was evaluated based on lesion length and using a disease rating scale (0 to 5). A significant two way inoculation method $\times$ isolate $(P \leq 0.05)$ affecting lesion length was observed. Additionally, the lesion length produced by the five D. aspalathi isolates varied based on the type of inoculation method used. The understanding of aggressiveness of $D$. aspalathi isolates will be useful to develop strategies for management of southern stem canker.

Characterization of Fusarium spp. isolates recovered from dry bean in Michigan

K. OUDMAN, J. L. Jacobs, M. Chilvers, Michigan State University, East Lansing, MI, USA

Root rot, damping-off, and seed rot are problems associated with dry bean stand establishment in Michigan. Significant losses can result when cool temperatures, wet soil conditions, and soil compaction occur after planting. It is common for growers to replant sections or whole fields due to poor stand establishment. In addition to increased cost of replanting, these diseases can lead to weakened root systems and delayed plant development and subsequent yield reductions. The objectives of this study were to identify which pathogens are associated with poor stand establishment due to seed rot, damping-off, and root rot of dry bean production in Michigan, determine which organisms are pathogenic, and identify the stage of plant development infected. A survey was conducted in 2014, 2015, and 2016 in fields with stand establishment issues to identify root rot pathogens. A total of 475 Fusarium spp. isolates were recovered from plant samples. Freeze-dried mycelium was used for DNA extraction using a CTAB protocol. Isolates were identified using partial translation elongation factor and intergenic spacer regions. Thus far, a total of 11 unique Fusarium spp. have been identified. Pathogenicity and aggressiveness were evaluated with a representative Fusarium spp. panel on red kidney and black bean in a seedling assay. Isolates belonging to Fusarium solani species complex were the most aggressive root rot pathogens.

\section{Screening inoculation methods and recombinant inbred lines for partial resistance to Phytophthora sojae in soybean} R. D. S. CHOWDHURY, SDSU, Brookings, SD, USA

Phytophthora root and stem rot (Phytophthora sojae Kaufmann and Gerdemann) is one of the most important diseases soybean (Glycine max) in the United States. In South Dakota, the diversity of $P$. sojae pathotypes has increased in South Dakota, and therefore, it is important to identify new resistance sources to manage this pathogen. To identify a suitable inoculation method for screening soybean genotypes with partial resistance to $P$. sojae, three methods (inoculum layer method, tray test) and rice grain inoculation method) were evaluated on Glycine. max cv. Williams and Glycine. max cv. Surge using two isolates (PS-14-F14 virulent on Rps7 and PS-15-TF3 virulent on all Rps differentials) in the greenhouse. Based on the recovery of $P$. sojae and correlation with lesion length caused by the $P$. sojae isolates at 7 days post inoculation, the inoculum layer method was selected. One hundred recombinant inbred lines (RILs) population derived from the cross between G. max cv. Surge and G. soja (PI 468916) were screened for partial resistance to PS-15-TF3 and PS-14-F14 using the inoculum layer method in the greenhouse. Upon comparing the response of RILs to PS-15-TF3 and PS-14-F14, 9 RILs had relatively shorter lesion length (lesion size $0-5 \mathrm{~mm}$ ) than G. soja and G. max cv. Surge. Our results suggest that the nine RILs could be a good resistance source for developing commercial soybean varieties with $P$. sojae resistance.

\section{Pathotypes of Phytophthora sojae from South Dakota soybean fields} R. D. S. CHOWDHURY, SDSU, Brookings, SD, USA

Phytophthora root and stem rot, caused by Phytophthora sojae Kaufmann and Gerdemann is one of the yield-limiting soybean (Glycine max) diseases in South Dakota. This study was undertaken to determine $P$. sojae pathotype diversity in South Dakota. Soil samples were collected from 384 commercial soybean fields in South Dakota during a three year survey (2013 to 2015), and 114 P. sojae isolates were recovered by soil baiting with the susceptible soybean cultivar Williams. From the 114 P. sojae isolates, 70 isolates were randomly selected for pathotyping, and each isolate was inoculated onto 13 soybean differentials carrying standard Rps genes using the hypocotyl inoculation method. Forty-four different virulence pathotypes were identified from the 70 isolates. About 96, 93, 87, 84, 84 and 79\% of the isolates of P. sojae were virulent on differential cultivars carrying Rps 7 , Rps $1 \mathrm{a}$, Rps $1 \mathrm{k}$, Rps $1 \mathrm{~b}$, $R p s 1 \mathrm{c}$ and $R p s 1 \mathrm{~d}$ genes. For the three years, the mean complexity measuring the number of Rps genes with which a specific isolate has a susceptible interaction ranged from 6.58 to 6.90, and the Shannon Diversity index which measures the relative differences in pathotype among the isolates ranged from 2.4 to 2.76. Our study suggests that the complexity of $P$. sojae pathotypes is continuing to increase in South Dakota, and farmers should combine use of partially resistant soybean cultivars with other strategies to manage Phytophthora root and stem rot in their field. 
In the United States, soybean sudden death syndrome (SDS) is caused by the soil borne ascomycete Fusarium virguliforme (Fv). Historically, corn has been grown in rotation with soybean; however, recent research revealed the asymptomatic host range of $F v$ included corn. Additionally, many early interactions between $F v$ and symptomatic and asymptomatic hosts remain to be elucidated within in planta or field samples. Herein, roots were collected from soybean and corn in $F v$-inoculated and infested field experiments at 1,2, 4, 8, 12, and 16 weeks after planting (WAP). DNA was extracted from ground roots for $F v$ quantification by a real-time qPCR assay. Pathogen DNA was detected in corn roots as early as 1 WAP within the inoculated soil, and $2 \mathrm{WAP}$ within infested soil. In soybean, $F v$ was detected $1 \mathrm{WAP}$ within infested treatments. Ongoing detection of $F v$ at $4,8,12$ and $16 \mathrm{WAP}$ will reveal if $F v$ can persist in corn over the growing season. Furthermore, to dissect early interactions, a growth pouch-based bioassay was developed with $F v$ macroconidia inoculated soybean and corn seedlings. In planta pathogen colonization was assessed using real-time qPCR and staining of root tissue over 14 days post-inoculation. Both soybean and corn were colonized by $F v$, and soybean developed root symptoms. These studies will establish a foundation to dissect the molecular signaling underpinning pathogen attachment, colonization and proliferation within symptomatic and asymptomatic hosts.

Towards sustainable solutions for Fusarium wilt: Ecology of putative pathogenicity factors in populations of Fusarium oxysporum from banana G. R. TESDALL, F. A. Magdama, M. D. M. Jimenez-Gasco, The Pennsylvania State University, University Park, PA, USA

Banana is a staple food crop in the developing world, where $85 \%$ of production is traded and consumed locally and exports generate $\$ 5$ billion USD annually. Fusarium wilt of banana, caused by Tropical Race 4 (FocTR4) of Fusarium oxysporum f. sp. cubense (Foc) is threatening Cavendish cultivars that dominate the export industry. To design sustainable solutions to manage FocTR4, for which no marketable resistant cultivars or chemical controls exist, scientists must understand the mechanisms of pathogenicity and the ecology of this organism, including the role of the pathogenicity-associated Secreted In Xylem (SIX) genes. In this research, SIX homologues were identified by PCR in endophytic isolates sampled from corms and roots of asymptomatic banana plants in Ecuador. Of 103 endophytes studied, $14.5 \%$ tested positive for SIX9, and 10.7\% for SIX2. SIX9 and SIX2 were also identified in all Foc isolates from Ecuador studied, in addition to SIX1, SIX4, SIX7, and SIX8a/b. SIX9 sequences found in twelve endophytic isolates from different banana cultivars, were identical to each other but phylogenetically distinct from those in Foc, and were more closely related to SIX 9 sequences in other formae speciales. Three putatively endophytic isolates carried SIX9 sequences identical to Foc. Furthermore, the role of SIX9 was investigated by comparing colonization patterns and phenotypes of banana plants inoculated with isolates carrying SIX9 and those lacking it.

The impact of pesticides on the turfgrass rhizosphere microbiome

E. BUCZKOWSI, M. Millican, P. L. Koch, University of Wisconsin-Madison, Madison, WI, USA

Pesticides are routinely applied to turfgrass landscapes, but the impact on the turf-associated microbiome is unclear. To evaluate how pesticides influence soil microbial biodiversity, turfgrass plots at the OJ Noer Turfgrass Research Station in Madison, WI were treated with a single pesticide application during the summer of 2016. Plots were treated with one of two pesticides from the herbicide, fungicide, or insecticide target groups and pesticides from each target group represented either a low or high toxicity product based on its acute oral LD50. Following application, the microbial response and recovery was monitored over the course of four weeks. Soil cores were removed at $0,1,7,14,21$, and 28 days and frozen at $-80^{\circ} \mathrm{C}$ until DNA extraction. Samples were thawed and washed to remove bulk soil and then placed in a sonicating water bath to remove the tightly adhered rhizosphere soil. DNA was extracted using a MoBio PowerSoil DNA extraction kit. Taxonomic marker regions were amplified using a KAPA polymerase system and universal primers specific to the V4 16S rRNA region. PCR products were pooled and sequenced using the Illumina MiSeq platform and raw sequencing data was processed using Mothur. As of this submission the final sequencing results have not been analyzed, but based on previous research in other systems we hypothesize that the microbial communities will initially undergo a structural change and over time return to a community of similar structure and diversity as prior to the pesticide application. This trial will be replicated in 2017.

\section{Mycobiome study reveals pathogens of Camassia in the Pacific Northwest}

G. M. FREED (1), T. C. Paulitz (2), F. M. Dugan (3), (1) Washington State University, Pullman, WA, USA; (2) USDA ARS, Pullman, WA, USA; (3) USDA ARS WRPIS, Pullman, WA, USA

Camassia (camas) is an endemic geophyte growing in wetland ecosystems of the Pacific Northwest and has cultural significance for Native American tribes. Concerted efforts are made by wetland restorationists to transplant camas bulbs, supplied by native plant nurseries, into restored sites. Our aim is to identify fungal bulb pathogens within the datasets of a mycobiome study. The purpose is to assist stakeholders in wetland restoration develop best management practices to prevent unintentional spread of pathogens to new locations and address the challenge of bulb storage rot. We profiled fungal taxa associated with camas sampled from six biogeographically distinct camas ecosystems using mycobiome analyses of camas root, tunic, basal plate, leaf, seed, rhizosphere, and proximal soil. Datasets of the Illumina MiSeq of ITS1 amplicons resulted in 3,077 operational taxonomic units ( $\geq 97 \%$ similarity). Koch's postulates were employed to test nine fungi isolated from symptomatic camas bulbs. Pathogenicity assays of inoculated (10 spores $/ \mathrm{ml}$ ) camas bulbs were incubated at two temperatures $\left(5^{\circ} \mathrm{C}\right.$ and $\left.23^{\circ} \mathrm{C}\right)$. Analyses of the rates of lesion expansion at inoculation sites were conducted using Dunnett's test of multiple comparison $(p<0.05)$. Our novel results identified Fusarium and Botrytis isolates pathogenic at $5^{\circ} \mathrm{C}$ and cold storage dramatically reduced lesion expansion of the Fusarium isolate. The Fusarium, Colletotrichum, and Trichoderma isolates tested were pathogenic at $23^{\circ} \mathrm{C}$.

\section{Successional dynamics of fungal and oomycete communities in herbicide-killed wheat roots} D. C. SCHLATTER, T. C. Paulitz, USDA ARS, Pullman, WA, USA

Dying roots of herbicide-killed weeds or volunteer plants can foster an increase in plant pathogens, such as Rhizoctonia and Pythium spp. and serve as a 'greenbridge' for pathogens infecting subsequent crops. To investigate the succession of fungal and oomycete communities after herbicide sprays, we tracked the communities in live wheat roots and killed roots following termination with Roundup (glyphosate) or SelectMax (clethodim). DNA was extracted from washed roots collected prior to herbicide application and at 3 days, 1 week, 2 weeks, and 3 weeks post-application. Fungal and oomycete ITS1 regions were PCR-amplified and sequenced (Illumina MiSeq). Fungal and oomycete communities varied significantly with sampling time and herbicide type. Myrmecridium sp. dominated fungal communities in roots at most time points in live and killed roots, but saprophytic species began to replace this taxon in herbicide-treated roots by three weeks. Pythium volutum and Lagena radicola dominated oomycete communities. $P$. volutum increased and $L$. radicola decreased in relative abundance after termination with glyphosate, but remained relatively stable in live roots or those killed with clethodim. Fungal and oomycete richness decreased over time in glyphosate-killed roots, but was relatively stable in other treatments. Oomycete pathogens in wheat roots quickly multiply after herbicide treatments, and saprophytic taxa displace pathogens over time.

\section{Potato crop yields and soil microbiome composition in response to nitrogen amendments in fumigated and non-fumigated soils} J. P. DUNDORE-ARIAS, L. K. Otto-Hanson, C. Rosen, L. L. Kinkel, University of Minnesota, St Paul, MN, USA

Soilborne pathogens are major constraints to potato production in the US. Fumigation is the primary practice used to manage soilborne pathogens in irrigated production systems. However, non-target effects of fumigation on indigenous soil microbiomes and selection for detrimental microbes are of concern. In this study, we investigated the interactive effects of fumigation and soil nitrogen amendments on potato diseases, yield, and reestablishment of indigenous soil microbial communities. Potatoes were planted in fumigated and non-fumigated field plots, with subplots composed of 5 doses of nitrogen $(\mathrm{N})$. Fumigation with either Vapam or Chloropicrin increased potato yields and reduced tuber diseases, but also reduced microbial activity 
measured as soil respiration. Additionally, higher $\mathrm{N}$ applications delayed senescence and reduced potato foliar diseases, but did not enhance yield relative to lower $\mathrm{N}$ doses. However, yields were significantly greater when higher $\mathrm{N}$ doses were applied at emergence in addition to the standard application at planting. Amplicon sequencing of soil bacteria and fungi are underway. Overall, this study advances our understanding of the relationships between fumigation, soil nutrient status, microbial community composition, and potato diseases, and of the post-fumigation assembly dynamics of soil microbiomes, and will contribute to optimized management for sustainable potato production systems.

Minimal impacts of repeated glyphosate use on wheat-associated bacterial microbiomes

D. C. SCHLATTER (1), C. Yin (2), S. H. Hulbert (2), I. Burke (3), T. C. Paulitz (1), (1) USDA ARS, Pullman, WA, USA; (2) Dept. of Plant Pathology, Washington State University, Pullman, WA, USA; (3) Dept. of Crop and Soil Science, Washington State University, Pullman, WA, USA

Glyphosate (Roundup) is the most widely used herbicide in the world and a critical tool for weed control in no-till wheat cropping systems. However, there are persistent concerns about non-target impacts of long-term glyphosate use on soil communities. We investigated the impacts of repeated glyphosate treatments on bacterial communities in the soil and rhizosphere of wheat across a geographic range of soils with and without a long-term history of glyphosate exposure. We cycled wheat in the greenhouse using soils from 4 paired fields under no-till (20+ years history of glyphosate) and no history of glyphosate. At each cycle, we terminated plants with glyphosate ( $2 \mathrm{x}$ field rate) or by removing the crown. After 4 cycles we sampled bulk and rhizosphere soil, extracted DNA, and characterized bacterial communities with Illumina MiSeq of $16 \mathrm{~S}$ rRNA genes. Location $\left(\mathrm{r}^{2}=0.13\right)$, cropping system $\left(r^{2}=0.05\right)$, year $\left(r^{2}=0.10\right)$, and proximity to the roots $\left(r^{2}=0.16\right)$ had much stronger effects on the bacterial communities than glyphosate, which only explained $2-5 \%$ of the variation. More taxa were affected by glyphosate in soil with a long history of use compared to soils with no history of use. Less than $1 \%$ of taxa were impacted by glyphosate, and more taxa increased vs. decreased in relative abundance with glyphosate. Thus, glyphosate has minimal impacts on soil and rhizosphere bacteria of wheat, though dying wheat roots after glyphosate application may favor some taxa.

The effects of penicillin injection on the bacterial phytobiome of citrus roots and petioles are dose and time dependent M. Ascunce (1), K. Shin (2), J. C. Huguet-Tapia (2), A. H. van Bruggen (2), E. M. GOSS (1), (1) Department of Plant Pathology, University of Florida, Gainesville, FL, USA; (2) University of Florida, Gainesville, FL, USA

The rise of antibiotic-resistant bacteria is a global threat, and although agricultural use of antibiotics is believed to contribute, little is known about the impact on the soil microbiome. A devastating plant pathogen worldwide is Candidatus Liberibacter asiaticus (Las), an uncultured $\alpha$-proteobacterium and causal agent of citrus greening or Huanglongbing (HLB). Trunk injections of antibiotics reduce Las titers and HLB symptoms in citrus. However, the effects of antibiotics on the bacterial phytobiome have not been addressed in detail, thus, we investigated the effects of penicillin injection ( 0 , 1000 and $6000 \mathrm{mg} \mathrm{L}^{-1}$ ) on the bacterial phytobiome of grapefruit trees. Petioles and roots from 16 HLB positive field trees and 18 HLB negative greenhouse trees were collected and their DNA extractions were subjected to $16 \mathrm{~S}$ rRNA high-throughput sequencing. Sequences were taxonomically assigned to 21 bacterial phyla with Proteobacteria accounting for almost $70 \%$ of the sequences. Principal components analysis based on weighted-UniFrac distances did not reveal differences in bacterial communities based on treatment in any of the sample sources. However, pairwise linear discriminant analysis between control trees and treated trees indicated significant differences in relative abundance of some taxa based on dose and time since treatment. This $16 \mathrm{~S}$ rRNA high-throughput sequencing approach revealed specific changes in the phytobiome in response to penicillin treatment.

The Effects of Cover Crops on the Soil Microbiome: A Metagenomics Study

J. HACKMAN (1), A. Y. Srour (2), R. Cook (3), J. P. Bond (4), A. M. Fakhoury (4), (1) Southern Illinois University Carbondale, Carbondale, IL, USA; (2) Southern Illinois Univ, Carbondale, IL, USA; (3) North Carolina State University, Raleigh, NC, USA; (4) Southern Illinois University, Carbondale, IL, USA

Seedling pathogens associated with corn and soybean cause extensive economic losses for growers worldwide. Different management practices, such as the use of fungicides, crop rotation, tillage and cover crops, are commonly used to mitigate the pathogens' impact in monoculture systems. However, not much is known about how these factors affect the microbial communities present in the soil. This metagenomics study attempts to investigate how (7) species of cover crops and two different types of tillage; conventional and no-till, affect soil microbial communities. The sequencing of samples was performed using the Illumina Mi-Seq platform, with a return of $14 \times 10^{6}$ bp reads in total, using 250 bp PE reads. Four different primer sets were used to generate libraries for the identification of fungi, bacteria, oomycetes, and fusaria. The goal of this analysis is to examine how cover crops along with other factors, such as tillage and crop rotation, suppress or support certain microbial profiles that are conducive to the incidence of seedling pathogens of corn and soybean.

\section{Metacoder: An R package for visualization and manipulation of community taxonomic diversity data} Z. FOSTER (1,2), T. Sharpton (2), N. Grunwald (1), (1) USDA ARS, Corvallis, OR, USA; (2) Oregon State University, Corvallis, OR, USA

High-throughput sequencing techniques such as metabarcoding are revolutionizing microbial ecology by avoiding the limits and biases of traditional culture-based approaches, but the complex hierarchical (e.g. taxonomic) data produced are difficult to plot and manipulate using conventional tools. The reliance on color to depict taxa in stacked bar charts and pie graphs limits the number of taxa displayed to the number of discernible colors. In addition, hierarchical data is more challenging to filter, sample, and subset than typical tabular data. Metacoder is an R package for visualization and manipulation of hierarchical data and in silico primer/barcode evaluation. Metacoder introduces novel tree-based plots called heat trees that use the color and size of nodes and edges to depict quantitatively up to 4 statistics distributed over a hierarchy. This allows for rapid exploration of data and information-dense, publication-ready graphics. Metacoder also provides intuitive functions to subset and sample hierarchical data based on the popular dplyr R package syntax. In addition, metacoder provides tools for evaluating potential primers and barcode loci. Metacoder has been used to analyze diverse data sets including the Human Microbiome Project, geographic voting data, and gene ontology hierarchies of gene expression data. Intuitive manipulation and plotting of large hierarchical data sets facilitates a better and faster understanding of complex data.

\section{A Vavilovian approach to the phytobiome: Impact of domestication on the common bean microbiome} G. IRIARTE, K. D. Broders, Colorado State University, Fort Collins, CO, USA

Wild progenitors of crop plants, when grown in situ, represent a valuable resource as an assays for beneficial co-evolved microbial associations lost during the processes of domestication and translocation from centers of origin. To explore this idea, we investigated genotypes belonging to the Mesoamerican pool of common bean (Phaseolus vulgaris) as both wild and cultivated forms of $P$. vulgaris can still be found growing in central Mexico, one of the centers of domestication of the species. Replicated common gardens of six wild accessions and six cultivated varieties were grown in five locations: three in situ in central Mexican and two ex situ in the northeastern U.S. Fungal communities of the soil, rhizosphere, and roots were characterized via amplican sequencing of the ITS regions. Sample type (bulk soil vs. rhizosphere vs. root) was associated with significant variation in microbial diversity across locations. Of particular interest was the varying compositions of microbial communities between rhizosphere and root samples, as such differences indicate the root colonization of fungi through either active or passive means in all locations. Finally, we identified and cultured potentially novel microbial species that associated with cultivated beans grown in situ in Mexico but were not found on cultivated beans when grown in the U.S. These organisms represent targets for future inoculation studies. 
Potential Pleiotropic Effects of 43 Leaf Rust Resistance Genes in Ozone Tolerance of Spring Wheat Variety "Thatcher" A. M. MASHAHEET (1,2), D. S. Marshall (3), R. Ullah (4), A. Abdelrhim (2,5), K. Burkey (3), (1) Damanhour University, Damanhour, EGYPT; (2) North Carolina State University, Raleigh, NC, USA; (3) USDA-ARS, Raleigh, NC, USA; (4) The University of Agriculture, Khyber Pakhtunkhwa, PAKISTAN; (5) Minia University, Minia, EGYPT

This study investigated the effects of 43 leaf rust resistance $(L r)$ genes on the ozone $\left(\mathrm{O}_{3}\right)$ response of spring wheat cultivar "Thatcher", using 43 near isogenic lines (NILs) with individual genes in the $\mathrm{O}_{3}$-sensitive Thatcher background. The tested hypothesis was that, the ability of containing and localizing hypersensitive responses -conferred by some rust resistance genes- will also limit $\mathrm{O}_{3}$-induced foliar lesion formation by restricting the formation of $\mathrm{O}_{3}$-induced Reactive Oxygen Species (ROS), therefore enhancing overall plant $\mathrm{O}_{3}$ tolerance. Seedlings of Thatcher and the NILs for $43 \mathrm{Lr}$ genes were grown in charcoal filtered (CF) greenhouse until 21-22 Zadoks. Plants were moved to outdoor plant environment chambers, where they were exposed to four $\mathrm{O}_{3}$ treatments (CF, 50,70 and $90 \mathrm{ppb} ; 12 \mathrm{hrs}$ daytime average), under controlled temperature, relative humidity and $\mathrm{CO}_{2}$. The study was repeated twice, and $\mathrm{O}_{3}$ injury on all leaves of the main stem were estimated. Ten NILs (Tc-Lr16, B, 15, 33, 39, 34, 52, 10, 28 and 3) consistently showed more tolerance to $\mathrm{O}_{3}$ than Thatcher, and nine NILs (Tc-Lr3ka, 11, 13, 18, 19, 24, 26, 29 and 60) were more tolerant than Thatcher in only one of the two experiments. Tc- $L r 16$ was consistently the most tolerant among the NILs tested. Tc- $L r 9$ and Tc- $L r 42$ were more sensitive to $\mathrm{O}_{3}$ than Thatcher in one of the two studies. These results suggest that leaf rust resistance and $\mathrm{O}_{3}$ tolerance may be genetically associated. Further genetic studies are needed to determine the nature of the $L r$ gene and ozone tolerance response.

Molecular mechanisms of resistance in alfalfa to bacterial stem blight caused by Pseudomonas syringae pv. syringae L. G. NEMCHINOV (1), J. Shao (2), O. Postnikova (3), D. A. Samac (4), (1) USDA ARS MPPL, Beltsville, MD, USA; (2) USDA, Beltsville, MD, USA; (3) USDA-ARS, Beltsville, MD, USA; (4) USDA ARS, St Paul, MN, USA

Bacterial stem blight of alfalfa caused by $P$. syringae pv. syringae is widespread in the central and western U.S. and yield losses from the pathogen can be as high as $50 \%$ of the first harvest. There are no cultivars selected for resistance to this disease and no known chemical control. Little is known about host-pathogen interactions and host defense mechanisms. There is an urgent need to address these issues and respond to the threat represented by this emerging pathogen. In this study, we identified key genes and processes involved in host resistance. Transcriptomic analysis of alfalfa genotypes resistant or susceptible to $P$. syringae pv. syringae strain ALF3 was performed and a total of 3,092,825,766 short reads were generated on an Illumina Hi-Seq 2500 platform. Strand-specific paired-end-reads were mapped onto the reference genome of cultivated alfalfa at the diploid level (CADL, $2 n=2 x$ $=16$ ) and independently onto the genome of a close relative, Medicago truncatula. Comparative bioinformatic analysis revealed a number of differentially expressed genes (DEGs) in resistant and susceptible genotypes. Among them were unique genes specific to the resistant genotypes. All DEGs were annotated in terms of their associated biological processes and their roles in disease resistance were proposed. Knowledge of the genetic mechanisms underlying resistance would facilitate selection of resistant parent populations for breeding commercial varieties, increase the percentage of resistant plants in released varieties, and advance future breeding strategies against bacterial stem blight.

Phenotypic characterization and transcriptomic analysis of apple root defense responses to apple replant soilborne pathogen Pythium ultimum Y. ZHU (1), M. Mazzola (1), G. Fazio (2), J. Shao (3), R. E. Davis (4), J. Zhao (5), Z. Zhou (6), (1) USDA ARS, Wenatchee, WA, USA; (2) Cornell Univ, Geneva, NY, USA; (3) USDA, Beltsville, MD, USA; (4) USDA ARS Molecular Plant Pathology Lab, Beltsville, MD, USA; (5) Hebei Academy of Forestry Science, Shijiazhuang, CHINA; (6) Institute of Pomology, Chinese Academy of Agricultural Sciences, Beijing, CHINA

Molecular defense responses against soilborne necrotrophic pathogens have not been well characterized in roots of perennial fruit trees. In this study, apple root phenotypes differing in resistance responses to infection by Pythium ultimum were carefully defined among diverse apple rootstock germplasms. Plant survival rate, root and shoot biomass reduction and patterns of progression of necrosis were systematically evaluated using age equivalent plants, which were generated by tissue culture-based micropropagation. A wide range of resistance levels, including survival rates from $8 \%$ to $95 \%$ and reductions in root biomass from $12 \%$ to $80 \%$, were observed. Distinguishable root necrosis progression patterns were found between resistant and susceptible germplasm lines. Global transcriptional reprogramming of the apple root due to $P$. ultimum infection was characterized by carrying out two consecutive RNA-seq based transcriptome analyses. The most intense transcriptomic changes specifically related to the defense response in apple root were observed at $48 \mathrm{hpi}$. Comparative transcriptomics of a resistant and susceptible line revealed that both preformed and induced molecular defense systems contributed to genotype-specific resistance responses. Substantial sections of the apple root transcriptome were suppressed in the susceptible line but not in the resistant line. Selected candidate genes are under functional analysis, including manipulation of their expression in planta.

Comparing root knot nematode (Meloidogyne spp.) effects on tomato (Solanum lycopersicum) and grapevine (Vitis spp.) metabolic profiles C. M. WALLIS, USDA ARS, Parlier, CA, USA

Root knot nematodes (Meloidogyne spp., RKN) can negatively impact both herbaceous annual and woody perennial hosts. RKN infestations also may increase plant host susceptibility to other stresses such as those imposed by water deficits or various diseases. However, little is known about direct or indirect effects of RKN feeding on plant physiology, and whether or not different plants types possess different resistance mechanisms. Therefore, this study assessed how metabolite profiles change in tomatoes (Solanum lycopersicum) or grapevines (Vitis spp.) infected with RKN over time. Preliminary results observed increased phenolic compounds in RKN infected tomatoes compared to controls, but no consistent changes in primary metabolites. Phenolic compounds also were increased in grapevines infected with RKN, although grapevines had many differences in phenolic profiles compared to tomatoes. Furthermore, grapevines infected with RKN had reduced fructose and glucose levels compared to controls. Taken together, these results demonstrated that RKN infections alter root physiology of both tomatoes and grapevines, with some distinct differences in host responses apparent. This study increases our knowledge of how host plants respond to RKN infections, which could ultimately lead to ways to exploit induced host resistance responses to control nematodes.

Below-ground attack by the root knot nematode Meloidogyne graminicola predisposes rice to blast disease T. Kyndt, H. YIMER, A. Haeck, R. Singh, D. De Vleesschauwer, S. Denil, T. De Meyer, M. M. Hofte, K. Demeestere, G. Gheysen, Ghent University, Gent, BELGIUM

Rice is the most important staple food grain and the second most widely produced cereal crop worldwide. However, the production of this cereal crop is constrained by several biotic and abiotic stress factors. The root-knot nematode Meloidogyne graminicola and the rice blast Magnaporthe oryzae are two of the most destructive pathogens in many rice growing areas, contributing to rice yield decline. However, potential interactions between these groups of plant parasites are undocumented. In this study, we show that nematode infection in rice roots can result in systemic manipulation of plant defense gene expression and hormonal imbalance in the above ground parts of the plant. This leads to an enhanced subsequent rice blast infection. Analysis of oxidative stress responses and hormone levels on M. graminicola infected rice plants revealed that above-ground plant tissues have disturbed oxidative stress level, with accumulation of $\mathrm{H}_{2} \mathrm{O}_{2}$. Furthermore, the use of an oxidative stress mutant and an auxin-deficient rice line in a double infection experiment indicates that the accumulation of auxin in the above-ground tissue plays a role in the blast-promoting effect of the root-knot nematode infection.

Functional study of rice 14-3-3 proteins GF14c and GF14f in resistance to Magnaporthe oryzae

P. SUTTIVIRIYA (1), C. H. Park (1), M. D. Bellizzi (1), G. L. Wang (1,2), (1) The Ohio State University, Columbus, OH, USA; (2) Institute of Plant Protection, Chinese Academy of Agricultural Sciences, Beijing, CHINA 
The hemibiotrophic fungal pathogen Magnaporthe oryzae causes rice blast disease and threatens stable rice production worldwide. Understanding the molecular mechanisms of the rice and M. oryzae interaction can lead to the development of novel strategies for breeding highly resistant cultivars. The blast resistance gene Piz-t confers broad-spectrum resistance to M. oryzae and is being used in rice breeding programs. In this study, we aimed to identify proteins associated with Piz-t (PAZs) that may function in the Piz-t signaling pathway in rice. Piz-t-HA plants were inoculated with the incompatible isolate P2-3 and compatible isolate Guy11. Total protein was isolated from infected leaves of both samples at 24 and 48 hours after inoculation and was subject to co-immunoprecipitation assays. The bands present in the resistant interaction but absent in the susceptible interaction were selected for mass spectrometry (MS) analysis to identify PAZs. We selected PAZ8 and PAZ9 for more characterizations because they encode defense-related 14-3-3 GF14f and GF14c, respectively, and interact with Piz-t in vitro. To study their roles in Piz-t-mediated resistance, PAZ8 and PAZ9 were over-expressed into both Nipponbare (NPB) and Piz-t-HA backgrounds. Blast inoculation of the transgenic lines showed that over-expression of $P A Z 8$ and $P A Z 9$ enhanced resistance to isolates RB22 and RO1-1 in both the NBP and Piz-t-HA backgrounds. These results suggest that $P A Z 8$ and $P A Z 9$ may play a role in both Piz-t-mediated immunity and basal defense against M. oryzae in rice.

Characterization of the structural basis of winter squash fruit age-related resistance to Phytophthora capsici S. A. ALZOHAIRY (1), R. Hammerschmidt PhD (2), M. K. Hausbeck (2), (1) Department of Plant Soil and Microbial Sciences, Michigan State University, East Lansing, MI, USA; (2) Michigan State University, East Lansing, MI, USA

Fruit rot caused by Phytophthora capsici limits the production of winter squash (Cucurbita sp.). Age-related resistance (ARR) to $P$. capsici was observed in $C$. moschata as the fruit matured. Wounding negated this effect suggesting that fruit exocarp is important for resistance. Five winter squash $(C$. moschata) cultivars were planted in the field and fruit harvested 10,14, 16, 18, and 21 days post pollination (dpp). Unwounded fruits were inoculated with P. capsici. Disease was assessed four days later for expression of ARR. Butternut squash expressed ARR at 14dpp and fruit of this variety was selected for analysis of exocarp cell wall changes across fruit development by scanning electron microscopy (SEM). Cross sections of unwounded fruits 7, 14, and $21 \mathrm{dpp}$ (nine replicates/age) were visualized using SEM. ImageJ software was used to measure the thickness of cell wall structures. Statistical analysis determined significant differences in cuticle and epidermal walls among the fruit ages. These same fruits were observed via SEM at time intervals post inoculation to investigate the effect of $P$. capsici infection and observe hyphal penetration. $P$. capsici caused complete degradation of the fruit cell wall within $48 \mathrm{hr}$ of inoculating $7 \mathrm{dpp}$, fruit. The 14 and $21 \mathrm{dpp}$ fruit remained unaffected.

A functional developmental genomics screen is identifying genes functioning within cells that function in plant to a root pathogen B. R. LAWAJU (1), B. McNeece (1), S. Pant (2), K. Sharma (1), P. Niraula (1), W. Aljaafri (1), G. W. Lawrence (3), K. S. Lawrence (4), V. Klink (3), (1) Mississippi State University, Starkville, MS, USA; (2) Texas A\&M AgriLife Research \& Extension Center, Texas A\&M University System, Weslaco, TX, USA; (3) Mississippi State University, Mississippi State, MS, USA; (4) Auburn University, Auburn, AL, USA

RNA has been isolated from Glycine max (soybean) root cells undergoing the process of defense to a root pathogen. The RNA has been used in gene expression analyses, leading to the identification of candidate resistance genes. A gene testing platform has been developed to functionally test these genes with the aim of determining if the genes function during the process of defense. The procedure has examined hundreds of genes with some functioning effectively in defense. The analysis has demonstrated the importance of various cellular processes to defense and has identified genes that previously had no known role in defense.

NPR1 interacts with TCP15 to regulate the transcription of PR5 in plant defense

M. LI, Z. Fu, H. Chen, J. Chen, University of South Carolina, Columbia, SC, USA

Plant defense hormone, salicylic acid (SA), regulates 2280 genes' expression, while 2248 of these genes' expression is dependent on NPR1 (NONEXPRESSER OF PR GENES 1), which is a master protein in systemic acquired resistance (SAR). NPR1 contains two conserved protein-protein interaction domains: BTB (Bric-a-brac, Tramtrack, Broad-complex) domain and ankyrin repeat domain. It is well known that NPR1 functions as a transcription co-factor, recruits TGA transcription factors to the promoter of PR1 (Pathogenesis-Related 1) and activates the transcription of PR1. The expression of $P R 1, P R 2$, and $P R 5$ are usually used as hallmarks for SAR. As a transcription co-factor, NPR1 may interact with novel transcription factors to regulate the expression of downstream genes. Using full-length NPR1 as bait and a comprehensive Arabidopsis transcription factor library as prey, we identified 8 TCP transcription factors that interacted with NPR1 in Yeast-Two Hybrid (Y2H) assay. Loss of TCP function resulted in decreased PR genes transcription and compromised plant immunity. So, TCP proteins may directly regulate $P R$ genes expression. Interestingly, a consensus TCP class I motif (GTGGGAC) is present in the promoter of $P R 5$ at $-788 \mathrm{bp}$. We found TCP15 showed the binding ability to the promoter of $P R 5$ in Yeast-One Hybrid assay. The interaction between NPR1 and TCP15 was confirmed again by Co-immunoprecipitation assay. We also found the expression of $P R 1$ and PR5 decreased in tcp8tcp 15 double mutant after SA application. Now, we are testing whether tcp8tcp 15 mutants compromise to SAR. We will use ChIP assay to further confirm the binding ability of TCP15 to the promoter of PR5. Our research will elucidate how NPR1 regulate the expression of PR5.

Group I and II Acidovorax citrulli strains elicit different gene expression profiles in susceptible melon seedlings Y. Zhang (1,2), M. Zhao (3), Y. Yang (1), W. Guan (1), W. Ji (2), Q. R. Bai (2), J. Gao (2), R. R. Walcott PhD (3), T. ZHAO (1,2), (1) Institute of Plant Protection, CAAS, Beijing, CHINA; (2) Jilin Agricultural University, Changchun, CHINA; (3) The University of Georgia, Athens, GA, USA

Bacterial fruit blotch (BFB) caused by Acidovorax citrulli is a severe bacterial disease of cucurbits. Currently there is no commercially available resistance to BFB and nothing is known about compatible host responses to $A$. citrulli infection. However, this is critical to understand the infection process, and eventually develop host resistance to BFB. The aim of this study was to analyze the transcriptomic responses of melon seedlings to infection by two genetically different $A$. citrulli strains. We used representative group I (pslb65) and group II (AAC00-1) strains and assessed the melon seedling transcriptome at 6 and 12 hours post inoculation (hpi). The transcriptomics data set was validated by measuring the expression of six genes by qRT-PCR. While melon seedlings developed BFB symptoms when inoculated with both A. citrulli strains, the transcriptome profile varied by strain. At 6 hpi, $66 \%$ and $54 \%$ of differentially expressed melon genes were down-regulated when infected with pslb65 and AAC00-1, respectively. Similarly, at 12 hpi, there were $62 \%$ and $52 \%$ down-regulated genes for pslb65 and AAC00-1 treatment, respectively. Pathway analysis revealed that melon genes such as $R b o h$, CaMCML, CDPK, and FLS2 that are involved in plant/pathogen interactions were down-regulated at $6 \mathrm{~h}$, suggesting that defense responses were suppressed. This data provides a foundation for further characterization of the compatible interaction between $A$. citrulli and melon seedling tissues.

\section{Disruption of the genome of Rice Blast to identify genes involved in production of Reactive Oxygen Species} J. COOPER, N. M. Donofrio, University of Delaware, Newark, DE, USA

Rice Blast disease, caused by the fungus Magnaporthe oryzae, causes devastating losses to crops worldwide, especially in South Asia. It is a virulent pathogen, which can cause complete loss when conditions are favorable. To better understand how to take advantage of basal immunity in rice against $M$. oryzae, we are performing forward genetic screens. Using a well characterized strain of $M$. oryzae 4091, previous work in our lab transformed a live sensor of ROS called Hyper-sensor (HS) gene into the fungus, which gives 4091 the ability to fluoresce when in the presence of reactive oxygen species. We are in the process of randomly disrupting the genome of 4091-HS by transformation with a DNA insert constructed with a gene for resistances to the drug Hygromycin (Hyg). This construct has two degenerate ends so that it will generate random insertions in the genome, disrupting as many genes as possible. This ability to bind anywhere would disrupt genes that pay a role in ROS production. Our forward genetic screen could potentially identify 
genes that play a role in ROS production, directly linked to basal immunity. Through screening the randomly mutagenized library of 4091-HS, we will be able to identify changes in ROS production using confocal microscopy. Currently we have 65 transformed candidates that are awaiting further analysis. Results will be presented on our screening strategy, and some preliminary data from a similar screen on a previously mutagenized population.

Understanding the mechanism of resistance breaking on tomato by Tomato mottle mosaic virus

X. Sui (1,2), M. SHAMIMUZZAMAN (1), Y. Zheng (3), A. Simmons (1), Z. Fei (3), Z. Wu (2), K. S. Ling (1), (1) USDA-ARS, Charleston, SC, USA; (2) Fujian Agriculture and Forestry University, Fuzhou, CHINA; (3) Boyce Thompson Institute, Ithaca, NY, USA

Tomato mottle mosaic virus (ToMMV) has broaden its distribution around the world. In our previous work, we observed a partial resistance breaking by ToMMV on tomato. To understand the mechanism of this resistance breaking, we carried out comparative analysis through Sanger sequencing, genotyping by sequencing and RNA sequencing. We confirmed the presence of $T m-2^{2}$ gene in both susceptible (S) and resistant (R) cv. 'B' plants and observed no sequence variations. Next, in Genotyping-by-sequencing of over $190 \mathrm{cv}$. 'B' plants showing resistance (175) or susceptibility (17), we also could not identify any SNPs in association with the resistance breaking. Then, through profiling of global gene expression using RNA-seq between the R and S cv. 'B' plants, only 42 differentially expressed genes (DEGs) were identified. Gene Ontology analysis revealed that DEGs were enriched for catalytic and binding functions, with major classes involved in pathogenesis, transcription factors and proteases. However, there was no clear correlation of any particular genetic element to resistance breaking. Finally, a temperature test was conducted to investigate whether the resistance breaking was caused by higher temperature. We observed an increasing number of ToMMV-inoculated cv. 'B' plants with resistance breaking as temperature increased from 25 to 30 or 35 . This resistance breaking by higher temperature might have a significant impact on food security due to global warming.

Inactivation of SnRK1 by Geminiviruses AC2/C2 Proteins Plays an Important Role in the Regulation of Plant Innate Immune Responses M. LOCKWOOD, G. Sunter, UT San Antonio, San Antonio, TX, USA

One important group of plant pathogens is the Geminiviridae. They are among the smallest viruses known and are transmitted exclusively by insect vectors. Existing control measures include the use of pesticides, but recent studies in animal tissues has found that pesticides can have a negative effect on brain development. Thus, less harmful ways of controlling Geminivirus are needed. Plant resistance to infection depends on interactions between the pathogen and the plant, which activates innate immune responses. RNA silencing is part of the PAM-triggered immunity response and studies have shown a potential role for Autophagy in RNA silencing. Geminivirus proteins (AC2/C2) can inactivate plant immune responses including RNA silencing by suppression of SnRK1 activity. Preliminary data obtained in the lab, showed AC2/C2-mediated inactivation of SnRK1 causes differential expression of autophagy related genes. Recent results by qPCR analysis of Autophagy genes $A T G 7, A T G 8, A T G 9$, and $A T G 10$ in response to BCTV, SCTV, and SCTV C2 protein showed up-regulation of those genes at 1 and 3dpi with fold changes in expression that range between 1.5 and 3 . Also, data in the lab indicates that Arabidopsis plants mutants for ATG genes when infected with SCTV exhibited a decrease in the Mean Latent Period. This decrease was up to two days in the case of the atg7-1 mutant. Therefore, our hypothesis is that Autophagy is necessary for plant defenses. The goal of this study is to understand the interactions between $\mathrm{AC} 2 / \mathrm{C} 2$, SnRK1, autophagy and RNA silencing in order to understand their role in plant defenses.

Psyllid haplotypes in Southern Idaho potato fields, 2012 to 2015, and incidence of 'Candidatus Liberibacter solanacearum' causing Zebra chip disease

J. DAHAN (1), E. J. Wenninger (2), B. Thompson (1), S. Eid (1), N. L. Olsen (2), A. V. Karasev (1), (1) University of Idaho, Moscow, ID, USA; (2) University of Idaho, Kimberly, ID, USA

Zebra chip (ZC) disease, a serious threat to the potato industry, is caused by the bacterium 'Candidatus Liberibacter solanacearum' (Lso). Two haplotypes (hapA and hapB) of this pathogen have been associated with ZC in potato. Both haplotypes are vectored and transmitted to solanaceaeous plants by the tomato/potato psyllid, Bactericera cockerelli (Šulc). Psyllids are native to North America, and four haplotypes have been identified and named based on their predominant geographic association: Northwestern, Central, Western and Southwestern. Psyllid samples collected in Idaho potato fields from 2012 to 2015 were used to clarify spatial and temporal patterns in distribution and abundance of psyllid and Lso haplotypes. A shift from hapA toward hapB population of Lso was revealed during these four seasons, indicating possible evolution of Lso in Idaho fields. Although we confirmed that Western psyllids were the most abundant by far during the four seasons of observation, we also observed changes in abundance of other haplotypes, including increased diversity of psyllid haplotypes during 2015. Seasonal changes observed for the Northwestern and Central haplotypes could potentially be linked to psyllid migration and/or habitat changes. South-central Idaho exhibited more diversity in psyllid haplotypes than Southwestern Idaho.

Metabarcoding pathogenic Fusarium spp. in soybean seeds R. PEDROZO (1), A. Jumpponen (2), C. R. Little (1), (1) Kansas State University, Manhattan, KS, USA; (2) Biology Department, Kansas State University, Manhattan, KS, USA

A total of nine seed samples, representing three soybean genotypes and five geographical locations, were used in this study. The soybean seedborne fungal DNA was extracted from five individual healthy seeds from each sample. Forward fITS7 and reverse ITS4-barcoded primers were used for the amplification of the fungal ITS2 region. After library construction, amplicons were sequenced using the Illumina MiSeq platform. Approximately 291,000 high-quality reads were obtained from all samples analyzed in this study. Overall, 66 OTUs representing 29 fungi genera were identified in this study. The BLAST search showed that the genus Fusarium, including known pathogens of soybean such as $F$. proliferatum and $F$. thapsinum, was observed among and within all of the soybean seed samples investigated. Overall, $F$. proliferatum (OTU02 with 44,429 reads) was the most abundantly amplified species followed by $F$. thapsinum (OTU03 with 11,820 reads), F. acuminatum (OTU08 with 4,609 reads), F. merismoides (OTU13 with 4,302 reads), F. solani (OTU35 with 254 reads), Fusarium sp. (OTU55 with 19 reads), and $F$. semitectum (OTU57 with 17 reads). Accurate information regarding the identity and frequency of the fungal constituents of seed lots is crucial for significant improvements towards seed and seedling disease management strategies, improvements in seed certification and quarantine programs, and as a basis for making decisions to protect the future of agriculture.

Understanding Fusarium species causing Fusarium root rot in corn, soybean, and wheat in Nebraska L. P. PARIKH, S. Kodati, M. Eskelson, A. O. Adesemoye, University of Nebraska Lincoln, North Platte, NE, USA

Fusarium species are a major group of soilborne pathogens causing root rot of important crops. Multiple species of Fusarium are involved in the disease complex attributed to the genus, leading to greater disease complexity and yield losses. The objective of this study was to conduct a survey of Nebraska to identify the most prevalent and pathogenic species of Fusarium causing root rot. Diseased seedlings of corn, soybean, and wheat were collected from 21 counties spreading across different ecological regions of the state from 2014 to 2016. Isolates were recovered and identified by using morphology and sequencing the internal transcribed spacer and $\beta$-tubulin regions using ITS $4 / 5$ and BT 2A/2B primers, respectively. Pathogenicity assays were conducted using rolled towel assay and greenhouse experiments to determine the most virulent strains. In the greenhouse trial, test strains were incubated on sorghum seeds, the seeds were grinded, and mixed with the growth medium. Rating of seedlings were done at three weeks after inoculation. A total of 114 isolates, which included 10 diverse species of Fusarium were isolated from seedlings. Results indicated that $F$. graminearum and $F$. oxysporum were the most prevalent and pathogenic species causing Fusarium root rots in the state and accounted for $25 \%$ and $30 \%$ respectively, of the isolates identified. The eight most virulent strains across all the three crops were selected for further studies. 
Genetic analysis of Pyrenopeziza brassicae, cause of light leaf spot of brassicas, in the European Union, Oceania, and North America S. CARMODY (1), K. King (2), B. Claassen (3), B. B. Fraaije (2), J. West (2), C. M. Ocamb (3), L. du Toit (1), (1) Washington State University, Mount Vernon, WA, USA; (2) Rothamsted Research, Hertfordshire, ENGLAND; (3) Oregon State University, Corvallis, OR, USA

Light leaf spot (LLS), caused by Pyrenopeziza brassicae, is an important disease of Brassica napus (canola and oilseed rape) and B. oleracea (vegetable brassicas) in Europe (EU) as well as New Zealand and Australia (Oceania, OC). LLS was first reported in North America (NA) on B. juncea, B. napus, and B. rapa in six counties in western Oregon in 2014; and on B. juncea cover crops and wild B. rapa in three counties in northwestern Washington in 2016. Multi-locus sequence analysis (ITS ribosomal DNA, beta-tubulin, and elongation factor 1- $\alpha$ sequences) and comparison of the mating type genes $(M A T 1-1$ and MAT1-2) grouped isolates from the EU $(\mathrm{n}=28)$ and OC $(\mathrm{n}=4)$ with the $P$. brassicae type specimen, IMI 204290, whereas isolates from NA $(\mathrm{n}=16)$ represented a novel genotype. Sexual compatibility of NA and EU strains of complementary MAT1-1 and MAT1-2 genotypes is being determined to assess if NA isolates represent a distinct evolutionary lineage or a cryptic sibling species. Fungicide resistance has been documented in some EU populations of $P$. brassicae, but none of the NA isolates possessed amino acid substitutions E198A and L240F in the beta-tubulin sequences that confer resistance to benzimidazole fungicides; comparison of these sequences for the NA isolates revealed $100 \%$ identity to wild type EU $P$. brassicae isolates and the closely related fungus Rhynchosporium commune; and 98 and 99\% identities to Sclerotinia sclerotiorum and Venturia inaequalis, respectively.

Morphological and molecular characterization of Mycosphaerella nawae isolates from Persimmon leaf

O. Hassan (1), J. Y. Jeon (1), J. S. Shin (1), N. K. Oh (1), T. CHANG PHD (2), (1) Kyungpook National University, Sangju, Korea, Republic of (South); (2) Kyungpook National University, Sangju, Gyungbuk, KOREA

Circular leaf spot (CLS) is the most important foliar disease of persimmon worldwide. However, the morphological and molecular characteristics of Mycosphaerella nawae Hiura \& Ikata, the causative agent of CLS, have not been well studied. Fifteen strains of M. nawae were isolated from persimmon leaves showing the typical symptoms of CLS in 2016. The isolates were initially identified by comparing colony morphology on potato dextrose agar (PDA) with that of previously described M. nawae strains. The rDNA ITS region was amplified with the primer pair ITS-1F/ITS4 and sequenced. Phylogenetic analysis based on the ITS gene using MEGA6 showed that our isolate clustered with M. nawae strains isolated in Spain, with $100 \%$ bootstrap support. The isolates grew very slowly, and growth was highly depend on temperature and the combination of temperature, light, and relative humidity (RH). Depending on incubation temperature, colonies were dark green, dark gray, and pink. Mycelia were dark gray and guttulate. After 40 days of culture on PDA medium at $25^{\circ} \mathrm{C}$ in the light, oval, short, cylindrical, guttulate, uniseptate conidia were detected. Pseudothecia were observed in the lesions on over-wintered diseased leaves. Microscopic analysis revealed that pseudothecia were black and oval, flask, and pear shaped. Asci were banana shaped and 30.70-51.42 × 8.11-10.4 $\mu \mathrm{m}$ (average, $40.65 \times 9.00 \mu \mathrm{m}$ ) in size. Ascospores were spindle-shaped, uniseptate (unequally), and $10.56-13.41 \times 2.93-4.65 \mu \mathrm{m}$ (average, $12.08 \times 3.85 \mu \mathrm{m}$ ). The results of this study will be helpful to plant pathologists for understanding the biology of M. nawae.

In vitro evidence for sexual reproduction in Venturia effusa, causal agent of pecan scab

N. D. CHARLTON (1), C. Mattupalli (1), C. H. Bock (2), C. A. Young (1), (1) The Samuel Roberts Noble Foundation, Ardmore, OK, USA; (2) USDA ARS, Southeastern Fruit and Tree Nut Research Laboratory, Byron, GA, USA

Venturia effusa is the causal agent of pecan scab, the most prevalent disease of pecan in the southeastern US. Venturia effusa is currently only known to reproduce asexually, yet the genetic diversity among populations of pecan scab suggest it is a sexually reproducing pathogen. Analysis of the mating type idiomorphs in a collection of 1203 isolates of $V$. effusa revealed a 1:1 equilibrium. Based on identification of the mating type idiomorphs, select isolates were crossed in pairwise combinations in triplicate on oatmeal agar and stored at $4 \mathrm{C}$ in the dark. After a four month cold treatment, immature sexual fruiting bodies were observed with pairings of opposite mating types. The pairings were subsequently incubated at $24 \mathrm{C}$ with a $12 \mathrm{~h}$ photoperiod to monitor maturation of the fruiting bodies. Pseudothecia were randomly selected from the zone between opposite mating types to evaluate the maturity of the ascocarp. Squash mounts of pseudothecia were examined to determine ascospore maturity. Asci and ascospores were typically observed after two weeks under a $12 \mathrm{~h}$ photoperiod at $24 \mathrm{C}$ following the four month cold treatment. This first report suggests that the fungus is capable of producing sexual spores in vitro, which emphasizes the need to revisit the disease cycle. Identification of the sexual stage in nature will provide improved understanding of the disease epidemiology, pathogen population genetics, and potentially provide insight into approaches to manage this disease.

Genotypic characterization of isolates of Golovinomyces magnicellulatus, the biotrophic powdery mildew pathogen of Phlox C. FARINAS (1), F. Peduto Hand PhD (2), (1) The Ohio State University, Columbus, OH, USA; (2) Ohio State Univ, Dept of Plant Pathology, Columbus, OH, USA

The native ornamental plant Phlox is appreciated worldwide for its long-lasting bloom that attracts pollinators. Unfortunately, the parasitic powdery mildew pathogen can thrive on Phlox, often rendering the plant unsightly and weakened. Growers rely on economically and ecologically costly fungicide applications to keep the pathogen at bay. Identifying the genetic diversity of powdery mildew populations infecting Phlox will build solid basis for epidemiological and resistance studies that may ultimately limit the use of fungicides for disease management. A collection of 42 powdery mildewinfected Phlox plants from commercial nurseries, retailer garden centers, and landscape sites, was made throughout Ohio during summer and autumn of 2016. A custom-made spore-collecting device was used to harvest conidia and mycelium from infected leaf tissues. A commercially available DNA extraction kit and a Chelex ${ }^{\circledR}$-based technique were used to compare DNA yield and quality. Sequence comparison of the ITS region of the rDNA led to the identification of two powdery mildew species: Golovinomyces magnicellulatus (75\%), and Podosphaera spp. (25\%). Sequence alignment allowed the identification of four Single Nucleotide Polymorphism (SNP) sites, each represented in several isolates. While ITS alone is not enough to assess genetic diversity of a population, these results indicate that $G$. magnicellulatus is highly diverse and its genetic characterization is worth further investigating.

\section{A 2-Year Analysis of Rust Fungi Intercepted at Arizona Ports of Entry}

D. C. SANDBERG, USDA-APHIS-PPQ, Nogales, AZ, USA

Rust fungi are highly specialized obligate parasites that cause significant damage to agricultural and forestry crops. Rusts are among the most destructive plant diseases affecting American agriculture, reducing yield, marketability and exports. Rusts that are not established in the United States, or that have limited distribution within the country, are considered quarantine significant by the USDA-APHIS-PPQ. Many of the rust fungi intercepted coming from Mexico are considered quarantine significant pests, and account for a substantial portion of the pathogens requiring phytosanitary action when identified at the border by PPQ personnel. USDA-APHIS-PPQ, alongside Customs and Border Protection Agriculture Specialists, work to ensure the prevention of any potential quarantine significant rust fungi from entering the United States. From January 1, 2015 to December 31, 2016, a total of 477 interceptions of rust fungi coming from various regions of Mexico were found at 5 different Arizona ports of entry. The 477 rust fungi were found to belong to 5 known rust families, 8 genera, and 21 species. Some of the rust fungi intercepted were identified only to the genus level, including 19 samples that were identified only to the anamorph genera Aecidium and Uredo. Interceptions of rust fungi found at Arizona ports of entry increased slightly from 207 in 2015 to 270 in 2016. Of the rust fungi that were intercepted, the most prevalent plant pathogen was Chrysanthemum White Rust (Puccinia horiana), which was found 268 times: 124 in 2015 and 144 in 2016. Chrysanthemum White Rust is an economically important disease in field and greenhouse production, and interceptions of this pathogen coming from Mexico have increased each year. 
Molecular phylogeny of Septoria isolates from tomato in Brazil

C. A. da Costa (1), V. LOURENÇO JR. (2), L. S. Boiteux (3), M. E. D. N. Fonseca (2), A. Reis (3), (1) Universidade Federal Rural de Pernambuco, Recife, BRAZIL; (2) Embrapa, Brasília, BRAZIL; (3) Embrapa, Brasilia, BRAZIL

Apparently, S. lycopersici is the only species associated with the Septoria leaf spot epidemics on tomatoes worldwide. However, no extensive studies were carried out thus far aiming to investigate the potentially cryptic diversity of Septoria isolates from tomatoes. The main objective of this study was to carry out the phylogenetic analysis of the translation elongation factor-1 $\alpha$ (TEF1- $\alpha$ ), $\beta$-tubulin ( $\beta \mathrm{t}$ ), and calmodulin (Cal) genes from 60, 75, and $11 S$. lycopersici isolates, respectively. For the phylogenetic analysis, TEF1- $\alpha, \beta \mathrm{t}$, and Cal sequences from other Septoria species were obtained from the GenBank. The phylogenetic analysis were conducted using the maximum likelihood method. The GTRGAMMA model was used for the search tree with 1000 replications. Even though, genetic variability was detected among the isolates, all of them clustered in the S. lycopersici clade. Five, nine, and three haplotypes were generated for $\beta$ t, TEF1- $\alpha$, and Cal genes, respectively. The mutation rates (per site/per sequence) from $\beta$ t, TEF1- $\alpha$, and Cal genes were $0.031 / 8.8,0.026 / 8.8$, and 0.009/2.5, respectively. Differences of total number of mutations were detected among $\beta \mathrm{t}$ (43), TEF1- $\alpha$ (44), and Cal (7) genes. Thus, $S$. lycopersici seems to be the sole species associated with Septoria leaf spot on tomatoes in Brazil. Additional analyses are being conducted with a larger group of Septoria isolates to confirm these initial observations.

\section{A phylogenomic marker based comparative studies of Pseudomonas syringae and its related species M. RANJAN, P. B. Patil, CSIR- IMTECH, chandigarh, INDIA}

Pseudomonas syringae is a complex and economically important major group of phytopathogenic bacteria. However, the species and its constituent pathovars underwent numerous taxonomic classifications because of limitation of traditional methods. In recent times, with advent of genomics era modern whole genome based criterion like Average Nucleotide Identity (ANI) and digital DNA-DNA hybridization (dDDH) have emerged as successful new age criterion to establish and resolve the taxonomic diversity of bacterial species. Interestingly, complete sequence of gene encoding RNA polymerase beta-subunit $(r р о B)$ had emerged and widely accepted as simple, an easy supplement to modern genome based criterions like ANI and $\mathrm{dDDH}$. In the present study, we show that rpoB is successful in establishing and resolving majority of the species group in $P$. syringae pathovars into six species groups. This study revealed possible limitation of modern ANI and dDDH based criteria. Hence, we propose $r p o B$ as an effective, easy, quick marker, as alternate supplement to genome based criterions, to establish phylogeny and also taxonomy of $P$. syringae and its pathovars. This understanding allowed us to study evolution of a significant hypervariable locus in $P$. syringae and its related species.

Stripe rust epidemics of wheat and barley and races of Puccinia striiformis identified in the United States in 2016

A. Wan (1), M. Wang PhD (2), X. CHEN (3), (1) Washington State University, Pullman, WA, USA; (2) Washington Sate University, Pullman, WA, USA; (3) USDA ARS, Pullman, WA, USA

Stripe rust of wheat, caused by Puccinia striiformis f. sp. tritici (Pst), occurred throughout the United States and caused severe yield losses in 2016. Stripe rust of barley, caused by $P$. striiformis f. sp. hordei (Psh), occurred in California, Oregon, Idaho, Utah, Washington, Minnesota, and Texas. For monitoring virulence changes of the pathogens, stripe rust samples collected from 26 states were tested on 18 wheat and 12 barley differentials to identify $P s t$ and $P s h$ races, respectively. Five previously existing Psh races were detected, of which PSH-33 (virulent on differentials Topper and Abed Binder 12) and PSH-48 (virulent only on Topper among the 12 barley differentials) were the predominant races with frequencies of $57 \%$ and $30 \%$, respectively. Of 61 Pst races identified, 26 were new with 7 identified from the eastern and 19 from the western U.S. All new races had very low frequencies (0.3-1.4\%). The top five predominant races were PSTv-52 (24\%), PSTv-37 (23\%), PSTv-198 (13\%), PSTv-53 (6\%), and PSTv-18 (4\%). The top three races were detected throughout the country, while PSTv-53 and PSTv-18 were almost restricted in the western U.S. Virulence frequencies to $Y r 6, Y r 7, Y r 8, Y r 9, Y r 17, Y r 27, Y r 43, Y r 44$, and $Y r E x p 2$ were high (62-93\%); and to $Y r 1, Y r 10, Y r 24, Y r 32, Y r S P$, and $Y r T r 1$ and $Y r 76$ were low (8-37\%). No virulence was detected to either $\operatorname{Yr} 5$ or $\operatorname{Yr} 15$. The results are useful for breeding resistant cultivars and control of stripe rust.

Diversity of Fusarium oxysporum f. sp. niveum from watermelon in Georgia A. PETKAR, P. Ji, University of Georgia, Tifton, GA, USA

Fusarium wilt has been an increasing concern in watermelon production in the southeastern U.S. in recent years. The disease is caused by Fusarium oxysporum f. sp. niveum (FON), a soilborne pathogen with different races based on their aggressiveness on watermelon plants. To determine diversity of FON in Georgia, isolates were collected from major watermelon producing counties and races of the isolates were determined by inoculation of a set of watermelon differentials. The isolates belonged to four races $(0,1,2,3)$ and the majority of the isolates were identified to be race 2 and race 3 . Assessment of sensitivity of the isolates to fungicides indicated that all isolates were sensitive to prothioconazole at $10 \mu \mathrm{g} / \mathrm{ml}$, while some isolates were resistant to thiophanate-methyl at $100 \mu \mathrm{g} / \mathrm{ml}$. Simple sequence repeats (SSR) markers were screened and polymorphic markers are being used for analysis of genetic diversity of the isolates. This is the first report of race 3 isolates of FON in the southeastern U.S. Results of the study indicate that FON populations in Georgia are diversified and the existence of the highly aggressive race 3 isolates is a challenge in management of Fusarium wilt in watermelon production.

Genetic variability among natural populations of Sugarcane mosaic virus (SCMV) in the Northern Guinea Savannah agroecological region of Nigeria

A. YAHAYA (1), D. B. Dangora (1), L. Gregg (2), M. D. Alegbejo (1), P. L. Kumar (3), O. J. Alabi (4), (1) Ahmadu Bello University, Zaria, NIGERIA; (2) Dept. of Plant Pathology and Microbiology, Texas A\&M AgriLife Research and Extension Center, Weslaco, TX, USA; (3) International Institute of Tropical Agriculture, Ibadan, NIGERIA; (4) Dept. of Plant Pathology \& Microbiology, Texas A\&M University, Weslaco, TX, USA

Sugarcane mosaic virus (SCMV) belongs to the genus Potyvirus (family Potyviridae) and is the most prevalent of the causative agents of sugarcane mosaic disease (SCMD). In this study, the genetic diversity and population genetics of 34 SCMV isolates from sugarcane $(n=33)$ and itch grass $(n=1)$ sampled from major sugarcane growing areas (Kaduna, Katsina and Kano states) in Nigeria was assessed relative to global SCMV isolates. DNA bands specific to the helper component protease (HC-Pro), cylindrical inclusion (CI) protein, and the nuclear inclusion replicase (NIb) gene were amplified by RT-PCR using published primer pairs, cloned, and sequenced. The results showed superior performance of primer pairs targeting the HC-Pro and NIb genomic segments compared to the CI-specific primers, with implications for virus diagnosis in epidemiological studies. Analysis of phylogenetic networks revealed complex non-tree-like reticulate networks among global SCMV isolates indicating fingerprints of recombination. Recombination analyses confirmed extensive influence of recombination on the evolution of natural SCMV populations than was previously realized. Phylogenetic analyses conducted with complete polyprotein and gene-specific global SCMV sequences revealed their segregation into five distinct phylogroups with some evidence of geography- and host-specific clustering patterns. These results represent the most comprehensive analysis of SCMV genetic diversity and population genetic to date and point to possible monophyletic origin for SCMV isolates from sub-Saharan African countries. The results have implications for the integrated and sustainable management of SCMD in cereal cropping systems.

Prevalence and pathogenicity of Rhizoctonia spp. from soybean in Nebraska

S. KODATI (1), N. Gambhir (2), S. E. Everhart (2), A. O. Adesemoye (1), (1) University of Nebraska Lincoln, North Platte, NE, USA; (2) University of Nebraska, Lincoln, NE, USA 
Rhizoctonia solani is a soilborne pathogen with a wide host range, causing damping off in seedlings and necrotic lesions on roots, seeds, and stems. $R$. solani is a species complex and varies in geographic distribution, morphology, host specificity, and pathogenicity. Rhizoctonia zeae is less studied as a pathogen of soybean. Our objectives were to survey Rhizoctonia spp. from soybean fields in Nebraska, identify predominant anastomosis groups (AGs) of $R$. solani, and assess pathogenicity on soybean. In 2015 and 2016, symptomatic seedlings and soil samples were collected in a Z-pattern from 15 fields located in 9 counties across NE soybean production regions. Isolates were recovered and identified based on morphology and sequencing of the ITS regions. Pathogenicity was determined for all 35 recovered Rhizoctonia isolates through a repeated rolled towel assay and greenhouse bioassay with visual ratings made 7 and 21 days after inoculation, respectively. Among the 35 isolates, 22 were identified as $R$. solani and 13 as $R$. zeae. The most prevalent AG of $R$. solani was AG-4 for which 14 isolates were recovered. Assay results indicated a wide range of virulence among isolates. The most virulent isolate was $R$. solaniAG-4 strain G1926, which exhibited cross-virulence to both corn and wheat. This isolate will be used in further studies to examine how cross-virulence to rotated crops may impact effectiveness of rotation in an integrated disease management system.

Genetic and morphological characterisation of Verticillium dahliae collected from cotton crops throughout NSW, Australia K. KIRKBY (1), T. Chapman (2), S. Roser (1), P. A. Lonergan (1), P. Dadd-Daigle (2), S. Harden (3), (1) Biosecurity and Food Safety, NSW Department Primary Industries, Narrabri, AUSTRALIA; (2) Biosecurity and Food Safety, NSW Department Primary Industries, Narellan, AUSTRALIA; (3) NSW Department Primary Industries, Tamworth, AUSTRALIA

The pathogen, Verticillium dahliae, causes disease in cotton and can be classified as defoliating (D) or non-defoliating (ND) pathotypes. Elsewhere in the world, disease symptoms from the ND pathotype are described as mild to severe but with no defoliation of the cotton plants. The pathotypes can be further categorised into vegetative compatibility groups (VCGs) with D pathotypes being assigned to VCG1 and the ND pathotypes assigned to VCG2 or VCG4. A selection of Australian isolates collected from cotton plants with symptoms varying from mild defoliation to complete defoliation were sent to Spain and characterised to pathotype and VCGs and subsequently used as reference isolates. The results of this molecular analysis showed that Australia had both D and ND pathotypes. The isolates classified as ND using this method were classified as D by traditional visual methods and were sourced from completely defoliated or dead plants. The traditional classification of isolates to pathotype level using the molecular method did not accurately describe the ND pathotype of Australian isolates. To overcome this, we scrutinised isolates from ND VCG2A and 4B and D VCG1A including the reference isolates for morphological and physiological parameters that could be used as diagnostic features to more accurately pathotype Australian Verticillium isolates.

Genome-wide informative microsatellite database and global population structure of Fusarium virguliforme G. CAI (1), T. J. Fleury (2), K. A. Wise (3), T. J. Hughes (4), (1) USDA-ARS / Purdue University, West Lafayette, IN, USA; (2) USDA-ARS, West Lafayette, IN, USA; (3) Purdue University, West Lafayette, IN, USA; (4) Monsanto Co, Chesterfield, MO, USA

Sudden death syndrome is one of the leading biotic stresses responsible for soybean yield loss in major soybean production areas. In North America, the disease is caused by the fungus Fusarium virguliforme, while in South America additional Fusarium species cause similar symptoms. Breeding for SDS resistance is being carried out by multiple academic and industrial sources. However, only limited information of the population structure of $F$. virguliforme is available, which hampers the breeding effort. The objective of current study is to build a genome-wide informative microsatellite database and use it to characterize the pathogen population structure in a global isolate collection. We developed a novel bioinformatics pipeline to scan the genome assemblies of multiple isolates of this fungus and created a database of informative microsatellite loci, thus bypassing / minimizing the step required to test the information content of microsatellite loci identified through sequencing of microsatellite-enriched library or genome scanning of a single assembly. Primers were designed for these loci. DNA is being extracted from a world-wide collection of 83 isolates. The microsatellite markers will be used to characterize the global population structure of this fungus.

\section{Genomic fingerprinting and phylogenetic analyses of Xanthomonas perforans strains provide insights into bacterial evolution and global} movement

S. TIMILSINA (1), P. Abrahamian (2), F. Iruegas-Bocardo (1), G. V. Minsavage Jr. (1), B. Kolaczkowski (3), F. F. White (4), B. Staskawicz (5), G. E. Vallad (2), E. M. Goss (1), J. B. Jones (1), (1) Department of Plant Pathology, University of Florida, Gainesville, FL, USA; (2) Gulf Coast Research and Education Center, University of Florida, Wimauma, FL, USA; (3) Department of Microbiology and Cell Science, University of Florida, Gainesville, FL, USA; (4) University of Florida, Gainesville, FL, USA; (5) University of California, Berkeley, CA, USA

Bacterial spot caused by Xanthomonas perforans is a major disease of tomato in Florida. Significant population changes have been observed since the discovery of $X$. perforans in Florida in 1991. Shifts in bacterial races and variation in effector profiles without any known selection pressure have challenged resistance-based disease management strategies. International trade and globalization may have led to the change in pathogen population. The objective is to identify the movement of $X$. perforans by developing a genomic fingerprint for sequenced genomes that can be used to trace bacterial strains. This study aims to reduce the spread of Xanthomonas strains in tomato by identifying sources of outbreaks and origins of new strains. We developed a core genome Multilocus Sequence Typing (cgMLST) scheme, built using $>100$ X. perforans genomes, representing strains isolated from different locations and at different time points. A total of 1,316 genes were identified as core genes, of which 783 genes were identical among all the genomes used in the study. Genetic fingerprints for strains were generated based on allelic variation in the core genes using $X$. perforans $91-118$ as the reference and effector profiles were also compared. Distinct genetic patterns were observed between $X$. perforans phylogenetic clusters. The cgMLST approach provides a high-resolution genetic profile for tracing distinct $X$. perforans strains.

\section{Characterization of microsatellites from a genome of Venturia carpophila}

C. Chen (1), P. M. Brannen (2), J. E. Adaskaveg (3), C. H. BOCK (1), (1) USDA ARS, Southeastern Fruit and Tree Nut Research Laboratory, Byron, GA, USA; (2) University of Georgia, Athens, GA, USA; (3) Department of Plant Pathology and Microbiology, University of California, Riverside, CA, USA

Microsatellites were mined from a genome of Venturia carpophila and 192 were selected to screen 39 isolates of the fungus collected from peach and nectarine in the southeastern USA. Reliable, polymorphic amplicons were produced by 32 of the selected primers. A preliminary analysis of the genetic diversity of the panel of isolates was performed. Allele number ranged from 2 to 9 , and the polymorphic information content from 0.097 to 0.792 . Shannon's information index was 0.914 , indicating substantial genetic diversity. Stoddart and Taylor's index of diversity and Simpson's index also indicated high diversity (32.4 and 0.969, respectively). Evenness within the sample was high ( 0.955$)$, but there was strong evidence for haploid linkage disequilibrium $(3.799(\mathrm{P}=0.001))$. Observations on diversity were supported by the dissimilarity analysis, which showed little affinity for clustering based on isolate source population, location or host. The microsatellites developed in this study should be useful in future research of the population genetic structure and dynamics of $V$. carpophila, and evaluating the risks posed by the ability of the pathogen to adapt on peach and possibly other stone fruit hosts in the USA and elsewhere in the world.

\section{Distribution and abundance of Macrophomina phaseolina in Paraguay}

H. D. LOPEZ-NICORA (1), C. Grabowski Ocampos (2), A. L. Orrego Fuente (2), L. M. Pedrozo (3), E. Hahn Villalba (4), T. I. Ralston (1), T. L. Niblack (1), (1) The Ohio State University, Columbus, OH, USA; (2) Facultad de Ciencias Agrarias, Universidad Nacional de Asunción, San Lorenzo, PARAGUAY; (3) Instituto Paraguayo de Tecnología Agropecuaria (IPTA), Caacupé, PARAGUAY; (4) Facultad de Ciencias Agropecuarias, Universidad Católica, Hohenau, PARAGUAY 
Paraguay ranks fifth among soybean-producing countries in the world and fourth in soybean exports. Soybean ranks first among Paraguayan field crops in planted area and is an important commodity for the country's economy. The fungus, Macrophomina phaseolina, has a wide host range and causes significant yield loss in soybean worldwide. Our objective was to determine the presence, distribution, and abundance of $M$. phaseolina from soybean fields in major agricultural regions of Paraguay. During the 2014/15 growing season, composite soil samples were collected from 300 soybean fields arbitrarily chosen across several departments in Paraguay. A fraction of each sample was processed for M. phaseolina colony forming units (CFU)/g soil and another sent for physical and chemical soil analyses. M. phaseolina was detected in all soil samples with levels ranging from 1 to more than 550 $\mathrm{CFU} / \mathrm{g}$ soil. The largest soybean producing regions had, on average, over $100 \mathrm{CFU} / \mathrm{g}$ soil. Other regions with less area planted to soybean had fungal levels below $50 \mathrm{CFU} / \mathrm{g}$ soil. Furthermore, a positive association was observed between abundance of $M$. phaseolina and several edaphic factors (e.g., clay, organic material, cation exchange capacity) except for soil $\mathrm{pH}$ which was negatively correlated with $\mathrm{M}$. phaseolina CFU/g soil. Information from this study highlights a potential threat for soybean production and may aid local growers in developing sustainable economic and agricultural systems.

Genetic diversity, aggressiveness, and fungicide sensitivity of Pythium irregulare isolated from soybean in Ohio J. HUZAR NOVAKOWISKI, A. E. Dorrance, The Ohio State University, Wooster, OH, USA

Numerous Pythium spp. can cause seed and seedling disease of soybean leading to reduction of plant stand and yield. Among all Pythium spp. that can affect soybean, $P$. irregulare is one of the most common species isolated in Ohio. Since P. irregulare is considered a cryptic species complex, the objective of this study was to characterize 54 isolates from 26 counties in Ohio for genetic diversity, aggressiveness and fungicide sensitivity. Genetic diversity was evaluated based on the sequence analysis of the internal transcriber spacer (ITS1-5.8s-ITS2) regions, and microsatellites markers. Preliminary analysis of the ITS region indicated that there were at least two distinct groups among the isolates. To evaluate aggressiveness, a seed plate and a cup assay were performed. In the seed plate assay, 51 and 54 isolates caused seed rot and completely inhibited seed germination of the cultivars 'Dennison' and 'Kottman', respectively. In the cup assay, significant differences $(P<0.05)$ among isolates were observed for emergence, root rot score, fresh root and shoot weight. In addition, all isolates were able to grow in potato carrot broth amended with metalaxyl at both 5 and $100 \mathrm{ug} \mathrm{ml}^{-1}$, indicating that this fungicide was not effective in reducing growth of $P$. irregulare. These results, combined with the genetic analysis, will help to identify key isolates for fungicide screening, to deploy host resistance, and to improve disease management recommendations.

Influence of sampling on detection of simple sequence repeat haplotypes of Aspergillus flavus associated corn

D. EDMUNDS (1), H. L. Mehl (2), P. J. Cotty (3), (1) University of Arizona, Tucson, AZ, USA; (2) Virginia Tech Tidewater AREC, Suffolk, VA, USA; (3) USDA-ARS, University of Arizona, Tucson, AZ, USA

Aspergillus section Flavi includes several species known to contaminate crops with aflatoxins. Aflatoxins are potent human carcinogens that may impair immune systems, retard development, cause liver disease, and reduce productivity of domestic animals. A. flavus is highly diverse with many vegetative compatibility groups and great variation in aflatoxin-producing ability. Atoxigenic $A$. flavus biocontrols successfully limit aflatoxin in crops in several regions. To precisely characterize etiology of contamination and identify atoxigenic genotypes well adapted to target agronomic systems, A. flavus populations must be analyzed. Efforts to reduce costs of sample collection, transportation, and analysis will facilitate development of biocontrol products. In the current study, 1,759 L-morphotypes of A. flavus were isolated from 10 Virginia maize samples for population analyses. Previously described Simple Sequence Repeats (SSRs) at 17 loci were characterized as PCR amplicons on an ABI3730 DNA analyzer. Haplotypes (598) were highly diverse with 398 haplotypes detected only once. A total of 32 common (detected in at least $40 \%$ of the samples) haplotypes were identified with the most frequent detected in $90 \%$ of the samples. Common haplotypes in target agroecosystems are considered the best candidates for biocontrol agents. The current work provides insight on optimization of methods to collect and identify A. flavus haplotypes most likely to be successful biocontrol agents.

I Think We're A Clone Now: Factors Influencing Inference Of Clonality In Diploid Populations Z. N. KAMVAR (1), S. E. Everhart (1), N. Grunwald (2), (1) University of Nebraska, Lincoln, NE, USA; (2) USDA ARS, Corvallis, OR, USA

The index of association is a measure of multilocus linkage disequilibrium, which reflects the deviation of observed genetic linkage among loci from expected. In sexual populations, where loci are randomly assorting due to recombination, the index of association is expected to be near zero. In clonal populations, where recombination is absent, meaning that loci are passed from parent to offspring as a copy, the index of association is expected to be significantly different from zero. This index is often used to provide support for the presence of clonal reproduction, but the statistical power to detect clonal reproduction has not been formally investigated. We build on previous work by investigating the effect of sample size, mutation rate, and clone correction on the power of the index of association to detect clonal reproduction in simulated data sets generated with microsatellite and genomic markers. Our findings suggest that power decreases with small sample sizes and low allelic diversity, and physical linkage in genomic markers does not affect power if permutation tests are performed across linkage groups. Our work provides novel insights for designing studies to distinguish clonal reproduction from sexual reproduction in natural populations.

AmpSeq: Use of a new genotyping tool to address practical questions in pathogen biology, population studies, and fungicide resistance B. KISSELSTEIN (1), L. E. Cadle-Davidson (2), W. Weldon (1), B. B. Forcelini (3), N. Peres (4), M. T. McGrath (5), B. A. Tadesse (6), A. Stensvand (7), D. M. Gadoury (1), (1) Cornell University, Geneva, NY, USA; (2) USDA Grape Genetics Research Unit, Geneva, NY, USA; (3) University of Florida, Wimauma, FL, USA; (4) Gulf Coast Research and Education Center; University of Florida, Wimauma, FL, USA; (5) Cornell University, Riverhead, NY, USA; (6) Norwegian Institute for Bioeconomy Research (NIBIO), Aas, NORWAY; (7) Norwegian Institute of Bioeconomy Research, Ås, NORWAY

Amplicon Sequencing (AmpSeq) is a broadly applicable and integrative platform for genome-wide marker discovery in a variety of pathosystems. This low-cost, next-generation technology requires computational analysis of large datasets that can be applied to many biological questions. For example, studies of baseline fungicide sensitivity in powdery mildews are often assumed to require single-spore subculturing of field-collected pathogen samples to ensure clonality. AmpSeq analyses of field-collected samples in four different powdery mildew pathosystems (Erysiphe necator (grape), Podosphaera macularis (hop), Podosphaera aphanis (strawberry), and Podosphaera xanthii (cucurbit)) indicated that pathogen samples collected on 1-cm squares of tape were frequently clonal, and mixed colonies could be detected by neutral markers. Furthermore, AmpSeq identified alleles known to be associated with resistance or sensitivity as well as novel mutations and copy number variants. AmpSeq can similarly be used to quantify, track, or map genes that are otherwise cryptic within a population. For example, AmpSeq can be used to map the frequency and distribution of mating types, obviating the need for more complex and time-consuming mating of isolates. Coupled with other next-generation technologies, AmpSeq allows for investigation of hundreds of genetic markers on thousands of environmental and experimental samples at a greatly reduced level of time and resources.

Cluster analysis of Anisogramma anomala isolates collected from the Pacific Northwest and New Jersey J. TOBIA (1), M. Muehlbauer (1), J. Honig (1), J. Pscheidt (2), T. J. Molnar (1), (1) Rutgers University, New Brunswick, NJ, USA; (2) Oregon State University, Corvallis, OR, USA

Absence of Anisogramma anomala, the causal agent of eastern filbert blight (EFB), in the Pacific Northwest (PNW) underpinned a century of hazelnut (Corylus avellana) cultivation in the region until its inadvertent introduction in the 1960s. To combat this devastating disease, breeding work in Oregon lead to introgression of a dominant resistance gene from the pollinizer 'Gasaway' into commercial hazelnut germplasm. This work revived the OR industry and supports the potential establishment of one in the eastern US. However, after initially providing resistance to A. anomala in New Jersey, 
'Gasaway' plants have begun to develop EFB; it is uncertain if this is due to fungal genetic variation, the environment, or both. The objectives of this study were to determine if genotypic differences exist between $A$. anomala isolates collected in NJ and the PNW, and if NJ isolates differ in plants with and without R-genes. Isolates, 116 from NJ and 63 from the PNW, were fingerprinted using 17 SSR markers. A genetic cluster analysis was performed. Initial results revealed that $\mathrm{NJ}$ isolates are genetically distinct from and more diverse than PNW isolates, with no clear pattern of genotype relating to Rgene presence, suggesting that genetically varied isolates are capable of causing infection. The PNW results support the premise of a single $A$. anomala introduction. These findings support hazelnut breeding efforts in developing durable resistance to EFB.

Population genetic analysis for a worldwide collection of Phytophthora palmivora

Y. GUO, G. A. Torres-Londono, M. K. Hausbeck, Michigan State University, East Lansing, MI, USA

Phytophthora palmivora (Butler) is a worldwide, destructive, tropical plant pathogen infecting roots, stems, branches, leaves, flowers and fruits on a wide range of plant species. The objective of this study was to identify the genetic structure of a worldwide collection of $P$. palmivora isolates in terms of host type, geographical distribution, mating-type, and morphological characters. A total of 95 P. palmivora isolates representing 4 continents, 15 countries, and 14 host species were used for mating-type identification and morphological characterization. We designed $>900$ primer pairs from 1,017 identified SSRs (simple sequence repeats) in the P. palmivora draft genome for the population genetics study. Evaluations of 70 primer pairs revealed that $>90 \%$ yielded expected amplification and $\sim 30 \%$ showed polymorphisms. Preliminary analyses of molecular variance (AMOVA) with 11 SSR markers from intergenic regions revealed significant genetic differences among Asia, South America and North America isolates. Higher Nei's genetic diversity was found in countries in North America and Asia while lower genetic diversity was found in countries in South America. AMOVA analyses indicated no significant differences from isolates collected from two major host species - African oil palm and cacao. Also, there were no significant differences among growth patterns or mycelial fluffiness. These results expanded the understanding of the genetic variation of this important pathogen.

Population biology and comparative genomics of Claviceps purpurea and other defensive mutualists in the Hypocreales S. WYKA, G. Wu, K. D. Broders, Colorado State University, Fort Collins, CO, USA

The genus Claviceps includes species that are ovarian parasites of plants in the Poaceae, causing the ergot disease symtoms. This genus recently experienced advances in the delimitation of cryptic species and existence of separate lineages with respect to geographic distribution and host preference. It was also recently discovered that Claviceps purpurea produces both cytokinins and auxins during infection. Current outbreaks of ergot on barley in relatively geographically isolated locations within the U.S. has spurred efforts to analyze the population structure, and study the evolution of phytohormone production in the agriculturally important $C$. purpurea. A phylogenetic analysis of five gene loci demonstrated the U.S. population is distinct from European isolates, but no subpopulations were observed. A comparative genomic analysis of species within the Hypocreales revealed orthologs of C. purpurea cytokinin biosynthesis genes (cpp450, cpipt-log, tRNA-ipt) and putative auxin genes in members of the closely related tribe Balansiae, which contains fungal endophytes and parasites that have been shown to provide mutualistic benefits to their host. These results indicate there is a distinct population of $C$. purpurea infecting barley and other grasses in the central plains, and the evolution of phytohormones may play an important role in the ecology and evolution of Claviceps and other species in the Hypocreales as defensive mutualists with broad host ranges.

Genetic diversity and population characterization of Erysiphe pulchra, the causal agent of dogwood powdery mildew, from the eastern United States

C. R. WYMAN (1), S. L. Boggess (1), D. Hadziabdic (1), T. A. Rinehart (2), A. S. Windham (3), P. A. Wadl (4), R. N. Trigiano (1), (1) University of Tennessee, Knoxville, TN, USA; (2) United States Department of Agriculture, Poplarville, MS, USA; (3) Soil, Plant, and Pest Center, Nashville, TN, USA; (4) US Vegetable Lab, Charleston, SC, USA

Erysiphe pulchra is an obligate fungal pathogen that causes powdery mildew in Cornus florida, commonly known as flowering dogwood. Disease management costs for dogwood nurseries increased substantially after 1995 with the sudden and swift spread of $E$. pulchra throughout the eastern United States. Initially, sexual reproduction was seen frequently, but in recent years, the teleomorph of E. pulchra has not been observed very often. This may indicate that the pathogen is only reproducing asexually by conidia and we hypothesized that these populations have become essentially clonal. A library of 237 microsatellite marker pairs were developed by de novo sequencing of the genome of E. pulchra using Ion Torrent technology. Fifteen microsatellite markers of 28 primer pairs examined were polymorphic and used to compare 187 samples from isolates collected from 10 eastern U.S. states. The objectives of the study were to assess genetic diversity and population structure of E. pulchra across different geographical regions in the eastern U.S. The original data set $(\mathrm{n}=174)$ was clone corrected $(\mathrm{n}=136)$ and therefore, all further analyses were performed using clone corrected data. Our results indicated a low genetic diversity among samples, scarce numbers of private alleles, and evidence of a population bottleneck. Our work implies a high probability that the pathogen is only reproducing asexually.

\section{Identification of promising bacterial root symbionts from switchgrass (Panicum virgatum) growing in the Prairies of Oklahoma} M. H. CHI (1), K. D. Craven (2), (1) The Samuel Roberts Noble Foundation, Ardmore, OK, USA; (2) Samuel Roberts Noble Foundation Inc, Ardmore, OK, USA

Switchgrass is a critical bioenergy crop in the US for cellulosic biofuel production. Improving productivity is essential to make switchgrass cultivation profitable such that a sustainable supply of feedstock can be supplied to biorefineries. Plant growth promoting rhizobacteria (PGPR) have shown tremendous potential as biofertilizers and phytostimulators. Thus, the exploration and use of naturally-occurring endophytic bacteria is a promising approach for the improvement of switchgrass productivity. Isolation of bacteria from surface-sterilized root samples of 214 switchgrass plants collected from the Prairies of Oklahoma during the spring, summer and fall of 2009 revealed the presence of diverse groups of endophytic bacteria. A total of 34 genera were identified from 16S rDNA sequences from 526 strains. The most frequent genera were Pseudomonas (55\%), Rhizobium (6.5\%), Bacillus (5.7\%), and Klebsiella (4.9\%). Using high-throughput screens including a Ca-phosphate solubilizing test, an ACC-deaminase test, and a presence/absence test for the NifH gene, a total of 113 strains were identified with at least one of these traits. One strain (Burkholderia sp.; NFPP32) was positive for all three traits, and turned out to be an auxin producer as well. Comparative genome analyses and in planta biomass gain tests using these beneficial candidates are currently underway.

\section{Metagenomic Sequencing Reveals Bacterial Community Composition of Native and cultivated Cranberry Bogs} G. EBADZADSAHRAI, A. Harrison, S. Soby, S. Gadagkar, Midwestern University, Glendale, AZ, USA

The bacterial communities of cultivated and fallow soils play a key role in promoting plant growth and suppressing plant disease. We used 16S rRNA V4 amplicon pyrosequencing to investigate the community structures of soil bacteria from cultivated and wild cranberry bogs. More than 17,000 valid reads indicated that the most abundant phyla across all soil samples were Proteobacteria, Acidobacteria, Bacterioidetes, and Verrucomicrobia. The taxonomic patterns of these dominant phyla were relatively uniform in all samples. However, the taxonomic patterns of lower abundance OTUs showed significant variation between samples taken from different sites: Chloroflexi, Cyanobacteria, Armatimonadetes, Nitrospirae, Spirochaetes, Elusimicrobia, and some Candidate Divisions correlated with micronutrients, whereas macronutrients affect the dominant four soil taxa. Principle component analysis (PCA) indicates that sampling location or soil type are more important than cultivation effects in determining bacterial community structure. 
Diversity and fungicide sensitivity of the cacao pathogens Moniliophthora perniciosa and $M$. roreri

M. G. Mariduena-Zavala (1), A. Freire-Penaherrera (1), M. Villavicencio-Vasquez (2), R. Espinoza-Lozano (2), J. CEVALLOS-CEVALLOS (3), (1) Escuela Superior Politecnica del Litoral, ESPOL, Guayaquil, ECUADOR; (2) ESPOL, Guayaquil, ECUADOR; (3) Escuela Superior Politecnica del Litoral, ESPOL, Guayaquil, FL, ECUADOR

Moniliophthora perniciosa and M. roreri are the two most devastating cacao pathogens worldwide. Controlling both pathogens is challenging, especially for producers of organic cacao. Knowing the molecular and phenotypic diversity of the pathogens and their sensitivity to organic fungicides would favor the development of control strategies. A total of 85 and 90 isolates of M. perniciosa and M. roreri were obtained from cacao trees in five provinces of Ecuador, the top fine-flavor cacao producer in the world. Molecular diversity of each pathogen was estimated by using RFLP, IRAP, REMAP, and SSR, as well as the sequencing of the ITS DNA region. Mycelial color and growth rate were evaluated as phenotypic characteristics of the pathogens. Sensitivity to organic fungicides was assessed and the IC50 values were determined in vitro. The average Nei's gene diversity values were 0.32 for $M$. perniciosa and 0.18 for M. roreri. Isolates developed into 14 different mycelial colour groups that correlated to growth rate. Growth of all pathogens fitted to a Gompertz model with a rate parameter index ranging from 0.08 to 0.13 and from 0.23 to 0.32 for $M$. pernicios $a$ and $M$. roreri, respectively. Both pathogens showed moderate sensitivity to organic fungicides with average IC50 doses of 1.5 to $2.0 \%$. Organic fungicides can be used to control both pathogens but additional disease control strategies for organic producers, including biocontrol agents should be evaluated.

Population biology of the downy mildew pathogen on tolerant and susceptible cucumber in Southeastern United States M. K. MANDAL PHD (1), J. Ikerd (2), E. Wallace (3), R. Grace (4), W. W. Turechek (5), L. Quesada-Ocampo (6), C. Kousik (2), (1) ORISE participant, US Vegetable Laboratory, USDA, ARS, Charleston, SC, USA; (2) U.S. Vegetable Laboratory, USDA, ARS, Charleston, SC, USA; (3) North Carolina State University, Raleigh, NC, USA; (4) U.S. Horticultural Research Laboratory, USDA, ARS, Fort Pierce, FL, USA; (5) USDA ARS SAA SPP, Fort Pierce, FL, USA; (6) Department of Plant Pathology North Carolina State University, Raleigh, NC, USA

Downy mildew (DM) caused by Pseudoperonospora cubensis can affect a wide variety of vegetable crops in the family Cucurbitaceae including cucumber, leading to significant yield loss in southeastern and other parts of the U.S.A. Cucumber cultivars with resistance to DM were available for over 30 years and were widely grown. After the breakdown of host resistance in 2004 by $P$. cubensis, intensive fungicide programs have been in use to limit the pathogen from completely destroying the crop. Our current research is focused on obtaining a better understanding of the population structure of P. cubensis across southeastern states on DM tolerant and susceptible cultivars. Large field plot trials with cultivar 'Expedition' (resistant to DM prior to 2004), a DM tolerant cultivar 'Peacemaker' (after 2004), and susceptible cultivar SMR-58 were conducted in Fort Pierce, FL and Charleston, SC. Disease developed very rapidly on SMR-58 at both locations. In FL, severe DM was observed on Peacemaker and Expedition by end of the season but the rate of disease development was much slower than on SMR-58. However, in SC no DM was observed on Expedition or Peacemaker in 2016 indicating a potential difference in the pathogen populations in FL and SC. Subsequent molecular marker analysis using 9 microsatellite primers showed distinct genetic diversity between the two populations. Further characterization of the prevalent DM strains will help appropriate deployment of DM tolerant cultivars.

Leaf spot fungi of no-till wheat in Oklahoma and evaluation of SSR loci for population biology of the tan spot fungus, Pyrenophora triticirepentis

S. SUAREZ, S. M. Marek PhD, R. M. Hunger, C. D. Garzon PhD, Oklahoma State University, Stillwater, OK, USA

Wheat is the third most cultivated crop in the US. In Oklahoma, an increasing use of no-till cultivation is resulting in a greater incidence of fungal leaf spot diseases, such as tan spot, caused by Pyrenophora tritici-repentis (PTR), and similarly appearing leaf spots caused by PTR-like Bipolaris spp. and pycnidial fungi Parastagonospora nodorum and Zymoseptoria tritici. To evaluate the relevance of PTR in Oklahoma wheat, a culture based approach was used to isolate fungi on semi-selective media from symptomatic leaves collected in the spring from 13 winter wheat fields across Oklahoma. Cultures were morphologically identified as 'PTR-like' or 'pycnidial fungi'. Of 755 cultures isolated, $565(75 \%)$ were PTR-like and 190 (25\%) were pycnidial fungi. Molecular identification of 500 single-spore PTR-like isolates using PTR-specific PCR primers indicated that $348(70 \%)$ of these were PTR. Based on these results, PTR appeared to cause almost half of the wheat leaf spot disease in Oklahoma no-till wheat, while $\sim 20 \%$ was caused by Bipolaris spp. and $\sim 30 \%$ by P. nodorum and Z. tritici. To study the genetic diversity of PTR in Oklahoma, twelve available simple sequence repeat (SSR) markers were assessed on a subset of 100 Oklahoma PTR isolates and nine of these SSR markers were found to be informative. Further studies will evaluate the genetic structure of PTR populations and production of cultivar-specific toxins A, B and C.

Temperature adaptation in Macrophomina phaseolina, the causal agent of charcoal rot on soybean V. ORTIZ LONDONO (1), K. A. Wise (2), M. Chilvers (1), (1) Michigan State University, East Lansing, MI, USA; (2) Purdue University, West Lafayette, IN, USA

Macrophomina phaseolina, a soil- and seed-borne fungal pathogen, infects more than 500 plant species and causes charcoal rot disease on soybean. Charcoal rot can result in severe yield losses under high temperatures and drought conditions. Therefore, charcoal rot affects soybean production mainly in tropical and subtropical areas, including the southern US. However, the incidence of charcoal rot disease in soybean has recently been increasing in the northern US. Temperature adaptation due to pathogen genetic divergence may be involved in the geographically broadening of charcoal rot disease. In this study, 80 M. phaseolina isolates, collected from soybean, dry bean and corn grown in the northern and southern US were identified to the species level, characterized for fitness at cold temperature and whole-genome sequenced. All 80 isolates were confirmed as M. phaseolina by Maximum Likelihood inference using ITS, EF-1 $\alpha$ and ACT loci. In vitro growth rate assays at 15 and $35^{\circ} \mathrm{C}$ were used to evaluate the influence of temperature on fungal growth. Isolates from the northern US grew significantly faster than those from the southern US at $15^{\circ} \mathrm{C}(\mathrm{p}<.0001)$. All isolates were wholegenome sequenced to $20 \mathrm{X}$ coverage using a 150 base-pair paired-end strategy on the Illumina HiSeq 4000 platform. Population and comparative genomics will be used to identify genomic regions and candidate genes involved in response to cold temperature.

\section{CRISPR/Cas sequence-based confirmation of an emergent population of Rathayibacter toxicus in South Australia} J. P. STACK (1), G. Y. Busot (1), M. Arif (2), (1) Kansas State University, Manhattan, KS, USA; (2) University of Hawaii, Oahu, HI, USA

Rathayibacter toxicus is a gram positive plant pathogenic bacterium found only in Australia and South Africa. The systematic, memory-based CRISPR/Cas defense mechanism in bacteria has been shown to provide spatial and temporal information with phylogenetic value. We determined that $R$. toxicus has the Escherichia coli Type I-E CRISPR/Cas system. In a previous MLST-based population genetic study, a newly emerged population (RT-I) of $R$. toxicus was detected at three locations in South Australia. To identify the geographic and genotypic source of that strain, comparative analyses of the CRISPR-Cas region were performed on representative strains from different population types (I-IV), host species (4), geographic locations (4), and collected over a forty-year period. The 7 Cas gene sequences, the CRISPR leader sequences, and concatenated CRISPR spacer sequences were identical for all RT-I strains, independent of the location of isolation. Phylogenetic relationships based on comparative analyses of the 7 Cas genes, the leader sequences, and the concatenated CRISPR spacer sequences were consistent with the MLST-based population genetic analyses. Based on several sequence analyses, the South Australia RT-I strains cluster close to strain CS36 from New South Wales. Yet, there was no homology between the RT-I concatenated spacer sequences and the CS36 spacer sequences. This suggests an independent origin and subsequent emergence of the RT-I population in South Australia. 
Characterization of Phytophthora infestans isolates from Indonesia

S. DANGI (1), D. Douches (2), P. S. Wharton (1), (1) University of Idaho, Aberdeen Research and Extension Center, Aberdeen, ID, USA; (2) Michigan State University, Department of Plant, Soil and Microbial Sciences, East Lansing, MI, USA

Late blight caused by Phytophthora infestans is a major constraint in all potato growing regions of the world. The genetic structure of $P$. infestans populations is dynamic. In recent years, the genetic structure of $P$. infestans population has been changing and new genotypes with different epidemiological characteristics have been emerging due to migration, mutation and sexual recombination. More isolates are becoming resistant to mefenoxam worldwide, and it is becoming a more common problem of crop failure in developing countries. Little is known about the genetic composition of $P$. infestans isolates in Indonesia. In this study, isolates were collected from infected potato leaves on FTA cards from cultivars Atlantic and Granola growing in Pangalengan, Indonesia and cards transported back to the lab at Aberdeen R\&E center in Idaho. Four isolates of $P$. infestans were successfully extracted and analyzed using 6 microsatellite markers. Allelic diversity was characterized using simple sequence repeats (SSR) at six loci and cluster population analysis obtained by Euclidean clustering analysis showed closeness of Indonesian isolates to US-8 and Blue 13. This was a preliminary study and more isolates and microsatellite markers will be used in the full study to determine the diversity of Indonesian isolates and the relatedness of these isolates with the European and North American isolates.

Phylogenetic analysis of Diaporthe spp. in highbush blueberries in Michigan

G. KOTAMRAJU, K. Clemens, A. C. Schilder, Michigan state university, East Lansing, MI, USA

Cane canker and twig blight are common in Michigan blueberry fields. To determine the causal agent(s), 20 fields were sampled in 2012 and 2013 and 6 additional fields (including in northern Indiana) in 2013. Diaporthe spp. represented 60\% and 58\% of isolated fungi in 2012 and 2013 , respectively. Diaporthe isolates (243 total) were identified by sequencing of the ITS region and comparison to published sequences in GenBank. Diaporthe vaccinii was predominant ( $89.3 \%$ of isolates), whereas D. eres made up $9.5 \%$ of the isolates, and D. melonis, D. stewartii, and D. cotoneastri together made up $1.2 \%$ of the isolates. $D$. eres was found in 11 fields of multiple varieties ranging in age from 4 to 75 years and has been previously reported from $V$. corymbosum in Chile and the Netherlands. Phylogenetic analysis was conducted by multiple sequence alignment using MUSCLE with 10 iterations. The subsequent alignment was analyzed using GUIDANCE 2 to obtain an alignment confidence score of 0.998 . A neighbor joining tree was constructed using MEGA 6.06 with 5000 bootstraps using D. viticola as an outgroup. Diaporthe vaccinii and $D$. eres separated into two distinct clades. The $D$. vaccinii clade showed little variability whereas the $D$. eres clade was more variable. Sequencing of the EF1- $\alpha$ and $\beta$-Tub genes and morphological characterization are in progress. The pathogenicity and virulence of $D$. eres in blueberry needs to be confirmed to determine the impact on blueberry production in Michigan.

Trade-offs and synergies in microbial mediated functions for potato plant health R. LANKAU, University of Wisconsin, Madison, WI, USA

The importance of diverse soil and rhizosphere microbial communities for plant health and production is well recognized, and new technologies have allowed an explosion in investigations of plant-associated microbiomes in recent years. We investigated how microbial community structure creates trade-offs or synergies between multiple functions for plant health; specifically, supporting plant growth under high or low nutrient conditions and suppression of the tuber blemish pathogen Streptomyces scabies, causal agent for potato common scab disease. We found that microbial communities that provided the strongest pathogen suppression also resulted in the lowest plant growth in the absence of disease, especially in well fertilized conditions. High phylogenetic diversity of the bacterial community correlated with high plant growth in all conditions, and high fungal diversity also promoted growth in low nutrients. Disease suppression was unrelated to microbial diversity, but instead responded to the presence of specific bacterial taxa. However, microbial diversity was the best predictor of ecosystem "multifunctionality" - the ability of a microbial community to provide both plant growth and disease suppressive services. Our results suggest that managing soil communities for multiple outcomes may be challenging due to inherent trade-offs between ecosystem functions; however, maintaining high microbial diversity may be the best approach for achieving multi-functional soils.

\section{The use of plant extracts for the management of post-harvest diseases of tomato in Kenya}

\section{G. M. KARIUKI, Kenyatta University, Nairobi, KENYA}

Tomato postharvest diseases pose a serious challenge in Kenya. Several pesticides have been used to control the postharvest decay of tomato fruits but with increasing consumer concern over pesticide residues, there is need to look for safer alternatives. Different crude plant extracts (ginger, neem and garlic) were used at different doses in vitro and in vivo for the control of pathogens causing post-harvest rot of tomato fruits. The pathogens that were tested in this study were Fusarium spp., Rhizopus spp., Geotrichum spp. and Erwinia spp. The growth media used was amended with the crude extracts and the pathogens introduced into the amended growth media. Different doses $(1 \mathrm{mg} / \mathrm{ml}, 2 \mathrm{mg} / \mathrm{ml}$, and $3 \mathrm{mg} / \mathrm{ml})$ of the crude plant extracts were used. Healthy tomato fruits were treated by dipping in the crude extracts $(3 \mathrm{mg} / \mathrm{ml}$ of each of the extract) and disease development evaluated. The experimental design was randomized complete block design replicated four times. Colony forming units were determined for the bacterial pathogen and compared with the untreated control. The inhibitory effect was proportional to the extract concentration. Radial growth of the fungal pathogens was measured at an interval of $24 \mathrm{hrs}$ after the second day for five days. All the doses for all the extracts had inhibitory effect against the test pathogens. Garlic extract was the most effective against all the pathogens while Fusarium spp. was the most susceptible. There was no rot development on the treated fruits. This study demonstrate that plant extracts have potential for the management of post-harvest diseases in tomato.

Aspergillus section flavi community structure impacts aflatoxin contamination in Zambia

P. W. KACHAPULULA (1,2), J. Akello (3), R. Bandyopadhyay (4), P. J. Cotty (5,6), (1) Univ of Arizona, Tucson, AZ, USA; (2) University of Zambia, Lusaka, ZAMBIA; (3) International Institute of Tropical Agriculture (IITA-Zambia, Lusaka, ZAMBIA; (4) International Institute of Tropical Agriculture, Ibadan, NIGERIA; (5) UNIVERSITY OF ARIZONA, Tucson, AZ, USA; (6) USDA-ARS, University of Arizona, Tucson, AZ, USA

Aflatoxins are carcinogenic, immunosuppressive toxins that contaminate maize and groundnut. Aspergillus flavus, A. parasiticus, and two unnamed taxa are frequently implicated as causal agents of contamination in Africa. However, Aspergillus populations associated with aflatoxin contamination in Zambia have not been adequately characterized. Relationships between fungal community composition and aflatoxin concentrations may suggest mitigation strategies. Aflatoxins were quantified in maize $(n=225)$ and groundnut $(n=137)$ with immunochromatographic assays. Fungi were isolated and assigned to morphological species, and relationships between frequency of fungi and aflatoxin concentration were determined using regression analyses. Seventeen percent of crops had aflatoxin concentrations above allowable limits in Zambia $\left(10 \mu \mathrm{g} \mathrm{kg}^{-1}\right)$. A. parasiticus and the L-strain morphotype of $A$. flavus were dominant on maize and groundnut ( $42 \%$ and $60 \%$ of Aspergillus section Flavi, respectively). Incidences of $A$. flavus L-morphotype were negatively related to aflatoxin in groundnut $\left(\log \mathrm{y}=2.4990935-0.09966 \mathrm{x}, \mathrm{R}^{2}=0.79, P=0.001\right)$, while $A$. parasiticus was related to aflatoxin concentration in both crops $\left(\log \mathrm{y}=0.1956034+0.510379 \mathrm{x}, \mathrm{R}^{2}=0.57, P<0.001\right)$. The results indicate that both $A$. parasiticus is an important causal agent of aflatoxin contamination in Zambia and there is potential for utilization of atoxigenic A. flavus-L morphotype genotypes for aflatoxin management.

Control of Rhizopus rot of tomato fruit by postharvest fungicide application

J. BARTZ (1), D. Spiceland (1), S. Sargent (1), A. Berry (1), M. T. Elkahky (2), G. E. Vallad (3), (1) University of Florida, Gainesville, FL, USA; (2) Mansoura University, Mansoura, EGYPT; (3) Gulf Coast Research and Education Center, University of Florida, Wimauma, FL, USA 
A severe outbreak of postharvest decay among fresh-market tomatoes accompanied heavy rainfall during the 2016 winter tomato season in Homestead, Florida. The most aggressive pathogen was Rhizopus stolonifer. The fungicide, Chairman ${ }^{\circledR}$ (Syngenta), containing fludioxinyl and propiconazole, is labelled for postharvest application to large- and medium-sized tomato fruit as an in-line dip/drench application for control of Rhizopus rot. To assess efficacy, we wounded mature green round tomatoes and applied $10 \mu \mathrm{L}$ of a $1 \times 10^{6}$ conidia/ml suspension to each wound, dried these fruit for $4 \mathrm{~h}$ and followed with a $5 \mathrm{sec}$ immersion in 430 or $860 \mathrm{ppm}$ a.i. of Chairman. Treated fruit were placed on foam depression sheets located on wet paper towels in 30 to $32 \mathrm{~L}$ plastic snap-lid containers. Lids were attached loosely and the fruit were exposed to 100-200 ppm ethylene at $20 \mathrm{C}$ for 5 days, per standard practice. Water soaking was observed on control fruit within $24 \mathrm{~h}$ and a "nest of mycelium" covered these fruit by 4 days. Disease incidence on day-12 of the subsequent $20-^{\circ} \mathrm{C}$ storage was 100,11 and $0 \%$ for the control, 430 and $860 \mathrm{ppm}$ treatments, respectively. In a second experiment, decay incidence on day- 15 was $100,1.3$ and $0 \%$, for those treatments respectively. Aerial mycelial development was not observed over wound sites on the Chairman treated fruit. Application of Chairman in packinghouses may provide a way to reduce or prevent postharvest losses during seasons that are favorable for Rhizopus rot.

Options to reduce costs of aflatoxin control in commercial cotton with area-wide programs utilizing atoxigenic biocontrol agents R. JAIME (1), L. Antilla (2), L. Liesner (2), P. J. Cotty (3), (1) University of Arizona, Tucson, AZ, USA; (2) Arizona Cotton Research and Protection Council, Phoenix, AZ, USA; (3) USDA-ARS, University of Arizona, Tucson, AZ, USA

Aflatoxin contamination, caused by fungi in Aspergillus section Flavi, is a serious concern for many crops. Several susceptible crops may be grown in the same area, therefore long-term and area-wide programs to limit aflatoxin contamination with biocontrol can improve both efficacy and value of applications. Biocontrol with atoxigenic genotypes of A. flavus is used on several crops, including cotton and maize, in Arizona. Biocontrol modifies population structures of $A$. flavus populations in order to reduce aflatoxin-producing potentials and, as a result, crop aflatoxin content. Atoxigenic genotypes of $A$. flavus used as active ingredients persist in treated soils resulting in some benefit to subsequent crops. However, incidences of atoxigenics decline over time and repeated treatment is necessary to ensure optimal aflatoxin management. Both toxigenic and atoxigenic $A$. flavus propagules also move between fields. Application of the biocontrol A. flavus AF36 Prevail ${ }^{\mathrm{TM}}$ to a single edge of cotton fields resulted in significant displacement of aflatoxin-producing fungi and lower aflatoxin levels compared to the crops produced in untreated opposite boarders and neighboring fields. The treatments also influence the population structure of $A$. flavus in untreated areas. Partial treatments might suffice to maintain levels of atoxigenic genotypes adequate to keep crop aflatoxin concentrations below maximum allowable levels with reduced application and material costs.

Molecular bases for loss of pectinase P2c activity in morphologically and geographically distinct populations of Aspergillus flavus S. SMITH (1), P. J. Cotty (2), (1) The University of Arizona, Tucson, AZ, USA; (2) USDA-ARS, University of Arizona, Tucson, AZ, USA

Aspergillus flavus is a cosmopolitan species of filamentous fungi. This opportunistic plant pathogen has two distinct morphotypes classified as the "S" and "L" strains. Both morphotypes are capable of producing aflatoxins, highly toxic and carcinogenic metabolites. Ingestion of contaminated foods can result in stunting, immune suppression and, at ppm levels, acute liver failure followed by death. P2c is an endopolygalacturonase produced by $A$. flavus and positively associated with virulence. Pectin degradation by $A$. flavus was visualized on pectin-agarose medium with $0.1 \%$ ruthenium red. Pectinase activity screening showed that 38 of 69 S \& 19 of $35 \mathrm{~L}$ isolates from the Southwestern and Southeastern United States were P2c deficient. The PecA gene, which encodes pectinase P2c, was sequenced to identify polymorphisms associated with lack of P2c activity. Two distinct polymorphisms were identified as molecular bases for the loss of P2c activity. These were an $\mathrm{A} \rightarrow \mathrm{T}$ transversion at nucleotide 308 resulting in an early stop codon present only in P2c deficient L-morphotype isolates, and an AG insertion after nucleotide 443 resulting in a frameshift mutation found only in P2c deficient Smorphotype isolates. The data suggests that functional pectinase P2c was lost twice through distinct molecular events during divergence of A. flavus genotypes, and that these loses were retained independently during clonal evolution of the $A$. flavus morphotypes.

\section{Characterization of atoxigenic Aspergillus parasiticus from peanuts in Malawi}

C. CHING'ANDA (1), J. Atehnkeng (2), R. Bandyopadhyay (3), P. J. Cotty (4), (1) University of Arizona, Tucson, AZ, USA; (2) International Institute of Tropical Agriculture, Lilongwe, MALAWI; (3) International Institute of Tropical Agriculture, Ibadan, NIGERIA; (4) USDA-ARS, University of Arizona, Tucson, AZ, USA

Aflatoxins often contaminate Malawi's food and feed where these toxic fungal metabolites may cause immune suppression, stunting, and cancer. $A$. flavus and A. parasiticus are the most often described causes of aflatoxin contamination. Biocontrol with atoxigenic genotypes of $A$. flavus is effective at reducing contamination through modification of fungal community structure so that aflatoxin producers are less common. Secondary modes of action include inhibition of aflatoxin production during co-infection. In some areas of Malawi A. parasiticus dominates Aspergillus section Flavi communities on groundnuts. During characterization of fungi associated with groundnut in Malawi, atoxigenic A. parasiticus was discovered. These atoxigenics did not accumulate o-methyl sterigmatocystin as previously described atoxigenic genotypes. Atoxigenic $A$. parasiticus was evaluated for ability to inhibit aflatoxin biosynthesis in mature peanut co-infected by one of three aflatoxin producers from Malawi at $24^{\circ} \mathrm{C}$ and $32^{\circ} \mathrm{C}$. At $24^{\circ} \mathrm{C}$ atoxigenic $A$. parasiticus reduced aflatoxin $\mathrm{B}_{1} 47 \%$ to $64 \%$, but was less effective at $32^{\circ} \mathrm{C}$. Results suggest possible development of strategies for utilizing atoxigenic A. parasiticus as active ingredients in biocontrol products targeted to regions where $A$. parasiticus dominates communities of aflatoxin-producing fungi. To assess the potential of atoxigenic $A$. parasiticus as biocontrols, competitiveness in the environment during crop production must be evaluated.

Evaluation of Academy ${ }^{\mathrm{TM}}$ postharvest fungicide to control blue mold of stored apple fruit caused by Penicillium expansum W. M. Jurick II PhD (1), V. L. Gaskins (1), M. W. Choi (2), K. D. COX (2,3), (1) USDA-ARS Food Quality Laboratory, Beltsville, MD, USA; (2) Cornell University, Geneva, NY, USA; (3) Cornell University, Plant Pathology\&Plant-Microbe Biology Section, Geneva, NY, USA

Blue mold, caused primarily by Penicillium expansum, is a major postharvest disease of apples. It not only causes economic losses, but produces mycotoxins that contaminate processed fruit products, which contributes to food waste. There are 3 postharvest fungicides labeled to control blue mold on stored apple fruit: $\operatorname{Mertect}^{\circledR}$ (a.i. thiabendazole), $\operatorname{Scholar}^{\circledR}$ (a.i. fludioxonil) and Penbotec ${ }^{\circledR}$ (a.i. pyrimethanil). However, resistance and reduced sensitivity have been found for all three compounds in P. expansum in the Mid Atlantic area. In 2016, a new postharvest fungicide (Academy ${ }^{\mathrm{TM}}$ ) was introduced to control blue mold on pome fruit containing difenoconazole and fludioxonil. Thus, we sought to investigate the impact of physical and chemical treatments on P. expansum inoculum levels on wooden bins and to evaluate blue mold control using Academy ${ }^{\mathrm{TM}}$-treated apple fruit. Wooden bins treated with steam, thyme guard, or Academy ${ }^{\mathrm{TM}}$ all had lower spore levels compared to untreated controls. Conidia in rinsate recovered from bins a week after treatment caused decay on wounded apples, albeit at lower incidence and severity, compared to the untreated control. Academy ${ }^{\mathrm{TM}}$ and Scholar ${ }^{\circledR}$ treated fruit had lower blue mold incidence and severity when applied as a curative treatment compared to the untreated check. Results from this study indicate that Academy ${ }^{\mathrm{TM}}$ can eliminate spores from bin surfaces and can reduce both blue mold incidence and severity on $P$. expansuminfected apple fruit.

Effect of humidity, temperature, and inoculum level on disease progression of sweetpotato black rot caused by Ceratocystis fimbriata M. STAHR (1), L. M. Quesada (2), (1) NCSU, Raleigh, NC, USA; (2) North Carolina State University, Raleigh, NC, USA

Ceratocystis fimbriata, causal agent of black rot in sweetpotato, is a reemerging post-harvest pathogen that has left sweetpotato growers and packers with losses of up to $\$ 150$ million in the past few years. While financial losses are large, not much is known about this pathogen, making preventative measures difficult to implement. The purpose of this study was to determine how environmental factors, such as temperature, humidity, and inoculum level, affect the growth and development of disease. Cured sweetpotatoes were surface sterilized, wounded with a toothpick, and inoculated with a spore 
suspension of $10^{4}, 10^{5}$, or $10^{6}$ spores $/ \mathrm{mL}$. Roots were separated by inoculum level and placed into clear sterile bins. Bins containing only roots maintained a relative humidity of approximately $80 \%$, while the addition of a petridish and $10 \mathrm{~mL}$ of water, or sterile paper towels and $15 \mathrm{~mL}$ of water maintained humidity levels of approximately 90 and $100 \%$, respectively. Roots were stored in dark growth chambers at $13,18,23,29$, or $35^{\circ} \mathrm{C}$ for 21 days with lesion and sporulation diameters being measured at 6 different time points. Results revealed that the pathogen was able to grow and sporulate at $18^{\circ} \mathrm{C}$, but not at $13^{\circ} \mathrm{C}$. Results also indicate that humidity level has no significant effect on growth and sporulation at low temperatures, but inoculum level significantly affects disease incidence. These findings can be applied to storage settings to help prevent future losses.

Control of sour rot (Geotrichum candidum) on tomato in a commercial packing house using a post harvest fungicide drip application C. COLLAZO-GONZALEZ (1), C. Weaver (2), (1) Syngenta Crop Protection, Vero Beach, FL, USA; (2) Syngenta Crop Protection, Visalia, CA, USA

Chairman ${ }^{\mathrm{TM}}$ Fungicide is a new post harvest product from Syngenta for control of storage rots on pineapple, stone fruit, cherries, and tomatoes. A premix of fludioxonil + propiconazole and formulated as a suspoemulsion (SE), Chairman applied via in-line drip can provide excellent control against diseases such as sour rot that have until now proved very difficult to manage. In order to confirm efficacy under commercial conditions, trials were conducted at a major packing house in Florida in December 2016. Tomato fruit were wound inoculated with a spore suspension of G. candidum. After incubation for 3 $\mathrm{h}$ under ambient conditions, inoculated fruit were treated on a packing line by dripping Chairman at a rate of 16 floz/gal of Decco Lustr 227 wax. The drip was calibrated to deliver 72 floz of Chairman + wax per hour, treating $c a 150,000 \mathrm{lb}$ of tomatoes. Fruit were then packed and gassed with ethylene $(100 \mathrm{ppm})$ for 5 days. Disease incidence was assessed 7 days after the Chairman treatment. Untreated fruit showed $100 \%$ decay, whereas fruit treated with Chairman showed no symptoms of decay. Additional studies are planned to optimize application procedures for storage rot control.

Prevalence and Incidence of Postharvest Diseases of Mandarin Fruit in California S. SAITO (1), C. L. Xiao (2), (1) USDA ARS, Parlier, CA, USA; (2) USDA-ARS, Parlier, CA, USA

In recent years, storing mandarin fruit in cold facilities has become a common practice to retain fruit quality and expand the marketing window. However, postharvest diseases can limit the storage of the fruit and cause significant economic losses if left uncontrolled. To determine major postharvest diseases affecting mandarins, decayed mandarin fruit were collected weekly during the storage seasons in 2015 and 2016 . In total, 20 and 46 grower lots (approximately 30 fruit per lot) were sampled either at pre-sorting or after cold storage in 2015 and 2016, respectively. Fungal isolation and identification were attempted for all decayed fruit sampled. Alternaria rot caused by Alternaria spp. was the most prevalent disease among non-stored fruit, accounting for $53.5 \%$ and $83.1 \%$ in 2015 and 2016, respectively. For stored fruit, green mold caused by Penicillium digitatum (36.3\%) was most prevalent followed by Mucor rot caused by primarily Mucor piriformis $(27.7 \%)$ and blue mold caused by P. italicum (23.3\%) in 2015 , while gray mold caused by Botrytis cinerea (29.7\%) was most prevalent followed by Mucor rot (27.1\%) and sour rot caused by Geotrichum spp. (18.7\%) in 2016. Percentage of each disease incidence in the total decayed fruit within a grower lot varied greatly from lot to lot. Our results indicate that in addition to green and blue mold and sour rot, gray mold and Mucor rot are two emerging postharvest diseases of mandarin fruit in California.

Unassembled transcriptome analysis to infer aflatoxin production in Aspergillus flavus strains infecting corn using EDNA A. ESPINDOLA (1), W. Schneider (2), H. A. Melouk (3), S. M. Marek PhD (1), K. F. Cardwell (1), C. D. Garzon PhD (1), (1) Oklahoma State University, Stillwater, OK, USA; (2) USDA ARS FDWSRU, Fort Detrick, MD, USA; (3) USDA ARS, Stillwater, OK, USA

Maize can be heavily infected by Aspergillus flavus under conducive conditions. Some A. flavus strains produce aflatoxin B1, a highly carcinogenic secondary metabolite. Currently, detection of aflatoxins is performed using chromatography or ELISA and molecular detection of aflatoxigenic A. flavus strains requires multiple PCR assays. Electronic (E-)probe Diagnostic Nucleic acid Analysis (EDNA) - a bioinformatic tool originally developed to detect microbial pathogens in plant metagenomes - was expanded to analyze transcriptomic data to identify active pathogens' molecular pathways. We used EDNA to detect genes associated with aflatoxin production in planta. Two strains of $A$. flavus, AF70 (toxigenic) and AF36 (atoxigenic), were grown on ground maize kernels (induces aflatoxin production) or potato dextrose broth (PDB) for 10 days at $31^{\circ} \mathrm{C}$ and total RNA isolated from cultures. Total RNA was poly-A selected and sequenced in an Illumina HiSeq 2500. Gene up-regulation was determined with DeSeq. Two hundred thirty-one eprobes were designed to detect up-regulated aflatoxin genes and used to perform EDNA on the transcriptomes. Hit frequencies (e-probes vs. transcriptomic reads) were significantly different $(\mathrm{p} \leq 0.05)$ between transcriptomes of AF70 grown on corn versus PDB and of AF70 versus AF36 grown on corn. This data suggests that EDNA can be used to infer the presence of aflatoxin in plant tissues or food products.

Fusarium ear rot and mycotoxin production by Fusarium subglutinans and $F$. temperatum on Bt and non-Bt maize infested with lepidopteran insect pests

D. A. MAYFIELD (1), F. E. Lanza (2), M. Sulyok (3), R. Krska (3), G. P. Munkvold (1), (1) Iowa State University, Ames, IA, USA; (2) CAPES Foundation, Ministry of Education of Brazil, Brasília, BRAZIL; (3) Department of Agrobiotechnology, University of Natural Resources and Life Sciences, Vienna, AUSTRIA

Fusarium subglutinans $(\mathrm{Fs})$ is one of the most common Fusarium spp. associated with Fusarium ear rot. However, there is limited information on the role of the closely related species F. temperatum ( $\mathrm{Ft}$ ), formally Fs group I, in Fusarium ear rot symptoms and mycotoxin contamination of grain. Field experiments were conducted in 2015 and 2016 including manual infestation with corn earworm (Helicoverpa zea) or European corn borer (Ostrinia nubilalis) and silk channel inoculations with Fs or Ft on Bt and non-Bt maize. Ear rot severity was estimated visually and a multi-mycotoxin analysis was performed on milled grain from each field plot. Ear rot severity was greater in the non-Bt hybrid in both years $(P \leq 0.0056)$ and inoculated treatments had significantly higher ear rot severity than non-inoculated controls $(P<0.0001)$. H. zea infestations increased ear rot severity in $2016(P=0.0452)$. In 2015, Ft inoculation resulted in higher levels of moniliformin than Fs inoculation $(P<0.0001)$. Grain from Ft-inoculated treatments had lower levels of fusaric acid than Fs treatments $(P<0.0001)$. There were large differences between $\mathrm{Ft}$ strains for moniliformin, fusaproliferin, and beauvericin levels. In Fs-inoculated treatments beauvericin levels were very low compared to Ft treatments. Fumonisins were primarily detected in non-inoculated treatments with the highest levels in non-Bt, insect-infested treatments. This is the first reported data of Ft causing Fusarium ear rot in North America.

Mexican maize landraces and their association with potentially toxigenic Fusarium spp.

L. M. VÁSQUEZ-SILLER, J. L. Herrera-Ayala, M. C. Vega-Sánchez, A. Muñóz-Urbina, S. Rodríguez-Herrera, Universidad Autónoma Agraria Antonio Narro, Saltillo, Coahuila, MEXICO

Mexico harvests around 23 million $\mathrm{t}$ of maize grain and $0.5 \%$ corresponds to maize native populations. Such grain may be infected by phytopathogenic fungi generators of mycotoxins that may affect human and animal health. This study estimated the association between their incidence and 18 different populations of maize in two states of Mexico: Mexico and Tlaxcala. They were microbiologically analyzed each, using the freeze blotter test with subsamples of 50 grains and four replications, coming from 10 ears, identifying and quantifying incidence of: Number of Phytopathogenic Fungi Genera (NPFG), Fusarium graminearum (FG) and Fusarium verticillioides (FV). Additionally, such populations were morphologically characterized and classified each as the respective landrace on samples of 20 ears, which were: Palomero, 4; Chalqueño, 2; Cacahuacintle, 4 and Cónico, 8. Principal Component Analysis (PCA) and Cluster Analysis (CA) with program Minitab 16 (Minitab, Inc.) were performed to associate fungi and landraces. The CA generated a dendrogram with seven clusters of landraces that responded differentially to the incidence of both Fusarium spp. The PCA defined that FV and FG were the most influential to generate such clustering, presenting a correlation between these two specie $(r=-0.467 *)$. The NPFG revealed that populations studied were 2-8 genera biodiverse. This work indicated its potential application in breeding programs and for studying mycotoxins contamination on maize for feed. 
Botrytis cinerea and B. prunorum associated with calyx-end rot in apples and pears in postharvest in the Maule Region, Chile E. E. FERRADA, M. A. Lolas, G. A. Diaz, Universidad de Talca, Talca, CHILE

Chile is one of the main exporters of fresh apples and pears in the Southern Hemisphere, with a cultivated area of 37,207 and 7,299 ha, respectively. The Maule Region (35 25' S) is the major area of the Chilean apple and pear industry. During the last season (2015-2016), symptoms of calyx-end rot on apples cv. Cripps Pink and Fuji ( $\mathrm{n}=271$ fruits) and pears cv. Beurre Bosc and Packham's Triumph ( $\mathrm{n}=53$ fruits) were observed in three commercial packinghouses. These fruits were obtained from ten localities in the Maule Region. The isolation from symptomatic fruits was performed in plates with PDA, showing that 244 isolates (91\%) were positive to Botrytis sensus lato. The level of species was determined by molecular analysis of the glyceraldehyde 3-phosphate dehydrogenase (G3PDH), heat-shock protein 60 (HSP60) and DNA-dependent RNA polymerase subunit II (RPB2) genes coding sequence alignments. Pathogenicity was demonstrated in apples and pears which were inoculated with conidia and mycelium and then stored at $20^{\circ} \mathrm{C}$ or $0^{\circ} \mathrm{C}$ for 30 days. In conclusion, B. cinerea and B. prunorum were identified and associated with calyx-end rot in postharvest. The most frequent species obtained from symptomatic fruit was $B$. cinerea.

Identification of Zearalenone degradation by Clonostachys rosea isolates, cloning and expression of $\boldsymbol{z h d} 101$ gene I. J. KANG, H. K. Shim, J. H. Roh, Y. Kim, S. Heu, National Institute of Crop Science, RDA, Suwon, Korea, Republic of (South)

The contamination of cereals with mycotoxins is a significant problem worldwide. Many strategies to eliminate mycotoxins have been applied and microbial biodegradation of mycotoxins may be the promising, effective, environmental friendly strategy so the biodegradation using microorganisms is now being widely examined. We isolated 20 Clonostachys rosea strains from soybean roots and identified the gene Zhd101 from all isolates. Then, all isolates were screened the level of degradation of Zearalnone using ELISA and TLS(thin layer chromatography). Almost all isolates degraded Zearalnone effectively and Quantitative reverse-transcription PCR revealed two-fold increase in expression zhd101 gene as Zearalenon increases up to $50 \mathrm{ppm}$. Also we described the clones of the zhd101 gene of 15-050 Clonostachys rosea strain and the genes size was identified. They was transformed into BL21 stains. The BL21 stains with zhd101 gene could be directly used for decontamination of cereal products.

\section{Aspergillus flavus, population biology of corn infectors}

J. A. REYES-PINEDA (1), K. E. Damann (2), (1) Louisiana State University, Baton Rouge, LA, USA; (2) Louisiana State University AgCenter, Baton Rouge, LA, USA

Aspergillus flavus is a threat to corn, cottonseed, peanuts and tree nuts due to contamination with carcinogenic aflatoxins. A. flavus subpopulations can be defined by vegetative compatibility groups (VCGs), morphotypes, chemotypes and mating type. Several studies have been conducted in the past where VCGs have been used as a measure of diversity and to which morphological and physiological features have been collated. Sweany et al, in 2011 identified 16 VCGs from soil and corn kernels in 11 Louisiana fields. Sclerotia morphotypes, levels of toxin produced, and mating type were more similar within VCG than among them. More important was that despite a high number of VCGs in the soil only a fraction of them colonized corn showing that certain VCGs have higher ability to infect. VCGs with ability to infect appeared to be predominantly mating type MAT1-2. Subsequent studies in our lab have also shown that MATI-2 isolates have higher growth rates in corn and produce more cyclopiazonic acid (a plant cell killing toxin) than MAT1-1 isolates, which also may indicate why they are more infectious. Research reported here involves assessing the population structure and diversity of A. flavus from 17 fields from Minnesota to Louisiana (8 states) along the Mississippi river to determine whether a correlation holds between VCG specificity and those characteristics associated with corn infection.

Interactions among active ingredients of a multi-isolate aflatoxin biocontrol product K. C. SHENGE (1), H. L. Mehl (2), P. J. Cotty (3), (1) USDA ARS, Tucson, AZ, USA; (2) Virginia Tech Tidewater AREC, Suffolk, VA, USA; (3) USDA-ARS, University of Arizona, Tucson, AZ, USA

The many vegetative compatibility groups (VCGs) of Aspergillus flavus vary widely in aflatoxin-producing potential. Indigenous atoxigenic VCGs are used to displace aflatoxin producers during crop production. Several biocontrol products have multiple atoxigenic $A$. flavus genotypes. The current study investigated competition among active ingredients in the FourSure ${ }^{\mathrm{TM}}$ field-use product. FourSure ${ }^{\mathrm{TM}}$ was manufactured by coating roasted sorghum kernels with a mixed spore suspension of A. flavus genotypes TC16-F, TC35-C, TC38-B and TC46-G. Similar products were manufactured each with only one of the four active ingredients. Products were incubated in $100 \% \mathrm{RH}$ at $10,20,30$, and $40^{\circ} \mathrm{C}$. Spores were harvested after 3,6 and 10 days and yields were quantified with a turbidimeter. DNA extracted from spores from multi-isolate treatments was used to estimate frequencies of each active ingredient in the mixture using quantitative Pyrosequencing. Overall, A. flavus genotypes did not grow at $10^{\circ} \mathrm{C}$ and maximum sporulation occurred at $30^{\circ} \mathrm{C}$. In products made with spore mixtures, frequencies of active ingredients in harvested spores were influenced $(\mathrm{P}<0.05)$ by temperature. The results suggest environmental conditions influence the dynamics of active ingredient release from multi-isolate biocontrol products. Further work is required to determine if manufacture of such products should utilized coating with single genotypes or genotype mixtures.

Influence of Bt maize (Zea mays (L.)) trait packages on aflatoxin contamination in South Texas maize production

G. L. SCHUSTER (1), D. Mays (2), M. Setamou (3), S. Nelson (4), (1) Texas A\&M Univ - Kingsville, Kingsville, TX, USA; (2) Texas A\&M AgriLife Extension Service, Brownfield, TX, USA; (3) Texas A\&M Kingsville Citrus Center, Weslaco, TX, USA; (4) Texas A\&M University Kingsville, Kingsville, TX, USA

Aflatoxin is produced by fungi in the Aspergillus genus, and the infection of maize by these fungi can be aided by insect damage. In the late 1990's Bacillus thuringiensis $(\mathrm{Bt})$ maize hybrids were introduced to minimize losses from Lepidopteran insect pests. Previous research showed variable results in the ability of Bt maize hybrids to reduce aflatoxin contamination. Field trials were conducted between 2014 and 2016 to evaluated the ability of newer $\mathrm{Bt}$ trait packages to reduce aflatoxin in maize grown in South Texas. Trait packages in the trials consisted of Genuity VT Double Pro, Round Up Ready Corn 2 only, Genuity Smart Staxx, Agrisure Viptera 3110 trait stack, Herculex 1, Optimum Intrasect Xtra, Optimum Leptra, and Optimum Intrasect. Aflatoxin levels among hybrids in 2014 ranged from 9.97 in DKC68-03 Genuity VT Double Pro to 89.85 in DKC66-97 Genuity VT Double Pro. In 2015 aflatoxin concentrations ranged from 0 ppb in P1319 Herculex 1 insect protection and DKC61-89 Genuity VT Double Pro to 7.15 ppb in DKC62-98 Genuity VT Double Pro. The mean concentration of aflatoxin in 2016 ranged from 0 ppb in P1319HR to 350.25 ppb in P1602 Optimum Intrasect. Significant $(P=0.05)$ differences were not observed among treatments in all three years, but large numerical differences were observed between certain treatments in both last two years of field trials. The results of these trials suggest that using Bt corn hybrids does not consistently reduce aflatoxin concentrations.

Interaction between Bt and non-Bt corn hybrids and Afla-Guard to reduce aflatoxin contamination D. MAYS (1), G. L. Schuster (2), M. Setamou (3), S. Nelson (4), (1) Texas A\&M AgriLife Extension Service, Brownfield, TX, USA; (2) Texas A\&M Univ - Kingsville, Kingsville, TX, USA; (3) Texas A\&M Kingsville Citrus Center, Weslaco, TX, USA; (4) Texas A\&M University Kingsville, Kingsville, TX, USA

Aflatoxins are produced by fungi in the genus Aspergillus, and it is estimated that mycotoxins, including aflatoxin, contaminate approximately 25 percent of the world's food crop. Recent research concluded that the use of atoxigenic strains of $A$. flavus for aflatoxin reduction can provide a net benefit between $\$ 8.00$ and $\$ 10.00$ per acre. A field trial was initiated in 2016 to assess the ability of a commercial atoxigenic strain, Afla-Guard, to reduce aflatoxin, when applied to Bacillus thuringiensis (Bt) and non-Bt corn hybrids at the VT (tassel) growth stage. Treatments included the Pioneer 
Hybrid P1319VYHR with and without Afla-Guard applied at the VT growth stage, and the Dekalb hybrid DKC66-94RR2 with and without Afla-Guard applied at the VT growth stage. Aflatoxin concentration in the Afla-Guard treatments averaged 1.0 parts per billion (ppb) and 0.0 ppb, in P1319VYHR and DKC66-94RR2, respectively. In the treatments that did not receive an application of Afla-Guard, the aflatoxin concentrations averaged $17.75 \mathrm{ppb}$ and 20.25 ppb in DKC66-94RR2 and P1319VYHR, respectively. Although aflatoxin differences between the Afla-Guard and non Afla-Guard treatments were not significantly $(P=0.05)$ different, there were large numerical reductions in aflatoxin concentrations following application of Afla-Guard at the VT growth stage.

Ecological diversity of Aspergilli community and aflatoxin prevalence in Mozambique

J. AUGUSTO (1), J. Atehnkeng (2), J. Akello (3), P. J. Cotty (4), R. Bandyopadhyay (5), (1) Intl Inst of Tropical Agriculture, Nampula,

MOZAMBIQUE; (2) International Institute of Tropical Agriculture, Lilongwe, MALAWI; (3) International Institute of Tropical Agriculture (IITAZambia, Lusaka, ZAMBIA; (4) USDA-ARS, University of Arizona, Tucson, AZ, USA; (5) International Institute of Tropical Agriculture, Ibadan, NIGERIA

Although high aflatoxin levels along the groundnut and maize value chains have been reported in Mozambique, aflatoxin prevalence and soil Aspergilli population and distribution in the field across agro-ecological zones (AEZ) in respect of cropping systems are not known. Groundnut is usually cultivated in lowlands $(<200 \mathrm{~m}$ of altitude) and midlands ( $200-600 \mathrm{~m}$ of altitude) while maize is mostly cultivated in midlands and highlands ( $>600 \mathrm{~m}$ of altitude). Georeferenced soil, maize and groundnut samples were arbitrarily taken from 900 farmers' fields at harvest in 2013, 2014 and 2015 in different AEZ and cropping systems to determine Aspergilli diversity and aflatoxin content. Aspergillus flavus $\mathrm{S}$ morphotype, A. flavus $\mathrm{L}$ morphotype, A. parasiticus and $A$. tamarii were isolated from different AEZ and cropping systems. The midlands had the highest Aspergillus biodiversity (Simpson's index of diversity $=0.8$ ) but $A$. parasiticus and A. flavus L morphotype were predominant. The lowlands had moderate Aspergillus biodiversity (Simpson's index of diversity=0.6) but $A$. flavus $\mathrm{L}$ morphotype followed by $A$. parasiticus and A. flavus $\mathrm{S}$ morphotype were predominant. The highlands had the lowest Aspergillus biodiversity (Simpson's index of diversity $=0.3$ ) and A. flavus L morphotype and A. parasiticus were predominant. Aflatoxin levels were very high $(0-6500 \mathrm{ppb})$ in lowland groundnut dominated cropping systems, moderate to high $(0-600 \mathrm{ppb})$ in midland maize and groundnut dominated cropping systems, and relatively low $(0-100 \mathrm{ppb})$ in highland maize dominated cropping systems. The results suggested that specific aflatoxin management practices may be needed for different agro-ecologies and cropping systems in Mozambique.

Effect of concentration of Pythium ultimum inoculum in soil, and on tubers at harvest, and storage temperature on disease incidence and severity

S. DANGI (1), A. Malek (2), J. Woodhall (3), K. Fairchild (2), P. S. Wharton (1), (1) University of Idaho, Aberdeen Research and Extension Center, Aberdeen, ID, USA; (2) University of Idaho, Aberdeen, ID, USA; (3) University of Idaho, Parma, ID, USA

Pythium leak caused by Pythium ultimum is a serious disease of potato in Pacific Northwest (PNW). In the PNW, Pythium leak is sometimes seen during harvest but most often in early storage. Pythium ultimum is of major concern to potato producers due to the large loses it can cause in storage. Infection of potatoes are favored by highly infested soil, high soil temperature at harvest, wounds and bruises during harvest and high humidity in storage. There is lack of understanding about the correlation between these factors and inoculum level in the soil. Therefore, an experiment was conducted to develop a model to predict the correlation between soil inoculum level and soil temperature during harvest to see the effect of these two factors on infection of tubers at two different storage temperatures. The inoculum concentration in the soil was tested before and after inoculation of $P$. ultimum. The tubers after harvest were incubated at $15^{\circ} \mathrm{C}, 20^{\circ} \mathrm{C}, 25^{\circ} \mathrm{C}$ and $30^{\circ} \mathrm{C}$ for 48 hours and stored at $8.8^{\circ} \mathrm{C}$ and $12.8^{\circ} \mathrm{C}$ for 35 days. The incidence and severity was recorded at 7 day's intervals. Levels of $P$. ultimum in infected tubers were quantified using species specific primers and qPCR. The results showed a high infection rate and inoculum concentration in tubers incubated at $20^{\circ} \mathrm{C}$ and $25^{\circ} \mathrm{C}$ and then stored at $12.8^{\circ} \mathrm{C}$. This is a preliminary experiment. In future, we will repeat the experiment and also investigate the minimum concentration of the sporangia/oospore required to infect the tubers at different temperatures and relative humidity. This project will develop a better understanding of pathogen-host-environment interactions and the underlying epidemiology of $P$. ultimum.

A comparative genomic analysis of cell wall degrading enzymes encoded proteins in Magnaporthe oryzae, Neurospora crassa, and Aspergillus sp. Q. NGUYEN (1,2), T. Ho (3), C. Nguyen (4), (1) Research Institute for Biotechnology and Environment, Ho Chi Minh, VIETNAM; (2) Nong Lam University, Ho Chi Minh, VIETNAM; (3) International University, Ho Chi Minh, VIETNAM; (4) Ho Chi Minh City Open University, Ho Chi Minh, VIETNAM

Filamentous fungi have a diverse array of cell wall degrading enzymes (CWDEs) to depolymerize the main structural polysaccharide of plant cell wall including cutin, cellulose, hemicellulose and pectin. The role of CWDEs and systemic analysis of these proteins have been previously reported in the rice blast fungus, Magnaporthe oryzae but a few studies have been demonstrated in filamentous fungi. In this study, a comparative genomic analysis of CWDEs in Magnaporthe oryzae, Neurospora crassa and Aspergillus s.p including Aspergillus niger and Aspergillus oryzae have been done. For M. oryzae, $N$. crassa, A. niger, A. oryzae respectively, we have identified 179, 79, 166 and 180 genes encoding CWDEs by crossing BLAST between CAZy database and Ensembl fungi database. Particularly, the function of 303 putative genes encoding CWDEs were revealed based on their conserved domain analysis and phylogenetic topology. The results of this study have been provided a comprehensive database resource on all CWDEs in $M$. oryzae, $N$. crassa, A. niger and A. oryzae to obtain further insight into the composition and evolutionary of CWDEs in filamentous fungi.

NGS of Monilinia causing Brown Rot of Fruit and Comparative Genomics for Diagnostics Development K. A. ZELLER, Y. Rivera, J. Sutherland, M. Galvez, Z. G. Abad, USDA-APHIS-PPQ-S\&T-CPHST, Beltsville, MD, USA

Monilinia fructicola, M. laxa, M. fructigena and M. polystroma cause brown rot of stone (e.g., peach, nectarine, cherry plum) and some pome (apple and pear) fruits. These fungi can cause twig and blossom blights, and significant fruit rot as the fruit ripen and their sugar content increases. M. fructicola and M. laxa are established within the USA, but M. fructigena and M. polystroma are currently not known from the USA. Morphological similarity among these species, and overlapping host ranges make differentiation between native and exotic species problematic. The PCR-based molecular diagnostics currently available for Monilinia species do not allow diagnosticians to unambiguously differentiate among these four species. We are using Next Generation Sequencing (NGS) technology to generate and assemble the genomes of each exotic and native Monilinia species of concern. Comparative genomics analyses among the species will help us identify species-specific gene targets that will be used to design improved diagnostic tests. Draft genomes for M. laxa and M. fructigena have been assembled from over 20 Million Illumina reads. The estimated genome sizes from M. laxa and M. fructigena are approximately $41 \mathrm{MB}$ each. PacBio NGS will be performed for selected isolates. Additional Illumina-based sequencing of all other Monilinia species will be done to permit comparative genomic analyses and identification of targets for species-specific diagnostics development.

Transcriptomic profiling of Soybean leaves reveals the programming language of glyceollin biosynthesis against Phakopsora pachyrhizi M. Z. HOSSAIN, University of Tsukuba, Tsukuba, JAPAN

Asian soybean rust (ASR), caused by the fungus Phakopsora pachyrhizi, is among the top twelve most virulent plant pathogens affecting up to $90 \%$ yield losses. Using fungicide is not sustainable solution to control ASR. Resistance loci (Rpp1-8) of soybean mountains opportunity to develop durable resistant variety but no genes have been cloned yet. Study on phenylpropanoid pathway intrigues continuously. B-14, B2-10, B3-12 and B4-4 are the near isogenic lines (NILs) having the Rpp1 to Rpp4 respectively. The expression profiling of these NILs on this pathway is not explored. To reveal this genetic framework, we generate RNA-seq data from transcriptomes of susceptible BRS-184 and resistance B3-12 inoculated with distilled water and 
ASR separately. A total of 5330 differentially expressed genes were annotated with different databases by similarity searching including Soybean knowledge Base (SoyKB) (3203, 60.09\%) Gene Ontology (GO) (2558, 47.99\%), Clusters of Orthologous Groups (KOG) $(1453,27.26 \%)$ and Kyoto Encyclopedia of Genes and Genomes Pathway (KEGG) $(2532,47.50 \%)$. The predominat genes are involved in basic biological process, oxidationreduction process, different ribosomal protein and catalytic activity. The isoflavonoid biosynthesis genes rank higher in KEGG. To validate these results, we conducted RT-qPCR expression profiling of 12 genes involved in isoflavonoid biosynthesis. Total RNA were extracted from susceptible BRS184 and other four NILs with ASR at 0, 12, 2448 and 96 hours after inoculation. These results demonstrate that soybean resistance resources reprogram their buttons to accumulate pterocarpan phytoalexins, glyceollins against ASR which will help for precision programming of these tools.

\section{Whole transcriptome analysis of Xylella fastidiosa grown inside microfluidic chambers identified specific calcium-regulated genes H. CHEN, L. De La Fuente, Auburn University, Auburn, AL, USA}

Microfluidic chambers (MC) allow constant microscopic observation of bacteria growing under media flow. This condition is a better representation of the natural habitat of xylem-limited insect-vectored pathogenic bacteria such as Xylella fastidiosa, as compared to batch culture systems. X. fastidiosa causes devastating plant diseases in several plant hosts in the Americas, as well as in Europe and Asia. Previous studies by our group showed that calcium $(\mathrm{Ca})$ enhanced virulence traits of $X$. fastidiosa, including biofilm formation and twitching motility. However, the specific mechanisms of these $\mathrm{Ca}$ effects are unclear. Previously, we conducted a transcriptome analysis of batch culture biofilm cells, and results indicated that the biofilm machinery was kept active longer under higher Ca concentrations -closer to those found in plant host xylem fluids. Here, RNA-Seq of 6-days old $X$. fastidiosa cells grown in $\mathrm{MC}$ with different $\mathrm{Ca}$ concentrations was performed to understand the role of $\mathrm{Ca}$ under flow conditions. Results indicate that a variety of genes were differentially expressed in response to Ca supplementation, including genes encoding transcriptional regulators, outer membrane adhesion proteins, pili-related proteins, toxin and others. Six genes were the same as those found in the batch culture study, but most of the genes found here were unique. We propose that MC is an ideal system for whole transcriptome analysis of bacteria growing under flow conditions in nature.

Pseudomonas syringae exhibits a genome-wide, bacteriophytochrome-mediated response to light and a stronger response to far-red than blue/red light

B. JANSSEN, H. Dong, G. A. Beattie, Iowa State University, Ames, IA, USA

Light is prevalent in the leaf environment, but the role of light signaling in foliar pathogen biology is poorly understood. Two photosensory proteins, a bacteriophytochrome (BphP1) and a blue light-sensing protein (LOV), modulate swarming motility and virulence through an integrated regulatory pathway in Pseudomonas syringae pv. syringae B728a. Here we report the results of transcriptome analyses aimed at characterizing the responses of B728a to light. Light functioned as a global signal in B728a, with far-red light impacting $26 \%$ of the pathogen's 5,220 genes and blue and red light affecting $23 \%$ and $13 \%$ of the genes, respectively. We also examined the transcriptome of mutants deficient in, and over-expressing, lov and $b p h P 1$. Whereas loss of lov affected the expression of very few genes (<4), loss of bphPl affected the expression of 600 to 1,300 genes, depending on the light treatment. BphP1 regulated, either directly or indirectly, $93 \%$ of the genes that were differentially expressed in the wild type in far-red light, and 87 and $80 \%$ of those in red and blue light, respectively, and did not detectably affect any genes in the dark. The functional categories that were over-represented among the BphP1-regulated genes included osmotic and oxidative stress tolerance functions. Collectively, these results demonstrate a genome-wide, bacteriophytochrome-mediated, coordinated response to light and other environmental cues in the foliar environment.

Genomic comparisons of Armillaria solidipes and A. altimontana - species with different ecological behaviors J. R. IBARRA CABALLERO (1), M. S. Kim (2), J. Hanna (3), N. B. Klopfenstein (3), J. Stewart (1), (1) Colorado State University, Fort Collins, CO, USA; (2) Kookmin University, Seoul, Korea, Republic of (South); (3) Rocky Mountain Research Station, USDA Forest Service, Moscow, ID, USA

Armillaria is a genus of basiodiomycota that includes primary tree pathogens, opportunistic pathogens, and saprophytes. To better understand species in the genus, high-quality PacBio genome assemblies of A. solidipes (primary pathogen) and A. altimontana (opportunistic pathogen/saprophyte) were produced and compared. The genome sizes were $55 \mathrm{Mbp}$ for $A$. solidipes and $73 \mathrm{Mbp}$ for A. altimontana. The genomes were structurally annotated with Maker and a functional annotation was performed with BlastP, InterProScan, and additional tools. More than $50 \%$ of the proteins encoded by each species grouped into orthologous clusters, while 6,717 and 11,099 were exclusive to A. solidipes or A. altimontana, respectively. Gene Ontology descriptions of the exclusive proteins were similar, but differences were found among the number of predicted secreted proteins. In A. solidipes, a larger number of encoded protein families, considered as pathogenicity related, were found including cell wall-degrading enzymes, proteases, and others. A larger number of repetitive sequences was found in A. altimontana ( $11,000,2.75 \mathrm{Mbp} ; 4,357$ corresponding to transposons) compared to A. solidipes ( 7,100,1.3 Mbp; 2,701 transposons). This information is important for identifying genes that contribute to potential pathogenic and/or saprophytic relationship between the fungi and tree host and understanding biology of Armillaria species.

Comparative whole genome analysis of the wheat eyespot pathogens, Oculimacula yallundae and $O$. acuformis H. SHENG, T. D. Murray, Washington State University, Pullman, WA, USA

Oculimacula yallundae and O. acuformis are the fungal pathogens of eyespot, an important disease of winter wheat in the Pacific Northwest, USA and other temperate wheat growing areas. The genomes of two O. yallundae isolates, OY90-45-7 (Mat1-1) and OY90-49-1 (Mat1-2), and one O. acuformis isolate, OA99-36-3 (Mat1-1), were sequenced using the PacBio RS II system and de novo assembled with HGAP 3 pipeline to compare genome structure and gene content. GenSAS and Blast2GO were used for the structural and functional annotations. Genome sizes of OY90-45-7, OY90-49-1 and OA9936-3 were estimated at 47.5, 46.9, and 45.1 Mb, and assembled into 91 (N50 1.25 Mb; Max contig 2.71 Mb; coverage 74x), 89 (N50 1.14 Mb; Max contig $2.64 \mathrm{Mb}$; coverage 71x), and 27 scaffolds (N50 2.36 Mb; Max contig $3.73 \mathrm{Mb}$; coverage 83x), respectively. When the assemblies were analyzed with BUSCO, each contained more than $97 \%$ fungal housekeeping genes. Each genome sequence predicted about 13,000 protein-coding genes of which nearly $50 \%$ were classified by functional annotation. Over 6,000 SSR loci were found in each genome. Polymorphic SSRs will be used to establish a genetic linkage map for $O$. yallundae with a progeny population developed between OY90-45-7 and OY90-49-1. Based on the number and location of telomeres, 19 to 22 chromosomes are predicted for these Oculimacula spp. This study provides the first whole genome sequence information of $O$. yallundae and $O$. acuformis.

Comparative genomics of Xanthomonas vasicola pv. vasculorum isolates recovered from maize G. WU, M. C. Ortiz, J. M. Lang, J. E. Leach, K. D. Broders, Colorado State University, Fort Collins, CO, USA

Xanthomonas vasicola pv. vasculorum (Xvv) is an emerging bacterial pathogen that causes bacterial leaf streak on maize. This bacterium was reported from maize in the USA in 2016, and has now reached epidemic proportion in Colorado, Kansas and Nebraska. While Xvv is known to infect sugarcane the only other report of this disease on corn was from South Africa, where the disease has been present since 1949, but rarely causes the extensive damage observed in the U.S. in 2016. Here, we report genome sequencing of multiple Xvv strains isolated from maize in the USA and South Africa. We delineate the phylogeny of these strains and previously sequenced related strains, including $X$. vasicola strains from maize, sugarcane, Tripsacum laxum and sorghum. The whole genome phylogeny revealed that isolates recovered from maize form a distinct phylogenetic clade within the $X$. vasicola group. By applying comparative genomics approaches, we identified several genes that are unique to Xvv that are recovered from maize, suggesting these genes might play a role in increased agressiveness on maize. We also profile virulence factors, including type III secreted effectors, in Xvv. This study not only demonstrates the distinct evolutionary history of this clade of Xvv strains recovered from maize, but also provides candidate genes for further molecular characterization to elucidate increased aggressiveness in isolates of Xvv recovered from maize in the U.S. 
Genome assembly and comparison of Macrophomina phaseolina isolates on strawberry and alfalfa A. BURKHARDT (1), K. L. Childs (2), M. Ramon (1), F. N. Martin (1), (1) USDA ARS, Salinas, CA, USA; (2) Michigan State University, East Lansing, MI, USA

Macrophomina phaseolina is a haploid fungus that typically causes charcoal rot on a wide range of hosts, but recently a group of strawberry host-specific isolates has been identified. To understand this host specificity, we sequenced M. phaseolina isolates from strawberry and from alfalfa using PacBio and Illumina paired-end and mate pair platforms. The genome assembly from the strawberry isolate using FALCON to assemble and PILON to error correct has an N50 of $3.3 \mathrm{Mb}$ with 89 contigs and $95 \%$ of the paired end reads mapping. About $90 \%$ of the genome is on 15 contigs, and the BUSCO completeness with the eukaryotic and fungal datasets was $98 \%$. The alfalfa isolate was also assembled and has an N50 of $4.3 \mathrm{Mb}$ with 27 contigs and $97 \%$ of the paired-end reads mapping. Over $96 \%$ of the genome was on the 12 longest contigs, and the BUSCO completeness for the fungal and eukaryotic datasets was $98 \%$. Genome annotation is underway for the strawberry isolate using the MAKER pipeline with RNA-Seq data which was collected under multiple conditions and assembled using Trinity. A MAUVE analysis shows that the predicted contigs between the two isolates are comparable, and additional comparative genomic analyses will be performed after the genome assemblies have been finalized using BioNano optical mapping. The ultimate goal of this project is to compare the final genome assemblies and to identify regions that are associated with host specificity on strawberry.

Functional genomics and detection of virulence factors in Clavibacter michiganesis subsp. nebraskensis J. C. HUGUET-TAPIA (1), B. Z. Fu (2), A. E. Robertson (3), S. Liu (4), F. F. White (1), (1) University of Florida, Gainesville, FL, USA; (2) Hubei Engineering Univ, Xiaogan Hubei, CHINA; (3) Iowa State University, Department of Plant Pathology, Ames, IA, USA; (4) Kansas State University, Manhattan, KS, USA

Clavibacter michiganensis subsp. nebraskensis (Cmn) is the agent of Goss's bacterial wilt and leaf blight in corn and has re-emerged as an important disease in North America. The bacteria colonize the vascular tissue in the plant, and infected leaves show water-soaked spots (freckles) and discolored vascular bundles. Genetic tools for Clavibacter species have been developed and used to elucidate the biology various species. However, the techniques are still limited and efficiency is low in comparison to other plant pathogens. We are using NextGen sequencing to improve our understanding of Cmn. Here, we report the complete genomes of three Cmn strains. Our results indicate that genomes of CMN061, a virulent isolate, and CMN7850, an avirulent isolate, show high identity with few single-nucleotide polymorphisms (SNPs), while CMNH4, also avirulent, is a distant related isolate. Comparative analyses are being used to identify candidate virulence genes for targeted mutagenesis. We were also generating a comprehensive chemical mutagenized library for CMN061 using deep sequencing (MUT-seq). Preliminary results show putative candidates to be investigated in further analysis, which will be presented and discussed.

High-quality genome of the peach scab pathogen, Venturia carpophila

C. Chen, C. H. BOCK, B. W. Wood, USDA ARS, Southeastern Fruit and Tree Nut Research Laboratory, Byron, GA, USA

Venturia carpophila causes peach scab, a disease that renders peach (Prunus persica) fruit unmarketable. We report a high-quality draft genome (36.9 $\mathrm{Mb}$ ) of $V$. carpophila from an isolate collected from a peach tree in central Georgia. The genome was sequenced by MiSeq using an Illumina paired-end library (a V3 kit, $2 \times 300$ cycles), which generated 40,041,052 raw reads consisting of 12,052,356,652 raw nucleotides. After trimming adaptors and removing low-quality reads, a total of 39,057,608 reads ( $\sim 97.54 \%)$, containing 10,111,608,273 nucleotides $(83.90 \%)$ passed robust error correction (EC) algorithms. The final assembly of the genome from EC reads included 657 scaffolds, accounting for 36,917,822bp. The average depth of each nucleotide was 263.47 and about $98.58 \%$ of the assembled bases had a PHRED-scale quality score $>=40$, indicating it is a very high-quality assembly. The longest contig is $1,454,817 \mathrm{bp}$ and the N50 length is $292,586 \mathrm{bp}$. BLASTN suggested 107 of the 657 scaffolds $(144,247 \mathrm{bp} \mathrm{long})$ were the mitochondrial sequences. The actual genome size of $V$. carpophila is unknown but estimated $\sim 38.9 \mathrm{Mb}$ (typical in the phylum Ascomycota), assuming this assembly has $95 \%$ genome coverage. The annotated genome sequence will be a useful resource for various studies of the pathogen and it's interaction with the host.

\section{Genome-wide characterization of alternative splicing patterns in sugarcane modulated during infection with smut pathogen, Sporisorium} scitamineum

R. BEDRE (1), S. Irigoyen (2), P. Schaker (3), C. Monteiro-Vitorello (4), K. K. Mandadi (5), (1) Texas A\&M AgriLife Research \& Extension Center, Weslaco, TX, USA; (2) Texas A\&M AgriLife Research and Extension Center, Texas A\&M University System, Weslaco, TX, USA; (3) University of São Paulo, Piracicaba, BRAZIL; (4) University of Sao Paulo, Piraciaba, BRAZIL; (5) Texas A\&M AgriLife Research, Weslaco, TX, USA

In eukaryotes, alternative splicing (AS) is a key posttranscriptional process that promotes transcriptome and proteome diversity during growth, development, and response to stress. Genome-wide studies of AS in sugarcane (Saccharum spp.) are lacking, mainly due to absence of a sequenced reference genome and its complex ploidy. Here, we analyzed sugarcane isoform-level transcriptome and AS landscapes, modulated during infection with smut fungus (Sporisorium scitamineum), using a combination of Sorghum bicolor reference-based and Trinity de novo mapping tools. Approximately 15,514 and 14,934 transcripts were detected ( $\geq 2$ FPKM) at 5 and 200 days after infection (DAI), respectively. Approximately 5000 (14\%) of the genes were found to be alternatively spliced. Among them, 415 (8\%), 235 (5\%), 435 (9\%), and $\sim 215$ (4\%) were intron retention, exon skipping, alternate acceptor and alternate donor type of splicing events, respectively. Differential splicing analysis of healthy and smut-infected sugarcane revealed $\sim 14$ and $\sim 506 \mathrm{AS}$ events modulated at 5 and $200 \mathrm{DAI}$, respectively. Gene-ontology and enrichment analysis revealed overrepresented functional categories such as cell-wall modification, defense signaling and oxidative stress among the differentially spliced genes. Together, our study provided new insights and bioinformatics tools to understand sugarcane AS landscapes altered during biotic stress.

Open pangenomes and recombination-generated diversity in Xanthomonas euvesicatoria and $X$ perforans

M. JIBRIN (1), P. D. Roberts (2), S. Timilsina (3), G. V. Minsavage Jr. (3), N. Potnis (4), G. E. Vallad (5), E. M. Goss (3), J. B. Jones (3), (1) University of Florida, Gainesville, FL, USA; (2) University of Florida, Immokalee, FL, USA; (3) Department of Plant Pathology, University of Florida, Gainesville, FL, USA; (4) Department of Entomology and Plant Pathology, Auburn University, Auburn, AL, USA; (5) Gulf Coast Research and Education Center, University of Florida, Wimauma, FL, USA

Xanthomonas euvesicatoria $(\mathrm{Xe})$ and $X$ perforans $(\mathrm{XP})$ are very closely related species- that cause bacterial spot disease on tomato and pepper. Multilocus, core genome, and effector sequence analyses have shown clear divisions between $X e$ and $X p$. However, new genomes also suggest the possibility for gene exchange between these taxa. In this study, we utilized $30 \mathrm{Xe}$ and $35 \mathrm{Xp}$ sequenced genomes, two of which are newly sequenced genomes of $X p$ and $X e$ strains from Nigeria, to delineate the pan and core genome evolution of $X e$ and $X p$ and estimate the contribution of recombination between strains on evolution. Our results show considerable heterogeneity within taxa, as indicated by bimodal distributions in sequential resampling of strains to delineate core genomes. Pangenome analysis also showed gradual increase in number of genes with each additional genome. For example, the Nigerian $X p$ strain increased the pangenome size of previously sequenced Florida $X p$ strains by more than 35 genes. Our analysis also showed that recombination is important in the evolution of these species, but more so in $X p$ than $X e$. We conclude that both $X e$ and $X p$ have open pangenomes, with sequencing of new strains expected to increase the gene profiles of new strains that may be important in pathogenicity and fitness. 
Morphological, Metabolic and Transcriptional Characterization of Sexual Fertilization in Aspergillus flavus

J. M. S. LUIS (1), I. Carbone (1), G. A. Payne (1), D. Bhatnagar (2), J. Cary (2), M. Lebar (2), G. Moore (2), P. Ojiambo (1), (1) North Carolina State University, Raleigh, NC, USA; (2) USDA-ARS, SRRC, New Orleans, LA, USA

Aspergillus flavus is a heterothallic fungus where sexual reproduction occurs between individuals of opposite mating types. The occurrence of sexual reproduction in A. flavus can have broad implications on the survival and genetic diversity of the fungus, but this process is not well understood. Additionally, there is limited information on the metabolic and transcriptional changes that occur during the fertilization of sclerotia. Two reciprocal crosses composed of sclerotia and conidia with different levels of female fertility were plated in mixed cereal agar and incubated at $30^{\circ} \mathrm{C}$ in continuous dark. Sclerotia from each reciprocal cross were harvested every two weeks for a total of eight weeks. Scanning electron micrographs showed distinct changes in sclerotia morphology over time and differences between high and low female fertility crosses. Ultra-performance liquid chromatography and mass spectrometry showed distinctive metabolite profiles associated with sclerotia from highly fertile cross. Transcriptional profiles revealed distinct clustering of genes according to fertility level based on backbone enzymes of the Aspergillus secondary metabolism gene clusters and genes involved in developmental stages of Aspergillus. Most of these genes were expressed progressively over time following fertilization. These results broaden our knowledge on sexual reproduction in A. flavus and could be useful in screening of atoxigenic strains of $A$. flavus with high sexual fertility.

Comparative genomic analysis of Clavibacter michiganensis subsp. nebraskensis isolates representing naturally occurring virulence diversity R. R. MCNALLY, D. K. Malvick, C. A. Ishimaru, University of Minnesota, St. Paul, MN, USA

Clavibacter michiganensis subsp. nebraskensis ( $\mathrm{Cmn}$ ) is a Gram-positive actinomycete causing Goss's wilt of maize. The specific genes required for pathogenicity are unknown and differ from those of other subspecies. Here we report complete genome sequences for six Cmn isolates representing naturally occurring virulence diversity. All isolates originated from maize tissues displaying symptoms of Goss's wilt. Isolates CIC313, CIC326, CIC328, and CIC396 were avirulent in subsequent maize inoculations while CIC395 and CIC292 induced typical Goss's wilt symptoms. PacBio long read sequencing was used to achieve a sequence depth per genome averaging $145 \mathrm{X}$ coverage over all isolates. Average $\mathrm{G}+\mathrm{C}$ content was $73 \%$, consistent with other C. michiganensis subspecies. Each genome contained one chromosome of $\sim 3.1 \mathrm{Mb}$. Only CIC292 contained extrachromosomal DNA with one plasmid of $65 \mathrm{~kb}$. Only CIC396 encoded a predicted genomic island of $\sim 14 \mathrm{~kb}$. With the exception on CIC 328 , chromosomes of Cmn isolates were highly syntenic. The genetic asynteny of CIC328 in combination with low relative $\mathrm{G}+\mathrm{C}$ content $(70 \%)$ and larger chromosome (3.5 Mb) suggests CIC328 may be a novel subspecies of C. michiganensis. An average of 3079 genes were predicted across these six isolates of Cmn. Comparative analysis of gene orthology revealed genes unique to virulent $\mathrm{Cmn}$. The unique genes represent candidates for identifying virulence factors and understanding $\mathrm{Cmn}$ infection of maize.

Gene expression profile of Acidovorax citrulli during the early stages of watermelon seed germination G. CHEN, R. R. Walcott PhD, The University of Georgia, Athens, GA, USA

Acidovorax citrulli is the causal agent of bacterial fruit blotch (BFB), an economically important disease of cucurbit crop species worldwide. A. citrulli is seed transmitted, and seed is the most important source of primary inoculum for BFB outbreaks. During the early stages of watermelon seed germination, A. citrulli colonizes the seed before seed-to-seedling transmission of BFB. A better understanding of this process may lead to more effective BFB management. Since a functional type 3 secretion system is not required for this process, we hypothesized that $A$. citrulli initially colonizes germinating watermelon seed as a saprophyte. To simulate the environment of germinating seeds, watermelon seeds were germinated for 24,48 and $120 \mathrm{~h}$, then crushed to generate seed homogenate (SH). A. citrulli strain AAC00-1 was grown in SH preparations for $24 \mathrm{~h}$ and RNA was extracted for RNA-Seq. In the transcriptome of $A$. citrulli cultured in $24 \mathrm{~h} \mathrm{SH}$, there were 451 and 407 differentially expressed genes (DEGs) compared to $48 \mathrm{~h}$ and $120 \mathrm{~h} \mathrm{SH}$, respectively. In contrast, there were only 257 DEGs when comparing 48 and $120 \mathrm{~h} \mathrm{SH}$. This indicates that the expression pattern is more distinct in $24 \mathrm{~h}$ $\mathrm{SH}$. Key genes including glutamine synthetase, nitrogen metabolism transcriptional regulator $n t r B$ and $n t r C$ were significantly up-regulated in $24 \mathrm{~h}$ SH. This suggests that nitrogen may be limiting for $A$. citrulli in the $24 \mathrm{~h} \mathrm{SH}$, and possibly during the early stages of watermelon seed germination.

\section{Survey of toxin-antitoxin system distribution and diversity among genomes of plant-associated bacteria} L. R. TRIPLETT, Connecticut Agricultural Experiment Station, New Haven, CT, USA

Toxin-antitoxin (TA) systems are diverse bacterial gene modules that encode an antibacterial toxin and a neutralizing antitoxin. Although TA systems are nearly ubiquitous and have been implicated in a variety of virulence, regulatory, and environmental fitness roles, little is known about the roles of TA systems in plant-associated lifestyles. Here, alignment and gene-arrangement based methods were used to analyze 194 genome assemblies representing 160 species of bacterial plant pathogens and symbionts for the presence of 23 families of TA systems. All genomes of free-living bacteria were found to encode predicted TA systems, ranging from three to 26 systems per genome. The majority of systems are encoded on the chromosome. Substantial variation was observed in the composition of TA systems between bacterial taxa, with some systems unique to or enriched in specific phyla. Toxin sequence phylogenetic groups largely separated according to bacterial taxon. These results demonstrate that TA systems are widespread and numerous throughout plant-associated bacteria, suggesting that they are functionally important in plant-associated lifestyles.

Using next generation sequencing tools to incorporate herbarium specimens in systematic research

E. C. WALLACE (1,2), J. A. Crouch (3), (1) ARS-ORISE Research Participation Program, Beltsville, MD, USA; (2) USDA-ARS, Beltsville, MD, USA; (3) USDA ARS, Beltsville, MD, USA

Molecular systematics research is crucial for examining the identity and evolutionary relationships between plant pathogens. When taxonomic revision is necessary, researchers must consult original descriptions and type specimens. Inclusion of old type specimens and other historic samples in molecular phylogenetic studies provides important information about the relationship between contemporary and historic pathogens, but obtaining sequence data from herbarium specimens may be complicated by several factors. DNA degradation, sample size limitations, contamination, and suboptimal storage conditions pose obstacles in performing PCR and generating quality sequences through traditional Sanger sequencing. Aspects of next generation sequencing (NGS) protocols seem suited for generating molecular data from herbarium specimens, as many platforms utilize short fragments and highcoverage reads. NGS technology has high-throughput capacity and works well with low levels of target material, nucleic acid degradation, and mixed samples. In this study, to generate sequences from plant-associated oomycete herbarium specimens, PCR primers were designed to amplify short overlapping segments spanning phylogenetically informative regions identified from reference genomes. The primers were validated for use in a targeted sequencing protocol on Illumina instruments. Assembly of these high-coverage sequences will provide complete sequences of phylogenetic regions in herbarium specimens.

Metagenomic analysis of virus and virus-like pathogens infecting pistachio in California M. AL RWAHNIH PHD (1), D. A. Golino (2), N. Westrick (3), K. Stevens (4,5), F. P. Trouillas (6), J. Preece (7), C. Kallsen (8), K. Farrar (9), A. Rowhani (2), (1) Department of Plant Pathology, University of California-Davis, Davis, Davis, CA, USA; (2) University of California, Davis, CA, USA; (3) Department of Plant Pathology, University of California-Davis, Davis, CA, USA; (4) Department of Evolution and Ecology, University of CaliforniaDavis, Davis, CA, USA; (5) Foundation Plant Services, University of California-Davis, Davis, CA, USA; (6) University of California, Davis, Davis, CA, USA; (7) USDA-ARS National Clonal Germplasm Repository, Davis, CA, USA; (8) Cooperative Extension Kern County, Bakersfield, CA, USA; (9) Foundation Plant Services, Davis, CA, USA 
Pistachio nurseries and growers have recently observed that both ungrafted UCB-1 and mature pistachio trees grafted into UCB-1 rootstocks have occasionally become stunted and expressed virus-like symptoms. From 2014 to 2016, high throughput sequencing (HTS) analysis was performed on 56 pistachio trees, Pistacia vera L. and clonal UCB-1, from selected growing regions in California and the National Clonal Germplasm Repository (NCGR) near Winters, CA. HTS analysis generated 18-75 million sequence reads per sample. Bioinformatics analysis identified contigs that shared homology with the plant virus family Closteroviridae. A new near-full genome virus, provisionally named "Pistachio Ampelovirus virus A" (PAVA), shows close similarity to viruses in the genus Ampelovirus, family Closteroviridae and was successfully graft transmitted to healthy UCB-1 seedlings and confirmed by RT-PCR testing from the original source and grafted plants. Further research is needed to determine the prevalence of PAVA in California and the effect of PAVA on tree performance and fruit quality. Analysis also revealed the presence of Hop stunt viroid, a cohort of mycoviruses, and contigs with close homology to plant viruses in the families Pospiviridae and Caulimoviridae. Sequence analysis and virus testing are in progress to identity other viruses found in this study.

Pantoea ananatis comparative genetics and in planta kinetics

S. STICE (1), B. Kvitko (1), B. Dutta (2), (1) University of Georgia, Athens, GA, USA; (2) University of Georgia, Tifton, GA, USA

Pantoea ananatis is a major causal agent of leaf blight and center rot of onion (Allium cepa L.), a significant issue for onion growers in Georgia. However, the virulence mechanisms of $P$. ananatis are currently unknown. To facilitate a mechanistic understanding of $P$. ananatis infection, we sequenced and annotated the draft genomes of 10 strains from diverse isolation sources; symptomatic onion foliage, asymptomatic weeds (epiphytic strain), and from tobacco and onion thrips. Pan-genomic analysis revealed a core genome comprised of 3,750 protein coding sequences (CDS) and an accessory genome consisting of 1,395 CDS which encompassed genes implicated in virulence, such as cell wall degrading enzymes. We also assessed the kinetics (CFU/distance/time) of $P$. ananatis infection when inoculated on onion foliage under controlled conditions. The center leaves of 6-8 week old onions (cv Century; $n=72$ ) were inoculated with the miniTn7Lux transformed (PNA 97-1 Rif) and kinetics of this strain was evaluated. Samples of leaf segments $\left(0.125 \mathrm{~cm}^{2}\right)$, were taken from $1,4,7$, and $10 \mathrm{~cm}$ below the inoculation site at 1,3,5, and 7 days post inoculation (DPI). The results indicate that $P$. ananatis $\left(8.9 \log _{10} \mathrm{CFU} / \mathrm{cm} 2\right)$ could be detected $7 \mathrm{~cm}$ below the inoculation point within 3 DPI, despite lacking visible symptoms. This data from Georgia isolates coupled with an optimization of bacterial infection kinetics in onion foliage will aid in elucidating pathogenicity determinants in $P$. ananatis.

Genetic characterization of cucurbit yellow vine disease strains of Serratia marcescens using whole genome sequencing Z. MATTEEN (1), E. L. Little (2), (1) University of Georgia, Department of Plant Pathology, Athens, GA, USA; (2) University of Georgia - Department of Plant Pathology, Athens, GA, USA

Serratia marcescens is a ubiquitous and genetically diverse bacterium. Some strains cause cucurbit yellow vine disease (CYVD) which results in a wilting and death of squash and watermelons plants. The mechanism for how CYVD strains are able to induce disease symptoms in cucurbit hosts is unknown and may be unique among bacterial plant pathogens. Based on previous molecular characterization work, strains causing CYVD form a closely related group that is genetically distinct from non-CYVD strains. In addition, CYVD strains were most closely related to two $S$. marcescens rice endophytic strains, R01-A and R02-A. This study provides the first full genomic comparison between CYVD strains and nonpathogenic $S$. marcescens strains. S. marcescens strains were sequenced and assembled, contigs were ordered against the complete reference genome of strain WW4, and draft genomes were annotated. CYVD strains were aligned with each other and with the endophytic rice strain R01-A using the genome alignment tool MAUVE and alignments were examined for sequence differences. The genomes of the CYVD strains were significantly reduced in size by approximately $0.8 \mathrm{Mb}$ compared to the rice endophyte strain, R01-A. In addition, unique insertions found only in the CYVD genomes were identified. Characterization of gene function within these regions may lead to insights on determinants of pathogenicity.

Deciphering protein-protein interaction network in the rice blast fungus, Magnaporthe oryzae H. Kim (1), Y. H. LEE (2), (1) Seoul National University, Seoul, Korea, Republic of (South); (2) Seoul National Univ, Seoul, Korea, Republic of (South)

Magnaporthe oryzae plays a role as a model organism in the study of molecular plant-microbe interactions. As multi-omics data are being available, genomic-level researches have been conducted to uncover the underlying biological process during the pathogenesis of $M$. oryzae. Deciphering proteinprotein interaction (PPI) network is one of omics-level approaches to understand signaling and regulatory pathways involved in fungal pathogenicity. However, existing biological network resources of are not sufficient to decipher molecular mechanisms of pathogenesis. PPI network analysis platform of M. oryzae, MagNet, was constructed with three methods: homology-based 'Interolog' search, co-expression network construction, and domaindomain interaction based prediction. Interologs within M. oryzae proteins which have orthologues in model species were predicted with six PPI repositories. Co-expression networks were built with RNA sequencing data from the infection and developmental stages. In addition, we gathered the information of domain-domain interactions from two integrated DDI databases, and predicted interactions between domain-annotated proteins. With three approaches, the pan-network with 6,600,976 interactions was generated including highly confident 215,731 interactions. Experimental data on $M$. oryzae PPIs demonstrated that our highly confident PPI network can predict PPIs with higher sensitivity $(89.65 \%)$ and specificity $(78.57 \%)$ compared to the previously constructed databases. MagNet would provide integrated biological network data which can help to understand the molecular mechanisms of the rice blast fungus and beyond.

Pan-Genome Analyses of Black Rot Pathogen of Crucifers, Xanthomonas campestris pv. campestris A. Larrea (1), A. M. Alvarez (1), J. P. Stack (2), M. ARIF (1), (1) University of Hawaii at Manoa, Honolulu, HI, USA; (2) Kansas State University, Manhattan, KS, USA

Family Brassicaceae (Cruciferae) has many genera, species and cultivars being raised for food production throughout the world. Black rot is one of the most destructive and economically important diseases of crucifers; Xanthomonas campestris pv. campestris is the causal agent of this systemic vascular and seed-borne disease. Nine genomes of Xcc from different geographical locations (United Kingdom, Italy, New Zealand, America and China) and one genome of the leafspot pathgen X. campestris pv. raphani (Xcr) were included in the analyses; genomes were downloaded from the NCBI GenBank database. The genome sizes and CDSs ranged from $4.91 \mathrm{MB}$ to $5.15 \mathrm{MB}$ and 42.2 to 4353 , respectively. The maximum number of singletons 138 were obtained with isolate B100 from Italy and least were from the Chinese isolates (4-10). A total of 5,054 pan-genome orthologous groups (POGs) were obtained from 42,344 CDSs; core size - 3,655 POGs (defined by 95\%). Genomic rearrangements were observed among the isolates and were grouped based on the geographical locations in phylogenomic analysis; Xcr (genome size $4.94 \mathrm{MB}$ ) was out-grouped. This comparative genomic analysis would enhance our understanding of genomic constituents of Xcc isolates important to disease development and thus improve integrated disease management strategies.

Tissue-specific transcriptional responses related to the horizontal and vertical transmission of a bacterial pathogen by its insect vector I. E. BADILLO-VARGAS, R. Bedre, G. Esparza-Diaz, C. Avila, K. Mandadi, Texas A\&M AgriLife Research, Weslaco, TX, USA

Bactericera cockerelli is the insect vector of the fastidious bacterium "Candidatus Liberibacter solanacearum" (Lso). This bacterial pathogen causes zebra chip disease of potato. Lso is transmitted in a persistent propagative manner by $B$. cockerelli where it infects and multiplies in the digestive track, reproductive organs, and salivary glands of its insect vector. Lso infection of the reproductive organs of $B$. cockerelli leads to transovarial transmission of the pathogen to the insect offspring. It has been previously shown that Lso has a detrimental effect on the fecundity and nymphal survival rate of $B$. cockerelli. To better understand the molecular bases of these biological consequences in the insect vector due to the bacterial infection, we have 
conducted a tissue-specific transcriptome analysis of $B$. cockerelli organs involved in the horizontal and vertical transmission of Lso. Total RNA was extracted from pools of dissected salivary glands and ovaries from non-infected and infected insects using three independent biological replicates. Libraries were prepared and sequenced using poly-A enriched RNA coupled with Illumina Hi-Seq technology. Bioinformatics analyses were conducted to identify the transcriptional changes in these insect tissues in response to the bacterial infection. Identification of a suite of candidate genes from $B$. cockerelli is allowing us to better understand this vector-bacteria interaction that has detrimental effects on the insect host.

\section{Effects of sublethal fungicide stress on genomes of Sclerotinia sclerotiorum}

N. GAMBHIR, Z. N. Kamvar, S. E. Everhart, University of Nebraska, Lincoln, NE, USA

Sublethal antifungal drug exposure is known to cause genomic instability in human fungal pathogens. This instability may facilitate emergence of genetic variation that confers fungicide resistance or other adaptive traits. The effects of sublethal fungicide exposure on fungal phytopathogens may be similar, but have not yet been fully characterized. We independently exposed five isolates of Sclerotinia sclerotiorum to sublethal doses of four commercially formulated fungicides with different modes of action (iprodione, dicarboximide; thiophanate-methyl, MBC; boscalid, SDHI; azoxystrobin, QoI) for twelve consecutive generations with experimental replication. Fungicide stress was introduced by growing isolates on a logarithmic concentration gradient, then sub-culturing from the zone with more than $50 \%$ inhibition. Whole-genome sequence data with $150 \mathrm{bp}$ paired-end reads were obtained for pre- and post-exposure individuals (55 in total). Genomes had an average read depth of 16.47X in the nuclear genome and 199.64X for mitochondria, with average coverage of $98.5 \%$ and $99.6 \%$, respectively. A total of 118,287 variants were called from mitochondrial and nuclear genomic data. After filtering for read depth, mapping quality, and intra-isolate polymorphism (at least one polymorphism across treatments and within an isolate), there were 2,358 high quality variants. In the future, we will characterize pre- vs. post-exposure variants within isolates. Results will help to determine if sublethal fungicide stress leads to random or directional mutations, and if fungicides with different modes of action generate similar mutational profiles.

Pseudomonas oleovorans strain KBPF-004 culture supernatants reduced seed transmission of CGMMV and PMMoV, and remodeled aggregation of $126 \mathrm{kDa}$ protein

H. S. LIM (1), N. G. Kim (2), C. N. Park (2), H. S. Park (2), I. H. Kim (1), J. K. Kim (1), E. Y. Seo (1), L. L. Domier (3), H. G. Kim (1), C. Jang (2), J. Hammond (4), (1) Applied Biology, Chungnam National University, Daejeon, Korea, Republic of (South); (2) Central Research Institute, Kyung Nong Co. Ltd, Gyeongju, KOREA; (3) USDAARS, Urbana, IL, USA; (4) USDA ARS Floral and Nursery Plant Research Laboratory, Beltsville, MD, USA

Several physical treatments have been examined for control of viral diseases in crop production. Unlike physical treatments, use of naturally-occurring microorganisms against plant viruses is not well known. We now report isolation of soil inhabiting bacteria Pseudomonas oleovorans strain KBPF-004 (developmental code KNF2016) which shows antiviral activity against tobamoviruses. Activity was evaluated against seed transmission of two tobamoviruses, Pepper mild mottle virus (PMMoV) and Cucumber green mottle mosaic virus (CGMMV). Seeds harvested from CGMMV-infected watermelon or PMMoV-infected pepper were treated with P. oleovorans strain KBPF-004 or strain ATCC 8062 before planting. Germinated seedlings were about 60\% CGMMV- or PMMoV-positive after treatment with water or supernatant of ATCC 8062 , but $<20 \%$ of seedlings from KBPF-004 treated seeds were CGMMV- or PMMoV-positive. Thus culture supernatants of $P$. oleovorans KBPF-004 significantly reduced seed transmission rates of both viruses. Supernatant of $P$. oleovorans KBPF-004 also remodeled subcellular localization of $126 \mathrm{kDa}$ protein and movement protein in Nicotiana benthamiana, diminishing the size of aggregates of the $126 \mathrm{kDa}$ protein and disrupting association of the movement protein with the microtubule network. In addition, P. oleovorans KBPF-004 treatment resulted in reduction of the amount of full-size coat protein (CP) of both CGMMV and $\mathrm{PMMoV}$, and a concomitant increase of $\mathrm{CP}$ degradation products

Survey and identification of viruses infecting tomato crops in Guam

R. L. SCHLUB (1), M. Marutani (2), C. Padmanabhan (3), Z. Fei (4), K. S. Ling (3), (1) Univ of Guam, Mangilao, AP, USA; (2) University of Guam, Mangilao, AP, USA; (3) USDA-ARS, Charleston, SC, USA; (4) Boyce Thompson Institute, Ithaca, NY, USA

The impact of viruses on Guam's two major tomato varieties 'Solar Set' and 'Season Red' has crippled production on the island. In 2007, viral diseaselike symptoms, including mosaic, leaf curl and chlorosis were associated with losses as high as $20 \%$ in some fields of Solar Set. In the spring of 2011 , severe symptoms of leaf curling, chlorosis and stunting were observed in Season Red, resulting in a total loss on some farms. In early 1980's, Tobacco mosaic virus (TMV), Tomato mosaic virus (ToMV) and Cucumber mosaic virus (CMV) were known to occur on Guam. Tomato samples tested in 2007 and 2011 identified a putative begomovirus. Further characterization in 2013 determined it to be a unique strain of Ageratum yellow vein virus (AYVV) with the highest nucleotide sequence identity of 90-91\% to several isolates of AYVV, from China (Accession no. FJ869908), Japan (AB306314), Taiwan (DQ866134), and Thailand (JN809821). Second highest identity was less than 90\% to Ageratum yellow vein China virus (AYVCNV) from China (AJ558120 and AJ849916) and the Philippines (EU487045). Additional analysis using deep sequencing of small RNAs and virus identification using samples collected in 2013 to 2015, identified the presence of Potato virus Y (PVY), Southern tomato virus (STV), Tobacco streak virus (TSV), Tomato bushy stunt virus (TBSV), and Tomato spotted wilt virus (TSWV). Our surveys reveal that in recent years a range of viruses were involved in causing losses to tomato crops in Guam.

\section{Influence of Beet necrotic yellow vein virus and freezing temperatures on sugar beet roots in storage} C. A. STRAUSBAUGH (1), I. Eujayl (2), (1) USDA ARS NWISRL, Kimberly, ID, USA; (2) USDA-ARS NWISRL, Kimberly, ID, USA

Rhizomania caused by Beet necrotic yellow vein virus (BNYVV) is a yield limiting sugar beet disease that was also observed to influence the roots ability to resist freezing in storage. Roots from 5 commercial sugar beet cultivars (1 susceptible and 4 resistant to BNYVV) were produced in fields under high and trace levels of rhizomania pressure and subjected to 5 temperatures ranging from 0 to $-4.4^{\circ} \mathrm{C}$ for $24 \mathrm{~h}$. After the freeze treatment, the 8 root samples were stored in a commercial indoor storage building (set point $1.1^{\circ} \mathrm{C}$ ) for $50 \mathrm{~d}$ in 2014 (repeated in 2015 at $57 \mathrm{~d}$ ). Mid-root temperature, frozen and discolored tissue, and moisture and sucrose loss were evaluated. The air temperature at $0,-1.1$, and $-2.2^{\circ} \mathrm{C}$ matched mid-root temperature, but mid-root remained near $-2.2^{\circ} \mathrm{C}$ when air temperature was dropped to -3.3 and $-4.4^{\circ} \mathrm{C}$. When comparing fields with the susceptible cultivar, frozen tissue increased $(P<0.01)$ from $0 \%$ up to 16 to $63 \%$ at $-3.3^{\circ} \mathrm{C}$ and from 13 to $27 \%$ up to 63 to $90 \%$ at $-4.4^{\circ} \mathrm{C}$ depending on year. Regardless of field, resistant cultivars only had elevated $(P<0.05)$ amounts of frozen tissue at $-4.4^{\circ} \mathrm{C}$ in 13 of 16 comparisons. Frozen tissue was related $(P<0.0001)$ to discolored tissue $\left(\mathrm{r}^{2}=0.91\right)$, weight loss $\left(\mathrm{r}^{2}=0.12\right.$ to 0.28$)$ and sucrose reduction $\left(\mathrm{r}^{2}=0.69\right.$ to 0.74$)$. Thus, rhizomania in sugar beet not only leads to yield and sucrose loss, but also increases frozen tissue as temperatures drop to $-3.3^{\circ} \mathrm{C}$ and below.

\section{Natural infection of Sorghum bicolor germplasm by Sugarcane yellow leaf virus in Florida}

W. BOUKARI (1), C. Wei (1), E. Mulandesa (1), M. Hincapie (1), D. S. Mollov (2), R. Beiriger (1), P. C. Rott PhD (1), (1) University of Florida, Belle Glade, FL, USA; (2) USDA, Beltsville, MD, USA

Sugarcane yellow leaf virus (SCYLV), the causal agent of sugarcane yellow leaf, is vectored by the aphid Melanaphis sacchari. Although sugarcane is the primary host of SCYLV, two new natural hosts were recently identified in Florida: the weed Columbus grass (Sorghum almum) and grain sorghum (Sorghum bicolor). Most sugarcane varieties grown in Florida are susceptible to infection by SCYLV, and resistance to this virus seems limited in sugarcane germplasm. We investigated the resistance of $S$. bicolor germplasm to infection by SCYLV. A field trial comprising 30 different sorghum lines was established at Belle Glade, FL in spring 2016. The planted crop produced 17 lines that were colonized by M. sacchari, whereas only 8 lines grew and were colonized by the aphids when ratooned. In the plant crop, out of 10 plants sampled per line, 2 to 10 tested positive for SCYLV by tissueblot immunoassay (TBIA). In the ratoon crop, out of 10 plants sampled per line, 5 to 10 tested positive. However, SCYLV was found in only 4 out of 11 
TBIA positive plants from ratoon crop by reverse transcription-polymerase chain reaction (RT-PCR). Disparity between TBIA and RT-PCR results suggest either serological cross-reactivity with an unknown virus or the presence of new genetic variants of SCYLV. This needs to be resolved before further screening of grain sorghum resistance to SCYLV is performed for potential use of sorghum resistance in sugarcane breeding and marker-assisted selection.

Taxonomic consideration and molecular characterization of a novel bipartite dsRNA mycovirus from Trichoderma atroviride NFCF005 J. Chun, H. E. Yang, M. Jo, S. Y. Choi, D. H. KIM, Chonbuk National University, Jeonju, Korea, Republic of (South)

Next-generation sequencing followed by traditional molecular cloning methods was applied to determine a novel mycoviral genome sequence. Here, we report near complete sequences of a novel bipartite double-stranded RNA (dsRNA) virus including dsRNA-1 (9,628 bp) and dsRNA-2 (1,072 bp), which were isolated from the filamentous fungus Trichoderma atroviride NFCF005. Genomic analysis revealed that 9,628 bp segment contained two open reading frames, ORF1 and ORF2. The deduced amino acid sequences of ORF1 and ORF2 showed high level of identity with the putative structural/gag proteins and mycoviral RNA-dependent RNA polymerase (RdRp) of Fusarium virguliforme dsRNA mycovirus 1 and 2, respectively, which indicated that this dsRNA might be a candidate for a new member of the family Fusagraviridae. The dsRNA-2 segment contained one open reading frame, potentially encodes fatty acid desaturase of Emilania huxleyi virus 86. In addition, virus curing experiments resulted in a virus-free isogenic T. atroviride NFCF005 isolate. Out of 36 screened isolates, only 13 isolates harbored both viral segments and, once cured, no revertant was observed. These combined efforts of mycoviral genome sequence and establishment of virus-free and -infected isogenic lines can help to further investigate the diversity of mycoviruses and consequences of mycoviral infection in Trichoderma spp.

Effect of single and mixed infections of Tomato severe rugose virus (ToSRV) and Tomato chlorosis virus (ToCV) on plant development and virus titer

D. BAMPI (1), G. Favara (2), J. A. M. Rezende (1), (1) University of São Paulo - ESALQ, Piracicaba, BRAZIL; (2) University of São Paulo, Piracicaba, BRAZIL

The begomovirus ToSRV and the crinivirus ToCV, in single and mixed infections, are very common in tomato crops in Brazil. Both viruses are transmitted by the whitefly Bemisia tabaci biotype B (MEAM1). The objective of this work was to analyze the interaction between ToSRV and ToCV in tomato plants cvs. St. Clara and Kadá. Plants at 30 and 45 days after germination (DAG) were inoculated with $30 \mathrm{~B}$. tabaci per plant. The following treatments were compared: plants inoculated with ToSRV, ToCV, ToSRV/ToCV, and healthy (control). The interaction between these viruses was analyzed by quantification of virus titer by qPCR and plant development based on dry weight of the aerial part. ToSRV titer increased in mixed infection, as compared to single infection, for both genotypes and ages of inoculation. ToCV titer, on the other hand, was slightly reduced on mixed infected plants, except for cv. Kadá inoculated at 45 DAG. Dry weight of tomato plants St. Clara infected with ToSRV, ToCV and ToSRV/ToCV, at 30 DAG, were 25, 36 and 33\% lower than the control. For cv. Kadá, reductions were 39, 48, and 40\%, respectively. When inoculated at 45 DAG, reductions of dry weight for St. Clara were 15, 16, and 29, respectively, and for Kadá were 13, 21, and 39, respectively. Dry weight of plants infected with ToSRV, ToCV, and ToSRV/ToCV did not differ.

State-wide surveys indicated widespread distribution of grapevine leafroll than red blotch in Washington vineyards J. ADIPUTRA (1), R. Naidu (2), (1) Washington State University, Prosser, WA, USA; (2) Washington State Univeristy, Prosser, WA, USA

Vineyard surveys were conducted between 2014 and 2016 seasons to document the relative distribution of grapevine leafroll (GLD) and red blotch (GRBD) diseases in Washington State vineyards. In red-berried wine grape (Vitis vinifera) cultivars, samples were collected from vines exhibiting typical symptoms of GLD and GRBD and from those showing GLD- or GRBD-like symptoms. In white-berried cultivars, samples were collected from individual vines randomly due to the absence of visual symptoms. Samples from 2,063 vines, consisting of 1,936 vines from 18 red-berried cultivars and 127 vines from 7 white-berried cultivars, were tested by molecular diagnostic assays for Grapevine leafroll associated virus-3 (GLRaV-3) and Grapevine red blotch-associated virus (GRBaV). Of the 2,063 samples tested, nearly $68 \%$ were positive for GLRaV-3, about $6 \%$ positive for GRBaV and $9 \%$ positive for both viruses. About $18.4 \%$ of samples were negative for both viruses and some of these samples tested positive for other viruses, such as GLRaV-1, -2, and -4. Overall, the survey results indicated that GLRaV-3 is more predominant than GRBaV in Washington vineyards. A multiplex PCR assay was optimized for simultaneous detection of GLRaV-3 and GRBaV. Phylogenetic analysis of the complete genome sequence of 42 isolates of $\mathrm{GRBaV}$ revealed segregation into two distinct groups independent of cultivar and geographic location, with 37 isolates clustering into one group and the others into a second group.

Epidemiology of Tobacco ringspot virus causing fanleaf degeneration and decline symptoms in wine grape (Vitis vinifera) cultivars N. NATRA (1), S. Akinbade (2), A. Schultz (3), R. Naidu (4), (1) Washington state university, Prosser, WA, USA; (2) Washington State Dept of Agriculture, Prosser, WA, USA; (3) Hattrup farms, Wapato, WA, USA; (4) Washington State University-Prosser, Prosser, WA, USA

Three red-berried wine grape (Vitis vinifera) cultivars planted in a commercial vineyard in Washington State were observed with severe decline and degeneration symptoms. Symptomatic vines showed stunted growth, produced small clusters containing uneven sized berries and displayed a wide range of foliar symptoms. In RT-PCR assays, samples from symptomatic vines tested positive only for Tobacco ringspot virus (TRSV, Comoviridae: Nepovirus). In host-range studies, Nicotiana occidentalis was identified as a suitable herbaceous indicator for biological indexing of TRSV. Using a combination of Sanger sequencing and next-generation sequencing, the complete sequence of RNA1 and RNA2 genome segments of TRSV was determined to be 7,519 nucleotides (nt) and 3,927 nt, respectively. Phylogenetic analysis of RNA1 and RNA2 sequences indicated that the TRSV isolate from Washington State is closely related to virus isolates infecting soybean from South Korea and USA. The spread of TRSV was demonstrated from symptomatic grapevines to healthy cucumbers and Cabernet Franc vines used as transmission bait plants. TRSV was detected by RT-PCR in dagger, but not in other types of nematodes, hand-picked from soil samples taken near symptomatic grapevines. Using morphological characteristics and sequence analysis of the 28S large ribosomal D2-D3 expansion segment the dagger nematode present in the vineyard soil was identified as Xiphinema rivesi.

Molecular characterization of Citrus tatter leaf virus and its detection using reverse transcription quantitative PCR

S. H. TAN (1), F. Osman (2), S. Bodaghi (1), T. Dang (1), S. Abuhajar (1), S. Hammado (1), G. Vidalakis (1), (1) University of California, Riverside, Riverside, CA, USA; (2) University of California, Davis, Davis, CA, USA

Citrus tatter leaf virus (CTLV) is one of the pathogens threating the citrus production worldwide. The full-length viral genome of $12 \mathrm{CTLV}$ isolates derived from diverse geographical locations preserved at the Citrus Clonal Protection Program was identified by next generation sequencing technologies. A phylogenetic analysis was performed comparing the 12 CTLV isolates with corresponding sequences of other closely related capilloviruses in the GenBank. A multiple sequence alignment was generated using all of the in house CTLV complete genomes and both full-length and CP gene sequences from the GenBank. A robust real-time reverse transcription quantitative PCR (RT-qPCR) assay was designed based on these alignments. The specificity and sensitivity of this RT-qPCR assay in detecting the virus was assessed using multiple virus isolates. The viral load, reproducibility and repeatability for the RT-qPCR assay was determined. This RT-qPCR can be used as a robust diagnostic tool for citrus germplasm screening programs as well as for citrus certification programs to ensure the availability of pathogen-tested materials for citrus propagation.

Stone Fruit survey efforts in Texas monitoring for Plum Pox Virus, European Stone Fruit Yellows \& Light Brown Apple Moth: 2016-2017 S. C. RHODES, K. Ong, Texas A\&M AgriLife Extension Service, College Station, TX, USA 
The Texas Plant Disease Diagnostic Lab continues participation in the National Stone Fruit Survey in FY16, monitoring for pathogens of concern for the stone fruit industry in Texas. The Texas A\&M stone fruit breeding plots and two (2) commercial peach orchards in two different counties in Texas were tested for the presence of Plum Pox Virus (PPV) and European Stone Fruit Yellows (ESFY) phytoplasma in 2016. A total of 488 foliar peach, plum and apricot samples were tested for PPV. For virus detection, each sample was tested by ELISA using Agdia ELISA kits and following USDA APHIS PPQ and National Plant Diagnostic Network protocols. All 488 samples tested negative for all strains of plum pox virus. 879 visual observations were collected from these same locations for ESFY and disease symptoms were not observed or detected in any of the surveyed peach orchards. Jackson traps were also set in every orchard to screen for the Light Brown Apple Moth (LBAM). A total of 31 traps were collected and LBAM was not observed on any of the traps. This survey effort will continue through 2017, focusing on PPV, ESFY and the LBAM. In addition, the presence of Phony Peach disease (Xylella fastidiosa) will be visually surveyed in 2017. This survey monitors for the possible introduction of these pests and allows for their early detection.

Effect of sequence variation in the constricta strain on the protein localization and interaction of Potato yellow dwarf virus C. JANG (1), R. Wang (1), J. Wells (1), F. Leon (1), M. L. Farman (1), J. Hammond (2), M. M. Goodin (1), (1) University of Kentucky, Lexington, KY, USA; (2) USDA ARS Floral and Nursery Plant Research Laboratory, Beltsville, MD, USA

The genome of constricta strain (CYDV) of Potato yellow dwarf virus (PYDV), type species of the genus Nucleorhabdovirus was determined and compared to that of the sanguinolenta strain (SYDV). The 12,792 nt antigenomic sequence of CYDV is organized into seven open reading frames (ORFs) which encode nucleocapsid(N), unknown $(\mathrm{X})$, phosphor(P), movement $(\mathrm{Y})$, matrix $(\mathrm{M})$, glyco(G), and RNA-dependent-RNA-polymerase(L) relatively in sequence. Except for $\mathrm{X}$ and $\mathrm{P}$, each ORF in the CYDV genome showed more than $80 \%$ amino acid similarity compare to the cognate proteins of SYDV. Each ORF, except L, was cloned into pSITE-2CA(GFP-fusion) and pSITE-BiFC (Bimolecular fluorescence complementation) vectors and was expressed in Nicotiana benthamiana. The protein localization patterns in infected and virus-free cells, and protein interactions of each strain were integrated into a strain specific protein interaction and localization map (PILM). The CYDV-PILM provides insight into the subcellular loci for replication, morphogenesis and movement. Phylogenetic analysis using L protein identified that CYDV forms a clade with other leafhoppertransmitted rhabdoviruses. Protein sequence comparisons revealed that CYDV X has greater similarity to the cognate protein of Eggplant mottle disease virus than to SYDV X.

\section{Rosa Species Resistance to Eriophyid Mite Populations}

K. SOLO (1), S. Collins (1), Q. Cheng (1), B. England (2), F. Hale (3), A. S. Windham (3), D. Byrne (4), N. Anderson (4), M. T. Windham (1), (1) University of Tennessee, Knoxville, TN, USA; (2) University of Tennessee, Crossville, TN, USA; (3) Soil, Plant, and Pest Center, Nashville, TN, USA; (4) Texas A\&M University, College Station, TX, USA

Rose rosette disease has become an epidemic in the United States, affecting nearly every variety of cultivated roses. Phyllocoptes fructiphilus, the eriophyid mite that vectors this disease, is only known to inhabit Rosa species. How this mite distributes itself on different varieties of Rosa hosts is unknown. This study evaluates mite populations on Rosa species to determine resistance to eriophyid mites (defined in this study as a lack of reproduction on host). Using a completely randomized design, 18 Rosa species were evaluated biweekly from May to September in 2016. Destructive sampling was used to extract mites. Mites were isolated through a series of sieves and counted using a stereomicroscope. The data was expressed as number of mites per gram of tissue. Mite data were converted into a binomial variable, where 0 meant the mite was not reproducing on the plant (included samples with less than 20 mites/g plant tissue) and where 1 meant the mite was reproducing on the plant (included samples with more than 20 mites/g plant tissue). There were five species ( $R$. arkansana, $R$. palustris $\mathrm{EB}, R$. clinophylla, $R$. nitida and $R$. wichuriana Basye ARE) that maintained low mite populations that were not statistically different from zero $(\mathrm{p}=.05)$. Differences in the eriophyid mites' ability to reproduce on the different accessions of $R$. wichuriana and $R$. palustris were tested, indicating intraspecific variability in the resistance to mite reproduction. This report uses data from the first year of a multiyear study.

\section{The ever-expanding range of tospoviruses: $A$ case of phytosanitary risk}

C. OLAYA (1), N. Y. Velasquez (2), M. Betancourt Vasquez (3), W. Cuellar (4), H. R. Pappu (1), (1) Department of Plant Pathology, Washington State University, Pullman, WA, USA; (2) Grupo de Investigación de Sanidad Vegetal (GISAVE), Universidad Católica de Oriente, Rionegro, COLOMBIA; (3) CORPOICA, Bogota, Cundinamarca, COLOMBIA; (4) International Center for Tropical Agriculture (CIAT), Cali, Valle del Cauca, COLOMBIA

Thrips-transmitted tospoviruses cause significant losses in numerous crops worldwide. Tomato spotted wilt virus (TSWV) and Chrysanthemum stem necrotic virus (CSNV) have been reported in Colombia. The goal of this project was to collect genomic information of the tospoviruses affecting solanaceous crops in Colombia. Tomato and bell pepper plants with symptoms of necrotic lesions, necrotic rings, veinal necrosis, and stem necrosis in grower fields in two states, Antioquia and Risaralda, were collected. Symptomatic leaves were tested for the presence of TSWV with DAS-ELISA and/or immunostrips. Positive samples were tested by RT-PCR with TSWV nucleoprotein-specific primers, however, no amplicons were obtained. Then, a set of degenerate primers from the tospoviral polymerase gene, and a set of generic primers from the small RNA of the tospoviral American clade were used. Amplicons of the expected size were obtained, cloned and sequenced. Sequence comparisons showed the presence of to identify Alstroemeria necrotic streak virus (ANSV). ANSV was previously reported infecting alstroemeria plants from Colombia, and this is the first time it was found naturally infecting tomato and bell pepper. The expanding host range of ANSV presents a phytosanitary risk for solanaceous and ornamental crops, stressing the need for constant surveys. Our findings emphasize the need to use species-specific detection tools and sequence comparisons for precise identification of tospoviruses.

Grapevine Pinot gris virus: An emerging virus in Napa Valley vineyards

M. AL RWAHNIH PHD (1), D. A. Golino (2), N. Westrick (3), A. Diaz Lara (3), M. Cooper (4), R. J. Smith (5), M. Battany (6), L. Bettiga (7), S. Zhuang (8), K. L. Arnold (2), K. Farrar (9), A. Rowhani (2), (1) Department of Plant Pathology, University of California-Davis, Davis, Davis, CA, USA; (2) University of California, Davis, CA, USA; (3) Department of Plant Pathology, University of California-Davis, Davis, CA, USA; (4) Univ of California, Napa, CA, USA; (5) Univ of California, Santa Rosa, CA, USA; (6) University of California, San Luis Obispo, CA, USA; (7) University of California, Salinas, CA, USA; (8) University of California Cooperative Extension - Fresno County, Fresno, CA, USA; (9) Foundation Plant Services, Davis, CA, USA

Grapevine Pinot gris virus (GPGV) is a new member of the genus Trichovirus, family Betaflexiviridae, first discovered 5 years ago by high-throughput sequencing (HTS) of Italian Pinot gris grapevines. Since then, GPGV has been reported in symptomatic and asymptomatic plants in many countries. In 2015, GPGV was detected in several commercial vineyards in Napa Valley, California. We conducted field surveys in 2016 throughout grape-growing regions in California to assess symptom enhancement by co-infection with other viruses, gain a better understanding of the incidence and distribution of GPGV, and molecularly characterize the California GPGV isolates. Thus far, from 1730 vines (1235 from Napa; 495 from other counties), we have only detected the virus in Napa and found that it infects many varieties of grape. Surveys throughout California will continue. While the putative symptoms associated with GPGV include leaf deformation and chlorotic mottling, the relationship between GPGV infection and symptoms remains complex. HTS analysis showed that all California GPGV isolates share 95-99\% homology with asymptomatic reference isolates in the genebank and when symptoms are observed in GPGV-positive vines, those vines were also infected with Grapevine fanleaf virus. Molecular characterization of the Californian virus isolates has enabled us to develop a new RT-qPCR detection assay which will facilitate monitoring the prevalence and natural spread of the virus. 
The Prevalence of Viruses in Ohio Wheat Fields

B. A. HODGE (1), L. R. Stewart (2), P. A. Paul (3), (1) The Ohio State University, Wooster, OH, USA; (2) USDA ARS, Wooster, OH, USA; (3) Ohio State Univ, Ohio Agricultural Research and Development Center, Dept of Plant Pathology, Wooster, OH, USA

The prevalence of viruses in wheat has been under-reported in Ohio, leaving growers with little information pertaining to the potential threat posed by these pathogens. In a 2016 survey, symptomatic plants were collected from 40 wheat fields in 20 counties from March to April. Additionally, samples were collected at 20 points along a transect across 37 of the fields surveyed. RNA from the symptomatic samples were subjected to RNA-Seq analysis, while the transect samples were subjected to a DAS-ELISA screen for 8 viruses. Thirty percent of the transect samples and $39 / 40$ fields tested positive for at least one virus. Barley yellow dwarf virus-PAV (detected from 25/40 fields) and Cereal yellow dwarf virus-RPV (33/40) were the most prevalent viruses, with incidence ranging from 5-45\% within fields. Other highly prevalent viruses included Wheat streak mosaic virus (20/40), at 5-75\% incidence, and Wheat spindle streak mosaic virus (25/40), at 10-100\% incidence. Less prevalent viruses included Brome mosaic virus (6/40), at 5-25\% incidence, Wheat mosaic virus (2/40) and Soil-borne wheat mosaic virus (1/40), at 5\% incidence. This study demonstrates that multiple viruses are present in Ohio wheat fields, and highlights the need to further assess and manage these pathogens in soft red winter wheat. Additionally, the survey suggests that the prevalence of certain viruses like Brome mosaic virus is higher than expected, potentially causing an economic threat in the future.

Effect of Bell pepper endornavirus to bell pepper (Capsicum annuum)

C. ESCALANTE GUARDADO (1), R. A. Valverde (1,2), (1) Louisiana State University Agricultural Center, Baton Rouge, LA, USA; (2) Dept of Plant Pathology and Crop Physiology, Louisiana State University Agricultural Center, Baton Rouge, LA, USA

Plant endornaviruses are capsid-less viruses with an RNA genome that ranges from 13.5 to $17.6 \mathrm{~kb}$ and do not cause visible symptoms. They have been reported to infect several economically important crops. Bell pepper endornavirus (BPEV) has been reported infecting many pepper (Capsicum annuum) cultivars; however, the effect on the plant has not been investigated. To address this, we developed near-isogenic lines (NIL) of the bell pepper cv. Marengo (one infected with BPEV and the other virus-free) and conducted comparative studies on the plant phenotype, seed germination, fruit yield, and total dry matter. The NIL were undistinguishable, suggesting that BPEV does not affect the plant phenotype. The BPEV-negative NIL consistently yielded higher percentage of seed germination, fruit weight, and total dry matter than the BPEV-positive line; however, only the fruit weight value was statistically significant. Although this data suggest that BPEV may not be beneficial to bell pepper, evaluations of the NIL under biotic and abiotic stresses may reveal a beneficial effect. It is possible that endornaviruses are beneficial to crops under some environmental conditions but detrimental under others.

\section{An endornavirus from cluster bean (Cyamopsis tetragonoloba)}

R. I. ALCALÁ-BRISEÑO (1), F. Herrera (2), R. A. Valverde (3), (1) University of Florida, Gainesville, FL, USA; (2) Louisiana State University Agricultural Center, Baton Rouge, LA, USA; (3) Dept of Plant Pathology and Crop Physiology, Louisiana State University Agricultural Center, Baton Rouge, LA, USA

The family Endornaviridae includes linear RNA viruses that infect plants, fungi, and oomycetes. Their genome ranges from 9.8 to $17.6 \mathrm{~kb}$, lack coat protein, and in plants, do not cause visible symptoms. They have been reported to infect economically important crops. Cluster bean (Cyamopsis tetragonoloba) is a drought-tolerant annual legume used as a vegetable for human consumption, cattle feed, oil-well drilling, and other industrial applications. We conducted investigations to characterize a dsRNA detected in an unidentified guar cultivar. Foliar tissues were used for dsRNA extractions. DsRNA was gel-purified and used for RNA sequencing. Sequencing was conducted by Illumina MySeq (pair-end $2 \times 250$ ). A total of 168,338 reads were assembled with Spades 3.8.0 producing a total of 355 contigs of up to 12,831 nt in length. BLASTx results showed similarities to sequences of members of the Endornavirus genus. The 12,831 nt contig contained an open reading frame from nucleotide 81 to 12,820 . The putative endornavirus was provisionally named Cluster bean endornavirus (CBEV). A search for conserved domains yielded three: methyltransferase, helicase, and RNA-dependent of RNA polymerase. Protein BLAST results showed that the closest endornavirus to CBEV was Hordeum vulgare endornavirus. Although the effect of endornaviruses to plants has not been elucidated, there is evidence that these viruses affect the host gene expression.

\section{Endornaviruses of Capsicum species}

R. I. Alcalá-Briseño (1), C. Escalante Guardado (2), R. A. VALVERDE (3), (1) University of Florida, Gainesville, FL, USA; (2) Louisiana State University Agricultural Center, Baton Rouge, LA, USA; (3) Dept of Plant Pathology and Crop Physiology, Louisiana State University Agricultural Center, Baton Rouge, LA, USA

Capsicum spp. (pepper) has been shown to be infected by several persistent viruses which include endornaviruses and partitiviruses. Endornaviruses do not cause symptoms and are transmitted only vertically through the gametes. Although endornaviruses are common in some pepper genotypes, the effect to this crop is not known. We have identified endornaviruses in two domesticated Capsicum species: C. frutescens (tabasco) and C. chinense (habanero). We have tentatively named these viruses Capsicum frutescens endornavirus (CfEV) and Capsicum chinense endornavirus (CcEV), respectively. We investigated their frequency in some genotypes and their relationship to reported endornaviruses of $C$. annumm. Viral dsRNAs were extracted from infected plant tissue, gel purified, and sequenced. Sequencing was conducted by Illumina MySeq (pair-end $2 \times 250$ ). Reads were de novo assembled with Spades 3.8.0 resulting in contigs of 15,002 (CfEV) and 14,939 (CcEV) nucleotides. Aminoacid sequence alignments between CfEV and CcEV resulted in $41.5 \%$ identity. A search with BLASTx showed homology to Bell pepper endornavirus (BPEV) and Hot pepper endornavirus (HPEV). Nucleotide sequence comparisons of CfEV and CcEV with other endornaviruses suggest that they are distinct endornavirus species, although closely related to BPEV and HPEV. Phylogenic analyses show that pepper endornaviruses cluster together, suggesting a common ancestor.

\section{Recovery from Ilarvirus symptoms in cranberry}

S. THOMAS-SHARMA, R. Page, T. L. German, P. S. McManus, University of Wisconsin-Madison, Madison, WI, USA

Two ilarviruses - Tobacco streak virus (TSV) and Blueberry shock virus (BIShV) - emerged recently as viruses associated with berry scarring symptoms on cranberry. For both pathogens, symptomatic cranberry shoots that were tagged in one year produced asymptomatic (but TAS-ELISA positive) berries in following years. Fruit set and berry weight on 'recovered' shoots were comparable to that on healthy shoots, suggesting that the viruses may have limited short-term economic impact. However, the recovered shoots remain infected and serve as inoculum sources via infected pollen and/or cuttings. In other pathosystems, recovery is explained as the onset of virus induced RNA silencing, reduction of virus titers, and consequent disappearance of symptoms. In leaves of cranberry shoots recovered from BlShV, sequencing small interfering RNA (siRNA) revealed viral sequences spanning the BIShV genome. This suggests that BIShV triggered RNA silencing. However, leaves from recovered uprights remained strongly positive by TAS-ELISA and northern blot analysis, suggesting that virus titers were still high. Further, negative strands of BlShV were detected by northern blot and siRNA sequencing, suggesting active virus replication in recovered shoots. These results suggest that the mechanism of recovery from symptoms in cranberry may differ from recovery mechanisms described in other systems.

A survey for grapevine viruses in Tennessee vineyards

N. SOLTANI (1), D. D. Hensley (1), D. Lockwood (2), M. Staton (1), K. L. L. Perry (3), M. R. Hajimorad (1), (1) University of Tennessee, Dept of Entomology \& Plant Pathology, Knoxville, TN, USA; (2) University of Tennessee, Dept of Plant sciences, Knoxville, TN, USA; (3) Cornell University, Plant Pathology \& Plant-Microbe Biology Section, Ithaca, NY, USA 
Viruses are considered one of the major pathogens of grapevine (Vitis vinifera L.). Tennessee (TN) has $\sim 900$ acres vineyards and grape production is a significant portion of its fruit industry. Despite the importance, no published study exists on the presence of viruses in TN vineyards. A survey for nine viruses was conducted during summer and fall 2016. Nineteen vineyards located in East and Middle of TN were visited, and tissue samples collected from vines exhibiting viral-like symptoms. A total of 315 vines representing cultivars of 'American', 'hybrids' and ' $V$. vinifera' were sampled. All samples were assayed by DAS-ELISA using polyclonal antibodies against the coat proteins of grapevine leafroll-associated viruses (GLRaV) - $1,-2,-3,-$ 4, arabis mosaic virus (ArMV), grapevine fanleaf virus (GFLV), tomato ringspot virus (ToRSV) and tobacco ringspot virus (TRSV). Selected ELISA positive vines with each of the viruses also were subjected to RT-PCR followed by Sanger sequencing. Additionally, tissues from 19 vines with suspected symptoms of infection with grapevine red blotch-associated virus (GRBaV) were assayed by PCR combined with Sanger sequencing. Viruses that were identified included GLRaV-1 (23\%), GLRaV-2 (8\%), GLRaV-3 (94\%) and ToRSV (21\%) while no infection with GLRaV4, ArMV, GFLV or TRSV was detected. The presence of GRBaV was also established in 9 vines. Sequence analysis of identified viruses with those reported elsewhere in the United States showed close genetic relationships. All the vines surveyed originated from outside of TN; hence, it is likely the identified viruses originated outside the state as well.

\section{Genetic diversity of pepper and tomato-infecting begomoviruses in Eastern Thailand} W. S. TSAI, L. T. Shen, National Chiayi University, Chiayi, TAIWAN

Begomovirus-causing diseases threaten to pepper and tomato production, especially in tropic and sub-tropic regions. In 2016, the viral diseases were observed in tomato and pepper fields in Eastern Thailand. The disease incidence ranged 30-100\% with symptoms of yellow leaf curling and mosaic was inspected in tomato fields, and ranged $10-100 \%$ with symptoms of yellow mottle, mosaic and leaf deformation in pepper fields. Forty-one symptomatic pepper samples from 8 provinces and 43 symptomatic tomato samples from 7 provinces were tested for presence of begomovirus by PCR using primer pair- PAL1v1978B/PAR1c715H. Begomovirus was detected positively in 19 pepper samples from 5 provinces and 28 tomato samples from 7 provinces. Nineteen PCR products from tomato samples and 7 from pepper samples were selected by province and sequenced. Based on the phylogenetic analysis and demarcation criteria ( $91 \%$ nucleotide sequence identity), those 26 sequences were grouped into 7 clusters. Viruses of clusters could be respectively considered as isolates of Ageratum yellow vein virus (AYVV), Eggplant golden mosaic virus (EGMV), Lidwgia yellow vein virus (LYVV), Pepper leaf curl virus (PepLCV), Pepper yellow leaf curl Thailand virus (PepYLCTHV), Tomato leaf curl Vietnam virus (ToLCVV) and Tomato yellow leaf curl Thailand virus (TYLCTHV). Two of those begomoviruses, LYVV and ToLCVV are first detected in Thailand, and the whole viral genomic DNA sequencing is ongoing for further characterization. However, our results reveal the increasing of begomovirus diversity in Thailand as well as Southeast Asia, and should be considered seriously as an important threating factor in releasing resistant cultivars in the area.

\section{Prevalence of viruses associated with Maize lethal necrosis (MLN) in Tanzania}

D. P. Massawe (1), L. R. STEWART (2), (1) The Ohio State University, Wooster, OH, USA; (2) USDA ARS, Wooster, OH, USA

Maize lethal necrosis (MLN) is caused by the synergistic interaction of two viruses: maize chlorotic mottle virus (Tombusviridae) and any of several maize-infecting viruses in the family Potyviridae. MLN is reported to cause 30-100\% yield loses in Kenya, Uganda and Rwanda; however, the distribution of maize-infecting viruses in Tanzania remains unknown. To assess the current distribution of viruses contributing to MLN in Tanzania, the survey was conducted in nine regions located in four major maize growing zones including the Lake zone, Northern Highland zone, Central/Eastern zone and Southern Highland zone. A transect method was used in collecting samples in 64 maize fields for incidence observation whereby 10 samples were collected at equidistant intervals in a diagonal transect across each field. The collected samples were subjected to DAS-ELISA for screening of potyviruses and maize chlorotic mottle virus (MCMV). Incidences of potyvirus and MCMV in analyzed samples were higher in Lake zone regions (46\% and $40 \%$ respectively) compared to other zones. Co-infection of MCMV and potyvirus that causes MLN were detected with high incidence in Lake zone and Northern Highland regions ( $20 \%$ and $15 \%$ respectively) but lower in Central/Eastern and Southern Highland zones. Our data indicate a north to south gradient of MCMV, consistent with its emergence and subsequence spread from Kenya and surrounding countries to the north. Containing and managing MLN is a major concern for food security in East Africa. Further research to understand the diversity of potyviruses and other viruses associated with MLN in Tanzania is ongoing.

\section{Molecular characterization of divergent Grapevine leafroll-associated virus 3 isolates in California, USA}

M. AL RWAHNIH PHD (1), V. Klaassen (2), K. Stevens (3), K. L. Arnold (4), H. J. Maree (5), N. Westrick (6), D. A. Golino (4), (1) Department of Plant Pathology, University of California-Davis, Davis, Davis, CA, USA; (2) Foundation Plant Services, Davis, CA, USA; (3) Department of Evolution and Ecology, University of California-Davis, Davis, CA, USA; (4) University of California, Davis, CA, USA; (5) Stellenbosch University, Stellenbosch, SOUTH AFRICA; (6) Department of Plant Pathology, University of California-Davis, Davis, CA, USA

Grapevines showing severe leaf rolling, rapid vine decline and death were observed in a vineyard in Santa Barbara County, California. Disease incidence was visually estimated at $80-90 \%$. In fall 2016 , leaves from symptomatic vines were randomly sampled, pooled into composite samples of four vines, and tested by RT-qPCR for common grapevine viruses. Forty-seven of the 56 composite samples were GLRaV-3 positive. Five individual vines from different positive composite groups were further characterized by high throughput sequencing. Analysis of sequence data identified a GLRaV-3 isolate in four samples, $99 \%$ identical to NZ2, a highly divergent GLRaV-3 isolate first identified in New Zealand. This is the first report of NZ2 in the US. In the same vineyard, a second divergent GLRaV-3 sequence was identified in one of the four samples. It has high identity to the partial sequence of GLRaV-3f which was first detected in Napa Valley (2011) and more recently in Kern County. Two nearly complete GLRaV-3f sequences from Santa Barbara and Kern Counties, with only $75-80 \%$ similarity to isolates in existing GLRaV-3 groupings, indicate GLRaV-3f may belong to a new divergent subgroup. This data suggests divergent GLRaV-3 isolates in California vineyards do not occur only at low frequency and are not restricted geographically. A larger scale survey of commercial vineyards is needed to determine the prevalence of these divergent isolates and evaluate the effect on vine performance.

\section{Grapevine vein clearing virus is prevalent in wild plant species}

C. KEITH, S. Petersen, W. Qiu, Missouri State University, Springfield, MO, USA

Grapevine vein clearing virus (GVCV) is a DNA virus that belongs to the Badnavirus genus in the family Caulimoviridae. The reference genome was published in 2011. Presence of the virus is associated with a severe disease that exhibits translucent vein-clearing, short internodes, deformed berries and vine decline symptoms on most infected grapevines. GVCV has been detected in cultivated grapevines in Missouri, Arkansas, Illinois, and Indiana vineyards of the Midwest. Prevalence of GVCV in wild plants had not been investigated. In this study, we collected samples from native plant species in the Vitis and Ampelopsis genera of the Vitaceae family in their habitats across three states. Samples were collected without regard to symptoms. We detected GVCV by using polymerase chain reaction (PCR) assay with two sets of specific primers. The results showed that 35 out of 113 samples ( $31 \%$ ) of $A$. cordata plants and two out of 49 samples (4\%) of wild Vitis plants tested positive for GVCV. Discovery of GVCV in $A$. cordata at a prevalence of $31 \%$ across a wide range indicates that the virus is spreading among wild plants in the Midwest, and supports the conclusion that wild plant species are a host reservoir of GVCV. These wild plants should be removed before planting a vineyard to help prevent infection of cultivated grapes.

Association of Grapevine leafroll-associated virus 2 with atypical symptoms on Pinot Noir grapevines in California H. E. MCCOWN (1), J. Vo (1), M. R. Sudarshana (2), (1) University of California, Davis, Davis, CA, USA; (2) USDA-ARS, Davis, CA, USA

In the fall of 2015, in Santa Barbara County, California, grapevines of Pinot Noir (PN) Swan clone (Vitis vinifera) on Riparia Gloire (V. riparia) rootstock were found to exhibit severe vine stunting and poor leaf development. The aforementioned symptoms did not resemble those of leafroll or 
fanleaf diseases, thus we examined a symptomatic grapevine by deep sequencing of cDNA libraries prepared from the total RNA and small RNA fractions of cambial scrapings from the affected trunk. Subsequent bioinformatics analysis revealed the presence of Grapevine leafroll-associated virus 2 $(\mathrm{GLRaV}-2)$ and Grapevine red blotch-associated virus $(\mathrm{GRBaV})$ in both libraries. Further qRT-PCR and qPCR analysis of nucleic acid extracts from 10 symptomatic and 10 asymptomatic grapevines indicated infection by GLRaV2 in all symptomatic grapevines, three of which also tested positive for GRBaV. We believe this is the first report of atypical symptoms associated with infection by GLRaV-2.

A new virus in Luteoviridae is associated with raspberry leaf curl disease

P. DI BELlO (1), A. Diaz Lara (2), R. R. Martin (3), (1) Oregon State University, Corvallis, OR, USA; (2) Foundation Plant Services, UC-Davis, Davis, CA, USA; (3) USDA ARS, Corvallis, OR, USA

To determine the etiology of Raspberry Leaf Curl Disease (RLCD), which causes leaf curling, leaf distortion, leaf chlorosis, shoot dwarfing, shoot proliferation in raspberries and can kill plants within three years, a next generation sequences approach was applied. Two red raspberry plants collected from native habitats in Wisconsin that exhibited symptoms of RLCD were used for the NGS analysis. Total nucleic acids were extracted, digested with DNase, and after removal of polyribosomal RNA, were subjected to next generation sequencing on the Illumina Miseq platform with paired end 150 bp. Two known viruses were found Black raspberry necrosis virus and Rubus yellow net virus, and two new viruses, one was Cytorhabdovirus-like and the other Luteoviridae-like. The new virus in Luteoviridae is related to viruses in the genera Enamovirus and Polerovirus. The luteo-like virus was found to be graft transmissible to raspberries and blackberries, and vectored by the large raspberry aphid, Amphorophora agathonica. In limited testing done to date, the virus was not found in commercial Rubus collected in Oregon and Washington, but was detected in symptomatic wild raspberry collected from eastern U.S.A. and Canada and in some accessions of native Rubus in the USDA-ARS NCGR Rubus collection. The full genome of this novel virus as well as a detection protocol will be presented.

Prevalence, Distribution and Characterization of Major Viral Diseases of Sugarcane from Southern India R. K. N (1), V. K. K. Kotamraju (2), S. S. UPPALA (3), (1) 1Acharya N G Ranga Agricultural University, Anakapalle, INDIA; (2) Acharya N G Ranga Agricultural University, Regional Agricultural Research Station, Anakapalle, INDIA; (3) Texas A\&M AgriLife Research, Beaumont, TX, USA

Yellow leaf disease (YLD) caused by Sugarcane Yellow Leaf Virus (ScYLV) and Mosaic disease (by Sugarcane Mosaic Virus, ScMV) are the major viral diseases affecting sugarcane productivity worldwide. The objective of our study was to enunciate the prevalence of these two diseases in Southern India, map risk and sensitive areas and to characterize the pathogens. Surveys were conducted from 2010-2011 to 2016-2017 growing seasons in nine districts in South India. Detection of pathogens was carried out from diseased leaf samples using direct antigen coating ELISA and PCR using pathogen specific primers. Further characterization was carried out using scanning electron microscopy. Average YLD incidence increased from 2010-2011 (22\%) to 2014-2015 (78\%), declined to 62\% during 2015-2016 (62\%) and increased to 69\% in 2016-2017. Average Mosaic incidence has shown a steady increase over the years from 2\% (2010-2011) to 41\% (2016-2017). Majority of surveyed areas witnessed mixed infection of both the viruses. Three Districts with high YLD and Mosaic were mapped as hot spot areas. DAC-ELISA absorbance values at $405 \mathrm{~nm}$ indicated the presence of ScMV and ScYLV in diseased samples. PCR studies also confirmed the presence of YLD and Mosaic pathogens. SEM studies revealed the presence of poty virus filamentous particles (ScMV) and Luteo virus particles (ScYLV) in diseased leaves. Overall, our studies indicated the upsurge of YLD and ScMV diseases in Southern India.

GC-rich structure interferes with Ralstonia solanacearum egl sequence amplification

A. Jeyaprakash (1), P. Srivastava (2), D. Jones (2), C. Lapaire Harmon (3,4), P. Harmon (5), A. Khan (6), D. J. Norman (7), X. SUN (1), (1) Florida Dept of Agric \& Consumer Service, Gainesville, FL, USA; (2) Florida Department of Agriculture and Consumer Services, Gainesville, FL, USA; (3) University of Florida, Gainesville, FL, USA; (4) UF-IFAS Dept. of Plant Pathology \& Plant Diagnostic Center, Gainsville, FL, USA; (5) Department of Plant Pathology, University of Florida, Gainesville, FL, USA; (6) University of Florida, Orlando, FL, USA; (7) Univ of Florida MREC, Apopka, FL, USA

Bacterial wilt caused by Ralstonia solanacearum (recognized as races and biovars) is a serious disease affecting many economically important crops all over the world, including several in Florida. This pathogen belongs to a species complex that are differentiated as phyllotypes and sequevars for proper identification that is critical for early detection of new races or biovars. This classification scheme has been developed by amplifying the internal transcribed spacer region, endoglucanase ( $\mathrm{egl}$ ) gene, and the helicase-related protein ( $\mathrm{rrpB}$ ) gene to identify these pathogens. Ralstonia solanacearum bacteria were recently isolated from several different locations in Florida. Efforts to amplify the egl sequences $(\sim 0.8 \mathrm{~kb})$ produced multiple DNA bands instead of one band. The egl gene sequence was closely examined and found to be GC-rich (68\%), which can generate hair-pin structures. A strong structure exists near the 5' ATG start site and this could have interfered with amplification. Therefore, a primer set was designed for the egl 3' region $(0.4 \mathrm{~kb})$ by excluding this structure. The new primer set consistently produced a single DNA band from all the $R$. solanacearum isolates collected. Selecting consistently amplifiable sequences for phylogenetic analysis and classification is essential for a clear pathogen identification. 